Definición de las pautas y condiciones de monitorización, encapsulado y fijación de sensores de fibra óptica para la medida de deformación y temperatura en estructuras 


\section{Colección Tesis Doctorales}

\section{Benjamín Torres Górriz}

(C) 2014, de la presente edición: Editorial Universitat Politècnica de València Telf.: 963877012 / www.lalibreria.upv.es

ISBN: 978-84-9048-835-5 (versión impresa)

Queda prohibida la reproducción, distribución, comercialización, transformación, y en general, cualquier otra forma de explotación, por cualquier procedimiento, de todo o parte de los contenidos de esta obra sin autorización expresa y por escrito de sus autores. 


\section{UNIVERSITAT POLITÈCNICA DE VALÈNCIA}

Departamento de Ingeniería de la Construcción y Proyectos de Ingeniería Civil.

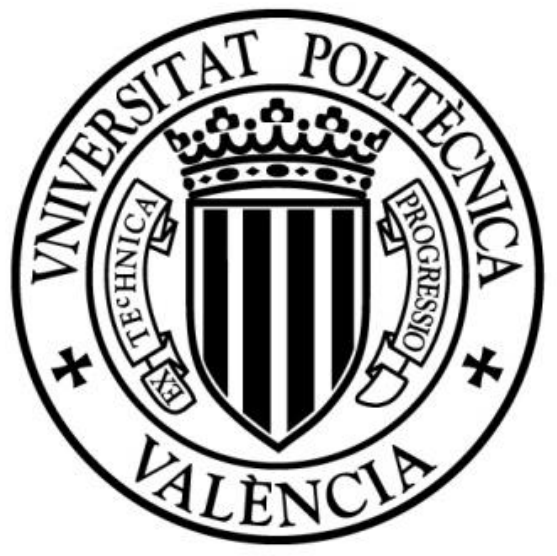

DEFINICIÓN DE LAS PAUTAS Y CONDICIONES DE MONITORIZACIÓN, ENCAPSULADO Y FIJACIÓN DE SENSORES DE FIBRA ÓPTICA PARA LA MEDIDA DE DEFORMACIÓN Y TEMPERATURA EN ESTRUCTURAS.

TESIS DOCTORAL

Autor:

Benjamín Torres Górriz.

Dirigida por:

Dr. Pedro A. Calderón García.

Dr. Ignacio Payá Zaforteza.

Valencia, Noviembre de 2012. 
Benjamín Torres Górriz.

ICITECH, Departamento de Ingeniería de la Construcción y de Proyectos de Ingeniería Civil. Universitat Politècnica de València, Camino de Vera s/n, 46071 Valencia, España

Tel.: +34 963877000 ext. 75619; fax: +34963877568

E-mail: bentorgo@upvnet.upv.es 
A mi mujer, Maria José.

A mis cuatro abuelos: Benjamín, Carmen, Pepe y Lile.

A mis padres: Benjamín y Cloti.

A mis hermanas y cuñados: Maria e Isabel, Jose Miguel y Fede.

A mis cuatro sobrinos: Isabelita, Mía, Carmen y Lucas.

"Son tardes en las que uno pone el corazón. Pones toda tu ilusión y toda tu esperanza. Entonces, cuando pasan esas tardes es verdad que el cuerpo se afloja, se pone en calma" (J. A. Morante de la Puebla) 



\section{AGRADECIMIENTOS}

A título personal me gustaría dejar constancia de mi gratitud a los directores de esta Tesis Doctoral, gracias a los que he podido formar parte de un grupo de investigación que destaca no sólo por su capacidad investigadora, sino por su calidad humana. Por un lado quiero agradecer al Dr. Pedro A. Calderón García su interés en el desarrollo de esta Tesis Doctoral. Pero sobre todo quiero darle las gracias por ser mi amigo, por preocuparse por mí, por mi formación y por su maravillosa personalidad. Por otro lado, Dr. Ignacio Javier Payá Zaforteza, quien me ha enseñado a trabajar con rigurosidad, con humildad y con seriedad, motivo por el que le estoy muy agradecido. Pero si de algo le estoy verdaderamente agradecido es por brindarme su amistad a lo largo de estos años.

Siguiendo con nombres propios me gustaría dejar patente la satisfacción y el honor de haber trabajado estrechamente con personas de tan alto nivel científico y a la vez humano como el Dr. Salvador Sales Maicas, Antonio Bueno Martínez, David Barrera y otros tantos del Instituto de Telecomunicaciones y Aplicaciones Multimedia (iTEAM). Gracias por su paciencia, ayudas desinteresadas y explicaciones sobre el manejo de los equipos ópticos.

Quiero manifestar mi agradecimiento al Dr. Daniele Zonta y a su equipo investigador de la Università degli Studi di Trento (Italia): Paolo Esposito, Davide Trapani, Marco Molignoni, Giuseppe Abbiati, Emiliano Debiasi, Anilo Ku. Durante mi estancia de 4 meses en Italia han sido mi grupo de investigación, mis amigos y mis referentes.

También quiero agradecer a mis compañeros del grupo de investigación sin el cual hubiese sido imposible desarrollar este trabajo: Juan José Moragues, Jose Miguel, Teresa, Joaquín, Julio y muy especialmente a Isabel y Yezid por ser compañeros en todos los sentidos que implica esta palabra.

No sería justo olvidarme de algunas personas con las que he compartido momentos, vivencias y situaciones que ya han quedado grabadas en mi memoria: Lucho, Erich, Andrés Caro, Samu, Andrés Núñez y muy especialmente Andreíta, Isabel y Yezid por compartir alegrías y penas, por saber escucharme cuando necesitaba hablar y por saber hablar cuando necesitaba oír.

Tengo que agradecer profundamente el apoyo de todos mis amigos de la Comissió Taurina Dona't Aire Meliana quienes han sido una válvula de escape durante estos años de trabajo.

Mis más sinceras gracias a mi familia que siempre han estado a mi lado en todo momento. Especialmente quiero agradecer a mi mujer Maria José, simplemente por todo: por ser, por estar y por existir. A mis padres, Benjamín y Cloti, por ser los mejores padres del mundo, por sus enseñanzas y sabios consejos que me han formado hasta el día de hoy y a quienes les debo cuanto soy. A mis cuatro abuelos, Benjamín, Carmen, Pepe y Lile, por quererme como lo hacen y ser los mejores abuelos del mundo. A mis hermanas y mis cuñados, María, Isabel, Jose Miguel y Fede por ser mis mejores amig@s y creer siempre en mí. A mis cuatro sobrinos, mi mayor hobbie, Isabelita, Mía, Carmen y Luquitas.

Por último, agradecer el apoyo económico de la Universitat Politècnica de València, así como la financiación del Proyecto SOPROMAC (P41/08) por parte del Ministerio de Fomento y del Proyecto BIA2011-27104 "Definición de condiciones de Monitorización, encapsulado y 
fijación de sensores ópticos para medir temperaturas y deformaciones en estructuras sometidas a altas temperaturas" por parte del Ministerio de Educación. Finalmente, agradecer también a la empresa constructora Sacyr-Vallehermoso la financiación del proyecto "Desarrollo de sensores ópticos aplicados a la instrumentación de estructuras para conocimiento de su comportamiento en tiempo real". Todos estos proyectos han permitido el desarrollo de esta Tesis Doctoral. 


\section{RESUMEN}

Acontecimientos como el colapso de las dos torres del Skyline Center Project en Virginia (1973), el colapso del puente I-35W sobre el río Mississipi en Mineapolis (2007) y, más recientemente, del puente colgante Kutai Kartanegara en Indonesia (2011) ponen de manifiesto la gran importancia y necesidad de monitorizar ciertas estructuras para poder evaluar su seguridad estructural en tiempo real.

El éxito de esta tarea depende fundamentalmente de dos aspectos. Por un lado, los sistemas y sensores empleados deben ser precisos y fiables y, a la vez, deben posibilitar una instrumentación segura, fácil y económica. Por otro lado, es necesario contar con pautas generales de monitorización de distintas tipologías estructurales que proporcionen una guía sobre cómo debe instrumentarse una estructura.

En este contexto general, esta Tesis Doctoral diseña y propone nuevos sensores para la monitorización estructural basados en la tecnología de la fibra óptica, dadas las ventajas comparativas que ofrece este material. La investigación se justifica por las limitaciones de las tecnologías actualmente disponibles y ha comprendido las fases siguientes:

- Desarrollo de un sensor óptico puntual basado en redes de Bragg (Fiber Bragg Gratting -o FBG- en inglés) para medir deformaciones y temperaturas.

- Desarrollo de un sensor óptico de longitud (long gauge sensor, en inglés) basado en redes de Bragg para medir deformaciones.

- Estudio y calibración de sensores distribuidos para medir deformaciones, basados en el fenómeno de dispersión de la luz (Stimulated Brillouin Scattering -o SBS-, en inglés).

- Ensayos con probetas en laboratorio de los sensores desarrollados.

- Comprobación y optimización del funcionamiento de los sensores mediante modelos numéricos realizados con el método de elementos finitos.

- Comprobación del comportamiento de los sensores en estructuras reales y mejora en su caso de su diseño. Las estructuras monitorizadas abarcan una gran variedad de materiales y tipologías como son el tablero de un puente empujado, una losa postesa de edificación, el encepado de cimentación de un puente atirantado de $318 \mathrm{~m}$. de luz o un falso túnel para una línea de tren de alta velocidad.

En esta Tesis Doctoral, sólo se muestran los trabajos realizados en la estructura del falso túnel de la línea de tren de Alta Velocidad, correspondiente al tramo de Mogente (Valencia). El resto de trabajos pueden observarse en una serie de artículos presentados en distintos congresos que se incluyen en los anejos.

Palabras clave: Fibra óptica, sensor puntual, sensor de longitud, sensor distribuido, Fiber Bragg Grating, Brillouin Scattering, transferencia de deformaciones, método de elementos finitos. 



\section{RESUM}

Esdeveniments com el col-lapse de les dos torres del Skyline Center Project en Virginia (1973), el col-lapse del pont I-35 sobre el riu Mississipi en Mineapolis (2007) i, més recentment, del pont penjant Kutai Kartanegara a Indonèsia (2011) posen de manifest la gran importància de monitoritzar certes estructures per a poder avaluar la seua seguretat en temps real.

L'èxit d'aquesta tasca depèn fonamentalment de dos aspectes. D'una banda, els sistemes i sensors empleats han de ser precissos i fiables al mateix temps que han de possibilitar una instrumentació segura, fàcil i econòmica. D’altra banda, és necessari comptar amb pautes generals de monitorització de distintes tipologies estructurals que proporcionen una guía sobre com s'ha d'instrumentar una estructura.

En aquest context general, la present Tesi Doctoral dissenya i proposa nous sensors per a la monitorització estructural basats en la tecnologia de la fibra òptica pels avantatges comparatius que ofereix aquest material. La investigació es justifica per les limitacions de les tecnologies actualment disponibles $i$ ha comprès les fases següents:

- Desenvolupament d'un sensor òptic puntual basat en xarxes de Bragg (Fiber Brag Gratting -o FBG- en anglès) per a mesurar deformacions i temperatures.

- Desenvolupament d'un sensor òptic de longitud (Long gauge sensor, en anglès) basat en xarxes de Bragg per a mesurar deformacions.

- Estudi i calibratge de sensors distribuïts per a mesurar deformacions, basats en el fenomen de dispersió de la llum (Stimulated Brillouin Scattering -o SBS- en anglès).

- Assajos amb provetes en laboratori dels sensors proposats.

- Comprovació i optimització del funcionament del sensors per mitjà de models numèrics realitzats amb el mètode d'elements finits.

- Comprovació del comportament dels sensors en estructures reals i millora del seu disseny si és el cas. Les estructures monitoritzades comprenen una gran varietat de materials i tipologies com són el tauler d'un pont espentat, una llosa posttesada d'edificació, l'encepat del fonament d'un pont atirantat de $318 \mathrm{~m}$. de llum o un fals túnel d’una línia de tren d’alta velocitat.

En aquesta Tesi Doctoral, només es mostren els treballs realitzats a l'estructura del fals túnel de la línea de tren d'Alta Velocitat, corresponent al tram de Moixent (València). La resta de treballs poden observar-se en una sèrie d'articles presentats en diversos congressos que s'engloben als anexes.

Paraules clau: Fibra òptica, sensor puntual, sensor de longitud, sensor distribuït, Fiber Bragg Gratting, Brillouin Scattering, transferència de deformacions, mètode dels elements finits. 



\section{SUMMARY}

Tragedies such as the collapse of both towers of the Skyline Center Project in Virginia (1973), the collapse of the I-35W bridge over the Mississippi River in Mineapolis (2007) and, more recently of the suspended bridge Kutai Kartanegara in Indonesia (2011), reveal the importance and necessity to monitor certain structures, in order to evaluate their structural safety in real time.

The success of this work depends fundamentally on two features. On the one hand, the systems and sensors applied in structural analysis must be precise and reliable, and at the same time they should provide a safe, easy-to-use and economic monitoring system. On the other hand, it is necessary to establish a general guideline and topologies for monitoring in order to monitor different structural typologies, as a guide to structure assessment.

Within this context, and due to the advantages offered by optical fibre, this doctoral thesis designs and proposes new sensors for structural monitoring based on this technology. The investigation is justified by the limitations of the technologies available to date, and has been carried out in the following phases:

- Development of a point fiber optic sensor based on Fiber Bragg Gratting (FBG), to measure strains and temperatures in structures.

- Development of a long gauge sensor based on Fiber Bragg Gratting (FBG) to measure strains in structures.

- Study and calibration of distributed sensors to measure strains, based on the phenomenon of light dispersion (Stimulated Brillouin Scattering, or SBS).

- Tests of the developed sensors with sample tests in the lab.

- Testing and optimization of the sensors via numerical models based on finite element analysis.

- Testing the behavior of the sensors on real structures, and the improvement of their design. The structures that are monitored cover a wide variety of materials and typologies such as the slab deck in an incrementally launched bridge, a postessed concrete slab, the foundation of a $318 \mathrm{~m}$ span suspended bridge or a section in a high speed railway tunnel.

In this Tesis, only the works carried out in the high speed railway tunnel near from Mogente (Valencia) are shown. The others can be found in different papers submitted to journals or conferences that are included in the appendices.

Key words: Optical fibre, point sensor, long gauge sensor, distribuited sensor, Fiber Bragg Gratting, Brillouin Scattering, strain transfer, finite element method. 



\section{INDICE GENERAL}

\section{INDICE DE FIGURAS}

INDICE DE TABLAS

\section{NOTACION Y ABREVIATURAS}

\section{CAPITULO 1. INTRODUCCIÓN Y OBJETIVOS.}

1.1. Introducción a la monitorización de estructuras.

1.2. Necesidades actuales en SHM. Identificación de la problemática.

1.2.1. Carencias en los sistemas de monitorización actuales.

1.2.2. Aspectos que deben reunir un nuevo sistema de monitorización estructural.

1.3. Objetivos, contribuciones y estructura de esta Tesis.

\section{CAPITULO 2. ESTADO DEL ARTE.}

2.1. Introducción.

2.2. La fibra óptica como elemento sensitivo.

2.2.1. La fibra óptica.

2.2.2. Tipos de sensores de fibra óptica.

a) El parámetro a medir.

b) Su aplicación.

c) El medio sensitivo.

2.2.3. Redes de Difracción de Bragg.

2.2.4. Principio de funcionamiento de los sensores de fibra óptica tipo FBG.

2.2.5. Compensación térmica de la fibra óptica.

2.2.6. Elementos que forman un sistema de monitorización óptico.

2.2.7. Extensometría clásica versus monitorización óptica. Ventajas e inconvenientes.

2.3. Estado del Arte sobre el diseño y estudio de sensores de fibra óptica.

2.3.1. Introducción. 
Monitorización estructural.

Aplicación con sensores de fibra óptica.

2.3.2. Estadísticas relativas al uso de distintos tipos de sensores de fibra óptica.

a) Lee (2003).

b) Hong-Nan et al. (2004).

2.3.3. Principales trabajos.

a) Encapsulado y calibración de sensores ópticos.

- Fei Luo et al. (1999).

- Moyo P. et al. (2005).

- Jun y Libo (2009).

- Kesavan et al. (2010).

- Calderón y Glisic (2012).

b) Señal del sensor de fibra óptica.

- Ramos et al. (2009).

- Biswas et al. (2010).

- Jochen y Tobías (2011).

c) Transferencia de deformaciones.

2.4. Estado del Arte sobre la aplicación e instrumentación estructural con fibra óptica.

2.4.1. Introducción.

2.4.2. Estadísticas relativas a la aplicación e instrumentación con fibra óptica.

a) $\mathrm{Ko} \mathrm{y} \mathrm{Ni}$ (2005).

b) Inaudi (2009).

2.4.3. Principales trabajos.

a) Aplicaciones SHM en el campo de la obra civil.

b) Aplicaciones SHM en el campo de la edificación.

c) Aplicaciones SHM en el campo del estudio de nuevos materiales aplicados a la ingeniería.

d) Otras investigaciones y aplicaciones SHM.

2.4.4. Pautas de monitorización estructural.

a) Introducción.

b) Mediciones obtenidas con sensores ópticos y su interpretación.

c) Pautas de monitorización de distintos elementos estructurales. 
- Tipología simple.

- Tipología paralela.

- Tipología cruzada.

- Tipología triangular.

2.4.5. Realizaciones

a) Monitorización de un edificio (Glisic e Inaudi 2007 y Zonta et al. 2011).

b) Monitorización del puente "South Lutrive" (Glisic e Inaudi 2007).

2.5. Conclusiones relativas al Estado del Arte.

\section{CAPITULO 3. OBJETIVOS PARTICULARES.}

3.1. Objetivos.

3.2. Organización de los capítulos.

CAPITULO 4. ESTUDIO DEL COMPORTAMIENTO, DESARROLLO Y DISEÑO DE UN SENSOR OPTICO PUNTUAL PARA LA OBTENCION DE DEFORMACIONES EN ESTRUCTURAS.

4.1. Introducción.

4.2. Caracterización de la respuesta de una FBG ante deformaciones y/o temperaturas impuestas.

4.2.1. Calibración frente a deformaciones impuestas.

4.2.2. Calibración frente a temperaturas impuestas.

4.3. Sensor FBG propuesto en la investigación.

4.4. Estudio numérico del sensor.

4.4.1. Introducción.

4.4.2. Condiciones de contorno, descripción de los elementos empleados y aplicación de la carga.

4.4.3. Resultados del estudio numérico.

a) Comportamiento general.

b) Estudios de sensibilidad del Módulo de Elasticidad y Coeficiente de Poisson del encapsulado, $E_{p}$ y $v_{p}$.

c) Análisis de sensibilidad del espesor de adhesivo (t).

d) Análisis de sensibilidad del Coeficiente de Poisson del adhesivo $v_{A}$.

e) Comparación del sensor propuesto con otros sistemas de encapsulado. 
Monitorización estructural.

Aplicación con sensores de fibra óptica.

4.5. Comportamiento del encapsulado frente a variaciones térmicas.

4.6. Conclusiones.

\section{CAPITULO 5. ESTUDIO DEL COMPORTAMIENTO, DESARROLLO Y DISEÑO DE UN SENSOR ÓPTICO DE LONGITUD PARA LA OBTENCIÓN DE DEFORMACIONES EN ESTRUCTURAS.}

\subsection{Introducción.}

5.2. Particularidades de los sensores de longitud.

5.3. Sensor de longitud FBG propuesto en la investigación.

5.4. Ventajas del sensor FBG de longitud propuesto en esta investigación.

5.5. Estudio experimental y numérico del sensor.

5.5.1. Introducción.

5.5.2. Estudio experimental.
a) Características de las probetas empleadas.
b) Propiedades de los materiales empleados.
c) Pórticos de ensayo.
d) Instrumentación.

5.5.3. Estudio numérico.
a) Descripción de los elementos finitos empleados, condiciones de contorno y aplicación de la carga.
b) Interfaz de contacto hormigón-adhesivo.
c) Estudio del pandeo.

5.6. Resultados.

5.6.1. Validez de los trabajos realizados y resultados obtenidos.

5.6.2. Comportamiento general.
a) Transferencia de deformaciones.
b) Rigidez a axil del elemento.

5.6.3. Modos de fallo del sensor.
a) Primer modo de fallo: Rotura por esfuerzo rasante.
b) Segundo modo de fallo: Pandeo del sensor. 
5.7. Conclusiones.

CAPITULO 6. APLICACIÓN DE LOS SENSORES DESARROLLADOS EN LOS CAPÍTULOS 4 Y 5 A LA MONITORIZACIÓN DE UNA ESTRUCTURA REAL. MONITORIZACIÓN DEL FALSO TUNEL DE LA LINEA DE ALTA VELOCIDAD DE LEVANTE A SU PASO POR MOGENTE (VALENCIA).

6.1. Introducción.

6.2. Estrategia de monitorización propuesta.

6.3. Trabajo experimental.

6.3.1. Descripción de la estructura objeto de estudio.

6.3.2. Operaciones monitorizadas y calendario de medidas.

6.3.3. Monitorización y captura de datos.

6.4. Estudio numérico.

6.4.1. Introducción.

6.4.2. Condiciones de contorno, descripción de los elementos empleados y aplicación de la carga.

a) Introducción de las temperaturas.

b) Introducción del empuje de tierras.

6.5. Resultados.

6.5.1. Introducción.

6.5.2. Compensación térmica de los sensores de longitud.

6.5.3. Mediciones continuas los días 26 y 27/09/2011.

a) Introducción.

b) Variaciones de temperaturas medidas.

c) Comparación de los resultados de los sensores de longitud.

d) Pauta general de comportamiento del falso túnel. Comparación de las mediciones con los resultados numéricos.

6.5.4. Mediciones instantáneas el día 14/05/2012.

a) Introducción.

b) Temperaturas y deformaciones medidas.

c) Comparación de las mediciones con los resultados numéricos. 
6.6. Conclusiones.

\section{CAPITULO 7. ESTUDIO, CALIBRACION Y APLICABILIDAD DE SENSORES DISTRIBUIDOS MEDIANTE LA DISPERSIÓN ESTIMULADA DE BRILLOUIN (SBS).}

7.1. Introducción.

7.2. Principio de funcionamiento de sensores distribuidos mediante la dispersión estimulada de Brillouin (Stimulated Brillouin Scattering, SBS).

7.3. Ventajas e inconvenientes del sensado distribuido.

7.3.1. Distancias de resolución del sensado distribuido de Brillouin.

7.4. Calibración del sensado distribuido frente a temperaturas.

7.4.1. Tramo de fibra de $20 \mathrm{~km}$.

7.4.2. Tramo de fibra de $1.5 \mathrm{~km}$.

7.4.3. Tramo de fibra de $2 \mathrm{~km}$.

7.4.4. Sensibilidades obtenidas.

7.5. Calibración del sensado distribuido frente a deformaciones.

7.5.1. Datos de las probetas empleadas en los ensayos y aplicación de la carga.

7.5.2. Instrumentación de las probetas.

7.5.3. Resultados obtenidos de los ensayos.
a) Comportamiento general del sensado distribuido de Brillouin.
b) Linealidad del sensado distribuido de Brillouin.
c) Sensibilidad de la deformación del sensado distribuido de Brillouin.
d) Comparación de los resultados obtenidos.

7.6. Conclusiones.

\section{CAPITULO 8. APLICACIÓN DE LOS SENSORES DISTRIBUIDOS DESARROLLADOS EN EL CAPITULO 7 A LA MONITORIZACION DE UNA ESTRUCTURA REAL. MONITORIZACION DEL FALSO TUNEL DE LA LINEA DE ALTA VELOCIDAD DE LEVANTE A SU PASO POR MOGENTE (VALENCIA).}

8.1. Introducción.

8.2. Instalación de los sensores ópticos distribuidos. 
8.3. Análisis de los datos.

8.3.1. Mediciones continuas el día 26/09/2011

a) Tramo B. Tramo de fibra adherida a la estructura con cinta adhesiva en determinados puntos.

b) Tramo C. Tramo de fibra adherida a la estructura con adhesivo.

8.4. Conclusiones.

\section{CAPITULO 9. CONCLUSIONES Y FUTURAS LÍNEAS DE INVESTIGACIÓN.}

9.1. Conclusiones generales.

9.2. Conclusiones particulares.

9.2.1. Respecto al estudio y diseño del sensor óptico puntual expuesto en el Capítulo 4.

9.2.2. Respecto al estudio y diseño del sensor óptico de longitud expuesto en el Capítulo 5.

9.2.3. Respecto a la aplicación de estos sensores ópticos propuestos en los Capítulos 4 y 5.

9.2.4. Respecto al estudio y calibración de los sensores distribuidos de Brillouin.

9.2.5. Respecto a la aplicación de los sensores distribuidos de Brillouin.

9.3. Producción científica.

9.3.1. Publicaciones.

9.3.2. Congresos.

9.3.3. Proyectos de investigación.

9.4. Futuras líneas de investigación.

\section{REFERENCIAS}

\section{APENDICES}

Apéndice I. Proyectos de investigación financiados por Administraciones Públicas o empresas privadas.

Apéndice II. Publicaciones en congresos y revistas. 



\section{INDICE DE FIGURAS}

Figura 1.1

Figura 1.2

Figura 1.3

Figura 2.1

Figura 2.2

Figura 2.3

Figura 2.4

Figura 2.5

Figura 2.6

Figura 2.7

Figura 2.8

Figura 2.9

Figura 2.10

Figura 2.11

Figura 2.12

Figura 2.13

Figura 2.14

Figura 2.15

Figura 2.16
Frontón Recoletos (1935). (a)-Modelo reducido a escala 1/10 (derecha).

(b)- Vista interior (Archivo Torroja-CEHOPU).

Colapso de un puente sobre el rio Missisipi en Mineapolis, USA. (www.nytimes.com)

Pilar instrumentado con galgas extensométricas (Adam 2007).

Vista de las capas de una fibra óptica (Kin-Tak et al. 2001)

Modo de viaje de la luz a través de la fibra óptica (www.Wikipedia.es).

Sensores localizados (Adaptado de Sunaryo et al. 2004).

Sensor distribuido Brillouin Scattering (Adaptado de Sunaryo et al. 2004).

Sensores multiplicados (Adaptado de Sunaryo et al. 2004).

Esquema de una Red de difracción (Barrera, 2008). Se representa el núcleo de la fibra óptica y en distinto color las perturbaciones en su índice de refracción.

Principio de funcionamiento de los sensores ópticos basados en FBG (Kin-Tak et al. 2001)

Variación en la longitud de onda reflejada por la Red de Bragg

(a)- Elementos que forman un sistema de monitorización óptico basado en FBG (Barrera 2008). (b)- Montaje durante un ensayo. Estrellas pasivas e interrogador.

FBG's en serie, sobre un mismo cable de fibra óptica.

Pérdida de potencia reflejada en el sensor rojo debido a suciedad en las conexiones. El sensor azul muestra una conexión limpia.

Doblez en el cableado de fibra óptica, y dificultad para la propagación de la luz (Gerard 2005).

Distribución de los estudios de diferentes tipos de sensores ópticos. Adaptado de Lee (2003).

Diseño del sensor propuesto por Fei Luo et al. (1999). Calibración entre la pérdida de potencia óptica reflejada y la deformación impuesta.

(a)-Diseño propuesto por Moyo et al. (2005) para un sensor óptico FBG.

(b)-Comparación de los resultados del sensor óptico FBG con las galgas extensométricas en un ensayo de flexión sobre vigas de hormigón.

(a)- Distintas geometrías y rugosidades propuestos para el encapsulado metálico del sensor óptico. (b)- Calibraciones del sensor propuesto en función de distintas longitudes de sensor. 
Monitorización estructural.

Aplicación con sensores de fibra óptica.

Figura 2.17 (a)- Imagen del sensor óptico compuesto con epoxi propuesto por Kesavan et al. (2010). (b)- Comparación entre los resultados de los sensores ópticos de deformación y galgas eléctricas en los ensayos de compresión sobre probetas cilíndricas.

Figura 2.18 Relación entre el error característico del sensor frente al ratio entre los Módulos de elasticidad del material que forma el sensor y el material que forma la estructura (Calderón y Glisic, 2012).

Figura 2.19 Espectro reflejado en función de la carga aplicada. Ramos et al. (2009)

Figura 2.20 (a)- distorsión en la forma del espectro de doble pico. (b)- Distorsión en la forma del espectro con picos "en cascada" (Biswas et al. 2010).

Figura 2.21 (a)- Espectro de luz sin birrefrigencia. (b)- Espectro de luz con birrefrigencia y fuerza transversal sobre la fibra óptica. Jochen y Tobías (2011)

Figura 2.22 Cortes al final de la fibra óptica y su influencia sobre el espectro de luz reflejado. Jochen y Tobías (2011).

Figura 2.23 Ratio de transferencia en función de la longitud de la fibra óptica (Dongsheng et al. 2006).

Figura 2.24 Relación entre el coeficiente de transferencia y la deformación del hormigón (Qingbin et al. 2003).

Figura 2.25 Coeficiente de transformación de la deformación para distintas longitudes de fibra y para distintos tipos de recubrimiento. Shiuh y Chih (2011)

Figura 2.26 Porcentaje de puentes estudiados con distintas técnicas de monitorización estructural. Adaptado de Ko y Ni (2005).

Figura 2.27 Distribución de los objetivos de un proyecto de monitorización en el tiempo (Inaudi, 2009)

Figura 2.28 (a)- Elemento instrumentado con tipología simple. (b)- Pilote enterrado instrumentado con una tipología simple (Glisic e Inaudi, 2007).

Figura 2.29 Tipología paralela en una célula de hormigón (Glisic e Inaudi, 2007).

Figura 2.30 Ejemplo de viga deformada instrumentada con tipología paralela. Cálculo de flechas (Glisic e Inaudi, 2007).

Figura 2.31 Tipología cruzada (Glisic e Inaudi, 2007).

Figura 2.32 Esquema de la tipología triangular (Glisic e Inaudi, 2007).

Figura 2.33 (a)- Sensores colocados en la armadura del pilar y caja de conexión. (b)Posición esquemática del sensor en el pilar (Zonta et al. 2011).

Figura 2.34 Evolución de las deformaciones en los pilares durante 7 años (Zonta et al. 2011).

Figura 2.35 Asentamiento de un pilar y evolución en sus deformaciones (Glisic e Inaudi, 2007).

Figura 2.36 Vista del puente Lutrive en Suiza. 
Figura 2.37

Figura 2.38

Figura 2.39

Figura 4.1

Figura 4.2

Figura 4.3

Figura 4.4

Figura 4.5

Figura 4.6

Figura 4.7

Figura 4.8

Figura 4.9

Figura 4.10

Figura 4.11

Figura 4.12

Figura 4.13

Figura 4.14

Figura 4.15 (a)- Sensores a lo largo del voladizo. (b)- Sensores instalados en la sección transversal (Glisic e Inaudi 2007).

Desplazamiento vertical del extremo del voladizo. (Glisic e Inaudi 2007)

Curvaturas obtenidas con los sensores ópticos. (Glisic e Inaudi 2007)

(a)- Sistema de posicionamiento milimétrico. (b)- Incrementos de longitud de onda en función del tiempo para incrementos de deformación.

Ajuste de la constante de sensibilidad.

Evolución de la temperatura ambiente, comparación y calibración de los sensores ópticos.

(a)- Cámara climática Feutron KPK 400. (b)- Probetas de hormigón y metálicas dispuestas en el interior de la cámara climática.

Ajustes térmicos para el sensor de temperatura. (a)- probeta de hormigón. (b)- probeta de acero.

Sensor propuesto en la investigación. Vista 3D del encapsulado y fibra óptica.

Variación en la longitud de onda de Bragg $\Delta \lambda_{B}$ durante el encapsulado del sensor.

(a)- Ensayo de tracción sobre una probeta fabricada con el material del ensapculado. (b)- Esquema que muestra la dirección de aplicación de la carga y la orientación de las galgas extensométricas. La probeta presenta forma de I, para facilitar una mejor aplicación de las cargas en cabeza.

Diseño habitual de sensores ópticos de deformación (Adaptado de Moyo et al. 2005).

(a)- Vista 3D del encapsulado sensor y adhesivo mostrando sus planos de simetría. (b)- Vista de una parte del MEF estudiado.

(a)- Elemento BRS3. (b)- Elemento HX20. (Lusas, 2010).

Mallas deformadas del modelo de elementos finitos para un espesor de adhesivo de (a)- $1000 \mu \mathrm{m}$, (b)- $100 \mu \mathrm{m}$.

Relación entre el gradiente de deformaciones $\Delta \varepsilon(\%)$ y el Módulo de Elasticidad del material del encapsulado $E_{p}$, para diferentes valores del Coeficiente de Poisson del encapsulado $v_{p}$. Espesor de adhesivo adoptado $400 \mu \mathrm{m}$. Coeficiente de Poisson del adhesivo $v_{A}=0.4$.

Relación entre el gradiente de deformaciones $\Delta \varepsilon(\%)$ y el Módulo de Elasticidad del material del encapsulado $E_{p}$, para diferentes valores del Coeficiente de Poisson del encapsulado $v_{p}$. Espesor de adhesivo adoptado $400 \mu \mathrm{m}$. Coeficiente de Poisson del adhesive $v_{A}=0.1$.

Sección transversal del sensor mostrando las deformaciones en el eje $\mathrm{X}$. Espesor de adhesivo $100 \mu \mathrm{m}$. Coeficiente de Poisson del adhesivo $v_{A}=0.4$. 
Monitorización estructural.

Aplicación con sensores de fibra óptica.

Figura 4.16 Relación entre el gradiente de deformaciones $\Delta \varepsilon(\%)$ y el espesor de adhesivo $\mathrm{t}(\mu \mathrm{m})$. Coeficiente de Poisson del adhesivo $v_{A}=0.4$.

Figura 4.17 Sección transversal del sensor adherido a una superficie. La imagen fue tomada con un microscopio de alta resolución StereoZoom SZ61 (Altra, 1999) y procesada con el software AnalySIS getIT Olympus (Olympus, 2006).

Figura 4.18 Relación exponencial entre el gradiente de deformaciones $\Delta \varepsilon(\%)$ y el coeficiente de Poisson del adhesivo $v_{A}$. Espesor de la capa de adhesivo $\mathrm{t}=$ $400 \mu \mathrm{m}$.

Figura $4.19 \quad$ Relación entre el gradiente de deformaciones $\Delta \varepsilon(\%)$ y el espesor de adhesivo $\mathrm{t}(\mu \mathrm{m})$ para diferentes valores del coeficiente de Poisson del adhesivo $v_{A}$.

Figura 4.20 Vista 3D del modelo de elementos finitos empleado para analizar el sensor genérico con dos capas.

Figura 4.21 Comparación entre el sensor propuesto en este trabajo y un sensor genérico de dos capas. Relación entre el gradiente de deformación $\Delta \varepsilon(\%)$ y el espesor de adhesivo $t(\mu \mathrm{m})$ ara diferentes valores del espesor de la capa inferior de encapsulado $(\mu \mathrm{m})$.

Figura 4.22 Ciclo de temperaturas aplicado sobre el sensor.

Figura 4.23 Respuesta del sensor encapsulado. Espectros de luz reflejados para diferentes temperaturas ambientes.

Figura 4.24 Aleatoriedad de los pelos de la fibra de vidrio sobre la red de difracción de Bragg del sensor.

Figura 4.25 Respuesta del sensor encapsulado con distribución uniforme de la fibra de vidrio. Espectros de luz reflejados para diferentes temperaturas ambientes

Figura 5.1 Recomendaciones acerca del empleo de los sensores ópticos puntuales o "long gauge" (Adaptado de Glisic e Inaudi 2007).

Figura 5.2 Representación de un sensor de longitud en un material no homogéneo (Glisic e Inaudi, 2007).

Figura 5.3 Elemento de hormigón instrumentado con sensores ópticos puntuales y de longitud (Glisic e Inaudi, 2007).

Figura 5.4 Esquema del sensor de longitud propuesto en esta Tesis Doctoral.

Figura 5.5 Campo de desplazamientos en dirección vertical en un elemento estructural de hormigón sometido a esfuerzos de compresión, obtenidos con el programa Lusas V.14.3-11 a) Sin la colocación de ningún elemento sensor en su superficie. b) Con un elemento sensor en su superficie entre los puntos $\mathrm{A}$ y $\mathrm{B}$ con una rigidez axil $E_{m} A_{m}=5000 \mathrm{KN}$. c) Con un elemento sensor en su superficie entre los puntos A y $\mathrm{B}$ con una rigidez a axil $E_{m} A_{m}=30000 \mathrm{KN}$.

Figura 5.6 Ensayo experimental. Pretensado inicial a una fibra óptica y pérdida de pretensado con el tiempo, en $\%$. 
Figura 5.7

Figura 5.8

Figura 5.9

Figura 5.10

Figura 5.11

Figura 5.12

Figura 5.13

Figura 5.14

Figura 5.15

Figura 5.16

Figura 5.17

Figura 5.18

Figura 5.19

Figura 5.20

Figura 5.21
Tres tipos de probetas empleadas en los ensayos. (a)- probetas de 0.4 metros de altura. (b)- probetas de 1 metro de altura. (c)- pilares de 3 metros de altura.

Elementos finitos empleados en los modelos (Lusas, 2010).

(a)- Deformaciones unitarias experimentales y numéricas en el hormigón y sensor en la dirección del eje Y. (b)- Modelo numérico de elementos finitos. $\varepsilon_{\mathrm{s}} / \varepsilon_{\mathrm{c}} \approx 0.75\left(\varepsilon_{\mathrm{s}}\right.$ es la deformación del sensor y $\varepsilon_{\mathrm{c}}$ es la deformación del hormigón).

Variación del ratio $\varepsilon_{S} / \varepsilon_{c}$ en función del módulo de elasticidad del adhesivo $E_{A}$. Valor de carga uniformemente repartida $21000 \mathrm{KN} / \mathrm{m}^{2}$.

(a)-Deformaciones unitarias experimentales y numéricas en el hormigón y sensor en eje Y. (b)- Modelo numérico de elementos finitos. ${ }^{\varepsilon_{\mathrm{s}}} / \varepsilon_{\mathrm{c}} \approx 1$

Variación del ratio $\varepsilon_{C, 0} / \varepsilon_{C}$ en función de la rigidez a axil del sensor $E_{m} A_{m}$. Valor de carga uniformemente repartida $21000 \mathrm{KN} / \mathrm{m}^{2}$. Valor del módulo de elasticidad del adhesivo adoptado $E_{A}=1310^{6} \mathrm{KN} / \mathrm{m}^{2}$

Campo de desplazamiento en dirección Y. a) Con un elemento sensor de rigidez a axil $E_{m} A_{m}=10000 \mathrm{KN}$. b) Sin ningún elemento sensor.

Resultados experimentales del sensor óptico puntual de referencia, de un sensor óptico de longitud y resultados teóricos obtenidos a través de un MEF 3D.

Elemento de hormigón ensayado. Para una mejor visualización, se muestran los resultados en la zona del elemento donde está instalado el sensor. Vista frontal de la probeta estudiada.

(a)- Imagen del despegue del sensor. (b)- Vista del modelo de elementos finitos de la interfaz de contacto entre hormigón y adhesivo donde se observa el valor de las tensiones tangenciales máximas alcanzadas $\left(12043 \mathrm{KN} / \mathrm{m}^{2}\right)$ en el instante en que se produce el despegue.

Variación de la tensión tangencial en el hormigón en función del valor del módulo de elasticidad del adhesivo $E_{A}$.

Tensiones tangenciales (valor máximo de $6148.6 \mathrm{KN} / \mathrm{m}^{2}$ ) en la interfaz de contacto entre hormigón y adhesivo.

Resultados del ensayo experimental.

Malla del elemento sensor sometido a compresión. Primer modo de pandeo.

Elemento sensor y modelización del inhibidor de pandeo mediante una rigidez $\mathrm{K}$. 
Monitorización estructural.

Aplicación con sensores de fibra óptica.

Figura 5.22 Cargas de pandeo en función de distintas rigideces relativas y longitudes de sensor.

Figura 6.1 Sección transversal instrumentada y principales dimensiones (Fuente: Anejo de Cálculo de Estructuras del Proyecto).

Figura 6.2 Sensores ópticos instalados a lo largo del perímetro de la sección de túnel.

Figura 6.3 Separación entre los sensores ópticos de longitud.

Figura 6.4 Posición de los sensores ópticos puntuales de temperatura.

Figura 6.5 (a)- Vista frontal del falso túnel instrumentado. (b)- Plano de la sección transversal del falso túnel.

Figura 6.6 Sección de túnel instrumentada indicada con una traza de color rojo discontinua.

Figura 6.7 Falso túnel sin relleno de tierras. Día 27/09/2011.

Figura 6.8 Relleno de tierras finalizado. Día 15/05/2012.

Figura 6.9 Numeración de los sensores de longitud dispuestos a lo largo de la bóveda del túnel.

Figura 6.10 Disposición de los sensores ópticos en el interior de la bóveda del túnel en la posición 2 .

Figura 6.11 (a)- Identificación de los sensores de temperatura dispuestos. (b)- Sensado distribuido.

Figura 6.12 Modelización de la sección del falso túnel de mayor altura de relleno de tierras. Movimientos impedidos en la base de los tacones y de la contrabóveda.

Figura 6.13 Elemento QPN8. (Lusas, 2010).

Figura 6.14 Ajuste realizado para las variaciones térmicas en los cuatro puntos instrumentados. Día 26/09/2011

Figura 6.15 Disposición de los sensores necesarios para realizar la compensación térmica.

Figura 6.16 Variaciones térmicas en los puntos instrumentados a lo largo del día 26/09/2011

Figura 6.17 Variaciones térmicas en los puntos instrumentados a lo largo del día $27 / 09 / 2011$.

Figura 6.18 Variaciones de temperatura en los puntos instrumentados durante los días 26 y 27/09/2011. Valores medidos e hipotéticos adoptados.

Figura 6.19 Variaciones de deformación entre los dos sensores de deformación de la posición 2 durante la toma de datos. (a)- El día 26/09/2011. (b) - El día $27 / 09 / 2011$.

Figura 6.20 Variaciones de deformación de los sensores durante la toma de datos de los días 26 y 27/09/2011. 
Figura 6.21

Figura 6.22

Figura 6.23

Figura 6.24

Figura 6.25

Figura 6.26

Figura 6.27

Figura 6.28

Figura 6.29

Figura 6.30

Figura 6.31

Figura 7.1

Figura 7.2

Figura 7.3

Figura 7.4

Figura 7.5

Figura 7.6

Figura 7.7

Figura 7.8

Figura 7.9
Evolución de la malla deformada en distintos instantes de tiempo.

Evolución de las deformaciones de la posición 1. Mediciones y resultados numéricos.

Evolución de las deformaciones de la posición 2. Mediciones y resultados numéricos.

Evolución de las deformaciones de la posición 3. Mediciones y resultados numéricos.

Evolución de las deformaciones de la posición 4. Mediciones y resultados numéricos.

Evolución de las deformaciones de la posición 5. Mediciones y resultados numéricos.

Evolución de las deformaciones de la posición 6. Mediciones y resultados numéricos.

Evolución de las deformaciones de la posición 7. Mediciones y resultados numéricos.

Reparaciones realizadas en la sección instrumentada.

Acciones sobre el modelo (a)- Variaciones de temperatura. (b)- Peso de las tierras. (c)- Empuje de las tierras.

Malla deformada. (a)- Variaciones térmicas. (b)- Peso de las tierras. (c)Empuje de las tierras.

Back-scattering de la luz (Página web de la compañía FOS\&S).

Análisis tridimensional basado en el Scattering de Brillouin (Luc et al. 2010).

Imagen del ensayo. Unidades de lectura de los sensores basados en FBG's y sensores distribuidos.

(a)-Montaje empleado para la evaluación del SBS. En la pantalla del analizador de redes aparece el pico de la zona de ganancia de Brillouin. (b)- Tramo de $20 \mathrm{~km}$ de fibra óptica en el interior de la cámara climática.

Frecuencia de Brillouin y temperaturas frente al tiempo. Tramo de fibra de $20 \mathrm{~km}$

Frecuencia de Brillouin frente a temperatura impuesta. Tramo de fibra de $20 \mathrm{~km}$.

Frecuencia de Brillouin y temperaturas frente al tiempo. Tramo de fibra de $1.5 \mathrm{~km}$.

Frecuencia de Brillouin frente a temperatura impuesta. Tramo de fibra de $1.5 \mathrm{~km}$.

Frecuencia de Brillouin y temperaturas frente al tiempo. Tramo de fibra de $2 \mathrm{~km}$. 
Monitorización estructural.

Aplicación con sensores de fibra óptica.

Figura 7.10 Frecuencia de Brillouin frente a temperatura impuesta. Tramo de fibra de 1.5 km. (a)- Rama ascendiente. (b)- Rama descendiente.

Figura 7.11 Ciclo de carga y descarga propuesto sobre la probeta de hormigón.

Figura 7.12 Imagen del refuerzo propuesto en las cabezas de las probetas.

Figura 7.13 (a)- Probeta instrumentada con sensores distribuidos. (b)- Montaje de una probeta instrumentada con los tres tipos de fibras.

Figura 7.14 Evolución de la frecuencia Brillouin a lo largo de la fibra óptica en el tiempo en el que han sido tomadas las medidas del ensayo. La leyenda muestra la carga (toneladas) sobre la probeta en cada instante.

Figura 7.15 Latiguillos que unen la fibra óptica con la unidad de lectura. Durante el ensayo sufrió radiación directa de la luz solar.

Figura 7.16 Variación de la frecuencia Brillouin en los 6 tramos de fibra adheridos a la estructura. La leyenda muestra la carga (toneladas) sobre la probeta en cada instante.

Figura 7.17 Evolución de la frecuencia Brillouin de las fibras instaladas en la probeta a lo largo del ensayo.

Figura 7.18 Evolución de la frecuencia Brillouin con la carga aplicada durante el ensayo.

Figura 7.19 Relación entre la frecuencia Brillouin y la deformación de la probeta medida a partir de un sensor óptico puntual de referencia.

Figura 7.20 Relación entre la frecuencia Brillouin y la deformación de la probeta medida a partir de un sensor óptico de longitud de referencia.

Figura 7.21 Deformación de la probeta en función de la longitud de la fibra óptica, medido por el sensado distribuido de Brillouin. (Deformaciones negativas corresponden con esfuerzos de compresión).

Figura 7.22 Deformación de la probeta en función de la longitud de la fibra óptica, medido por el sensado distribuido de Brillouin, para el tramo desde los 12 hasta los 26 metros. (Deformaciones negativas corresponden con esfuerzos de compresión).

Figura 7.23 Deformación medida por el sensor puntual, de longitud y distribuidos durante el ensayo.

Figura 8.1 Variación de la frecuencia Brillouin a lo largo de la longitud de la fibra óptica.

Figura 8.2 Sección del falso túnel instrumentada con distintas tecnologías.

Figura 8.3 (a)- Sección transversal instrumentada, ubicación de los equipos de medida. (b)- Vista tridimensional de la estructura instrumentada con los tramos B y C de Brillouin.

Figura 8.4 Disposición aproximada de la fibra que cuelga sobre la estructura del falso túnel. 
Figura 8.5

Figura 8.6

Figura 8.7

Figura 8.8

Figura 8.9

Figura 8.10

Figura 8.11

Figura 8.12

Figura 8.13

Figura 8.14 Evolución de las deformaciones para la posición 4. Comparación entre la solución teórica, sensores puntuales, sensores de longitud y sensores distribuidos.

Figura 8.15 Evolución de las deformaciones para la posición 5. Comparación entre la solución teórica, sensores puntuales, sensores de longitud y sensores distribuidos.

Figura 8.16 Evolución de las deformaciones para la posición 6. Comparación entre la solución teórica, sensores puntuales, sensores de longitud y sensores distribuidos. 



\section{ÍNDICE DE TABLAS}

Tabla 1.1 Aplicaciones de los sistemas de monitorización actuales más comunes.

Tabla 2.1 Cuadro resumen con los diferentes tipos de sensores.

Tabla 2.2 Sensibilidades obtenidas por Torres (2008).

Tabla 2.3 Recopilación de investigaciones sobre el estudio de sensores ópticos. (FBGFiber Bragg Gratting; BOTDR- Brillouin Optical Time Domain Refelctomery. LG- Long Gauge).

Tabla 2.4 Sensibilidades de los sensores ópticos obtenidos por Moyo et al. (2005).

Tabla 2.5 Aplicaciones SHM en el campo de la obra civil.

Tabla 2.6 Aplicaciones SHM en el campo de la edificación.

Tabla 2.7 Aplicaciones SHM en el campo del estudio de nuevos materiales aplicados a la ingeniería.

Tabla 2.8 Otras investigaciones y aplicaciones SHM.

Tabla 4.1 Especificaciones técnicas del adhesivo

Tabla 4.2 Propiedades mecánicas del material compuesto por fibra de vidrio y resina de poliéster empleado en los ensayos.

Tabla 4.3 Propiedades mecánicas del adhesivo adoptadas en el MEF (Fuente: HBM, 2006).

Tabla 5.1 Valores de resistencia a compresión y Módulo de Elasticidad. Probetas 0.1 X $0.1 \times 0.4$

Tabla 5.2 Valores de resistencia a compresión y Módulo de Elasticidad. Probetas de dimensiones $0.2 \times 0.2 \times 1$ y $0.3 \times 0.3 \times 3$.

Tabla 5.3 Propiedades asignadas a la superficie de contacto entre hormigón y adhesivo.

Tabla 5.4 Principales resultados numéricos del modelo.

Tabla 5.5 Principales resultados numéricos del modelo.

Tabla 6.1 Calendario de medidas.

Tabla 6.2 Ajustes que representan las variaciones térmicas en los puntos instrumentados. Día 26/09/2011.

Tabla 6.3 Ecuaciones que representan los valores de temperatura hipotéticos adoptados.

Tabla 6.4 Variación de temperatura entre la puesta a cero y la fecha de medición en el sensor de la clave interior del túnel.

Tabla 6.5 Valores de deformación entre la puesta a cero y la fecha de medición.

Tabla 6.6 Valores de deformación medidos y teóricos entre la puesta a cero y la fecha de medición. 
Monitorización estructural.

Aplicación con sensores de fibra óptica.

Tabla 6.7 Porcentajes sobre la deformación total originados por el relleno de tierras y por las variaciones térmicas.

Tabla 7.1 Coeficientes de sensibilidad obtenidos en los ensayos y coeficientes $\mathrm{R}^{2}$. (RdRama descendente, $R a$-Rama ascendente)

Tabla 7.2 Coeficientes de ajuste $\mathrm{R}^{2}$ para cada tramo de fibra.

Tabla 7.3 Coeficientes de ajuste $R^{2}$ para cada tipo de fibra y según sensor puntual y de longitud.

Tabla 7.4 Coeficientes de sensibilidad de la deformación para el sensado distribuido de cada tipo de fibra óptica. Ajustes según el sensor óptico puntual, de longitud y valor medio.

Tabla 9.1 Información de los proyectos de investigación.

Tabla A1 Información de los proyectos de investigación. 


\section{NOTACIÓN Y ABREVIATURAS}

A, B Puntos que delimitan la longitud del sensor a partir de sus coordenadas $X_{B}-X_{A}$

$A_{i, t} \quad$ Área de la sección transversal de la celda i en el instante t. (Glisic e Inaudi, 2007).

$A_{i 1, r}, A_{i 2, r} \quad$ Puntos de desplazamientos conocidos, en una tipología triangular.

$A_{m}$

Área de la sección transversal del elemento que forma el sensor de longitud.

$\mathrm{C}$

Punto medio del sensor de coordenadas $X_{C}=\left(X_{A}+X_{B}\right) / 2$

$c_{s} \quad$ Cohesión en la superficie de contacto entre hormigón y adhesivo según el criterio de rotura Mohr-Coulomb.

$C_{s, i} \quad$ Punto medio de una celda instrumentada con tipología simple (Glisic e Inaudi, 2007).

$c_{1}, c_{2} \quad$ Constantes de integración de la curvatura.

$d_{B} \quad$ Distancia de resolución del sensado distribuido de Brillouin.

$d_{i 1} \quad$ Canto de la célula i en una tipología paralela.

E Empuje de tierras.

E Módulo de elasticidad del núcleo de la fibra óptica.

$E_{A} \quad$ Módulo de Elasticidad del adhesivo.

$E_{C} \quad$ Módulo de elasticidad del hormigón.

$E_{i, t} \quad$ Módulo de elasticidad del material que forma la celda i. (Glisic e Inaudi, 2007).

$E_{m} \quad$ Módulo de elasticidad del material que forma el sensor de longitud.

$E_{N}^{S} \quad$ Módulo de elasticidad perpendicular al plano del contacto entre hormigón y adhesivo.

$E_{p} \quad$ Módulo de elasticidad del material que forma el encapsulado del sensor puntual.

$E_{P}^{S} \quad$ Módulo de elasticidad contenido en el plano del contacto entre hormigón y adhesivo

$E_{T} \quad$ Módulo de Elasticidad del material de relleno del falso túnel.

$f_{t} \quad$ Tensión límite de arrancamiento según el criterio 3-D Elasto-Plastic Interface Model implementado en Lusas V14.3-11.

$f_{y k} \quad$ Límite elástico de las armaduras de las probetas.

$f_{y r} \quad$ Límite elástico del refuerzo de las cabezas de las probetas. 
$G_{i, t} \quad$ Módulo de rigidez transversal de la célula i, en el instante t, en una tipología cruzada.

$G_{N}^{S} \quad$ Módulo de rigidez transversal perpendicular al plano de contacto entre hormigón y adhesivo.

$G_{P}^{S} \quad$ Módulo de rigidez transversal contenido en el plano del contacto entre hormigón y adhesivo.

$h_{i} \quad$ Distancia entre el sensor traccionado y comprimido en la célula $\mathrm{i}$, en una tipología paralela.

$I_{i, t}$

$I_{m}$

$\mathrm{K}$

$K_{o}$

$k_{i, E m, t}$

$k_{l g}$

$k_{m, t}(x)$

$k_{s 1}, k_{s 2}$

$K_{T}$

$K_{\varepsilon}$

$K_{\varphi} f_{\varphi}$

$L_{\text {cell }, j}$

$L_{s, i}$

$M_{i, t}$

$N_{C_{s, i, t}}$

$N_{i, t}$

$\mathrm{n}$

$\mathrm{n}^{\prime}$

$n_{\text {eff }}$

$n_{i, t}$

$p_{11}$ у $p_{12}$

Momento de inercia de la sección transversal de la célula i, en el instante t, en una tipología paralela.

Momento de inercia del elemento que forma el sensor de longitud.

Rigidez que impone el inhibidor de pandeo sobre el sensor de longitud.

Coeficiente de empuje al reposo del material de relleno del falso túnel.

Curvatura elástica en la célula i, en el instante t, en una tipología paralela.

Curvatura obtenida con sensores de longitud.

Distribución de las curvaturas elásticas en el instante t.

Curvaturas obtenidas con sensores puntuales.

Coeficiente de sensibilidad en temperatura de la FBG (según Torres, 2008).

Coeficiente de sensibilidad en deformación de la FBG (según Torres, 2008).

Parámetros de fluencia (según CEB-FIP, 1990).

Longitud de la celda j en la tipología simple. (Glisic e Inaudi, 2007).

Longitud del sensor S instalado en la célula i en una tipología paralela.

Momento flector en la célula i, en el instante t, en una tipología paralela.

Axil en la celda $C_{s, i}$ en el instante t en una tipología simple. (Glisic e Inaudi, 2007).

Esfuerzo axil en la célula i, en el instante t, en una tipología paralela.

Índice de refracción de la cubierta de la fibra óptica (según Kin Tak et al. 2001).

Índice de refracción del núcleo de la fibra óptica (según Kin Tak et al. 2001).

Índice de refracción efectivo del núcleo de la fibra óptica.

Número de fisuras en la parte traccionada de la célula i en el instante t.

Coeficientes fotoelásticos de Pockels (según Bertholds y Dandliker, 1986). 


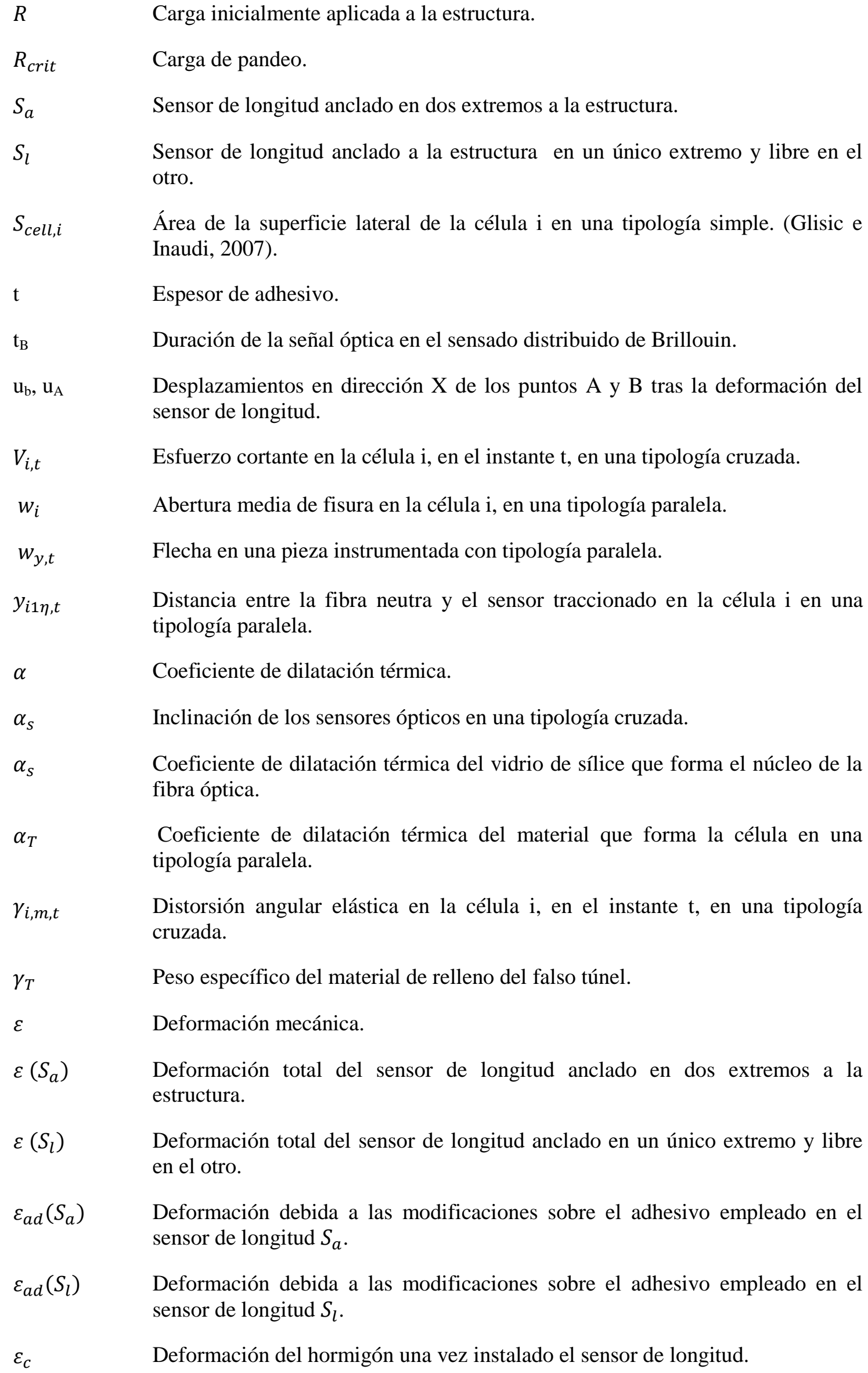

$\varepsilon\left(S_{a}\right) \quad$ Deformación total del sensor de longitud anclado en dos extremos a la estructura.

$\varepsilon\left(S_{l}\right) \quad$ Deformación total del sensor de longitud anclado en un único extremo y libre en el otro.

$\varepsilon_{a d}\left(S_{a}\right) \quad$ Deformación debida a las modificaciones sobre el adhesivo empleado en el sensor de longitud $S_{a}$.

$\varepsilon_{a d}\left(S_{l}\right) \quad$ Deformación debida a las modificaciones sobre el adhesivo empleado en el sensor de longitud $S_{l}$.

$\varepsilon_{c} \quad$ Deformación del hormigón una vez instalado el sensor de longitud. 
Monitorización estructural.

Aplicación con sensores de fibra óptica.

$\varepsilon_{C, 0}$

$\varepsilon_{\text {comp }}$

$\varepsilon_{C, S}$

$\varepsilon_{E}$

$\varepsilon_{f}$

$\varepsilon_{f i b r a}$

$\varepsilon_{h}$

$\varepsilon_{h}\left(S_{a}\right)$

$\varepsilon_{i, E m, t}$

$\varepsilon_{i 0, E m, t}$

$\varepsilon_{i 1, E m+\varphi m, t}$

$\varepsilon_{i 1, m, t}$

$\varepsilon_{i 2, m, t}$

$\varepsilon_{j, m, t}$

$\varepsilon_{m}\left(S_{l}\right)$

$\varepsilon_{n}\left(S_{a}\right)$

$\varepsilon_{n}\left(S_{l}\right)$

$\varepsilon_{P}$

$\varepsilon_{S}$

$\varepsilon_{s h}$

$\varepsilon_{T}$

$\varepsilon_{\text {tot }}$

$\varepsilon_{u}^{+}$

Deformación del hormigón antes de instalar el sensor de longitud.

Deformación compensada del sensor de longitud.

Deformación media medida por el sensor de longitud atribuida al punto medio C.

Componente elástica de la deformación total (Según Glisic e Inaudi 2007).

Deformación de la fibra óptica.

Deformación de la fibra a compensar en un sensor de longitud.

Deformación de la superficie de la estructura monitorizada.

Deformación compensada en los sensores de longitud.

Deformación elástica en una celda instrumentada con tipología simple (Glisic e Inaudi 2007).

Deformación elástica del centro de gravedad de la célula i en el instante t, en una tipología paralela.

Deformación elástica y de fluencia en la célula $\mathrm{i}$, en el instante $\mathrm{t}$, en una tipología paralela.

Deformación mecánica del sensor traccionado instalado en la célula i, en el instante $\mathrm{t}$, en una tipología paralela.

Deformación mecánica del sensor comprimido instalado en la célula i, en el instante $\mathrm{t}$, en una tipología paralela.

Deformación mecánica de la celda j en el instante de tiempo t en una tipología simple. (Glisic e Inaudi, 2007).

Deformación debida a la dilatación del material que forma el encapsulado del sensor de longitud $S_{l}$.

Deformación debida a la variación del índice de refracción de la fibra óptica en el sensor de longitud $S_{a}$.

Deformación debida a la variación del índice de refracción de la fibra óptica en el sensor de longitud $S_{l}$.

Componente plástica de la deformación total. (Según Glisic e Inaudi 2007).

Deformación del sensor de longitud.

Componente de retracción de la deformación total. (Según Glisic e Inaudi 2007).

Componente térmica de la deformación total. (Según Glisic e Inaudi 2007).

Deformación total medida por un sensor de fibra óptica.

Deformación última de tracción del material instrumentado en una tipología paralela. 
$\varepsilon_{x, s}(X) \quad$ Distribución de deformaciones a lo largo del eje $\mathrm{X}$ del sensor.

$\varepsilon_{x x}, \varepsilon_{y y}, \varepsilon_{z z} \quad$ Deformaciones mecánicas según los tres ejes X, Y, Z.

$\varepsilon_{x y} \quad$ Deformación de cortante entre dos planos X, Y.

$\varepsilon_{x z} \quad$ Deformación de cortante entre dos planos X, Z.

$\varepsilon_{y z} \quad$ Deformación de cortante entre dos planos Y, Z.

$\varepsilon_{\varphi} \quad$ Componente de fluencia de la deformación total. (Según Glisic e Inaudi 2007).

$\lambda \quad$ Valores propio asociado a cada modo de pandeo según el módulo Eigenvalue extraction for buckling. Linear buckling analysis implementado en Lusas V14.3-11.

$\lambda_{1} \quad$ Menor valor propio asociado a un modo de pandeo según el módulo Eigenvalue extraction for buckling. Linear buckling analysis implementado en Lusas V14.3-11.

$\lambda_{B} \quad$ Longitud de onda reflejada de Bragg.

$\lambda_{i} \quad$ Longitud de onda inicial.

$\lambda_{f} \quad$ Longitud de onda final.

$\sigma_{x x}, \sigma_{y y}, \sigma_{z z}$ Tensiones mecánicas según los tres ejes $\mathrm{X}, \mathrm{Y}, \mathrm{Z}$.

$\tau_{C_{s, i-1}-C_{S, i},} \quad$ Tensión tangencial lateral entre dos células $C_{s, i-1}$ y $C_{s, i}$ en una tipología simple. (Glisic e Inaudi, 2007).

$\tau_{\text {hormigón }} \quad$ Tensión tangencial en el hormigón en la zona de contacto con el sensor de longitud.

$\tau_{\text {interfaz }} \quad$ Tensión tangencial en la interfaz de contacto entre hormigón y adhesivo.

$v \quad$ Coeficiente de Poisson del núcleo de la fibra óptica.

$v_{A} \quad$ Coeficiente de Poisson del adhesivo.

$v_{N}^{S} \quad$ Coeficiente de Poisson perpendicular al plano del contacto entre hormigón y adhesivo.

$v_{p} \quad$ Coeficiente de Poisson del material que forma el encapsulado del sensor puntual.

$v_{P}^{S} \quad$ Coeficiente de Poisson contenido en el plano del contacto entre hormigón y adhesivo.

$v_{T} \quad$ Coeficiente de Poisson del material de relleno del falso túnel.

$\phi_{S} \quad$ Ángulo de rozamiento en una superficie de contacto según el criterio de rotura de Mohr-Coulomb.

$\phi \quad$ Vector propio asociado a cada modo de pandeo según el módulo Eigenvalue extraction for buckling. Linear buckling analysis implementado en Lusas V14.3-11. 
Monitorización estructural.

Aplicación con sensores de fibra óptica.

$\phi_{T} \quad$ Coeficiente de rozamiento del material de relleno del falso túnel.

$\Delta \mathrm{L}_{\mathrm{cell}, \mathrm{j}} \quad$ Incremento de longitud de la celda $\mathrm{j}$ en una tipología simple (Glisic e Inaudi, 2007).

$\Delta n \quad$ Variación del índice de refracción del núcleo de una fibra óptica.

$\Delta T \quad$ Incremento de temperatura.

$\Delta T_{i 1, m, t} \quad$ Incrementos de temperatura en la fibra del material donde está instalado el sensor traccionado en la célula i, en el instante t, en una tipología paralela.

$\Delta T_{i 2, m, t} \quad$ Incrementos de temperatura en la fibra del material donde está instalado el sensor comprimido en la célula i, en el instante t, en una tipología paralela.

$\Delta v_{C S_{i}-C S_{n}} \quad$ Desplazamiento relativo entre dos celdas $C_{S, i}$ y $C_{s, n}$.

$\Delta w_{d, i} \quad$ Cambio de dimensiones en el intervalo limitado por $X_{B}-X_{A}$ debido a abertura de fisuras, cambios en los tamaños de las inclusiones de aire y otras heterogeneidades, en dirección del eje X tras la deformación. (Glisic e Inaudi 2007).

$\Delta \varepsilon(\%) \quad$ Error relativo del sensor puntual de deformación.

$\Delta \lambda_{B} \quad$ Variación de la longitud de onda reflejada en una red de difracción de Bragg.

$\Lambda$

Periodo espacial de las perturbaciones inducidas en el índice de refracción del núcleo de la fibra óptica.

$\sum_{j} w_{i, j, t} \quad$ Suma de todas las aberturas de fisuras en la parte traccionada de la célula i, en el instante $t$ en una tipología paralela.

BOTDA Brillouin Optical Time Domain Analysis.

BOTDR Brillouin Optical Time Domain Reflectomery.

CFRP Carbon Fiber Reinforced Polymer.

FBG Fiber Bragg Grating.

FOS's $\quad$ Fiber Optic Sensor's.

FRP Fiber Reinforced Polymer.

LG Long Gauge.

LGFOS Long Gauge Fiber Optic Sensor.

NDE Non Destructive Evaluation.

OTDR Optical Time Domain Reflectomery.

SBS $\quad$ Stimulated Brillouin Scattering.

SHM Structural Health Monitoring.

SHMBM Structural Health Monitoring Bridge Management. 


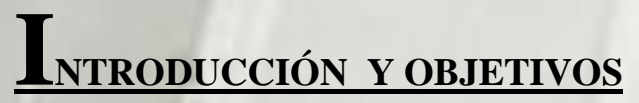

En la introducción de esta Tesis Doctoral se presenta una visión general del proceso de monitorización estructural. Tras analizar sus antecedentes e importancia, se expondrán los sistemas más empleados en la actualidad para llevarlo a cabo. Se discutirán seguidamente las carencias que presentan estos sistemas, lo que permitirá plantear las necesidades actuales en la monitorización de estructuras y las cuestiones básicas a investigar que motivan esta Tesis Doctoral. 



\subsection{Introducción a la monitorización de estructuras.}

La monitorización de estructuras (structural health monitoring -o SHM- en inglés) es una rama de la ingeniería estructural dedicada al desarrollo de sistemas que proporcionen información sobre cualquier cambio significativo o daño que se produzca en una estructura (www.ishmii.org).

El establecimiento formal de esta disciplina es relativamente reciente. Por ejemplo, la International Society for Structural Health Monitoring of Intelligent Infrastructure fue fundada en el año 2003 y el primer número de la revista más antigua dedicada íntegramente a esta disciplina, el "Journal of Intelligent Material Systems \& Structures “, apareció en el año 1990. No obstante, el interés por conocer el comportamiento real de las estructuras es muy anterior. Ya los ingenieros ingleses como Thomas Telford, Isambard Brunel y Robert Stephenson aplicaron en el S. XIX métodos experimentales para diseñar y comprobar la seguridad de estructuras, cuyo comportamiento exacto eran incapaces de predecir por las limitaciones de los métodos de cálculo existentes (Billington, 1985). En España, probablemente sea Eduardo Torroja el gran pionero de la instrumentación estructural. Su deseo por desarrollar nuevas formas con estructuras laminares como la cubierta del Mercado de Algeciras y la cubierta del Frontón Recoletos en Madrid (Figura 1.1), le llevó a crear modelos reducidos de las mismas, y en el segundo caso, incluso a instrumentar también la obra real.

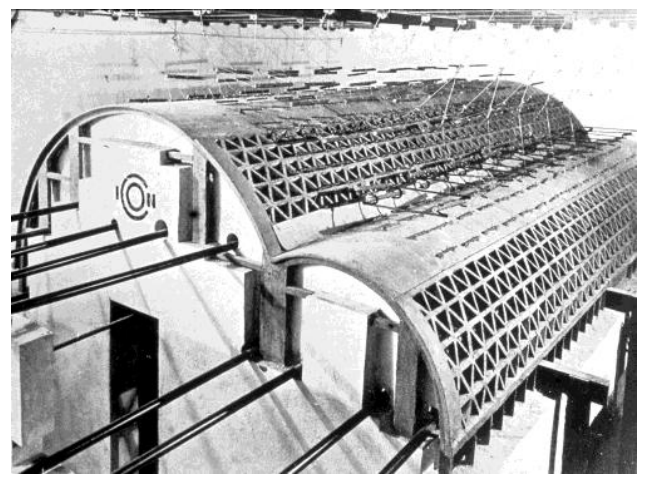

(a)

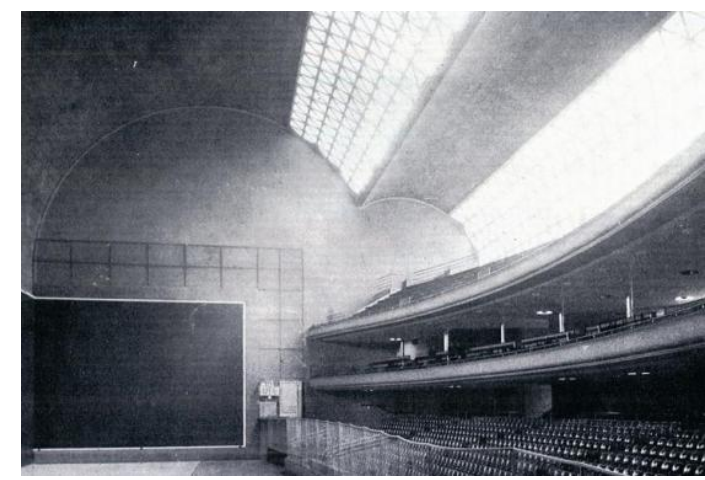

(b)

Figura 1.1 Frontón Recoletos (1935). (a)-Modelo reducido a escala 1/10 (derecha). (b)- Vista interior. (Archivo Torroja-CEHOPU).

La monitorización estructural tiene una gran importancia y desarrollo hoy en día. Algunas de las causas de este auge son:

- Conforme aumenta el nivel de desarrollo de los países tienen menos infraestructuras nuevas que construir y más infraestructuras antiguas que mantener y conservar. Los SHM permiten disponer de datos que ayuden a optimizar la gestión de infraestructuras y garantizar su seguridad.

- Los avances científicos y tecnológicos hacen que hoy en día sea posible proyectar y construir estructuras cada vez más complejas. En estos casos, los datos proporcionados por los SHM permiten comprobar que el comportamiento real de las estructuras se corresponde con el previsto en proyecto. De este modo, se garantiza tanto la seguridad de la estructura como los grados de confort requeridos por el propietario. 
Monitorización estructural.

Aplicación con sensores de fibra óptica.

- Los accidentes recientes de algunas estructuras como el colapso del puente metálico en Minneapolis (USA) el 2 de agosto del año 2007 (Figura1.2), han sensibilizado a la opinión pública sobre la importancia de invertir en el mantenimiento y gestión de las infraestructuras.
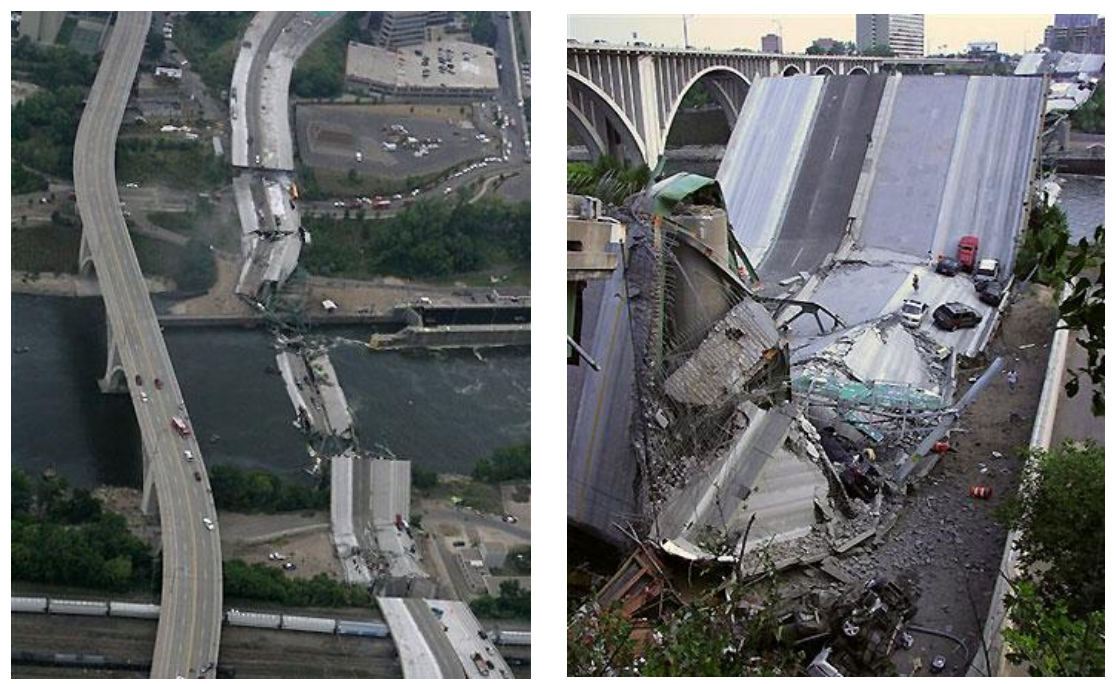

Figura 1.2 Colapso de un puente sobre el rio Missisipi en Mineapolis, USA. (www.nytimes.com)

Un sistema de monitorización se compone de los siguientes elementos: Transductores, sistemas de procesado y acondicionado de la señal, líneas de transmisión, y sistemas de procesado y registro de datos. Existe actualmente una amplia variedad de sistemas de monitorización que son aplicables a la instrumentación y evaluación de las estructuras. Los más conocidos y empleados son:

- Inspección visual.

- Métodos topográficos.

- Métodos basados en radar (Radio Detection And Ranging).

- Métodos basados en rayos X

- Métodos acústicos.

- Métodos fotogramétricos.

- Métodos basados en termografía.

- Métodos basados en flujos magnéticos.

- Sensores elastomagnéticos.

- Métodos GPS.

- Galgas eléctricas.

- Fibra óptica.

Un estudio detallado de todas estas técnicas puede encontrarse en Torres (2008), León (2009) y Molignoni (2011), teniendo cada una de ellas un campo de aplicación característico. La Tabla 1.1 muestra las principales aplicaciones que se pueden llevar a cabo con las distintas técnicas enumeradas: 
Inspección visual

Métodos topográficos

Métodos basados en radar

Métodos basados en rayos $\mathrm{X}$

Métodos acústicos

Métodos fotogramétricos

Métodos basados en termografía

Métodos basados en flujos magnéticos

Sensores elastomagnéticos.

\section{Método GPS}

Galgas eléctricas

Fibra óptica
Problemas estructurales que impliquen grandes deformaciones o patologías en avanzado estado de degradación (Vurpillot et al. 1998).

Deformaciones relativamente grandes, del orden de milímetros (Inaudi, 1997).

Fisuración, exploración de anclajes, localización de armaduras, detección de objetos en el suelo, instalaciones enterradas y cimentaciones (Otsuka et al. 2003).

Localización de diámetro y distribución de armaduras, inspección de cables de pretensado, fisuración y homogeneidad del hormigón (Joachim, 2003).

Fisuración del hormigón (Yuyama et al. 2007, Baifeng y Weiliam 2008).

Desplazamientos, deformaciones y aberturas de fisuras (Whiteman et al. 2002, Lange et al. 2006, Torres et al. 2010).

Fisuración, coqueras y otros defectos en el hormigón (Clark et al. 2003, Carosena 2006).

Estado de las armaduras en una estructura de hormigón armado $\mathrm{y}$, en general, de aquellos materiales que puedan ser magnetizados por la acción de un campo magnético, como el caso del acero (Villalba, 2006).

Fuerza en elementos lineales metálicos (Molignoni, 2011).

Grandes desplazamientos en estructuras (Knecht y Manetti 2001, Inaudi 1997).

Deformaciones (www.hbm.com).

Temperaturas, deformaciones, aceleración, desplazamientos (Moyo et al. 2005).

Tabla 1.1 Aplicaciones de los sistemas de monitorización actuales más comunes.

Por ejemplo, la inspección visual sirve para detectar problemas que impliquen grandes deformaciones, a pesar de sus evidentes limitaciones. Los métodos basados en el empleo del radar o de rayos $\mathrm{X}$ ofrecen ventajas para la inspección de una zona local de una estructura, como el estado de las armaduras, anclajes o la fisuración en un determinado punto. Los métodos acústicos, fotogramétricos y basados en termografía han ofrecido buenos resultados bajo condiciones muy controladas y por tanto, son muy aptos para su aplicación en trabajos y ensayos en laboratorio. Las galgas eléctricas son el método más empleado en la actualidad para la obtención de deformaciones. Sin embargo, su naturaleza eléctrica impide su aplicación en determinados escenarios. 


\subsection{Necesidades actuales en SHM. Identificación de la problemática.}

Como se ha indicado en el apartado anterior, existen técnicas que permiten realizar el seguimiento y la evaluación de un parámetro estructural. Sin embargo, el estudio de las mismas ha permitido comprobar que:

- Algunas de estas técnicas como los métodos basados en el radar o los rayos X sirven para la inspección local de una zona de la estructura y se limitan a realizar mediciones instantáneas, no pudiendo funcionar como un sistema de monitorización continuo en el tiempo (Inaudi, 1997).

- Algunas de estas técnicas no son aplicables en determinados escenarios y por tanto no satisfacen algunas necesidades actuales (Inaudi, 1997). Por ejemplo, las galgas extensométricas no son válidas para su aplicación en líneas electrificadas como las de los trenes de alta velocidad debido a su interacción con las radiaciones electromagnéticas.

\subsubsection{Carencias en los sistemas de monitorización actuales.}

Algunos de los sistemas de monitorización actuales no responden totalmente a las expectativas de sus usuarios. En ocasiones, los resultados obtenidos inspiran poca confianza debido fundamentalmente a afecciones de todo tipo sobre los sistemas de medición. Así, algunas de las principales quejas planteadas por los usuarios de distintos sistemas de monitorización son (Inaudi, 1997):

- Complejidad en su uso, lentitud e ineficiencia. Esto obliga a los operadores del sistema a alcanzar cierta experiencia en el manejo del sistema y en la interpretación de sus medidas.

- Dificultad para llevar a cabo mediciones remotas y a grandes distancias.

- Necesidad de calibración y recalibración del sistema de medida cada vez que se realiza una toma de datos.

- Elevada sensibilidad frente a ciertos factores ambientales. Los de mayor importancia son la humedad y la temperatura. Éstos pueden llegar a alterar considerablemente las mediciones obtenidas. Por tanto, resulta de vital importancia el empleo de sensores cuya respuesta ante este tipo de acciones esté totalmente calibrada.

- Algunos sistemas de medida son muy sensibles a la radiación solar y a cambios en la iluminación sobre el objeto a medir. Este hecho limita su aplicación a ensayos donde todos estos factores se encuentran altamente controlados.

- En general, los sistemas de medida actuales presentan un mal comportamiento en ambientes vibratorios, presentando muy poca estabilidad en sus medidas.

- Elevada sensibilidad a las interferencias electromagnéticas producidas por tormentas y líneas eléctricas. Esta sensibilidad afecta de forma notable a aquellos sistemas basados en señales eléctricas, llegando a hacer totalmente inapropiado su uso.

- Algunos sistemas son muy sensibles a la corrosión. Este fenómeno puede llegar a dejar fuera de servicio el sistema de medida. 
- En ocasiones, se trata de sistemas de gran tamaño, lo que dificulta la instrumentación de puntos o zonas de difícil acceso.

- Elevados costes de operación y de mantenimiento de los sistemas de monitorización.

- Necesidad de estudiar y conocer la interacción de los elementos sensores con el comportamiento de la estructura. El hecho de que los sensores deban ir encapsulados en un material huésped y en ocasiones embebidos en el material que forma la estructura causa una perturbación en el campo de deformaciones que afecta a la exactitud de la medición. En este aspecto, Calderon y Glisic 2012 identificaron los parámetros que afectan a la precisión y a los resultados de la medida de deformaciones empleando sensores ópticos de longitud. Calderón y Glisic 2012 observaron que existen una serie de parámetros cuya afección sobre las mediciones son muy importantes, como son la transferencia de deformaciones desde los anclajes del sensor con la estructura, las dimensiones de estos anclajes, la longitud del sensor y el ratio entre las propiedades mecánicas del sensor y del material de la estructura. Por este motivo, es importante que las características de los nuevos sensores sean compatibles con el medio que los alberga.

- Necesidad de mejorar la transferencia de deformaciones entre la estructura, el material huésped que forma el sensor y el sensor. En el caso de la tecnología de la fibra óptica, ésta no puede ser instalada sin ser encapsulada previamente debido a su fragilidad. En este aspecto, algunos investigadores ( $\mathrm{Li}$ et al. 2006, Glisic e Inaudi 2007, Majumder et al. 2008, Torres et al. 2010) han estudiado distintas técnicas de encapsulado para protegerlas. Es habitual asumir que los valores de deformación medidos por la fibra óptica son los valores de deformación reales de la estructura. Sin embargo no siempre es así. En la mayoría de los casos, la fibra óptica se embebe en un material huésped y posteriormente se instala sobre la estructura. En ocasiones, puede aparecer una discrepancia entre las mediciones y los valores reales, que están asociadas a algunos parámetros como son el espesor del material sobre el que se embebe la fibra óptica, el espesor y propiedades del adhesivo empleado en la instalación. Por este motivo, es importante el diseño de nuevos sensores que tengan en cuenta estas consideraciones.

- Necesidad de integrar las variaciones de deformación en materiales fisurables o heterogéneos a través de sensores de longitud o sensores distribuidos. En ocasiones, el empleo de sensores puntuales pueden proporcionar valores incorrectos en cuanto a la obtención de deformaciones. En el caso de materiales heterogéneos como el hormigón, es necesario el empleo de sensores que sean capaces de integrar en la medida los defectos puntuales como las oclusiones de aire, coqueras o fisuración. En algunos escenarios, la monitorización de estructuras de hormigón a partir de sensores puntuales puede proporcionar resultados totalmente incorrectos y del lado de la inseguridad.

Un nuevo sistema de monitorización estructural debería resolver, al menos, algunos de estos inconvenientes y problemas para tener éxito frente a las tecnologías ya existentes. Ello posibilitaría aplicaciones de los sistemas de monitorización como las siguientes:

- Geoestructuras. Cada vez más, y debido a recientes accidentes ocurridos en obras subterráneas (Calderón, 2010), éstas deben ser instrumentadas para controlar su comportamiento estructural. 
- Puentes de ferrocarril y túneles. Debido a la presencia de campos electromagnéticos resulta muy costoso llevar a cabo la monitorización de este tipo de estructuras. En estos casos, es necesario emplear sistemas y métodos que sean inmunes a las radiaciones electromagnéticas.

- Presas. Las presas son estructuras que suelen estar instrumentadas con sistemas de distinta tipología. Algunos de estos sistemas podrían ser reemplazados por otros más eficientes permitiendo ciertas ventajas como una mayor precisión o la captura remota y a distancia de los datos.

- Campañas experimentales de laboratorio. El diseño de sistemas de monitorización sencillos de implementar y más económicos que los actuales permitiría incrementar su aplicación en los ensayos de elementos estructurales que se realizan en laboratorio con fines de investigación o para probar y homologar elementos estructurales. A modo de ejemplo, la figura 1.3 muestra la instrumentación de un pilar reforzado con presillas y angulares de acero realizada en el contexto de la tesis doctoral de Adam (2007). Cada una de las galgas extensométricas dispuestas para medir deformaciones precisó de un cable, lo que hizo compleja la instrumentación. Un sistema que, manteniendo o mejorando la precisión de las galgas, simplificara el cableado necesario sería mucho más recomendable.

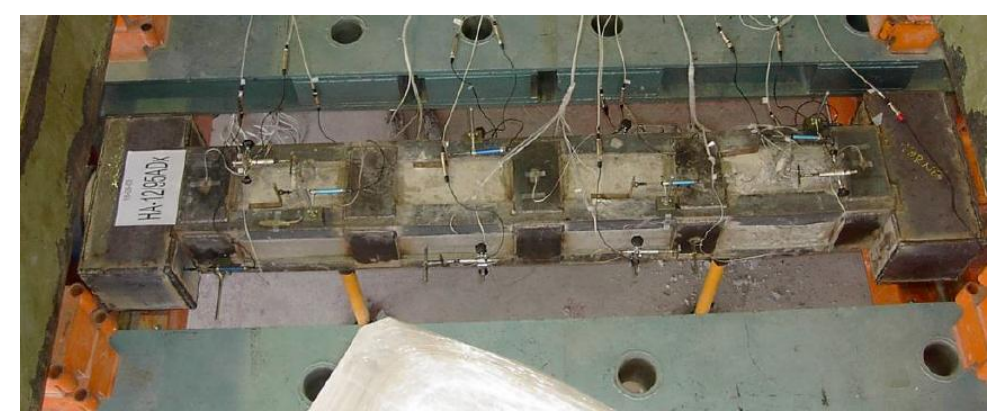

Figura 1.3 Pilar instrumentado con galgas extensométricas (Adam 2007).

Existe un gran número de estructuras que no se encuentran suficientemente instrumentadas debido a la falta de un sistema de instrumentación eficaz (Inaudi, 1997). Por un lado, este hecho pone de manifiesto la necesidad de desarrollar nuevas técnicas que aventajen a las ya existentes. Pero por otro lado, este hecho manifiesta indirectamente que la monitorización estructural no se encuentra todavía totalmente integrada como parte del proceso proyecto-construcción en la ingeniería estructural.

\subsubsection{Aspectos que deben reunir un nuevo sistema de monitorización estructural.}

Las evidentes limitaciones de algunos sistemas de monitorización citados en el apartado 1.1 ha impulsado el desarrollo de nuevas tecnologías que aporten importantes ventajas frente al resto de técnicas (Glišić, 2000). Todo nuevo sistema de monitorización debe cumplir las condiciones siguientes:

\section{- Seguridad.}

Un nuevo sistema de monitorización deberá proporcionar información fiable que garantice una explotación segura de la estructura y una intervención inmediata en caso necesario. Existen dos factores determinantes para cubrir el aspecto de la seguridad: la estrategia de monitorización, y el funcionamiento del sistema de monitorización. Mientras que el diseño de la estrategia de 
monitorización depende del ingeniero responsable de la seguridad de la estructura, el funcionamiento del sistema depende de la tecnología en la que se base.

- Rendimiento y aplicabilidad.

Aprovechando la existencia de las nuevas tecnologías emergentes, un nuevo sistema de monitorización deberá ofrecer mayores rendimientos que los actuales (mayores precisiones y resoluciones, más durabilidad, menores dimensiones...) y deberá ser aplicable en escenarios donde los sistemas actuales no son operativos.

\section{- Economía.}

La economía hace referencia al ratio entre la inversión realizada para la implantación del sistema de monitorización en la estructura y los beneficios económicos obtenidos con su implantación.

Existen dos enfoques de instrumentación que tienen una repercusión directa sobre el aspecto económico: Inspecciones periódicas y monitorización permanente.

Las inspecciones periódicas se realizan por grupos de expertos que analizan algún parámetro de la estructura y toman decisiones sobre posibles reparaciones o refuerzos, en caso de ser necesarios. En este caso, una intervención sobre la estructura (reparación, refuerzo o rehabilitación) sólo puede realizarse tras una inspección. Tanto los costes de la intervención como los costes de la inspección pueden llegar a ser muy elevados por dos motivos (1) porque la intervención no se realiza a tiempo y el daño se ha propagado y (2) porque en la inspección se emplea algún sistema de monitorización que necesite ser dirigido por personal especializado. De acuerdo a lo anterior, distintos autores (Frangopol et al. 1998, Radojicic et al. 1999, Glišić 2000) han llegado a la conclusión de que la inversión en el mantenimiento de una estructura empleando inspecciones periódicas como medio de control podría llegar a ser superior al coste de construcción de una nueva estructura.

En el caso de las monitorizaciones permanentes, la estructura se encuentra monitorizada y se puede conocer la evolución de los parámetros instrumentados (deformación, temperatura, desplazamientos...) en tiempo real. En este caso, es posible una intervención inmediata sobre la estructura, evitando los problemas derivados de la inspección periódica. Los sistemas de monitorización alcanzan su máximo rendimiento cuando se encuentran en funcionamiento durante toda la vida útil de la estructura y durante este periodo, el ingeniero es el responsable de proponer y llevar a cabo el calendario de medidas. Distintos autores han afirmado que la monitorización permanente puede llegar a reducir los costes de mantenimiento de una estructura hasta en un $10 \%$ (Glišić 2000, Bertoncini y Hammerschlag 2000).

\section{- Estética.}

Existen estructuras que forman parte del patrimonio histórico y cultural de la sociedad. La mayoría de ellas no se encuentran instrumentadas, pero existen casos en los que es necesario un seguimiento de las mismas (Del Grosso et al. 2000, Inaudi et al. 2001, Glišić e Inaudi 2007). En caso de que una estructura antigua sea instrumentada, un nuevo sistema de monitorización no deberá afectar a su apariencia estética y deberá ser lo más transparente posible para el usuario.

Análogamente, este aspecto debe ser aplicado a estructuras en general que tengan un importante valor estético. 


\subsection{Objetivos, contribuciones y estructura de esta Tesis.}

La visión global de los apartados anteriores muestra que existe una necesidad real de nuevos sistemas de monitorización estructural que permitan nuevas aplicaciones y abran nuevas posibilidades en el campo de la SHM.

Dentro de los numerosos parámetros medibles (tensiones, deformaciones, velocidades, aceleraciones, desplazamientos, inclinaciones, temperaturas, etc), la presente Tesis Doctoral se ha centrado en el estudio de las deformaciones y temperaturas ya que se ha considerado que representan mejor el comportamiento del material y permiten, a partir de él, componer el comportamiento de la estructura. En este contexto, los objetivos principales de esta Tesis Doctoral son:

- Estudiar y diseñar nuevos elementos sensores para la monitorización de las temperaturas y deformaciones en estructuras, de los tipos siguientes:

- Sensor puntual para la monitorización de estructuras de acero, materiales homogéneos o zonas de estructuras de hormigón no fisuradas.

- Sensor de longitud para la monitorización de estructuras de hormigón o materiales heterogéneos.

- Sensor distribuido para la monitorización de grandes longitudes en estructuras.

- La aplicación de estos nuevos elementos sensores a la instrumentación de estructuras reales y su validación como herramienta en la disciplina de la SHM.

Los nuevos sensores diseñados y estudiados en esta Tesis Doctoral están basados en el empleo de fibra óptica, por ser ésta una nueva tecnología con numerosas ventajas como se muestra en el Capítulo 2. Ya existen actualmente estructuras instrumentadas con distintas variantes de esta tecnología. Sin embargo, existen algunos aspectos que requieren ser estudiados en mayor profundidad y que permiten establecer las principales contribuciones de esta Tesis que son:

- El diseño y desarrollo de sensores ópticos de deformación (puntuales, de longitud y distribuidos) validados experimentalmente y con modelos numéricos de comportamiento. Los diseños y desarrollos de estos sensores ópticos están basados a partir de los siguientes criterios:

$\checkmark$ Robustez ante cualquier manipulación de puesta en obra. En ocasiones el proceso de instalación de los sensores puede provocar unos resultados incorrectos. Este efecto se ha minimizado con el diseño de los sensores propuestos en esta Tesis Doctoral.

$\checkmark$ Conocimiento de la transferencia de deformación entre el material huésped y la fibra óptica.

$\checkmark$ Compatibilidad con las características mecánicas del material, estudiando y conociendo la interacción estructura-sensor.

$\checkmark$ Capaz de compensar la influencia de la temperatura en la medición.

$\checkmark$ Además, estos diseños permiten:

$\checkmark$ La medición de la retracción del hormigón empleando el sensor puntual (Véase apéndice II, Torres et al. 2010). 
$\checkmark$ El desarrollo futuro de sensores ópticos capaces de medir temperaturas y deformaciones a muy altas temperaturas como las producidas durante un incendio, tomando como base el sensor de longitud estudiado en esta Tesis Doctoral. Este planteamiento representa una de las futuras líneas de investigación que se planteará en el Capítulo 9.

- La aplicación y validación del comportamiento de estos sensores en obras reales.

El diseño de los sensores se inició con fases experimentales donde se realizaron ensayos para validar su comportamiento. Estas campañas experimentales contemplaron ensayos sobre probetas de hormigón y metálicas, sometidas a distintos casos de carga (compresión, tracción y flexión simple). Las campañas experimentales se apoyaron en estudios numéricos del conjunto sensor-probeta con el método de los elementos finitos. Esta fase numérica tuvo un triple objetivo. En primer lugar, se estudió la afección que sobre el sensor tienen distintos parámetros que afectan a su instalación en la estructura. En segundo lugar, se estudió el comportamiento de los sensores frente a factores externos como la temperatura o parámetros de fabricación (espesores, propiedades mecánicas de adhesivos, rigideces de los materiales empleados...). Finalmente, esta fase permitió optimizar el diseño y validar el comportamiento del sensor.

Los sensores desarrollados han sido instalados en estructuras reales en el marco de diferentes proyectos de investigación (véase Apéndice I). Estos proyectos han permitido comprobar el funcionamiento de los prototipos en condiciones reales de obra y, en caso necesario, ajustar su diseño. Las estructuras instrumentadas tienen una tipología muy diversa que incluye obra nueva y ya existente. Los elementos analizados fueron losas postesas, encepados de pilas de puentes, forjados de un edificio durante su rehabilitación, un viaducto metálico empujado, edificios de varias alturas y un túnel.

En algunos casos fue posible comparar los resultados de los sensores con los de otros sistemas de medida, con modelizaciones numéricas y/o con resultados teóricos. Esta comparativa permitió alcanzar tres objetivos. En primer lugar, se validaron de los sensores ópticos propuestos para su empleo en la monitorización de estructuras. En segundo lugar, sirvió de apoyo en la investigación sobre el comportamiento de los sensores en las condiciones reales que impone el sector de la construcción. Finalmente, en ocasiones posibilitó detectar algún funcionamiento anómalo del sensor y redefinir los diseños inicialmente propuestos.

El contenido de esta Tesis está organizado de la forma siguiente. El Capítulo 2 contiene un estado del arte de la monitorización estructural empleando la tecnología óptica con un especial énfasis en:

- El principio de funcionamiento de la fibra y de los sensores ópticos más importantes.

- Las principales investigaciones acerca del comportamiento y diseño de los sensores ópticos.

- Las principales aplicaciones de la tecnología óptica en el campo de la monitorización estructural.

El Capítulo 3 presenta una descripción detallada de los objetivos particulares de la investigación, expuestos siguiendo un orden cronológico de evolución. 
Monitorización estructural.

Aplicación con sensores de fibra óptica.

El Capítulo 4 presenta el estudio y diseño de un sensor óptico puntual de deformación y su calibración. El trabajo correspondiente a este capítulo ha sido publicado en la revista Engineering Structures (Torres et al. 2011a).

En el Capítulo 5 se presenta el estudio y diseño de un nuevo sensor óptico de longitud. Su diseño hace que las discontinuidades en el material instrumentado (fisuración, coqueras o inclusiones de aire) no afecte a las medidas. Este diseño está basado en campañas experimentales y modelización numérica.

El Capítulo 6 muestra los trabajos de monitorización de una estructura real. Concretamente, este Capítulo expone los trabajos de monitorización de la estructura del falso túnel de la Línea de Alta Velocidad de Levante a su paso por Mogente (Valencia). En este capítulo se han comparado los dos tipos de sensores estudiados en los Capítulos 4 y 5 (sensores puntuales y sensores de longitud).

Una vez calibrados, aplicados y validados los sensores puntuales y de longitud estudiados en los capítulos anteriores, el Capítulo 7 recoge los resultados de los ensayos experimentales para la calibración de sensores distribuidos. A diferencia de los anteriores diseños basados en FBG's, estos sensores distribuidos están basados en el fenómeno de la dispersión de Brillouin (en inglés, Brillouin Scattering).

Siguiendo con la evolución de la Tesis Doctoral, el Capítulo 8 recoge los trabajos de aplicación de los sensores distribuidos estudiados en el Capítulo 7. Su aplicación se ha llevado a cabo en la estructura del falso túnel de la Línea de Alta Velocidad de Levante a su paso por Mogente (Valencia).

Las principales conclusiones del trabajo realizado, así como las futuras líneas de investigación se detallan en el Capítulo 9.

Finalmente se recogen varios Apéndices que contienen información que complementa la investigación desarrollada. Estos apéndices profundizan en temas tratados en la Tesis Doctoral, y facilitan el entendimiento de la misma al lector.

- Resumen de los proyectos de investigación que han financiado esta investigación

- Las publicaciones que engloban parte del trabajo de esta Tesis.

- Otras publicaciones que se han realizado en distintos congresos y que guardan una clara coherencia con la línea de investigación planteada. 


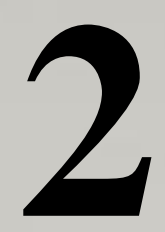

\section{$\underline{\text { Estado del arte }_{\text {ste }}}$}

Una parte esencial de toda Tesis Doctoral es la identificación de aquellos aspectos relacionados con el tema estudiado y que hayan sido tratados en investigaciones anteriores. Esta parte recibe el nombre de Estado del Arte o revisión bibliográfica y permite tanto establecer la idoneidad de un tema como enfocarlo correctamente.

Este capítulo contiene el Estado del Arte del tema objeto de esta Tesis y está estructurado en tres apartados. Inicialmente se expone una visión general acerca de la fibra óptica como elemento capaz de medir parámetros físicos relevantes en una estructura (Apartado 2.2). A continuación, se resumen las aplicaciones más importantes en el desarrollo y estudio de sensores de fibra óptica para la monitorización estructural (Apartado 2.3). Finalmente, se presentan las aportaciones más relevantes sobre monitorización estructural con fibra óptica (apartado 2.4). 



\subsection{Introducción.}

En la monitorización de estructuras es necesario el empleo de sensores y sistemas de medida que proporcionen información relativa a un parámetro con el que poder evaluar su estado. En la actualidad existen numerosas técnicas para llevarla a cabo y fueron expuestas en el Capítulo 1. De todas ellas, la fibra óptica se trata de una tecnología reciente y que presenta muchas ventajas que radican en su naturaleza, sus dimensiones y sus prestaciones.

En estos sensores, la fibra óptica encargada de realizar la medición puede ir embebida entre capas de materiales para asegurar su integridad. En ocasiones puede ser instalada sobre un elemento auxiliar y a su vez, este elemento ser instalado en la estructura. En algunos escenarios y según los objetivos de la monitorización pueden ir embebidas en el interior del hormigón. En cualquier caso, la fibra óptica debe ir encapsulada. Este encapsulado puede ser origen de discrepancia entre las mediciones y los valores reales y por este motivo es necesario estudiar, por un lado, el diseño y la configuración de los sensores empleados para conocer la transferencia de deformaciones, y por otro, la interacción de los elementos sensores con el comportamiento de la estructura.

En este capítulo, Estado del Arte, se exponen los antecedentes y estado actual de conocimiento que motivan el desarrollo de la presente Tesis Doctoral, revisando las aportaciones de otros autores relativas a:

1) El diseño de sensores de fibra óptica para medir parámetros estructurales.

Son numerosas las investigaciones que hacen referencia a este aspecto, sin embargo, se ha evidenciado la falta de pautas de validación definidas con claridad sobre el diseño de nuevos sensores. Además, es necesario contar con pautas para el uso de los distintos tipos de sensores ópticos (puntuales, de longitud o distribuidos), dependiendo del material y de los parámetros a medir. En el caso que ocupa la presente Tesis Doctoral, el estudio se ha centrado en la medición de las deformaciones ya que para evaluar el estado de una estructura, la medida de la deformación es uno de los parámetros más relevantes. También se ha estudiado la medición de las temperaturas, ya que el sensado óptico exige una compensación térmica como se verá en el apartado correspondiente.

2) La aplicación de estos sensores para evaluar el comportamiento estructural.

Los sensores empleados en la monitorización de una estructura deben proporcionar una información relevante, completa y fiable sobre el comportamiento estructural. Es muy importante tener en cuenta algunos aspectos como la tipología de los sensores o sus longitudes dependiendo del tipo de material, así como una correcta disposición en la estructura para obtener, según se trate, un esfuerzo u otro (momentos flectores o torsores, cortantes o axiles).

Para ello, tal y como hace el capítulo, en primer lugar se describe las propiedades de la fibra óptica para la medida de parámetros estructurales, junto con las bases de diseño de un sensor de fibra óptica y los principales tipos de sensor. A continuación se recopila, sintetiza y comenta las principales investigaciones relativas al diseño de sensores de fibra óptica aplicados a la monitorización estructural (apartado 2.3). El siguiente apartado recopila y comenta las principales aportaciones relativas a la aplicación de sensores de fibra óptica para la evaluación de estructuras. En él se comentan tanto las pautas básicas de monitorización como las principales realizaciones contenidas en la literatura. 


\subsection{La fibra óptica como elemento sensitivo.}

\subsubsection{La fibra óptica.}

La fibra óptica es un medio de transmisión empleado habitualmente en redes de datos. Se trata de un hilo muy fino de material transparente, vidrio o materiales plásticos por el que se envían ondas o pulsos de luz que representan los datos a transmitir. La fibra es capaz de guiar una potencia óptica (lumínica) normalmente introducida por un láser o por un LED (Light-Emitting Diode).

La fibra óptica está constituida por tres capas (Figura 2.1): núcleo, cubierta y recubrimiento. El núcleo central está hecho de cuarzo puro al que se le añaden pequeñas cantidades de dopantes (óxido de silicio y germanio). El núcleo tiene un alto índice de refracción (cociente entre la velocidad de la luz en el vacío, $c$, y la velocidad de la luz en ese material, v) y un diámetro de $10 \mu \mathrm{m}$. En cambio, la cubierta (de un material similar) presenta un índice de refracción algo menor para satisfacer la Ley de Snell, limitando de este modo la propagación de la luz sólo a lo largo del núcleo. Tiene un diámetro de $125 \mu \mathrm{m}$. La última capa exterior de la fibra óptica, llamada recubrimiento (hecha de acrilato) da a la fibra resistencia mecánica y la protege frente a posibles daños y humedades. Presenta un diámetro de $250 \mu \mathrm{m}$.

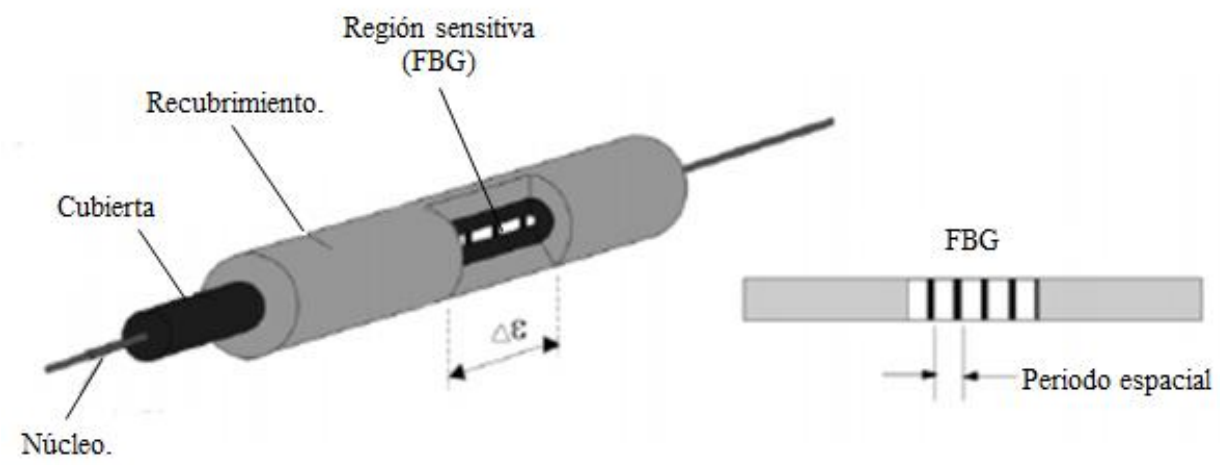

Figura 2.1 Vista de las capas de una fibra óptica (Kin-Tak et al. 2001)

La luz viaja a lo largo de la longitud de la fibra quedando atrapada en el interior del núcleo. Este modo de propagación de la luz cumple la Ley de Refracción de Snell a partir de:

- Las relaciones entre el ángulo de incidencia de la luz y el ángulo crítico (ángulo de incidencia para el que el ángulo de refracción alcanza $90^{\circ}$ ).

- Las relaciones entre los coeficientes de refracción del núcleo y cubierta (Figura 2.2).
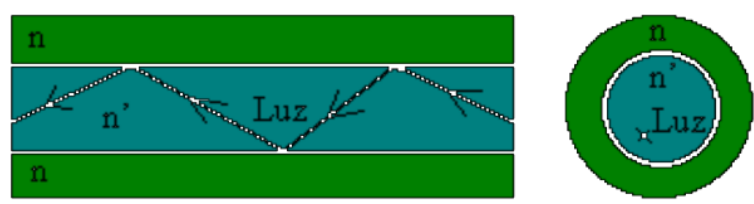

Figura 2.2 Modo de viaje de la luz a través de la fibra óptica (www.Wikipedia.es). 


\subsubsection{Tipos de sensores de fibra óptica.}

Los sensores de fibra óptica se pueden clasificar en función de varios criterios (Tabla 2.1). Sin embargo, todos ellos basan su funcionamiento en el estudio de las propiedades de la luz que viaja a su través.

\begin{tabular}{c|cc}
\hline \multicolumn{1}{c|}{ Criterio } & Tipología & Aplicación \\
\hline $\begin{array}{c}\text { Parámetro } \\
\text { a medir } \\
\text { intensidad }\end{array}$ & $\begin{array}{c}\text { Interferométricos } \\
\text { Deformación, temperatura, } \\
\text { humedad, radiación }\end{array}$ & $\begin{array}{c}\text { Deformación, temperatura, } \\
\text { rotaciones, vibraciones }\end{array}$ \\
& Espectométricos & Deformación y temperatura \\
& Polarimétricos & Uso casi nulo o muy limitado. \\
Aplicación & Distribuidos & Medida puntuales \\
& Multiplicados & Medidas distribuidas \\
\hline $\begin{array}{c}\text { Medio } \\
\text { sensitivo }\end{array}$ & Intrínsecos & Fibra medio sensitivo \\
& Extrínsecos & Fibra medio de transporte \\
\hline
\end{tabular}

Tabla 2.1 Cuadro resumen con los diferentes tipos de sensores.

Los distintos tipos de sensores ópticos se exponen a continuación clasificados según distintos criterios (Villalba Herrero, 2006):

a) El parámetro a medir:

Dependiendo de las propiedades de la luz que se desee modular, los sensores se pueden clasificar como (Ansari, 2007):

- Moduladores de intensidad. Es el tipo de sensor que mide cualquier parámetro que pueda producir pérdidas de la potencia óptica de la luz guiada. Los principales fenómenos que producen atenuación de la luz son microcurvaturas, temperatura, radiación ionizada... Utilizando este tipo de sensores se puede llegar a medir deformación, temperatura, curvatura o humedad.

- Sensores interferométricos. El cambio de fase es monitorizado mediante el uso de este tipo de sensores. Son los sensores ópticos más precisos y sensibles. Los principales parámetros que 
pueden medir estos sensores son deformaciones, temperaturas, vibraciones y rotaciones. Los sensores interferométricos más usuales son los llamados sensores Fabry-Perot.

- Sensores espectométricos. Este tipo de sensores controlan los cambios en la longitud de onda de la luz. Los sensores espectométricos más usuales son los Bragg-Grating. Su sensibilidad es menor que la de los sensores interferométricos pero su configuración, instalación y procesado de datos es mucho más simple.

- Sensores polarimétricos. Sensores basados en la polarización de la luz guiada. La sensibilidad de estos sensores es menor que la de los sensores interferométricos debido al ruido intrínseco del sistema, pero en la actualidad, gracias a los avances en el sector se está incrementando su sensibilidad. Son los que menor uso y sensibilidad presentan.

- Moduladores de la dispersión. Son los sensores que miden las variaciones de la dispersión de la luz provocadas por pequeñas imperfecciones en el material. En este grupo se engloban los sensores distribuidos basados en fenómenos de la dispersión de Rayleigh, Brillouin o Raman. $\mathrm{Su}$ uso es muy apropiado para grandes longitudes de monitorización donde la precisión no supone un factor importante. Son aptos para medir deformaciones y temperaturas. Ejemplos típicos de aplicaciones con este tipo de sensores son la monitorización de tuberías, cables de puentes colgantes, o vigas de gran luz.

\section{b) Su aplicación:}

- Localizados. Se trata de sensores preparados para realizar medidas puntuales. La figura 2.3 muestra una sección longitudinal de un elemento de hormigón sobre el que aparecen 3 fisuras y está instrumentado con tres sensores de deformación puntuales localizados. Mientras que los sensores instalados próximos a las fisuras miden poca deformación, el sensor instalado justo sobre la fisura central proporciona un valor de deformación elevado. Para la aplicación de estos sensores, hay que conocer previamente los puntos de estudio. Esto puede ser su inconveniente (Ansari 2007, Inaudi et al. 1996).
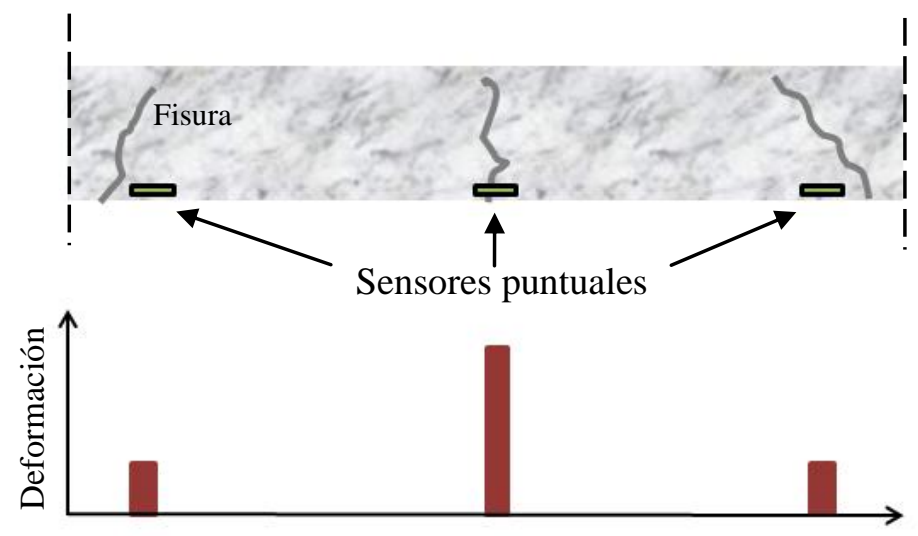

Longitud de la viga

Figura 2.3 Sensores localizados (Adaptado de Sunaryo et al. 2004). 
- Distribuidos. Se trata de sensores preparados para realizar una medida continua a lo largo de una fibra y por tanto, obtener una lectura continua de la medida. La principal ventaja de su aplicación es que con un solo cable de fibra óptica se puede medir en cualquier punto usando una única fuente de luz y un sistema de procesado. No obstante, la precisión y la sensibilidad son mucho menores que la de los localizados (Ansari 2007, Gu et al. 2000).

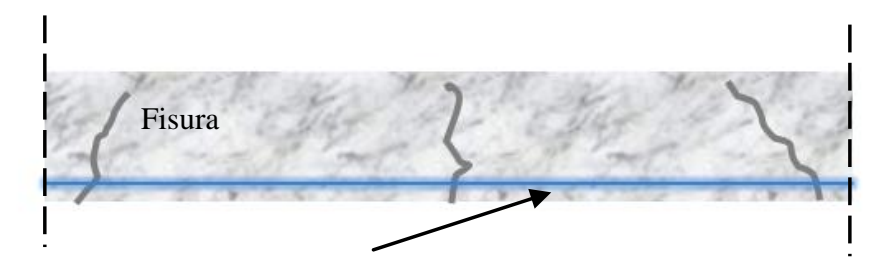

Sensor distribuido (BOTDR)

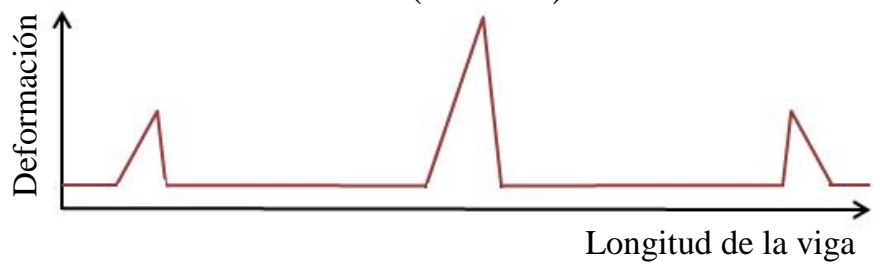

Figura 2.4 Sensor distribuido Brillouin Scattering (Adaptado de Sunaryo et al. 2004).

En la figura 2.4 se muestra la misma sección de viga de hormigón anterior instrumentada con un sensor distribuido continuo. El resultado es una ley de deformaciones continua. A partir del BOTDR (Brillouin Optical Time Domain Reflectomery) el sensor detecta la fisuración que se produce sobre el elemento estructural.

- Multiplicados o multiplexed. Su funcionamiento es parecido al de los sensores distribuidos. Normalmente se construyen mediante una combinación de varios sensores individuales (Ansari 2007, Davis et al. 1997, Maaskant et al. 1997). Los sensores ópticos multiplicados más empleados son los sensores Bragg-Grating empleados en gran parte de este trabajo.

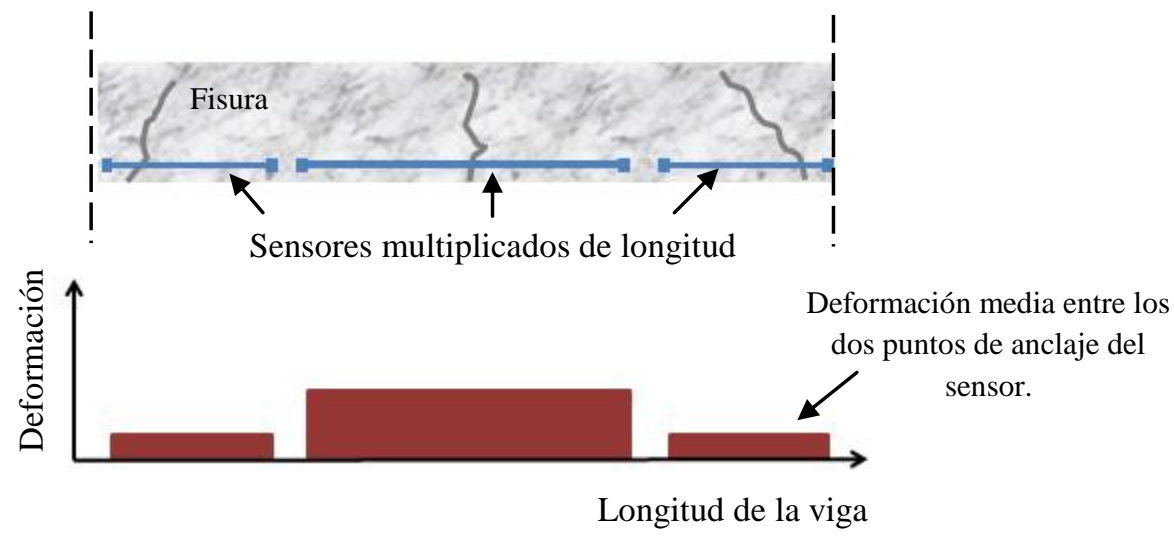

Figura 2.5 Sensores multiplicados (Adaptado de Sunaryo et al. 2004).

En la Figura 2.5 se muestra la misma viga, pero esta vez instrumentada con sensores multiplicados de longitud. En este caso, el sensor proporciona el valor medio de las deformaciones entre los puntos de anclaje del sensor. 
Monitorización estructural.

Aplicación con sensores de fibra óptica.

c) El medio sensitivo:

- Sensores intrínsecos. La medida se realiza sobre la fibra óptica, y las variaciones se producen sobre las propiedades de la fibra óptica. Por tanto, la fibra es empleada como medio de transporte de la luz y como medio sensitivo.

- Sensores extrínsecos. La fibra óptica es usada puramente como medio de transporte desde la fuente a la parte sensitiva. Por lo tanto la luz no sufre ningún cambio a lo largo de la fibra óptica.

En esta Tesis Doctoral se van a estudiar los sensores de fibra óptica llamados FBG (Fibber Bragg Gratting) y los sensores distribuidos llamados SBS (Stimulated Brillouin Scattering). Como se indicó en el apartado 2.1 el estudio se ha centrado en la medición de las deformaciones ya que, la medida de la deformación es uno de los parámetros más relevantes a la hora de evaluar el comportamiento estructural.

\subsubsection{Redes de Difracción de Bragg.}

Una red de difracción es un dispositivo óptico que se crea al alterar, de forma periódica o cuasiperiódica, el índice de refracción del núcleo de una fibra óptica. Estas perturbaciones del índice de refracción son producidas por la exposición de la fibra óptica a un patrón de interferencia óptica intenso. La luz que se propaga por el interior de la fibra óptica se encuentra con múltiples discontinuidades en el medio que provocan que parte de la luz incidente sea transmitida y otra parte sea reflejada debido a las sucesivas reflexiones (Figura 2.6).

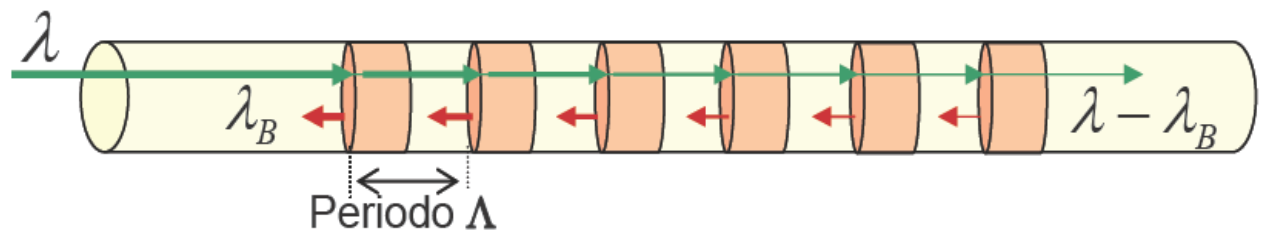

Figura 2.6 Esquema de una Red de difracción (Barrera, 2008). Se representa el núcleo de la fibra óptica y en distinto color las perturbaciones en su índice de refracción.

\subsubsection{Principio de funcionamiento de los sensores de fibra óptica tipo FBG.}

Una FBG (Fibre Bragg Gratting) es en un tipo de reflector constituido en un breve segmento de fibra óptica capaz de reflejar diferentes longitudes de onda de la luz (Alan et al. 1997). Esto se logra inscribiendo en su núcleo una red de difracción de Bragg (Figura 2.7). 

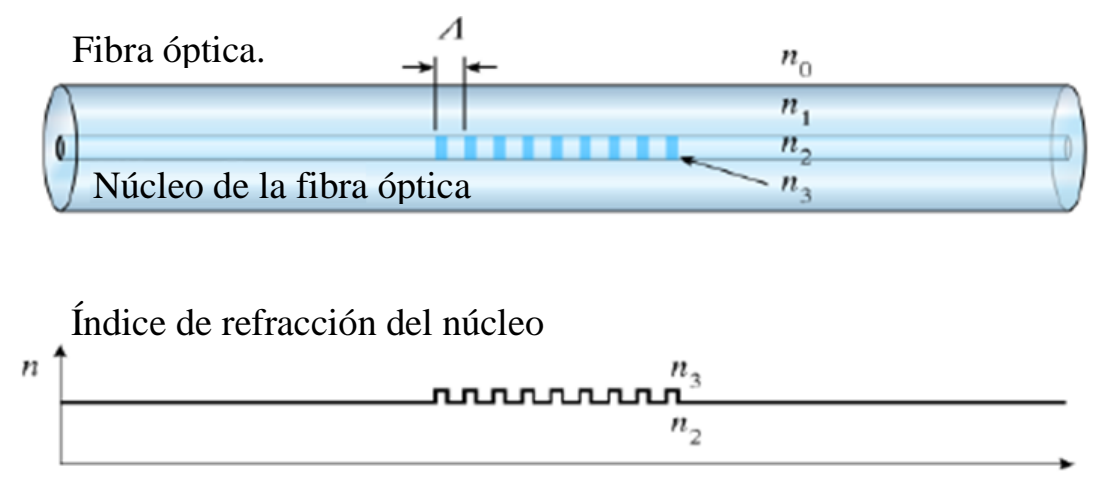

Respuesta espectral

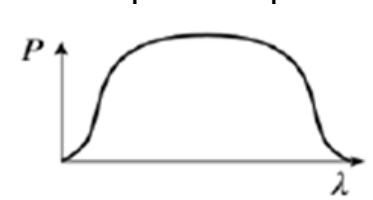

Entrante

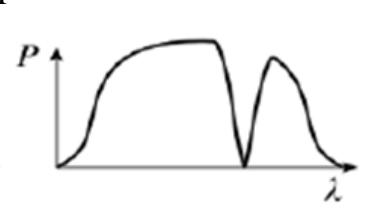

Transmitido

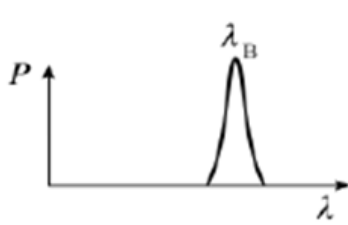

Reflejado

Figura 2.7 Principio de funcionamiento de los sensores ópticos basados en FBG (Kin-Tak et al. 2001)

Se puede demostrar (véase p. ej. Barrera 2008) que en una red de difracción de Bragg la longitud de onda del máximo de reflectividad, o mínimo de transmisividad (Longitud de onda de Bragg, $\lambda_{B}$ ), viene determinada por la ecuación 2.1:

$\lambda_{B}=2 n_{\text {eff }} \Lambda$

Donde $n_{\text {eff }}$ es el índice de refracción efectivo del núcleo y $\Lambda$ es el periodo espacial de las perturbaciones inducidas en el índice de refracción del núcleo de la fibra óptica.

La longitud de onda del máximo de reflectividad se puede desplazar debido a variaciones de temperatura o bajo la influencia de deformaciones aplicadas en la región donde se encuentra la FBG. Teniendo en cuenta únicamente los efectos termo-mecánicos, la variación de la longitud de onda $\left(\Delta \lambda_{B}\right)$ en función del incremento de la temperatura y de la deformación axial en una red de difracción de Bragg, se puede expresar mediante la ecuación 2.2:

$\Delta \lambda_{B}=K_{\varepsilon} \varepsilon+\lambda_{B} \xi_{0} \Delta T$

Donde $\varepsilon$ es la deformación unitaria en la región donde se encuentra la FBG, y $K_{\varepsilon}$ es un coeficiente de sensibilidad en deformación de la FBG que se obtiene de forma experimental. La segunda parte de la expresión determina la deriva del máximo de reflectividad debido al incremento de temperatura.

Incrementos y decrementos de la longitud de onda reflejada por la FBG indican que el periodo de la perturbación aumenta y disminuye respectivamente. Esto significa que el elemento instrumentado se tracciona en el primero de los casos, y se comprime en el segundo. (Figura 2.8). 


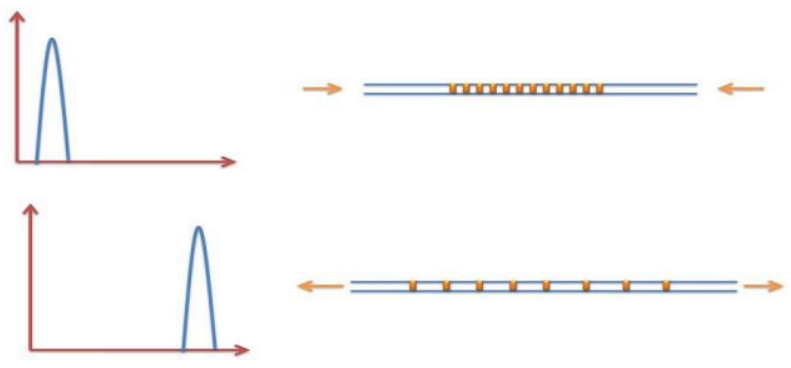

Figura 2.8 Variación en la longitud de onda reflejada por la Red de Bragg

\subsubsection{Compensación térmica de la fibra óptica.}

Tal y como se comentó en el apartado 2.1.3, la longitud de onda del máximo de reflectividad en una red de difracción se desplaza debido a variaciones de deformación y temperatura. Para separar estas respuestas es necesario realizar una compensación térmica.

Para ello, si se deriva la expresión 2.1en función de la deformación longitudinal $\varepsilon$ y la temperatura T, se obtienen las expresiones (2.3) y (2.4) que definen el comportamiento de las redes de difracción como sensores:

$$
\begin{aligned}
& \Delta \lambda_{B}=\frac{\partial \lambda_{B}}{\partial \varepsilon} \varepsilon+\frac{\partial \lambda_{B}}{\partial T} \Delta T \\
& \Delta \lambda_{B}=2\left[\left(\frac{\partial n_{\text {eff }}}{\partial \varepsilon} \Lambda+n_{\text {eff }} \frac{\partial \Lambda}{\partial \varepsilon}\right) \varepsilon+\left(\frac{\partial n_{\text {eff }}}{\partial T} \Lambda+n_{\text {eff }} \frac{\partial \Lambda}{\partial T}\right) \Delta T\right]
\end{aligned}
$$

Teniendo en cuenta las ecuaciones constitutivas de Hooke (ecuaciones 2.5, 2.6, 2.7 y 2.8):

$\Delta n=\frac{n^{3}}{2}\left[p_{12}-v\left(p_{11}-p_{12}\right)\right] \Delta \varepsilon$

$\varepsilon_{x x}=\frac{1}{E}\left(\sigma_{x x}-v\left(\sigma_{y y}+\sigma_{z z}\right)\right) \quad \varepsilon_{x y}=\frac{(1+v)}{E} \sigma_{x y}$

$\varepsilon_{y y}=\frac{1}{E}\left(\sigma_{y y}-v\left(\sigma_{x x}+\sigma_{z z}\right)\right) \quad \varepsilon_{y z}=\frac{(1+v)}{E} \sigma_{y z}$

$\varepsilon_{z z}=\frac{1}{E}\left(\sigma_{z z}-v\left(\sigma_{y y}+\sigma_{x x}\right)\right) \quad \varepsilon_{x z}=\frac{(1+v)}{E} \sigma_{x z}$

Se obtiene la ecuación (2.9):

$$
\Delta \lambda_{B}=\lambda_{B}\left\{\left[1-\left(\frac{n^{2}}{2}\right)\left[p_{12}-v\left(p_{11}+p_{12}\right)\right]\right] \Delta \varepsilon+\left[\left(\frac{n^{2}}{2}\right)\left(p_{11}+2 p_{12}\right) \alpha_{s}+\frac{1}{n}\left(\frac{\partial n}{\partial T}\right)\right] \Delta T\right\}
$$

Donde: 
- $p_{11}$ y $p_{12}$ son los coeficientes foto-elásticos, conocidos también como constantes de Pockels, que fueron determinados experimentalmente por Bertholds y Dandliker (1986). Valores habituales para estos coeficientes son $p_{11}=0.121 \mathrm{y} p_{12}=0.270$.

- $\alpha_{s}$ es el coeficiente de expansión térmica del vidrio de sílice que forma la fibra óptica. Para el caso de una fibra libre sin estar adherida a ningún material su valor es $\alpha_{s}=0.5510^{-6}{ }^{\circ} \mathrm{C}^{-1}$.

- $(\partial n / \partial T) / n$ representa la variación del índice de refracción efectivo debido a la variación de

temperatura. El valor de esta expresión no depende del material sobre el que se encuentre adherida la fibra óptica y tiene un valor aproximado de $6.810^{-6}{ }^{\circ} \mathrm{C}^{-1}$.

Observando la ecuación 2.9 se observa que un incremento de temperatura $\Delta T$ tiene una doble afección sobre la fibra óptica:

- La fibra óptica sufre un incremento de su longitud provocado por el coeficiente de dilatación térmica del vidrio de sílice $\alpha_{s}$.

- Se modifica el índice de refracción n de la fibra óptica debido al término $\frac{(\partial n / \partial T)}{n}$

- Teniendo en cuenta los valores adoptados anteriormente y si se comparan entre sí los sumandos que acompañan a $\Delta T$ en la ecuación 2.9 , se observa la importancia relativa de

$$
\frac{(\partial n / \partial T)}{n} \text { frente al término }\left(\frac{n^{2}}{2}\right)\left(p_{11}+2 p_{12}\right) \alpha_{s}\left(\left(\frac{(\partial n / \partial T)}{n} /\left(\frac{n^{2}}{2}\right)\left(p_{11}+2 p_{12}\right) \alpha_{s}\right)=21\right) \text {. }
$$

A su vez, Torres (2008) reescribió la ecuación (2.9) mediante el uso de dos coeficientes de sensibilidad (ecuación 2.10):

$\Delta \lambda_{B}=K_{\varepsilon} \Delta \varepsilon+K_{T} \Delta T$

A partir de la ecuación (2.10) anterior, Torres (2008) obtuvo experimentalmente los coeficientes de deformación y temperatura (Tabla 2.2), según el material sobre el que se encuentran adheridos los sensores. Esta distinción permite separar ambas respuestas (temperatura y deformación) en función del material que forme la estructura estudiada.

\begin{tabular}{cccccc}
\hline \multirow{2}{*}{$\begin{array}{c}\text { Sensibilidades sensores ópticos de } \\
\text { deformación y temperatura }\end{array}$} & \multicolumn{2}{c}{ Sensor de deformación } & \multicolumn{2}{c}{ Sensor de temperatura } \\
\cline { 2 - 5 } & Hormigón & Acero & Hormigón & Acero \\
\hline Coeficiente de deformación $(\mathrm{pm} / \mu \varepsilon)$ & 1,15 & 1,15 & 0 & 0 \\
Coeficiente de temperatura $\left(\mathrm{pm} /{ }^{\circ} \mathrm{C}\right)$ & 17.5 & 20.5 & $9-10$ & $9-10$ \\
\hline
\end{tabular}

Tabla 2.2 Sensibilidades obtenidas por Torres (2008). 
Monitorización estructural.

Aplicación con sensores de fibra óptica.

\subsubsection{Elementos que forman un sistema de monitorización óptico.}

Un sistema de monitorización óptico basado en FBG's está compuesto por los siguientes elementos (véase también la Figura 2.9):

- Una red o conjunto de sensores y sus conexiones.

Son los elementos encargados de medir los parámetros relevantes de la estructura monitorizada. Se instalan en los puntos de la estructura donde se desea conocer información.

- Un sistema de procesado de datos.

Es el sistema encargado de transmitir, adquirir y almacenar los mismos. Suele ser un PC.

- Una fuente óptica.

Es la encargada de transmitir las ondas de luz. Dependiendo de la fibra óptica utilizada, se empleará como fuente óptica de luz:

$\checkmark$ Láser: Fuente suministradora de ondas de banda estrecha (luz formada por sólo una longitud de onda específica -luz monocromática-), para fibras ópticas que sólo propagan un modo de onda (fibras tipo monomodo).

$\checkmark$ LED (Light-Emitting Diode): Fuente suministradora de ondas de banda ancha (Luz formada por diferentes longitudes de onda -Luz policromática o luz blanca-), para fibras ópticas tipo multimodo.

- Circulador.

El circulador permite el paso de la luz en un sentido, desde la fuente óptica hacia las estrellas pasivas. A su vez, impide que el espectro de luz reflejado llegue de nuevo a la fuente óptica y sea dirigido hacia el analizador de espectros ópticos (OSA, Optical Spectrum Analyzer).

- OSA (Optical Spectrum Analyzer).

Es el encargado de la obtención del espectro de luz y sus variaciones en el tiempo.

Actualmente y debido a los avances que en los últimos años ha gozado el campo del sensado óptico, la fuente óptica de luz, el circulador y el OSA se engloban en un único elemento que recibe el nombre de Interrogador (Figura 2.9). Este elemento es muy cómodo, fácil de transportar y de reducido tamaño. Además, es muy común el empleo de estrellas pasivas. Las estrellas pasivas son elementos que acoplan distintas FBG's. Estos elementos tienen un número determinado de canales de entrada y una única salida. Las estrellas pasivas se emplean en aquellas FBG's que no pueden conectarse en serie unas con otras. 


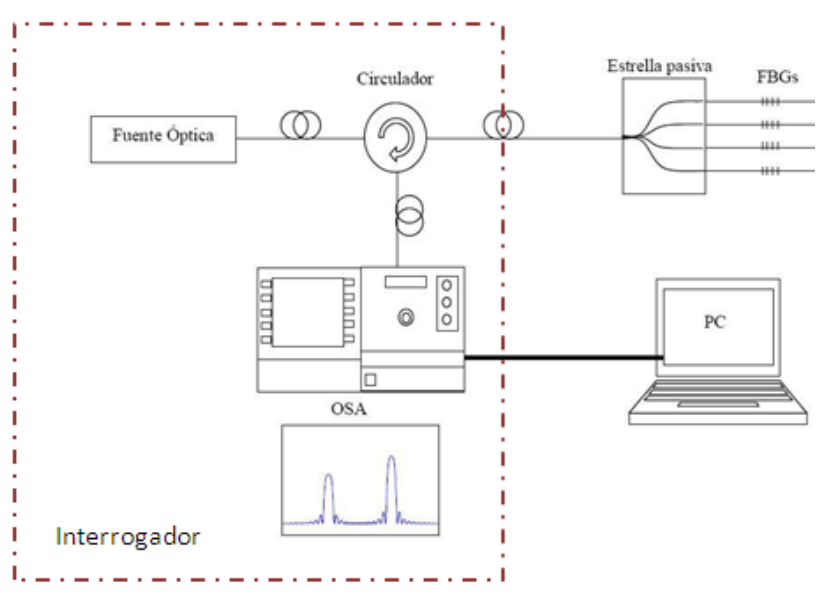

(a)

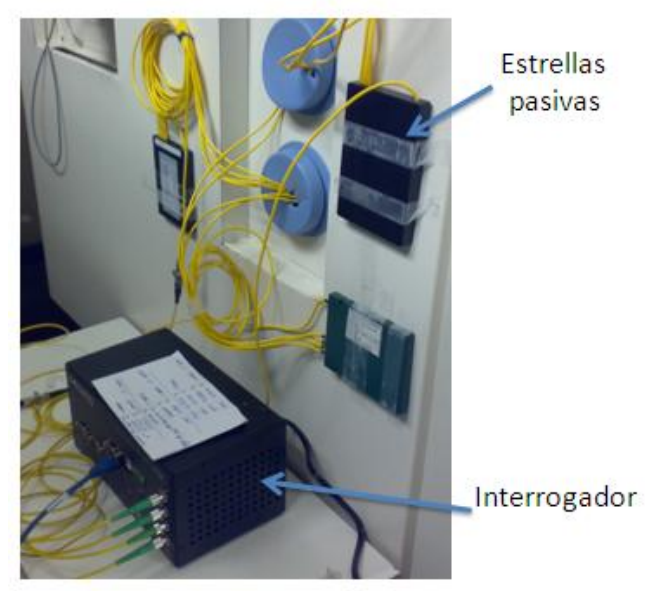

(b)

Figura 2.9 (a)- Elementos que forman un sistema de monitorización óptico basado en FBG (Barrera 2008). (b)- Montaje durante un ensayo. Estrellas pasivas e interrogador.

\subsubsection{Extensometría clásica versus Monitorización óptica. Ventajas e inconvenientes.}

Desde hace algunos años, la electrónica y la óptica han sido tecnologías que han competido en el campo de los elementos sensitivos. Obviamente, la historia industrial de los sensores eléctricos es bastante más extensa que la historia industrial de los sensores ópticos puesto que su tecnología y dispositivos se han desarrollado durante mucho más tiempo. Los precios son más asequibles y éste es uno de los motivos por los que su uso es más frecuente.

Sin embargo y en la actualidad, tanto las fibras ópticas como los láseres han alcanzado un gran desarrollo y compiten cada vez más con los sensores eléctricos al tener cada vez unos costes más reducidos. Esto, unido a sus ventajas comparativas, hace que su uso se esté extendiendo considerablemente.

Algunas ventajas importantes de los sensores ópticos frente a los sensores eléctricos se detallan a continuación:

a) El sensor óptico es un elemento pasivo. Esto significa que no requiere energía eléctrica en el punto a medir. Por este motivo, no se genera calor y por tanto, no existe riesgo eléctrico ni peligro de incendio.

b) El sensor óptico presenta una elevada sensibilidad. Es capaz de detectar variaciones muy pequeñas.

c) El sensor óptico se caracteriza por su pequeño tamaño y su flexibilidad, por lo que pueden ser instalados en superficies complejas.

d) Son completamente inmunes frente a las interferencias electromagnéticas y frecuencias de radio (EMI/RFI) puesto que la información no se transfiere como señal eléctrica.

e) Los sensores ópticos destacan por sus altos rangos de temperatura de trabajo. En caso de que el núcleo de la fibra óptica sea de zafiro, es posible alcanzar valores de $1500^{\circ} \mathrm{C}$. 
Monitorización estructural.

Aplicación con sensores de fibra óptica.

f) Los sensores ópticos presentan ventajas importantes trabajando en ambientes corrosivos, siendo totalmente fiables frente a estas patologías en las estructuras.

g) La multiplexación de los sensores permite instalar varios sensores en un único cableado de fibra óptica, por lo que con un único cable se pueden obtener datos de puntos separados largas distancias (Figura 2.10). Este es uno de los motivos por el que los sensores FBG se están convirtiendo en preferidos para muchas aplicaciones de ingeniería civil.

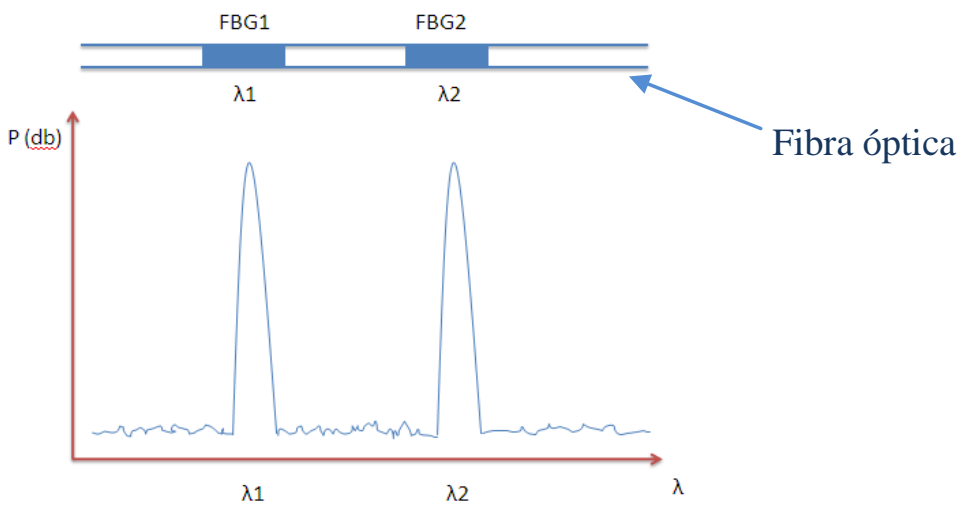

Figura 2.10 FBG's en serie, sobre un mismo cable de fibra óptica.

h) Presentan ciclos de vida largos, soportando cargas elevadas en tracción, con alargamientos de hasta un $5 \%$, es decir, $50.000 \mu \varepsilon$.

i) Las pérdidas de señal en el cable de fibra óptica son despreciables, hasta un límite de $5 \mathrm{~km}$ de longitud.

j) El sensor óptico presenta la capacidad de ser compensado térmicamente.

No obstante, existen algunos aspectos que deben tratarse con especial atención y precaución:

a) Se debe extremar la precaución con la suciedad. Es importante mantener las conexiones limpias, exentas de polvo. En caso contrario la detección del sensor resulta complicada (Figura 2.11).

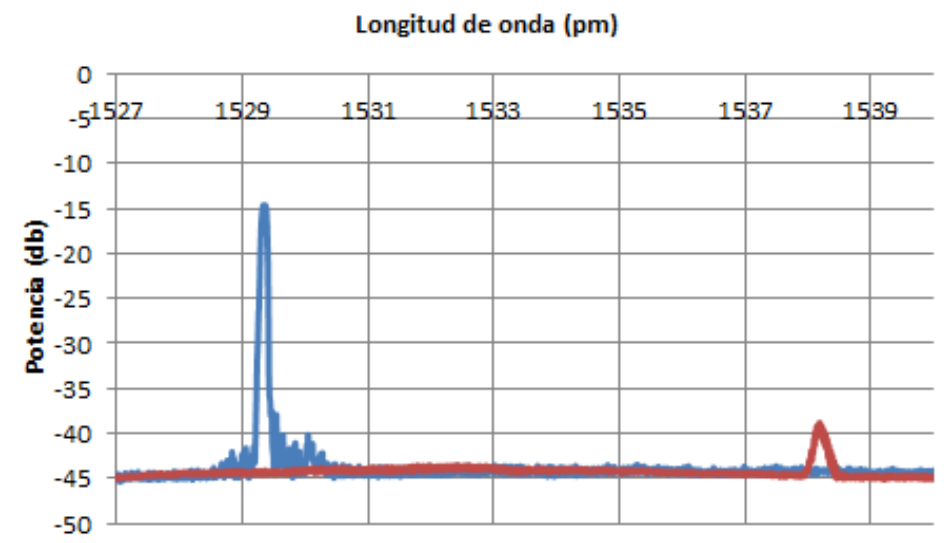

Figura 2.11 Pérdida de potencia reflejada en el sensor rojo debido a suciedad en las conexiones. El sensor azul muestra una conexión limpia. 
b) La fibra óptica es un elemento débil y frágil. El sector de la construcción representa un medio hostil donde abundan los tajos superpuestos, la maquinaria pesada y la suciedad. En este aspecto, es muy importante extremar las precauciones durante la instalación para evitar así roturas inesperadas.

c) Se debe extremar la precaución con los dobleces del cableado. Un doblez muy pronunciado impide la propagación de la luz y puede impedir la detección del sensor (Figura 2.12).

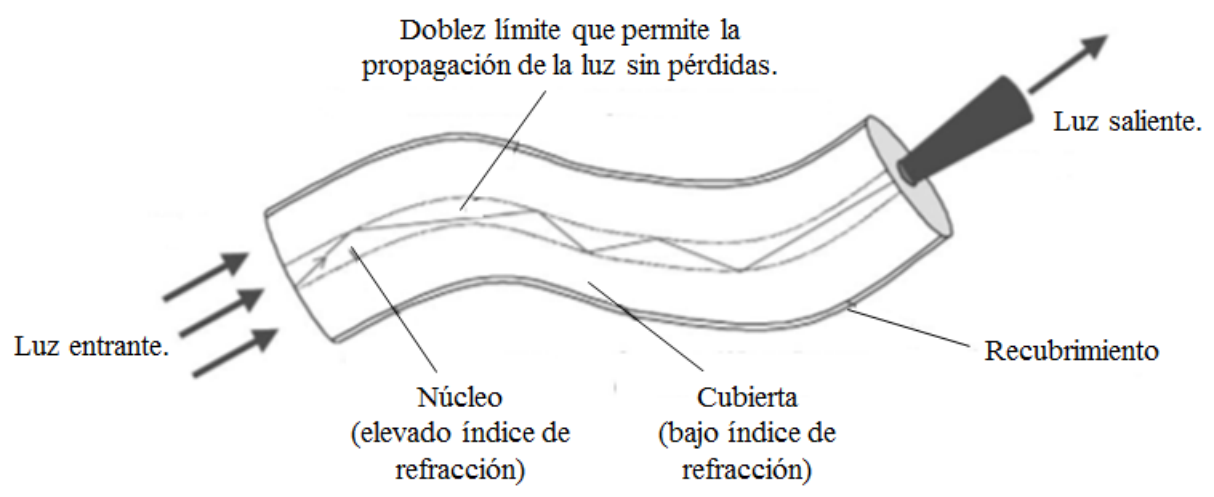

Figura 2.12 Doblez en el cableado de fibra óptica, y dificultad para la propagación de la luz (Gerard 2005).

\subsection{Estado del Arte sobre el diseño y estudio de sensores de fibra óptica.}

\subsubsection{Introducción.}

Cuando surge la necesidad de evaluar el comportamiento o estado de una estructura, uno de los parámetros más relevantes a monitorizar es la deformación del material. Existen distintas técnicas para su medición y en el Capítulo 1 se expusieron las más importantes para realizarlo. De todas ellas la más empleada en la actualidad son las galgas extensométricas.

Esta técnica se encuentra muy extendida en diversos países europeos, asiáticos y americanos como demuestra el que el 95\% de los 40 puentes de gran luz estudiados por Ko y Ni (2005) estaban siendo instrumentados con galgas.

La fibra óptica, a pesar de ser una técnica que aventaja en muchos aspectos a la extensometría clásica, no se encuentra tan extendida como la anterior. Como se apuntó anteriormente, la historia industrial de los sensores eléctricos es bastante más extensa que la historia industrial de los sensores ópticos y este es el motivo principal de esta diferencia. Por este motivo no se ha estudiado en profundidad el comportamiento y diseño de sensores ópticos y no son muy abundantes los trabajos relacionados con este aspecto.

En este apartado se enumerarán los principales trabajos sobre el estudio del comportamiento y diseño de sensores ópticos, y se detallarán aquellos más relevantes. 


\subsubsection{Estadísticas relativas al uso de distintos tipos de sensores de fibra óptica.}

Distintos investigadores han recopilado datos relativos al estudio del comportamiento y diseño de sensores ópticos. Estos datos reflejan el crecimiento en la importancia de los métodos ópticos aplicados a la instrumentación estructural.

a) Lee (2003).

De acuerdo con los resultados publicados por Lee (2003), el tipo de sensor de fibra óptica que más estudios y aplicaciones recoge es el sensor óptico FBG (44.2\%), seguido por los sensores interferométricos $(11.1 \%)$ y los distribuidos basados en fenómenos no lineales como el Scattering $(10 \%)$.

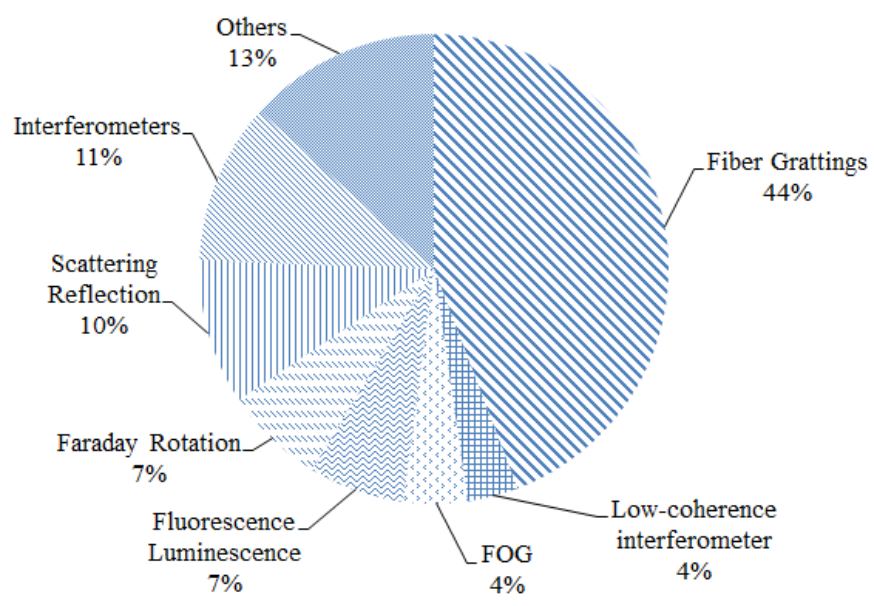

Figura 2.13 Distribución de los estudios de diferentes tipos de sensores ópticos. Adaptado de Lee (2003).

Lee (2003) señala en su estudio que a pesar de no ser los sensores que mayor precisión presentan, los FBG sí son los que mayores prestaciones ofrecen, dado que su principio de funcionamiento, instalación y procesado de datos es mucho más simple.

A su vez, en este mismo estudio se señalan los parámetros más estudiados empleando sensores ópticos FBG: deformaciones (23\%), temperatura (17\%) y, por último, presiones (15.2\%). El resto de aplicaciones responden a otros parámetros no estructurales, tales como pérdida de potencia o atenuaciones.

b) Hong-Nan et al. (2004).

Hong-Nan et al. (2004) publicaron un artículo de revisión sobre construcciones monitorizadas. En su trabajo, Hong-Nan Li et al. instrumentaron estructuras muy variadas como edificios, cimentaciones de pilotes, puentes, tuberías, túneles o presas. En todas ellas destacaron, en general, un buen comportamiento de los sensores de fibra óptica. Sin embargo, también destacaron algunos aspectos a tener en cuenta:

- "Strain and temperature discrimination". Hong-Nan et al. (2004) observaron que la temperatura juega un papel fundamental en la medición con fibra óptica. Para compensar estos efectos, proponen la colocación de una fibra óptica aislada de la deformación de la estructura, que recoja únicamente los efectos térmicos. No obstante, la investigación acerca de la 
compensación de temperatura es una línea que se encuentra muy activa actualmente y abierta a múltiples discusiones.

- "Ingrees and egrees problems". Hong-Nan et al. (2004) observaron que en ocasiones los puntos de entrada y salida del cableado a la estructura eran puntos críticos, puesto que se producía la rotura del cable. Por ese motivo, proponen una protección adicional en estos puntos que evite no sólo la rotura, sino suavizar el trazado del cable sin transiciones bruscas que suponen una pérdida de la señal del sensor.

- "FOS's packaging". Este trabajo señala el encapsulado del sensor como un área muy importante de estudio. Los autores afirman que un correcto diseño del sensor debe englobar un encapsulado que permita, por un lado una correcta transferencia de deformaciones entre fibra óptica y estructura, y por otro, una correcta detección de la señal del sensor.

El siguiente apartado expondrá los trabajos más relevantes al respecto y que recogen algunos aspectos aquí mencionados. La mayoría de ellos se centran en campañas experimentales sobre elementos de hormigón para verificar el comportamiento de los sensores ópticos propuestos.

Por el contrario, son escasos los estudios numéricos que reproduzcan el comportamiento del sensor y que permitan conocer la afección que distintos parámetros que lo definen tienen en su respuesta.

\subsubsection{Principales trabajos.}

La Tabla 2.3 resume las investigaciones más relevantes orientadas al estudio del diseño y del comportamiento de sensores de fibra óptica. Los trabajos más importantes o novedosos se detallan en profundidad en apartados posteriores.

\begin{tabular}{|c|c|}
\hline Autor (año) & Estudios sobre sensores ópticos. \\
\hline Fei et al. (1999) & Sensor óptico FBG basado en curvaturas (microbend sensor) \\
\hline Jin et al. (2000) & Sensor óptico FBG para medición de vibraciones. \\
\hline Yuan et al. (2001) & $\begin{array}{l}\text { Sensor interferométrico embebido en hormigón. Campañas } \\
\text { experimentales. }\end{array}$ \\
\hline Quintela et al. (2002) & $\begin{array}{c}\text { Sensor FBG autocompensado en temperatura. Campañas } \\
\text { experimentales. }\end{array}$ \\
\hline Qingbin et al. (2003) & Afección recubrimiento de plástico en la medición de fibra óptica. \\
\hline Hong-Nan et al. (2004) & Estudio y comparativa de FOSs: FBG, BOTDR y LG. \\
\hline López et al. (2005) & Fabricación sensor FBG y campañas experimentales. \\
\hline Moyo et al. (2005) & $\begin{array}{l}\text { Desarrollo sensor FBG para aplicación en ingeniería civil. } \\
\text { Campañas experimentales. }\end{array}$ \\
\hline Yeo et al.(2006) & Sensor de humedad y absorción mediante FBG. \\
\hline $\begin{array}{l}\text { Dongsheng et al. } \\
\qquad(2006)\end{array}$ & Influencia del recubrimiento de la fibra óptica en las mediciones. \\
\hline Kister et al. (2007a) & $\begin{array}{l}\text { Evaluación de adhesivos y protecciones empleados en FOS's. } \\
\text { Campañas experimentales. }\end{array}$ \\
\hline Dawood et al. (2007) & Comportamiento de la fibra óptica embebida en GFRP \\
\hline Fresvig et al.(2008) & Comparativa sensores ópticos y galgas extensométricas. \\
\hline
\end{tabular}


Monitorización estructural.

Aplicación con sensores de fibra óptica.

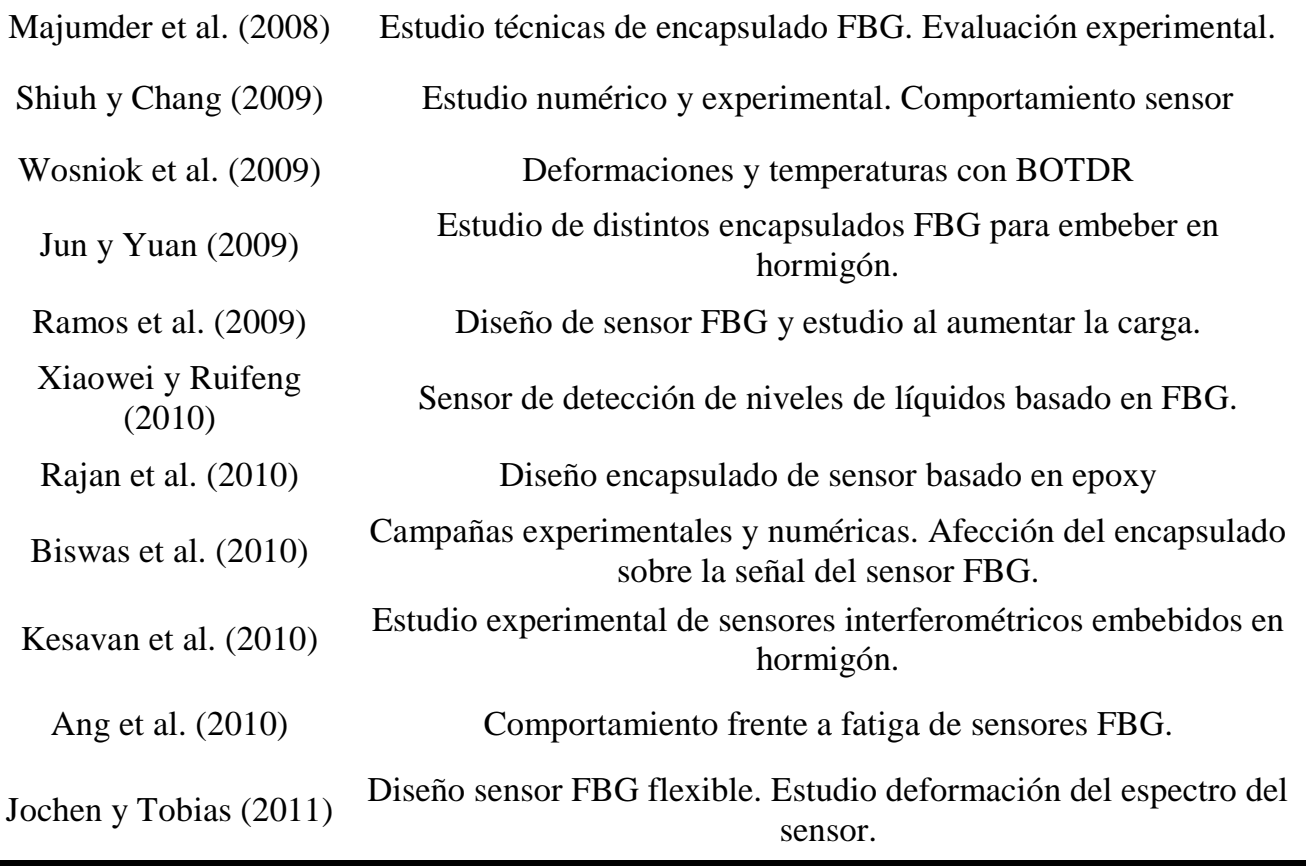

Tabla 2.3 Recopilación de investigaciones sobre el estudio de sensores ópticos. (FBG-

Fiber Bragg Gratting; BOTDR-Brillouin Optical Time Domain Refelctomery. LG- Long Gauge).

Como se observa en la tabla anterior, existen multitud de estudios experimentales que tienden a calibrar los sensores ópticos y a comprobar su funcionamiento a partir de comparaciones con otros sistemas de medida de referencia. Sin embargo, son escasos los modelos numéricos que reproducen el comportamiento de un sensor y que permiten su estudio avanzado y la optimización de su diseño.

Los principales trabajos pueden clasificarse en tres grupos que se describen en los apartados a) b) y c).

a) Encapsulado y calibración de sensores ópticos.

El estudio del encapsulado del sensor ha sido objeto de múltiples investigaciones por parte de la comunidad científica como se observa en la Tabla 2.3. Los principales aspectos a investigar han sido el diseño del sensor y su calibración frente a temperaturas y deformaciones impuestas. Estos estudios se han basado fundamentalmente en campañas experimentales para validar el funcionamiento de los sensores.

- Fei Luo et al. (1999).

El sensor óptico FBG pertenece a la tipología de los sensores denominados espectométricos) y este nombre se debe a que proporcionan la información a partir de variaciones en el espectro de luz reflejado.

Sin embargo y en función de su modo de encapsulado, los sensores FBG pueden funcionar en base a otro parámetro. Son los llamados moduladores de intensidad, y el parámetro objeto de estudio (Deformación, temperatura...) se obtiene a partir de variaciones en la potencia óptica de luz reflejada por el sensor. 
En esta línea trabajó Fei Luo et al. (1999) proponiendo un sensor óptico puntual de deformación, la cual era medida a partir de variaciones en la potencia reflejada por el sensor. El diseño propuesto por Fei Luo et al. (1999) se muestra en la Figura 2.14.a:

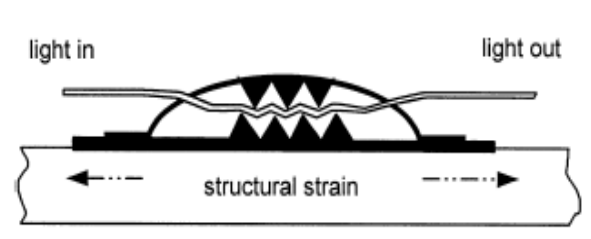

(a)

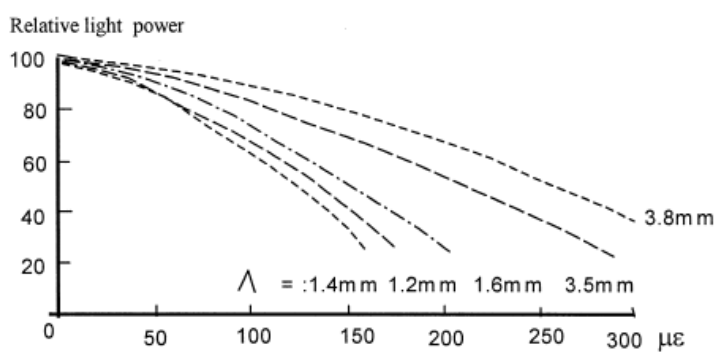

(b)

Figura 2.14 (a)- Diseño del sensor propuesto por Fei Luo et al. (1999). Calibración entre la pérdida de potencia óptica reflejada y la deformación impuesta.

El diseño está formado por un encapsulado inferior plano y otro superior curvo. En su interior se coloca la fibra óptica entre unas mordazas. Cuando la superficie a monitorizar se deforma las mordazas infieren una serie de microcurvaturas a lo largo de la fibra óptica, produciendo una pérdida de potencia de la luz que pasa a su través (Figura 2.14.b). Fei Luo et al. (1999) calibró la pérdida de potencia con la deformación que sufría la estructura y para distintas longitudes de onda. Sin embargo, únicamente planteó ensayos de carga sobre elementos sometidos a tracción. En caso de esfuerzos de compresión, Fei Luo et al. (1999) propone dotar a la fibra de una deformación previa a partir de las mordazas indicadas en la Figura 2.14.a.

- Moyo P. et al. (2005).

Moyo et al. (2005) estudiaron el encapsulado de un sensor óptico tipo FBG y su aplicación para la medida de deformaciones bajo cargas estáticas y dinámicas. El estudio se basó, una vez propuesto el diseño del sensor, en su calibración y posterior campañas experimentales para validar su funcionamiento.

En cuanto al diseño, Moyo et al. (2005) proponen el sensor de la Figura 2.15.a, donde la fibra óptica queda embebida entre dos capas de material que forma el encapsulado.

Moyo et al. (2005) estudiaron la calibración de los sensores frente a deformaciones y variaciones de temperatura impuestas. Las sensibilidades obtenidas en los ensayos se muestran en la Tabla 2.4. Sin embargo, Moyo et al. (2005) no distinguieron entre materiales sobre los que puede estar adherido el sensor, obteniendo un único coeficiente de sensibilidad a la temperatura del sensor de deformación.

\begin{tabular}{ccc}
\hline Sensibilidades & Deformación impuesta $(\mathbf{p m} / \boldsymbol{\mu \varepsilon})$ & Temperatura impuesta $\left(\mathbf{p m} /{ }^{\circ} \mathrm{C}\right)$ \\
\hline Sensor de deformación & 1.06 & 13.74 \\
Sensor de temperatura & - & 11.67 \\
\hline
\end{tabular}

Tabla 2.4 Sensibilidades de los sensores ópticos obtenidos por Moyo et al. (2005). 
Las campañas experimentales englobaron ensayos de tracción sobre probetas metálicas, ensayos estáticos y dinámicos sobre vigas rectas de hormigón armado de sección rectangular, y ensayos térmicos durante el proceso de curado del hormigón. Los resultados obtenidos con los sensores ópticos fueron comparados con los resultados obtenidos a partir de galgas extensométricas (Figura 2.15.b) y termopares, arrojando resultados satisfactorios.

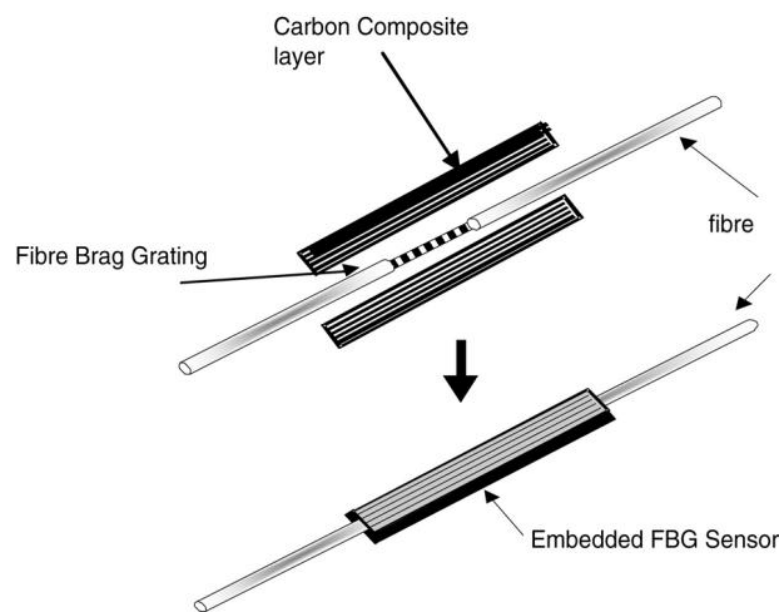

(a)

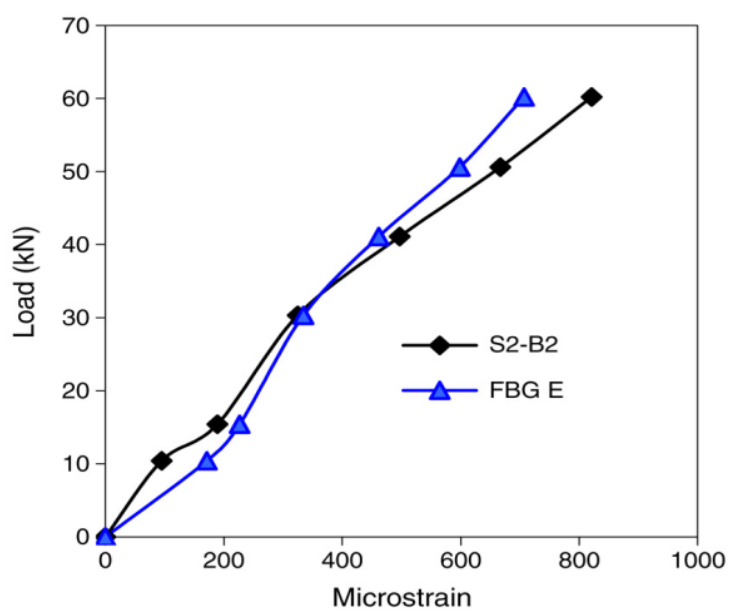

(b)

Figura 2.15 (a)-Diseño propuesto por Moyo et al. (2005) para un sensor óptico FBG. (b)-Comparación de los resultados del sensor óptico FBG con las galgas extensométricas en un ensayo de flexión sobre vigas de hormigón.

Tras finalizar su trabajo, Moyo et al. (2005) concluyeron que los sensores de fibra óptica FBG ofrecen una gran fiabilidad para su empleo en la monitorización de estructuras. Sin embargo, Moyo et al. (2005) señalan que es necesaria y muy importante una adecuada protección de los mismos.

- Jun y Libo (2009).

Jun y Libo (2009) estudiaron un diseño de un sensor óptico basado en interferometría para su instalación en el interior del hormigón. En su trabajo, Jun y Libo (2009) estudiaron distintas tipologías de encapsulados, tanto en forma como en rugosidades para mejorar la adherencia con el hormigón y por tanto su transferencia de deformaciones (Figura 2.16.a).

Jun y Libo (2009) resaltan la importancia de una cuidadosa y protectora instalación del sensor óptico en el interior del hormigón, haciendo especial hincapié en cuanto a los dobleces con el cableado de fibra óptica por posibles pérdidas de potencia lumínica, proponiendo radios de curvatura no inferiores a $3 \mathrm{~cm}$. Además, Jun y Libo (2009) proponen un procedimiento para la instalación de los sensores ópticos en el interior del hormigón. Este procedimiento está basado en garantizar la supervivencia del sensor durante el hormigonado del elemento y propone emplear un elemento de conexión cerámico a la salida del cableado de la estructura para evitar su doblez pronunciada.

De una forma similar a Moyo P. et al. (2005), Jun y Libo (2009) realizan una calibración del sensor óptico propuesto para distintas longitudes. Los resultados obtenidos por Jun y Libo (2009) muestran que para sensores de mayor longitud se consigue una mejor transferencia de deformaciones en un mismo tipo de encapsulado. La Figura 2.16.b indica por ejemplo que, para sensores de $30 \mathrm{~mm}$ de longitud cuando la deformación de la estructura es aproximadamente $8000 \mu \varepsilon$ el sensor indica una deformación en torno a $5000 \mu \varepsilon$. 
La máxima longitud del sensor estudiada es de $180 \mathrm{~mm}$. En este estudio no se ofrecieron datos acerca de la colocación de la fibra óptica en el interior del encapsulado metálico.

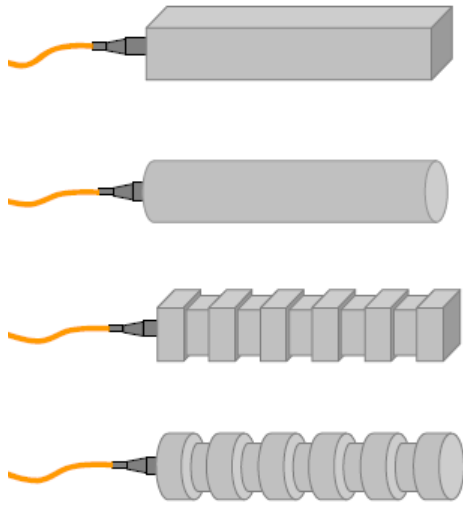

(a)

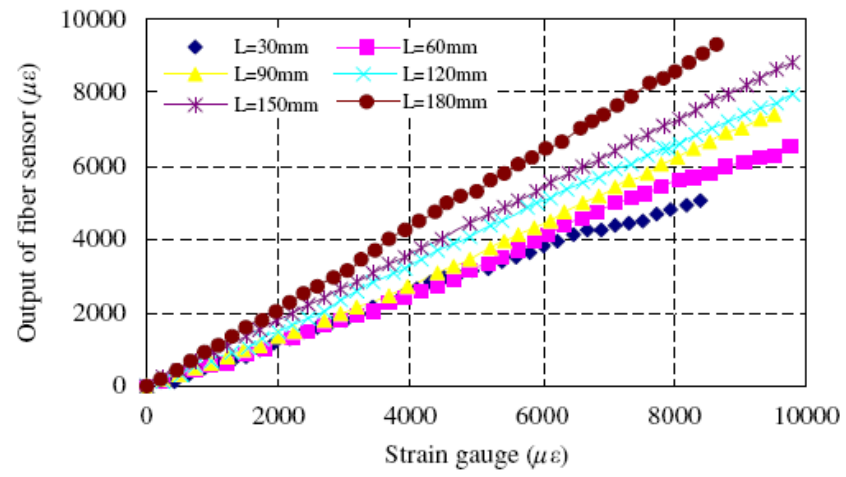

(b)

Figura 2.16 (a)- Distintas geometrías y rugosidades propuestos para el encapsulado metálico del sensor óptico. (b)- Calibraciones del sensor propuesto en función de distintas longitudes de sensor.

- Kesavan et al. (2010).

De una manera similar, Kesavan et al. (2010) realizó un estudio experimental de sensores ópticos embebidos en el hormigón. En su trabajo, Kesavan et al. (2010) afirmó la necesidad de desarrollar un encapsulado del sensor para la protección de la fibra óptica en el interior del hormigón, debido a la fragilidad de la misma. Para ello, Kesavan et al. (2010) propuso dos tipos distintos de encapsulados. El primero de ellos consiste en embeber la fibra sobre dos capas de material compuesto (Figura 2.17.a). El segundo de ellos consiste en un encapsulado metálico en forma de "hueso de perro". En ambos casos, Kesavan et al. (2010) resalta la importancia de una total transferencia de deformaciones entre el hormigón y la fibra óptica.

En este estudio se realizaron campañas experimentales sobre probetas cilíndricas sometidas a compresión simple y sobre vigas de hormigón sometidas a flexión y cargas cíclicas. En los ensayos a compresión simple Kesavan et al. (2010) estudió la transferencia de deformaciones entre hormigón y fibra óptica, mientras que en los ensayos sobre vigas de hormigón, estudió el comportamiento de los sensores ópticos bajo cargas de fatiga. En ambos ensayos, los resultados obtenidos con los sensores ópticos son comparados con los valores de 4 galgas extensométricas dispuestas en la misma sección que el sensor óptico (Figura 2.17.b).

Entre las conclusiones más importantes de este estudio, Kesavan et al. (2010) observó un mejor funcionamiento del encapsulado de material compuesto que en el encapsulado metálico. Sobre el encapsulado formado por material compuesto observó mayores diferencias en las medidas a mayores cargas, llegando a obtener diferencias del orden de un 6\%. En cuanto a las cargas de fatiga, Kesavan et al. (2010) observó un buen comportamiento de los sensores ópticos propuestos, coincidiendo las medidas entre los sensores ópticos y las galgas extensométricas con un buen grado de ajuste. 


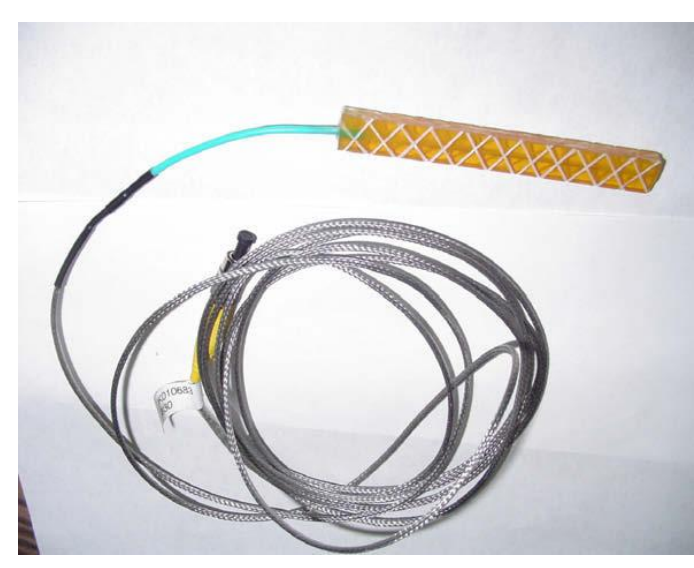

(a)

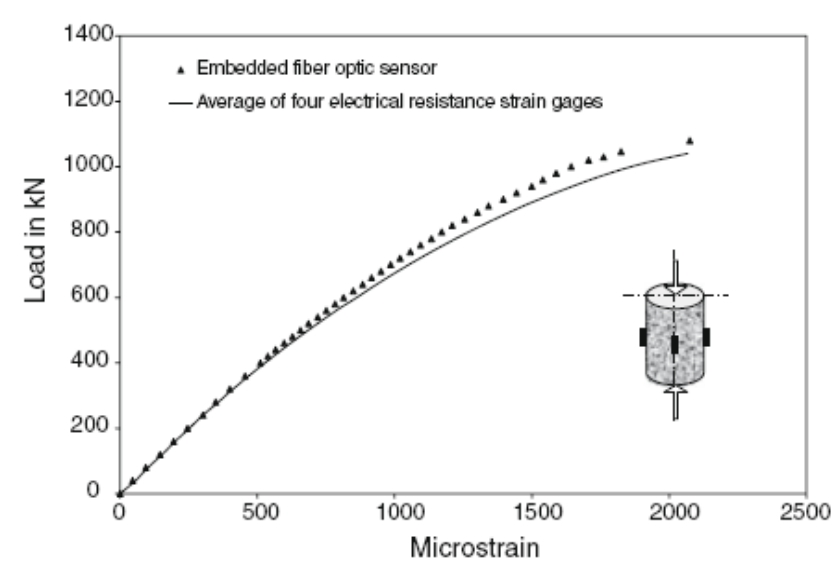

(b)

Figura 2.17 (a). Imagen del sensor óptico compuesto con epoxi propuesto por Kesavan et al. (2010). (b) Comparación entre los resultados de los sensores ópticos de deformación y galgas eléctricas en los ensayos de compresión sobre probetas cilíndricas.

\section{- Calderón y Glisic (2012).}

Como se ha explicado en apartados anteriores, la fibra óptica encargada de medir debe ir encapsulada en un material huésped y en ocasiones embebidos en la estructura. Este hecho puede causar una perturbación en el campo de deformaciones que afecta a la exactitud de la medición.

En este aspecto, Calderón y Glisic (2012) llevaron a cabo un estudio donde identificaron los parámetros críticos que afectan a la medición de la deformación empleando sensores ópticos de longitud, para conocer su afección sobre la exactitud de la medida y dar recomendaciones acerca de su uso. Este estudio fue llevado a cabo a través de modelos numéricos de elementos finitos y su calibración re realizó a partir de resultados experimentales previamente realizados.

Calderón y Glisic 2012 observaron que existen una serie de parámetros cuya afección sobre las mediciones son muy importantes, como son la transferencia de deformaciones desde los anclajes del sensor con la estructura, las dimensiones de estos anclajes, la longitud del sensor y el ratio entre las propiedades mecánicas del sensor y del material de la estructura.

De estos parámetros, Calderón y Glisic (2012) observaron que la rugosidad de las cabezas de anclaje es la que más importancia presenta en la exactitud de los resultados, siendo muy importante la rugosidad de estos elementos y una buena interacción con el material de la estructura. Otro factor clave es el ratio entre el Módulo de Young del material que forma el sensor y el Módulo de Young del material de la estructura. A ratios más pequeños, los resultados obtenidos serán más precisos (Figura 2.18). En el caso de que este ratio sea elevado, sería necesario el empleo de cabezas de anclajes del sensor de mayores dimensiones. 


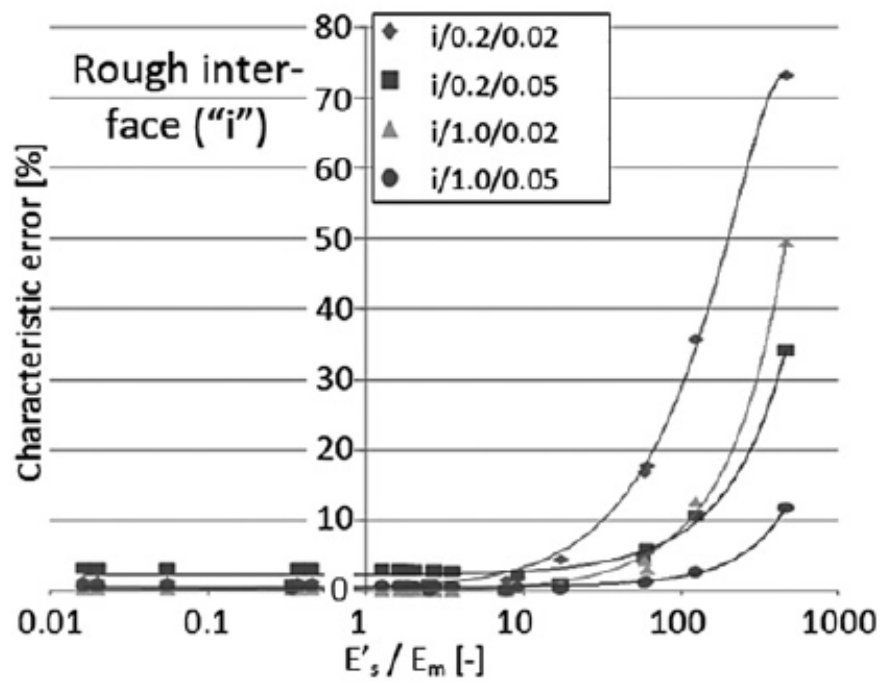

Figura 2.18 Relación entre el error característico del sensor frente al ratio entre los Módulos de elasticidad del material que forma el sensor y el material que forma la estructura (Calderón y Glisic, 2012).

b) Señal del sensor de fibra óptica.

El diseño de un sensor óptico FBG debe de satisfacer unas condiciones que garantice:

1) Una correcta transferencia de deformaciones entre sensor y estructura.

2) Aportar a la fibra resistencia frente agresiones medioambientales y mecánicas.

3) Garantizar una correcta señal del sensor que permita su detección.

En los sensores ópticos FBG, esta señal es el espectro de luz reflejado por la red de difracción, y éste debe cumplir unas condiciones:

1) La forma del espectro debe ser en campana de Gauss.

2) Debe detectarse una sola longitud de onda reflejada (longitud de onda del máximo de reflectividad).

3) El espectro debe tener potencia lumínica $(\mathrm{db})$ suficiente para ser detectado.

Una señal que no cumpla algunas de las condiciones anteriores podría aportar resultados incorrectos que se traducirían en evaluaciones estructurales incorrectas y potencialmente del lado de la inseguridad.

- Ramos et al. (2009).

Ramos et al. (2009) estudiaron dos diseños de sensores ópticos basados en FBG formados por fibra de vidrio y fibra de carbono. En su trabajo centró especial atención al proceso de encapsulado para controlar las alteraciones de la señal de luz reflejada por el sensor. Posteriormente comparó el comportamiento de los mismos con galgas extensométricas realizando ensayos de carga, al mismo tiempo que estudió cómo afectaba la carga aplicada sobre la evolución del espectro de luz reflejado por el sensor (Figura 2.19). 
Monitorización estructural.

Aplicación con sensores de fibra óptica.

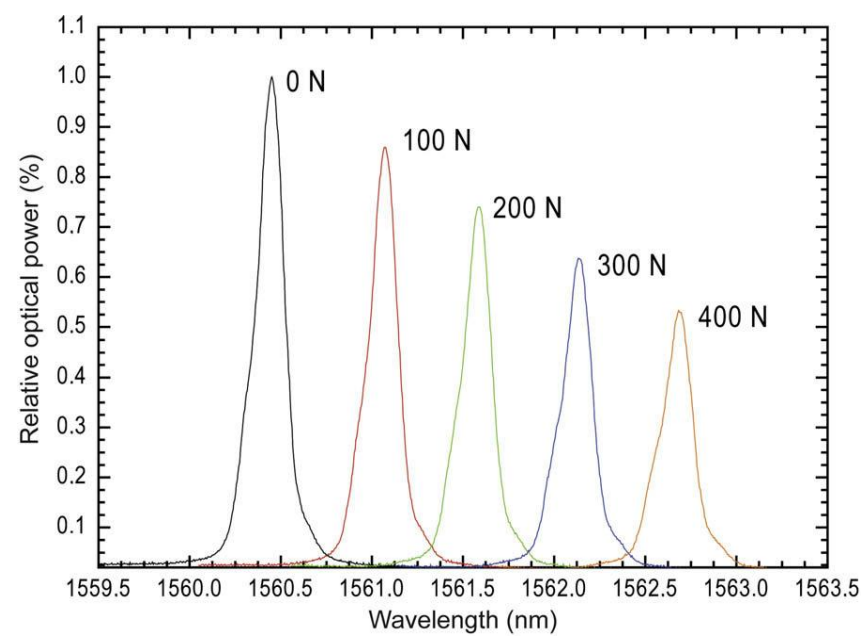

Figura 2.19. Espectro reflejado en función de la carga aplicada. Ramos et al. (2009)

Entre las conclusiones más importantes del estudio, Ramos et al. (2009) destacaron que la señal del sensor FBG perdía intensidad a medida que aumentaba la carga del ensayo, aspecto que no debe permitirse en el diseño de un sensor óptico.

Por otro lado, observó que el sensor encapsulado con fibra de carbono indicaba unas deformaciones inferiores a las obtenidas con galgas extensométricas. Esta diferencia fue justificada por la gran rigidez del encapsulado propuesto.

\section{- Biswas et al. (2010).}

Una de las causas que puede alterar la señal del sensor es el proceso de encapsulado, por lo que éste ha sido un aspecto estudiado por varios investigadores entre los que destaca el trabajo de Biswas et al. (2010). Este equipo estudió numérica y experimentalmente el efecto que sobre la señal del sensor provoca una distribución de deformaciones no uniforme, debido a imperfecciones durante el curado del material que forma el encapsulado del sensor óptico.

Biswas et al. (2010) observaron que para un proceso de curado normal, la modificación de la señal de la FBG es repetitiva y sin distorsión en su forma, pero en ocasiones el espectro de luz reflejado experimenta unas modificaciones que alteran su forma en campana de Gaus. Estas modificaciones son debidas a distribuciones de deformaciones no uniformes sobre la zona donde se encuentra escrita la FBG.

Fundamentalmente, el espectro sufre dos tipos de distorsiones en su forma. La primera de ellas (Figura 2.20.a), cuando surgen dos picos principales y el espectro queda partido en dos, debido a una distribución sinusoidal de deformaciones sobre la fibra óptica. En la segunda de ellas (figura 2.20.b), se producen muchos picos secundarios "en cascada", debido a una distribución parabólica de segundo orden de deformaciones sobre la fibra óptica. 


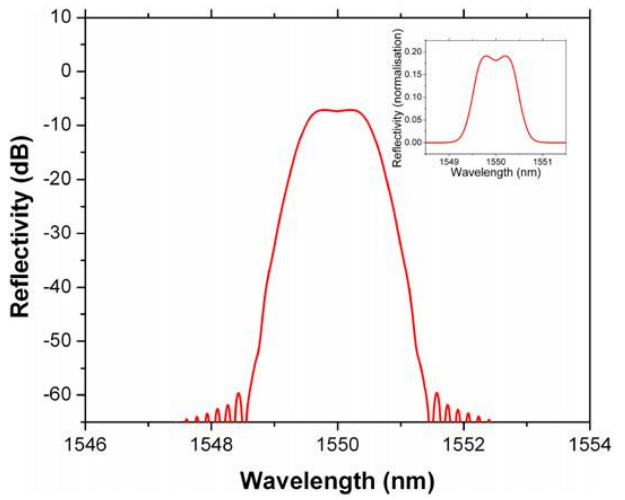

(a)

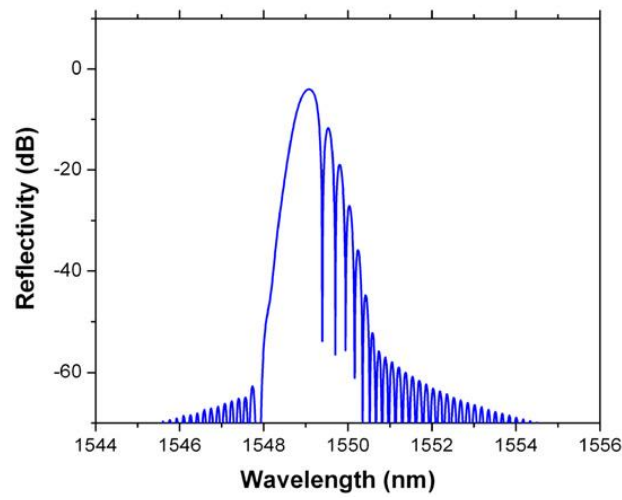

(b)

Figura 2.20 (a)- distorsión en la forma del espectro de doble pico. (b)- Distorsión en la forma del espectro con picos "en cascada" (Biswas et al. 2010).

Biswas et al. (2010) estudiaron la importancia de ambas deformaciones sobre la señal del sensor, identificó las causas que las provocan y puso de manifiesto la necesidad de tomar precauciones durante el proceso de encapsulado del sensor óptico FBG.

- Jochen y Tobías (2011).

Jochen y Tobías (2011) también estudiaron las causas que motivaban anomalías en el espectro de luz del sensor. Según Jochen y Tobías (2011), estudiar las causas que provocan una distorsión del espectro de luz reflejado es fundamental en el diseño de un sensor óptico, ya que una anomalía en éste conllevaría una detección errónea del valor de la longitud de onda reflejada y por tanto un falseo en las medidas.

Jochen y Tobías (2011) se centraron en el estudio de la birrefrigencia (aparición de dos picos sobre el espectro de luz reflejado). Según Jochen y Tobías (2011), la causa más común que provoca la birrefrigencia es la existencia de una fuerza en dirección transversal a la dirección de la fibra óptica. Esta fuerza en dirección transversal provoca que la sección de la fibra pase de ser circular a ser elíptica y por tanto el índice de refracción varía, siendo distinto en dos direcciones (Figura 2.21).

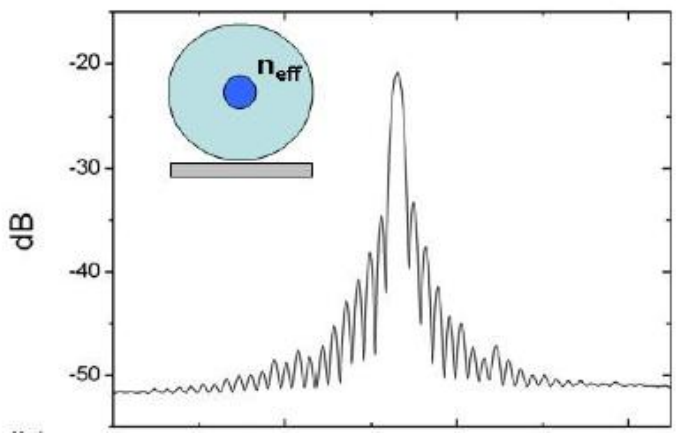

(a)

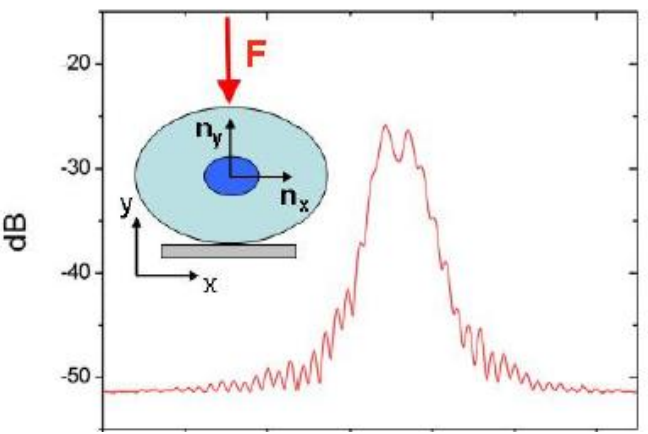

(b)

Figura 2.21 (a)- Espectro de luz sin birrefrigencia. (b)- Espectro de luz con birrefrigencia y fuerza transversal sobre la fibra óptica. Jochen y Tobías (2011)

La aparición de los dos picos en el espectro de luz reflejado se debe a una variación en el índice de refracción de la fibra óptica, de tal modo que Jochen y Tobías (2011) lo expresaron así: 
Monitorización estructural.

Aplicación con sensores de fibra óptica.

$\lambda_{B}^{i}=2 n_{e f f}^{i}(F / L) \Lambda \quad(i=x, y)$

Y definieron la birrefrigencia como la diferencia entre los dos índices de refracción (ecuación 2.12):

$\beta=n_{e f f}^{X}-n_{e f f}^{y}$

Con este parámetro $\beta$, Jochen y Tobías (2011) estimaron directamente la distancia en longitud de onda entre picos:

$\Delta \lambda_{B}(F / L)=2 \Lambda \beta(F / L)$

Jochen y Tobías (2011) afirmaron que la birrefrigencia puede estar motivada por distintas causas, entre las que destaca una modificación en las propiedades del adhesivo a lo largo de la longitud de la fibra óptica. Otra de las causas que motivan la birrefrigencia es la aplicación de una presión sobre en la cara superior de la fibra, por ejemplo durante el proceso de la instalación.

Además, Jochen y Tobías (2011) observaron que los cortes al final de una fibra óptica tienen una incidencia en la potencia del espectro de luz reflejado, tal y como indica la Figura 2.22.

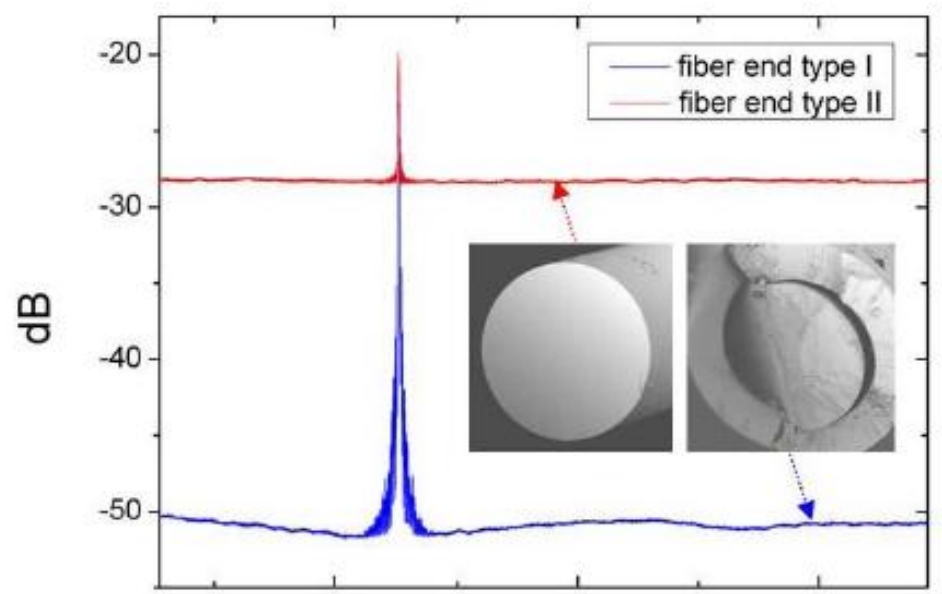

Figura 2.22 Cortes al final de la fibra óptica y su influencia sobre el espectro de luz reflejado. Jochen y Tobías (2011).

Cuando el corte es perfectamente perpendicular a la directriz de la fibra óptica, la luz tiene una reflexión perfecta (puesto que el corte es un espejo perfecto) y esta reflexión se superpone a la longitud de onda reflejada de Bragg, teniendo como consecuencia una disminución en la potencia de la señal. Cuando el corte es totalmente irregular, no se produce ninguna interferencia con la señal de medida y la señal no pierde potencia.

c) Transferencia de deformaciones.

Como se ha visto en la Tabla 2.3 del apartado 2.3.3, muchos autores han propuesto distintos tipos de encapsulados para formar el sensor óptico y permitir su instalación segura en la estructura a 
monitorizar. Sin embargo, la transferencia de deformaciones entre la estructura y la fibra óptica es un aspecto muy poco tratado hasta el momento.

Inicialmente este efecto se consideró despreciable, como publica Ansari y Libo (1998) en su trabajo "Mechanics of bond and interface shear transfer in optical fiber sensors" y se consideró que ambas deformaciones (la que medía el sensor, y la de la estructura) eran iguales.

Sin embargo, en ocasiones la fibra óptica es instalada en elementos robustos metálicos o embebida entre capas de material, de forma que la fibra óptica no mide la totalidad de la deformación que experimenta la estructura. Esto provoca diferencias entre las deformaciones medidas por la fibra y las deformaciones reales de la estructura.

Los primeros investigadores que estudiaron este efecto fueron Duck y Leblanc (2000) y Leung et al. (2000). Sus estudios se basaron en campañas experimentales en las que embebieron FBGs en láminas de materiales compuestos por fibra de carbono y resina de poliéster. Estos materiales compuestos fueron sometidos a carga, y analizaron que existía una transferencia de deformaciones hacia la fibra óptica, sin llegar a cuantificarla.

Otros autores como Dongsheng et al. (2006), Kai Tai et al. (2008) y Li et al. (2009) han estudiado este fenómeno mediante campañas experimentales y modelizaciones numéricas. Todos ellos coinciden en que la transferencia de deformaciones hasta la fibra óptica es función de una serie de parámetros como son la longitud de fibra que se encuentre en contacto con la estructura (Figura 2.23) y los espesores existentes en la instalación de la FBG en la estructura.

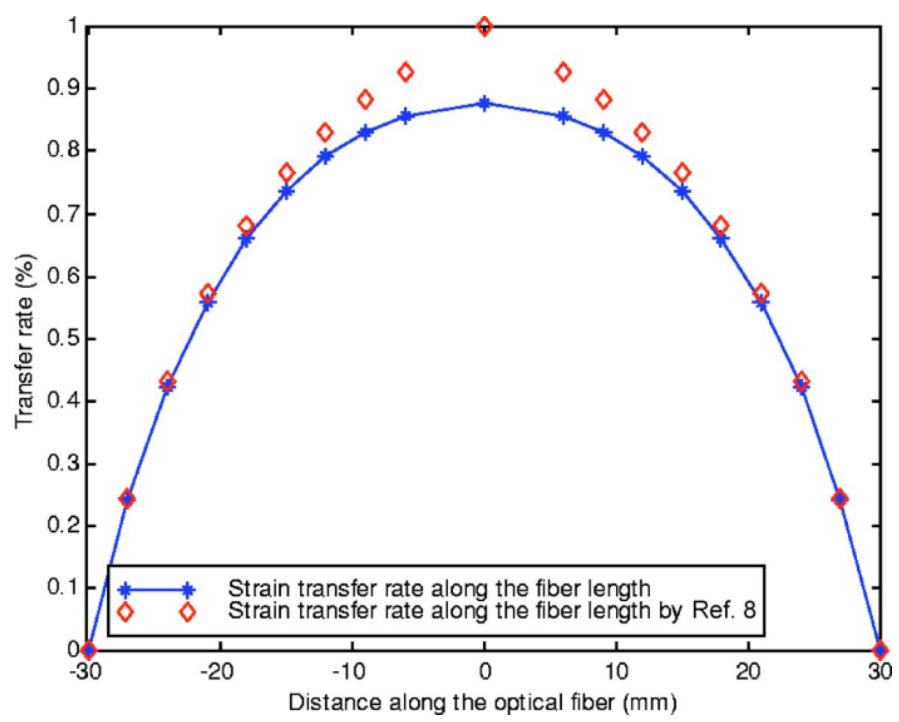

Figura 2.23 Ratio de transferencia en función de la longitud de la fibra óptica (Dongsheng et al. 2006).

Además, otros autores como Qingbin et al. (2003) han estudiado otros factores que afectan a la transferencia de deformaciones hasta la fibra óptica como la influencia en las mediciones del recubrimiento de plástico de la fibra óptica. Para ello realizaron un modelo teórico donde estudiaron la transferencia de deformaciones a través de esta capa, asumiendo un comportamiento elástico y lineal para la matríz de hormigón y el núcleo de fibra óptica, y un comportamiento elasto-plástico para el recubrimiento de la fibra óptica. Además, adoptaron como hipótesis que no existía desplazamiento relativo entre las capas que se encuentran en contacto, esto es, hormigón con recubrimiento y recubrimiento con núcleo de fibra óptica. 
Qingbin et al. (2003) concluyeron que debido a este comportamiento de la fibra óptica, es necesario corregir la deformación obtenida por un coeficiente de transferencia, de modo que se obtenga la deformación del hormigón. Este coeficiente de transferencia es variable con la deformación del hormigón (Figura 2.24), lo que dificulta su aplicación directa.

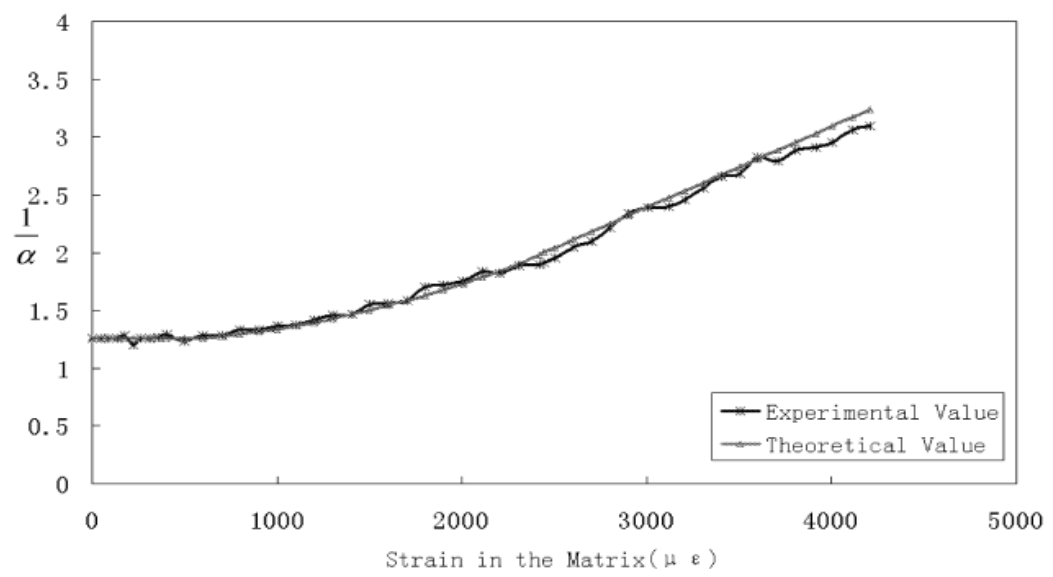

Figura 2.24 Relación entre el coeficiente de transferencia y la deformación del hormigón (Qingbin et al. 2003).

Sin embargo, ninguno de ellos considera en sus modelos un sensor encapsulado adherido a la estructura ni aspectos como las propiedades mecánicas de los materiales que intervienen en el proceso de fabricación e instalación o las distintas configuraciones posibles de sensores.

La cuestión sobre la transferencia de deformaciones entre la estructura a monitorizar y la fibra óptica es un campo que se encuentra actualmente en estudio. Algunas publicaciones como Sans et al. (2011), o Shiuh y Chih (2011) lo manifiestan. En este estudio, Shiuh y Chih (2011) demostraron que el recubrimiento de la fibra óptica presenta una afección sobre la obtención del valor de las deformaciones que no puede ser despreciada. En esta investigación, se estudió la interacción entre el recubrimiento de la fibra óptica y el material de la estructura a partir de modelos numéricos de elementos finitos y posteriormente se realizaron ensayos experimentales de laboratorio.

Los modelos numéricos revelaron que la transferencia de deformaciones era mayor cuanto mayor era la longitud de la fibra óptica en contacto con el material instrumentado, y cuando más rígido era el material que formaba el recubrimiento de la fibra óptica. Por este motivo, Shiuh y Chih (2011) afirmaron que la medida de la fibra óptica debe ser corregida por un factor que depende de la longitud de fibra adherida y de las propiedades mecánicas del material que forme el recubrimiento (Figura 2.25). Esto mismo fue observado por las campañas experimentales, donde la deformación medida por la fibra óptica era menor que la deformación real del elemento ensayado. 


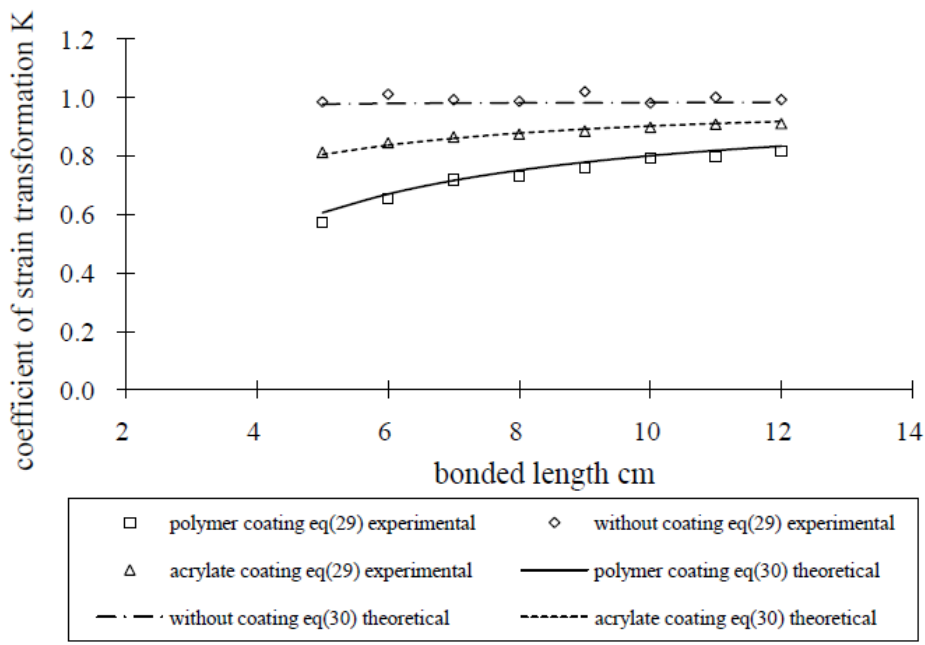

Figura 2.25. Coeficiente de transformación de la deformación para distintas longitudes de fibra y para distintos tipos de recubrimiento. Shiuh y Chih (2011)

\subsection{Estado del Arte sobre la aplicación e instrumentación estructural con fibra óptica.}

\subsubsection{Introducción.}

Durante los últimos años, han sido principalmente tres las áreas de trabajo en el campo de la investigación y la aplicación de la fibra óptica para la monitorización de estructuras: la obra civil (y muy especialmente los puentes de grandes luces), la edificación y el empleo de materiales compuestos tipo FRP en el refuerzo de estructuras. Se detallan a continuación los trabajos más relevantes en estas áreas en relación con esta Tesis.

\subsubsection{Estadísticas relativas a la aplicación e instrumentación con fibra óptica.}

Distintos investigadores han realizado estudios que recogen datos relativos al empleo de la fibra óptica en la SHM. El crecimiento en la importancia de esta nueva disciplina se observa en la información estadística que se describe a continuación.
a) Ko y Ni(2005).

En el trabajo publicado por Ko y Ni (2005) se recopila información acerca 40 puentes de grandes luces (todos ellos disponían una longitud de vano mínima de 100 metros) instrumentados con distintas técnicas. Algunos de los puentes objeto del estudio son el Great Belt Bridge en Dinamarca, el Confederation Bridge en Canadá, el Tsin Ma Bridge en Hong Kong, el Commodore Barry Bridge en Estados Unidos, el Akashi Kaikyo en Japón y el Seohae Bridge en Korea.

La Figura 2.26 muestra los porcentajes de puentes del estudio instrumentados con las distintas tecnologías que se enumeran. 


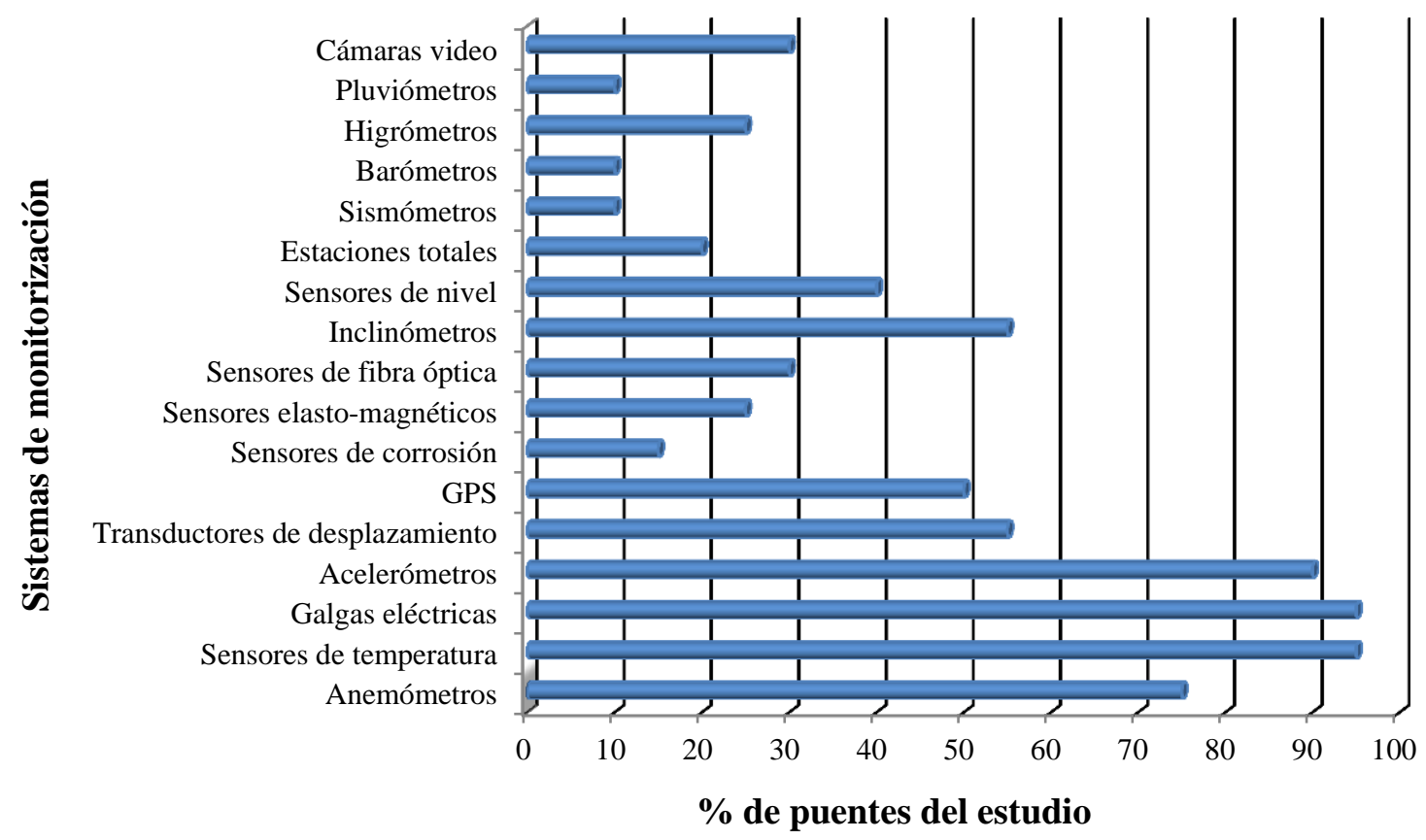

Figura 2.26 Porcentaje de puentes estudiados con distintas técnicas de monitorización estructural. Adaptado de Ko y Ni (2005).

Como se observa, la tecnología de la fibra óptica se emplea en un 30\% de los puentes que engloban el estudio de Ko y Ni (2005) mientras que las galgas extensométricas se emplean en un $95 \%$ de los puentes objetos del estudio.

Estos datos ponen de manifiesto que la tecnología óptica se encuentra en una clara fase de expansión, y que todavía muchos aspectos de la misma están siendo objeto de estudios y mejoras.

\section{b) Inaudi (2009).}

Inaudi (2009) recopiló datos acerca de proyectos de monitorización con fibra óptica de 40 puentes durante los últimos 12 años llevados a cabo por la empresa suiza SMARTEC S.A. creada en el año 1996 y centrada en los proyectos de monitorización estructural con fibra óptica. Inaudi (2009) clasificó esta información atendiendo a distintos criterios: ubicación de cada puente, tipología estructural, fase de proyecto en que se encontraban, objetivos que se perseguía en cada proyecto de monitorización y presupuesto disponible. Sin embargo, una de las clasificaciones más importantes probablemente sea la de la evolución de los objetivos del proyecto de montorización estructural en función del tiempo (Figura 2.27).

Ésta clasificación demuestra que el empleo de la fibra óptica para incrementar la seguridad extendiendo la vida útil del puente es uno de los motivos principales más investigados en los últimos 10 años. Por el contrario, la demostración del funcionamiento de la tecnología óptica ha empezado a decrecer considerablemente con el paso de los años debido a la confirmación, poco a poco, de su adecuación en la monitorización estructural. 


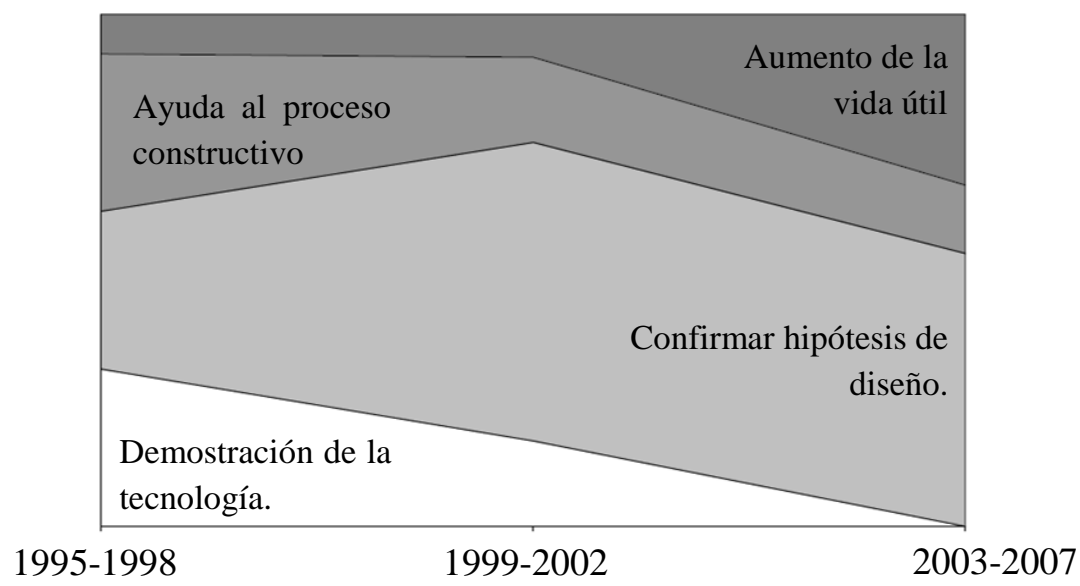

Figura 2.27 Distribución de los objetivos de un proyecto de monitorización en el tiempo (Inaudi, 2009)

\subsubsection{Principales trabajos.}

Multitud de publicaciones hacen referencia a las 3 líneas de trabajo citadas anteriormente en el apartado 2.3.1. Las más relevantes se han recogido en las Tablas 2.5, 2.6, 2.7 y 2.8, clasificándolas según 4 grupos:

- Aplicaciones SHM en el campo de la obra civil.

- Aplicaciones SHM en el campo de la edificación.

- Aplicaciones SHM en el campo del estudio de nuevos materiales aplicados a la ingeniería.

- Otras investigaciones y aplicaciones SHM.

En las Tablas 2.5, 2.6, 2.7 y 2.8 se ha empleado la siguiente notación:

- $\quad$ SHM- Structural Health Monitoring.

- LGFOS- Long Gauge Fiber Optic Sensors.

- SHMBM-Structural Health Monitoring Bridge Management.

- BOTDR-Brillouin Optical Time Domain Reflectomery.

- FBG-Fiber Bragg Gratting.

- FOS's- Fiber Optic Sensor's.

- FRP-Fiber Reinforced Polymer.

- CFRP-Carbon Fiber Reinforced Polymer.

- NDE-Non Destructive Evaluation. 
Monitorización estructural.

Aplicación con sensores de fibra óptica.

SHM en obra civil con sensores ópticos.

\begin{tabular}{|c|c|c|c|c|c|c|c|c|}
\hline Autor (año) & $\begin{array}{c}\text { Puentes: } \\
\text { tablero, } \\
\text { aparatos de } \\
\text { apoyo, cables. }\end{array}$ & $\begin{array}{c}\text { Cimentaciones } \\
\text { profundas }\end{array}$ & Pilas & Tuberías & Túneles & Presas & Carreteras & $\begin{array}{c}\text { Construcciones } \\
\text { históricas }\end{array}$ \\
\hline $\begin{array}{l}\text { Inaudi et al. } \\
\text { (1999a) }\end{array}$ & $\begin{array}{c}\text { Interacción } \\
\text { hormigones } \\
\text { distintas edades. } \\
\text { Deformada del } \\
\text { tablero, } \\
\text { desplazamientos. } \\
\text { LGFOS }\end{array}$ & - & - & - & - & - & - & - \\
\hline
\end{tabular}




\begin{tabular}{|c|c|c|c|c|c|c|c|c|}
\hline Autor (año) & $\begin{array}{c}\text { Puentes: } \\
\text { tablero, } \\
\text { aparatos de } \\
\text { apoyo, cables. }\end{array}$ & $\begin{array}{c}\text { Cimentaciones } \\
\text { profundas }\end{array}$ & Pilas & Tuberías & Túneles & Presas & Carreteras & $\begin{array}{c}\text { Construcciones } \\
\text { históricas }\end{array}$ \\
\hline Vurpillot (1999) & $\begin{array}{l}\text { SHM puente de } \\
\text { vigas continuas. } \\
\text { Fisuración. } \\
\text { LGFOS }\end{array}$ & - & - & - & - & - & - & - \\
\hline $\begin{array}{l}\text { Glišić et al. } \\
\text { (1999) }\end{array}$ & - & - & - & - & - & $\begin{array}{l}\text { SHM presa arco } \\
\text { de hormigón. . } \\
\text { Comparativa } \\
\text { extensómetros } \\
\text { de cuerda. } \\
\text { Deformaciones } \\
\text { al variar el nivel } \\
\text { del embalse. } \\
\text { Sensores } \\
\text { interferométricos }\end{array}$ & - & - \\
\hline $\begin{array}{l}\text { Inaudi et } \\
\text { al.(2001) }\end{array}$ & $\begin{array}{l}\text { SHM puente } \\
\text { arco desde } \\
\text { construcción. } \\
\text { Afección de la } \\
\text { temperatura. }\end{array}$ & - & - & - & - & - & - & $\begin{array}{l}\text { SHM cúpula de } \\
\text { iglesia. } \\
\text { Evolución de la } \\
\text { abertura de } \\
\text { fisura. LGFOS }\end{array}$ \\
\hline $\begin{array}{c}\text { Inaudi y Glišić } \\
\text { (2002) }\end{array}$ & - & $\begin{array}{l}\text { SHM pilotes. } \\
\text { Fisuración y } \\
\text { propiedades } \\
\text { geotécnicas del } \\
\text { terreno. LGFOS. }\end{array}$ & - & - & - & - & - & - \\
\hline
\end{tabular}


Monitorización estructural.

Aplicación con sensores de fibra óptica.

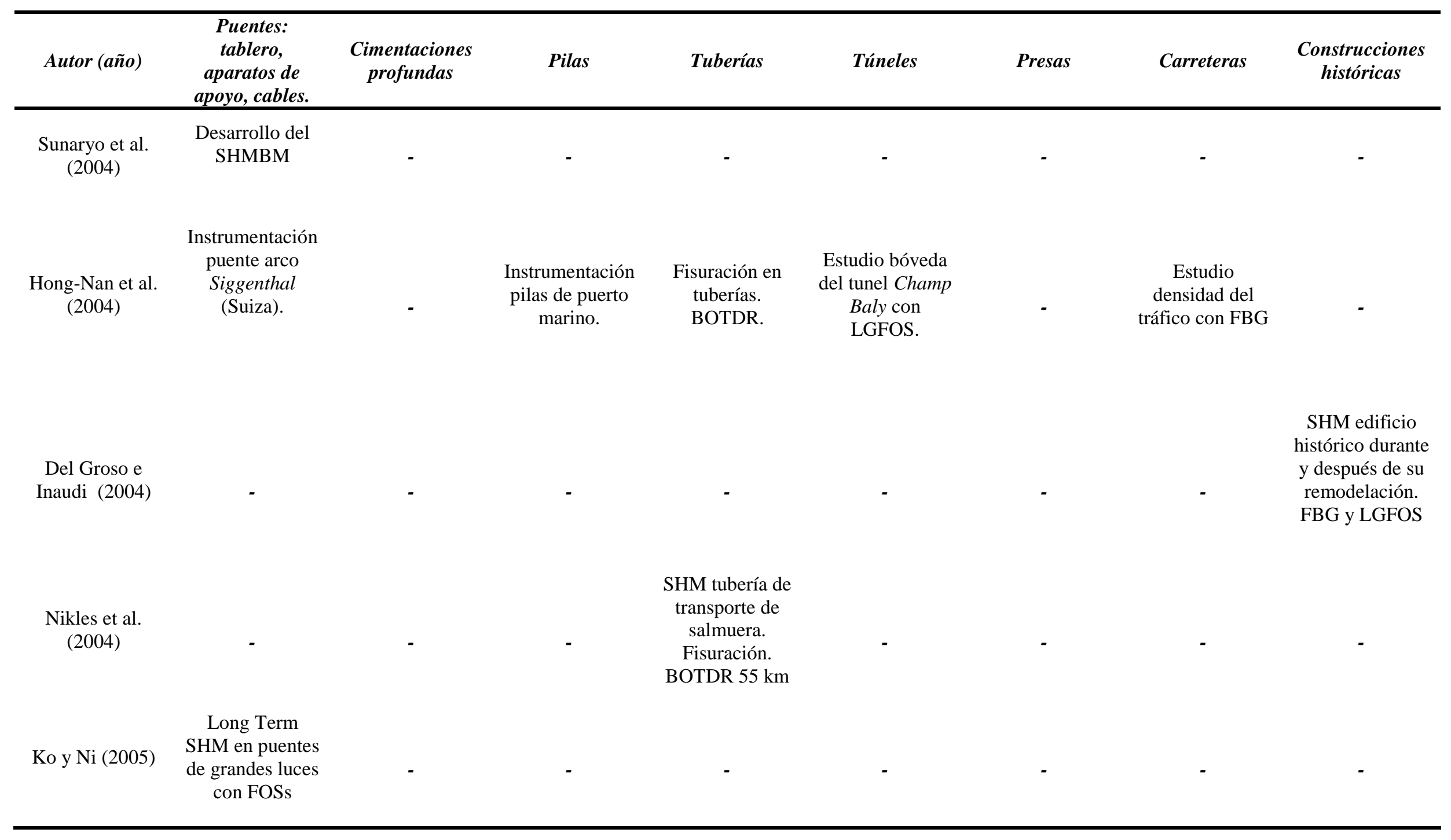




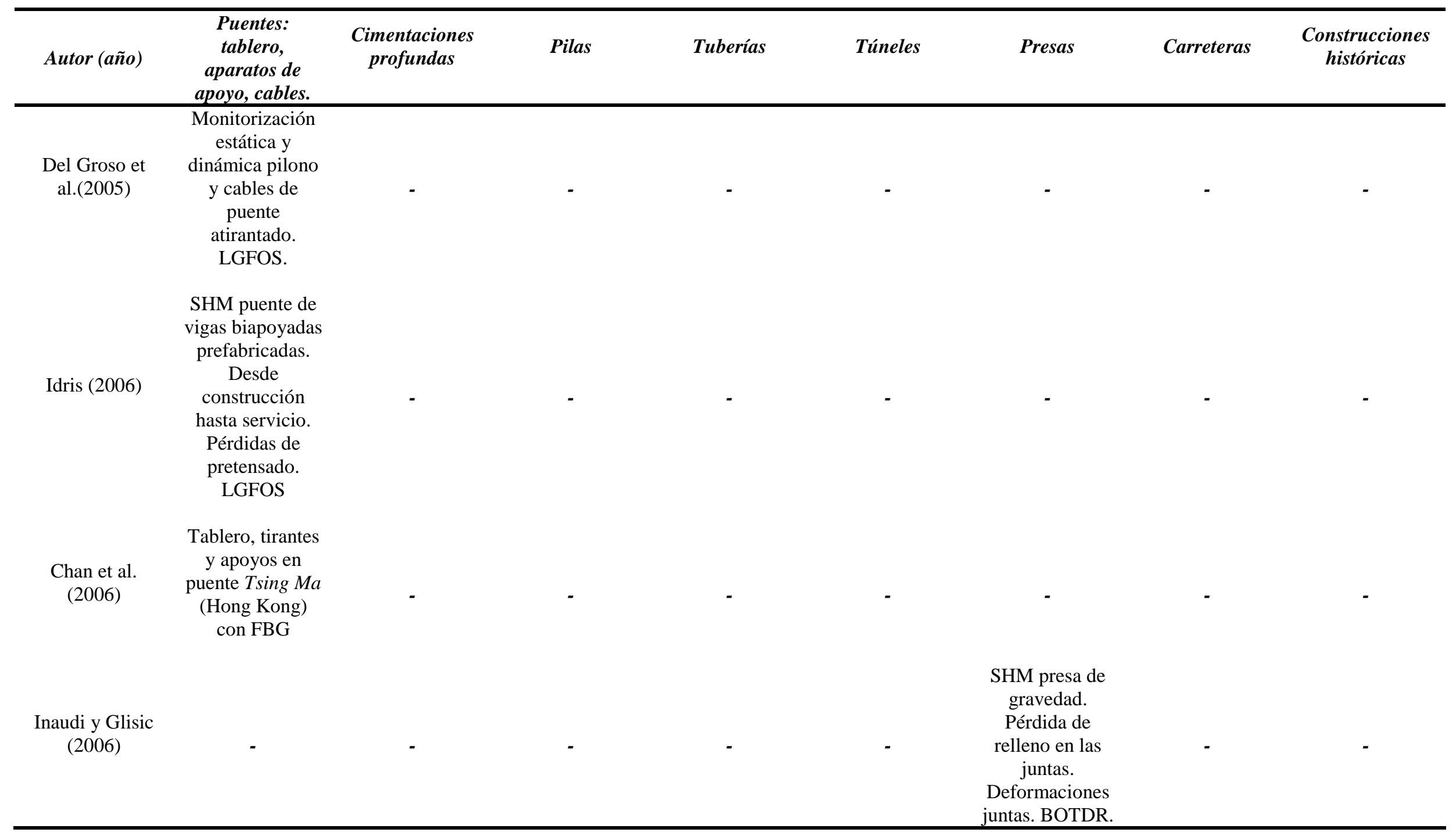


Monitorización estructural.

Aplicación con sensores de fibra óptica.

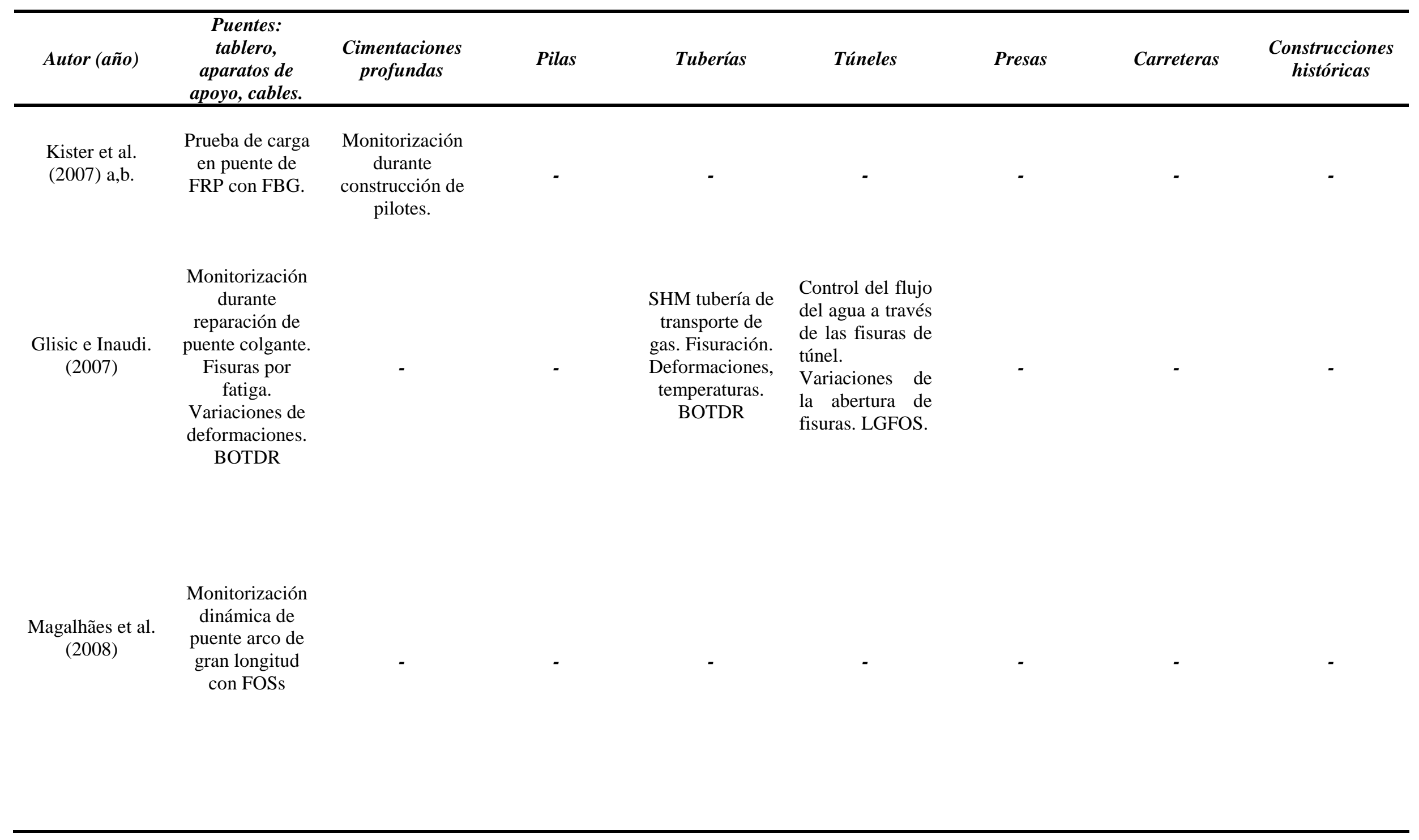




\begin{tabular}{|c|c|c|c|c|c|c|c|c|}
\hline Autor (año) & $\begin{array}{c}\text { Puentes: } \\
\text { tablero, } \\
\text { aparatos de } \\
\text { apoyo, cables. }\end{array}$ & $\begin{array}{c}\text { Cimentaciones } \\
\text { profundas }\end{array}$ & Pilas & Tuberías & Túneles & Presas & Carreteras & $\begin{array}{c}\text { Construcciones } \\
\text { históricas }\end{array}$ \\
\hline Chuan (2008) & - & - & - & - & $\begin{array}{c}\text { Deformaciones } \\
\text { térmicas en } \\
\text { túnel con FBG. } \\
\text { Campaña } \\
\text { experimental y } \\
\text { modelo } \\
\text { numérico. }\end{array}$ & - & - & - \\
\hline $\begin{array}{l}\text { Wonseok et al. } \\
\text { (2008) }\end{array}$ & $\begin{array}{l}\text { Ensayos sobre } \\
\text { vigas } \\
\text { pretensadas de } \\
\text { puentes. FBG y } \\
\text { LGFOS. }\end{array}$ & - & - & - & - & - & - & - \\
\hline $\begin{array}{l}\text { Samir et al. } \\
\text { (2009) }\end{array}$ & $\begin{array}{l}\text { Instrumentaicón } \\
\text { durante } 4 \text { años } \\
\text { cajón metálico } \\
\text { Puente Star City }\end{array}$ & - & - & - & - & - & - & - \\
\hline
\end{tabular}


Monitorización estructural.

Aplicación con sensores de fibra óptica.

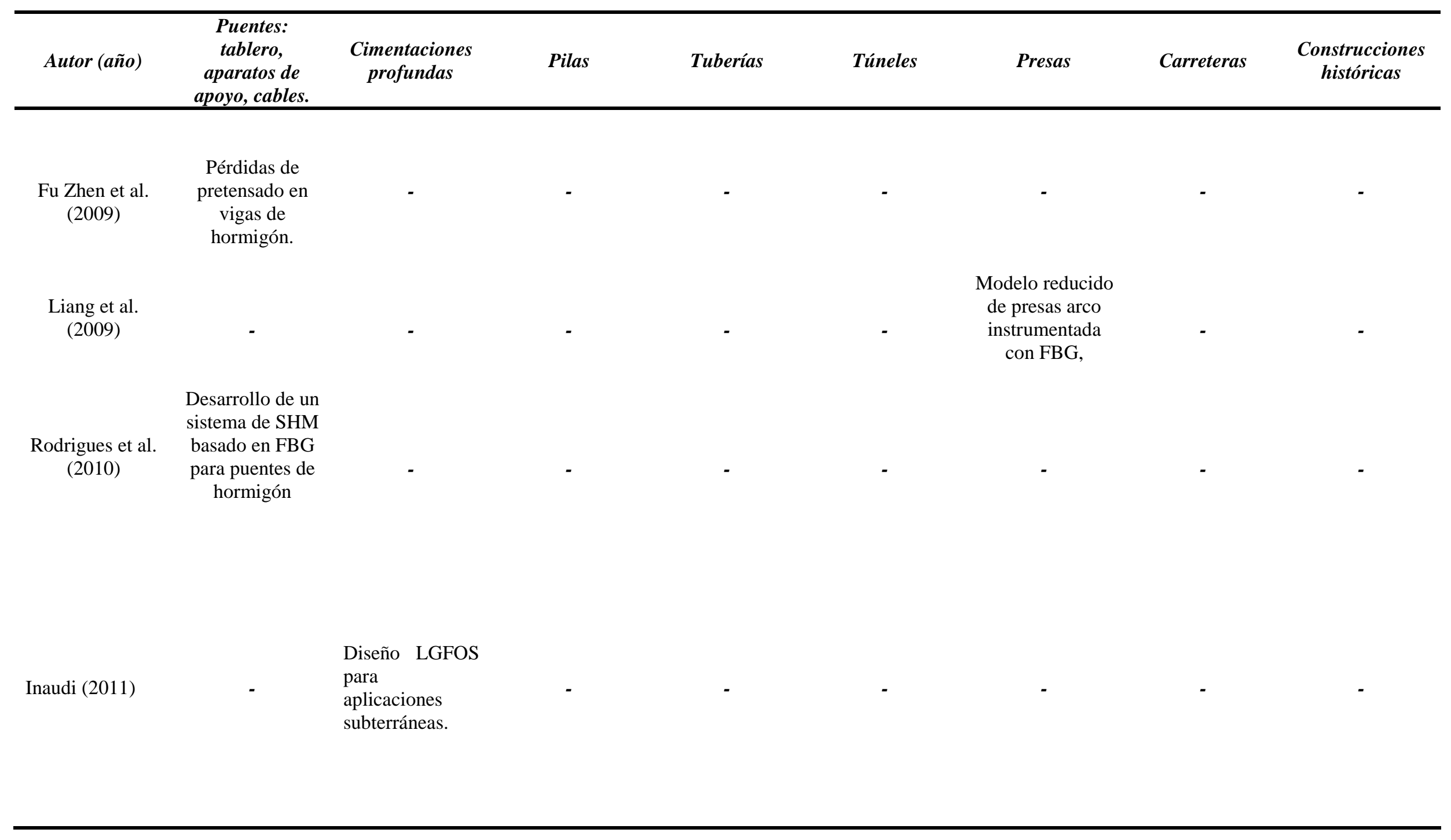




\begin{tabular}{|c|c|c|c|c|c|c|c|c|}
\hline Autor (año) & $\begin{array}{c}\text { Puentes: } \\
\text { tablero, } \\
\text { aparatos de } \\
\text { apoyo, cables. }\end{array}$ & $\begin{array}{c}\text { Cimentaciones } \\
\text { profundas }\end{array}$ & Pilas & Tuberías & Túneles & Presas & Carreteras & $\begin{array}{c}\text { Construcciones } \\
\text { históricas }\end{array}$ \\
\hline
\end{tabular}

Tabla 2.5 Aplicaciones SHM en el campo de la obra civil. 
Monitorización estructural.

Aplicación con sensores de fibra óptica.

SHM en elementos estructurales de edificación.

Autor (año)

Pantallas, núcleos

Forjados, vigas

Pilares.

Glisic et al.. (2002)

Glisic et al. (2005)

SHM nucleos de hormigón en edificios. Obtención de

Glisic e Inaudi (2007)

Majumder et al. (2008) deformaciones de torsión, cortante y compresión. LGFOS y FBG
SHM de pórticos de hormigón para aumentar el conocimiento sobre su comportamiento estructural. LGFOS

\section{SHM pilares con LGFOS. \\ Identificación de esfuerzos \\ en pilares: compresión centrada, flexión. \\ SHM de pilares de un rascacielos desde su construcción hasta fase explotación. Identificación terremotos. Afección de las variaciones térmicas. LGFOS.}

Monitorización de pilares durante fase constructiva y en servicio. Estudio de sus solicitaciones: flexión simple o compuesta, compresión centrada. LGFOS

Instrumentación elementos sometidos a flexión con

FOSs. Obtención de curvaturas, deformaciones $\mathrm{y}$ flechas.

Tabla 2.6 Aplicaciones SHM en el campo de la edificación.

Autor (año)

Estudio de nuevos materiales aplicados a la ingeniería.

Yang y Ansari (2002) Sensor interferométrico long gauge. Estudio materiales compuestos FRP

Yung et al. (2005)

Hormigón a edades tempranas en tablero de puente de carretera.

Shin y Chiang (2006)

Fatiga en materiales compuestos empleando FBG

Zhishen et al. (2006)

Eficacia del refuerzo FRP en hormigón con FBG y BOTDR

Majumder et al. (2008)

Retracción, humedad y absorción a edades tempranas con FOSs.

Shu et al. (2011)

Monitorización del ciclo de vida de estructuras CFRP sometidas a impactos. BOTDR

Tabla 2.7 Aplicaciones SHM en el campo del estudio de nuevos materiales aplicados a la ingeniería. 
Autor (año)

Leng y Asundi (2002)

Chang et al. (2003)

Junqi et al. (2006)

Wendy (2006)

Takeda et al. (2007)

Majumder et al. (2008)

Donghoon y Wonseok (2009)

Rodrigo y López (2009)

Zhi et al. (2010)

Kuang et al. (2011)

Junqi et al. (2011)
Otras investigaciones y aplicaciones

NDE con sensores ópticos vibracionales

NDE sensores interferométricos.

Monitorización tendones de pretensado mediante BOTDR

Daños en carriles de ferrocarril con FBG

SHM en alas de aviones durante ensayos de fatiga con FBG
Deformación, curvaturas y desplazamientos en carriles de ferrocarril. Campañas experimentales con FBG

SHM de estructuras marinas con FBG

Monitorización de estructuras de hielo (ice structures) con FOSs.

Monitorización de geotextiles con FOSs.

Monitorización de corrosión en estructuras de hormigón con FBG

Tabla 2.8 Otras investigaciones y aplicaciones SHM. 
Monitorización estructural.

Aplicación con sensores de fibra óptica.

\subsubsection{Pautas de monitorización estructural.}

a) Introducción:

El éxito de un buen trabajo de monitorización depende de una serie de tareas que engloban el proceso de monitorización estructural. Glisic e Inaudi (2007) profundizaron y sintetizaron las tareas que exige llevarlo a cabo:

- Selección de la estrategia de monitorización. La estrategia de monitorización es una tarea que exige un amplio conocimiento de la obra instrumentada. Esta tarea engloba a su vez otras actividades:

- Establecer el objetivo de la monitorización.

- Identificar y seleccionar los parámetros a ser monitorizados.

- Seleccionar unos adecuados sistemas de monitorización.

- Diseñar la red de sensores. Esta actividad depende las características de la obra, de su tipología y de su forma de trabajo.

- Establecer el programa de monitorización.

- Gestión de los datos.

- Coste de la monitorización.

- Instalación del sistema de monitorización.

- Mantenimiento del sistema de monitorización.

- Gestión e interpretación de los datos.

- Actividades de cierre, en caso de interrupción o finalización del proceso de monitorización. Esta tarea engloba el desmontaje de los equipos, cableado y sensores en caso de haber sido instalados superficialmente.

De los anteriores, Glisic e Inaudi (2007) destacan la importancia de una adecuada planificación de la estrategia de monitorización. A su vez, en esta fase existe una actividad que cobra especial importancia y consiste en las pautas de monitorización del elemento estudiado.

\section{b) Mediciones obtenidas con sensores ópticos y su interpretación:}

Según Glisic e Inaudi (2007) la deformación total de una fibra de un material es la suma de los siguientes términos:

$\varepsilon_{t o t}=\varepsilon_{E}+\varepsilon_{P}+\varepsilon_{T}+\varepsilon_{\varphi}+\varepsilon_{s h}$

Siendo $\varepsilon_{t o t}$ la deformación total medida, $\varepsilon_{E}$ la deformación elástica, $\varepsilon_{P}$ la deformación plástica, $\varepsilon_{T}$ la deformación de temperatura, $\varepsilon_{\varphi}$ la deformación de fluencia (en caso de materiales como hormigón o madera) y $\varepsilon_{s h}$ la deformación de retracción (en caso de hormigón).

$\varepsilon_{t o t}, \varepsilon_{T}$ y $\varepsilon_{s h}$ son las componentes de la deformación que se obtienen a través de la medición con sensores ópticos. $\varepsilon_{\varphi}$ se estima a través de las normativas existentes (por ejemplo, CEB-FIP, 1990). Las componentes $\varepsilon_{E} y \varepsilon_{P}$ se obtienen de la ecuación 2.11 a partir del resto de valores conocidos. Una vez 
conocidos los valores de la deformación que se deben exclusivamente a las cargas actuantes, se deben obtener los valores de las tensiones en el material y los esfuerzos asociados, para así obtener el coeficiente de seguridad.

\section{c) Pautas de monitorización de distintos elementos estructurales.}

Glisic e Inaudi (2007) estudiaron la instrumentación de distintos elementos estructurales y propusieron unas pautas generales para su instrumentación. Glisic e Inaudi (2007) proponen inicialmente 4 tipologías de instrumentación (simple, paralela, cruzada y triangular) que hacen referencia a la posición de los sensores en el interior de la estructura.

Para ello, Glisic e Inaudi (2007) proponen un procedimiento que se resume en los siguientes apartados:

1) Dividir la estructura en celdas.

2) Instrumentar cada celda según una tipología.

3) Analizar de acuerdo a las mediciones el comportamiento de la celda.

4) Extrapolar el comportamiento de la celda para conocer el comportamiento de la estructura.

Cada celda en que se divide una estructura puede estar instrumentada con una tipología concreta (simple, paralela, cruzada o triangular). La tipología dependerá del tipo de parámetro que se desee estudiar (deformación, deformación de cortante, curvatura, desplazamiento relativo...)

\section{- Tipología simple.}

Glisic e Inaudi (2007) definieron como tipología simple a un solo sensor instalado en la dirección principal de deformación del elemento estructural. Esta tipología se emplea para instrumentar elementos lineales sujetos a compresión o tracción centrada, combinado con esfuerzos longitudinales de cortante y en general, con cargas paralelas a la directriz de la pieza, como pilares (Figura 2.28.a).

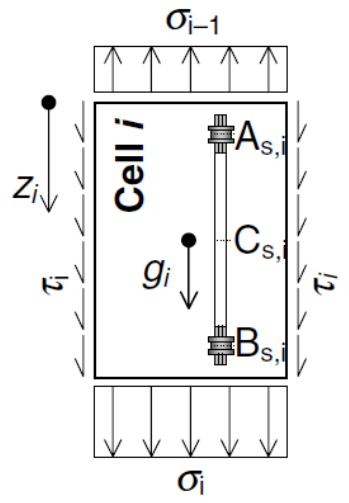

(a)

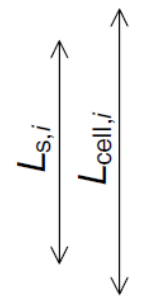

(b)

Figura 2.28 (a)- Elemento instrumentado con tipología simple. (b)- Pilote enterrado instrumentado con una tipología simple (Glisic e Inaudi, 2007). 
Glisic e Inaudi (2007) proponen una formulación para la monitorización estructural de este tipo de elementos: Conocidas las deformaciones en una celda i $\left(\varepsilon_{i, E m, t}\right)$ en su punto medio $C_{s, i}$ (Figura 2.28.b), obtiene el desplazamiento relativo del elemento a partir de la ecuación 2.15:

$\Delta v_{C S_{i}-C S_{n}}=\sum_{j=i+1}^{n} \Delta \mathrm{L}_{\mathrm{cell}, \mathrm{j}}=\sum_{j=i+1}^{n}\left(\varepsilon_{j, m, t} L_{c e l l, j}\right)$

Conocido el valor $\varepsilon_{i, E m, t}$, la obtención del esfuerzo axil es inmediata:

$N_{C_{s, i, t}}=E_{i, t} A_{i, t} \varepsilon_{i, E m, t}$

Por último, los esfuerzos de cortante longitudinales (por ejemplo, fricción en caso de pilotes enterrados) se obtiene a través de un equilibrio de fuerzas en el interior de cada célula:

$\tau_{C_{s, i-1}-C_{s, i,}}=2 \frac{N_{C_{s, i, t}}-N_{C_{s, i-1, t}}}{S_{c e l l, i}+S_{c e l l, i-1}}$

Donde $S_{c e l l, i}$ es la superficie perimetral de la célula i.

\section{- Tipología paralela.}

Esta tipología se emplea para instrumentar células en las que se divide una estructura que estén sometidas a flexión, como el caso de vigas. Consiste en dos sensores iguales, situados paralelos a la fibra neutra de la viga e instalados uno a cada lado de la misma (Figura 2.29).
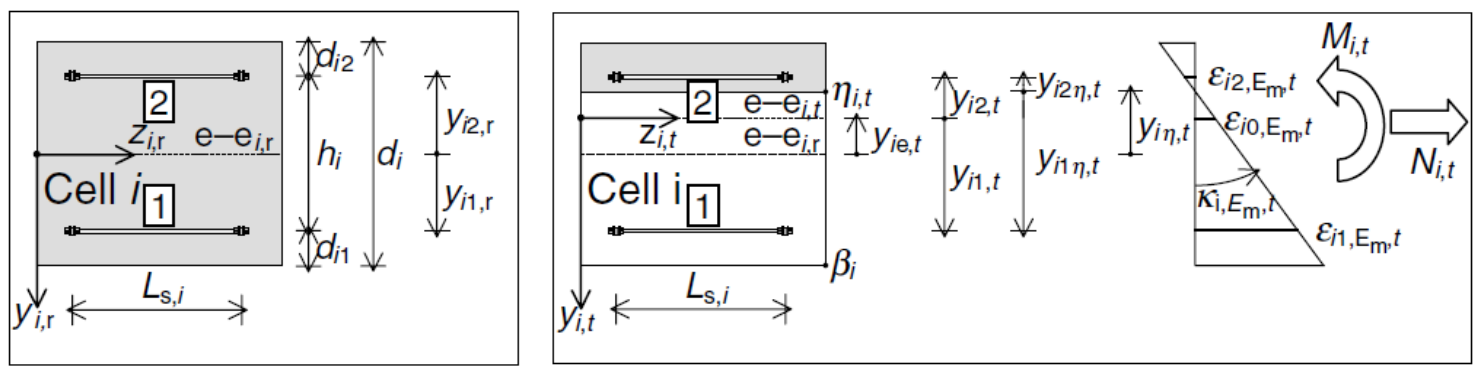

Figura 2.29 Tipología paralela en una célula de hormigón (Glisic e Inaudi, 2007).

Glisic e Inaudi (2007) proponen una formulación para obtener esfuerzos, aberturas de fisura y flechas mediante esta tipología de instrumentación. La formulación se basa fundamentalmente en tres aspectos:

1) La obtención del esfuerzo axil (ecuación 2.18) y momento flector (ecuación 2.19) a partir de la deformación del centro de gravedad de la pieza y de su curvatura (ecuación 2.20)

$$
N_{i, t}=E_{i, t} A_{i, t} \varepsilon_{i 0, E m, t}
$$




$$
\begin{aligned}
& M_{i, t}=E_{i, t} I_{i, t} k_{i, E m, t} \\
& k_{i, E m, t}=\frac{\left(\varepsilon_{i 1, m, t}-\varepsilon_{i 2, m, t}\right)-\alpha_{T}\left(\Delta T_{i 1, m, t}-\Delta T_{i 2, m, t}\right)}{h_{i}\left(1+K_{\varphi} f_{\varphi}\right)}
\end{aligned}
$$

Siendo $K_{\varphi} f_{\varphi}$ los parámetros de fluencia que indica la normativa CEB-FIP, 1990.

2) La obtención de la abertura media de fisura en la parte traccionada, a partir de las ecuaciones 2.21 y 2.22 :

$$
\begin{aligned}
& \sum_{j} w_{i, j, t}=\left[\varepsilon_{i 1, E m+\varphi m, t}-\varepsilon_{u}^{+}\left(1+K_{\varphi} f_{\varphi}\right)\right] \frac{y_{i 1 \eta, t}+d_{i 1}}{y_{i 1 \eta, t}} L_{s, i} \\
& w_{i}=\frac{\sum_{j} w_{i, j, t}}{n_{i, t}}
\end{aligned}
$$

3) La obtención de las flechas de la pieza (Figura 2.30), a partir de la integración doble de las curvaturas obtenidas según la ecuación $\mathrm{Z}$ (ecuación 2.23). En esta ecuación, $c_{1}$ y $c_{2}$ son constantes de integración.

$$
w_{y, t}=\int\left(\int k_{m, t}(x) d x\right)+c_{1} z+c_{2}
$$

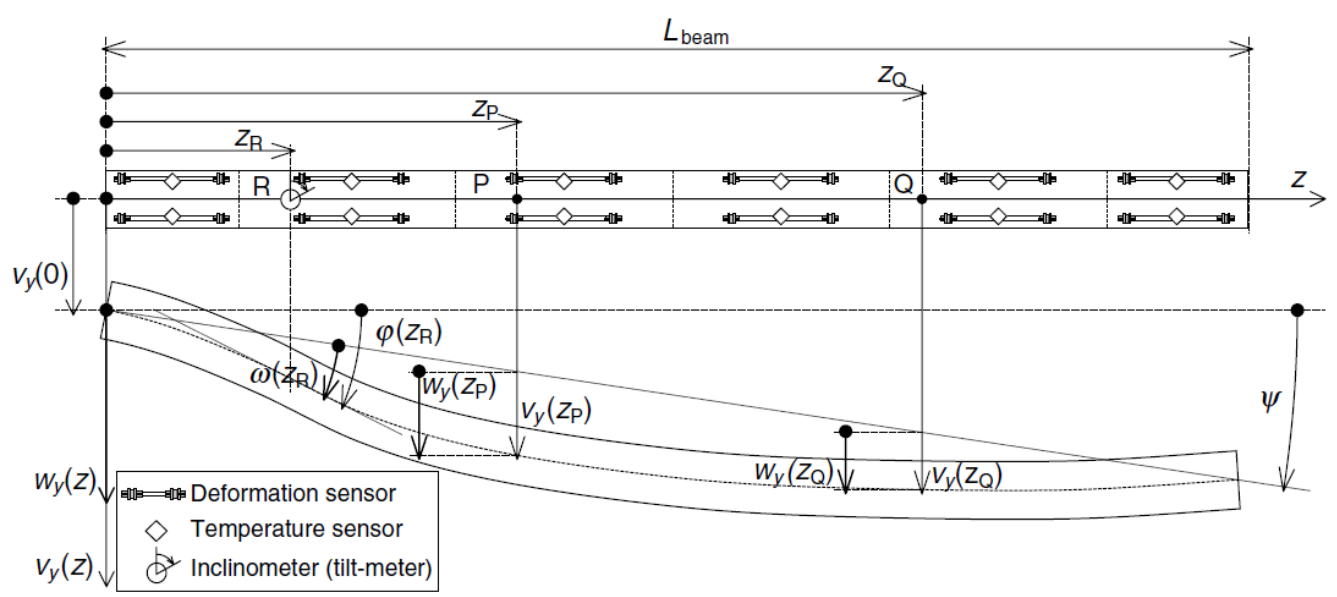

Figura 2.30 Ejemplo de viga deformada instrumentada con tipología paralela. Cálculo de flechas (Glisic e Inaudi, 2007). 
Monitorización estructural.

Aplicación con sensores de fibra óptica.

\section{- Tipología cruzada}

La tipología cruzada consiste en dos sensores cruzados instalados con un ángulo predefinido entre ellos respecto a la dirección principal de deformación. El objetivo de esta tipología es el de cuantificar la distorsión angular en el plano de los sensores. Los sensores deben tener la misma longitud e iguales ángulos de inclinación, pero de signo opuesto (Figura 2.31).

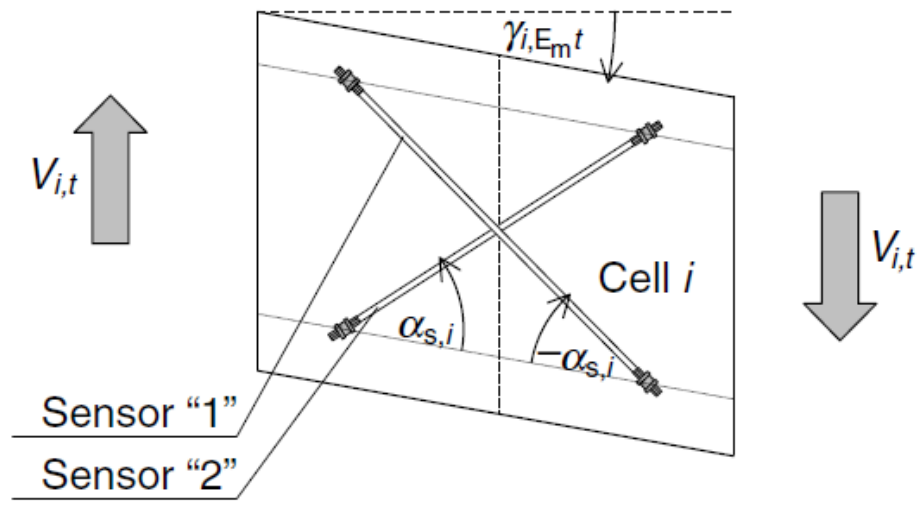

Figura 2.31 Tipología cruzada (Glisic e Inaudi, 2007).

La distorsión angular medida por la tipología cruzada vendrá dada por la ecuación 2.24:

$\gamma_{i, m, t}=\frac{\varepsilon_{i 1, m, t}-\varepsilon_{i 2, m, t}}{2 \sin \alpha_{s, 1} \cos \alpha_{s, 1}} \frac{1}{\left(1+K_{\varphi} f_{\varphi}\right)}$

Con la distorsión angular medida, el esfuerzo cortante se obtendrá de acuerdo a la ecuación 2.25:

$V_{i, t}=G_{i, t} k_{i, t}^{\gamma} I_{i, t} \gamma_{i, E m, t}$

Donde $G_{i, t}$ es el módulo de rigidez transversal del material.

Esta tipología debe instalarse en las zonas donde se estimen importantes esfuerzos cortantes.

\section{- Tipología triangular.}

Glisic e Inaudi (2007) proponen esta tipología para instrumentar células sometidas a distintos tipos de cargas, de forma que la deformación plana que se produce puede ser explicada a partir de desplazamientos relativos de varios puntos. Típicos ejemplos de aplicación de esta tipología son los arcos de fábrica, muros de piedra o estructuras con fisuración importante.

El parámetro principal que se monitoriza en esta tipología es el desplazamiento relativo de un punto y para ello, Glisic e Inaudi (2007) proponen dos sensores que tienen un punto en común, y los otros dos puntos se disponen formando un triángulo (Figura 2.32). 


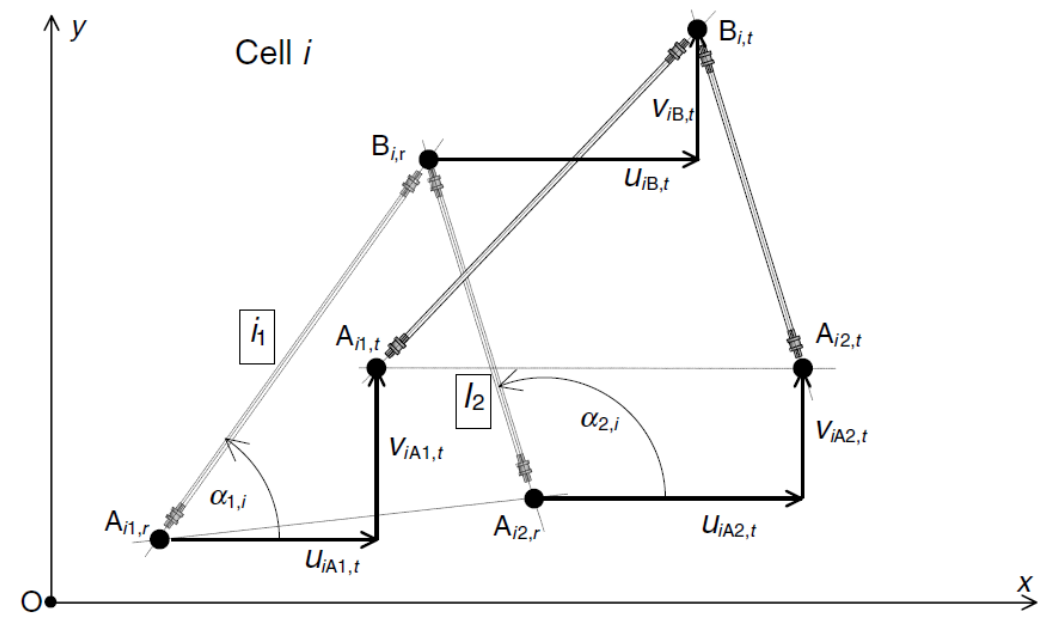

Figura 2.32 Esquema de la tipología triangular (Glisic e Inaudi, 2007).

A partir de relaciones geométricas y trigonométricas en la Figura 2.32 se obtiene el desplazamiento relativo del punto común a ambos sensores (punto B) a partir de los desplazamientos conocidos de otros dos puntos, $A_{i 1, r}$ y $A_{i 2, r}$. Glisic e Inaudi (2007) proponen escoger estos dos puntos estratégicamente de forma que sus desplazamientos sean conocidos, por ejemplo, el caso de puntos fijos con desplazamientos nulos.

\subsubsection{Realizaciones.}

En este apartado se mostrarán los trabajos de monitorización de distintos elementos estructurales, empleando las pautas generales de monitorización que fueron definidas en el apartado 2.4.4.

a) Monitorización de un edificio. (Glisic e Inaudi 2007 y Zonta et al. 2011).

Glisic e Inaudi (2007) han instrumentado multitud de construcciones empleando las estrategias y técnicas descritas previamente. En este trabajo, Glisic e Inaudi (2007) embebieron sensores en los pilares de la planta baja de un edificio de 19 plantas (Figura 2.33.a). Los objetivos de este proceso de instrumentación fueron variados:

- Aumento de la seguridad durante el proceso constructivo.

- Verificar comportamiento de los pilares.

- Aumentar el conocimiento acerca de elementos sometidos a compresión.

- Optimización de los costes de mantenimiento de la estructura.

- Evaluación de la condición de la estructura tras un terremoto, impacto o acto terrorista. 


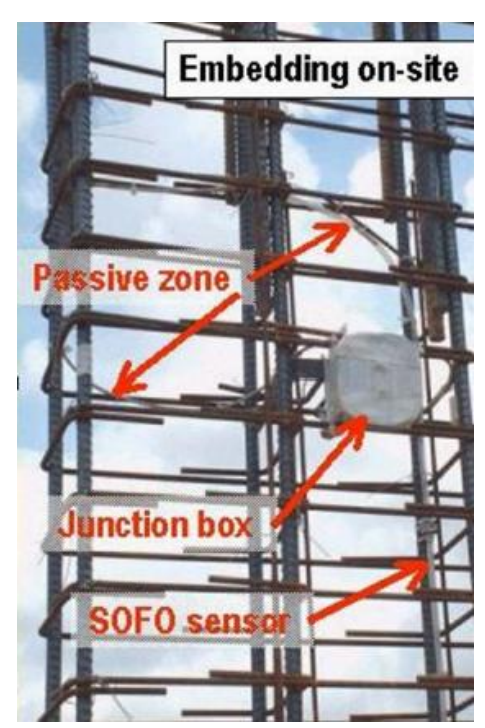

(a)

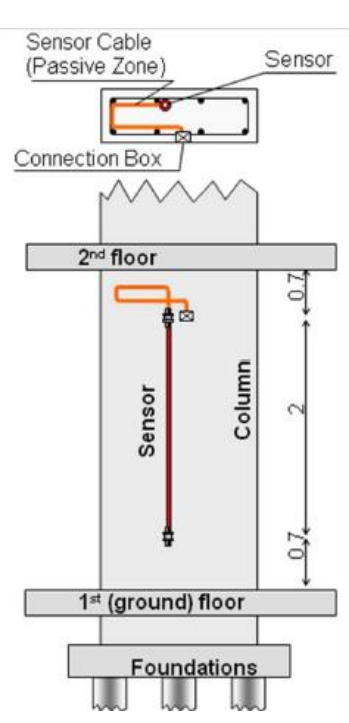

(b)

Figura 2.33 (a)- Sensores colocados en la armadura del pilar y caja de conexión. (b)- Posición esquemática del sensor en el pilar (Zonta et al. 2011).

Glisic e Inaudi (2007) consideran que la carga dominante sobre el pilar es fundamentalmente una carga de compresión, suponiendo que la influencia de la flexión en la deformación es despreciable. Por este motivo, Glisic e Inaudi (2007) proponen una estrategia de monitorización instrumentando cada pilar con un único sensor, instalado paralelo al eje y no necesariamente en el centro de gravedad de la sección (Figura 2.33.b).

Gracias al proceso de monitorización estructural, un comportamiento anómalo de los pilares fue detectado (Figura 2.34). Uno de los pilares, Col. 3, mostró una historia de deformaciones inferior al resto de pilares, e inferior a la que se predijo a partir de modelos teóricos.

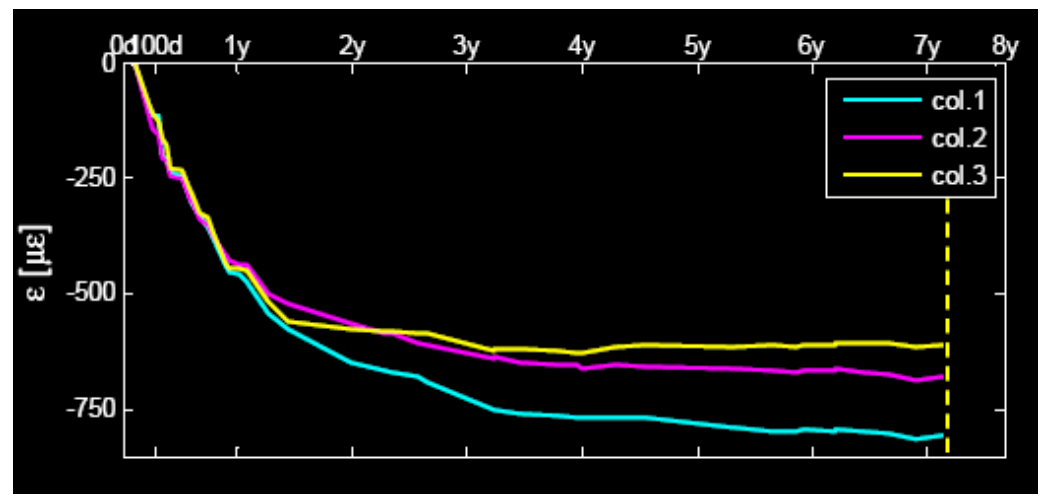

Figura 2.34 Evolución de las deformaciones en los pilares durante 7 años (Zonta et al. 2011).

Inicialmente, Glisic e Inaudi (2007) achacaron este comportamiento anómalo a las deformaciones térmicas del pilar. Sin embargo, tras analizar las temperaturas concluyeron que estas variaciones térmicas no eran lo suficientemente importantes como para producir un comportamiento tan anómalo (Zonta et al. 2011). 
Tras llevar a cabo un estudio del terreno afirmaron que esta tendencia anómala era debida a un asiento del terreno bajo este pilar (figura 2.35):

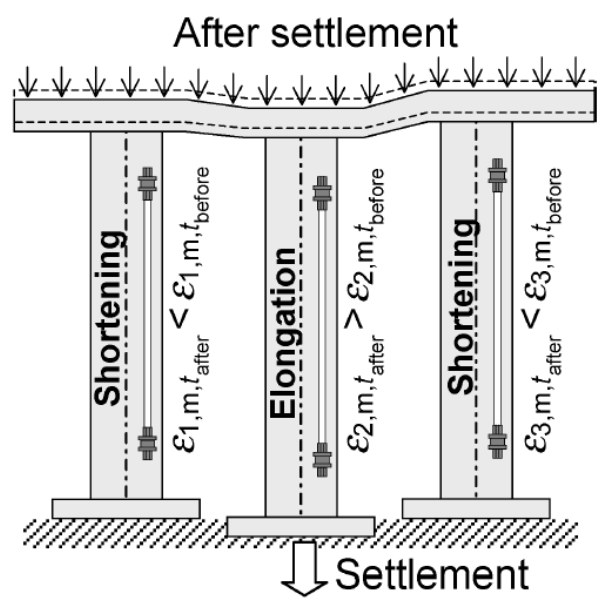

Figura 2.35 Asentamiento de un pilar y evolución en sus deformaciones (Glisic e Inaudi, 2007).

Tras analizar los resultados, Glisic e Inaudi (2007) enumeraron posibles causas que pueden ocasionar diferencias entre valores medidos y teóricos:

- Sobrecargas sobre los pilares debido a cargas desconocidas.

- Evolución de la retracción y fluencia.

- Asentamientos diferenciales de cimientos y de pilares vecinos.

- Inclinación y giro de las plantas superiores.

b) Monitorización del puente "South Lutrive". (Glisic e Inaudi 2007).

En este apartado se resume el trabajo de Glisic e Inaudi (2007) sobre la monitorización y evaluación estructural de un puente de voladizos compensados.

Un puente de voladizos compensados consiste en dos voladizos simétricos con su empotramiento en una pila común. Habitualmente, ambos voladizos tienen la misma longitud, de forma que el momento flector ocasionado por cargas gravitatorias queda compensado en el empotramiento con las pilas. La geometría de este tipo de puente se asemeja a la ley de flectores en cada voladizo. (Figura 2.36).

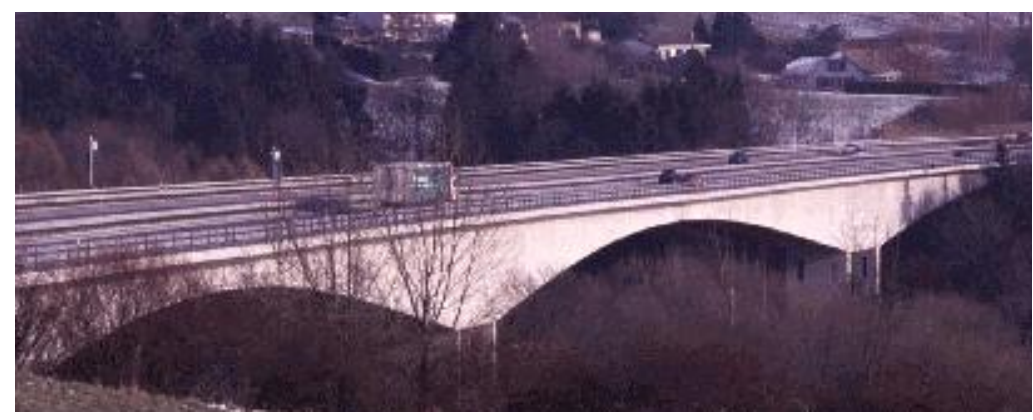

Figura 2.36 Vista del puente Lutrive en Suiza. 
En el caso que ocupa este trabajo, Glisic e Inaudi (2007) estudiaron el comportamiento del puente "South Lutrive" en Suiza. Se trata de dos puentes de carretera paralelos construido en el año 1972, con una ligera curvatura en planta $($ radio $=1000 \mathrm{~m})$ y 385 metros de longitud entre los cuatro vanos.

Glisic e Inaudi (2007) plantean una estrategia de monitorización a partir de las recomendaciones mostradas en los anteriores apartados. Concretamente, empleó distintas estrategias para la instrumentación de distintos elementos de este puente:

1) Tipología paralela a lo largo de la directriz de la viga con el objetivo de obtener las flechas (Figura 2.37).

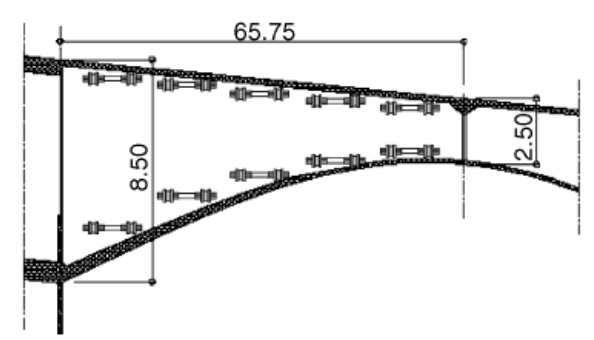

(a)

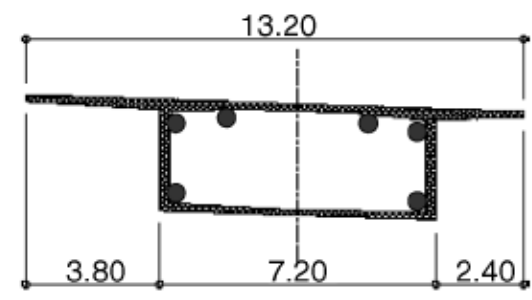

(b)

Figura 2.37 (a)- Sensores a lo largo del voladizo. (b)- Sensores instalados en la sección transversal (Glisic e Inaudi 2007).

2) Tipología cruzada en la zona de empotramiento con la pila, para evaluar los esfuerzos cortantes y los momentos torsores.

3) Dado que el paso de vehículos genera momentos descompensados en su empotramiento con la pila, instala una tipología paralela de sensores en la parte alta de la pila.

4) Para obtener una mayor información del comportamiento del extremo libre del voladizo, propone su instrumentación con una mayor densidad de sensores de menor longitud, en tipología paralela.

5) Tipología cruzada en el extremo libre del voladizo, para conocer así su desplazamiento relativo.

6) Por último, Glisic e Inaudi (2007) instalan inclinómetros en el empotramiento y en el extremo libre del voladizo para conocer las rotaciones en el plano vertical del puente.

Una vez implementada la estrategia de monitorización y en noviembre de 1997, el Instituto de Hormigón armado y pretensado de Suiza (IBAP) realizó una prueba de carga estática. La carga fueron dos camiones de 25 toneladas, combinados en distintos casos de carga en función de la posición de los camiones.

Los desplazamientos verticales del extremo libre del voladizo fueron medidos empleando un sistema de topografía convencional, y éstos fueron comparados con los resultados que se obtuvieron a partir de las mediciones de los sensores ópticos (Figura 2.38). Para ello, las rotaciones obtenidas con los inclinómetros fueron empleadas como condiciones de contorno en la integración de la curvatura. La Figura 2.38 muestra la equivalencia de los dos métodos, con un error máximo de un $6.5 \%$ entre ambos sistemas de medida. 


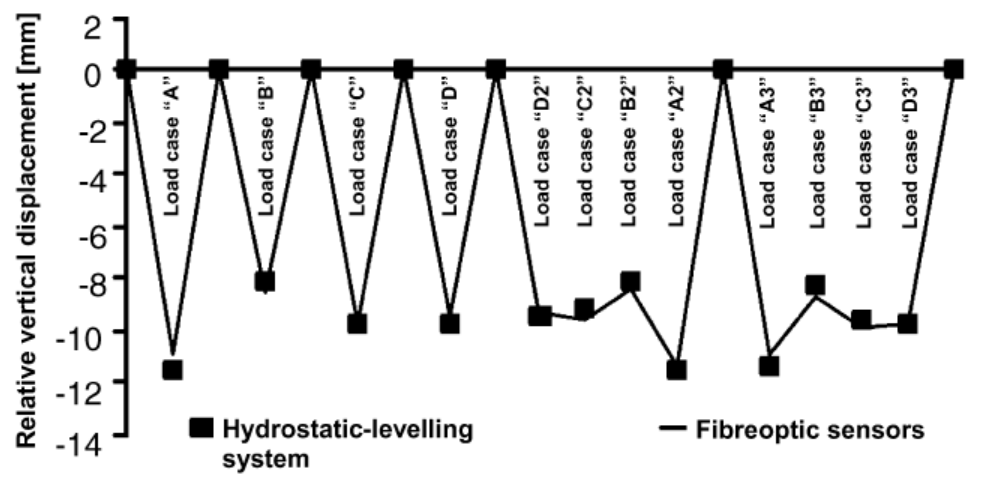

Figura 2.38 Desplazamiento vertical del extremo del voladizo. (Glisic e Inaudi 2007)

Por otro lado, también se obtuvieron las curvaturas durante el paso de vehículos (la mayoría de ellos pesados) durante 24 horas (Figura 2.39). El cálculo de las curvaturas mostró que se producía una concentración de paso de vehículos desde las 06:00 hasta las 15:00. La mayoría de los valores fueron positivos, indicando flexiones negativas sobre el voladizo.

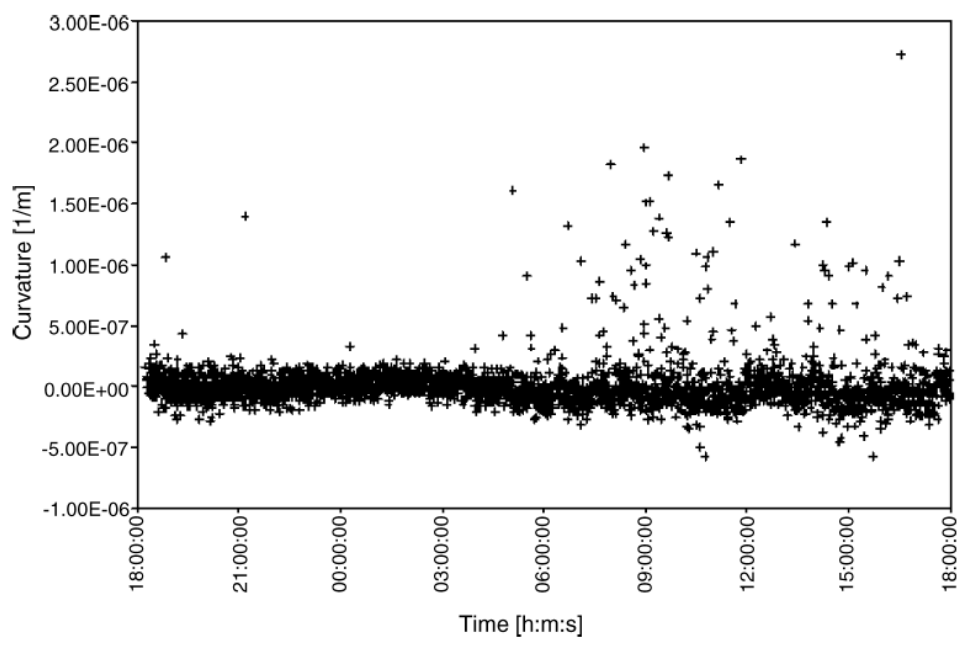

Figura 2.39 Curvaturas obtenidas con los sensores ópticos. (Glisic e Inaudi 2007)

Sin embargo, existían algunos valores negativos que indicaron flexiones positivas que pudieron ser provocadas por dos situaciones: Por un lado, un rebote debido al paso de un vehículo sobre el vano instrumentado o por otro, una flexión negativa de un vano contiguo que provoca flexiones positivas en el vano instrumentado. Además, la presencia de algunos valores anómalos indicaron el paso de camiones pesados de doble eje (40 toneladas) o el paso simultáneo de dos camiones a la vez.

\subsection{Conclusiones relativas al Estado del Arte.}

Tal y como se observa en el epígrafe 2.3 del presente capítulo, son numerosas las investigaciones que hacen referencia al desarrollo de un sensor óptico y su encapsulado. Sin embargo se ha evidenciado la falta de pautas de validación definidas con claridad sobre el diseño de nuevos sensores (puntuales, de longitud, distribuidos), dependiendo del material y de los parámetros a medir. Uno de los aspectos objeto de mayor número de investigaciones consiste en la transferencia de deformaciones hacia la fibra óptica, como consecuencia fundamentalmente del encapsulado propuesto y de la interacción 
Monitorización estructural.

Aplicación con sensores de fibra óptica.

entre el sensor y la estructura. A pesar de que sean escasas, este tipo de investigaciones son fundamentales en todo sistema óptico de sensado y el hecho de no tener en cuenta este aspecto, pueden traer consigo algunas consideraciones incorrectas o poco exactas como:

a) Adopción de la deformación medida por el sensor como la deformación real de la estructura.

b) Calibración de los sensores únicamente en base a resultados experimentales.

c) Inexistencia de modelos teóricos que reproduzcan el comportamiento de los sensores ópticos ante una variación de cualquier parámetro que influya en su fabricación o instalación en la estructura (Fijaciones, espesores, propiedades mecánicas, longitudes...).

Otro aspecto que ha sido objeto de investigaciones ha sido la integración de la fibra óptica en su encapsulado y en la afección sobre la señal del sensor. Algunos autores han estudiado las causas que provocan una distorsión en la forma de la señal para evitar su aparición y otros han cuantificado esa distorsión para evaluar su afección sobre los resultados obtenidos.

A partir de esta situación, en esta Tesis Doctoral se han desarrollado nuevos sensores ópticos a partir de campañas experimentales y numéricas que ofrecen diversas ventajas sobre los ya existentes, que serán comentadas en los capítulos correspondientes. Su funcionamiento ha sido validado a través de ensayos de laboratorio a pequeña escala y de su empleo en estructuras reales bajo las condiciones que impone el sector de la construcción. Aunque las estructuras instrumentadas tienen una tipología muy diversa, esta Tesis Doctoral incluye únicamente la monitorización de la estructura del falso túnel de la línea de Alta Velocidad de Levante en Mogente. En este trabajo se ha llevado a cabo una comparativa entre los tipos de sensores estudiados en esta Tesis Doctoral y se ha validado y discutido el funcionamiento y aplicación de los sensores propuestos.

El resto de trabajos de monitorización realizados se adjuntan en el Apéndice II. Este Apéndice recoge las distintas publicaciones que han sido presentadas en revistas o congresos nacionales $\mathrm{e}$ internacionales:

- Benjamín Torres, Ignacio Payá-Zaforteza, Pedro A. Calderón, Jose M. Adam. “Analysis of the strain transfer in a new FBG sensor for Structural Health Monitoring". Engineering Structures 33 (2011) 539-548.

- Antonio Bueno, Benjamín Torres, David Barrera, Pedro Antonio Calderón, José Manuel Lloris, María José López, Salvador Sales. "Optical fiber sensors embedded in concrete for measurement of temperature in a real fire test". Optical Engineering, Volume 50, Issue 12, pp. 124404-124404-7 (2011)

- Benjamín Torres, Ignacio Payá-Zaforteza, Antonio Bueno, Salvador Sales, Jose M. Adam, Pedro A. Calderón. "Early age concrete temperature and shrinkage monitoring by using Fiber Bragg Grattings “. XXXVII IAHS World Congress on Housing Science. Santander, Spain. (2010).

- Antonio Bueno, Benjamín Torres, David Barrera, Salvador Sales. "Monitoring of a steel incrementally launched bridge construction with strain and temperature FBGs sensors ". SPIE Photonics Europe. Brussels, Belgium. (2010). 
- Benjamín Torres, Ignacio Payá Zaforteza, Antonio Bueno, Salvador Sales, Pedro Calderón, Raquel López. "Aplicaciones de sensores de fibra óptica desarrollados por la U.P.V. para la medida de deformaciones y temperaturas en estructuras". V Congreso ACHE. Barcelona 2011.

- Antonio Bueno, Benjamín Torres, David Barrera, Pedro Calderón, José Manuel Lloris, María José López, Salvador Sales. "Fiber Bragg grating sensors embedded in concrete samples for a normalized fire test", 21st International Conference on Optical Fiber Sensors. Ottawa, Canada. May 15-19 (2011). 



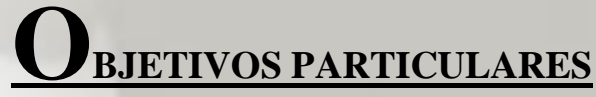

Este apartado definirá los objetivos de esta Tesis Doctoral. Entre ellos y como objetivo principal, se tratará de profundizar en el estudio, diseño y aplicaciones de nuevos sensores ópticos aplicados a la instrumentación de estructuras para conocimiento de sus deformaciones y temperaturas. 



\subsection{Objetivos.}

La presente Tesis Doctoral tiene dos objetivos principales:

(1) El estudio y diseño de nuevos sensores ópticos basados en Redes de Bragg (FBG en inglés, Fiber Bragg Gratting) y en el fenómeno de dispersión de Brillouin (en inglés Brillouin Scattering) para la medida de deformaciones y temperaturas en estructuras.

(2) El estudio de la aplicación de los sensores a la instrumentación de obras reales.

Tanto el estudio y el diseño de los sensores como su aplicación a la instrumentación de obras reales se han basado en campañas experimentales y numéricas.

Estos objetivos se recogen en un total de 5 capítulos. Uno de ellos está basado en un artículo publicado en la revista Engineering Structures situada en el cuartil Q1 de la relación publicada por el Journal of Citation Reports. De los otros cuatro capítulos que siguen la misma línea de investigación planteada, uno de ellos (Capítulo 5) se encuentra pendiente de publicación debido a que sus resultados se encuentran bajo un proceso de posible patente.

De esta forma, los objetivos particulares de este trabajo y que se expondrán en los próximos capítulos son los siguientes:

1) Caracterización de la respuesta de una FBG ante deformaciones y/o temperaturas impuestas.

2) Desarrollo de un encapsulado que forme el sensor óptico puntual de deformación para su instalación en estructuras.

3) Estudio del sensor óptico puntual a partir de modelizaciones numéricas empleando el programa de elementos finitos Lusas V.14.3-11. En este apartado se realiza un estudio de sensibilidad de los factores que afectan a su medición (espesores, propiedades mecánicas de los materiales que intervienen en la configuración, variaciones térmicas), y se comparan distintas configuraciones de sensores ópticos.

4) Desarrollo y diseño de un nuevo sensor de longitud basado en FBG para la medida de deformaciones en estructuras de hormigón o materiales heterogéneos.

5) Estudio del sensor óptico de longitud a partir de modelizaciones numéricas empleando el programa de elementos finitos Lusas V.14.3-11. En este apartado se acota el diseño del sensor, se estudian sus distintos modos de fallo y se realiza un estudio de sensibilidad de los parámetros que intervienen en su diseño (longitud, rigidez a axil, fijaciones...).

6) Aplicación de los anteriores sensores a la instrumentación de la estructura del falso túnel del tramo de Alta Velocidad de Levante a su paso por Mogente (Valencia) para discutir su funcionamiento y comparar sus resultados.

7) Caracterización de la respuesta de sensores ópticos distribuidos basados en el fenómeno no lineal de Brillouin Scattering. Estudio de su respuesta ante variaciones de temperatura y deformación.

8) Instalación y aplicación de los sensores ópticos distribuidos estudiados en el punto 7) anterior, a la instrumentación del falso túnel del tramo de Alta Velocidad de Levante, a su paso por 
Mogente (Valencia). Comparación de sus resultados a los de los otros tipos de sensores colocados.

\subsection{Organización de los capítulos.}

Se presentan brevemente a continuación el resto de capítulos de esta Tesis Doctoral que se corresponden con los objetivos particulares enunciados en el apartado anterior. Así pues, los capítulos de la Tesis Doctoral son:

Capítulo 4. Estudio del comportamiento, desarrollo y diseño de un sensor óptico puntual para la obtención de deformaciones en estructuras.

Capítulo 5. Estudio del comportamiento, desarrollo y diseño de un sensor óptico de longitud para la obtención de deformaciones en estructuras.

Capítulo 6. Aplicación de los sensores desarrollados en los Capítulos 4 y 5 a la monitorización de una estructura real. Monitorización del falso túnel de la línea de Alta Velocidad de Levante a su paso por Mogente (Valencia).

Capítulo 7. Estudio, calibración y aplicabilidad de sensores distribuidos mediante la dispersión estimulada de Brillouin (SBS).

Capítulo 8. Aplicación de los sensores distribuidos desarrollados en el Capítulo 7 a la monitorización de una estructura real. Monitorización del falso túnel de la línea de Alta Velocidad de Levante a su paso por Mogente (Valencia).

Capítulo 9. Conclusiones y propuestas de nuevas investigaciones.

Por tanto, a partir del siguiente capítulo se expondrá la metodología empleada, los procedimientos adoptados, resultados y conclusiones, justificando que la temática de los mismos es coherente con los objetivos investigadores propuestos. 


\section{$\underline{\text { Estudio del comportamiento, }}$}

\section{DESARROLLO Y DISEÑO DE UN SENSOR}

\section{ÓPTICO PUNTUAL PARA LA OBTENCIÓN DE}

\section{DEFORMACIONES EN ESTRUCTURAS.}

El Capítulo 4 presenta un nuevo diseño de sensor óptico puntual FBG para ser fijado a la superficie de la estructura a monitorizar o embebido en el hormigón.

Para ello, se han realizado campañas experimentales y un modelo numérico de elementos finitos 3D donde se ha estudiado la influencia de (1) el espesor y propiedades mecánicas del adhesivo empleado y (2) la configuración del encapsulado del sensor en la sensibilidad del mismo. Además, se ha realizado una comparación entre el diseño propuesto en el artículo y las configuraciones habituales de sensores ópticos puntuales existentes en el mercado. El modelo numérico permite conocer la afección de las variables sobre la medición del sensor y poder llevar a cabo un estudio de sensibilidad que permite un diseño óptimo del sensor.

Los resultados obtenidos del modelo numérico mostraron que existe una diferencia inferior a un $2.5 \%$ entre la deformación medida por el diseño propuesto y la deformación de la superficie monitorizada, debido al hecho de adoptar una configuración asimétrica del sensor con una única capa de material compuesto.

El trabajo que corresponde a este capítulo ha sido publicado en la revista Engineering Structures, que pertenece al cuartil Q1 en la relación publicada por el Journal of Citation Report:

Benjamin Torres, Ignacio Payá-Zaforteza, Pedro A. Calderón, Jose M. Adam. Analysis of the strain transfer in a new FBG sensor for Structural Health Monitoring. Engineering Structures 33 (2011) 539-548. 



\subsection{Introducción.}

Las FBG's son elementos muy frágiles y de reducido tamaño que no pueden ser instaladas directamente en una estructura debido a las duras y hostiles condiciones que impone el sector de la construcción. Estas condiciones propiciarían la rotura de la fibra óptica y afectarían a su durabilidad y comportamiento.

Para evitarlo, muchos encapsulados de protección han sido desarrollados por distintos investigadores (Moyo et al. 2005, Li et al. 2006, Glisic e Inaudi 2007, Majumder et al. 2008), como se expuso en el capítulo 2. Estos encapsulados varían según el tipo de sensor óptico utilizado: Puntuales, de longitud o distribuidos, embebidos en el hormigón o de instalación en superficie. Cada tipo de sensor óptico presenta un encapsulado distinto que deberá garantizar, por un lado la protección de la fibra óptica, y por otro su correcta medición.

Es habitual considerar que la deformación medida por el sensor corresponde con la deformación real que sufre la estructura, pero esta consideración no siempre es correcta. En la mayoría de los casos, la FBG se embebe primeramente en un material compuesto o se fija a un elemento rígido y posteriormente se instala sobre la estructura empleando para ello un adhesivo. En esta situación puede aparecer una discrepancia entre deformaciones medidas y reales de la estructura, y esta discrepancia depende del espesor del material sobre el que se embebe la FBG o del elemento rígido sobre el que se instala, y muy fuertemente de las propiedades geométricas y mecánicas del adhesivo empleado.

En este capítulo de la Tesis se aborda el diseño, desarrollo y estudio del comportamiento de un sensor puntual de deformaciones para su instalación en la superficie de la estructura. Como punto de partida, se ha considerado que el encapsulado de la FBG debe:

1. Permitir una correcta transferencia de deformaciones entre sensor y estructura.

2. Garantizar una correcta señal del sensor que permita su identificación.

3. Aportar a la fibra resistencia frente agresiones medioambientales y mecánicas.

4. No alterar el campo de deformaciones del material sobre el que está instalado, es decir, debe existir una relación entre las propiedades mecánicas del sensor y del material que forma la estructura que minimice esta alteración.

En el diseño del sensor se ha prestado especial atención a las dos primeras consideraciones anteriormente indicadas. Y para ello, a diferencia de la mayoría de sensores puntuales existentes donde la deformación medida por el sensor difiere de la de la superficie sobre la que está instalado, se ha desarrollado un encapsulado en el que las diferencias entre deformaciones reales y medidas es despreciable. Una vez estudiado y diseñado este sensor, se ha comprobado su robustez en pruebas experimentales de laboratorio y en obra.

\subsection{Caracterización de la respuesta de una FBG ante deformaciones y/o temperaturas impuestas.}

El diseño de un nuevo sensor óptico debe tener como precedente el estudio de la respuesta de la fibra óptica frente a deformaciones y temperaturas impuestas. 
Monitorización estructural.

Aplicación con sensores de fibra óptica.

Dado que un filtro FBG se trata de un sensor espectométrico (Capítulo 2, apartado 2.1.7.3.c), éste proporciona variaciones en la longitud de onda de la luz reflejada o transmitida por el sensor. La calibración del mismo y por tanto su respuesta, consistirá en la obtención de unos coeficientes, llamados coeficientes de sensibilidad, que permitan transformar los datos de longitud de onda en variables ingenieriles como deformación (microstrain, $\mu \varepsilon$ ) y temperatura (grado centígrado, ${ }^{\circ} \mathrm{C}$ ).

Estos coeficientes han sido obtenidos experimentalmente imponiendo a la fibra óptica deformaciones y temperaturas, y registrando la variación de longitud de onda que experimenta. Ambas deberían ser proporcionales y por tanto, un coeficiente de ajuste relacionaría ambos parámetros obteniendo el coeficiente de sensibilidad de la deformación, y el coeficiente de sensibilidad de la temperatura, respectivamente.

\subsubsection{Calibración frente a deformaciones impuestas.}

Las deformaciones impuestas han sido aplicadas a la FBG de forma precisa empleando para ello un sistema de posicionamiento milimétrico (Figura 4.1.a).

Al mismo tiempo que se aplican incrementos de deformación de valor conocido, se registra la variación de longitud de onda que experimenta la FBG (Figura 4.1.b).

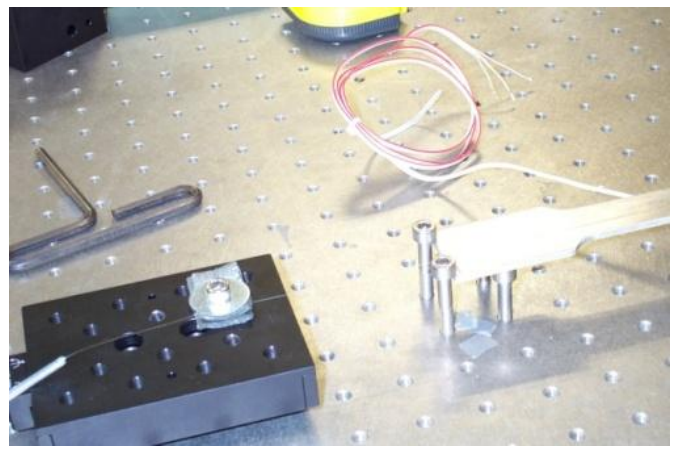

(a)

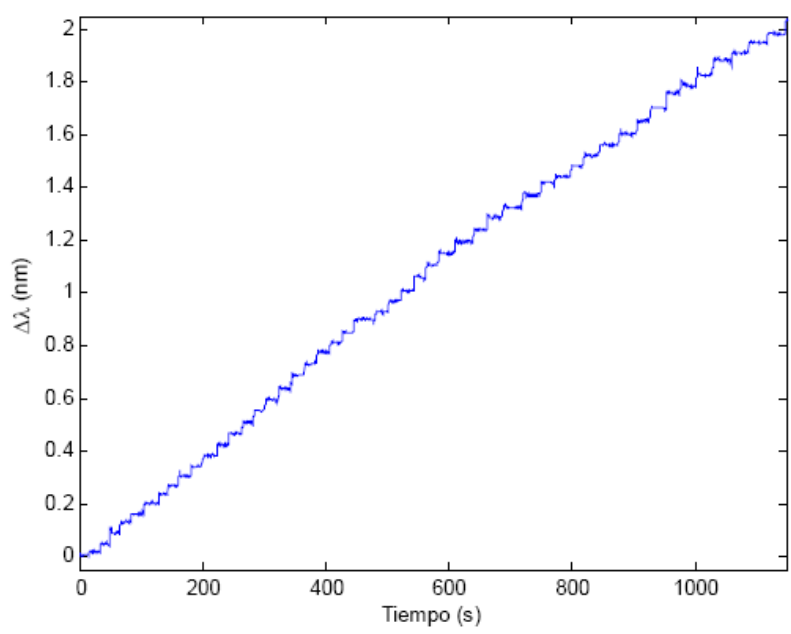

(b)

Figura 4.1 (a)- Sistema de posicionamiento milimétrico. (b)- Incrementos de longitud de onda en función del tiempo para incrementos de deformación.

Posteriormente se realiza un ajuste entre ambos parámetros para obtener el coeficiente que permita obtener la deformación a partir de la variación de longitud de onda (Figura 4.2). 


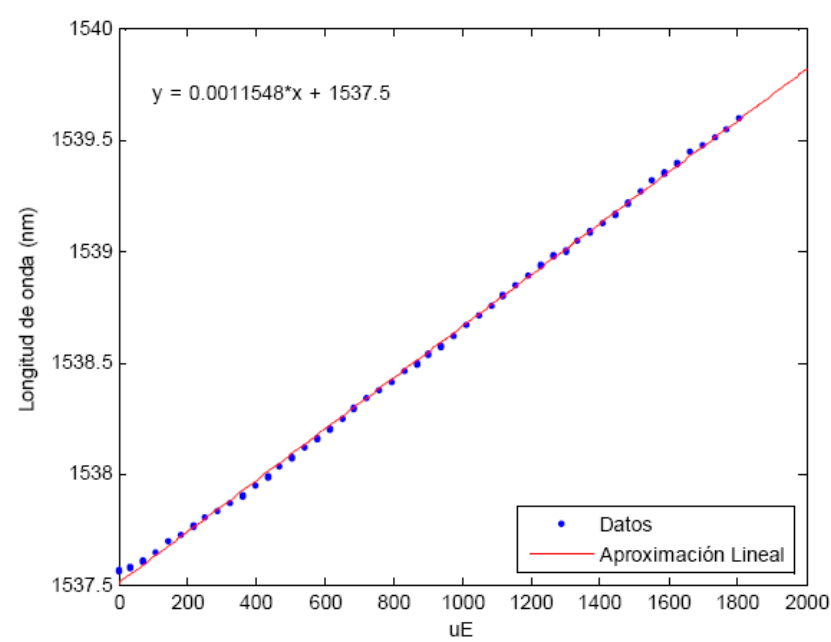

Figura 4.2 Ajuste de la constante de sensibilidad.

De esta forma, el coeficiente de sensibilidad de la deformación (pendiente de la recta de ajuste de la Figura 4.2) resulta ser aproximadamente de $1,15 \mathrm{pm} / \mu \varepsilon$.

Con este coeficiente de sensibilidad obtenido se llevaron a cabo campañas experimentales sobre elementos de hormigón sometidos a compresión y flexión, y sobre elementos metálicos sometidos a tracción. Los resultados demostraron la correcta obtención del coeficiente de sensibilidad de la deformación y su aptitud para la aplicación como sensor de deformación. Para mayor información sobre los ensayos y resultados obtenidos, véase Torres 2008 y Torres 2011 (véase Apéndice II).

\subsubsection{Calibración frente a temperaturas impuestas.}

Para la obtención del coeficiente de sensibilidad de la temperatura se registró la evolución de la longitud de onda al mismo tiempo que la evolución de la temperatura.

Inicialmente, estos ensayos se realizaron a partir de la temperatura ambiente que fue registrada empleando un termopar (Figura 4.3). El ajuste entre las curvas de la Figura 4.3 fue posible empleando un coeficiente de sensibilidad de $9 \mathrm{pm} /{ }^{\circ} \mathrm{C}$.

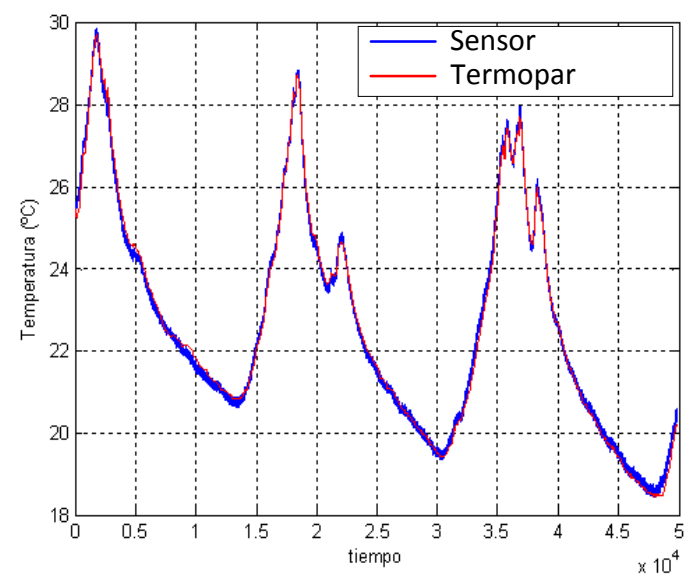

Figura 4.3 Evolución de la temperatura ambiente, comparación y calibración de los sensores ópticos. 
Monitorización estructural.

Aplicación con sensores de fibra óptica.

Pero en una segunda fase, las temperaturas impuestas fueron aplicadas a la FBG mediante el uso de una cámara climática de la marca Feutron (Figura 4.4), para abarcar así un mayor rango de temperaturas aplicadas.

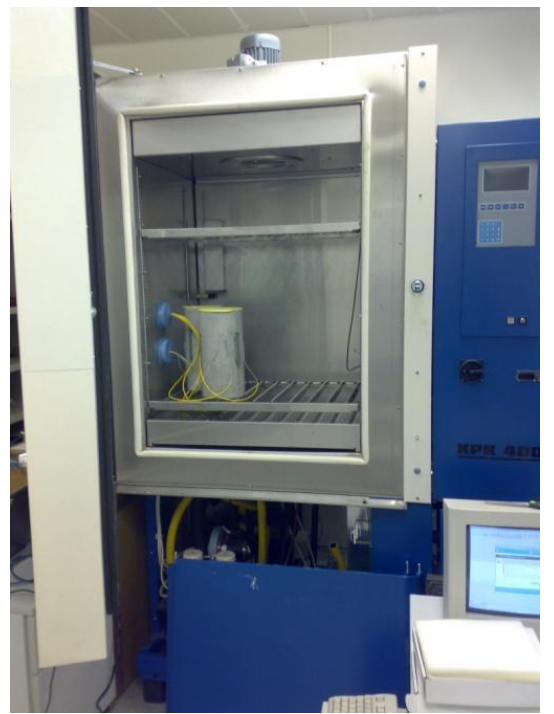

(a)

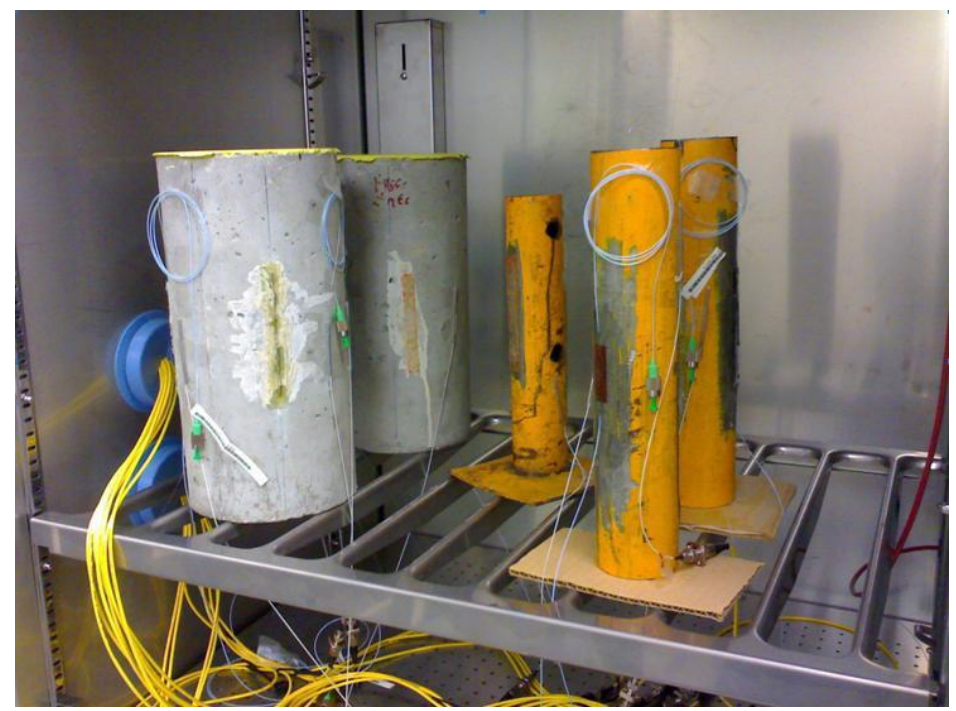

(b)

Figura 4.4 (a)- Cámara climática Feutron KPK 400. (b)- Probetas de hormigón y metálicas dispuestas en el interior de la cámara climática.

En el interior de la cámara climática se alojaron probetas de hormigón y metálicas, y se instalaron sensores de temperatura en ellas. Empleando el coeficiente de sensibilidad obtenido en la anterior prueba, se determinó la temperatura en el hormigón (Figura 4.5.a) y en el acero (Figura 4.5.b), y su resultado fue comparado con el termopar dispuesto en el interior de la cámara climática.

El coeficiente de sensibilidad obtenido de $9 \mathrm{pm} /{ }_{\mathrm{C}} \mathrm{C}$ proporciona un buen grado de ajuste para la obtención de las temperaturas en hormigón y acero.

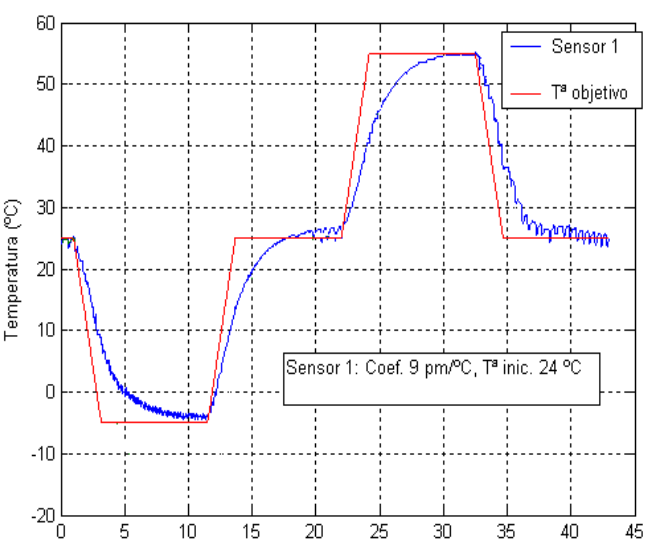

(a)

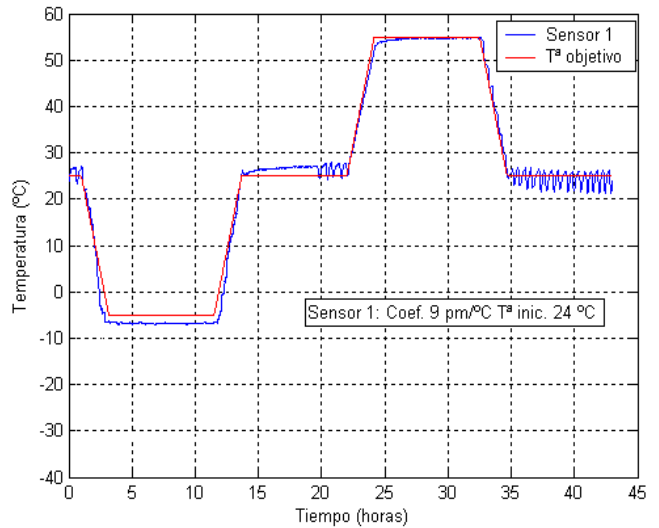

(b)

Figura 4.5 Ajustes térmicos para el sensor de temperatura. (a)- probeta de hormigón. (b)- probeta de acero. 
Dada la distinta naturaleza de los materiales ensayados, se observa un decalaje en la curva de temperatura del sensor instalado sobre la probeta de hormigón, debido a su inercia térmica.

Para mayor información sobre los ensayos y los resultados obtenidos, véase Torres 2008 y Torres 2011.

\subsection{Sensor FBG propuesto en la investigación.}

El sensor propuesto en la presente Tesis Doctoral consiste en una FBG protegida por capas de material compuesto en base de fibra de vidrio y resina de poliéster, con un peso de $300 \mathrm{~g} / \mathrm{m}^{2}$. (Figura 4.6). El sensor presenta unas dimensiones aproximadas de $100 \mathrm{~mm}$ de longitud, $10 \mathrm{~mm}$ de ancho y $0.6 \mathrm{~mm}$ de espesor, y se adhiere a la superficie de la estructura a monitorizar con una muy fina capa de adhesivo cuyas propiedades mecánicas se muestran en la Tabla 4.1.

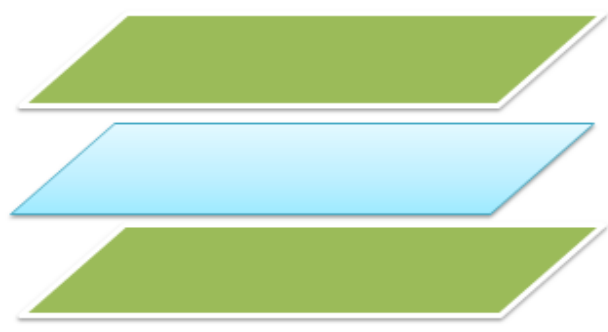

Resina de Poliéster.

Fibra de vidrio $\left(300 \mathrm{~g} / \mathrm{m}^{2}\right)$.

Resina de Poliéster.

Fibra óptica.

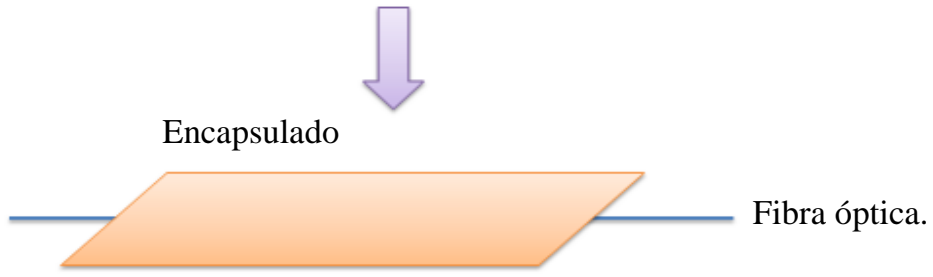

Figura 4.6 Sensor propuesto en la investigación. Vista 3D del encapsulado y fibra óptica.

\begin{tabular}{l|cc}
\hline \multicolumn{1}{c|}{ Propiedades } & Unidades & Valores \\
\hline Coeficiente de expansión térmica, $\alpha$ & ${ }^{0} \mathrm{C}^{-1}$ & $3,5-410^{-6}$ \\
Conductividad térmica & $\mathrm{Kcal}$ & $0.17\left(\mathrm{entre} 0 \ldots 50^{\circ} \mathrm{C}\right)$ \\
Módulo de elasticidad, tras endurecimiento. & $\mathrm{N} / \mathrm{mm}^{2}$ & 13000 \\
Resistencia eléctrica & $\Omega$ & $>10^{15}$ \\
Retracción durante el curado, depende proporciones & $\%$ & \\
$1: 4$ & \multicolumn{2}{|c}{4} \\
$1: 2$ & \multicolumn{2}{c}{10} \\
$1: 1$ & ${ }^{\circ} \mathrm{C}$ & $-200 \ldots+80$ \\
Límites térmicos para medida de deformaciones: & & \\
\hline
\end{tabular}

Tabla 4.1 Especificaciones técnicas del adhesivo

Es importante tener en cuenta las siguientes consideraciones:

1. La FBG se sitúa en una de las caras del encapsulado, para asegurar que se encuentra lo más cercana posible de la estructura a monitorizar. 
2. Durante el curado de la resina de poliéster se produce un fenómeno exotérmico que desprende calor. Este incremento de temperatura produce una variación en la longitud de onda de Bragg $\Delta \lambda_{B}$ reflejada por la FBG. Para el sensor propuesto y estudiado en esta Tesis Doctoral, esta variación tras de 130 minutos de curado de la resina es de un valor máximo de 100 pm (Figura 4.7). Este valor representa sólo un $2 \%$ de la distancia entre dos longitudes de onda consecutivas (este valor se encuentra alrededor de 5000pm) que pueden reflejar dos sensores colocados en serie. Además, este valor no tiene ninguna afección sobre el comportamiento y precisión del sistema.

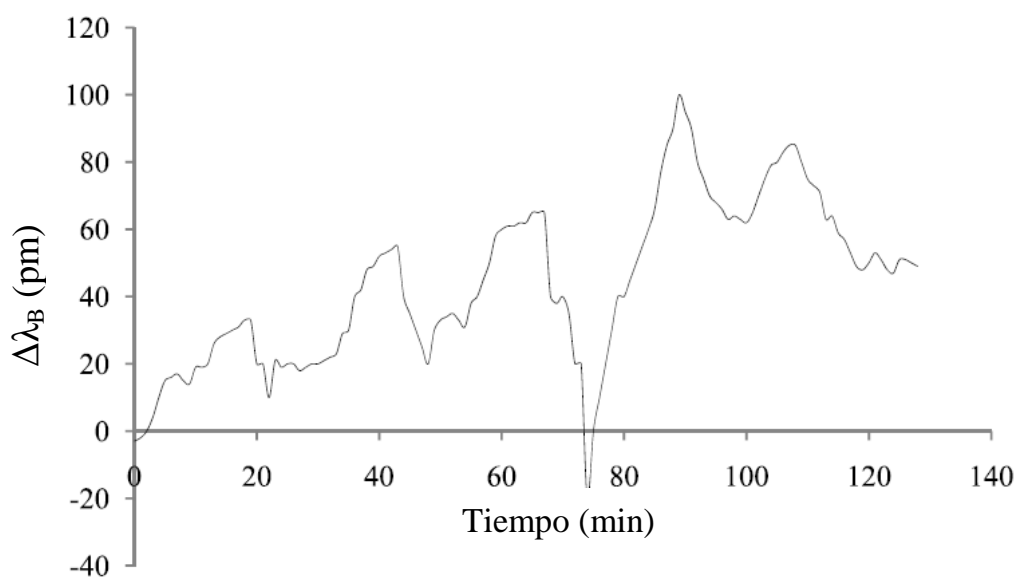

Figura 4.7 Variación en la longitud de onda de Bragg $\Delta \lambda_{B}$ durante el encapsulado del sensor.

3. El diseño del encapsulado minimiza la variación del coeficiente de sensibilidad de la deformación debido al diseño propuesto.

La validez del sensor propuesto fue comprobada con ensayos experimentales comparando las medidas de los sensores ópticos con las medidas que proporcionaban galgas extensométricas situadas junto a los anteriores. Todos los sensores fueron instalados en la superficie de elementos metálicos o elementos de hormigón, y fueron sometidos a esfuerzos de tracción, compresión y flexión. Las lecturas de los sensores FBG fueron muy similares a las que ofrecieron las galgas extensométricas, con una diferencia máxima de un 3\% entre ambas. Una descripción más detallada sobre los ensayos experimentales y sus resultados han sido descritos en Barrera 2007, Torres 2008 y Torres 2011 y sus resultados no se presentan en este trabajo.

Las propiedades mecánicas del material que forma el encapsulado del sensor, especialmente su Módulo de Elasticidad $\left(E_{p}\right)$ y coeficiente de Poisson $\left(v_{p}\right)$, son necesarias para llevar a cabo el análisis numérico que se expondrá más adelante. Sin embargo, estas propiedades no son conocidas inicialmente, ya que dependen de las características de la fibra de vidrio y la resina de poliéster empleadas en el encapsulado.

Para conocerlas se ensayaron seis probetas con la geometría indicada en la Figura 4.8.a y cada una de ellas, de dimensiones $200 \mathrm{~mm}$ X $20 \mathrm{~mm}$ X $0.5 \mathrm{~mm}$, fue ensayada a tracción hasta la rotura de la misma. Las deformaciones verticales y horizontales que experimentó la probeta fueron medidas empleando galgas extensométricas dispuestas según la Figura 4.8.b. Además, también se registró la carga aplicada sobre las muestras. 


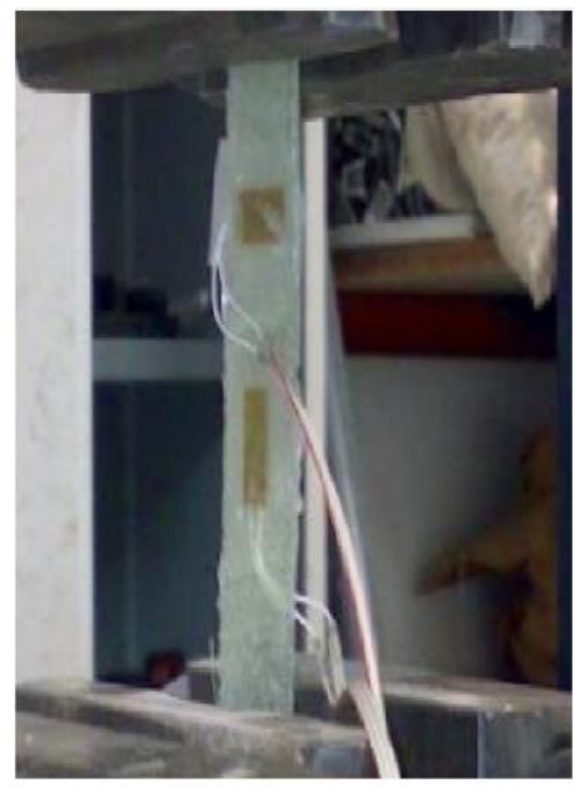

(a)

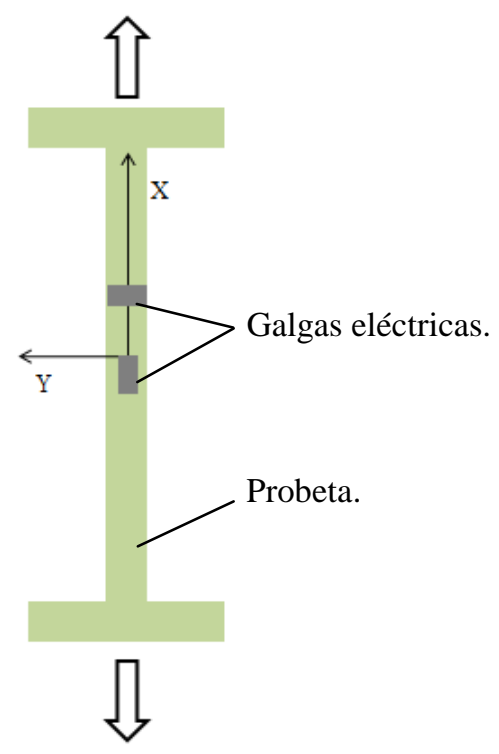

(b)

Figura 4.8 (a)- Ensayo de tracción sobre una probeta fabricada con el material del ensapculado. (b)-

Esquema que muestra la dirección de aplicación de la carga y la orientación de las galgas extensométricas. La probeta presenta forma de I, para facilitar una mejor aplicación de las cargas en cabeza.

La pendiente del tramo recto inicial de la curva tensión-deformación proporciona el valor del Módulo de Elasticidad $E_{p}$, mientras que el Coeficiente de Poisson ha sido obtenido a partir de las ecuaciones 4.1 y 4.2 (Véase ASTM 2004a y ASTM 2004b).

$\varepsilon_{y}=\frac{1}{E}\left\{\sigma_{y}-v\left(\sigma_{x}+\sigma_{z}\right)\right\}$

$v=-\frac{\varepsilon_{y}}{\varepsilon_{x}}$

La Tabla 4.2 proporciona los valores de $E_{p}$ y $v_{p}$ obtenidos a partir de los ensayos realizados. Estos valores se encuentran en el orden de magnitud de los encontrados en la literatura existente (Exequiel, 2003), a pesar de que la probeta número 1 proporcionó un valor del módulo de elasticidad superior al resto. Este hecho se debió a una mayor cantidad de resina de poliéster en esta probeta.

\begin{tabular}{c|cc}
\hline Probeta & Módulo de Elasticidad $\boldsymbol{E}_{\boldsymbol{p}}(\boldsymbol{G P a})$ & Coef. Poisson $\boldsymbol{v}_{\boldsymbol{p}}$ \\
\hline 1 & 16.6 & $0.36-0.39$ \\
2 & 13.4 & $0.29-0.4$ \\
3 & 12.8 & $0.31-0.38$ \\
4 & 11.3 & $0.33-0.4$ \\
5 & 13.1 & $0.29-0.36$ \\
6 & 12.7 & $0.33-0.4$ \\
\hline
\end{tabular}

Tabla 4.2 Propiedades mecánicas del material compuesto por fibra de vidrio y resina de poliéster empleado en los ensayos. 
Para llevar a cabo el análisis numérico, se han adoptado valores de $E_{p}$ y $v_{p}$ de 11 GPa y 0.4 respectivamente. Estos valores adoptados son los más desfavorables para el análisis efectuado, tal y como se justifica en un apartado posterior.

\subsection{Estudio numérico del sensor.}

\subsubsection{Introducción:}

Los datos obtenidos por un sensor fijado a una estructura a través de una capa de adhesivo pueden verse afectados por el espesor y las propiedades mecánicas del adhesivo, y por este motivo es importante cuantificar esta afección sobre las medidas.

Mediante un estudio experimental exhaustivo sería posible analizar la influencia de estos parámetros sobre las mediciones del sensor. Sin embargo, en este caso se requerirían unas inversiones temporales y económicas considerables, ya que el control de estos parámetros en laboratorio supondría una tarea que no resultaría fácil.

En el trabajo que engloba esta Tesis Doctoral se ha estudiado un modelo numérico de elementos finitos (en adelante MEF) que modeliza el elemento sensor y el adhesivo. En este modelo, se impone una deformación en la superficie monitorizada, y se estudia la transferencia de deformaciones en:

1. A través del adhesivo hasta la fibra óptica, en el caso del sensor óptico descrito en este trabajo.

2. A través del adhesivo y de la capa inferior de encapsulado hasta la fibra óptica, cuando el modelo numérico se emplea para estudiar la configuración del sensor donde la fibra óptica se encuentra embebida entre dos capas de material (Figura 4.9).

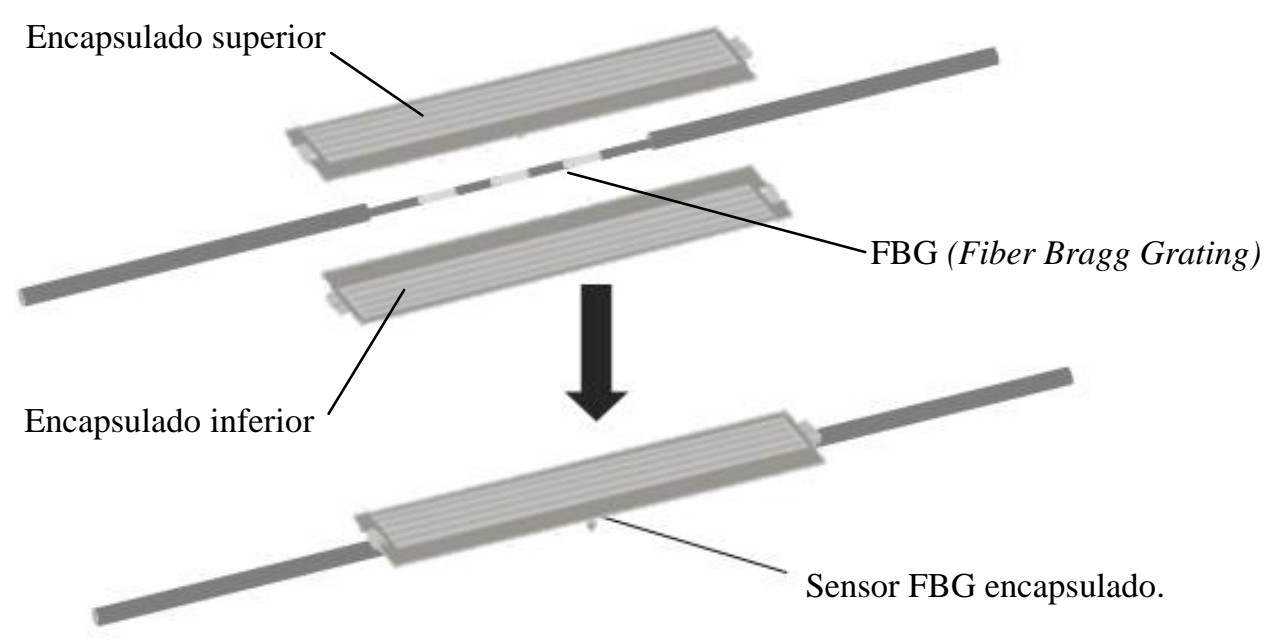

Figura 4.9 Diseño habitual de sensores ópticos de deformación (Adaptado de Moyo et al. 2005).

\subsubsection{Condiciones de contorno, descripción de los elementos empleados y aplicación de la carga:}

El modelo numérico fue estudiado empleando el programa de elementos finitos Lusas V.14. (Lusas, 2010). Debido a la simetría de cargas aplicadas y geometría, se modelizó un cuarto de elemento sensor para reducir de esta forma el coste computacional del cálculo. La Figura 4.10.a muestra los planos de simetría considerados y el modelo estructural de cálculo (Figura 4.10.b). Además de las condiciones 
de contorno derivadas de la simetría, se impidió el movimiento vertical en la cara inferior del adhesivo.

(a)

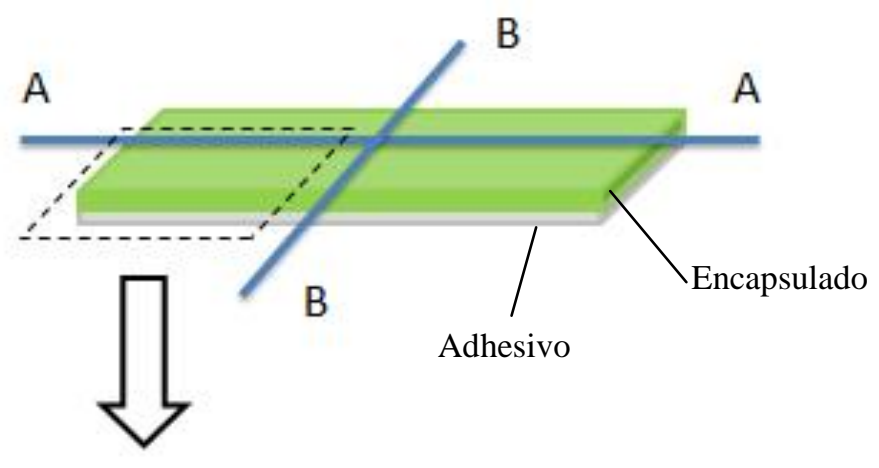

(b)

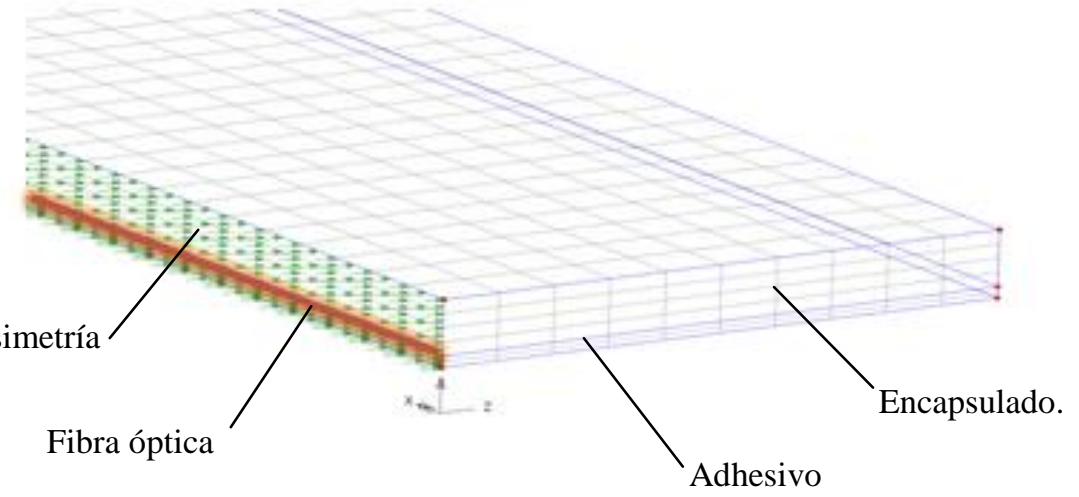

Figura 4.10 (a)- Vista 3D del encapsulado sensor y adhesivo mostrando sus planos de simetría. (b)Vista de una parte del MEF estudiado.

La fibra óptica que forma el sensor se modeliza mediante elementos lineales tipo BRS3 (Figura 4.11.a), los cuales están formado por tres nodos, con 3 grados de libertad por nodo (traslaciones en $X$, $\mathrm{Y}, \mathrm{Z})$.

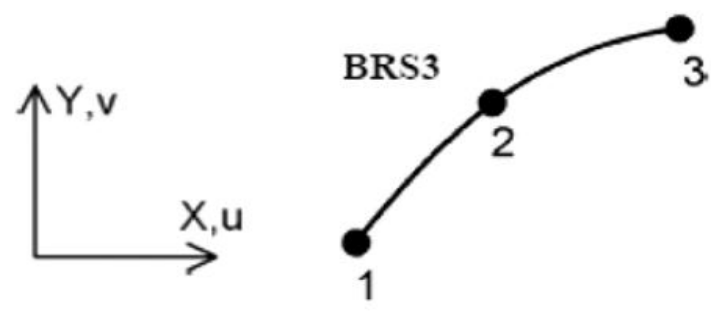

(a)

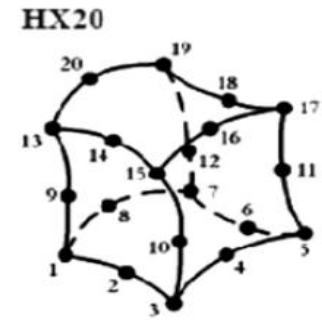

(b)

Figura 4.11 (a)- Elemento BRS3. (b)- Elemento HX20. (Lusas, 2010).

El adhesivo y el encapsulado están modelizados mediante elementos sólidos 3D HX20 (Figura 4.11.b), formado por 20 nodos, con 3 grados de libertad por nodo (traslaciones en X, Y, Z). Todos los elementos modelados tienen un comportamiento elástico y lineal.

El contacto entre la superficie del encapsulado y la superficie del adhesivo se modeliza como contacto fijo (coincidiendo geométricamente los nodos de ambos elementos). No se han considerado elementos 
Monitorización estructural.

Aplicación con sensores de fibra óptica.

de contacto en esta superficie puesto que no se producen movimientos relativos en ella. Esta hipótesis se corresponde con lo observado en el laboratorio, en el que no se producía despegue en esa zona.

Para estudiar la transferencia de deformaciones entre la estructura instrumentada y la fibra óptica, se aplica una deformación en la cara inferior del adhesivo, en contacto con la estructura. Esta deformación se impone aplicando un incremento de temperatura de acuerdo a la ecuación 4.3.

$\varepsilon=\alpha \Delta T$

Donde $\alpha$ es el coeficiente de expansión térmica del material y $\varepsilon$ la deformación impuesta.

Para que la deformación impuesta afectara sólo al eje longitudinal del sensor, el adhesivo se ha considerado como un material ortótropo con las propiedades indicadas en la Tabla 4.3, considerando el mismo Módulo de Elasticidad en las tres direcciones X, Y y Z, y el coeficiente de expansión térmica sólo en la dirección longitudinal X.

\begin{tabular}{c|cc}
\hline Propiedades. & Unidades & Valor \\
\hline Módulo de Elasticidad X & $\mathrm{GPa}$ & 13 \\
Módulo de Elasticidad Y & $\mathrm{GPa}$ & 13 \\
Módulo Rigidez Transversal XY & $\mathrm{GPa}$ & 0 \\
Coeficiente Poisson XY & - & 0.2 \\
Ángulo de ortotropía & $\circ$ & 0 \\
Densidad & $\mathrm{GN} / \mathrm{m}^{3}$ & $210^{-5}$ \\
Coeficiente dilatación térmica X & ${ }^{0} \mathrm{C}^{-1}$ & $0.03510^{-4}$ \\
Coeficiente dilatación térmica Y & ${ }^{0} \mathrm{C}^{-1}$ & 0 \\
Coeficiente dilatación térmica Z & ${ }^{0} \mathrm{C}^{-1}$ & 0 \\
\hline
\end{tabular}

Tabla 4.3 Propiedades mecánicas del adhesivo adoptadas en el MEF (Fuente: HBM, 2006).

Dado que el fabricante del adhesivo no proporciona datos relativos al Coeficiente de Poisson $v_{A}$, se ha llevado a cabo un estudio de sensibilidad de este parámetro (Apartado 4.4.3.b).

\subsubsection{Resultados del estudio numérico:}

\section{a) Comportamiento general.}

Las mallas deformadas de todos los modelos mostraron la misma pauta general de comportamiento, existiendo un gradiente de deformaciones entre la superficie instrumentada y el plano que contiene la fibra óptica. A mayor espesor de adhesivo adoptado, mayor gradiente se produce (Figura 4.12) y se evalúa a partir del parámetro $\Delta \varepsilon(\%)$ (ecuación 4.4$)$ :

$\Delta \varepsilon(\%)=\frac{\varepsilon_{h}-\varepsilon_{f}}{\varepsilon_{h}} * 100$

En la ecuación 4.4 anterior, $\varepsilon_{h}$ y $\varepsilon_{f}$ representan las deformaciones de la superficie de la estructura instrumentada y de la fibra óptica, respectivamente. $\Delta \varepsilon(\%)$ representa una medida del error relativo del sensor de deformación, de forma que valores cercanos a 0 indican una perfecta transferencia de deformaciones entre la estructura y la fibra óptica. 

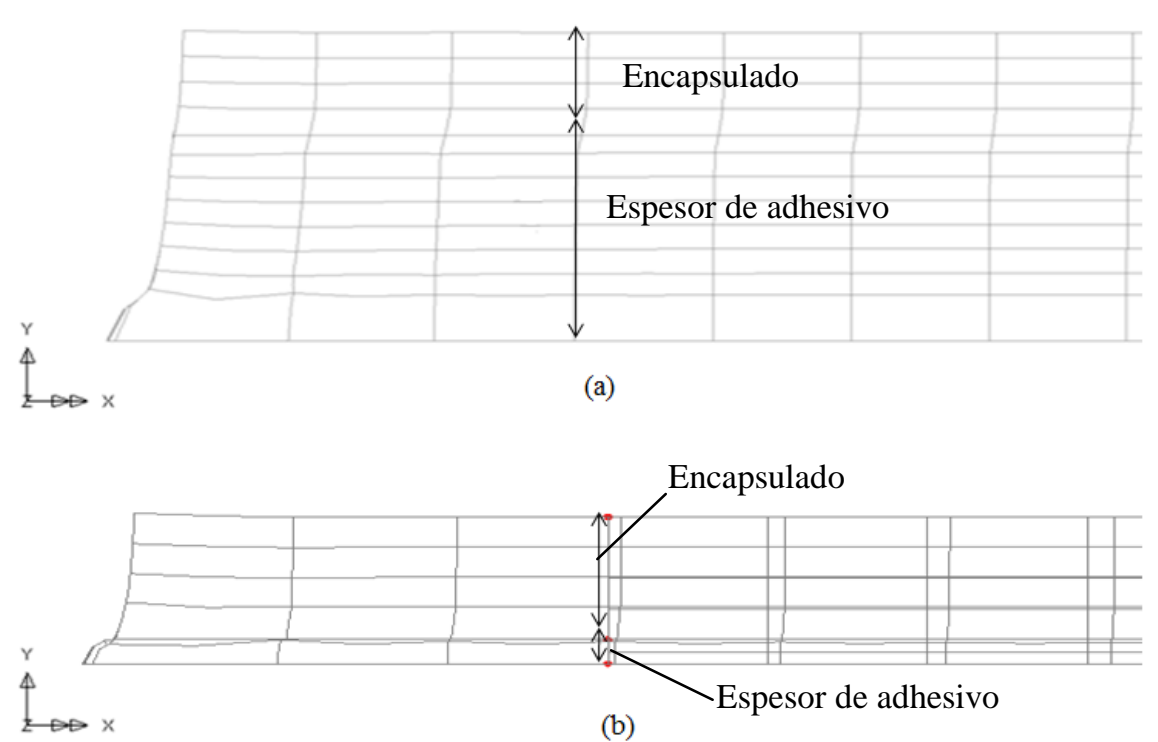

Figura 4.12 Mallas deformadas del modelo de elementos finitos para un espesor de adhesivo de (a)- 1000 $\mu \mathrm{m},(\mathrm{b})-100 \mu \mathrm{m}$.

b) Estudios de sensibilidad del Módulo de Elasticidad y Coeficiente de Poisson del encapsulado, $E_{p} y v_{p}$

Como los resultados de los ensayos experimentales sobre el material compuesto que se indican en la Tabla 4.2 arrojaron dispersión en sus valores, se llevó a cabo un análisis de sensibilidad que consideró valores de $E_{p}$ comprendidos entre 11 y $17 \mathrm{GPa}$, valores de $v_{p}$ comprendidos entre 0.29 y 0.4 y dos posibles valores del coeficiente de Poission del adhesivo $v_{A}(0.1$ y 0.4$)$

El objetivo de este estudio fue obtener los valores de estos parámetros a adoptar para los posteriores estudios numéricos que se llevaron a cabo. De acuerdo a esto, se adoptaron valores de $E_{p}$ y $v_{p}$ igual a $11 \mathrm{GPa}$ y 0.4 puesto que son aquellos que maximizan el parámetro de error relativo $\Delta \varepsilon$ (\%). Las Figuras 4.13 y 4.14 resumen los resultados obtenidos. 


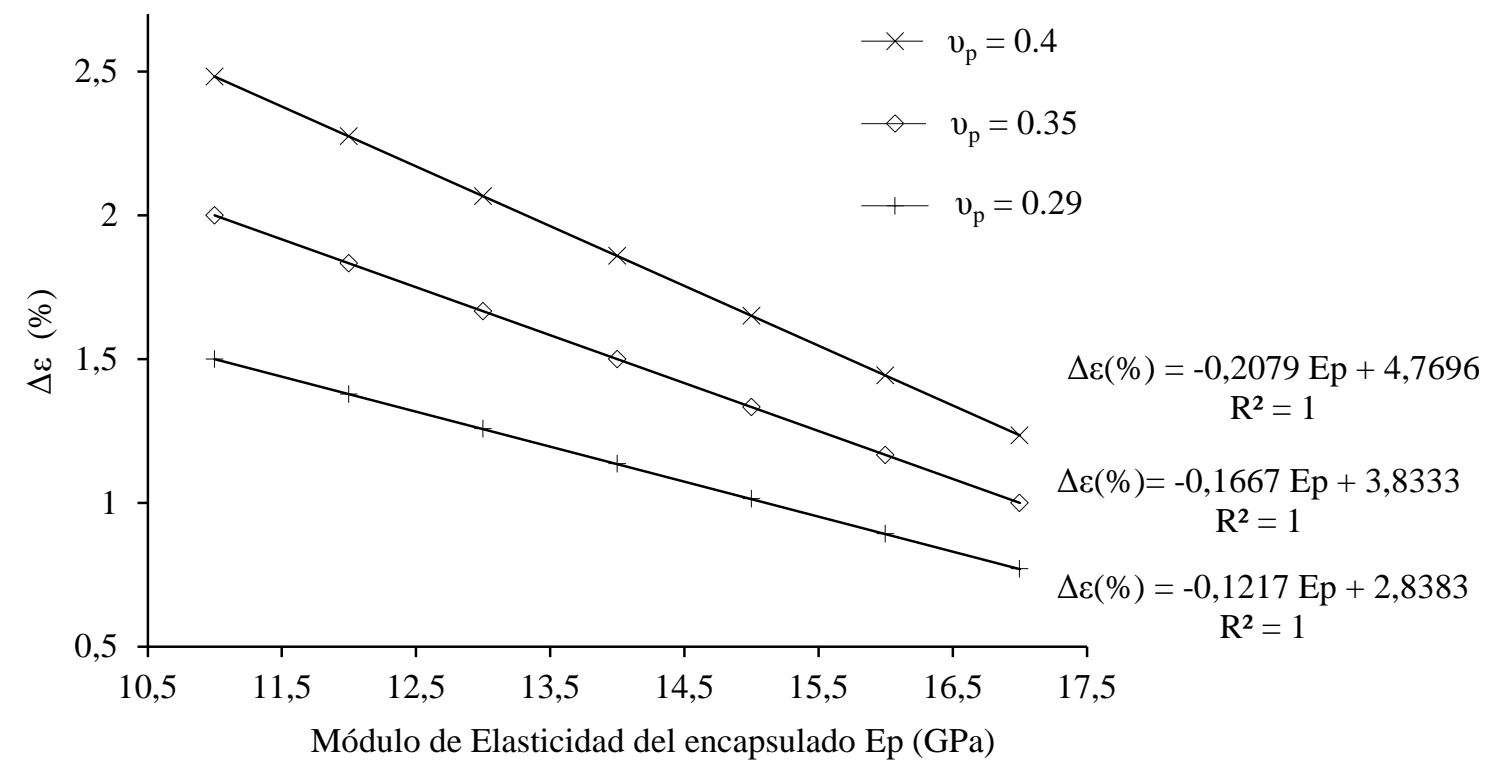

Figura 4.13 Relación entre el gradiente de deformaciones $\Delta \varepsilon$ (\%) y el Módulo de Elasticidad del material del encapsulado $E_{p}$, para diferentes valores del Coeficiente de Poisson del encapsulado $v_{p}$. Espesor de adhesivo adoptado $400 \mu \mathrm{m}$. Coeficiente de Poisson del adhesivo $v_{A}=0.4$.

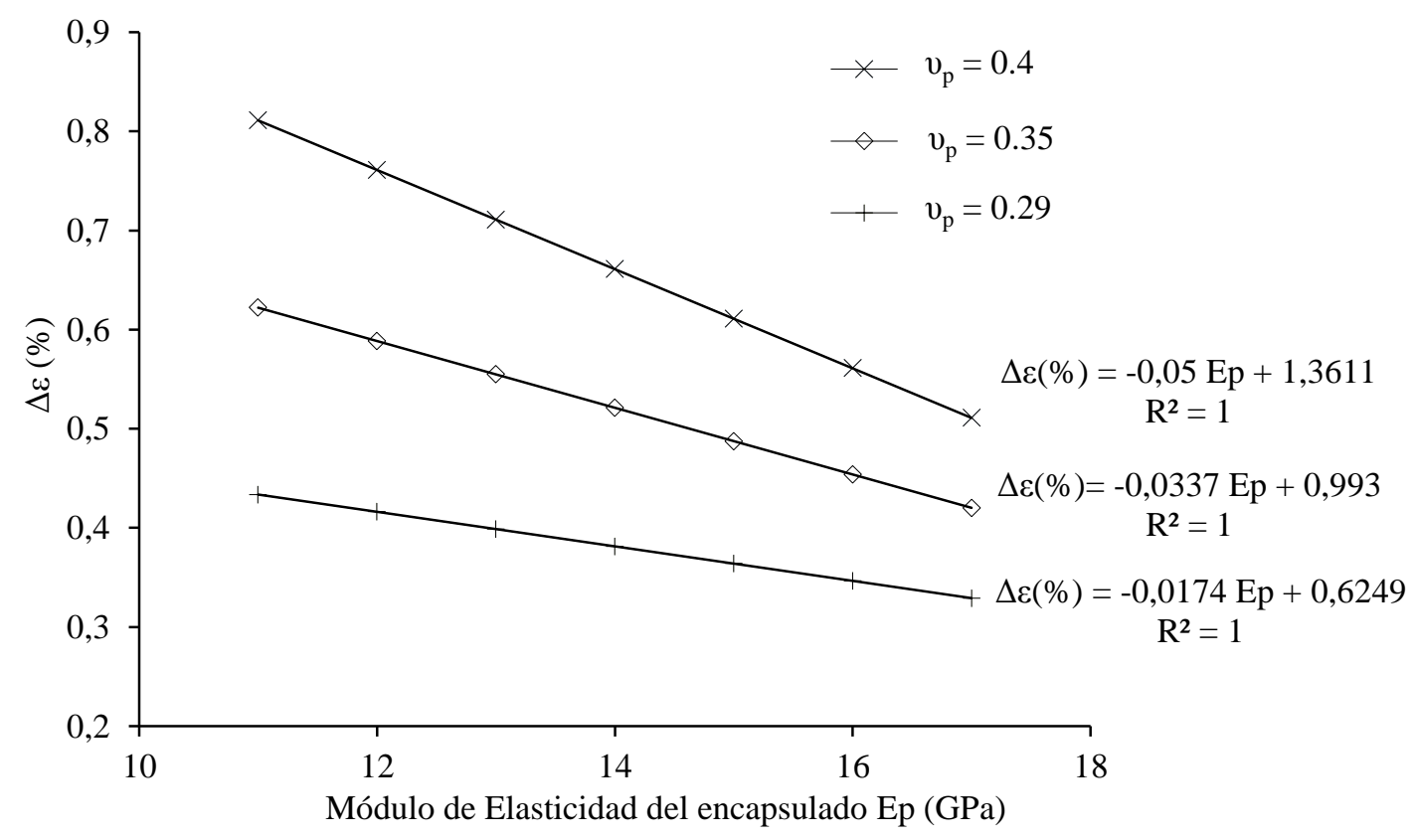

Figura 4.14 Relación entre el gradiente de deformaciones $\Delta \varepsilon$ (\%) y el Módulo de Elasticidad del material del encapsulado $E_{p}$, para diferentes valores del Coeficiente de Poisson del encapsulado $v_{p}$.

Espesor de adhesivo adoptado $400 \mu \mathrm{m}$. Coeficiente de Poisson del adhesive $v_{A}=0.1$. 
c) Análisis de sensibilidad del espesor de adhesivo (t).

Para estudiar la influencia del espesor de adhesivo sobre las medidas del sensor se llevó a cabo un estudio de sensibilidad de este parámetro.

Por un lado la Figura 4.15 muestra, en una sección transversal, la deformación en la dirección X longitudinal a lo largo del sensor, correspondiente a un espesor de adhesivo de $100 \mu \mathrm{m}$.

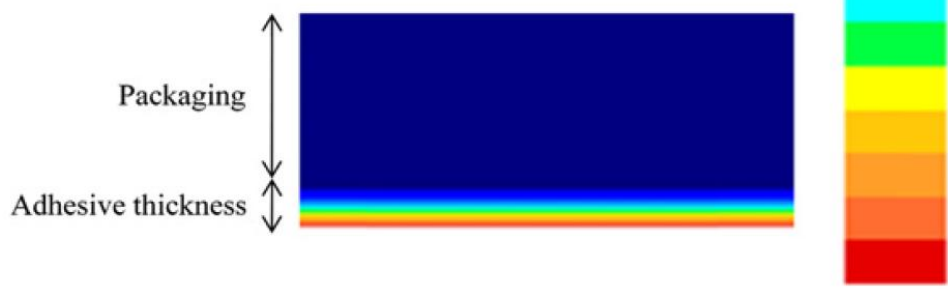

Figura 4.15 Sección transversal del sensor mostrando las deformaciones en el eje X. Espesor de adhesivo $100 \mu \mathrm{m}$. Coeficiente de Poisson del adhesivo $v_{A}=0.4$.

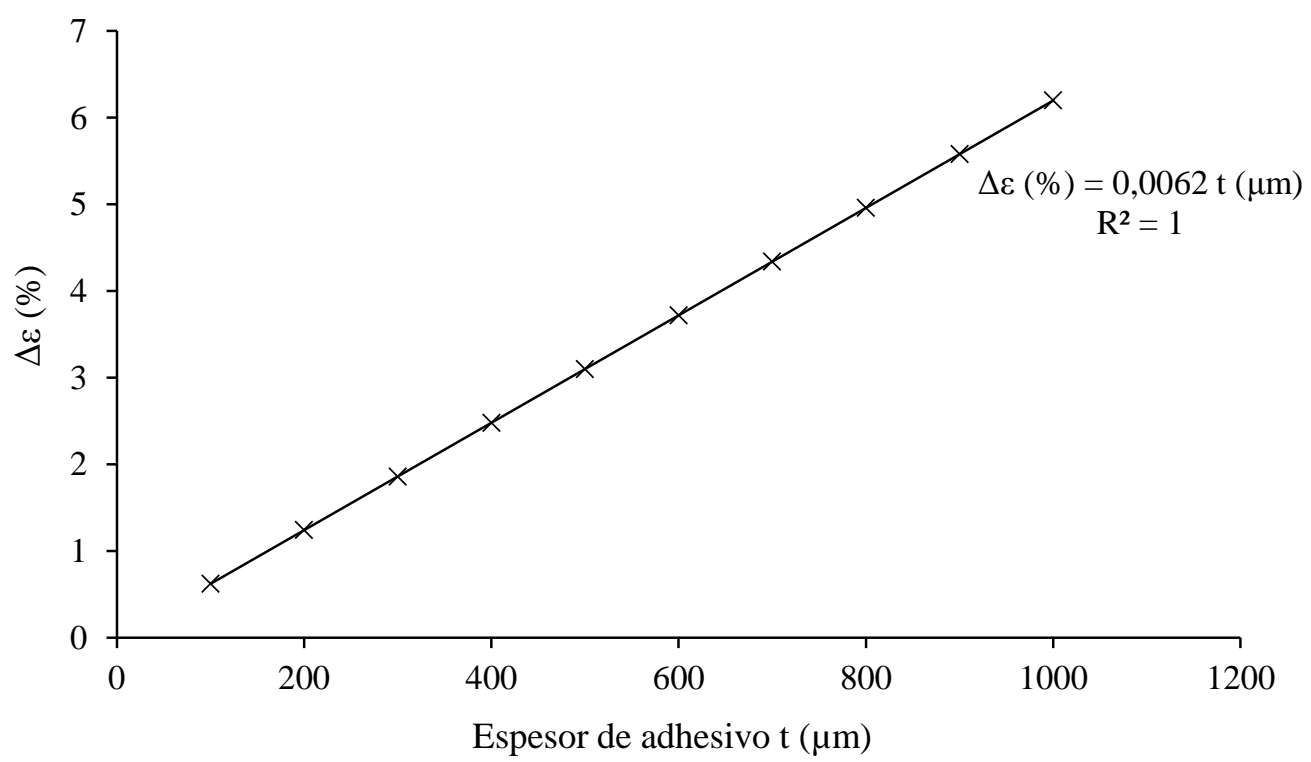

Figura 4.16 Relación entre el gradiente de deformaciones $\Delta \varepsilon$ (\%) y el espesor de adhesivo $\mathrm{t}(\mu \mathrm{m})$. Coeficiente de Poisson del adhesivo $v_{A}=0.4$.

Por otro lado, la Figura 4.16 representa el valor del parámetro $\Delta \varepsilon(\%)$ en función del espesor de adhesivo. Tal y como se observa, el error relativo que se asume empleando el sensor propuesto en este trabajo es muy pequeño. Por ejemplo, para valores habituales de espesor de adhesivo de $400 \mu \mathrm{m}$ (Figura 4.17), se observa que el error cometido es inferior a un 3\%. Es importante destacar que, incluso con espesores de adhesivo mucho mayores $(1000 \mu \mathrm{m})$, el error que se asume con el sensor propuesto en esta investigación (menos que un 6.5\%) sigue siendo muy inferior si se compara con el 
Monitorización estructural.

Aplicación con sensores de fibra óptica.

error que se comete al emplear un sensor de dos capas (la fibra óptica queda embebida entre dos capas de material). Este resultado se presentará en un apartado posterior.

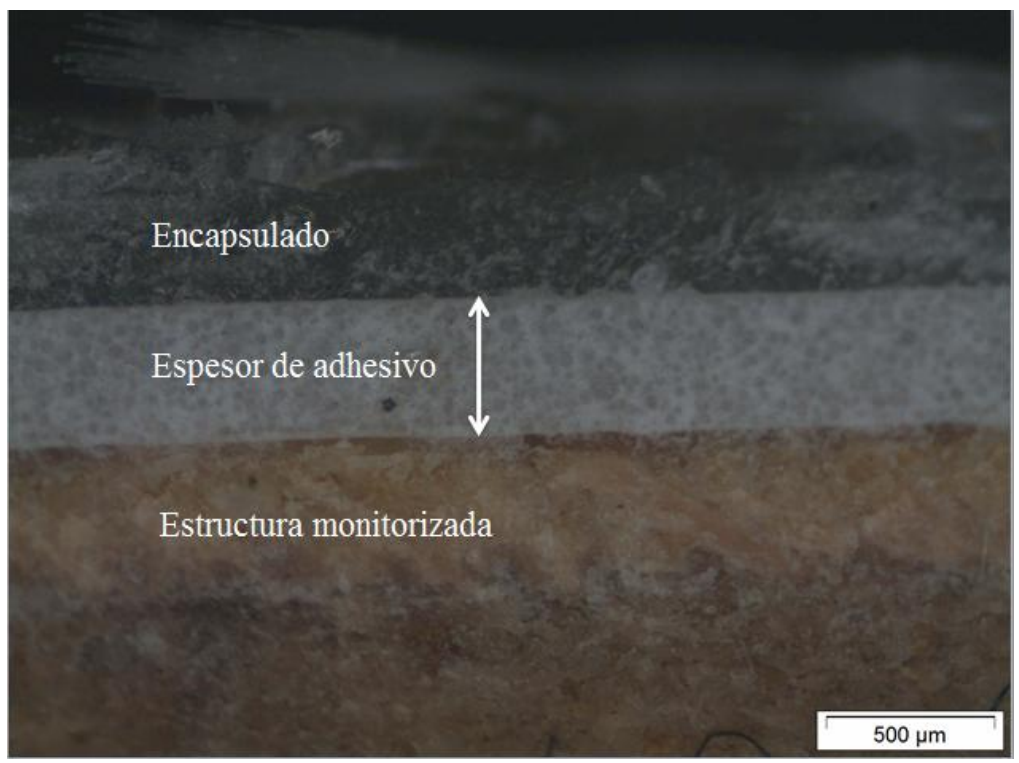

Figura 4.17 Sección transversal del sensor adherido a una superficie. La imagen fue tomada con un microscopio de alta resolución StereoZoom SZ61 (Altra, 1999) y procesada con el software AnalySIS getIT Olympus (Olympus, 2006).

Es importante destacar que valores de espesores de adhesivo de $1000 \mu \mathrm{m}$ indican una deficiente operación de pegado del sensor, y en estos casos, es preferible su retirada y proceder a una nueva instalación.

\section{d) Análisis de sensibilidad del Coeficiente de Poisson del adhesivo $v_{A}$.}

Tal y como se indicó anteriormente, la Tabla 4.1 contiene las características técnicas del adhesivo empleado en este estudio (HBM, 2006). Como el fabricante no proporciona el valor del Coeficiente de Poisson del adhesivo, se ha llevado a cabo un análisis de sensibilidad para estudiar la influencia de este parámetro en el error relativo $\Delta \varepsilon(\%)$

La Figura 4.18 muestra la variación del error relativo $\Delta \varepsilon(\%)$ en función del parámetro $v_{A}$ para un espesor de la capa de adhesivo de $400 \mu \mathrm{m}$. La ecuación 4.5 proporciona una relación exponencial obtenida de un análisis de regresión, que ajusta con los datos con un coeficiente de correlación de 0.9818 , indicando de este modo un muy buen ajuste con los datos.

$\Delta \varepsilon(\%)=0.5702 e^{3.7322 v_{A}}$ 


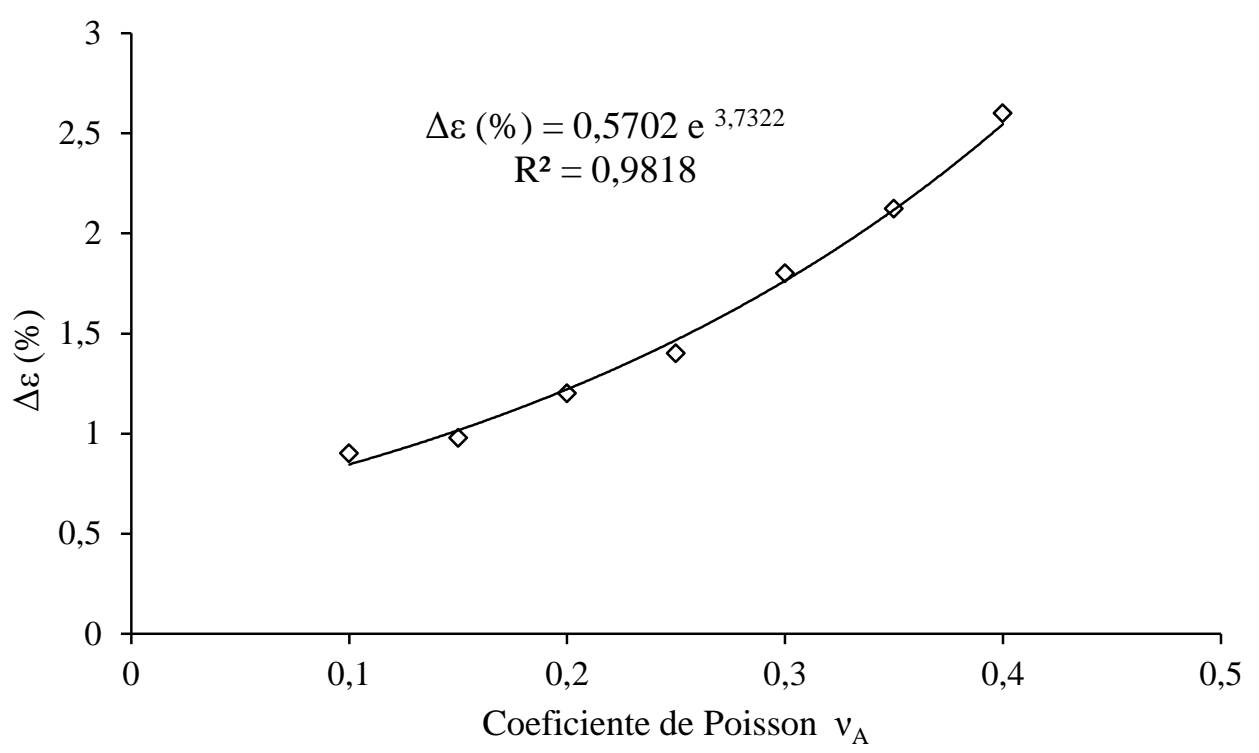

Figura 4.18 Relación exponencial entre el gradiente de deformaciones $\Delta \varepsilon$ (\%) y el coeficiente de Poisson del adhesivo $v_{A}$. Espesor de la capa de adhesivo $\mathrm{t}=400 \mu \mathrm{m}$.

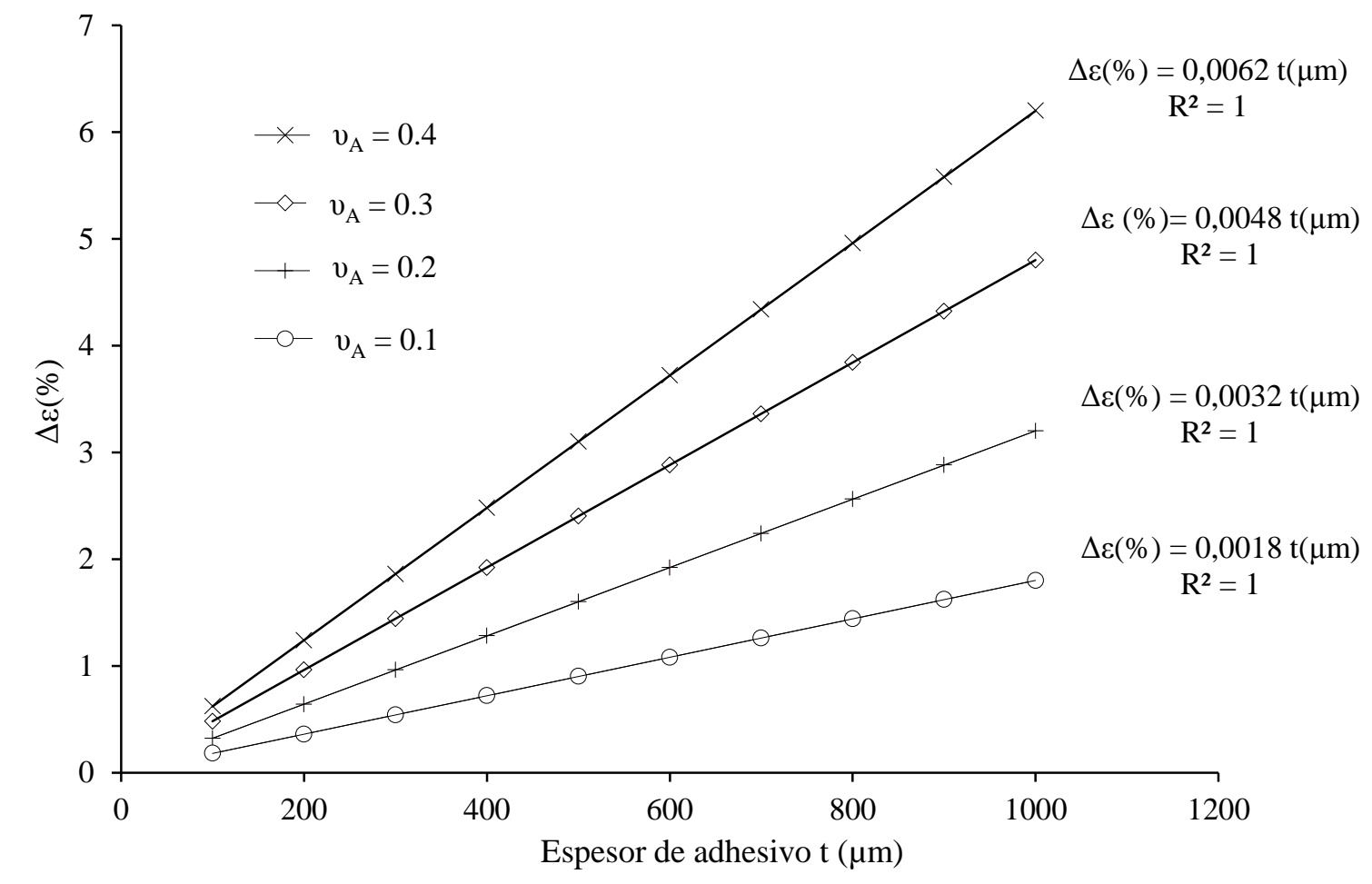

Figura 4.19 Relación entre el gradiente de deformaciones $\Delta \varepsilon$ (\%) y el espesor de adhesivo $\mathrm{t}(\mu \mathrm{m})$ para diferentes valores del coeficiente de Poisson del adhesivo $v_{A}$.

La Figura 4.19 muestra la variación del error $\Delta \varepsilon(\%)$ en función del espesor de adhesivo $\mathrm{t}$ (se han empleado los más frecuentemente empleados en las operaciones de instalación de sensores) y de diferentes Coeficientes de Poisson del adhesivo $v_{A}$. El valor máximo de $\Delta \varepsilon(\%)$ en la situación más desfvorable y para un espesor de adhesivo habitual de $400 \mu \mathrm{m}$ no excede el valor de $3 \%$. 
Monitorización estructural.

Aplicación con sensores de fibra óptica.

e) Comparación del sensor propuesto con otros sistemas de encapsulado.

En un encapsulado habitual la fibra óptica se encuentra embebida entre dos capas de material (Moyo et al 2005, Majumder et al. 2008), y por este motivo, se consideró importante estudiar la transferencia de deformaciones hasta la fibra óptica en esta configuración. Para llevar a cabo este apartado, se estudió un nuevo modelo numérico de un sensor genérico con dos capas de material empleando para ello los elementos y las propiedades mecánicas descritas en la sección 4.3 (Figura 4.20).

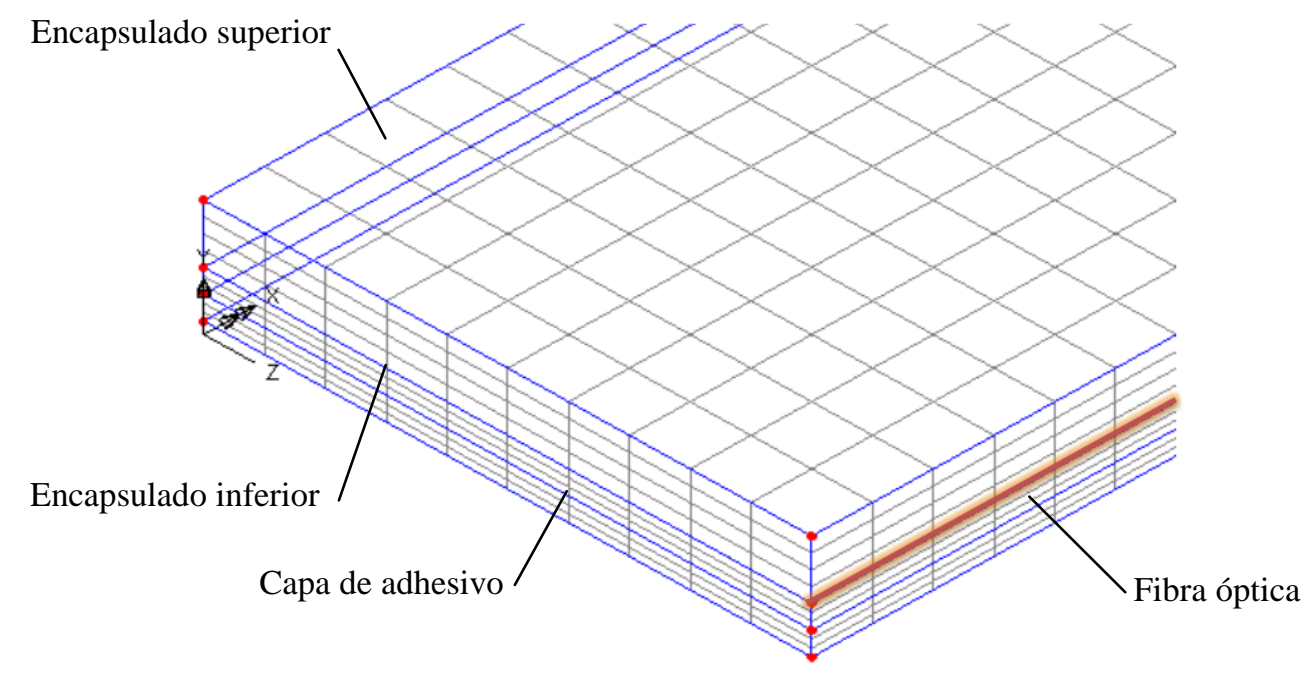

Figura 4.20 Vista 3D del modelo de elementos finitos empleado para analizar el sensor genérico con dos capas.

En este caso, se estudiaron varios modelos numéricos con valores del espesor de adhesivo $\mathrm{t}$ comprendidos entre 100 y $1000 \mu \mathrm{m}$, y valores de la capa inferior de encapsulado comprendidos entre 100 у $700 \mu \mathrm{m}$.

Como se observa en la Figura 4.21, en este caso los resultados del error relativo $\Delta \varepsilon$ (\%) son muy superiores que en el caso del sensor descrito en el apartado 4.3. Por ejemplo, para un espesor de adhesivo de $400 \mu \mathrm{m}$, las lecturas del sensor genérico de dos capas modelizado darían errores relativos de $3.82 \%, 7.71 \%$ y $15.46 \%$ para espesores de la capa inferior de encapsulado de 100, 400 y $700 \mu \mathrm{m}$ respectivamente. Estos valores son un $35.1 \%, 67.8 \%$ y $83.9 \%$ más que los obtenidos con el diseño del sensor objeto de este trabajo. Es importante destacar que las medidas en el sensor genérico de dos capas estarán fuertemente influenciadas por las propiedades de la capa inferior de encapsulado (por ejemplo, cambios en su coeficiente de Poisson debido a variaciones de temperatura). En el caso del sensor propuesto en la presente Tesis Doctoral, esta capa inferior de encapsulado es inexistente, por lo que automáticamente se elimina esta fuente de error. 


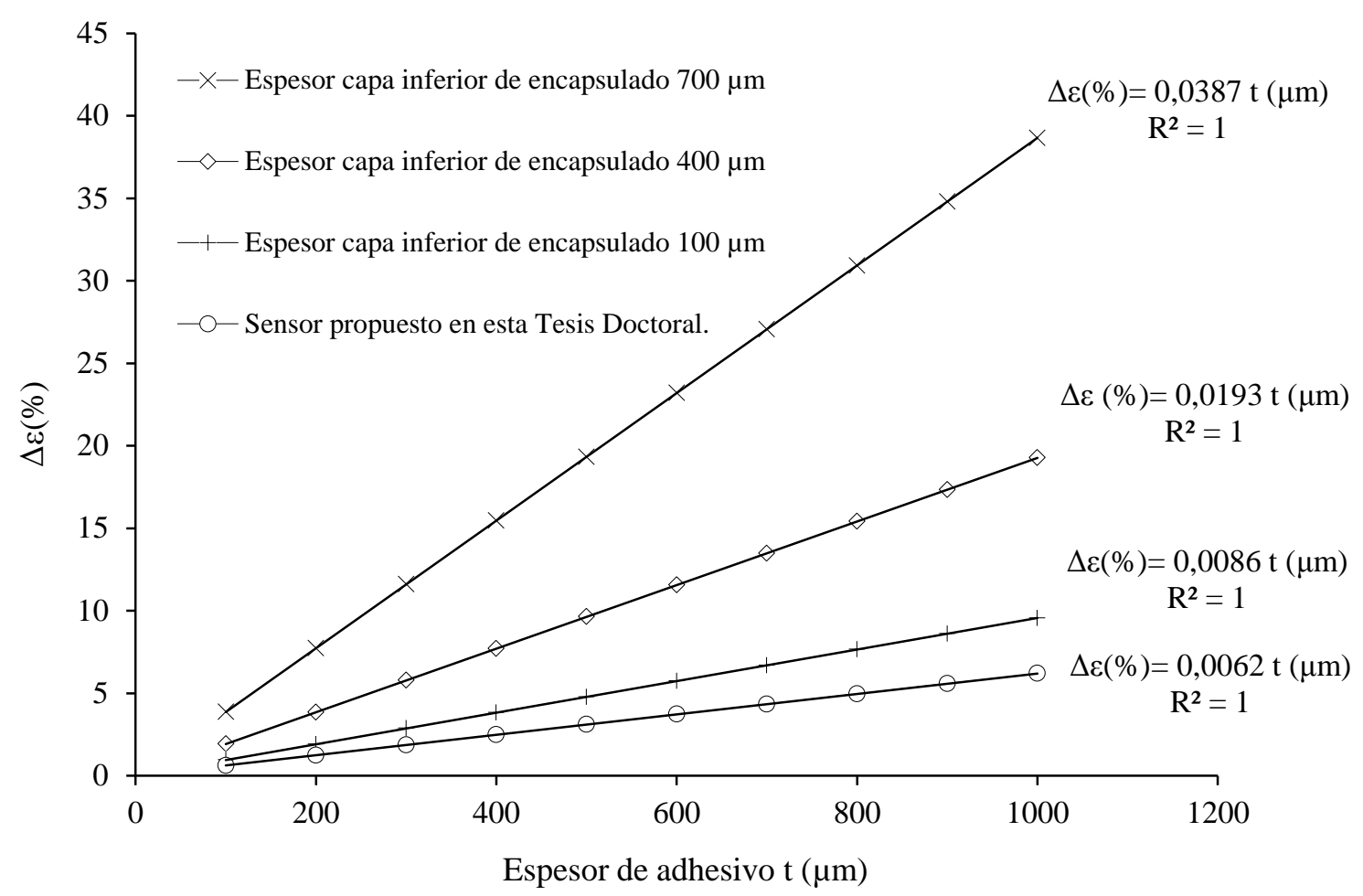

Figura 4.21 Comparación entre el sensor propuesto en este trabajo y un sensor genérico de dos capas. Relación entre el gradiente de deformación $\Delta \varepsilon(\%)$ y el espesor de adhesivo t $(\mu \mathrm{m})$ ara diferentes valores del espesor de la capa inferior de encapsulado $(\mu \mathrm{m})$.

\subsection{Comportamiento del encapsulado frente a variaciones térmicas.}

El proceso de encapsulado de una FBG es crucial para la correcta medición de las deformaciones, debido a que un encapsulado deficiente puede provocar un espectro de luz reflejado con dos o más picos. Esto conllevaría una medición incorrecta por parte del sensor y por tanto, evaluaciones estructurales que en ocasiones se quedarían del lado de la inseguridad.

Dado que el sensor va a estar expuesto a variaciones térmicas a lo largo de la vida útil de la estructura a la cual va a estar adherido, su comportamiento durante este tipo de acciones resulta especialmente importante.

De esta forma, se llevó a cabo un estudio sobre el espectro de luz reflejado por el sensor FBG al mismo tiempo que se aplicó un ciclo de variaciones térmicas. Estos ensayos consistieron en obtener la respuesta del sensor (espectro reflejado) ante variaciones térmicas controladas y observar la evolución de su forma durante el ensayo. El ciclo de temperaturas propuesto en los ensayos fue el indicado en la Figura 4.22. 
Monitorización estructural.

Aplicación con sensores de fibra óptica.

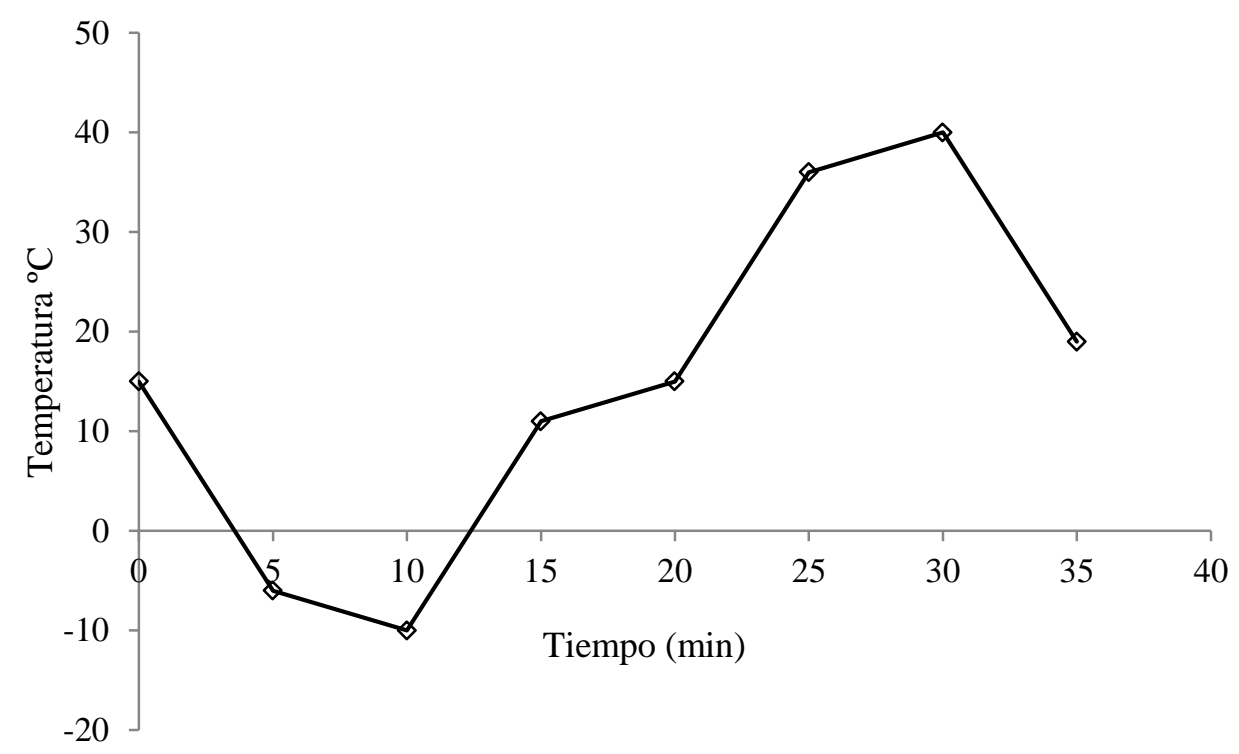

Figura 4.22 Ciclo de temperaturas aplicado sobre el sensor.

Para ello se introdujo en el interior de la cámara climática Feutron KPK 400 del apartado 4.2.2 uno de los sensores propuestos en esta investigación. Sobre este sensor se aplicó el ciclo de temperaturas indicado en la Figura 4.22 y se captó la respuesta del sensor en cada uno de los puntos de temperatura indicados, esto es, a los $15,-5,-10,11,15,36,40$ y $19^{\circ} \mathrm{C}$.

Los resultados obtenidos se indican en la Figura 4.23. En esta figura se observa que al disminuir las temperaturas, la respuesta del sensor es altamente indeseable: el espectro de luz reflejado sufre modificaciones importantes en su forma.

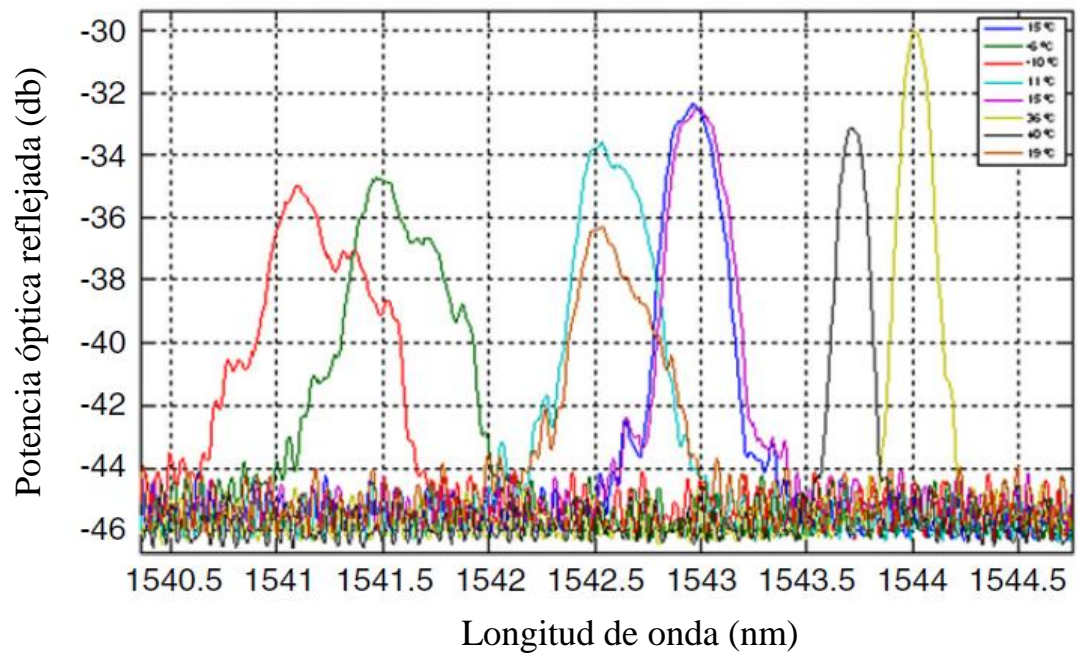

Figura 4.23 Respuesta del sensor encapsulado. Espectros de luz reflejados para diferentes temperaturas ambientes. 
Las principales modificaciones que se han observado y que son importantes destacar son:

- Una importante disminución de la potencia del espectro. Cuando se alcanza la temperatura más baja del ciclo, $-10^{\circ} \mathrm{C}$, la potencia del espectro ha experimentado una disminución de casi $-6 \mathrm{db}$.

- Un ensanchamiento en la base del espectro, lo cual complica excesivamente su identificación como sensor y por tanto su lectura (espectros de color rojo y verde en la Figura 4.23).

- Distorsión en la forma del espectro y aparición de más de un pico (espectros de color rojo y verde en la Figura 4.23). Este aspecto dificulta excesivamente la detección del sensor y en caso de ser posible, sus resultados sembrarían muchas dudas al respecto.

Sin embargo, al aumentar la temperatura este efecto desaparece. La respuesta del sensor es mucho más limpia que en el caso anterior, el espectro de luz reflejado sufre un estrechamiento de su base y su forma describe la deseada campana de Gauss con la identificación de un único pico (espectro de color pistacho de la figura 4.23).

Durante el ensayo se observó que se producía un cambio en la forma del sensor. Al disminuir las temperaturas se producía una flexión del sensor y dado que éste está formado por una capa de resina de poliéster y fibra de vidrio, la aleatoriedad de la fibra de vidrio (Figura 4.24) introducía una modificación de la red de difracción del sensor, produciendo la modificación en la forma del espectro reflejado por el sensor.

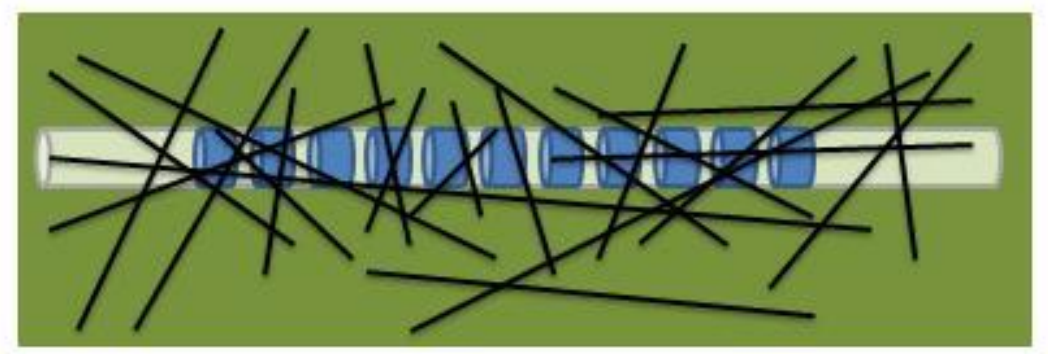

Figura 4.24. Aleatoriedad de los pelos de la fibra de vidrio sobre la red de difracción de Bragg del sensor.

Por otro lado, la Figura 4.25 muestra el mismo ensayo en el que el material de encapsulado del sensor garantiza una distribución uniforme de la fibra de vidrio. En esta figura se observa como, a pesar del ciclo de temperaturas propuesto, la respuesta del sensor es la misma. En ningún instante se produce ensanchamiento en la base del espectro del sensor ni pérdida en su potencia, además de garantizarse en todo instante la detección de un único pico. 


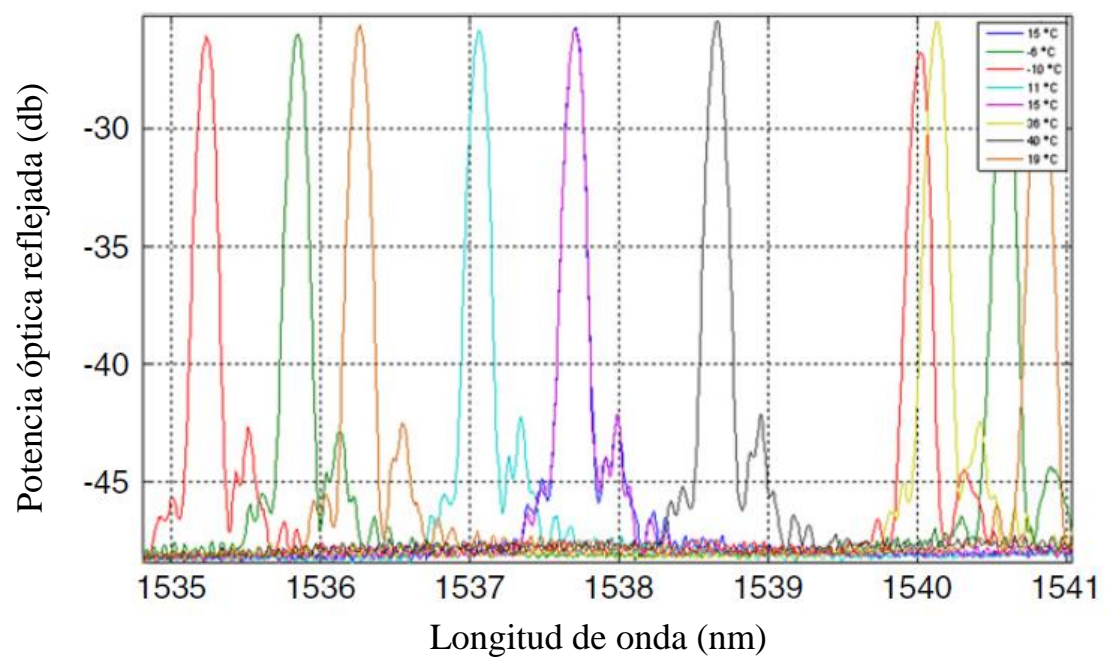

Figura 4.25 Respuesta del sensor encapsulado con distribución uniforma de la fibra de vidrio. Espectros de luz reflejados para diferentes temperaturas ambientes.

\subsection{Conclusiones.}

Establecida la necesidad de estudiar en profundidad el comportamiento de un sensor óptico puntual de instalación en la superficie del material para la obtención de deformaciones, se han mostrado los resultados de un modelo numérico de comportamiento del sensor propuesto y la afección que sobre las medidas ejercen distintos parámetros presentes en su instalación y fabricación.

El sensor propuesto sólo presenta una capa de encapsulado, por lo que la fibra óptica se encuentra prácticamente en contacto con la capa de adhesivo empleada en la instalación del sensor en la estructura.

Con los modelos numéricos presentados se ha caracterizado el comportamiento del sensor y obtiene los errores en las medidas $\Delta \varepsilon(\%)$ asociados a parámetros como el espesor de adhesivo y propiedades mecánicas, que se encuentran sujetas a incertidumbre.

Además, el modelo numérico fue adaptado para caracterizar el comportamiento de un sensor genérico de dos capas, con el objetivo de comparar su comportamiento con el diseño propuesto. Tras estos estudios realizados, se pueden extraer las siguientes conclusiones:

1) La configuración del sensor propuesto en este trabajo está fuertemente influenciada por el espesor de adhesivo empleado para su instalación en la estructura. Valores habituales en los espesores de adhesivo empleados $(400 \mu \mathrm{m})$ han dado valores de $\Delta \varepsilon$ (\%) por debajo de $2.5 \%$. Elevados valores de espesor de adhesivo, alrededor de $1000 \mu \mathrm{m}$, en el rango de lo que sería considerada una "instalación inadecuada", proporcionan errores relativos de $6.5 \%$.

2) Pequeños valores del coeficiente de Poisson del adhesivo $v_{A}$, (del orden de 0.1 ), mejoran la transferencia de deformaciones debido a que aumenta el valor del Módulo de Rigidez transversal G. En caso contrario, elevados valores de $v_{A}$ aumentan los errores relativos. En cualquier caso y en la situación más desfavorable $\left(\mathrm{t}=1000 \mu \mathrm{m}, v_{A}=0.4\right)$ el error máximo asumido por el sensor resulta inferior a $6.5 \%$. 
3) La existencia de una capa inferior de encapsulado tiene una influencia negativa en su comportamiento. Por ejemplo, para un espesor de esta cada de $400 \mu \mathrm{m}$ se pueden inducir errores superiores a un 15\%. La precisión en el sensor genérico de dos capas se encuentra muy fuertemente influenciada por efectos de fabricación y variaciones en las propiedades de esta capa, por ejemplo, variaciones del coeficiente de Poisson debidas a variaciones de temperatura. Esto indica que es preferible el empleo de un sensor con una sola capa, como el propuesto en la presente Tesis Doctoral.

4) Además, también se ha estudiado la importancia de las variaciones térmicas sobre el espectro de luz reflejado por el sensor. El empleo de un material adecuado mostró un espectro capaz de mantener su forma durante el ciclo de variaciones térmicas, y garantizar de esta forma una correcta medición del sensor. 



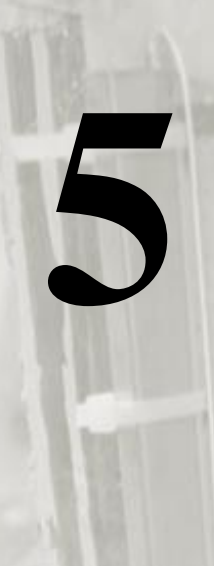

Estudio del comportamiento,

DESARROLLO Y DISEÑO DE UN SENSOR

ÓPTICO DE LONGITUD PARA LA OBTENCIÓN

DE DEFORMACIONES EN ESTRUCTURAS.

El quinto capítulo de este documento presenta un nuevo diseño de sensor óptico de longitud basado en FBG's para ser fijado a la superficie de la estructura a monitorizar. A diferencia del sensor propuesto en el Capítulo 4 que medía las deformaciones puntuales, este nuevo diseño proporciona la deformación media entre los puntos que delimitan el longitud del sensor.

El diseño propuesto en este capítulo difiere en su comportamiento respecto de los sensores de longitud existentes en el mercado. La mayoría de éstos basan su funcionamiento en una fibra óptica anclada en dos puntos y que por tanto, requiere ser pretensada para su correcto funcionamiento. En el caso del diseño propuesto en este capítulo, la fibra óptica no necesita ser pretensada.

El diseño del sensor propuesto se ha validado mediante campañas experimentales y se ha optimizado mediante modelos de elementos finitos 3D que han permitido analizar la influencia de parámetros que intervienen en su funcionamiento (fijaciones, longitudes del sensor, rigideces y propiedades mecánicas de los materiales...).

Los resultados obtenidos demuestran el correcto funcionamiento de los sensores ópticos de longitud propuestos para la medida de deformaciones. 



\subsection{Introducción.}

En el Capítulo 4 se estudió el comportamiento y diseño de un nuevo sensor óptico puntual de deformación y su aplicación a la monitorización de una obra real, obteniendo unos resultados muy satisfactorios. Sin embargo, los sensores puntuales no cubren la totalidad de los objetivos de la monitorización estructural.

El planteamiento de este proceso puede ser enfocado atendiendo a dos objetivos: La monitorización a nivel local y la monitorización a nivel general (Glisic e Inaudi, 2007).

En el primero de los casos, la instrumentación va orientada a determinar el comportamiento local del material bajo cargas o agentes externos ambientales. En este caso, es recomendable el empleo de sensores de corta longitud o puntuales (también llamados short-gauge) como el estudiado en el Capítulo 4. En caso de emplear un elevado número de sensores instalados sobre distintos puntos de la estructura sería posible obtener información sobre el comportamiento de la estructura completa a partir de medidas locales (Glisic e Inaudi, 2007). Algunos elementos estructurales como ménsulas cortas, nudos entre barras o zonas de aplicación de las cargas son susceptibles de ser monitorizados con varios sensores puntuales.

En el caso de la monitorización general, el objetivo es estimar el comportamiento de la estructura en su conjunto. Para ello, es recomendable el empleo de sensores ópticos de longitud, también llamados long-gauge, para evitar que las heterogeneidades locales de la estructura afecten a las medidas. Las longitudes de estos sensores se extienden desde unos pocos centímetros hasta varios metros, siendo posible obtener información de las deformaciones que sufre la estructura en su conjunto y extrapolar el comportamiento global de la misma. Este hecho constituye la diferencia fundamental con la práctica habitual, que considera que un número reducido de puntos instrumentados con sensores puntuales proporciona el comportamiento general del elemento estructural. Las vigas o cables de las grandes estructuras civiles como puentes, túneles o presas son susceptibles de ser monitorizados con varios sensores de longitud.

A partir de estos dos enfoques, Glisic e Inaudi (2007) clasificaron el empleo de sensores puntuales o de longitud atendiendo a los criterios que indica la Figura 5.1:

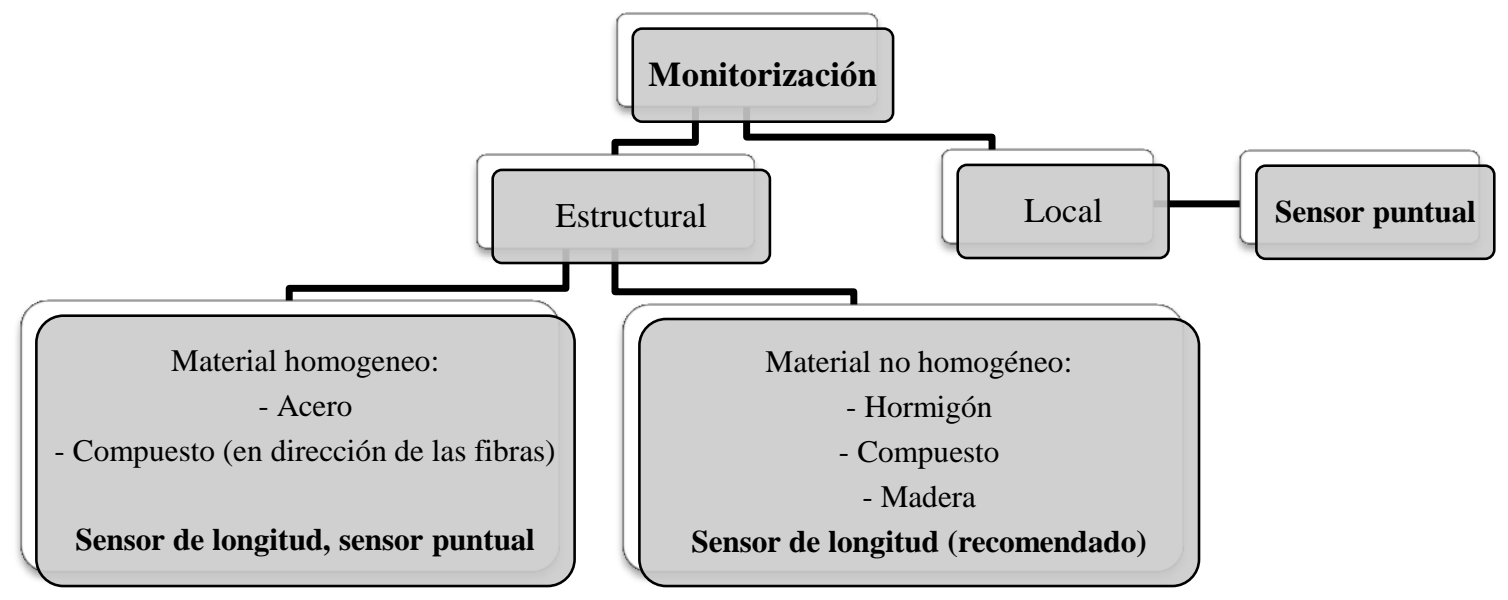

Figura 5.1 Recomendaciones acerca del empleo de los sensores ópticos puntuales o "long gauge" (Adaptado de Glisic e Inaudi 2007). 
Por lo tanto, existen dos enfoques en la monitorización estructural que dependerán de los objetivos de la monitorización (comportamiento estructural o comportamiento a nivel local) y del material instrumentado (homogéneo o no homogéneo). Cada uno de estos enfoques será cubierto empleando sensores puntuales o sensores de longitud.

\section{2- Particularidades de los sensores de longitud.}

Algunos materiales de construcción como el hormigón, la madera o los materiales compuestos presentan defectos locales tales como inclusiones de aire o fisuras. Estos defectos introducen discontinuidades en sus propiedades mecánicas y los convierten en materiales no homogéneos (Glisic e Inaudi, 2007) lo que resulta problemático desde el punto de vista de la monitorización. El empleo de sensores de longitud permite obviar este problema, pues el valor de deformación por ellos medido representa la deformación media a lo largo de la longitud del sensor, y se atribuye a su punto medio tal y como indican la Figura 5.2 y la ecuación 5.1:

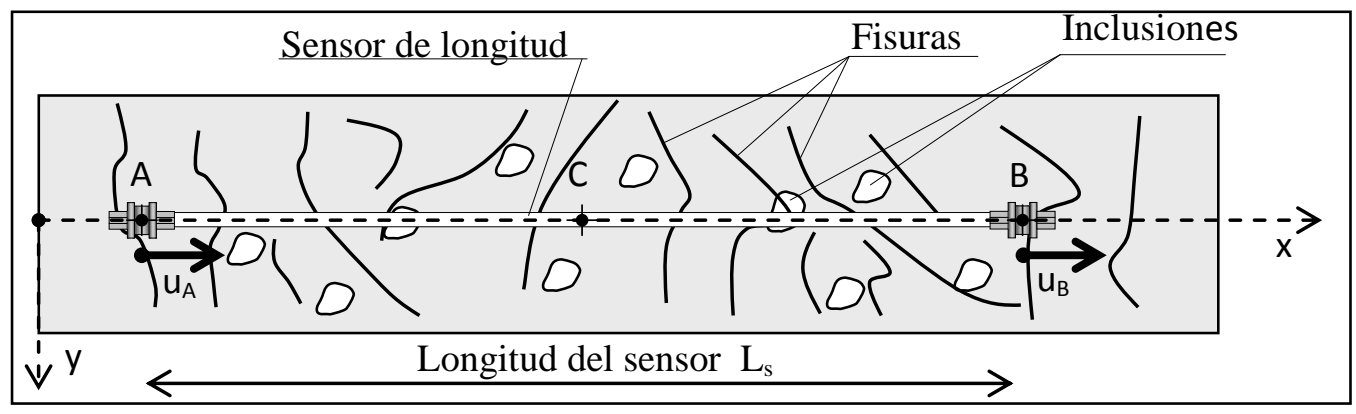

Figura 5.2 Representación de un sensor de longitud en un material no homogéneo (Glisic e Inaudi, 2007).

$$
\varepsilon_{C, s}=\frac{\Delta L_{s}}{L_{s}}=\frac{u_{B}-u_{A}}{x_{B}-x_{A}}=\frac{1}{L_{s}} \int_{x_{A}}^{x_{B}} \varepsilon_{x, s}(x) \mathrm{d} x+\frac{1}{L_{s}} \sum_{i} \Delta w_{d, i}
$$

Donde:

- A, B- Puntos que delimitan la longitud del sensor a partir de sus coordenadas $X_{B}-X_{A}$

- C- Punto medio del sensor de coordenadas $X_{C}=\left(X_{A}+X_{B}\right) / 2$

- $\quad L_{S}=X_{B}-X_{A}$ es la longitud del sensor, antes de ser deformado.

- $\mathrm{u}_{\mathrm{b}}, \mathrm{u}_{\mathrm{A}}$, son los desplazamientos en dirección X de los puntos A y B tras la deformación.

- $\varepsilon_{C, S}$ es la deformación media medida por el sensor atribuida al punto medio C.

- $\varepsilon_{x, s}(X)$ es la distribución de deformaciones a lo largo del eje $X$ del sensor.

- $\Delta w_{d, i}$ engloba el cambio de dimensiones en el intervalo limitado por $X_{B}-X_{A}$ debido a abertura de fisuras, cambios en los tamaños de las inclusiones de aire y otras heterogeneidades, en dirección del eje X tras la deformación. 
La Figura 5.3 (Glisic e Inaudi, 2007) muestra la importancia del empleo de sensores de longitud en la monitorización estructural. Esta figura representa un elemento de hormigón sometido a flexión simple. El elemento se instrumenta con dos sensores ópticos de longitud $s_{t}$ y $s_{b}$ a lo largo de la longitud del elemento en sus caras superior e inferior, y cuatro sensores ópticos puntuales, $g_{1 t}, g_{2 t}, g_{1 b}, g_{2 b}$, instalados en sus fibras superiores e inferiores en dos secciones transversales, 1-1 y 2-2 respectivamente.

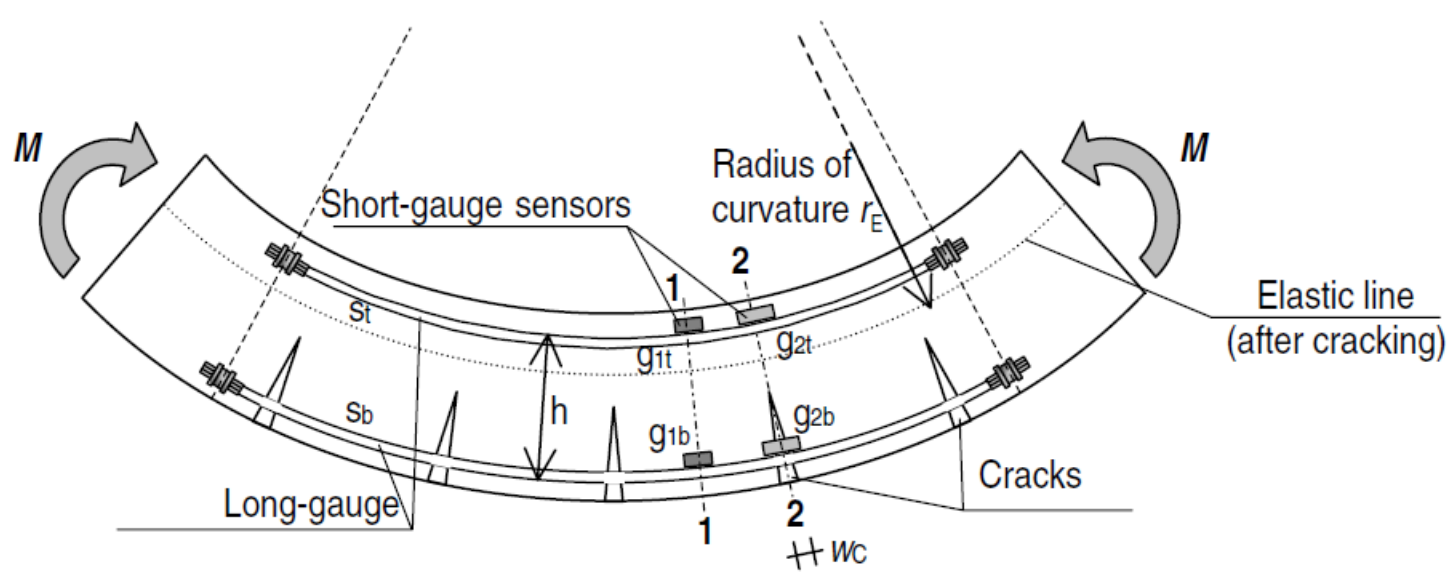

Figura 5.3 Elemento de hormigón instrumentado con sensores ópticos puntuales y de longitud (Glisic e Inaudi, 2007).

Con la instrumentación propuesta se desea obtener la curvatura de la sección para estimar su momento flector, de tal forma que las curvaturas obtenidas con los sensores de longitud, y con los puntuales de las secciones 1 y 2 se llamarán, respectivamente $k_{l g}, k_{s 1}, k_{s 2}$.

Sobre la sección 2-2 se abre una fisura en la misma posición del sensor puntual $g_{2 b}$, por lo que la estimación de la curvatura $k_{s 2}$ en esta sección es muy superior al valor de la curvatura media real del elemento estructural.

La curvatura obtenida con la instrumentación de la sección $1, k_{s 1}$, se encuentra muy infravalorada dado que a ambos lados del sensor inferior $g_{1 b}$ han aparecido fisuras.

La curvatura obtenida con los sensores ópticos de longitud, $k_{l g}$, ofrece una buena estimación para la evaluación del elemento estructural. Con esta disposición, se obtiene la curvatura media del elemento, integrando en su medida las discontinuidades, fisuras y heterogeneidades (recogidas con el término $\frac{1}{L_{s}} \sum_{i} \Delta w_{d, i}$ en la ecuación 5.1) que no se engloban con los sensores ópticos puntuales.

De esta forma se tiene que $k_{S 2} \gg k_{l g} \gg k_{s 1}$, dado que en ambas secciones, 1-1 y 2-2, los sensores puntuales se ven fuertemente influenciados por los defectos locales propios del hormigón.

En este caso, el funcionamiento de un sensor óptico de longitud exige que la fibra óptica se encuentre pretensada para garantizar su correcto funcionamiento frente a esfuerzos de compresión (por ejemplo el sensor $s_{t}$ instalado en la fibra superior de la Figura 5.3). Smartec o FOS\&S son algunas empresas que comercializan este tipo de sensor óptico y el funcionamiento de los mismos ha sido probado con eficacia (Glisic e Inaudi, 2007). 
No obstante, el hecho de tener que pretensar la fibra antes de cada instalación es una operación cargada de incertidumbre, ya que se debe decidir el nivel de pretensado sobre la fibra. Si se sobrepasa este nivel de pretensado, el sensor de longitud no mediría en caso de esfuerzos de compresión.

Ante esta situación, en este capítulo se muestra el estudio numérico y experimental de un sensor óptico FBG de deformación de longitud, que no precisa de un pretensado previo para su funcionamiento. Ambas fases, experimental y numérica, se combinan para converger en un proceso que tiene como conclusión un diseño óptimo y eficaz del elemento sensor.

\section{3- Sensor de longitud FBG propuesto en la investigación.}

El diseño del sensor de longitud propuesto en la presente Tesis Doctoral se muestra en la Figura 5.4. La fibra óptica se encuentra anclada a un elemento lineal, y este elemento lineal se encuentra adherido a la estructura a monitorizar en sus puntos extremos.

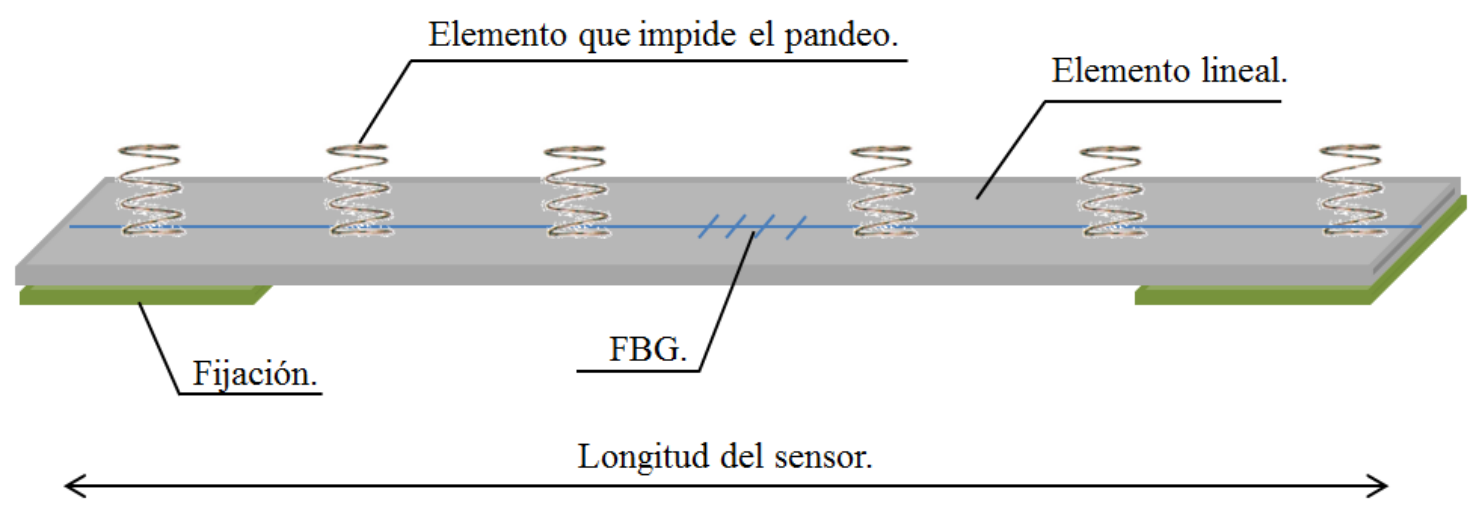

Figura 5.4 Esquema del sensor de longitud propuesto en esta Tesis Doctoral.

El diseño del elemento sensor debe tener en cuenta una serie de aspectos:

1. El elemento lineal debe sufrir las mismas deformaciones que la estructura monitorizada. Por tanto, la transferencia de deformaciones desde la estructura hasta el elemento que forma el sensor debe ser adecuada. Para ello, el diseño de los anclajes y las propiedades del material que forma el adhesivo son fundamentales, tal y como se estudió en el Capítulo 4.

2. La instalación de este elemento supone una modificación local en la estructura alrededor de la zona monitorizada (Figura 5.5). Esta afección debe ser mínima, por lo que en el diseño de este elemento se debe tener en cuenta su rigidez axil $E_{m} A_{m}$, y su rigidez a flexión $E_{m} I_{m}$, (siendo $E_{m}$ el módulo de elasticidad del material que forma el sensor, $A_{m}$ su sección transversal e $I_{m}$ el momento de inercia de la sección). 


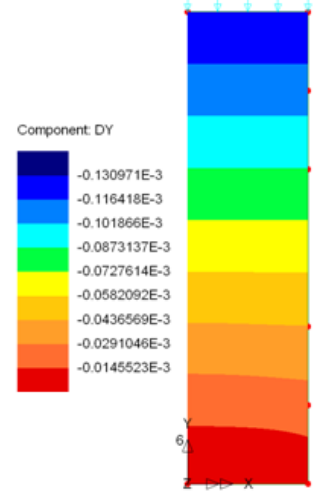

(a)

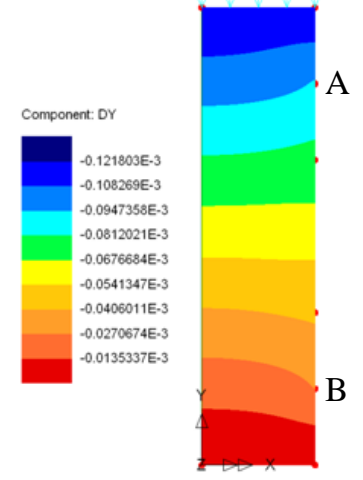

(b)

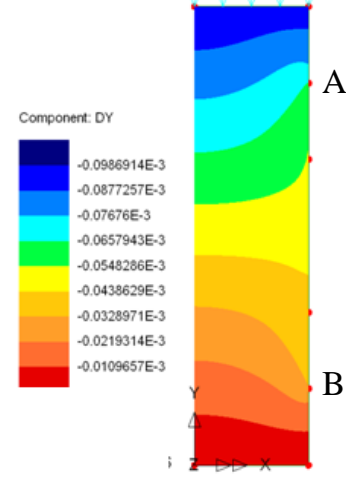

(c)

Figura 5.5 Campo de desplazamientos en dirección vertical en un elemento estructural de hormigón sometido a esfuerzos de compresión, obtenidos con el programa Lusas V.14.3-11 a) Sin la colocación de ningún elemento sensor en su superficie. b) Con un elemento sensor en su superficie entre los puntos A y B con una rigidez axil $E_{m} A_{m}=5000 \mathrm{KN}$. c) Con un elemento sensor en su superficie entre los puntos A y B con una rigidez a axil $E_{m} A_{m}=30000 \mathrm{KN}$.

3. El elemento que forma el sensor es prismático, de sección transversal rectangular y de longitud variable. En este trabajo se han ensayado sensores de distintas longitudes, que oscilan desde los 0.2 metros hasta 1 metro. La selección de una u otra longitud dependerá de las características de la estructura y del interés del estudio de la zona instrumentada. Glisic e Inaudi (2007) proporcionan unas recomendaciones para definir esta longitud.

4. Finalmente, la medida obtenida con el diseño propuesto, según la ecuación 5.1, nos proporciona directamente el valor de la deformación media entre los dos puntos de anclaje del sensor. La FBG está anclada al punto medio del elemento y se considera que la deformación es constante a lo largo de toda la longitud del elemento lineal.

\section{4- Ventajas del sensor FBG de longitud propuesto en esta investigación.}

Tal y como se explicó en los apartados anteriores, el pretensado de la fibra es una operación cargada de incertidumbre y que exige una muy cuidadosa manipulación. El sensor óptico de longitud propuesto en la presente Tesis Doctoral no exige el pretensado de la fibra óptica lo que supone una clara ventaja.

El hecho de pretensar la fibra introduce algunas complicaciones e inconvenientes sobre el funcionamiento de los sensores ópticos de longitud como son:

1. En la mayoría de situaciones se desconoce el nivel de pretensado que hay que introducir en la fibra, pudiendo causar pretensados erróneos errores de medición.

2. El pretensado es una operación que necesita de una cuidadosa manipulación debido a la posibilidad de rotura de la fibra óptica.

3. Las variaciones térmicas pueden provocar pérdida de este valor de pretensado, de acuerdo con la expresión $\Delta \varepsilon=\alpha \Delta T$; siendo $\Delta \varepsilon$ el incremento de la deformación en la fibra óptica, $\alpha$ el 
coeficiente de dilatación térmica del material que forma la fibra óptica y $\Delta T$ el incremento de temperatura que sufre la fibra. La Figura 5.6 muestra el resultado de un ensayo experimental realizado por el autor de esta tesis y por D. Antonio Bueno sobre una FBG. El ensayo consistió en anclar los dos extremos de un tramo de fibra que contenía una FBG y, mediante un sistema de posicionamiento milimétrico, pretensar la fibra hasta alcanzar un valor de 2000 $\mu \varepsilon$. Esta operación fue monitorizada, y con el paso del tiempo se observó una pérdida de pretensado debida a los incrementos de temperatura ambiente y a deslizamientos de la fibra sobre los anclajes que alcanzó un valor del 7.5\% del pretensado inicial a las 14 horas de iniciado el ensayo.

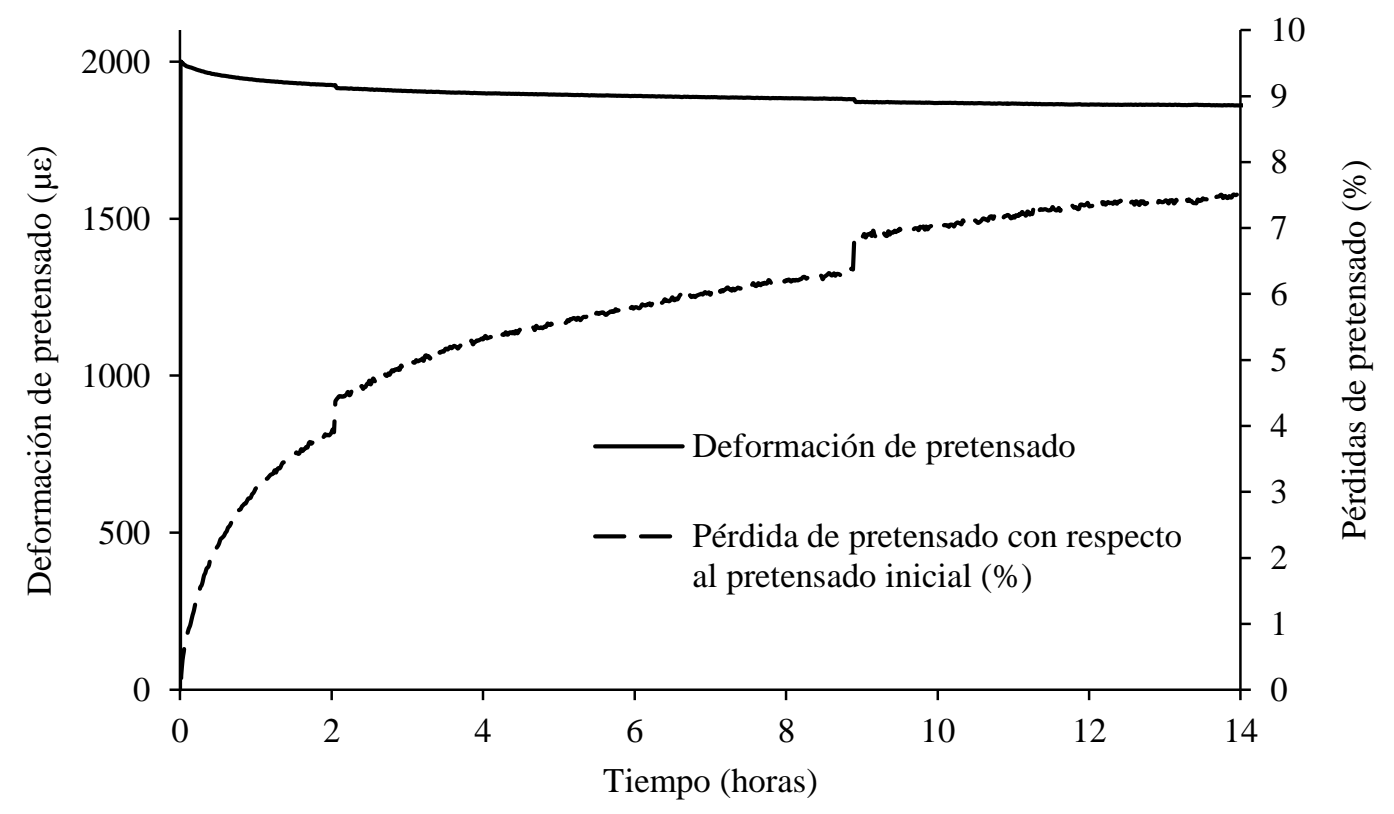

Figura 5.6 Ensayo experimental. Pretensado inicial a una fibra óptica y pérdida de pretensado con el tiempo, en $\%$.

4. Además, un incremento de temperatura supone también una afección sobre los sistemas de pretensado y anclajes de la fibra, siendo otra posible causa de pérdidas de pretensado. Para evitarlo, un pretensado sobre la fibra óptica debería disponer unos elementos de anclaje muy precisos que eviten cualquier deslizamiento relativo de la fibra.

5. El diseño de un sensor de longitud que no necesite de un pretensado de la fibra óptica es el único sistema que puede permitir la medición de las deformaciones en estructuras de hormigón sometidas a elevadas temperaturas como es el caso de incendios.

\section{5- Estudio experimental y numérico del sensor.}

\subsubsection{Introducción:}

De una manera similar a como se procedió en el Capítulo 4, el diseño y optimización de un nuevo sensor óptico de longitud se ha basado en campañas experimentales y numéricas que se detallan a continuación. 


\subsubsection{Estudio experimental.}

a) Características de las probetas empleadas.

Para probar el funcionamiento de los sensores propuestos se han llevado a cabo unas campañas experimentales sobre probetas de hormigón sometidas a compresión. Se han fabricado distintos tipos de probetas, con el objetivo de realizar los ensayos para distintas longitudes de sensores:

1. Probetas prismáticas de hormigón en masa de sección cuadrada de $0.10 \mathrm{~m}$ de lado y con una altura de $0.40 \mathrm{~m}$ (Figura 5.7.a).

2. Probetas prismáticas de hormigón armado de sección cuadrada de $0.20 \mathrm{~m}$ de lado y de $1.2 \mathrm{~m}$ de altura. (Figura 5.7.b).

3. Pilares de hormigón armado de sección cuadrada de $0.30 \mathrm{~m}$ de lado y de $3 \mathrm{~m}$ de altura (Figura 5.7.c).

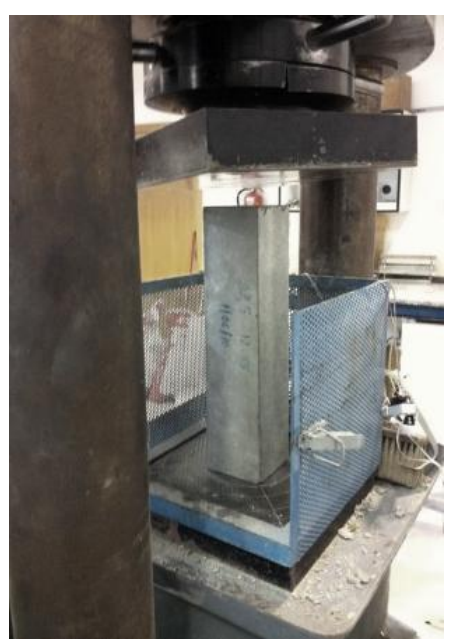

(a)

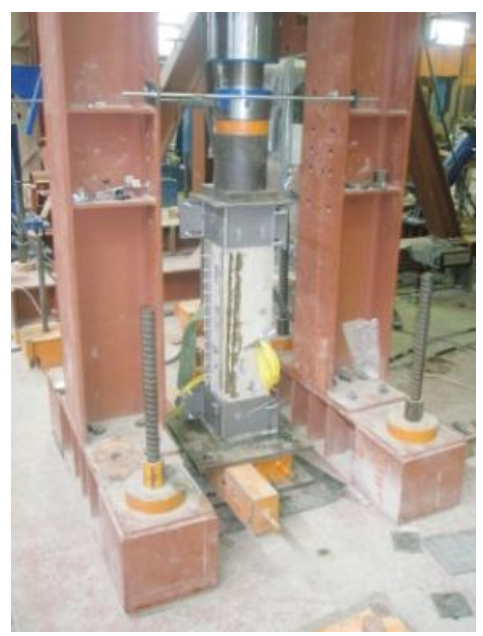

(b)

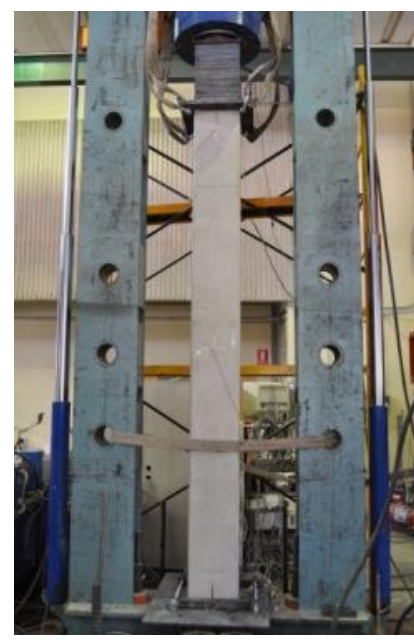

(c)

Figura 5.7 Tres tipos de probetas empleadas en los ensayos. (a)- probetas de 0.4 metros de altura. (b)probetas de 1 metro de altura. (c)- pilares de 3 metros de altura.

En los pilares de las Figuras 5.7.b y 5.7.c ha sido necesario un refuerzo en las cabezas (zona de aplicación de la carga) para evitar la rotura de estas zonas por compresión localizada. Los refuerzos se han planteado en base a un encamisado metálico que confina al hormigón en esa zona. Los incrementos y decrementos de carga han sido aplicados de forma lenta y progresiva en todos los ensayos hasta producir la rotura del hormigón.

\section{b) Propiedades de los materiales empleados.}

Las probetas de hormigón que se presentaron en el apartado 5.5.2.a pertenecían a hormigones distintos, ya que los distintos ensayos no fueron realizados simultáneamente sino que se realizaron en momentos distintos. 
En los tres casos, tanto la resistencia a compresión del hormigón como su módulo de elasticidad se determinaron con ensayos de compresión simple sobre probetas cilíndricas normalizadas de $0.15 \mathrm{~m}$ de diámetro y $0.3 \mathrm{~m}$ de altura (Norma UNE, 1996).

La probeta de la Figura 5.7.a. (dimensiones $0.1 \mathrm{X} 0.1 \mathrm{X}$ 0.4) se trata de un hormigón de alta resistencia, que arrojó un valor promedio de su resistencia a compresión de 100.4 MPa y un valor de Módulo de Elasticidad promedio estimado en $34116 \mathrm{MPa}$, a los 28 días, momento en que se realizaron los ensayos. La Tabla 5.1 presenta los valores obtenidos en los ensayos sobre 3 probetas cilíndricas normalizadas.

\begin{tabular}{ccc}
\hline \multirow{2}{*}{ Probeta } & Resistencia compresión (MPa) & Módulo Elasticidad E (MPa) \\
& & 35350 \\
2 & 106.6 & 33824 \\
3 & 98.2 & 33176 \\
Promedio & 96.5 & 34116 \\
\hline
\end{tabular}

Tabla 5.1. Valores de resistencia a compresión y Módulo de Elasticidad. Probetas 0.1 X 0.1 X 0.4

En el caso de las probetas de las Figuras 5.7.b y 5.7.c, el hormigón empleado se trata de un hormigón convencional que ha arrojado una resistencia a compresión media de 37.1 MPa y un valor de Módulo de Elasticidad promedio de $29450 \mathrm{MPa}$. La Tabla 5.2 presenta los valores obtenidos en los ensayos obtenidos sobre tres probetas cilíndricas normalizadas. Nótese que el valor de resistencia a compresión en la tercera probeta no pudo obtenerse debido a un problema con el sistema de adquisición de datos.

\begin{tabular}{ccc}
\hline Probeta & Resistencia compresión (MPa) & Módulo Elasticidad E (MPa) \\
\hline 1 & 37.4 & 30330 \\
2 & 36.8 & 29664 \\
3 & - & 28356 \\
Promedio & 37.1 & 29450 \\
\hline
\end{tabular}

Tabla 5.2. Valores de resistencia a compresión y Módulo de Elasticidad. Probetas de dimensiones 0.2 X 0.2 X 1 y $0.3 \times 0.3 \times 3$.

El límite elástico del acero de las armaduras fue $f_{y k}=500 \mathrm{MPa}$ y el del encamisado metálico de refuerzo de las cabezas $f_{y r}=265 \mathrm{MPa}$.

c) Pórticos de ensayo.

Los ensayos se han llevado a cabo en el laboratorio del Instituto y Ciencia de Tecnología del Hormigón (ICITECH) de la Universitat Politècnica de València (U.P.V.).

Las probetas de la Figura 5.7.a se han ensayado en una prensa de la marca ICON. El sistema de control es automático, y es posible alcanzar una carga máxima de 250 toneladas. La prensa está conectada a un equipo informático que permite la toma y almacenamiento de los datos del ensayo.

Las probetas de la Figura 5.7.b se han ensayado en un pórtico metálico equipado con un gato hidráulico con capacidad de carga de 250 toneladas. La probeta se coloca sobre un apoyo que se supone infinitamente rígido, materializado por tres planchas metálicas macizas de $0.10 \mathrm{~m}$ de canto. La carga se aplica en la cabeza de la estructura, hasta producir la rotura de la probeta. 
Las probetas la Figura 5.7.c se han ensayado sobre otro pórtico metálico de 3.5 metros de altura. Del mismo modo que en el caso anterior, los pilares se asientan sobre apoyos que se suponen infinitamente rígidos y se aplica una carga vertical en cabeza hasta la rotura. La carga máxima del gato es de 500 toneladas.

\section{d) Instrumentación.}

En todos los ensayos se han utilizado los sensores puntuales de fibra óptica desarrollados en el Capítulo 4 de esta Tesis Doctoral para la validación de los resultados obtenidos. Estos sensores, que servirán de referencia, se colocan muy próximos a los nuevos sensores ópticos de longitud objeto de estudio.

El sistema de interrogación empleado en la instrumentación de las deformaciones está compuesto por un interrogador de sensores tipo FBG de la marca Micron Optics, modelo sm125-500 y por un ordenador portátil en el que se ejecutaba un software desarrollado específicamente para esta aplicación (Bueno, 2008). Este software fue desarrollado por personal del Instituto de Telecomunicaciones y Aplicaciones Multimedia (ITEAM) de la Universitat Politècnica de València en el lenguaje de programación LabVIEW ${ }^{T M}$ versión 8.5. El interrogador de sensores ópticos sm125-500 está compuesto por una fuente láser sintonizable Fabry-Perot con un rango en longitud de onda de 1510 a $1590 \mathrm{~nm}$ y 4 canales ópticos de salida. La frecuencia máxima de captura de datos es de $1 \mathrm{~Hz}$ y la precisión de la medida es de $1 \mathrm{pm}$. El ordenador portátil utilizado fue un Intel Pentium 4 a $2.4 \mathrm{GHz}$ y con $768 \mathrm{MB}$ de memoria RAM.

Para el registro de la carga aplicada por el gato hidráulico se han empleado células de carga. El tratamiento de estos datos se realiza en tiempo real a través de un ordenador Pentium $I V$, equipado con el programa CEAD programado en $L a b V I E W^{T M}$. El equipo de adquisición de datos es de la marca Hewlett Packard, modelo HP 3852A.

\subsubsection{Estudio numérico.}

Se han realizado modelos numéricos de elementos finitos 3D del conjunto probeta-sensor con el programa Lusas V14 (Lusas, 2010). Estos modelos numéricos se han calibrado a partir de los ensayos experimentales y han permitido valorar distintas pautas de comportamiento del sensor y proponer mejoras en su diseño.

Además, con la ayuda de un modelo numérico de elementos finitos 3D se ha realizado un estudio avanzado del contacto entre hormigón y adhesivo (apartado 5.6.2.a) y del comportamiento del sensor sometido a compresión (apartado 5.6.2.b). Se describen seguidamente los aspectos más significativos del modelo numérico empleado.

a) Descripción de los elementos finitos empleados, condiciones de contorno y aplicación de la carga.

Se han modelado los distintos elementos que intervienen en el estudio: hormigón, adhesivo, elemento sensor y fibra óptica (Figura 5.8).

El hormigón, el elemento sensor y el adhesivo han sido modelados empleando elementos finitos hexaédricos de 20 nodos y tres grados de libertad por nodo (elemento HX20 del Lusas). La fibra óptica se modeliza como se expuso en el capítulo 4, con elementos lineales BRS3, con tres nodos y 
tres grados de libertad por nodo (traslaciones a lo largo de los tres ejes X, Y, Z). Todos los elementos empleados presentan un comportamiento elástico.

La probeta de hormigón se encuentra apoyada sobre apoyos metálicos macizos. Para modelizar este elemento, supuesto infinitamente rígido, se impide en los nudos de la base de la probeta la traslación en cualquiera de los ejes.
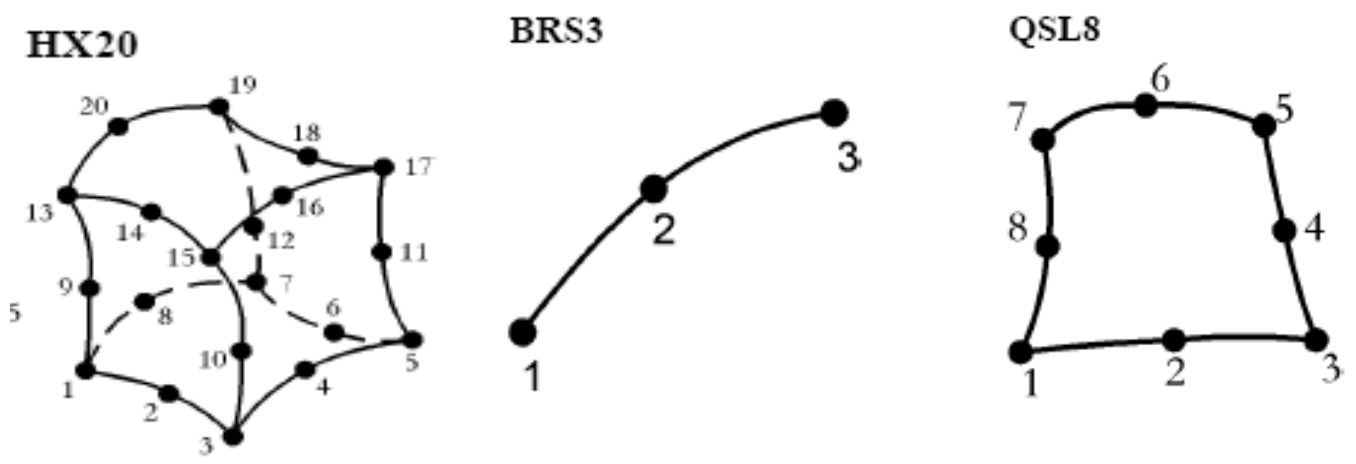

Figura 5.8 Elementos finitos empleados en los modelos (Lusas, 2010).

La carga se aplica en la cara superior de la probeta, incrementándose hasta llegar al valor alcanzado en los distintos ensayos.

\section{b) Interfaz de contacto hormigón-adhesivo}

El contacto entre el hormigón y el adhesivo ha sido simulado empleando elementos hexahédricos HX20 (Figura 5.8). Para este contacto, el modelo constitutivo empleado ha sido 3-D Elasto-Plastic Interface Model (Adam et al. 2010), implementado en Lusas V.14.3-11 el cual permite considerar un plano de fricción entre dos cuerpos 3D. Las propiedades elásticas (E, G y v) se definen para dos ejes, uno contenido en el plano de contacto y otro perpendicular al mismo (Tabla 5.3). El comportamiento no lineal de este contacto viene gobernado por dos criterios:

- Para la tensión tangencial se emplea el criterio de rotura de Mohr-Coulomb.

- Para la tensión normal se define una tensión normal límite de arrancamiento $f_{t}$. Si se alcanza este valor de $f_{t}$, se produciría el fallo de contacto entre ambos materiales.

Las propiedades que se asignan a la interfaz de contacto entre ambos elementos se resumen en la Tabla 5.3. En esta tabla se indica el módulo de elasticidad, módulo de rigidez transversal y coeficiente de Poisson de la interfaz (llamados $E_{P}^{S}, E_{N}^{S}, G_{P}^{S}, G_{N}^{S}, v_{P}^{S}$ y $v_{N}^{S}$ respectivamente) tanto en el plano del contacto -subíndice " $P$ "'- como en la dirección perpendicular al mismo -subíndice " $N$ "-. También se indica el valor de los parámetros necesarios para el criterio de rotura Mohr-Coulomb, esto es, la cohesión de la superficie de contacto $c_{s}$ y su ángulo de rozamiento $\phi_{s}$. Finalmente, este módulo de Lusas necesita el valor de $f_{t}$, llamado tensión normal límite. En caso de alcanzarse este valor, se produciría el despegue del sensor por tensiones normales. 


\begin{tabular}{|c|c|c|c|c|c|c|c|c|c|}
\hline $\begin{array}{c}\text { Superficie } \\
\text { contacto }\end{array}$ & $\begin{array}{c}E_{P}^{S} \\
(M P a)\end{array}$ & $\begin{array}{c}E_{N}^{S} \\
(M P a)\end{array}$ & $\begin{array}{c}G_{P}^{S} \\
(M P a)\end{array}$ & $\begin{array}{c}G_{N}^{S} \\
(M P a)\end{array}$ & $v_{P}^{S}$ & $v_{N}^{S}$ & $\begin{array}{c}c_{s} \\
(M P a)\end{array}$ & $\begin{array}{l}\phi_{s} \\
\left(^{\circ}\right)\end{array}$ & $\begin{array}{c}\text { Tensión } \\
\text { normal } \\
\boldsymbol{f}_{t} \\
(\mathrm{MPa})\end{array}$ \\
\hline $\begin{array}{l}\text { Hormigón } \\
\text {-adhesivo }\end{array}$ & $21.2510^{3}$ & $21.2510^{3}$ & $8.1710^{3}$ & $8.1710^{3}$ & 0.3 & 0.3 & $10-15$ & 0 & 35 \\
\hline
\end{tabular}

Tabla 5.3 Propiedades asignadas a la superficie de contacto entre hormigón y adhesivo.

Respecto a la Tabla 5.3, es importante destacar que:

- Se ha considerado el mismo valor de las propiedades elásticas (E, G y v) para el plano de contacto y para la dirección perpendicular al mismo (Adam et al. 2010).

- Los valores asignados a la interfaz de contacto se obtienen como la media de los valores de los dos elementos que están en contacto (Adam et al. 2010), esto es, hormigón y adhesivo.

- El valor adoptado para la cohesión (c) es de $10 \mathrm{MPa}$. Este valor representa la resistencia a cizallamiento del adhesivo comercial empleado, proporcionado por el fabricante.

- El valor del ángulo de rozamiento $\left(\phi_{S}\right)$ asumido es 0 . Los resultados del modelo numérico no dependen de este valor, puesto que no hay fuerzas normales a la superficie de contacto.

- Para el valor $f_{t}$ (tensión normal de arrancamiento) se ha adoptado el valor que proporciona el fabricante, de $35 \mathrm{MPa}$.

\section{c) Estudio del pandeo.}

Para el estudio del pandeo, el elemento sensor ha sido modelizado con elementos tipo lámina QSL8 (Figura 5.8), con 8 nodos y:

- Tres grados de libertad (traslaciones según los ejes X, Y, Z) en los nodos de las esquinas del elemento.

- Cinco grados de libertad (tres traslaciones y dos giros) en los nodos situados en los lados del elemento.

El método empleado para su estudio ha sido Eigenvalue extraction for buckling. Linear buckling analysis implementado en Lusas V.14.3-11 (Lusas, 2010)

Eigenvalue extraction for buckling. Linear buckling analysis es una técnica que puede ser aplicada a estructuras para estimar la carga máxima que pueden soportar sin pandear o colapsar. Lusas V.14.3-11 resuelve el problema a partir de la ecuación 5.2:

$(K+\lambda K) \phi=0$

Donde $\lambda$ son los valores propios que multiplicados a la carga inicial proporcionan las cargas de pandeo (ecuación 5.3), y $\phi$ son los vectores propios asociados a cada modo de pandeo. 
Monitorización estructural.

Aplicación con sensores de fibra óptica.

$R_{\text {crit }}=\lambda_{1} R$

Siendo $\lambda_{1}$ es el menor valor propio y $\mathrm{R}$ es la carga inicialmente aplicada.

\section{6- Resultados.}

\subsubsection{Validez de los trabajos realizados y resultados obtenidos.}

A lo largo del apartado 5.6 se presentan los resultados obtenidos para el desarrollo y diseño del sensor óptico de longitud expuesto en el epígrafe 5.3 de este capítulo. El estudio de este sensor está basado en campañas experimentales y numéricas, y el alcance de los trabajos y sus resultados se centran en las siguientes consideraciones:

1) El estudio llevado a cabo se refiere a ensayos y pruebas sobre probetas de hormigón endurecido y por tanto, los resultados obtenidos son de aplicación bajo estas condiciones. Este estudio no se ha llevado a cabo sobre probetas metálicas debido a que su naturaleza homogénea permite su monitorización en base a sensores puntuales.

2) El estudio llevado a cabo acota los valores del producto EA del sensor para que permita una correcta medición de las deformaciones. De la misma forma, se ha estudiado cómo afecta la longitud de los sensores en la obtención de los resultados y exactitud en la medida obtenida por el sensor. Por lo tanto, se han estudiado las propiedades mecánicas y geométricas del sensor y su repercusión sobre los resultados obtenidos.

3) En este estudio se han llevado a cabo ensayos sobre tres probetas de dimensiones distintas y con propiedades mecánicas que oscilan entre un valor de módulo de elasticidad de 29450 MPa y $34116 \mathrm{MPa}$. A pesar de que no se ha estudiado la relación entre las propiedades mecánicas y geométricas del sensor con las propiedades mecánicas del material huésped (material que forma la probeta instrumentada), es importante destacar que:

a. Los resultados mostrados son válidos para el rango de módulos elásticos que abarcaría un hormigón endurecido.

b. Los resultados mostrados son válidos para el rango de dimensiones habituales empleadas en columnas de hormigón.

\subsubsection{Comportamiento general.}

\section{a) Transferencia de deformaciones.}

El sensor se ha instalado en la superficie de probetas de hormigón apoyado en dos puntos que delimitan su longitud mediante un adhesivo bicomponente. Las probetas se sometieron a un ciclo de carga y descarga, comparándose los valores de deformación medidos por el sensor óptico puntual de referencia con los valores del nuevo sensor óptico de longitud diseñado. La Figura 5.9.a muestra los resultados numéricos y experimentales de la deformación de la probeta y del sensor, así como el ciclo de cargas al que ha sido sometida la probeta (Figura 5.9). 


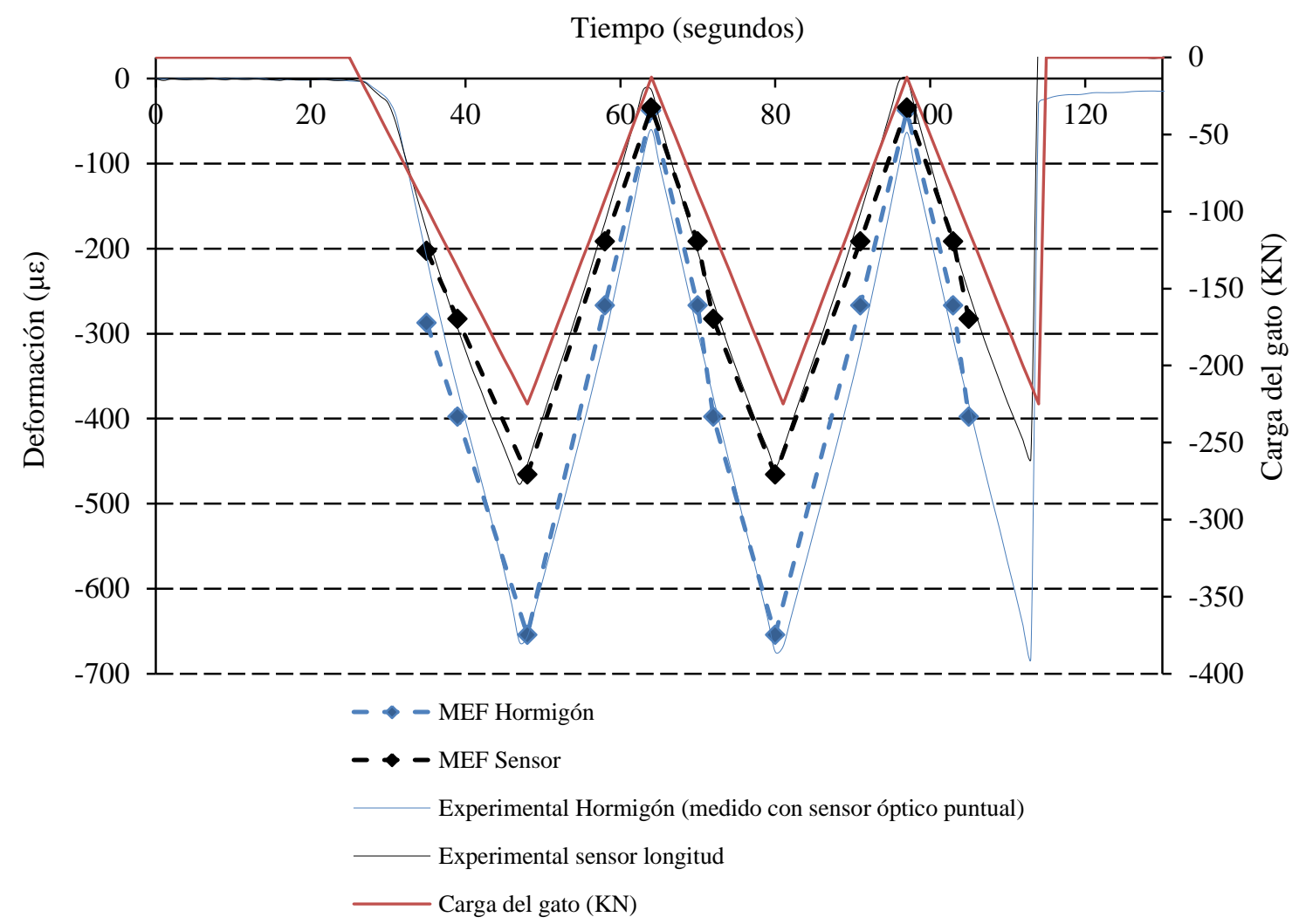

(a)
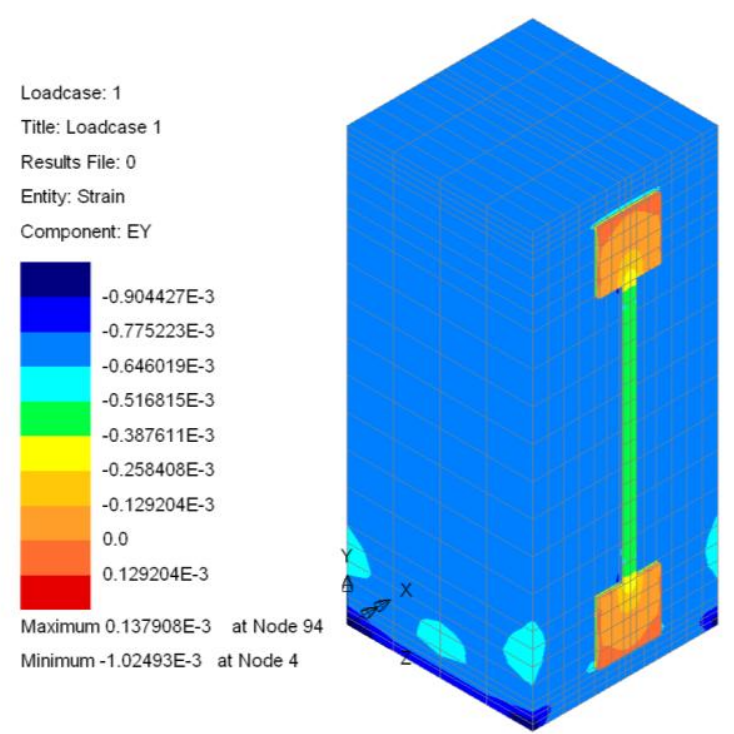

(b)

Figura 5.9 (a)- Deformaciones unitarias experimentales y numéricas en el hormigón y sensor en la dirección del eje Y. (b)- Modelo numérico de elementos finitos. ${ }^{\varepsilon_{\mathrm{s}}} / \varepsilon_{\mathrm{c}} \approx 0.75\left(\varepsilon_{\mathrm{s}}\right.$ es la deformación del sensor y $\varepsilon_{\mathrm{c}}$ es la deformación del hormigón).

La Figura 5.9.a muestra el valor de las deformaciones experimentales del hormigón (medidas con el sensor puntual de referencia) y del sensor óptico de longitud objeto de estudio, ambas representadas en trazas contínuas de color azul y negro. Además también muestra el valor de las deformaciones teóricas 
Monitorización estructural.

Aplicación con sensores de fibra óptica.

del hormigón y del sensor de longitud obtenidas a partir de un modelo numérico 3D de elementos finitos (con marcadores y en traza discontinua). Finalmente, en traza continua de color granate se indica el ciclo de cargas propuesto, alcanzándose un valor máximo de carga de $225 \mathrm{KN}$.

A la vista de los resultados observados en la Figura 5.9.a, se produce una incompleta transferencia de deformaciones entre el hormigón y el sensor (gráficamente se observa en la Figura 5.9.b) pues el sensor de longitud mide deformaciones inferiores a las del hormigón (el ratio entre las deformaciones del sensor y del hormigón $\varepsilon_{s} / \varepsilon_{c}$ se encuentra en torno a 0.75 , lo que supone un $25 \%$ de diferencia entre las medidas). En el modelo numérico se observa que estas diferencias son debidas a las propiedades mecánicas del adhesivo. La propiedad del adhesivo que tiene una mayor influencia sobre el comportamiento del sensor es su módulo de elasticidad $E_{A}$.

A partir del anterior modelo numérico, se ha estudiado la afección del módulo de elasticidad del adhesivo $E_{A}$ sobre el ratio $\varepsilon_{S} / \varepsilon_{C}$, siendo $\varepsilon_{S}$ la deformación del elemento sensor y $\varepsilon_{C}$ la deformación del hormigón (Figura 5.10). Los resultados muestran que existe un valor mínimo de $E_{A}$ igual a 400 $\mathrm{MN} / \mathrm{m}^{2}$ a partir del cual el ratio $\varepsilon_{S} / \varepsilon_{c}$ es prácticamente 1 y se produce una total transferencia de deformaciones entre hormigón y elemento sensor.

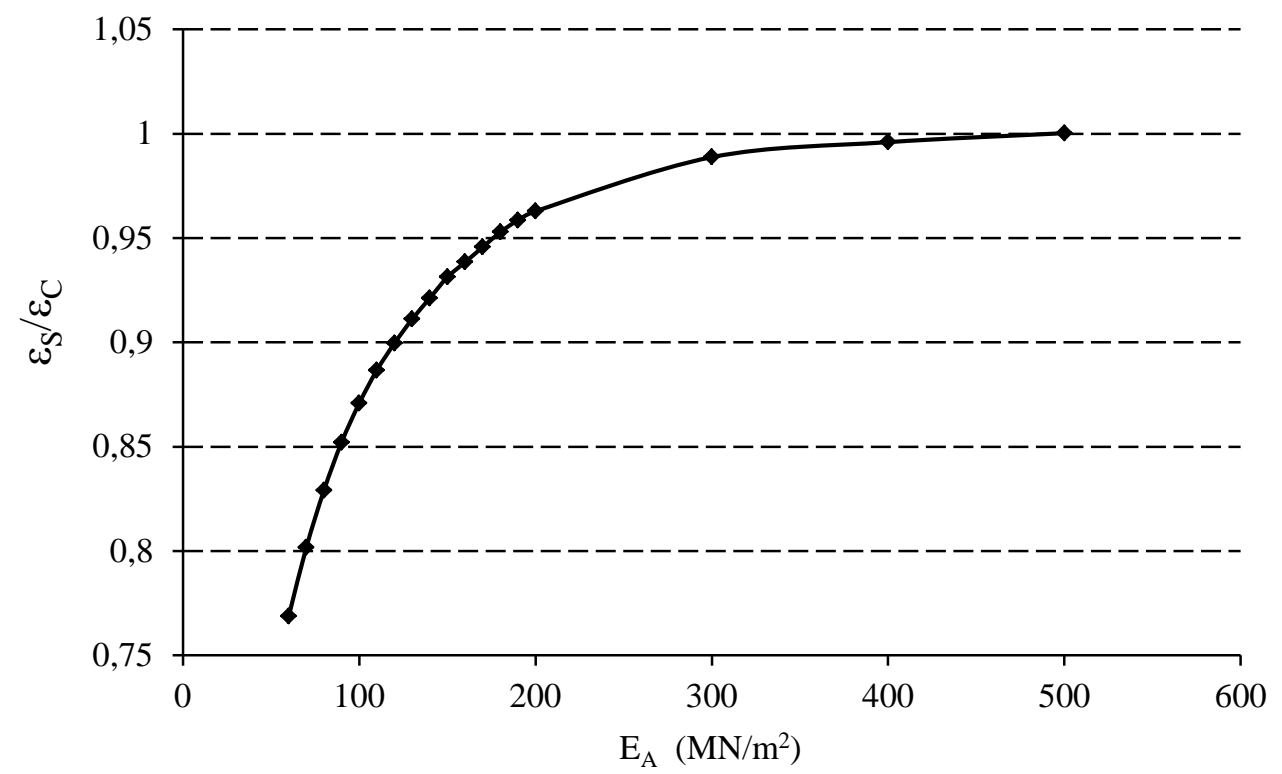

Figura 5.10 Variación del ratio $\varepsilon_{S} / \varepsilon_{c}$ en función del módulo de elasticidad del adhesivo $E_{A}$. Valor de carga uniformemente repartida $21000 \mathrm{KN} / \mathrm{m}^{2}$.

Sin embargo, para valores pequeños de $E_{A}$ (en torno a $90 \mathrm{MPa}$ ) se produce una incompleta transferencia de deformaciones, con ratios $\varepsilon_{S} / \varepsilon_{c}$ próximos a 0.75 , que supone un $25 \%$ en la diferencia de los resultados.

Descubierta la importancia de las propiedades del adhesivo en la transferencia de las deformaciones entre el hormigón y el sensor, se planteó una nueva campaña de ensayos experimentales con los mismos escalones de carga pero con un adhesivo con $\mathrm{E}_{\mathrm{A}}$ igual a $13000 \mathrm{MPa}$. Los resultados de la misma se proporcionan en la Figura 5.11 y confirman la total trasferencia de deformaciones entre hormigón y sensor (ratio $\varepsilon_{s} / \varepsilon_{c}$ muy cercano a 1) y, por tanto, el correcto funcionamiento del sensor. 
De una forma similar a la Figura 5.9.a, la Figura 5.11.a indica los resultados numéricos y experimentales de la probeta y del sensor de longitud, así como el ciclo de cargas propuesto en el ensayo.

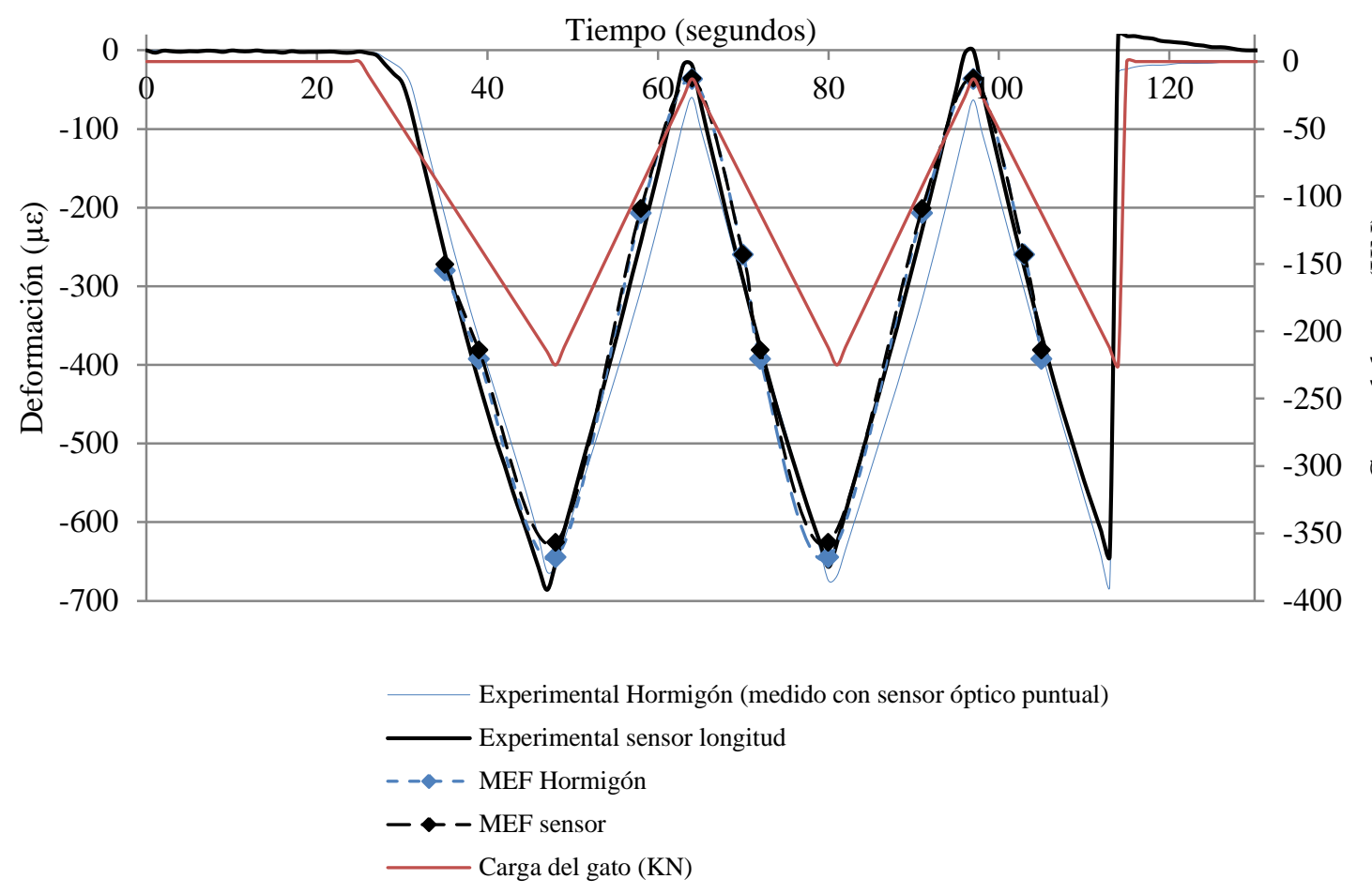

(a)
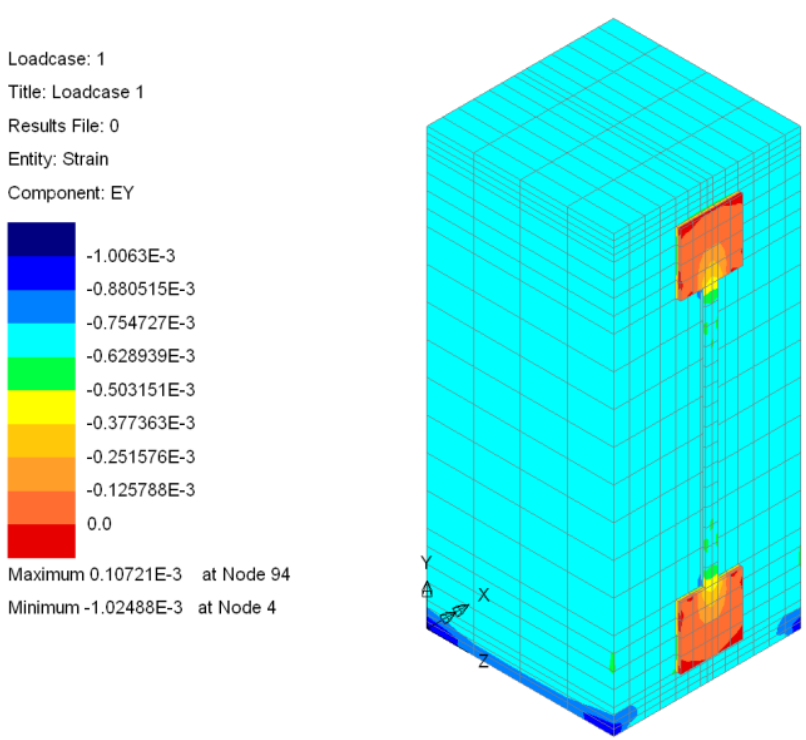

(b)

Figura 5.11 (a)-Deformaciones unitarias experimentales y numéricas en el hormigón y sensor en eje Y. (b)Modelo numérico de elementos finitos. $\varepsilon_{\mathrm{s}} / \varepsilon_{\mathrm{c}} \approx 1$ 
Monitorización estructural.

Aplicación con sensores de fibra óptica.

\section{b) Rigidez a axil del elemento.}

El elemento que forma el sensor óptico de longitud debe tener una rigidez a axil $E_{m} A_{m}$, siendo $E_{m}$ el módulo de elasticidad del material que forma el sensor y $A_{m}$ su sección transversal, adecuada, de modo que la existencia del sensor no suponga una coacción a la deformación del hormigón. La influencia de este valor se ha evaluado a partir del ratio entre las deformaciones en el hormigón sin sensor $\varepsilon_{C, 0}$ y la deformación del hormigón una vez colocado el sensor $\varepsilon_{C}$. La Figura 5.12 muestra la relación entre la rigidez a axil del elemento $E_{m} A_{m}$, el ratio $\varepsilon_{C, 0} / \varepsilon_{C}$, y el error (\%), obtenido como (1 $\left.\varepsilon_{C, 0} / \varepsilon_{C}\right)$, para sensores de distintas longitudes.

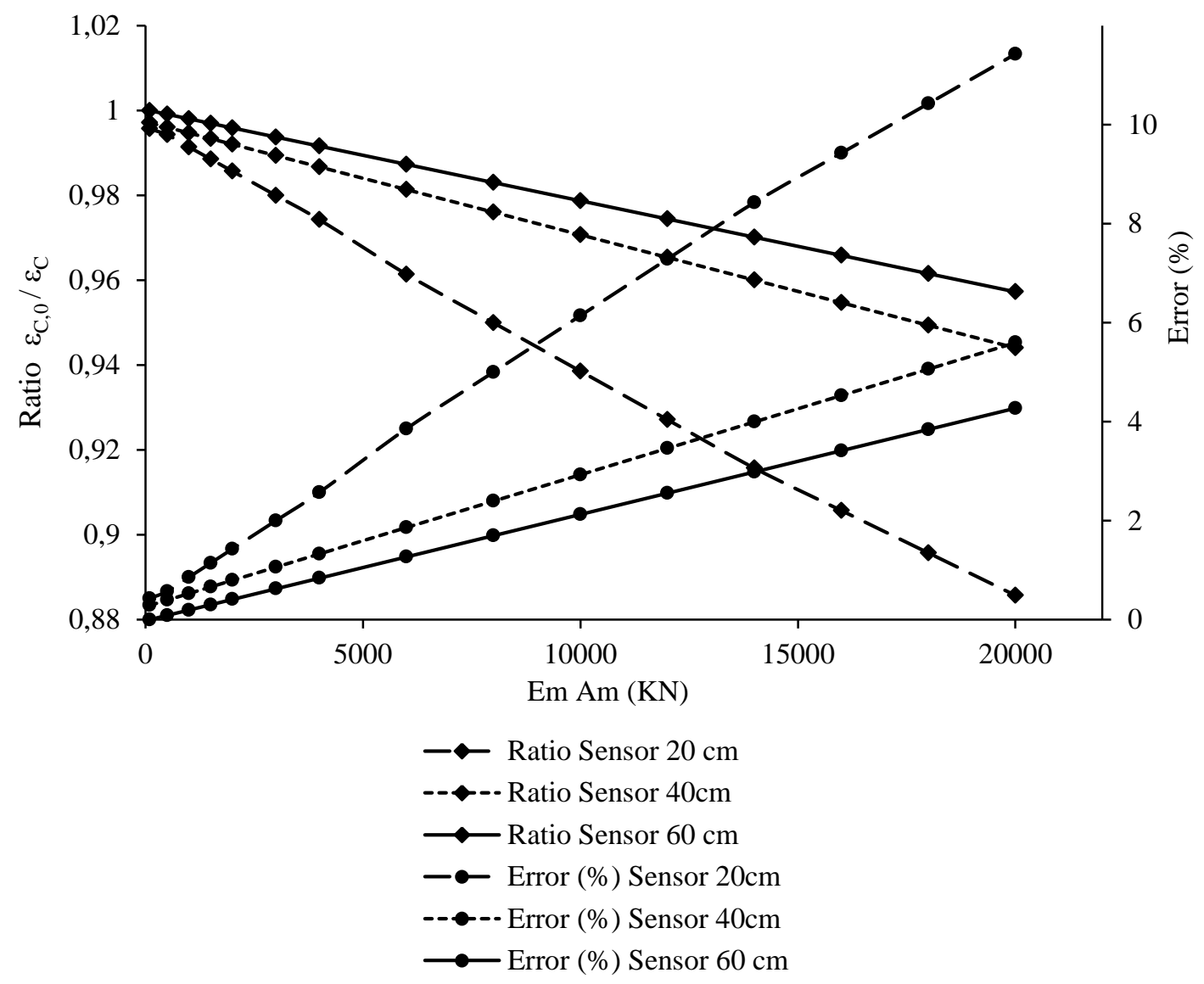

Figura 5.12 Variación del ratio ${ }^{\varepsilon_{C, 0}} / \varepsilon_{c}$ en función de la rigidez a axil del sensor $E_{m} A_{m}$. Valor de carga uniformemente repartida $21000 \mathrm{KN} / \mathrm{m}^{2}$. Valor del módulo de elasticidad del adhesivo adoptado $E_{A}=1310^{6} \mathrm{KN} / \mathrm{m}^{2}$

Como se observa en la Figura 5.12, la influencia de la rigidez a axil del elemento sensor es menor para sensores con mayores longitudes. La influencia de este factor se puede evaluar también gráficamente a partir del campo de desplazamientos en dirección Y (eje de la aplicación de la carga) que sufre el hormigón (Figura 5.13). En esta figura (5.13.a), se observa una clara influencia del elemento sensor debido a que su rigidez a axil $E_{m} A_{m}$ es elevada. En este caso, las isolíneas de igual desplazamiento en la probeta dejan de ser horizontales. En la Figura 5.13.b no existe ningún elemento sensor en superficie y las isolíneas de igual desplazamiento son horizontales. 

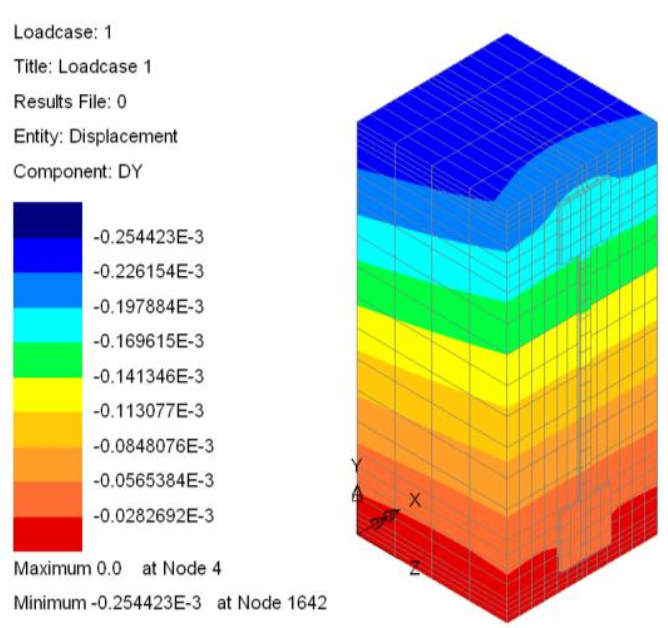

(a)

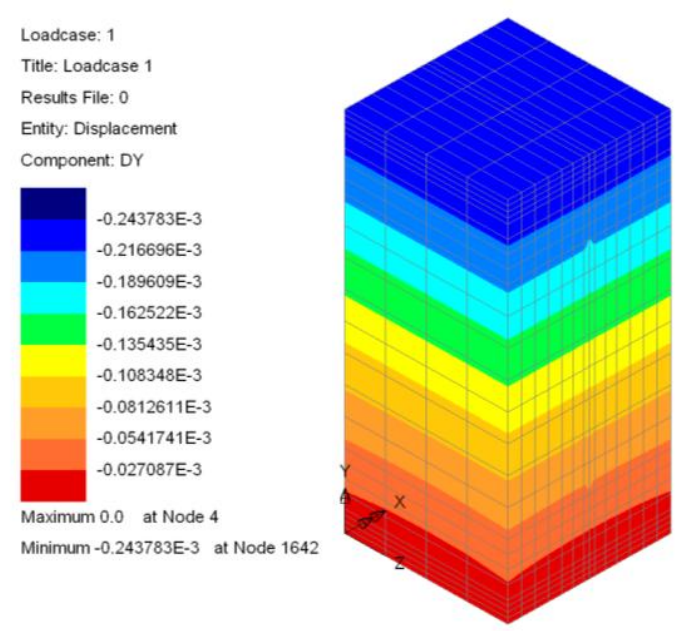

(b)

Figura 5.13 Campo de desplazamiento en dirección Y. a) Con un elemento sensor de rigidez a axil $E_{m} A_{m}=10000 \mathrm{KN}$. b) Sin ningún elemento sensor.

\subsubsection{Modos de fallo del sensor.}

Las campañas experimentales han puesto de manifiesto la existencia de dos posibles modos de fallo del sensor:

a) El despegue del sensor del hormigón por las tensiones rasantes en la zona de unión del elemento sensor y el hormigón,

b) El pandeo del sensor durante su funcionamiento.

Estos modos de fallo y la forma de corregirlos se han analizado mediante un estudio especial con un modelo numérico de elementos finitos 3D implementado en Lusas cuyos resultados se proporcionan a continuación.

a) Primer modo de fallo: Rotura por esfuerzo rasante.

El sensor propuesto produce un aumento local de las tensiones tangenciales en la interfaz de contacto entre hormigón y sensor. Si estas tensiones tangenciales alcanzan un valor límite (definido por el criterio de rotura Mohr-Coulomb), se produciría la rotura y en este caso el despegue del elemento sensor.

La Figura 5.14 muestra los resultados numéricos y experimentales de un ensayo de compresión simple sobre el pilar de 3 metros de altura indicado en el apartado 5.5.2. En esta figura se representan las deformaciones experimentales del hormigón y del sensor, y la deformación teórica del hormigón y sensor obtenida a partir de un modelo 3D de elementos finitos. En esta figura se observa que se produce el despegue entre el elemento sensor y el hormigón alrededor del minuto 28.8 del ensayo (Figuras 5.14 y 5.16.a). Hasta el instante del fallo, el funcionamiento del sensor de longitud ha sido comparado con el valor de la deformación del hormigón (obtenida a partir del sensor puntual de referencia), y su comportamiento guarda un elevado grado de ajuste. A medida que aumenta el valor de la carga se producen mayores diferencias entre los valores experimentales y teóricos. Esto puede 
Monitorización estructural.

Aplicación con sensores de fibra óptica.

ser debido a posibles flexiones sobre el pilar debido a que el centrado de la carga aplicada en su cabeza no es perfecto.

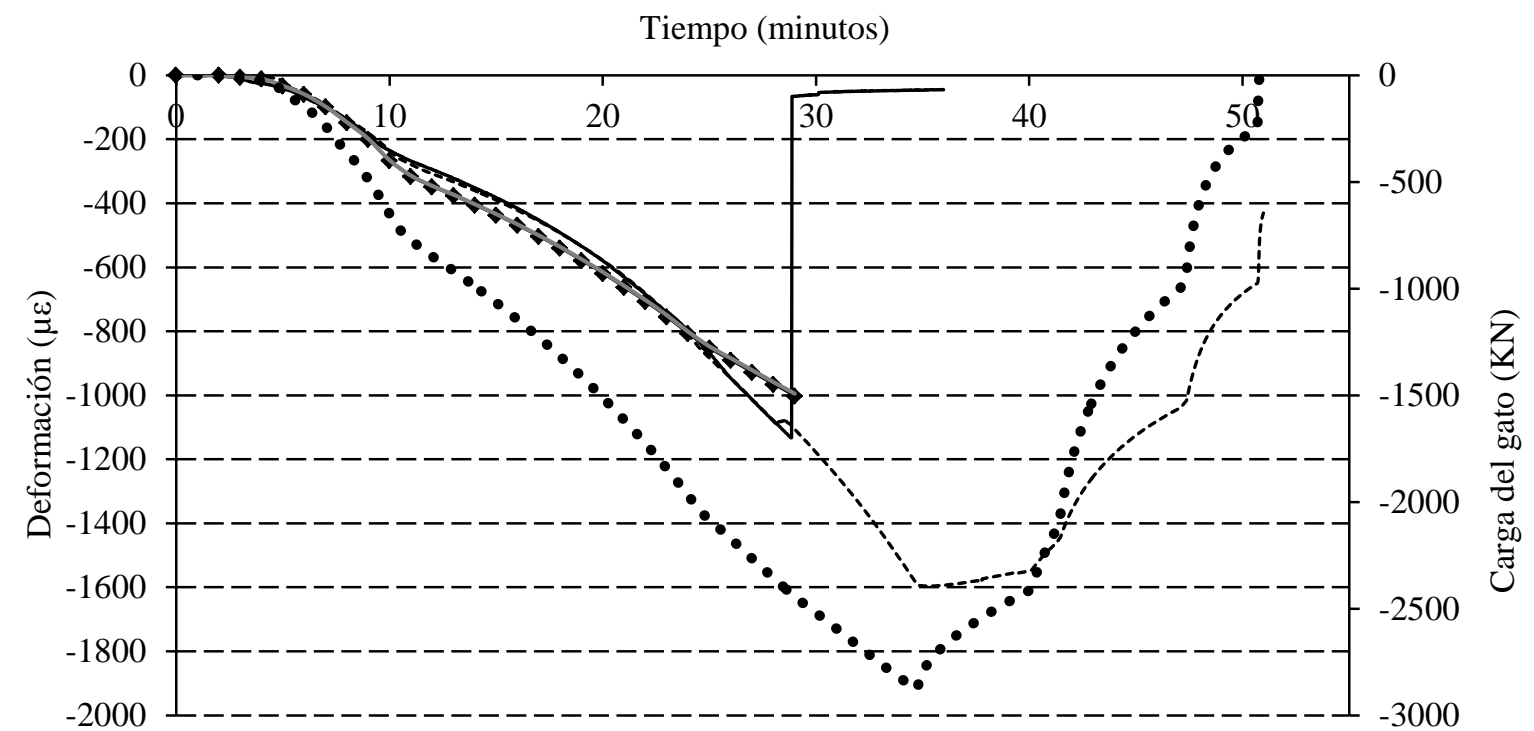

- Sensor óptico de longitud

------- Deformación hormigón

$\longrightarrow$ - Sensor óptico de longitud (MEF)

- Deformación hormigón (MEF)

-... Carga del gato $(\mathrm{KN})$

Figura 5.14 Resultados experimentales del sensor óptico puntual de referencia, de un sensor óptico de longitud y resultados teóricos obtenidos a través de un MEF 3D.

Además, el ajuste del modelo numérico de elementos finitos con los resultados experimentales (Figura 5.14) ha permitido su calibración. De este modo, se han podido determinar las tensiones tangenciales que se alcanzan en la interfaz de contacto. En la Tabla 5.4 se indican los principales datos del sensor y resultados numéricos obtenidos como la longitud de sensor ensayado $L_{\text {sensor }}$, el módulo de elasticidad del adhesivo adoptado $E_{A}$, el área de la sección transversal del sensor $A_{m}$, la tensión tangencial en la interfaz de contacto entre hormigón y adhesivo $\tau_{\text {interfaz }}$, la deformación del hormigón en dirección de la aplicación de la carga $\varepsilon_{\text {hormigón,MEF }}$ y la deformación del sensor $\varepsilon_{\text {sensor, } M E F}$.

\begin{tabular}{cccccc}
\hline$L_{\text {sensor }}(\mathrm{m})$ & $\boldsymbol{E}_{\boldsymbol{A}}(\mathrm{MPa})$ & $\boldsymbol{A}_{\boldsymbol{m}}\left(\mathrm{cm}^{\mathbf{2}}\right)$ & $\boldsymbol{\tau}_{\text {interfaz,MEF }}\left(\boldsymbol{K N} / \boldsymbol{m}^{2}\right)$ & $\varepsilon_{\text {hormigón,MEF }}(\mu \varepsilon)$ & $\varepsilon_{\text {sensor,MEF }}(\mu \varepsilon)$ \\
\hline 1 & 13000 & 0.2 & 12043 & -1001.9 & -994.7 \\
\hline
\end{tabular}

Tabla 5.4 Principales resultados numéricos del modelo.

La Figura 5.15 muestra el elemento ensayado con el sensor de longitud en superficie. La Figura 5.16.a muestra un detalle del sensor y de la probeta ensayada en el momento en el que se produjo el despegue del sensor. La Figura 5.16.b muestra las tensiones tangenciales obtenidas en la interfaz de contacto entre el hormigón y el adhesivo empleado. En la situación de carga que produce el despegue del sensor, se observa que las tensiones tangenciales alcanzadas en la interfaz de contacto tienen un valor 
de $12043 \mathrm{KN} / \mathrm{m}^{2}$. Este valor se alcanza en las esquinas superiores de la interfaz de contacto, tal y como indica la Figura 5.16.b.
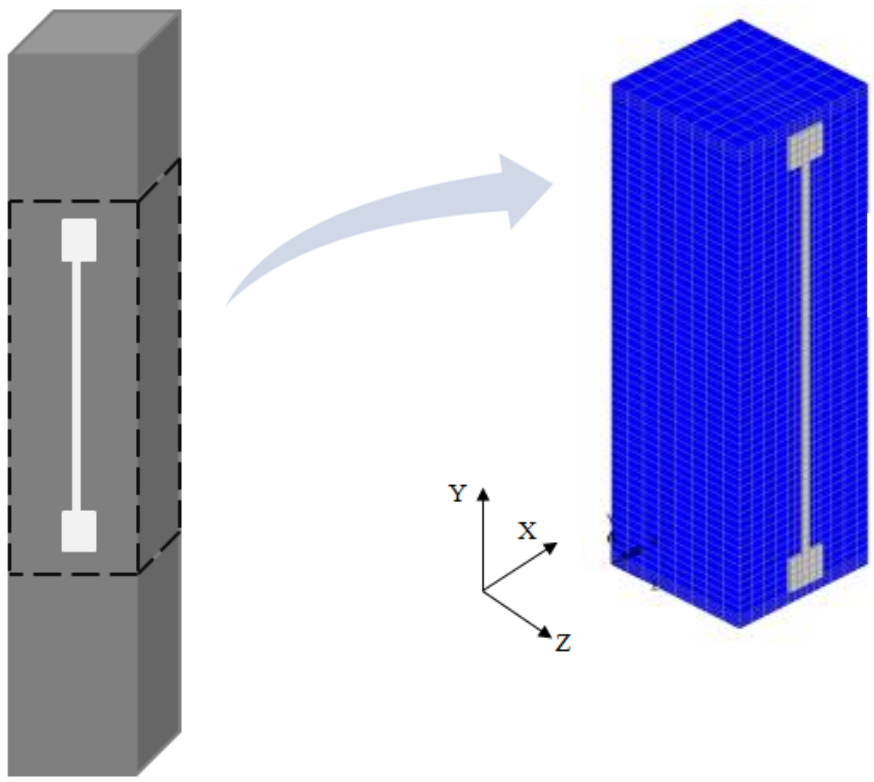

Figura 5.15 Elemento de hormigón ensayado. Para una mejor visualización, se muestran los resultados en la zona del elemento donde está instalado el sensor. Vista frontal de la probeta

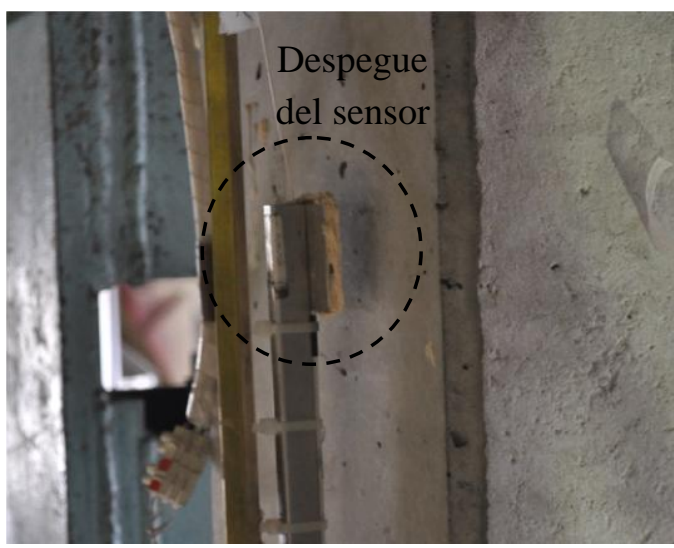

(a)

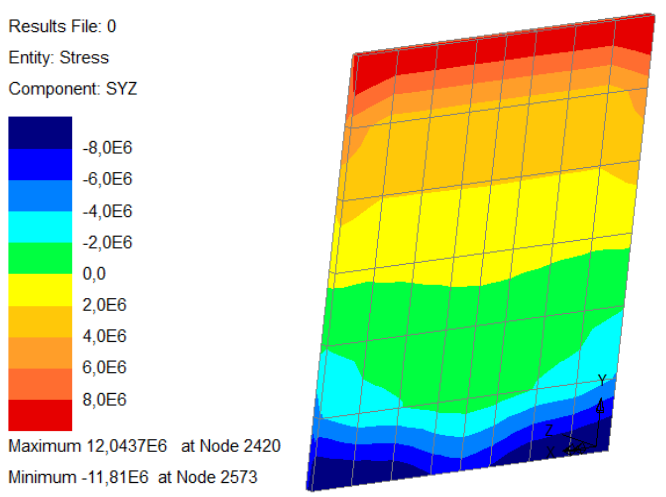

(b)

Figura 5.16 (a)- Imagen del despegue del sensor. (b)- Vista del modelo de elementos finitos de la interfaz de contacto entre hormigón y adhesivo donde se observa el valor de las tensiones tangenciales máximas alcanzadas $\left(12043 \mathrm{KN} / \mathrm{m}^{2}\right)$ en el instante en que se produce el despegue.

El valor de la tensión tangencial alcanzada en la interfaz de contacto $\left(12043 \mathrm{KN} / \mathrm{m}^{2}\right)$ es superior al valor límite dado por el valor de la resistencia a cizallamiento del adhesivo y que se ha introducido en el modelo numérico como el valor de la cohesión $\left(\mathrm{c}=10.000 \mathrm{KN} / \mathrm{m}^{2}\right.$, Apartado 5.5.3.b). 
Monitorización estructural.

Aplicación con sensores de fibra óptica.

Es importante explicar que el modelo empleado para simular la interfaz de contacto se trata de un modelo elasto-plástico. Sin embargo, la rotura producida ha sido repentina, por lo que el modelo numérico adoptado sólo es válido hasta el momento en que se produce el despegue, para de esta forma estimar el valor de las tensiones tangenciales en la interfaz de contacto.

Tras observar los anteriores resultados y para garantizar un funcionamiento correcto del sensor de longitud, se hace necesario adoptar alguna medida para la reducción de las tensiones tangenciales. Para ello se estudian dos alternativas:

1) Estudio del módulo de elasticidad del adhesivo $E_{A}$.

La evolución de las tensiones tangenciales está en relación directa con el valor de $E_{A}$ adoptado. El modelo numérico muestra claramente como a mayor valor de $E_{A}$ se generan mayores tensiones tangenciales en la unión entre ambos elementos (Figura 5.17).

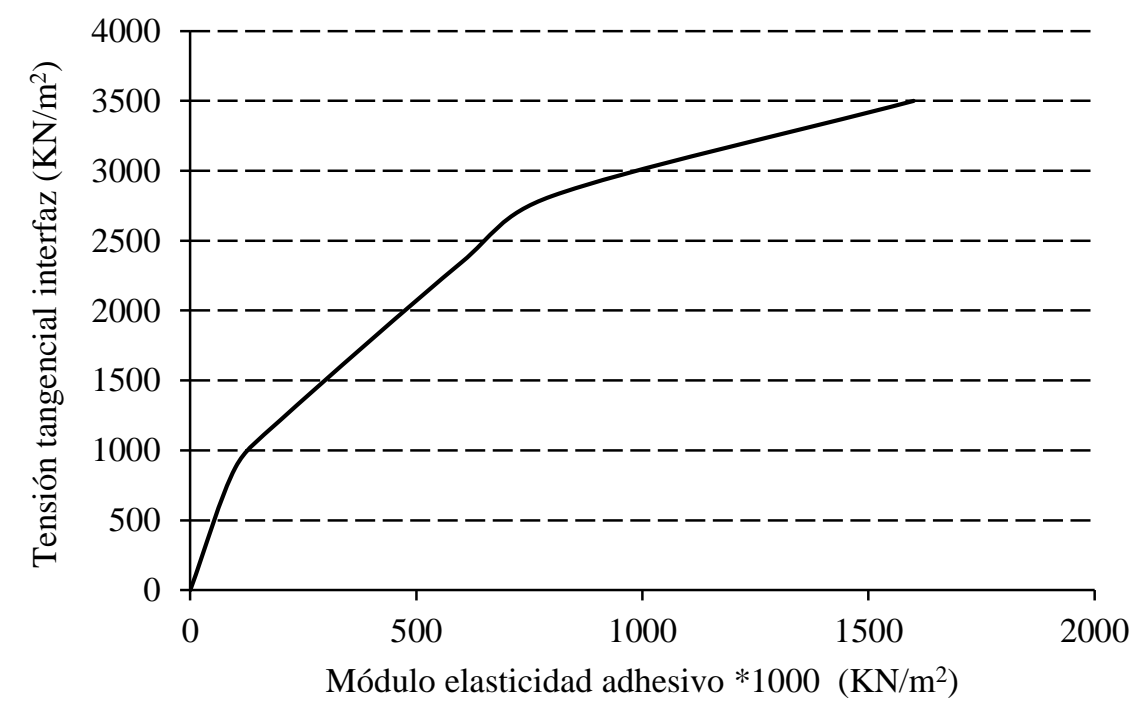

Figura 5.17 Variación de la tensión tangencial en el hormigón en función del valor del módulo de elasticidad del adhesivo $E_{A}$.

No obstante, la reducción del módulo de elasticidad del adhesivo adoptado $E_{A}$ presenta los inconvenientes que se indicaron en el apartado 5.6.1.a "Resultados. Comportamiento general. Transferencia de deformaciones" por lo que su aplicación no garantiza una buena solución.

2) Reducción de la sección transversal del elemento sensor $A_{m}$.

Una menor área transversal del elemento sensor implica una menor solicitación tangencial sobre la unión entre el elemento sensor y hormigón. De este modo, se ha reducido la sección transversal del elemento sensor en un 50\% y, mediante un MEF 3D, se han estudiado las tensiones tangenciales que se producen en la zona de contacto.

La Tabla 5.5 resume los principales resultados numéricos obtenidos. La Figura 5.18 muestra el valor de las tensiones tangenciales en el hormigón, en la misma situación de carga que producía el fallo de la Figura 5.14. 


\begin{tabular}{cccccc}
\hline $\begin{array}{c}\boldsymbol{L}_{\text {sensor }} \\
(\boldsymbol{m})\end{array}$ & $\boldsymbol{E}_{\boldsymbol{A}}(\mathbf{M P a})$ & $\boldsymbol{A}_{\boldsymbol{m}}\left(\mathbf{c m}^{2}\right)$ & $\boldsymbol{\tau}_{\text {interfaz,MEF }}\left(\boldsymbol{K N} / \boldsymbol{m}^{2}\right)$ & $\boldsymbol{\varepsilon}_{\text {hormigón,MEF }}(\mu \varepsilon)$ & $\boldsymbol{\varepsilon}_{\text {sensor,MEF }}(\mu \varepsilon)$ \\
\hline 1 & 13000 & 0.1 & 6148.6 & -979.5 & -970.3 \\
\hline
\end{tabular}

Tabla 5.5 Principales resultados numéricos del modelo.
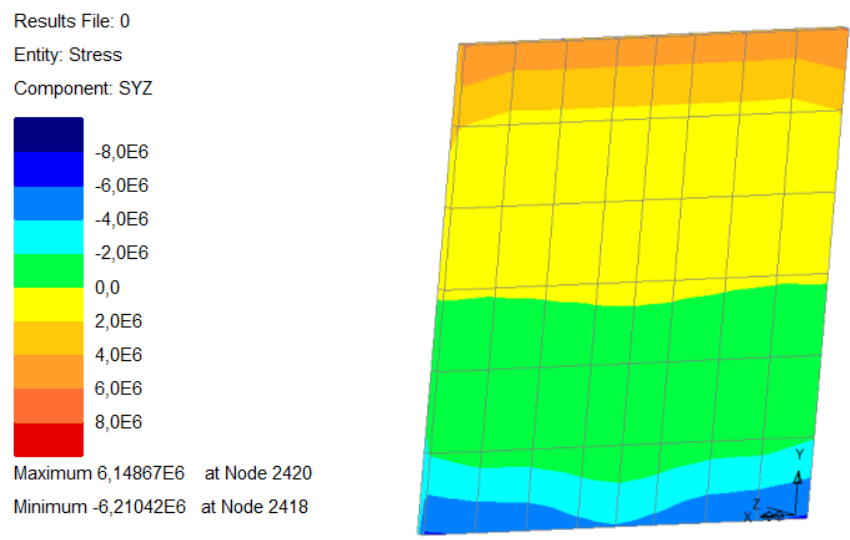

Figura 5.18 Tensiones tangenciales (valor máximo de $6148.6 \mathrm{KN} / \mathrm{m}^{2}$ ) en la interfaz de contacto entre hormigón y adhesivo.

Tras una reducción en el 50\% de su sección transversal, el valor de la tensión tangencial máxima en la interfaz de contacto, para una deformación del hormigón de $-979.5 \mu \varepsilon$, es de $6148.6 \mathrm{KN} / \mathrm{m}^{2}$ (Figura 5.18), frente al valor anterior de $12043 \mathrm{KN} / \mathrm{m}^{2}$. De esta forma se ha mejorado notablemente el funcionamiento de la unión entre sensor y hormigón, no debiendo producirse el despegue entre ambos elementos ya que este nuevo valor de tensión tangencial $\left(6148.6 \mathrm{KN} / \mathrm{m}^{2}\right)$ es inferior al valor límite que produciría su despegue (Tabla 5.3, Apartado 5.5.3.b).

Una vez estimado numéricamente el valor de las tensiones tangenciales alcanzadas en los ensayos experimentales, éstos se han repetido adoptando las precauciones siguientes para evitar que se produzca el fallo del elemento sensor:

- Se ha aumentado la rugosidad de las superficies de contacto, lo que supone un aumento considerable en la tensión tangencial última al producirse un aumento de su cohesión (c).

- Se ha disminuido la sección transversal del elemento sensor para disminuir las tensiones tangenciales.

- No se ha llevado ninguna actuación sobre el área de contacto entre hormigón y sensor debido a que un aumento de esta área no implica una disminución de las tensiones tangenciales. Esto se debe a que la concentración de las tensiones se produce en una zona localizada y el área que eficazmente trabaja no aumenta al aumentar el área de contacto entre sensor y hormigón. 
Monitorización estructural.

Aplicación con sensores de fibra óptica.

Los resultados obtenidos se muestran en la Figura 5.19, donde la deformación obtenida con el sensor de longitud se compara con la deformación del hormigón (obtenida con un sensor puntual de referencia), colocados muy próximos entre sí.

En este ensayo la rotura del hormigón se ha producido sobre el minuto 4.45 y con una deformación del hormigón del $2 \%$, lógica puesto que la probeta se ensaya a compresión simple. Alrededor del minuto $3.4 \mathrm{y}$, por tanto, antes de alcanzarse la rotura del hormigón se ha producido una descarga con la finalidad de detectar una posible histéresis asociada a la carga y descarga sobre el elemento sensor. Durante el ensayo no se produce el despegue del elemento sensor y éste proporciona la misma deformación que el hormigón, que ha sido medida empleando un sensor óptico puntual de referencia (Figura 5.19). Por este motivo los resultados presentan un buen grado de ajuste y el comportamiento del sensor de longitud se considera muy satisfactorio.

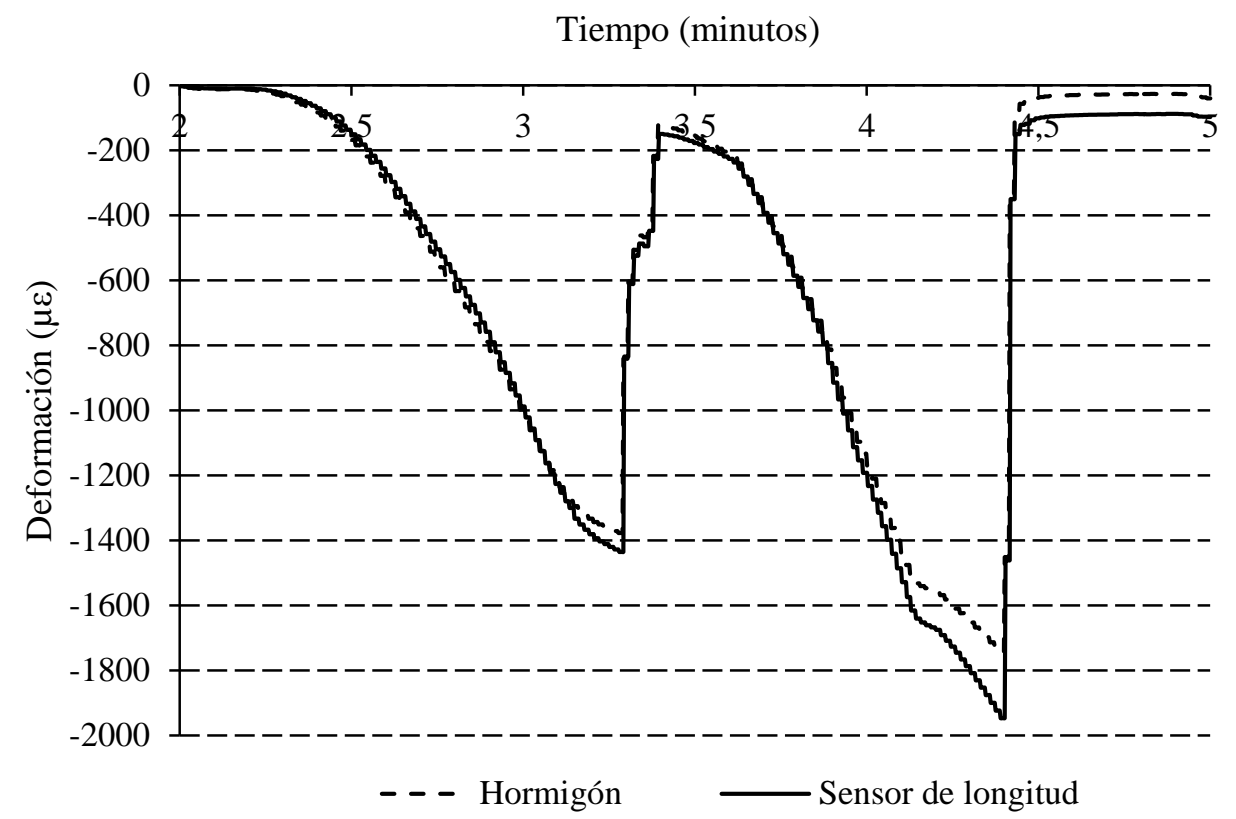

Figura 5.19 Resultados del ensayo experimental.

\section{b) Segundo modo de fallo: Pandeo del elemento sensor.}

El sensor propuesto es un elemento que podría pandear ante solicitaciones de compresión transmitidas al elemento sensor a través de las uniones entre la estructura monitorizada y el sensor.

Una posible medida para evitar el pandeo del sensor sería un aumento del producto $E_{m} I_{m}$, donde $E_{m}$ representa el valor del módulo de elasticidad del material que forma el sensor e $I_{m}$ el momento de inercia de su sección. Sin embargo, el aumento de $E_{m} I_{m}$ lleva asociado un aumento de $E_{m} A_{m}$, y esto tiene los inconvenientes que se expusieron en el apartado "5.6.1.b Rigidez a axil del elemento". Por ello, en esta Tesis Doctoral se ha propuesto un elemento que proporciona un aumento del valor $E_{m} I_{m}$ del sensor, pero no modifica su valor $E_{m} A_{m}$. Para el diseño de este elemento y dado que el pandeo del sensor ha sido motivo de fallos en los ensayos experimentales, ha sido necesario un estudio especial con modelos de elementos finitos. 
El estudio del pandeo también se ha realizado empleando el programa Lusas V.14.3-11, tal y como se explicó en el apartado 5.5.3.c. En él se ha modelado el sensor como un conjunto de elementos finitos tipo QSL8 (Lusas, 2010) y se ha estudiado la carga a la que se produce el pandeo. Como el correcto funcionamiento del sensor debe impedir su pandeo, sólo se ha estudiado el primero de todos los modos (Figura 5.20).

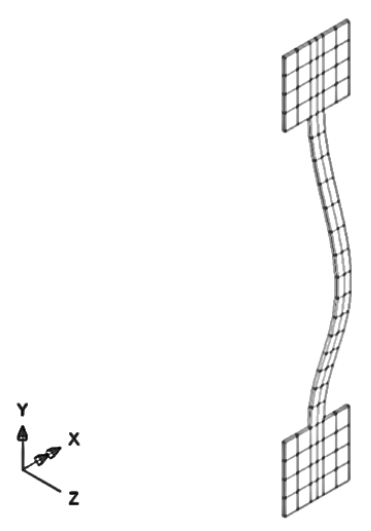

Figura 5.20 Malla del elemento sensor sometido a compresión. Primer modo de pandeo.

Dado que el sensor es un elemento esbelto (debido a las recomendaciones de los apartados anteriores) su pandeo se produciría con pequeñas cargas. Para evitar esto se ha diseñado un elemento que impide esta inestabilidad. Tal y como se explicó anteriormente, este elemento, llamado inhibidor de pandeo, no contribuye a aumentar el valor de $E_{m} A_{m}$ del sensor, pero sí su producto $E_{m} I_{m}$.

El diseño de este elemento se ha llevado a cabo considerando que impone sobre el sensor una coacción de rigidez K, que actúa sobre el sensor cuando éste tiende a pandear (Figura 5.21). El valor de estas rigideces se ha obtenido a partir de una modelización del inhibidor de pandeo, imponiendo una fuerza $\mathrm{F}$ unidad y obteniendo sus desplazamientos $d$ en dirección de la carga. La rigidez $\mathrm{K}$ del inhibidor se obtiene a partir del cociente entre ambos $F / d$.

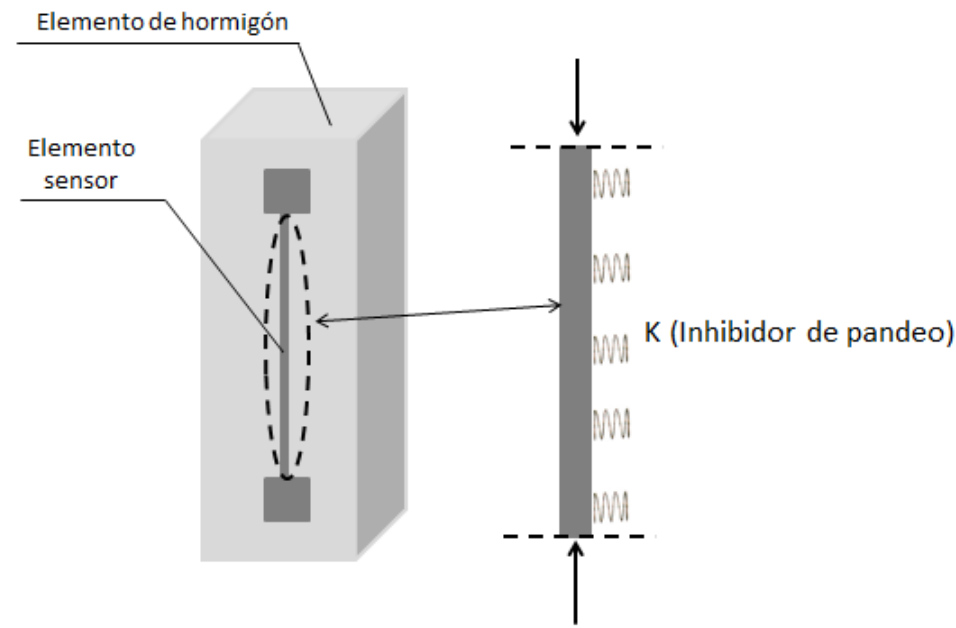

Figura 5.21 Elemento sensor y modelización del inhibidor de pandeo mediante una rigidez K. 
La carga de pandeo del sensor con distintos inhibidores de pandeo se compara con el valor de carga al que debe estar sometido el sensor, cuando el hormigón le transfiere su deformación máxima correspondiente a un 3.5\% en flexión compuesta (Figura 5.22). Este valor se llamará "carga de pandeo del sensor". De esta forma se ha estudiado la relación existente entre la longitud del sensor, la rigidez $E_{m} I_{m}$ del inhibidor y las cargas a las que se produce el pandeo. A partir de la Figura 5.22 se obtiene el mínimo valor de $E_{m} I_{m}$ que deberá tener el inhibidor de pandeo que garantice un buen funcionamiento del sensor.

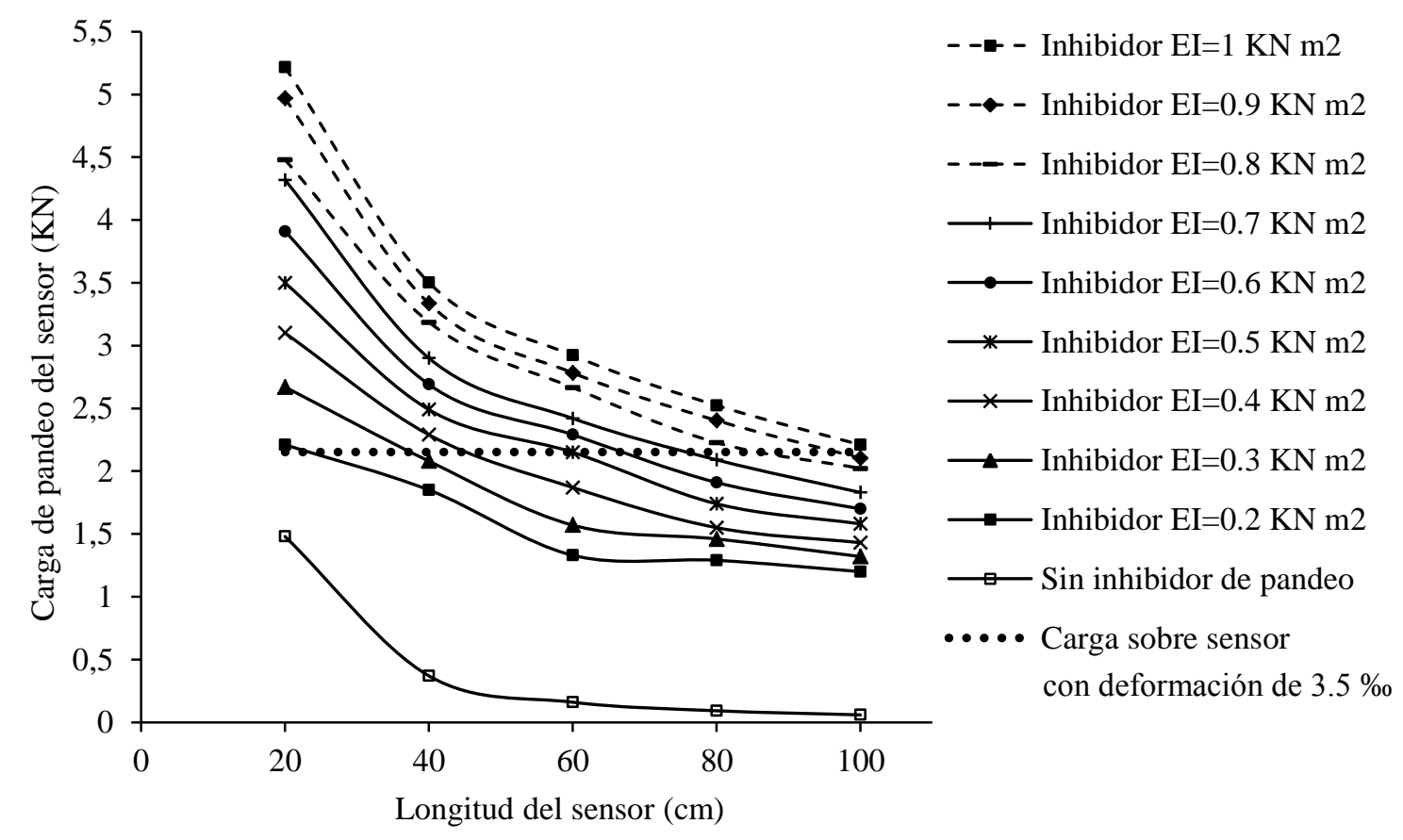

Figura 5.22 Cargas de pandeo en función de distintas rigideces relativas y longitudes de sensor.

A partir de los resultados obtenidos en la Figura 5.22 se observa que:

- Sin emplear ningún inhibidor de pandeo el sensor no funciona correctamente ante esfuerzos de flexocompresión en los que la deformación máxima a transmitir al sensor sería del 3.5 \%.

- A medida que aumenta la longitud del sensor, es necesario emplear inhibidores de pandeo que proporcionen mayores valores de $E_{m} I_{m}$. Por ejemplo, empleando un inhibidor de pandeo de $0.4 \mathrm{KN} \mathrm{m}^{2}$ se resuelve el problema del pandeo para sensores de hasta $40 \mathrm{~cm}$. Sin embargo, este inhibidor no es suficiente si queremos emplear un sensor de $60 \mathrm{~cm}$ de longitud. En este caso sería necesario emplear un inhibidor cuyo valor $E_{m} I_{m}$ sea igual a $0.6 \mathrm{KN} \mathrm{m}^{2}$.

- Mediante el empleo de un inhibidor del pandeo con un valor de $E_{m} I_{m}$ igual a $1 \mathrm{KNm}^{2}$ queda resuelto este modo de fallo para sensores de hasta 1 metro de longitud. 


\section{7- Conclusiones.}

La monitorización de estructuras exige en ocasiones el empleo de sensores que no se vean influenciados por los defectos puntuales de los materiales. Los sensores de longitud, (también llamados long gauge en inglés) ofrecen estas posibilidades pero su funcionamiento exige un pretensado previo de la fibra óptica, y ésta es una operación delicada y cargada de incertidumbre.

Establecida esta necesidad, se han presentado los resultados experimentales y numéricos sobre el diseño y comportamiento de un nuevo sensor óptico de longitud, al que no es necesario dotarle de un pretensado previo para su funcionamiento. Ambas fases se han empleado para calibrar su comportamiento y definir un diseño óptimo. El diseño y los modos de fallo han sido estudiados a partir de modelos numéricos 3D empleando para ello el programa Lusas V.14.3-11.

Antes de mostrar las principales conclusiones obtenidas, es importante destacar que el alcance de los trabajos y sus resultados se centran en las siguientes consideraciones:

1) Los resultados obtenidos son de aplicación para la monitorización de estructuras de hormigón endurecido.

2) Los resultados mostrados son válidos para el rango de módulos elásticos que abarcaría un hormigón endurecido. En este estudio se han ensayado hormigones con módulos elásticos que abracan entre unos valores de 29450 y $34116 \mathrm{MPa}$.

3) Los resultados mostrados son válidos para el rango de dimensiones habituales empleadas en columnas de hormigón. En este estudio se han ensayado probetas de base cuadrada con dimensiones mínimas de $0.1 \mathrm{~m}$ de lado y con dimensiones máximas de $0.3 \mathrm{~m}$ de lado.

Establecidos los alcances de los trabajos realizados y su campo de aplicación, las principales conclusiones obtenidas han sido las siguientes:

1) El diseño de los anclajes del sensor a la estructura debe permitir una correcta transferencia de deformaciones entre el material huésped (estructura) y el sensor. En este apartado, se ha estudiado la influencia de las propiedades del adhesivo empleado, acotando los valores que ofrecerán resultados satisfactorios. En este sentido, un adhesivo que disponga de un módulo de elasticidad $E_{A}$ superior a $400 \mathrm{MPa}$ permitirá una correcta transferencia de deformaciones con ratios $\varepsilon_{S} / \varepsilon_{c}$ próximos a 1 . Por el contrario, valores de $E_{A}$ en torno a $100 \mathrm{MPa}$ proporcionarán unas diferencias entre las deformaciones reales de la estructura y las medidas por el sensor de un $20 \%$.

2) El valor del producto $E_{m} A_{m}$ del sensor debe ser lo suficientemente bajo como para permitir una correcta medición. Elevados valores de $E_{m} A_{m}$ ofrecen resultados incorrectos porque suponen un refuerzo que se opone a la deformación de la estructura. Sensores con valores de $E_{m} A_{m}$ inferiores a $5 \mathrm{MN}$ proporcionarán unas diferencias entre las deformaciones reales y medidas inferiores a un $4 \%$.

3) Además, tal y como se mostró en la Figura 5.12, la influencia de la rigidez axil del elemento sensor es menor para sensores con mayores longitudes. Por ejemplo, para sensores de $20 \mathrm{~cm}$ de longitud y con un valor de $E_{m} A_{m}$ de $20 \mathrm{MN}$ se ha obtenido que el ratio entre la deformación del hormigón antes de instalar el sensor y una vez instalada, ${ }^{\varepsilon_{C, 0}} / \varepsilon_{C}$, está en 
torno a 0.89 , lo que supone un error en la medida en torno a un $11 \%$. Por el contrario, el mismo sensor (valor de $E_{m} A_{m}$ de $20 \mathrm{MN}$ ) pero de $60 \mathrm{~cm}$ de longitud presenta un ratio ${ }^{\varepsilon_{C, 0}} / \varepsilon_{C}$ de aproximadamente 0.96 , o lo que es lo mismo un error estimado en la medida de $4 \%$.

4) En el estudio presentado en este capítulo no se ha considerado la relación entre las propiedades mecánicas y geométricas del sensor propuesto con el módulo elástico del material huésped. Sin embargo, los rangos de aplicación de los resultados obtenidos han quedado acotados teniendo en cuenta el alcance de los trabajos.

5) Por otro lado, elevados valores de $E_{m} A_{m}$ provocan elevadas tensiones tangenciales en los anclajes del sensor al hormigón, llegando a producir su despegue. Para evitar este modo de fallo, se ha propuesto el empleo de un sensor con un valor de ${ }_{m} A_{m}=700 \mathrm{KN}$ y se ha aumentado la rugosidad de la cara de contacto, para aumentar de este modo la cohesión en la interfaz de contacto entre hormigón y sensor.

6) La adopción de un adhesivo con un elevado módulo de elasticidad también provoca elevadas tensiones tangenciales en la interfaz de contacto entre hormigón y sensor. Para evitarlo, debería escogerse un adhesivo con un bajo módulo de elasticidad. Sin embargo, la reducción del módulo de elasticidad del adhesivo presenta el inconveniente de una transferencia incompleta de las deformaciones y por tanto, no se recomienda esta alternativa.

7) Dado que el sensor propuesto en este capítulo es un elemento sometido a compresión, otro modo de fallo es el pandeo del sensor. Para evitarlo, se ha propuesto el empleo de un elemento que inhibe esta inestabilidad, aumentando el producto $E_{m} I_{m}$ del sensor y sin colaborar con su rigidez $E_{m} A_{m}$. Un elemento longitudinal con una rigidez a flexión de $1 \mathrm{KNm}^{2}$ solucionaría este modo de fallo para sensores de hasta 1 metro de longitud y para esfuerzos de flexocompresión, donde la deformación máxima del hormigón a compresión es de 3.5\%.

Por tanto, el diseño de sensor propuesto tiene las características siguientes:

- Elemento prismático de $0.1 \mathrm{~cm}^{2}$ de sección transversal.

- Valor de $E_{m} A_{m}=700 \mathrm{KN}$.

- Valor de $E_{m} I_{m}=58.310^{-6} \mathrm{KN} \mathrm{m}^{2}$.

- Módulo de elasticidad del adhesivo empleado $E_{A}=13000 \mathrm{MPa}$.

- Longitud del sensor comprendida desde 20 hasta $100 \mathrm{~cm}$.

Y permite medir con gran precisión las deformaciones de la estructura. 


\section{A \\ PLICACIÓN DE LOS SENSORES DESARROLLADOS}

EN LOS CAPÍTULOS 4 Y 5 A LA MONITORIZACIÓN DE

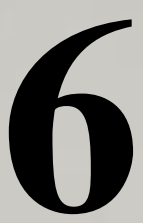

UNA ESTRUCTURA REAL.

MONITORIZACIÓN DEL FALSO TÚNEL DE LA LÍNEA DE

ALTA VELOCIDAD DE LEVANTE A SU PASO POR

MOGENTE (VALENCIA).

El Capítulo 6 de este documento presenta la aplicación de los sensores ópticos puntuales y de longitud estudiados en los Capítulos 4 y 5 a la instrumentación de una estructura real de hormigón. La estructura en cuestión es un falso túnel de la línea de Alta Velocidad de Levante a su paso por Mogente (Valencia).

Para ello, se instrumentó una sección completa del arco del túnel (concretamente la sección más solicitada por ser aquella que poseía mayor altura de tierras sobre ella) con los sensores ópticos diseñados en los capítulos anteriores. Los resultados obtenidos de la monitorización fueron comparados con los resultados teóricos obtenidos con un modelo numérico simplificado de elementos finitos $2 \mathrm{D}$.

Esta comparación de resultados ha permitido validar el funcionamiento de los sensores, ya que éstos marcaron la pauta general de comportamiento del falso túnel incluso estando sometido a cargas muy pequeñas originadas por las variaciones térmicas. Los resultados numéricos y experimentales mostraron, en general, un buen ajuste entre ellos.

Con este trabajo se ha estudiado el comportamiento de los sensores ópticos propuestos bajo la influencia de las condiciones reales que impone el sector de la construcción y ha permitido comparar el funcionamiento de ambos tipos de sensores. 



\subsection{Introducción.}

En el Capítulo 5 se ha mostrado el estudio y diseño de un nuevo sensor óptico de longitud que permite obtener las deformaciones en estructuras. Este sensor presenta una importante ventaja sobre los ya existentes, y es que no necesita de un pretensado previo en la fibra óptica. Las campañas experimentales y numéricas llevadas a cabo han mostrado su correcto funcionamiento. Llegados a este punto, el siguiente paso consiste en la aplicación de este nuevo sensor óptico de longitud a la instrumentación de una obra real: un falso túnel en la línea de Alta Velocidad de Levante a su paso por Mogente (Valencia).

En esta estructura se ha instalado un total de cuatro tipos distintos de sensores ópticos:

- Sensores puntuales de deformación y temperatura expuestos en el Capítulo 4.

- Sensores de longitud de deformación propuestos en el Capítulo 5.

- Sensores distribuidos de deformación basados en el fenómeno de dispersión de Brillouin (en inglés, Brillouin Scattering), expuestos en los capítulos 7 y 8 . Esta última aplicación se ha llevado a cabo en colaboración con la Universidad Pública de Navarra (UPNA).

La instalación de los sensores se ha realizado una vez finalizada la construcción de la estructura de hormigón. Concretamente se llevó a cabo 3 meses después de su hormigonado. El motivo de este retraso fue la existencia de importantes coqueras y defectos en el hormigón, que fue necesario reparar antes de iniciar el relleno de tierras. La toma de datos se ha realizado en instantes anteriores y posteriores al relleno de las tierras, para conseguir varios objetivos:

1. Conocer el comportamiento y las prestaciones de los sensores de longitud propuestos bajo las condiciones reales que impone el sector de la construcción.

2. Comparar el comportamiento de los sensores ópticos de longitud propuestos frente a los puntuales.

3. Estudiar la aplicabilidad de los sensores distribuidos propuestos frente a deformaciones y temperaturas. Los trabajos y resultados ligados a este objetivo se exponen en el Capítulo 8.

4. Con las mediciones realizadas por los sensores ópticos, mostrar la pauta general de comportamiento de la estructura ante las acciones de:

a. Las variaciones térmicas existentes, a partir de mediciones continuas a lo largo de varias horas donde existen importantes gradientes de temperatura.

b. El peso y empuje de las tierras, a partir de mediciones instantáneas una vez finalizada la operación de relleno de tierras.

Es importante destacar que estas son las dos principales acciones que influirán sobre el comportamiento de la estructura y sobre las mediciones realizadas con los sensores ópticos. Dado que los trabajos en la estructura se llevaron a cabo pasados más de tres meses desde su hormigonado, y que las tomas de datos se han realizado en periodos relativamente cortos, se ha despreciado los efectos de la retracción y fluencia en la obtención de los valores teóricos con el modelo simplificado de elementos finitos $2 \mathrm{D}$. 
Los valores medidos han sido comparados con los resultados teóricos obtenidos a partir de un modelo numérico simplificado 2D de elementos finitos empleando el programa Lusas V.14.3-11 (Lusas, 2010). El objetivo de este modelo numérico simplificado es el de obtener una pauta general de comportamiento del falso túnel, y poderla comparar con la obtenida a partir de las mediciones con los sensores ópticos. La comparación entre los resultados medidos y numéricos ha presentado, en general, un buen ajuste y justifica de esta forma el buen comportamiento de los sensores propuestos en los Capítulos 4 y 5.

\subsection{Estrategia de monitorización propuesta.}

Para llevar a cabo el proceso de monitorización en el falso túnel se han tenido en cuenta una serie de consideraciones:

- Se ha monitorizado una sección transversal de túnel. Concretamente, se ha instrumentado la sección sobre la que descansa una mayor altura de relleno de tierras, correspondiente a 20.3 metros medidos desde la base de la bóveda del túnel (Figura 6.1).

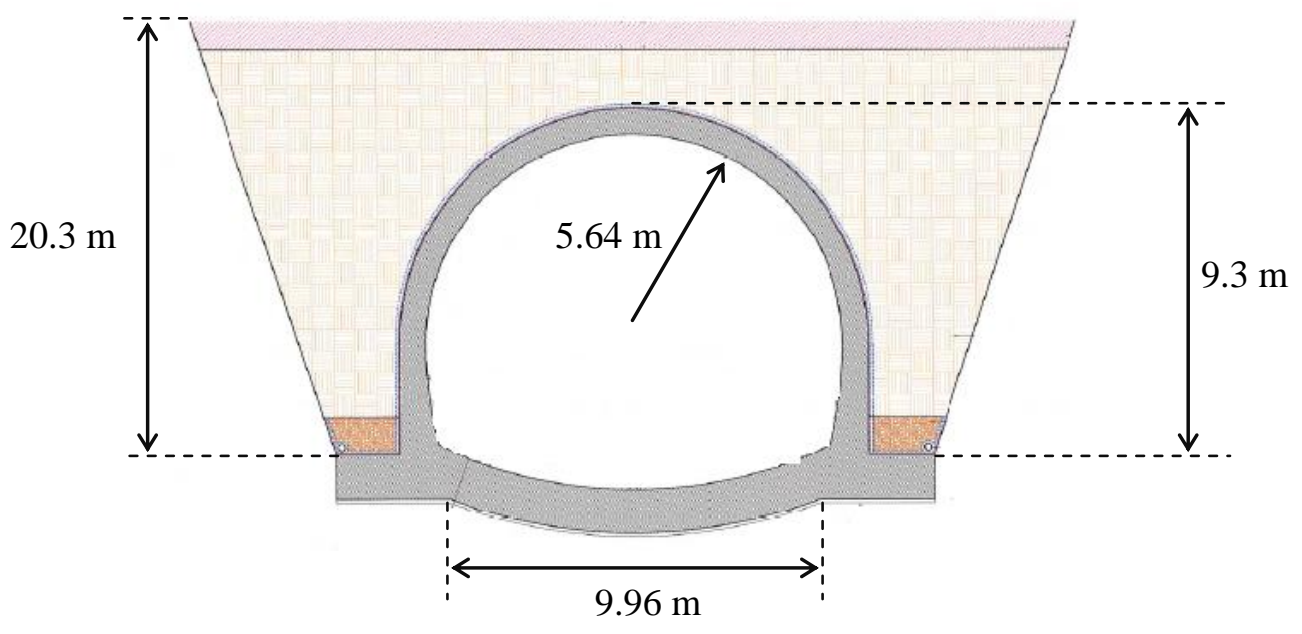

Figura 6.1 Sección transversal instrumentada y principales dimensiones (Fuente: Anejo de Cálculo de Estructuras del Proyecto).

- Todos los sensores ópticos de deformación han sido instalados siguiendo el perímetro interior de la sección del túnel. No ha sido posible la instalación de sensores de deformación en el perímetro exterior debido a incompatibilidades con el desarrollo de los trabajos de la obra.

- En una posición concreta del falso túnel se instalaron sensores ópticos de dos longitudes distintas ( 1 y 0.6 metros) en la superficie del hormigón y situados uno junto al otro para poder comparar sus resultados (Figura 6.2). En esta figura se representa la sección del falso túnel y los sensores ópticos instalados en las posiciones indicadas (líneas de colores negras). Las líneas de color azul indican la conexión en serie entre los distintos sensores.

- En cada posición de los sensores de longitud se ha instalado un sensor puntual de deformación y las mediciones entre ambos tipos de sensores han sido comparadas (Figura 6.2). 


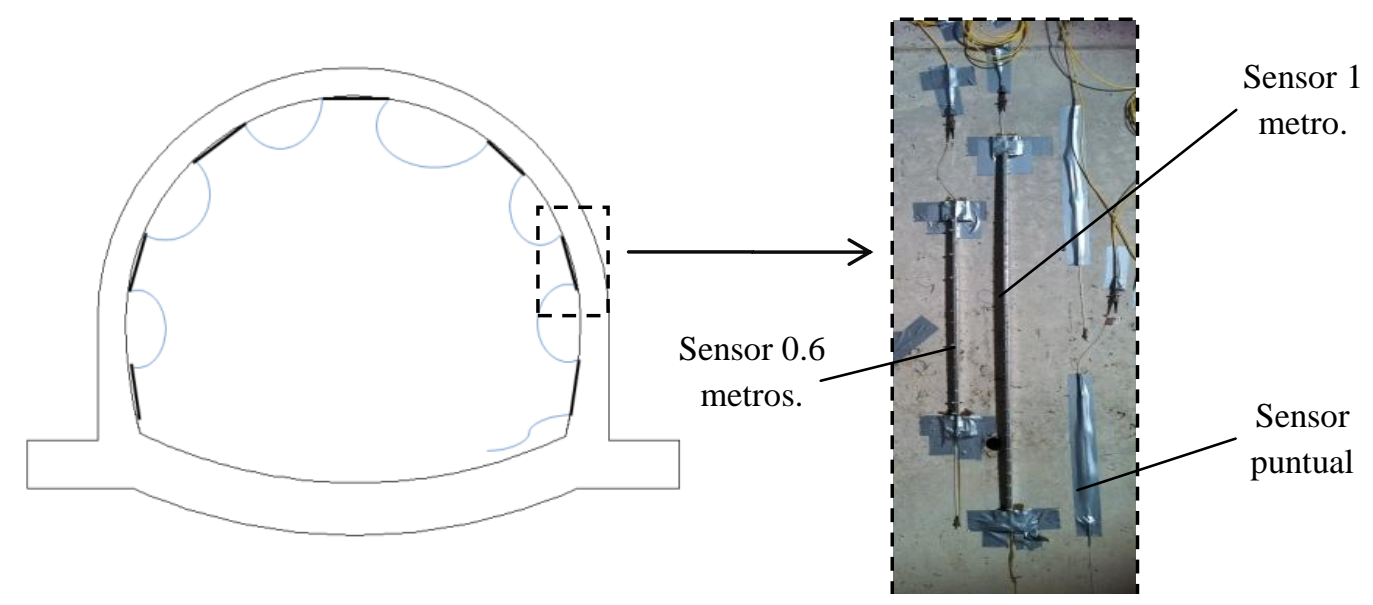

Figura 6.2 Sensores ópticos instalados a lo largo del perímetro de la sección de túnel.

- Como se observa en la Figura 6.2 anterior, todos los sensores han sido instalados en serie, por lo que con un único cable de fibra óptica ha sido posible la lectura de todos ellos.

- Los sensores ópticos de longitud se instalaron espaciados entre sí dos metros aproximadamente, tal y como indica la Figura 6.3.

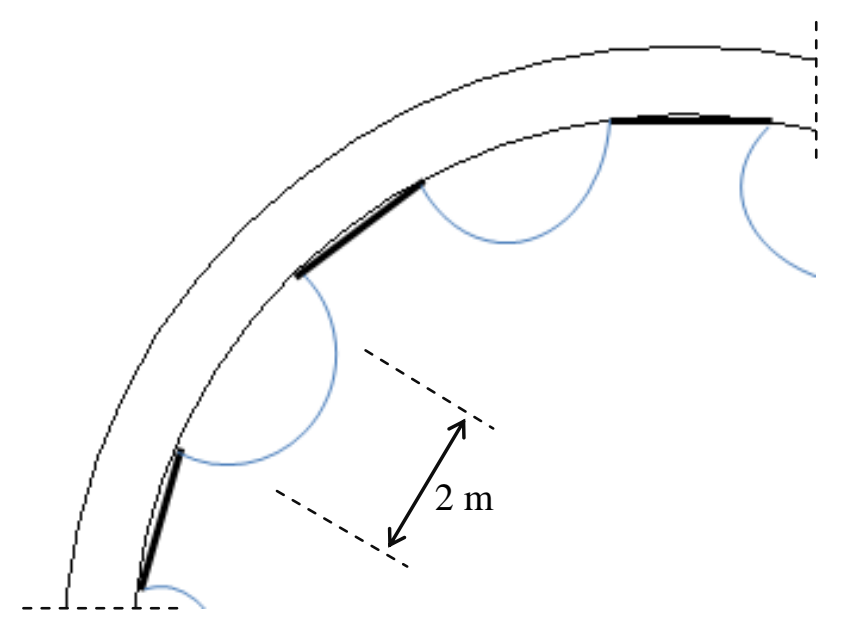

Figura 6.3 Separación entre los sensores ópticos de longitud.

- Además, se han instalado sensores ópticos puntuales de temperatura en el interior y en el exterior de la estructura en las secciones A-A y B-B de la bóveda indicadas en la Figura 6.4. No se ha instalado ningún sensor de temperatura en el hastial derecho del túnel en la Figura 6.4 ya que en ningún instante del día le incide la radiación solar y por tanto, no se encuentra sometida a importantes variaciones térmicas. 
Monitorización estructural.

Aplicación con sensores de fibra óptica.

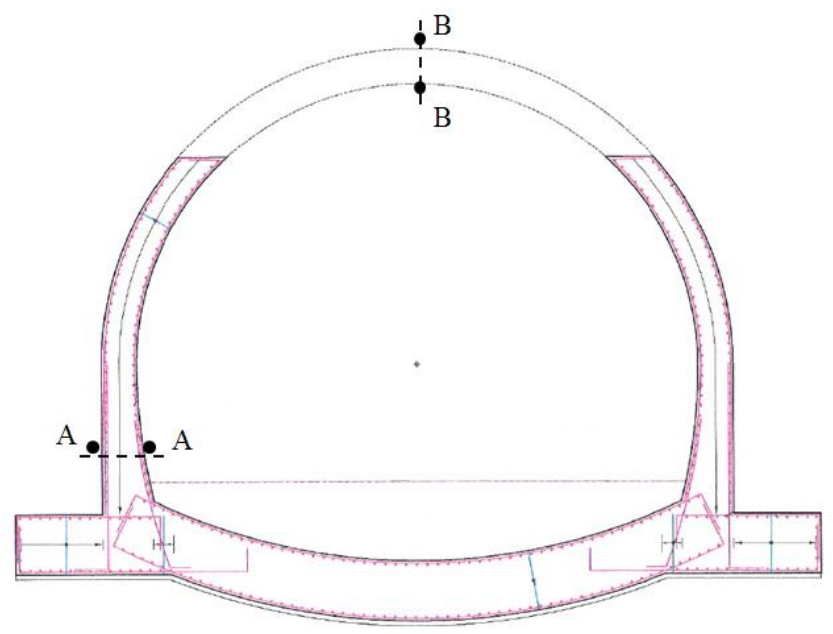

Figura 6.4 Posición de los sensores ópticos puntuales de temperatura.

\subsection{Trabajo experimental.}

\subsubsection{Descripción de la estructura objeto del estudio.}

La monitorización que se presenta en este capítulo se ha realizado en la estructura del túnel artificial situado sobre el emboquille del túnel de Mogente, correspondiente al "Proyecto de Construcción del nuevo acceso ferroviario de alta velocidad de Levante: Madrid-Castilla La Mancha-Comunidad Valenciana-Región de Murcia. Tramo: Nudo de la Encima-Mogente".

La estructura en cuestión consiste en una bóveda circular de 0.7 metros de canto y 5.64 metros de radio interior. Los hastiales son rectos, de canto variable entre 0.7 y 1.04 metros, con una altura de 3 metros. La cimentación está resuelta mediante una contrabóveda de 12.35 metros de radio y un canto constante de 1.2 metros (Figura 6.5).

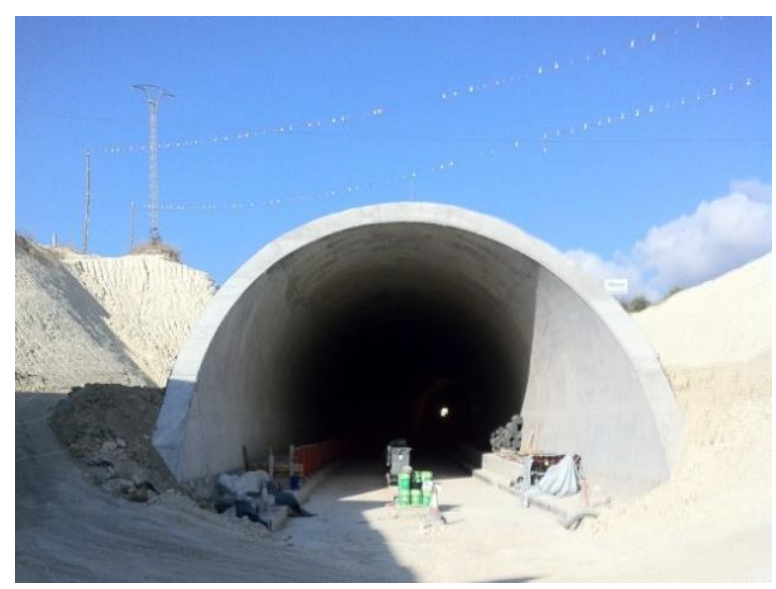

(a)

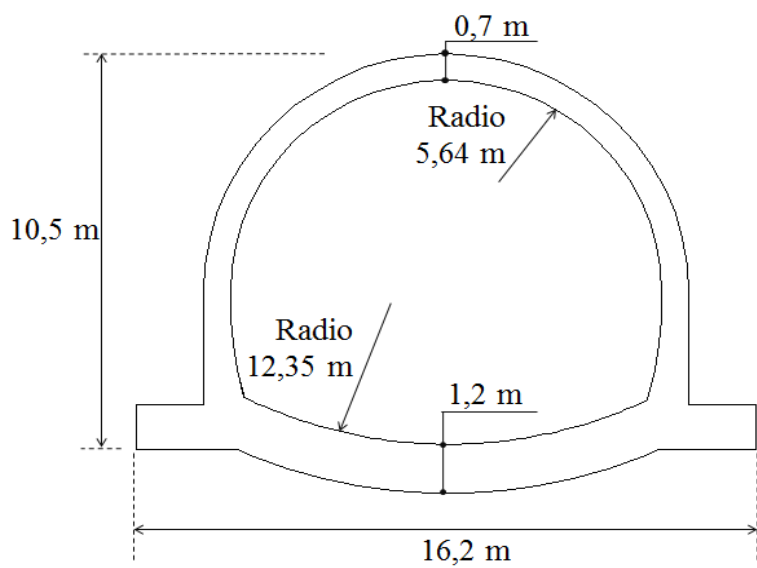

(b)

Figura 6.5 (a)- Vista frontal del falso túnel instrumentado. (b)- Plano de la sección transversal del falso túnel.

La estructura completa está ejecutada con hormigón armado, cuyos valores de proyecto son los siguientes: 
- En capas de nivelación y limpieza: HM-15.

- En cimentaciones: HA-30/B/20/IIa.

- En alzados: HA-30/B/20/IIb.

- Acero para todos los elementos: B-500-SD.

El material de relleno del túnel se trata de una arcilla margosa cuyos valores de proyecto son:

- Peso específico $\gamma_{T}=20 \mathrm{KN} / \mathrm{m}^{3}$

- Módulo de elasticidad $E_{T}=100 \mathrm{MPa}$

- Coeficiente de Poisson $v_{T}=0.33$

- Ángulo de rozamiento interno $\phi_{T}=30^{\circ}$

- Coeficiente de empuje al reposo $K_{o}=0.5$

Este material de relleno ha sido extendido por tongadas de 2 metros de altura alternando a ambos lados del túnel. Una vez que las tierras han alcanzado la cota de la bóveda se ha rellenado hasta la altura máxima del terreno, que para la sección instrumentada ha sido de 20.3 metros contados a partir de la base de la bóveda.

La Figura 6.6 muestra la sección instrumentada del falso túnel, una vez finalizada su construcción y antes del relleno con tierras.

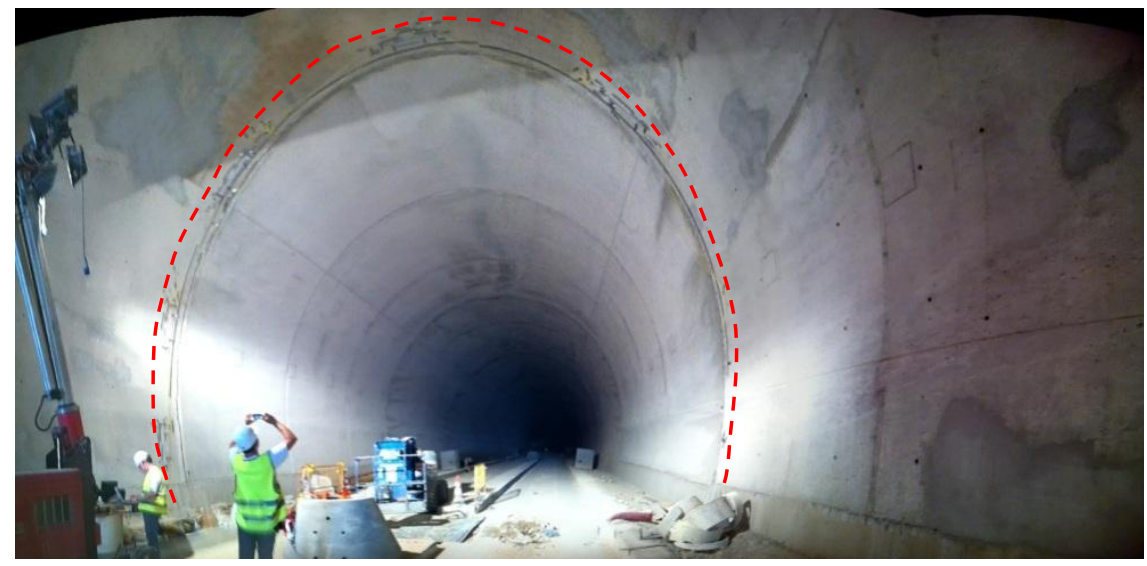

Figura 6.6 Sección de túnel instrumentada indicada con una traza de color rojo discontinua.

\subsubsection{Operaciones monitorizadas y calendario de medidas.}

Como se detalló en el apartado anterior, el relleno de las tierras se realizó por tongadas de 2 metros de altura, alternativamente a ambos lados del túnel. Esta operación tuvo una duración aproximada de tres meses y medio, iniciándose el 1 de octubre y finalizando el 20 de enero de 2012.

Durante este intervalo de tiempo se han realizado la toma de datos, resultando ser el calendario de medidas el indicado en la Tabla 6.1: 
Monitorización estructural.

Aplicación con sensores de fibra óptica.

\begin{tabular}{cc}
\hline Fecha & Operación monitorizada. \\
\hline $23,24,25 / 08 / 2011$ & Instalación de los sensores de deformación y temperatura. \\
$26 / 09 / 2011$ & Toma de datos continua. No existe relleno de tierras. \\
$27 / 09 / 2011$ & Toma de datos continua. No existe relleno de tierras. \\
$14 / 05 / 2012$ & Toma de datos instantánea. Altura de relleno 20.3 metros. \\
\hline
\end{tabular}

Tabla 6.1 Calendario de medidas.

Durante los días 26 y 27 de septiembre se realizó una toma de medidas continuada a lo largo de varias horas del día:

- 26/09/2011: La toma de datos inició a las 09:34 de la mañana y finalizó a las 16:58 de la tarde.

- 27/09/2011: La toma de datos inició a las 09:03 de la mañana y finalizó a las 13:23 de la tarde.

Durante estos dos días no existía relleno de tierras sobre la estructura (Figura 6.7). Las mediciones realizadas y las variaciones de deformación medidas fueron debidas, por tanto, fundamentalmente a las variaciones térmicas que se registraron a lo largo de estas horas.
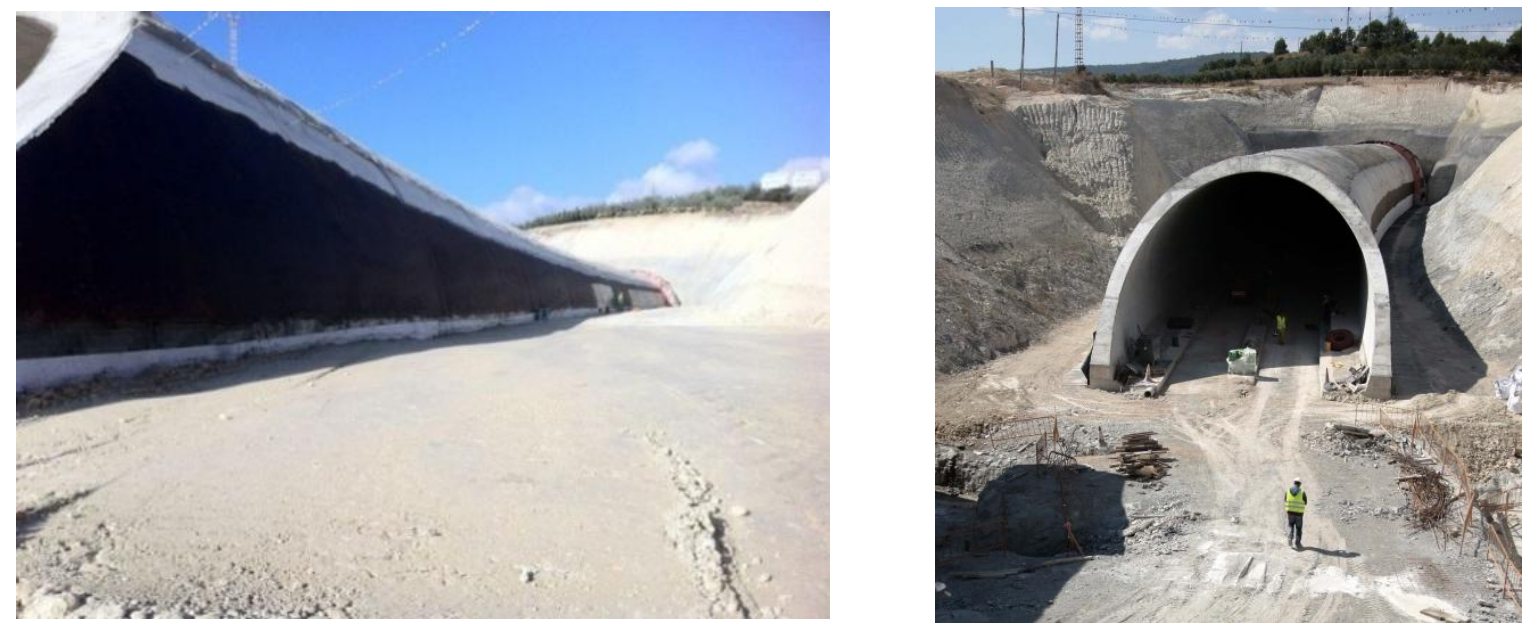

Figura 6.7 Falso túnel sin relleno de tierras. Día 27/09/2011.

El día 14 de mayo de 2012 se realizó una toma de datos instantánea. La toma de datos comenzó a las 09:09 y finalizó a las 09:50. El relleno de tierras ya había alcanzado su cota máxima, de 20.3 metros medidos desde la base de la bóveda (Figura 6.8). 


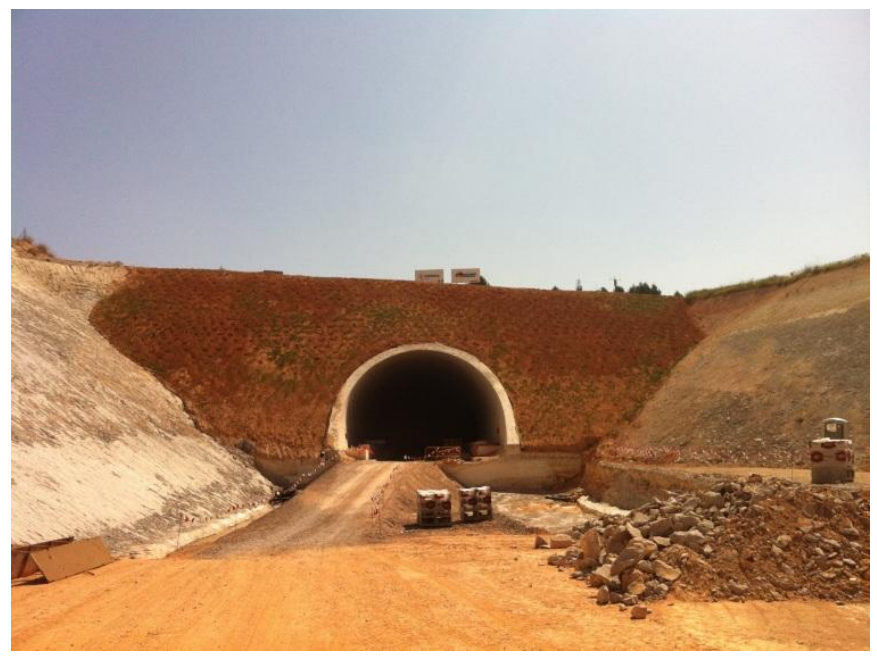

Figura 6.8. Relleno de tierras finalizado. Día 15/05/2012.

\subsubsection{Monitorización y captura de datos.}

Como se ha comentado en los apartados anteriores, se instrumentó la sección transversal de falso túnel con mayor altura de relleno de tierras. Todos los sensores ópticos de deformación se instalaron siguiendo el perímetro de la sección.

La instalación se dispuso de la siguiente forma:

- 7 sensores ópticos de deformación de 1 metro de longitud. Cada uno de los sensores de longitud estaban separados entre sí, aproximadamente dos metros en dirección perimetral de la sección de falso túnel. (apartado 6.2).

- Los sensores se numeraron en sentido horario (véase la Figura 6.9).

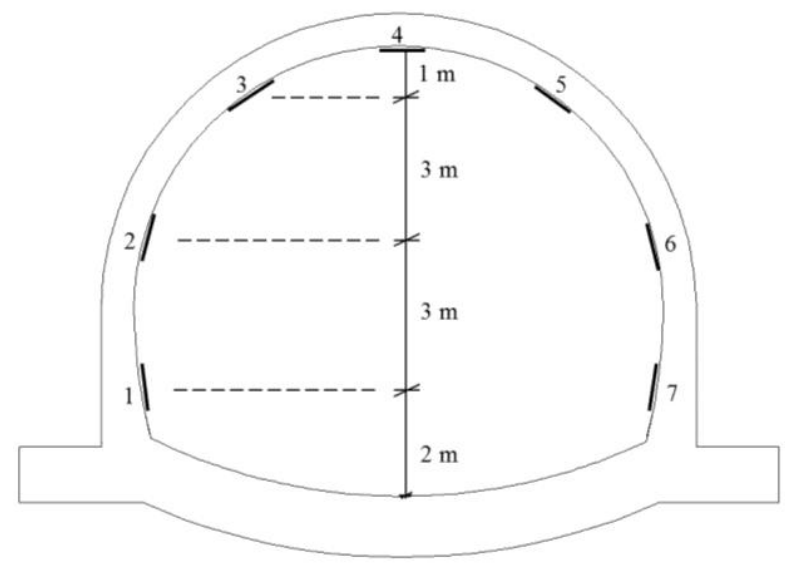

Figura 6.9 Numeración de los sensores de 1 metro de longitud dispuestos a lo largo de la bóveda del túnel. Cotas.

- 7 sensores ópticos puntuales de deformación, instalados próximos a los sensores de longitud para comparar sus resultados (Figura 6.10).

- En la posición 2 se instalaron dos sensores de longitudes de 1 y 0.6 metros. El objetivo de esta instalación fue la de comparar los resultados obtenidos entre uno y otro. 
Monitorización estructural.

Aplicación con sensores de fibra óptica.

- El sensor de la posición 4 se encuentra anclado sólo en uno de sus extremos y libre en el otro. Con esta disposición se obtiene la afección de la temperatura sobre los sensores de longitud y se pueden corregir los efectos de la misma en las mediciones de los sensores.

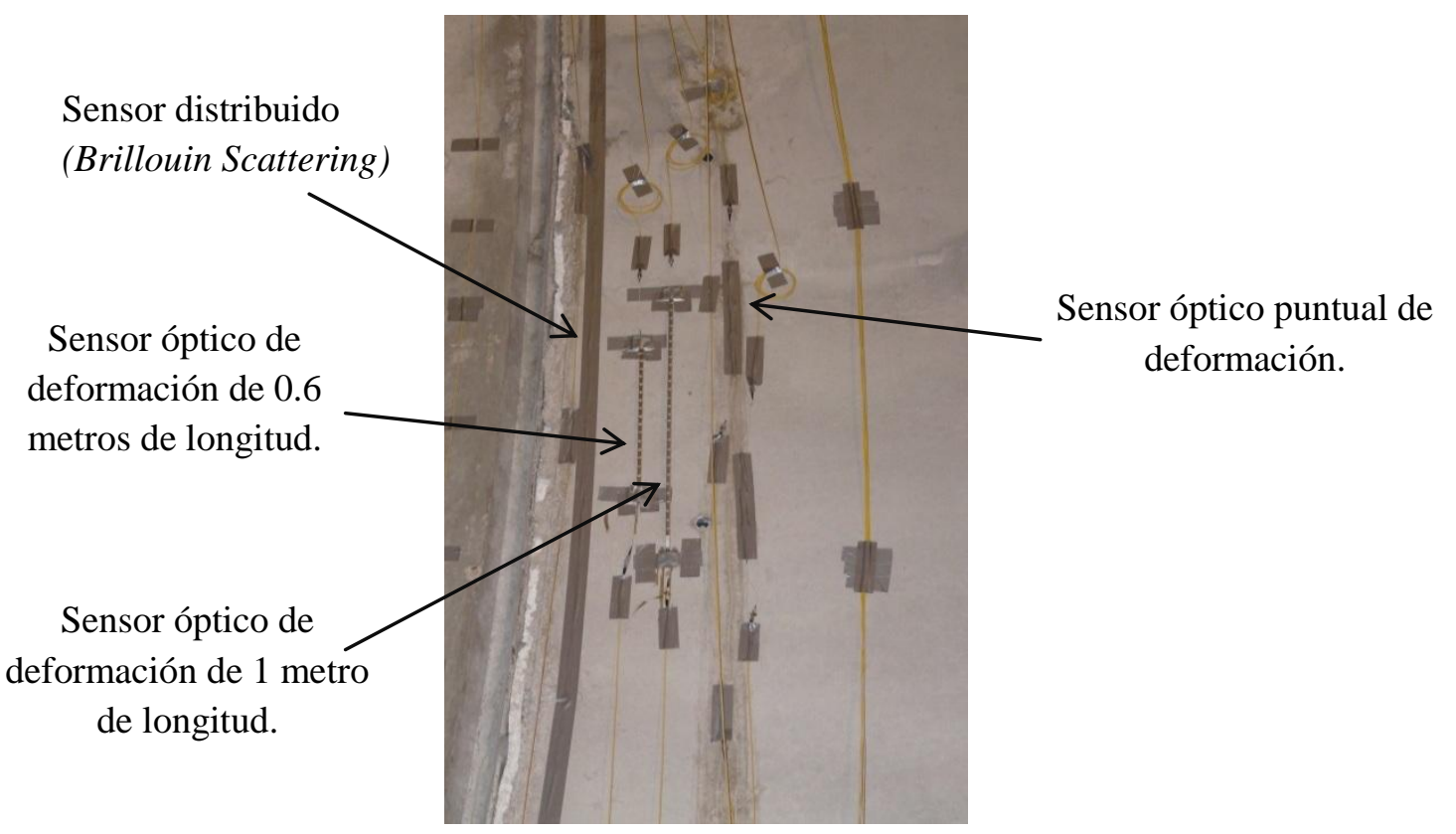

Figura 6.10 Disposición de los sensores ópticos en el interior de la bóveda del túnel en la posición 2.

- Los sensores ópticos puntuales de temperatura se encuentran instalados en las secciones indicadas en la Figura 6.4 anterior. Su identificación se ha realizado en base a colores como los indicados en la figura 6.11.a:

1. En color rojo, la temperatura exterior en la clave de la bóveda.

2. En color verde, la temperatura interior de la clave de la bóveda

3. En color azul, la temperatura exterior en el hastial izquierdo.

4. En color negro, la temperatura interior en el hastial izquierdo.

Estos sensores ópticos de temperatura tienen una doble misión: Por un lado permiten conocer los incrementos de temperatura a los que está sometida la estructura. Por otro lado, son necesarios para llevar a cabo la compensación térmica que exige el sensado óptico.

- El sensado distribuido de Brillouin (Capítulo 8) se ha instalado siguiendo el perímetro del túnel de la forma indicada en la Figura 6.11.b, con un color naranja. 


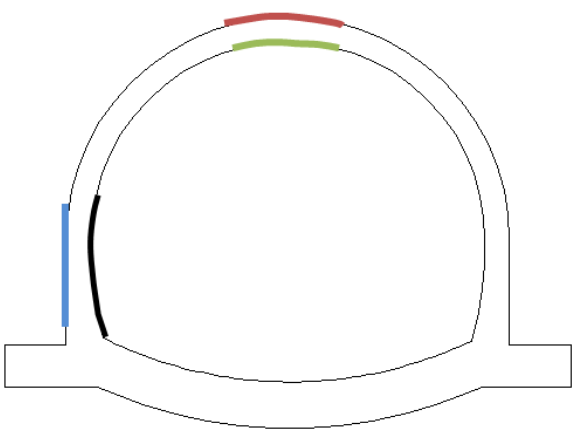

(a)

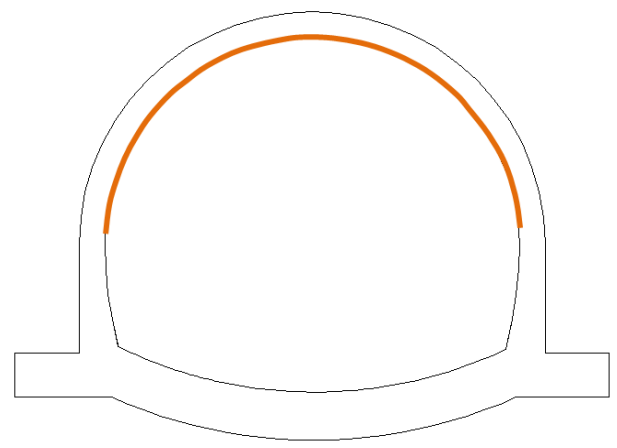

(b)

Figura 6.11 (a)- Identificación de los sensores de temperatura dispuestos. (b)- Sensado distribuido.

Para la instalación de todos los sensores ópticos de deformación fue necesaria la preparación previa de la superficie mediante una exhaustiva limpieza de la misma y posterior eliminación de la lechada superficial mediante un pulido.

El sistema de interrogación empleado en la instrumentación es el mismo que el empleado en los Capítulos 4 y 5. Está compuesto por un interrogador de sensores tipo FBG de la marca Micron Optics, modelo sm125-500 y por un ordenador portátil en el que se ejecutaba un software desarrollado específicamente para esta aplicación (Bueno, 2008). Este software fue desarrollado por personal del Instituto de Telecomunicaciones y Aplicaciones Multimedia (ITEAM) de la Universitat Politècnica de València en el lenguaje de programación LabVIEW ${ }^{\mathrm{TM}}$ versión 8.5. El interrogador de sensores ópticos sm125-500 está compuesto por una fuente láser sintonizable Fabry-Perot con un rango en longitud de onda de 1510 a $1590 \mathrm{~nm}$ y 4 canales ópticos de salida. La frecuencia máxima de captura de datos es de $1 \mathrm{~Hz}$ y la precisión de la medida es de $1 \mathrm{pm}$. El ordenador portátil utilizado fue un Intel Pentium 4 a $2.4 \mathrm{GHz}$ y con $768 \mathrm{MB}$ de memoria RAM.

\subsection{Estudio numérico.}

\subsubsection{Introducción.}

La instalación de los sensores descrita en los apartados anteriores permitió la obtención de las temperaturas y de las deformaciones en la bóveda del falso túnel.

Para validar estas medidas y por tanto el comportamiento de los sensores ópticos propuestos se ha llevado a cabo un modelo numérico simplificado 2D de elementos finitos. En él, se ha modelado una sección transversal de falso túnel con los elementos que lo forman, esto es, contrabóveda, hastiales, tacones y bóveda y se ha estudiado las variaciones de deformación que experimenta la estructura debido a las acciones que actúan sobre ella:

1. Las variaciones de temperatura existentes.

2. El peso y empuje de las tierras.

Es importante destacar que con el modelo numérico simplificado se pretende mostrar la pauta general de comportamiento de la estructura. Conocida esta pauta general de comportamiento se compara con la obtenida a partir de las mediciones de los sensores ópticos, y se observa si ambas pautas generales de comportamiento son similares para validar el empleo de los sensores propuestos en la monitorización de una estructura real. 


\subsubsection{Condiciones de contorno, descripción de los elementos empleados y aplicación de la carga:}

El modelo numérico ha sido estudiado empleando el programa de elementos finitos Lusas V.14.3-11 (Lusas, 2010). A pesar de que geométricamente la estructura presenta simetría, no sucede lo mismo con las cargas aplicadas sobre ella como se mostrará posteriormente. Por este motivo, se ha modelado la totalidad de la sección transversal.

La estructura se encuentra apoyada en el terreno y dado que los tacones y la contrabóveda están enterrados, se considera impedido el desplazamiento horizontal. Por tanto, en los nodos que definen la contrabóveda y los tacones se han impedido los desplazamientos horizontales y verticales (Figura $6.12)$.

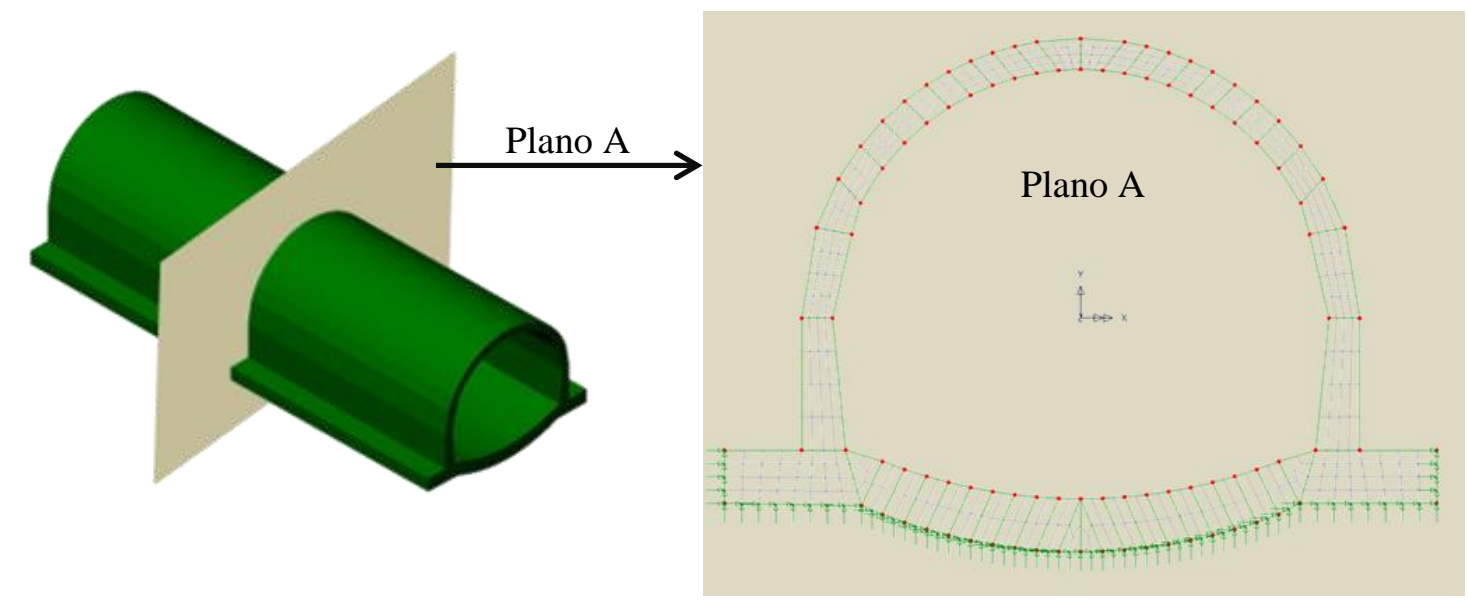

Figura 6.12 Modelización de la sección del falso túnel de mayor altura de relleno de tierras. Movimientos impedidos en la base de los tacones y de la contrabóveda.

Los elementos finitos empleados para la modelación de la estructura son elementos continuos 2D tipo QPN8 (Figura 6.13), los cuales están formados por 8 nodos, y dos grados de libertad por nodo (traslaciones en $\mathrm{X}, \mathrm{Y}$ ).

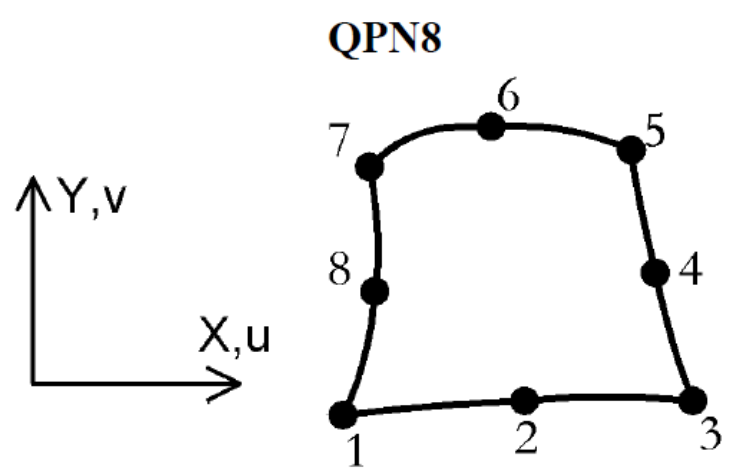

Figura 6.13 Elemento QPN8. (Lusas, 2010). 
a) Introducción de las temperaturas.

La introducción de las variaciones térmicas en el programa se ha llevado a cabo a partir de la opción Load Curve implementada en Lusas V14.3-11. Esta opción permite introducir cargas variables en el tiempo y por tanto sólo pueden emplearse con análisis no lineales o transitorios. Estas curvas se definen a partir de parejas de valores "tiempo - factor de curva". El factor de curva será el parámetro que multiplicará al valor de carga asignado al elemento estructural, en este caso, un valor unidad.

Las variaciones de temperatura a las que está sometida la estructura han sido introducidas en el programa como leyes lineales y parabólicas de segundo y tercer grado (Figura 6.14), ya que éstas presentan un muy buen grado de ajuste con las mediciones obtenidas (Tabla 6.2).

En la Figura 6.14 se muestra el ajuste realizado para la evolución de las temperaturas en los distintos puntos instrumentados del falso túnel, a lo largo del día 26/09/2011. Este mismo procedimiento se ha repetido para el resto de días.

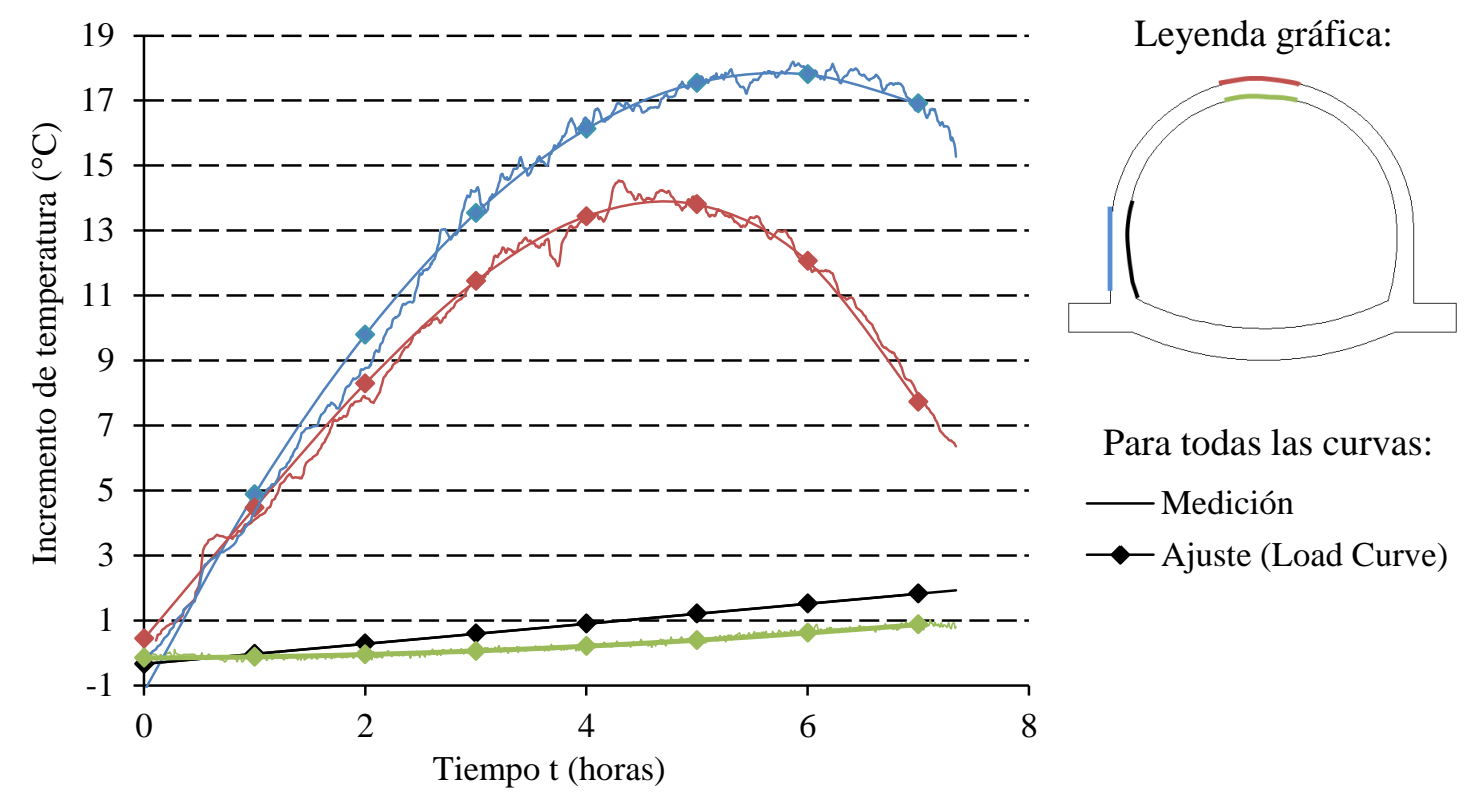

Figura 6.14 Ajuste realizado para las variaciones térmicas en los cuatro puntos instrumentados. Día $26 / 09 / 2011$

\begin{tabular}{ccc}
\hline Punto instrumentado & Ecuación del ajuste & $\boldsymbol{R}^{2}$ \\
\hline Hastial exterior & $\Delta T\left({ }^{\circ} \mathrm{C}\right)=-0.5809 t^{2}+6.6514 t-1.1904$ & 0.9898 \\
Clave exterior & $\Delta T\left({ }^{\circ} \mathrm{C}\right)=-0.0796 t^{3}+0.1391 t^{2}+3.9675 t+0.4356$ & 0.9827 \\
Hastial interior & $\Delta T\left({ }^{\circ} \mathrm{C}\right)=0.3085 t-0.3396$ & 0.9766 \\
Clave interior & $\Delta T\left({ }^{\circ} \mathrm{C}\right)=0.0186 t^{2}+0.0166 t-0.1585$ & 0.9584 \\
\hline
\end{tabular}

Tabla 6.2 Ajustes que representan las variaciones térmicas en los puntos instrumentados. Día 26/09/2011. 
Monitorización estructural.

Aplicación con sensores de fibra óptica.

b) Introducción del empuje de tierras.

La introducción del empuje de tierras en el programa se ha llevado a cabo a partir de la opción Face Load, implementada en Lusas V14.3-11. Este tipo de carga es aplicada en los bordes de los elementos finitos 2D, o en las caras de los elementos finitos 3D.

El valor del empuje de tierras se ha introducido de acuerdo a la ecuación 6.1:

$E=K_{o} \gamma_{T} h$

Siendo E el valor del empuje en $\mathrm{KN} / \mathrm{m}^{2}, K_{o}$ el coeficiente de empuje a reposo definido en el apartado 6.3.1., $\gamma_{T}$ el peso específico de las tierras sobre la estructura, y h la altura del relleno de tierras en el punto considerado.

\subsection{Resultados.}

\subsubsection{Introducción.}

La instrumentación descrita en los apartados 6.2 y 6.3 .3 se empleó para medir las deformaciones y temperaturas en la bóveda del túnel durante las fechas indicadas en la Tabla 6.1 anterior que define el calendario de medidas.

Para obtener las deformaciones de la estructura, es necesario previamente realizar una compensación térmica de las mediciones de los sensores. A continuación se describe el procedimiento empleado para realizar la compensación térmica, las mediciones experimentales obtenidas y resultados numéricos.

\subsubsection{Compensación térmica de los sensores de longitud.}

Las medidas obtenidas con los sensores de longitud necesitan ser corregidas debido a una serie de consideraciones:

- Las variaciones térmicas afectan el índice de refracción de la fibra óptica, que a su vez, supone una afección sobre la longitud de onda reflejada por la red de difracción de Bragg que forma el sensor, y por tanto sobre la deformación medida.

- Las variaciones térmicas presentan una afección sobre el adhesivo empleado para la fabricación del sensor de longitud.

Ambas consideraciones introducen efectos en la medición que es necesario compensar. Para llevar a cabo esta operación, se ha planteado un esquema de sensores donde (Figura 6.15):

- El sensor de longitud anclado en dos extremos a la estructura $\left(S_{a}\right)$ será el encargado de medir la deformación de la estructura.

- El sensor anclado en un único extremo y libre en el otro $\left(S_{l}\right)$ servirá para medir los efectos sobre el material que forma el encapsulado, la fibra óptica y el adhesivo empleado en la fabricación del sensor. 
Material que forma el

encapsulado del sensor

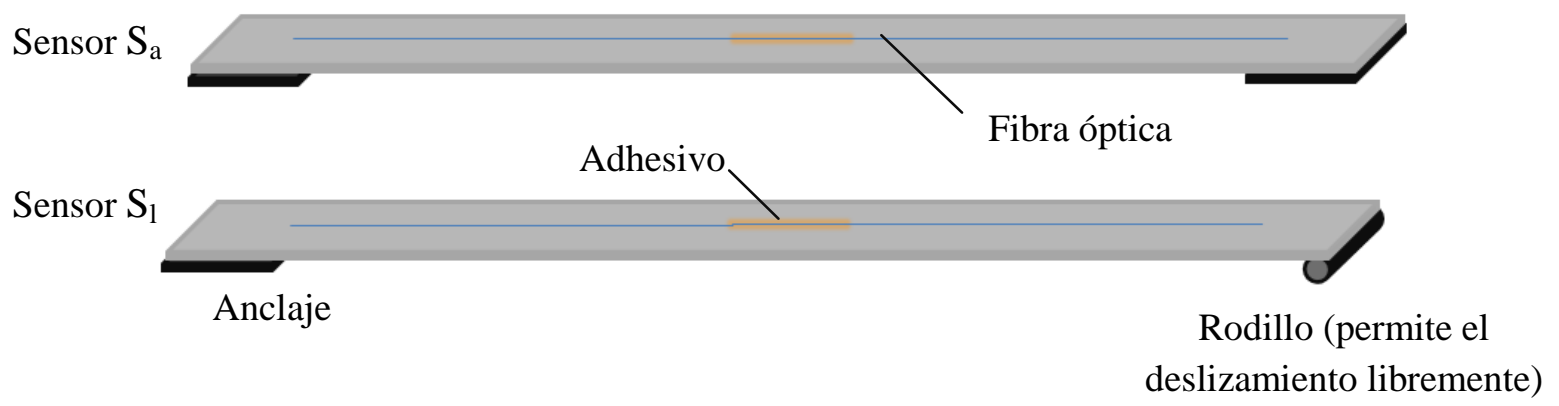

Figura 6.15 Disposición de los sensores necesarios para realizar la compensación térmica.

A partir de la disposición mostrada en la Figura 6.15, los sensores recogen los siguientes efectos:

- Una deformación debida a la variación del índice de refracción de la fibra óptica en los sensores $S_{a}$ y $S_{l}, \varepsilon_{n}\left(S_{a}\right)$ y $\varepsilon_{n}\left(S_{l}\right)$ respectivamente.

- Una deformación debida a las modificaciones sobre el adhesivo empleado en los sensores $S_{a}$ y $S_{l}, \varepsilon_{a d}\left(S_{a}\right)$ y $\varepsilon_{a d}\left(S_{l}\right)$ respectivamente.

- Una deformación debida a la dilatación del material que forma el encapsulado del sensor, únicamente en el sensor $S_{l}, \varepsilon_{m}\left(S_{l}\right)$ (el sensor $S_{a}$ se encuentra anclado a la estructura en sus dos extremos).

- Un término debido a la deformación de la estructura medida por el sensor $S_{a}, \varepsilon_{h}\left(S_{a}\right)$. Este término es el que es necesario obtener tras la compensación térmica para estimar la deformación de la estructura.

Estos efectos pueden escribirse de la forma que indican las ecuaciones 6.2 y 6.3 :

$\varepsilon\left(S_{a}\right)=\varepsilon_{n}\left(S_{a}\right)+\varepsilon_{a d}\left(S_{a}\right)+\varepsilon_{h}\left(S_{a}\right)$

$\varepsilon\left(S_{l}\right)=\varepsilon_{n}\left(S_{l}\right)+\varepsilon_{a d}\left(S_{l}\right)+\varepsilon_{m}\left(S_{l}\right)$

La deformación debida a la dilatación del material que forma el encapsulado del sensor, $\varepsilon_{m}\left(S_{l}\right)$, es conocida a partir del coeficiente de dilatación térmica del material que forma el encapsulado del sensor $\alpha_{m}$ y las variaciones térmicas medidas $\Delta T$, de forma que $\varepsilon_{m}\left(S_{l}\right)=\alpha_{m} \Delta T$.

Teniendo en cuenta la proximidad de los sensores $S_{a}$ y $S_{l}$ se puede considerar que $\varepsilon_{n}\left(S_{l}\right)=\varepsilon_{n}\left(S_{a}\right)$ y que $\varepsilon_{a d}\left(S_{l}\right)=\varepsilon_{a d}\left(S_{a}\right)$. A partir de las anteriores ecuaciones, fácilmente se obtiene el valor de la deformación de la estructura como:

$\varepsilon_{h}\left(S_{a}\right)=\varepsilon\left(S_{a}\right)-\left(\varepsilon\left(S_{l}\right)-\alpha_{m} \Delta T\right)$

De esta forma se han estimado las deformaciones de la estructura en cada uno de los sensores de longitud dispuestos a lo largo del perímetro de la bóveda del falso túnel. 
Monitorización estructural.

Aplicación con sensores de fibra óptica.

\subsubsection{Mediciones continuas los días 26 y 27/09/2011.}

a) Introducción.

El día 26/09/2011 se inició la toma de datos a las 09:34 de la mañana, y finalizó a las 16:58 de la tarde. A lo largo de este día no hubo relleno de tierras sobre el falso túnel. Sin embargo, sí existieron gradientes térmicos importantes entre el interior y el exterior de la bóveda (provocados por las variaciones térmicas ambientales), y éstos fueron registrados por la red de sensores de temperatura instalados sobre la estructura.

De esta forma, la red de sensores de deformación instalada en la cara interior de la bóveda registró variaciones de deformación. Estas variaciones en la deformación de la estructura sólo pudieron ser debidas a estas variaciones térmicas, que debido a la fecha de los trabajos (septiembre), la ubicación de la estructura (Mogente) y el horario de medida (de 09:34 a 16:58) fueron importantes.

De una forma similar sucedió con el día 27. La toma de datos inició a las 09:03, finalizaba a las 13:23 y las variaciones de temperatura ambiente provocaron modificaciones en la deformación de la estructura. A lo largo de este día, tampoco hubo relleno de tierras sobre el falso túnel.

\section{b) Variaciones de temperaturas medidas.}

La Figura 6.16 muestra las variaciones térmicas que se han medido a lo largo del día 26/09/2011. Como se observa, existen importantes variaciones térmicas en los dos puntos monitorizados del exterior de la bóveda, situados en el hastial izquierdo y en la clave, con gradientes térmicos máximos de $18.17{ }^{\circ} \mathrm{C}$ y $14.08{ }^{\circ} \mathrm{C}$ respectivamente. En los dos puntos interiores también existen variaciones térmicas pero de menor importancia, con valores máximos de $2.77{ }^{\circ} \mathrm{C}$ en el hastial izquierdo y $0.85{ }^{\circ} \mathrm{C}$ en la clave.

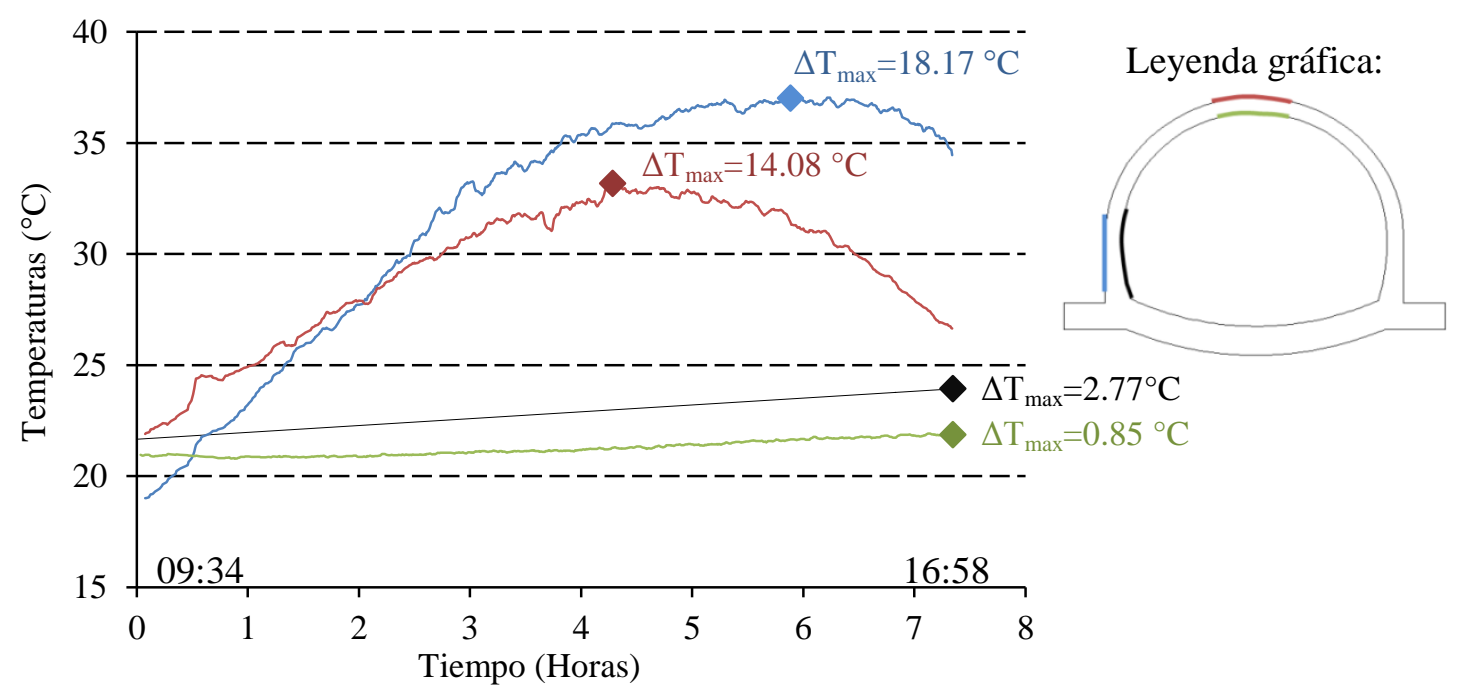

Figura 6.16 Variaciones térmicas en los puntos instrumentados a lo largo del día 26/09/2011.

De una forma similar, la Figura 6.17 muestra las variaciones térmicas en la estructura durante la jornada del 27/09/2011. Nótese que el eje horizontal empieza sobre la hora 23.5 que coincide con las 09:03 de este día, instante en que se volvió a tomar medidas el día 27/09/2011. La toma de datos finalizó a las 13:23, que corresponde con el instante de tiempo 27.8 horas. 


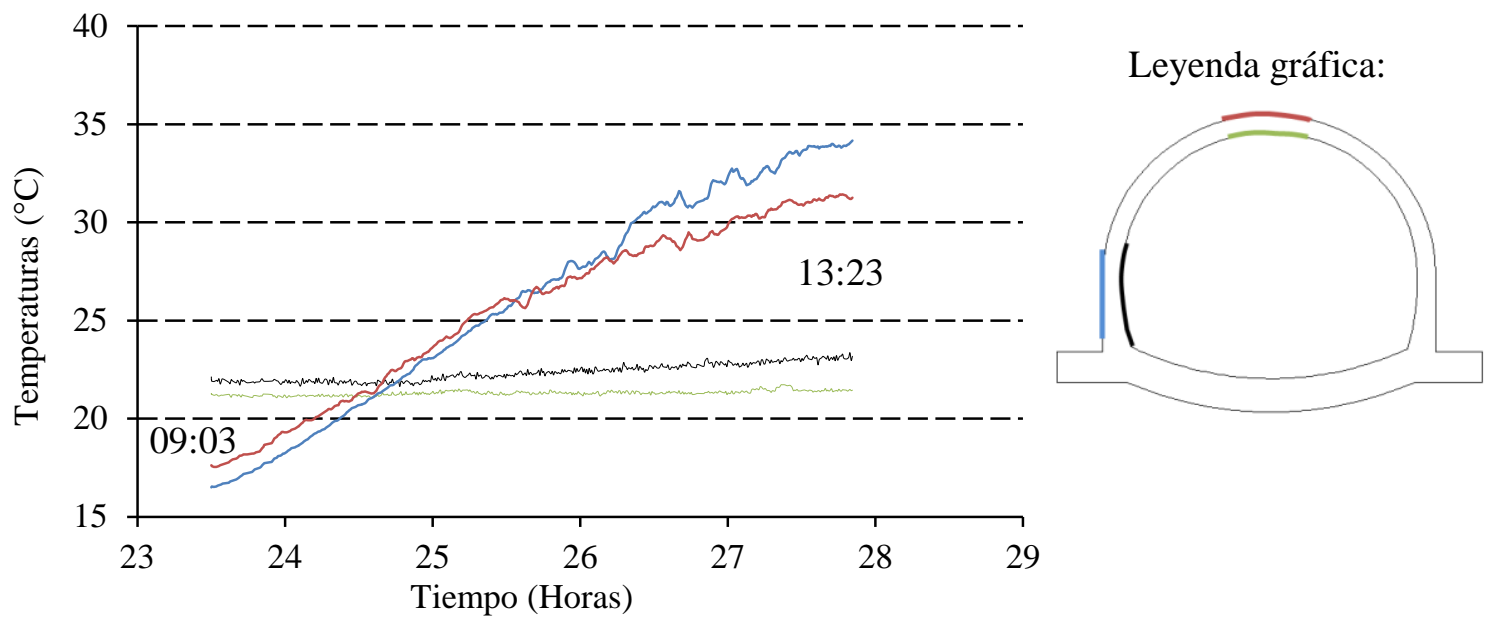

Figura 6.17 Variaciones térmicas en los puntos instrumentados a lo largo del día 27/09/2011.

Para el análisis numérico de la estructura y teniendo en cuenta las tendencias anteriores, se han adoptado leyes de temperaturas polinómicas para los intervalos de tiempo en que no se disponen datos, esto es, entre las 16:58 del día 26/09/2011 y las 09:03 del día 27/09/2011. Este ajuste de temperaturas se ha realizado teniendo en cuenta los valores iniciales y finales de los tramos en que no se disponen datos, así como las pendientes de las curvas en esos puntos.

Finalmente, la Figura 6.18 muestra las leyes de temperatura consideradas para los puntos instrumentados.

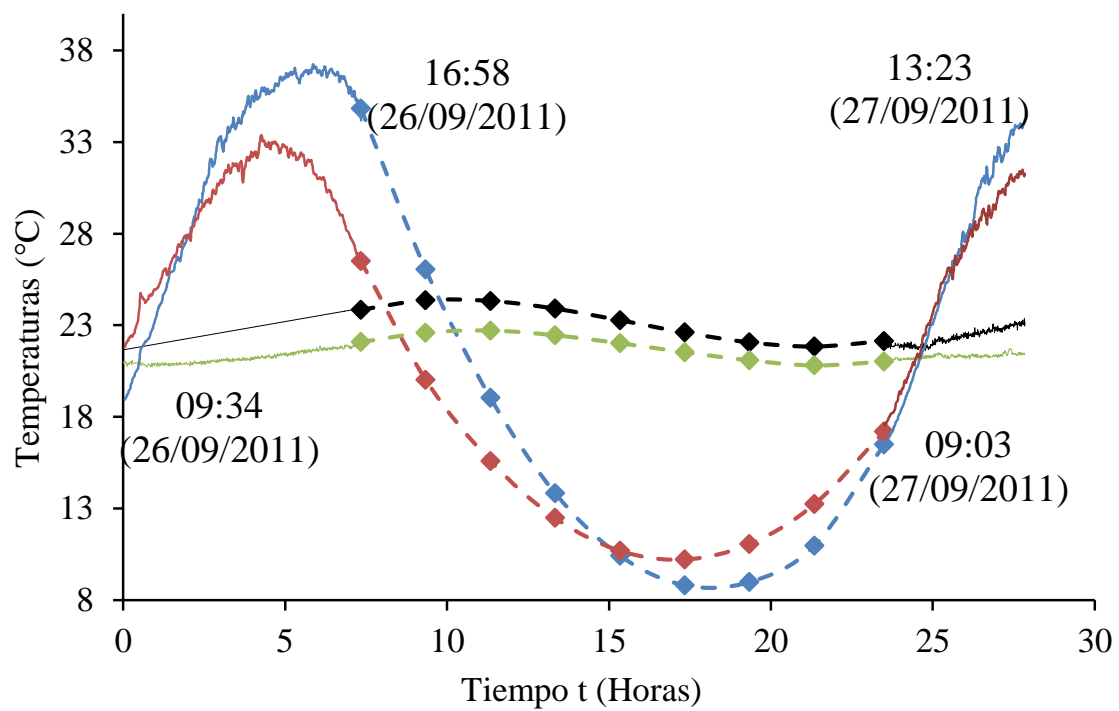

Leyenda gráfica:

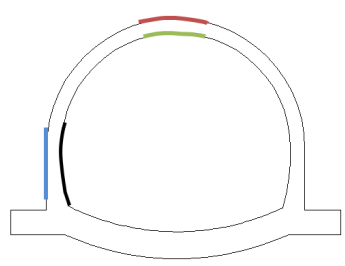

Para todas las curvas:

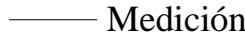

$-\rightarrow$ - Hipótesis

Figura 6.18 Variaciones de temperatura en los puntos instrumentados durante los días 26 y 27/09/2011. Valores medidos e hipotéticos adoptados.

Las ecuaciones que definen los tramos intermedios cuyos valores han sido adoptados se muestran en la Tabla 6.3: 
Monitorización estructural.

Aplicación con sensores de fibra óptica.

\begin{tabular}{cc}
\hline Punto instrumentado & Ecuación del ajuste \\
\hline Hastial exterior & $\Delta T\left({ }^{\circ} \mathrm{C}\right)=0.2244 t^{2}-8.1407 t+82.49$ \\
Clave exterior & $\Delta T\left({ }^{\circ} \mathrm{C}\right)=0.165 t^{2}-5.628 t+58.203$ \\
Hastial interior & $\Delta T\left({ }^{\circ} \mathrm{C}\right)=0.0036 t^{3}-0.1704 t^{2}+2.3462 t+14.38$ \\
Clave interior & $\Delta T\left({ }^{\circ} \mathrm{C}\right)=0.0023 t^{3}-0.1133 t^{2}+1.6122 t+15.14$ \\
\hline
\end{tabular}

Tabla 6.3 Ecuaciones que representan los valores de temperatura hipotéticos adoptados.

c) Comparación de los resultados de los sensores de longitud.

Como se indicó en la Figura 6.10 del apartado 6.3.3, en la posición 2 se han instalado dos sensores de longitud, de 1 y 0.6 metros. Los resultados de ambos sensores han sido coincidentes entre ellos con un buen grado de ajuste aunque con pequeñas diferencias entre ellos que serán justificadas a continuación. Por este motivo, en apartados sucesivos sólo se mostrará el resultado del sensor de 1 metro de longitud.

Las Figuras 6.19.a y 6.19.b muestran las variaciones de deformación que se producen en el punto indicado para sensores de deformación de 1 y 0.6 metros de longitud, durante los días 26 y 27/09/2011. La Figura 6.20 muestra ambas mediciones sobre la misma gráfica.

Es importante señalar que a partir de este apartado y en lo que sigue, las deformaciones que se presentan ya han sido compensadas térmicamente según las expresiones del apartado anterior 6.5.2.

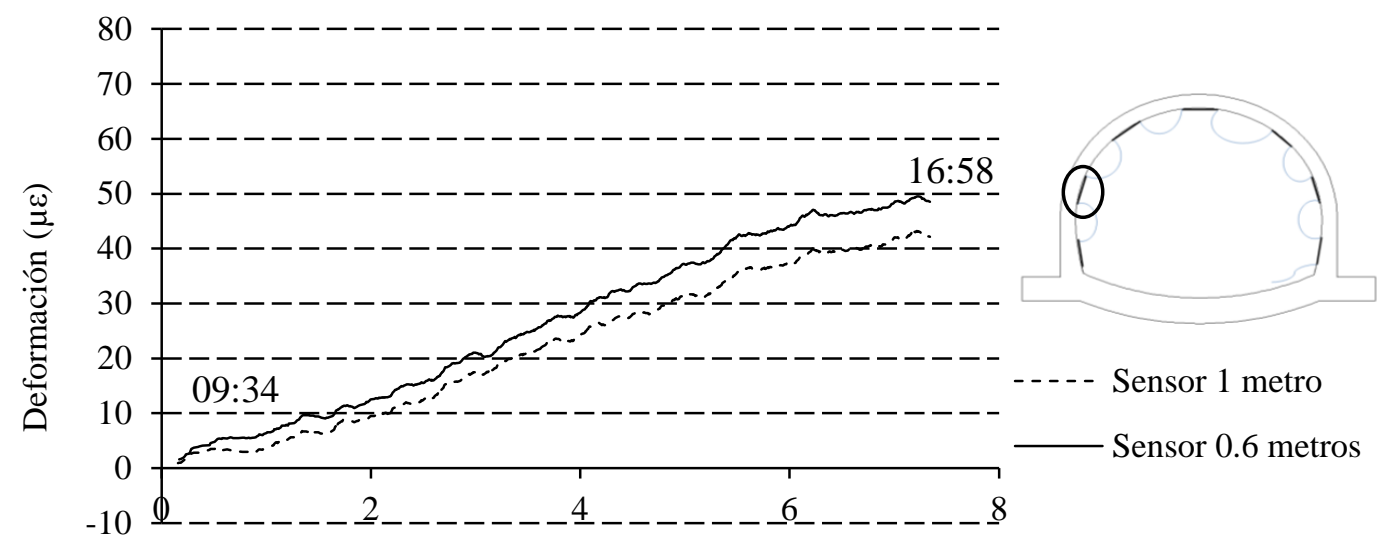

Tiempo t (horas)

(a) 


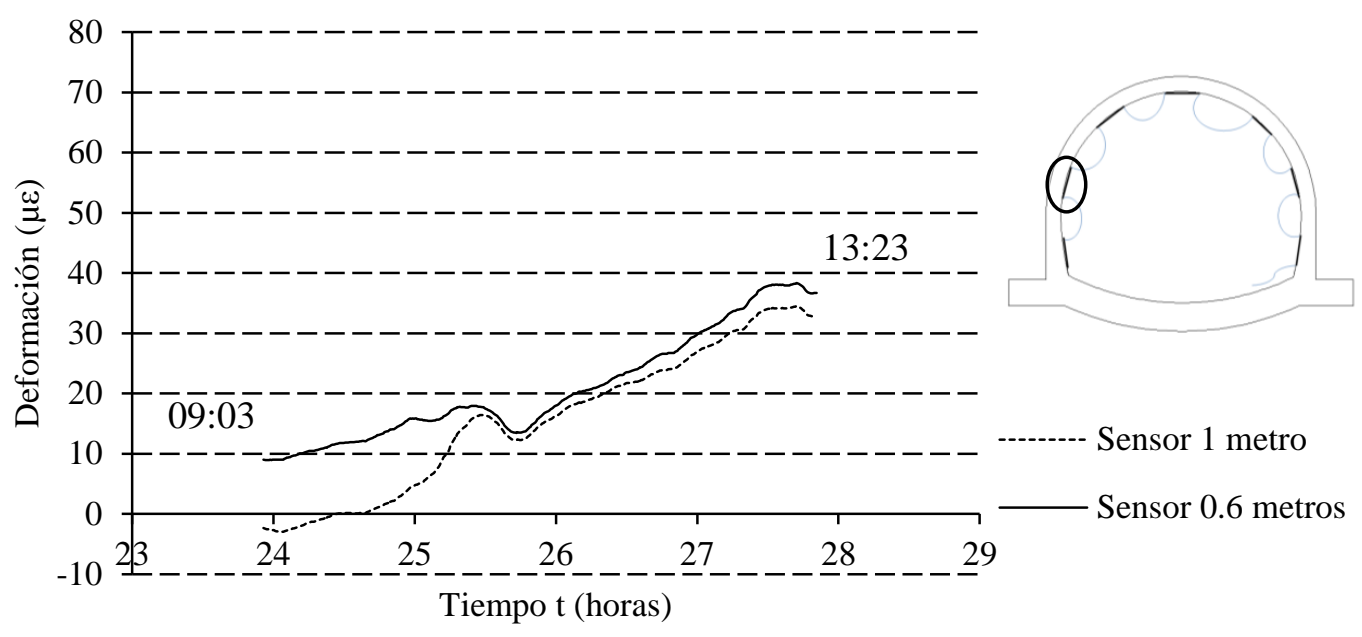

(b)

Figura 6.19 Variaciones de deformación entre los dos sensores de deformación de la posición 2 durante la toma de datos. (a)- El día 26/09/2011. (b) - El día 27/09/2011.

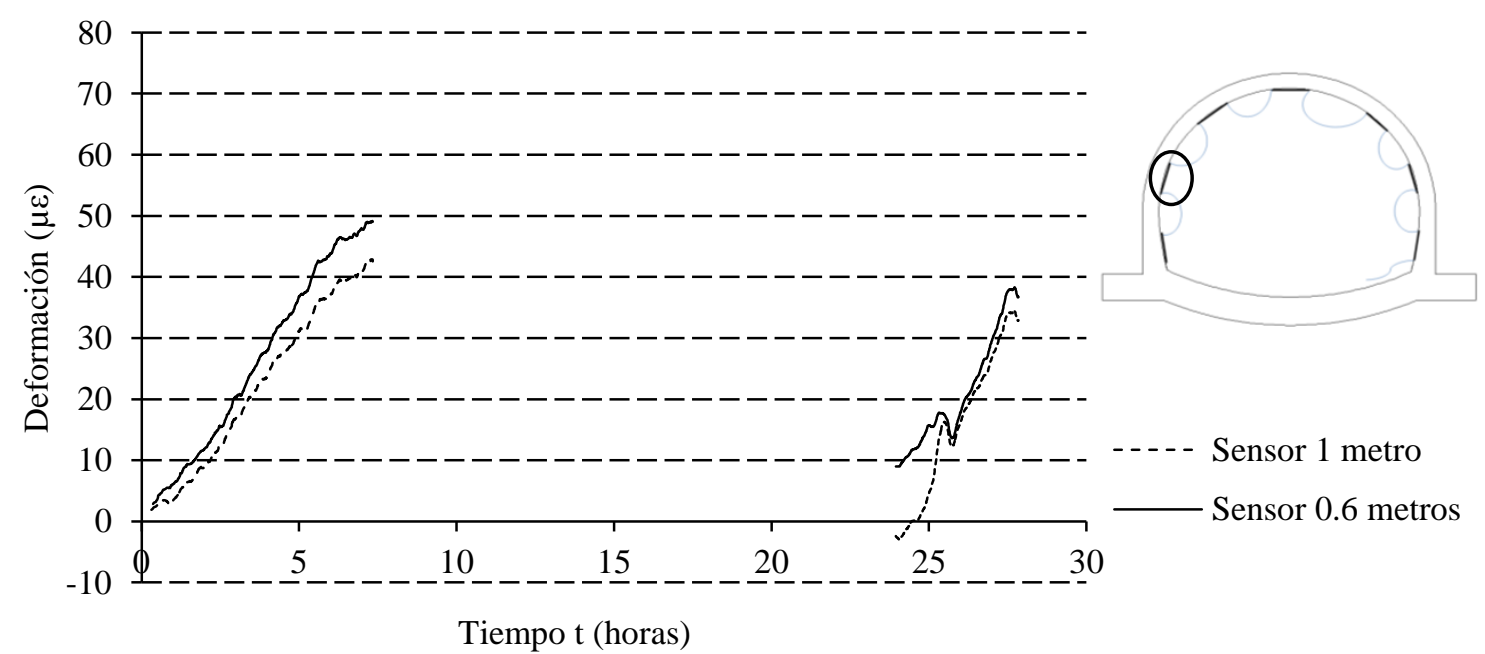

Figura 6.20 Variaciones de deformación de los sensores durante la toma de datos de los días 26 y 27/09/2011.

Existe una pequeña diferencia entre los resultados de ambos sensores y puede ser debido, fundamentalmente, al error que se deriva de la compensación térmica.

Es importante señalar que una correcta compensación térmica exigiría una fibra de compensación por cada sensor de deformación. En el presente trabajo esto no ha sido posible, y la compensación térmica se ha llevado a cabo empleando el sensor de la posición 4 que se indicó en el apartado 6.3.3. No obstante, las variaciones de temperaturas en el interior del falso túnel han sido relativamente pequeñas (incremento máximo de $2.77^{\circ} \mathrm{C}$ en el hastial) a lo largo del tiempo en que se tomaron medidas, como se indica en las figuras 6.16 y 6.17 del apartado 6.5.3.b, por lo que el error cometido puede ser asumible. 
Monitorización estructural.

Aplicación con sensores de fibra óptica.

d) Pauta general de comportamiento del falso túnel. Comparación de las mediciones con los resultados numéricos.

Tal y como se citó en el apartado 6.5.2, las variaciones térmicas existentes en el falso túnel han provocado deformaciones en la estructura. A partir de un modelo numérico simplificado 2D de elementos finitos se han obtenido unos resultados teóricos que serán contrastados con las mediciones de los sensores, con el objetivo de comparar la pauta general de comportamiento. Una vez contrastados estos resultados se podrá validar el correcto funcionamiento de los sensores.

La respuesta del falso túnel ante las variaciones térmicas expuestas anteriormente sigue una ley homóloga a la que describen las variaciones térmicas dadas por la Figura 6.18: ante incrementos y decrementos de temperatura, la bóveda del túnel responde traccionándose y comprimiéndose respectivamente (Figura 6.21).

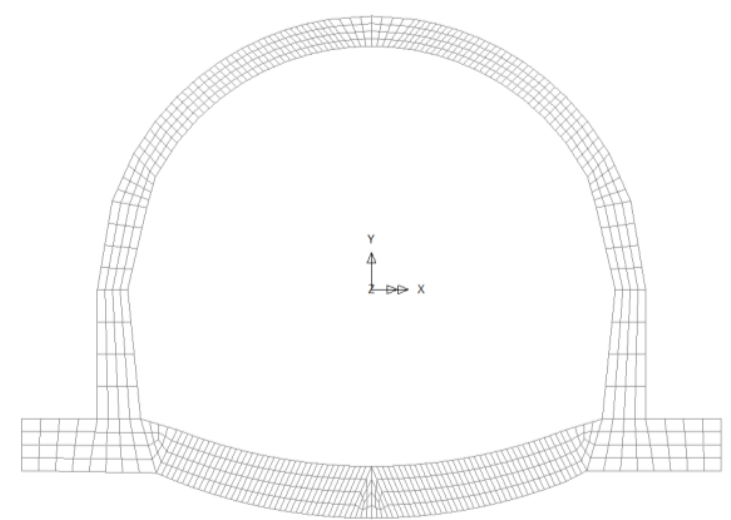

$\mathrm{t}=0 \mathrm{~h}$

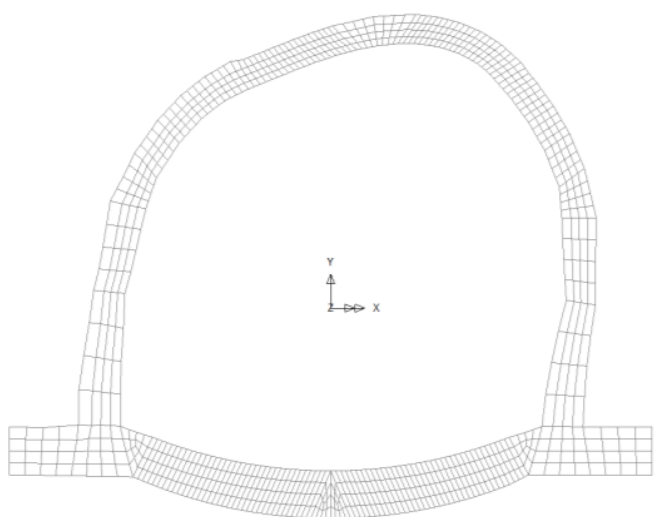

$\mathrm{t}=7 \mathrm{~h}$

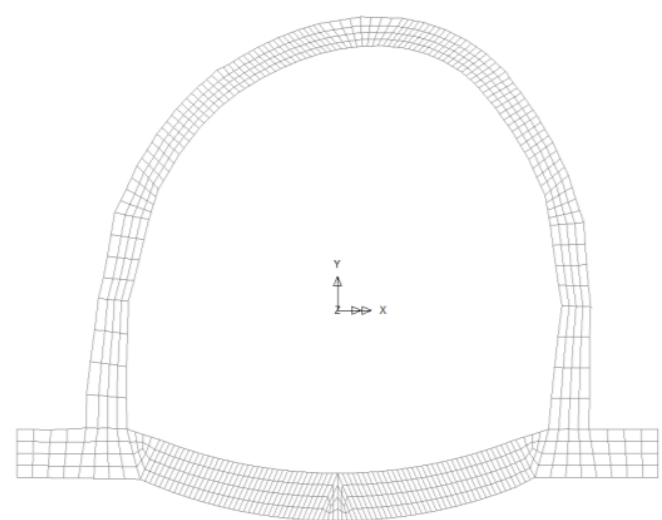

$\mathrm{t}=2 \mathrm{~h}$

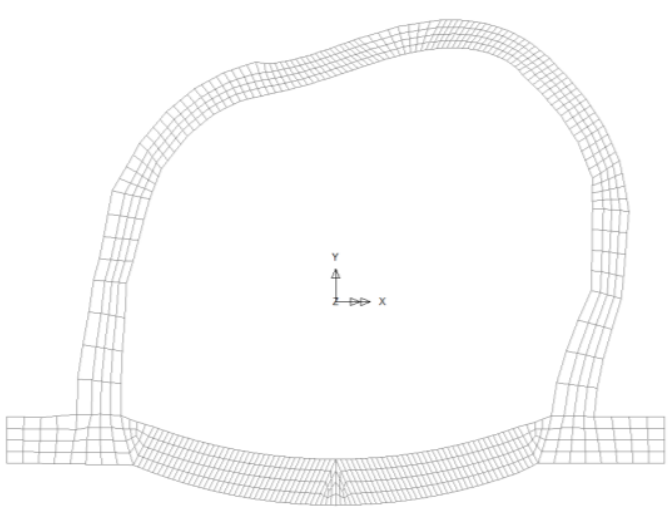

$\mathrm{t}=8.5 \mathrm{~h}$ 


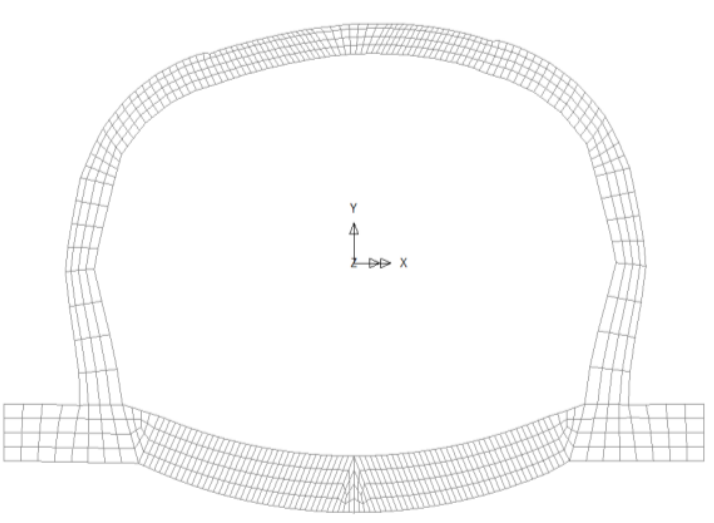

$\mathrm{t}=14 \mathrm{~h}$

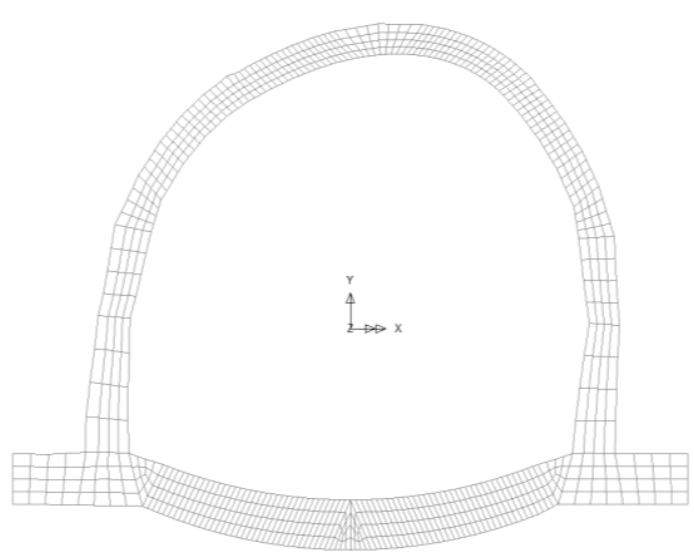

$\mathrm{t}=27 \mathrm{~h}$

Figura 6.21 Evolución de la malla deformada en distintos instantes de tiempo.

Las variaciones de temperaturas diurnas y nocturnas imponen a la estructura del túnel una ley de deformaciones ascendente y descendente. Las figuras que se muestran a continuación (Figuras 6.22 a 6.28) comparan los resultados numéricos y las mediciones realizadas con sensores puntuales y de longitud. En las siguientes figuras, las deformaciones positivas corresponden con deformaciones de tracción.

La solución teórica (con marcadores) responde al ciclo completo de temperaturas indicado en la Figura 6.18 del apartado 6.5.2.b. Sin embargo, las mediciones de los sensores (en traza continua y discontinua de color negro) corresponden únicamente a los horarios de la toma de medida.

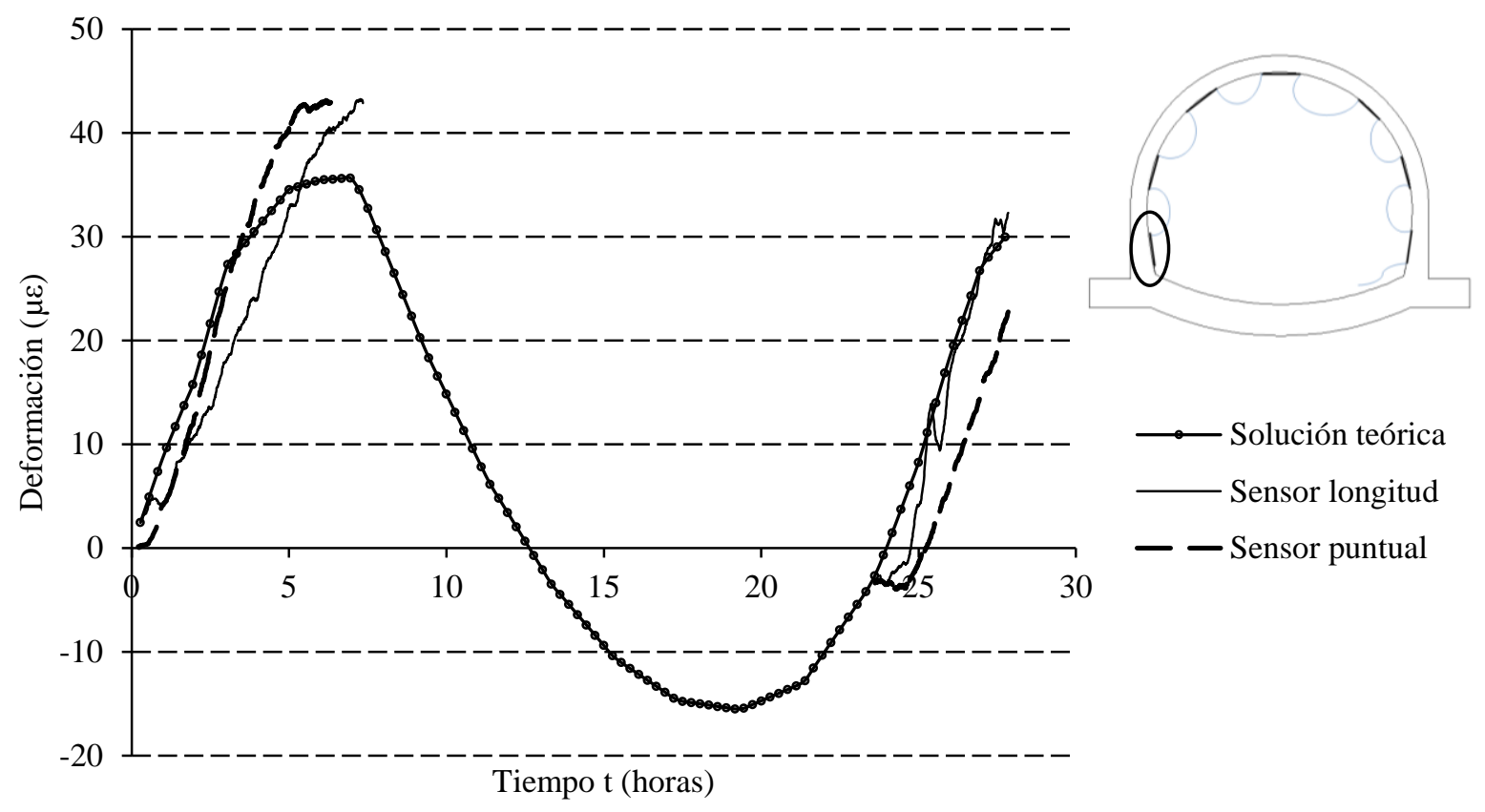

Figura 6.22 Evolución de las deformaciones de la posición 1. Mediciones y resultados numéricos. 
Monitorización estructural.

Aplicación con sensores de fibra óptica.

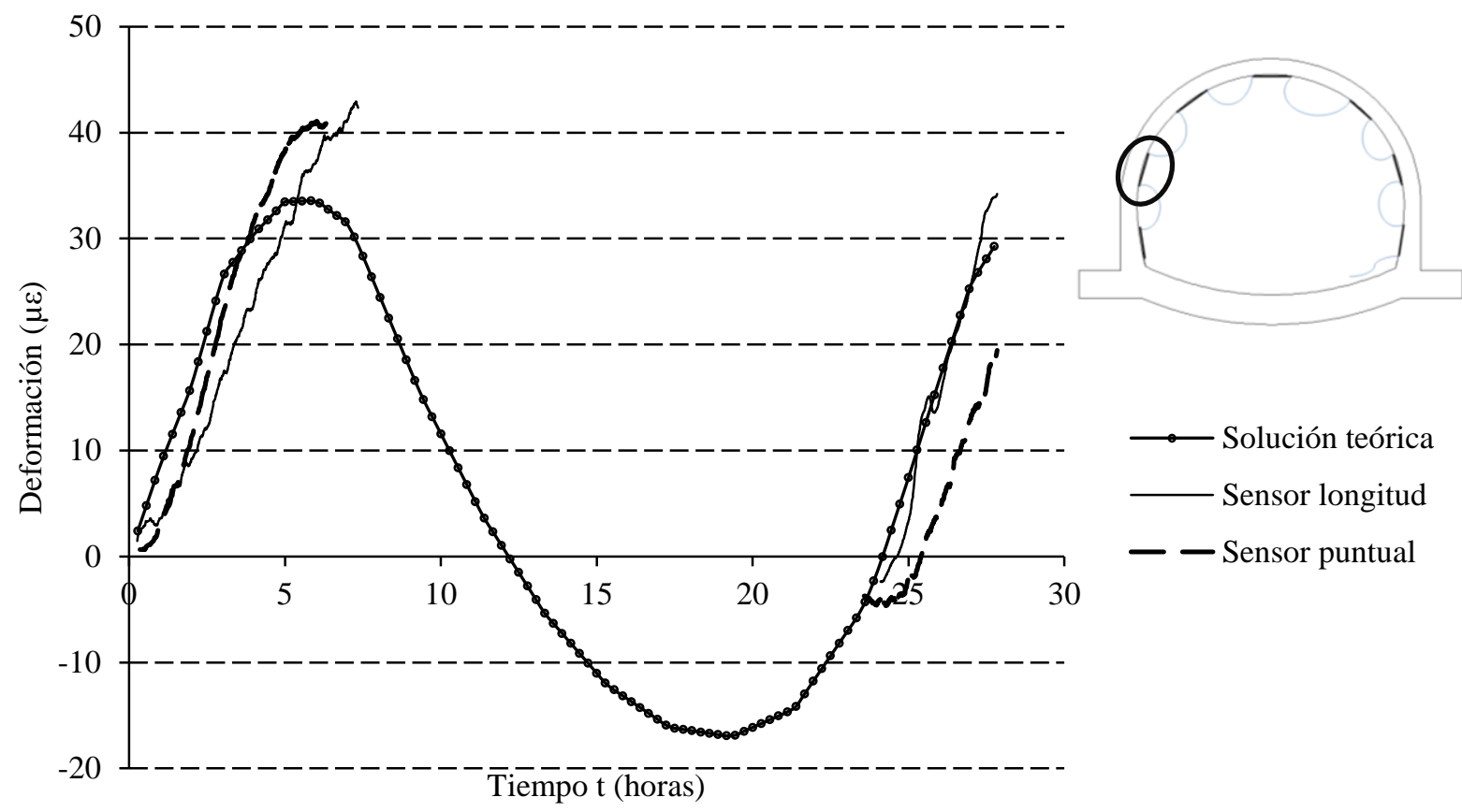

Figura 6.23 Evolución de las deformaciones de la posición 2. Mediciones y resultados numéricos.

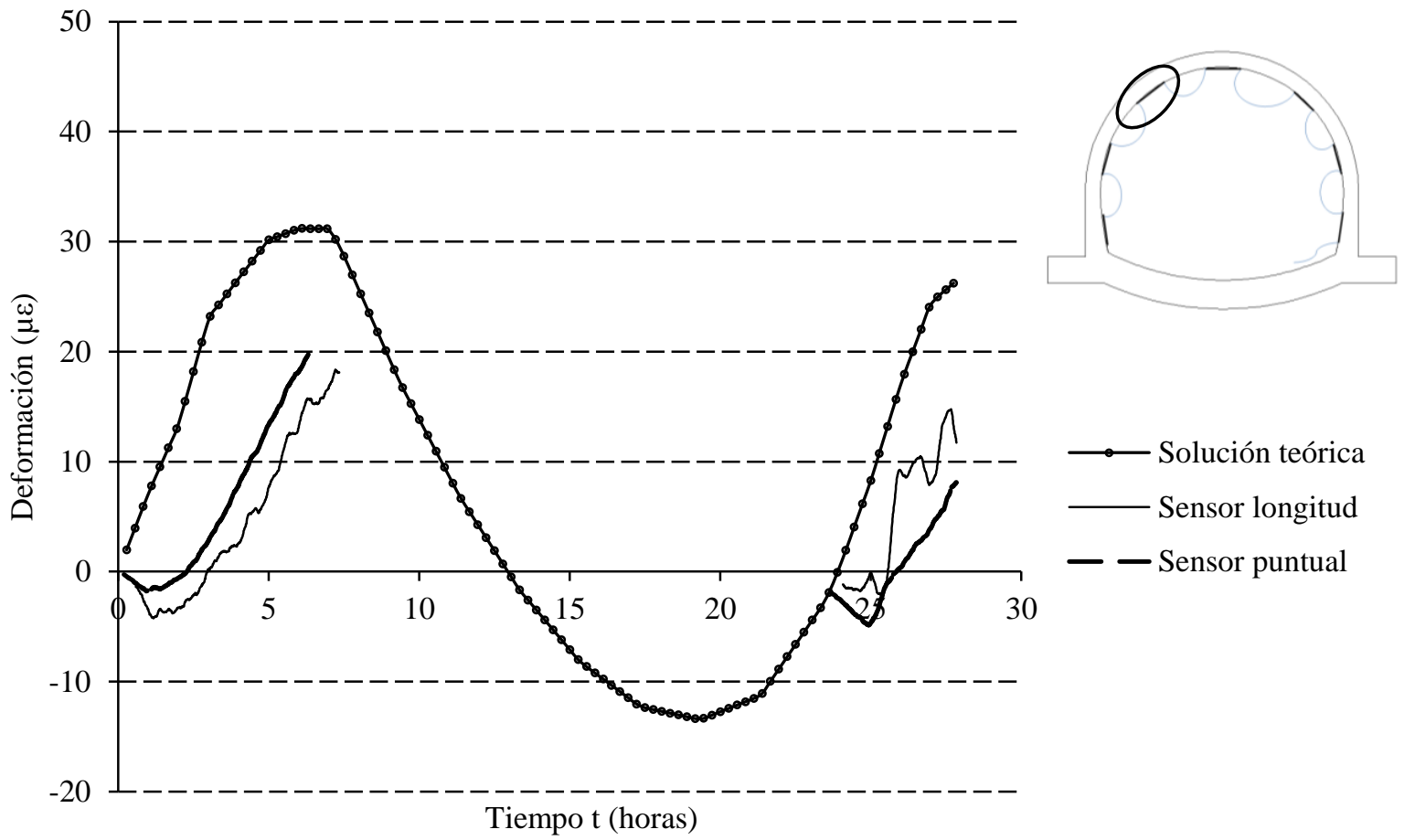

Figura 6.24 Evolución de las deformaciones de la posición 3. Mediciones y resultados numéricos. 


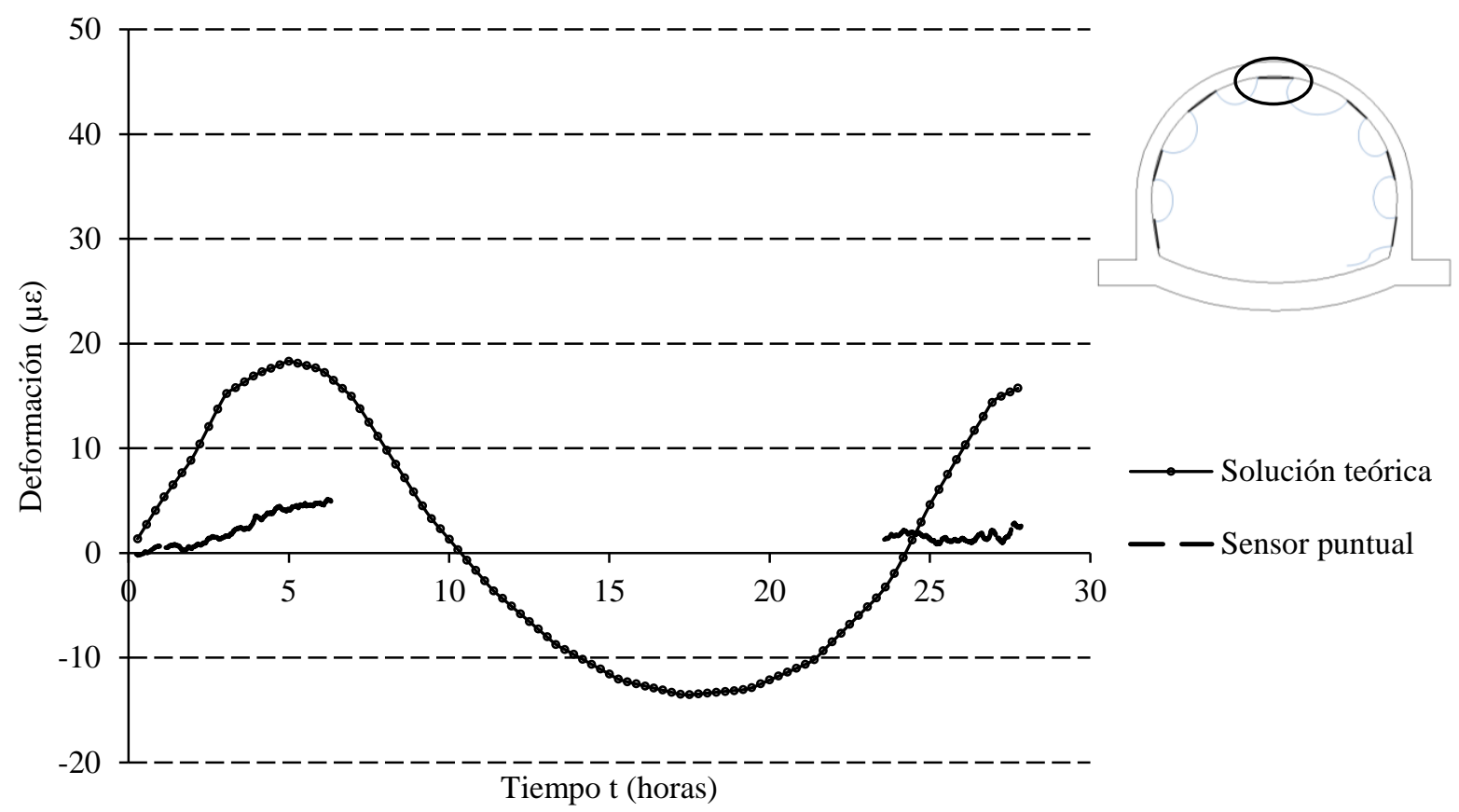

Figura 6.25 Evolución de las deformaciones de la posición 4. Mediciones y resultados numéricos.

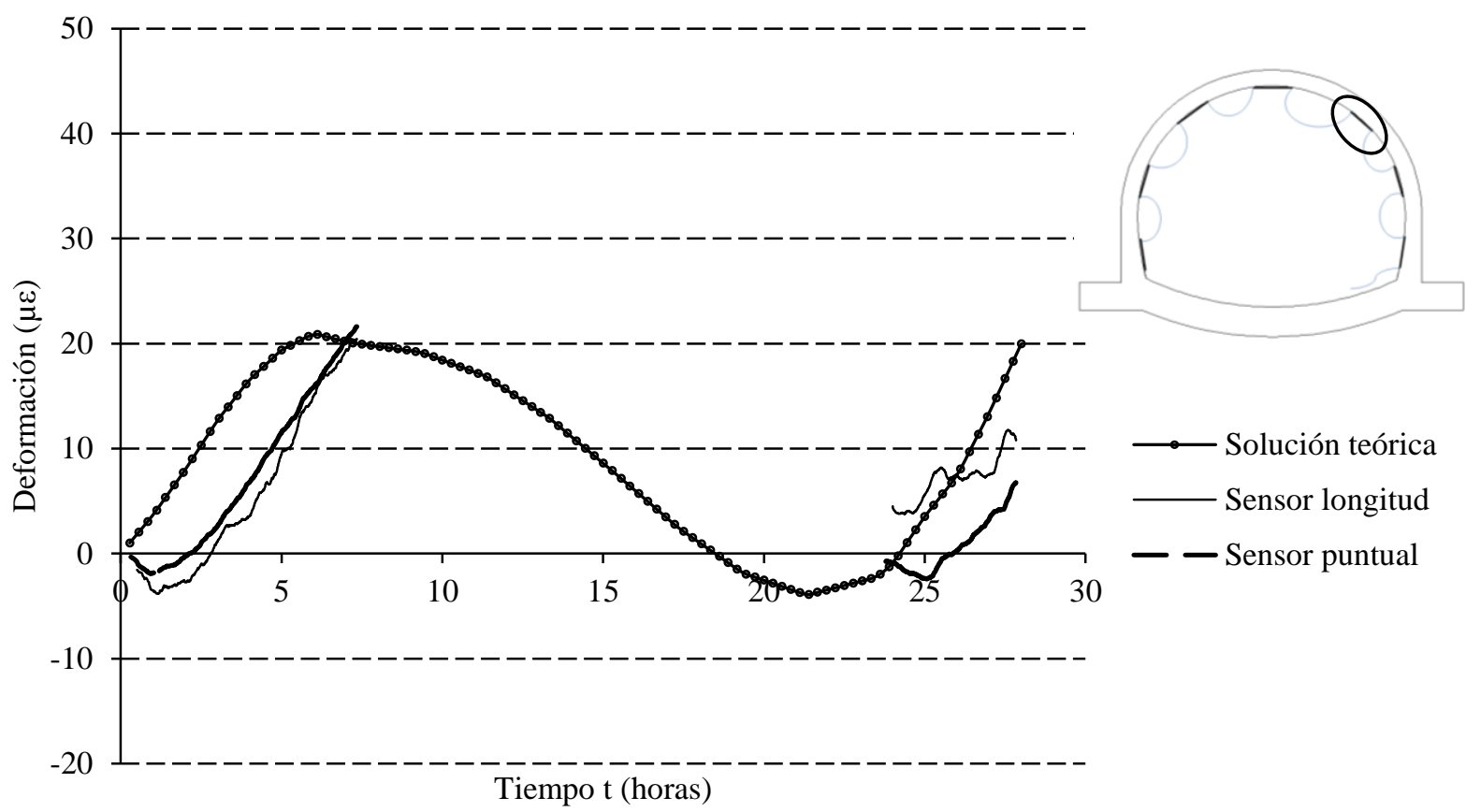

Figura 6.26 Evolución de las deformaciones de la posición 5. Mediciones y resultados numéricos. 
Monitorización estructural.

Aplicación con sensores de fibra óptica.

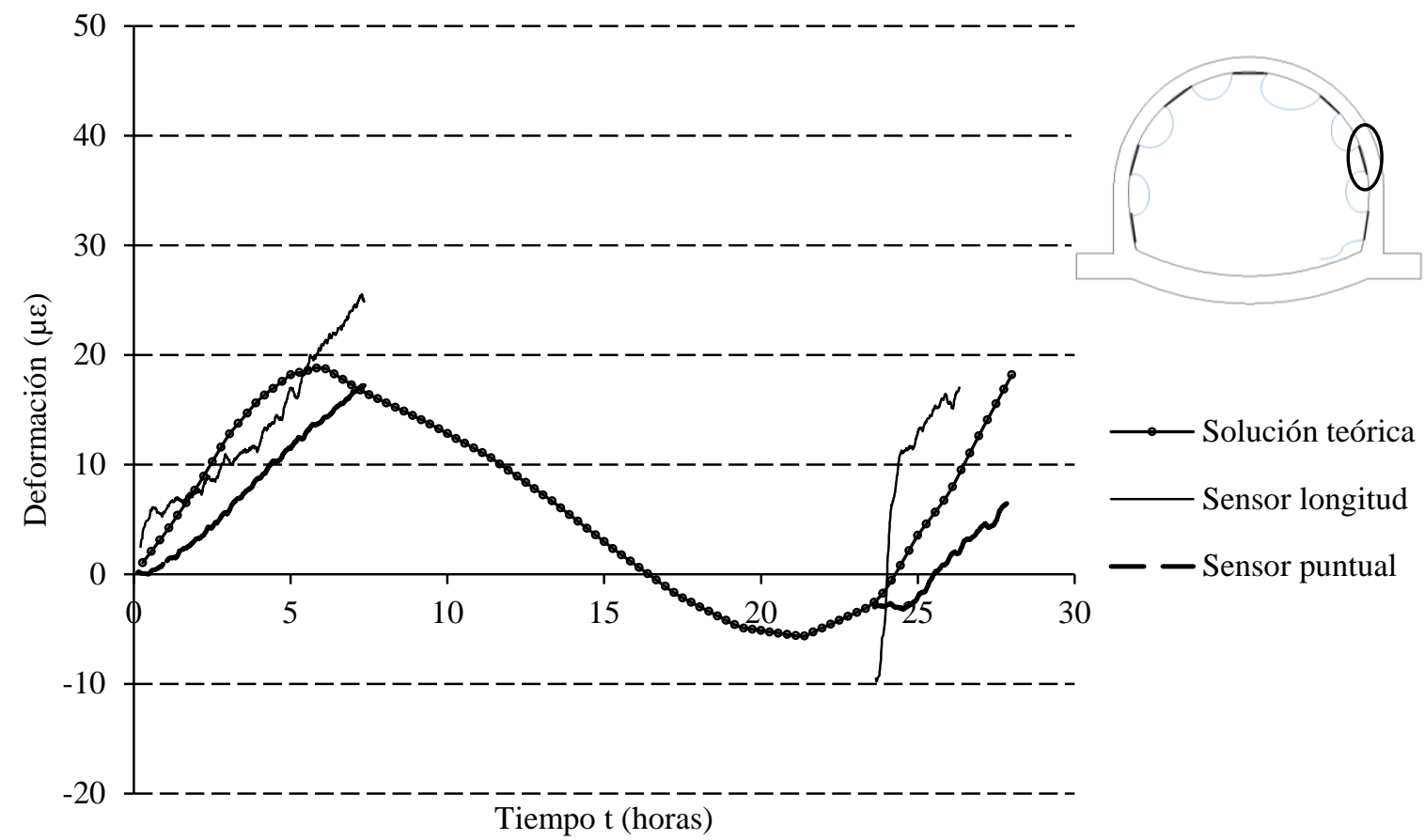

Figura 6.27 Evolución de las deformaciones de la posición 6. Mediciones y resultados numéricos.

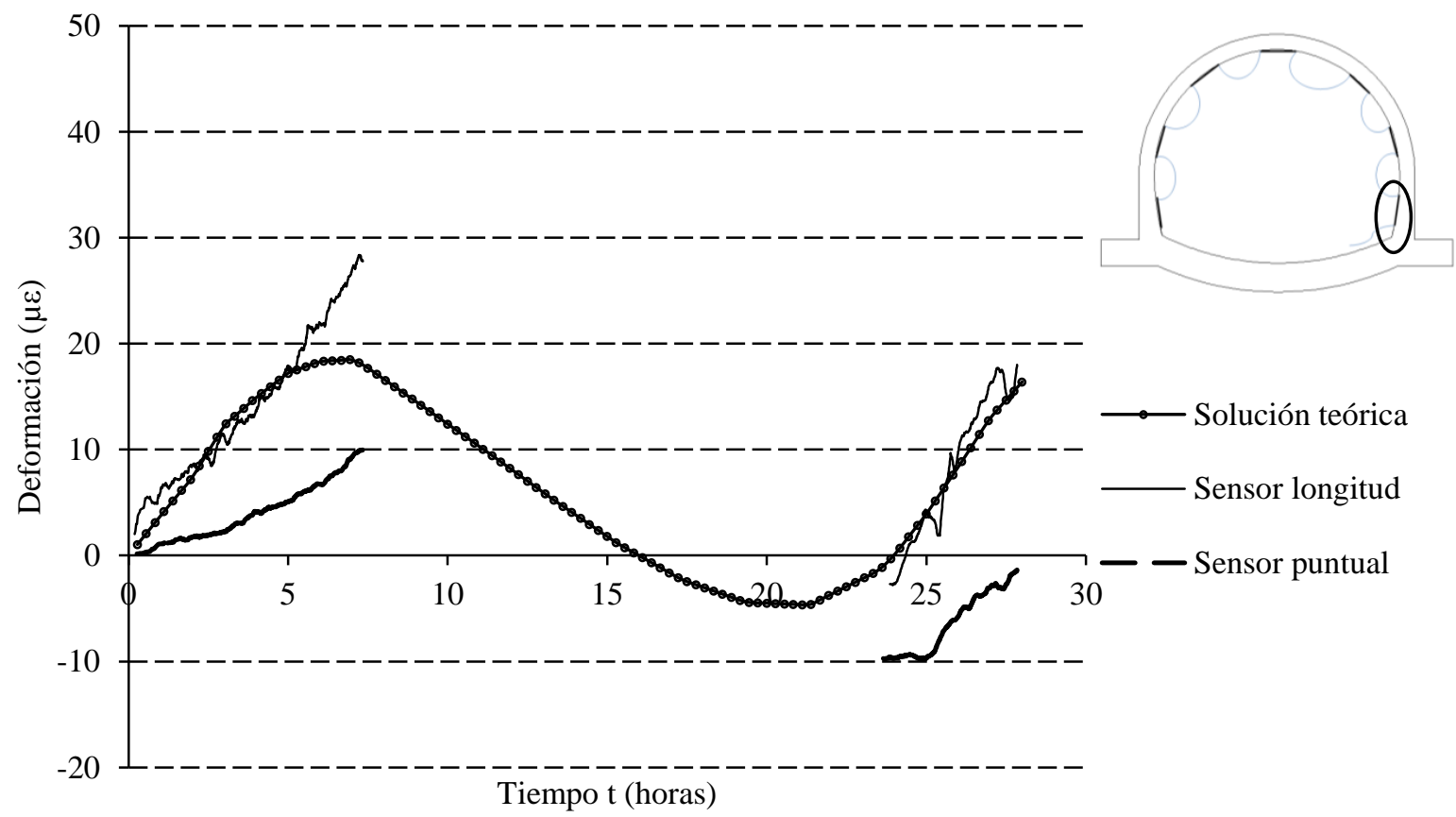

Figura 6.28 Evolución de las deformaciones de la posición 7. Mediciones y resultados numéricos. 
Tal y como se observa en las anteriores figuras, los sensores ópticos han reproducido, en general, notablemente bien la pauta general de comportamiento que indica la solución obtenida del modelo numérico simplificado. Sin embargo, existen algunas diferencias entre las soluciones teóricas y las medidas con los sensores puntuales y de longitud. Algunos aspectos relativos a estas diferencias son:

- Es importante destacar que la estructura está trabajando en niveles de deformación muy pequeños. A niveles tan bajos de deformación, la compensación térmica realizada puede introducir algunos errores durante su aplicación, debido a que todos los sensores de deformación se han compensado con un único sensor, el situado en la clave de la estructura.

- Los resultados teóricos están obtenidos a partir de un modelo numérico simplificado de elementos finitos. Sólo se han medido las temperaturas en 4 puntos instrumentados, y a partir de estos valores se ha interpolado el valor de las temperaturas a lo largo de toda la sección, por lo que los resultados teóricos también presentan un margen de incertidumbre.

- Tal y como se indicó en el Capítulo 5, los sensores puntuales presentan el inconveniente que se encuentran fuertemente influenciados por el punto en cuestión donde se instalen. A pesar de que los niveles de deformación medidos son inferiores a la deformación última de fisuración del hormigón, las irregularidades puntuales derivadas de la propia naturaleza del hormigón pueden ser motivo de algunas diferencias entre las mediciones con sensores puntuales y de longitud. Además, es importante destacar que la sección instrumentada se encontraba reparada (Figura 6.29) debido a la existencia de múltiples coqueras, aumentando más todavía el grado de heterogeneidad del material.

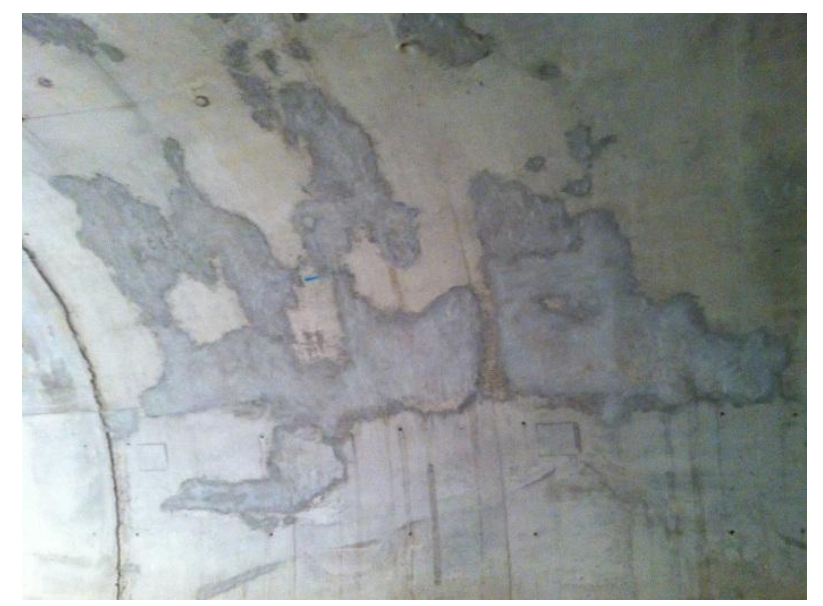

Figura 6.29. Reparaciones realizadas en la sección instrumentada.

- No ha sido posible la comparativa entre sensores de longitud y puntuales en el punto 4, debido a que en este punto el sensor de longitud ha sido empleado para realizar la compensación térmica. En este punto es donde existen las mayores diferencias entre valores teóricos y medidos. Probablemente esto ha sido debido a que el sensor puntual ha sido instalado justo sobre una de las reparaciones sobre la bóveda o que en este punto, el modelo numérico simplificado no haya reproducido bien las deformaciones.

En general y a pesar de las diferencias lógicas debidas a las incertidumbres y simpleza del modelo teórico, las pautas generales de comportamiento observadas coinciden notablemente bien con las que predice el modelo teórico. 
Monitorización estructural.

Aplicación con sensores de fibra óptica.

\subsubsection{Mediciones instantáneas el día 14/05/2012.}

\section{a) Introducción.}

La siguiente toma de datos se realizó el día 14/05/2012. En este instante de tiempo ya se había finalizado la operación de relleno de tierras, existiendo una altura de relleno de tierras de 20.3 metros sobre la base de la bóveda, y 11 metros sobre la clave (Figura 6.8).

Para llevar a cabo el estudio teórico con el modelo numérico simplificado se han considerado las acciones que han influido desde la toma de datos inicial, correspondiente al día 26/09/2011 a las 09:34. Estas acciones a tener en cuenta son las variaciones térmicas y el peso y empuje de las tierras a ambos lados del túnel. Es importante tener en cuenta las siguientes consideraciones:

- En este instante de tiempo la estructura se encuentra enterrada, por lo que se ha supuesto que las variaciones de temperatura, desde el día 26/09/2011, han sido las mismas en las superficies interiores y exteriores del túnel. Ha sido necesario adoptar esta hipótesis -en principio razonable- debido a que los sensores de temperatura del exterior del túnel dejaron de funcionar durante las operaciones de movimiento de tierras sobre ellos. El sensor de temperatura instalado en el hastial interior del túnel también dejó de funcionar, probablemente debido a su rotura durante la operación de construcción de aceras y bordillos en ese margen.

- En este caso, las temperaturas han sido introducidas en el modelo numérico simplificado como incrementos absolutos, sin emplear la opción Load Curve implementada en el software ya que se desconoce la evolución continuada en el tiempo de la temperatura.

- El peso y empuje de las tierras se han introducido en el modelo como fuerzas por unidad de superficie sobre las caras exteriores de los elementos finitos. El empuje considerado ha sido el empuje al reposo.

Durante la toma de datos no hubo ninguna modificación en la altura de tierras ya que esta operación había finalizado, motivo por el que se ha realizado una medición instantánea.

\section{b) Temperaturas y deformaciones medidas.}

Como se ha explicado en el apartado anterior, se han obtenido las variaciones de temperatura y deformación entre la fecha inicial de medición (26/09/2011 a las 09:34) y la fecha de toma de datos $(14 / 05 / 2012$ a las 09:15).

Debido a la rotura de tres de los cuatro sensores de temperatura dispuestos, la variación de temperatura medida ha sido obtenida con el sensor de temperatura dispuesto en la clave interior del túnel. Esta variación de temperatura resulta ser de $-4.94{ }^{\circ} \mathrm{C}$ (Tabla 6.4):

\begin{tabular}{cccc}
\hline Sensor & $\lambda_{\boldsymbol{i}}(\mathrm{nm})(\mathbf{2 6 / 0 9 / 2 0 1 1})$ & $\lambda_{f}(\mathrm{~nm})(\mathbf{1 4 / 0 5 / 2 0 1 2})$ & $\Delta T$ \\
\hline Clave interior & 1543.141 & 1543.1012 & -4.94 \\
\hline
\end{tabular}

Tabla 6.4 Variación de temperatura entre la puesta a cero y la fecha de medición en el sensor de la clave interior del túnel. 
Donde $\lambda_{i}$ es la longitud de onda inicial del sensor de temperatura, en nanómetros el día 26/09/2011, $\lambda_{f}$ es la longitud de onda final del sensor de temperatura, en nanómetros el día 14/05/2012 y $\Delta T\left({ }^{\circ} \mathrm{C}\right)$ es el incremento de temperatura obtenido a partir de las dos longitudes de onda definidas previamente.

Como se observa en la Tabla 6.4, la variación de temperatura obtenida ha sido negativa, lo que indica que la temperatura ha sido inferior en la fecha de toma de datos (14/05/2012) que en el día 26/09/2011. Este hecho es evidente debido a los decrementos de la temperatura ambiente ligados a los meses en que se tomaron los datos.

Tal y como se explicó en el apartado anterior y debido a la rotura de los sensores de temperatura, se ha considerado que toda la bóveda de la estructura ha sufrido el mismo decremento de temperatura. Este razonamiento se ha realizado teniendo en cuenta que la estructura se encuentra totalmente enterrada.

Tras aplicar la compensación térmica en la forma descrita en el apartado 6.5.2. se ha obtenido las variaciones de deformación en los sensores dispuestos que muestra la tabla 6.5.

\begin{tabular}{cccccccc}
\cline { 2 - 7 } & \multicolumn{7}{c}{ Ubicación de los sensores } \\
\cline { 2 - 7 } & $\mathbf{1}$ & $\mathbf{2}$ & $\mathbf{3}$ & $\mathbf{4}$ & $\mathbf{5}$ & $\mathbf{6}$ & $\mathbf{7}$ \\
\cline { 2 - 7 } $\begin{array}{c}\text { Sensor de } \\
\text { longitud }(\mu \varepsilon)\end{array}$ & 24.1 & -245.3 & -165.0 & - & -118.1 & -305.3 & -32.7 \\
$\quad \begin{array}{c}\text { Sensor } \\
\text { puntual }(\mu \varepsilon)\end{array}$ & - & -198.9 & -106.6 & -78.3 & -146.6 & - & -106.8 \\
\hline
\end{tabular}

Tabla 6.5 Valores de deformación entre la puesta a cero y la fecha de medición.

Respecto a los valores de la Tabla 6.5 es importante destacar los siguientes aspectos:

- No ha sido posible la medición de los sensores puntuales 1 y 6 , probablemente debido a roturas de los mismos. Tal y como se indicó en los apartados anteriores, el sensor de longitud 4 no proporciona las deformaciones de la estructura puesto que ha sido empleado para llevar a cabo la compensación térmica.

- En un mismo punto considerado, existen diferencias entre los valores medidos por los sensores de longitud y los sensores puntuales. En este aspecto es importante destacar que los sensores puntuales se ven fuertemente influenciados por los defectos locales que presente el material, en este caso un material heterogéneo como es el hormigón. Sin embargo, en la mayoría de puntos instrumentados existe una clara pauta general de comportamiento marcada por ambos tipos de sensores.

- El sensor puntual de la posición 7 es el que mayor dispersión presenta en relación a la medición obtenida con los sensores de longitud. La causa más probable de esta dispersión es un funcionamiento anómalo del sensor. Hay que tener en cuenta que en esta zona se han llevado a cabo los trabajos de construcción de sobrelevaciones sobre la contrabóveda, aceras y bordillos y es muy probable que estas operaciones hayan dañado el sensor. Esta hipótesis está apoyada por el hecho de que en el sensor puntual de la posición 1, simétrica a la posición 7, no fue posible su toma de datos debido a que se encontraba dañado. 
Monitorización estructural.

Aplicación con sensores de fibra óptica.

- En general, la estructura muestra un comportamiento bastante simétrico:

- Los sensores de longitud 1 y 7, instalados en los hastiales de la estructura, indican pequeñas deformaciones. El punto 7 indica una deformación de compresión de valor $32.7 \mu \varepsilon$, mientras que el punto 1 indica una deformación de tracción de valor $24.1 \mu \varepsilon$. Este valor de tracción puede ser debido a un error derivado de la propia compensación térmica. En cualquier caso, ambas deformaciones son pequeñas en comparación con el resto de valores.

- Los puntos 2 y 6 son los que mayor deformación de compresión alcanzan, con valores de -245.3 y $-305.3 \mu \varepsilon$. El sensor puntual de deformación del punto 2 indica un valor de compresión de -198.9

- En los puntos 3 y 5 se han obtenido deformaciones de compresión inferiores a las obtenidas en los puntos 2 y 6 , y superiores a la considerada en los puntos 1 y 7 que coinciden con los hastiales.

- Finalmente, en el punto instrumentado 4 se ha obtenido una deformación de compresión de valor $-78.3 \mu \varepsilon$ con el sensor puntual.

\section{c) Comparación de las mediciones con los resultados numéricos.}

Una vez obtenidas las mediciones realizadas, éstas han sido comparadas con los valores teóricos obtenidos a partir del modelo numérico simplificado 2D de elementos finitos.

Sobre este modelo se han aplicado los incrementos de temperatura medidos que fueron expuestos en el apartado anterior 6.5.3.b. y el empuje y peso de tierras. Los incrementos de temperatura se han asignado a las caras interiores y exteriores de la bóveda del falso túnel, y el empuje de tierras correspondiente a 20.3 metros de altura sobre la totalidad de la bóveda (Figura 6.30).

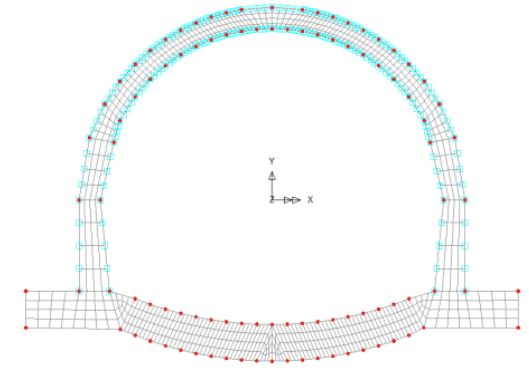

(a)

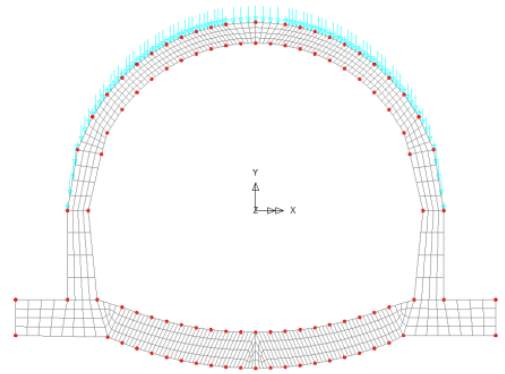

(b)

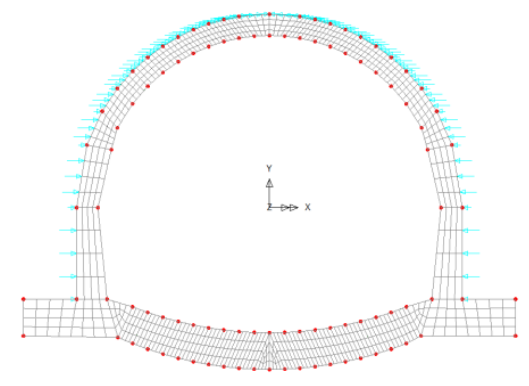

(c)

Figura 6.30. Acciones sobre el modelo (a)- Variaciones de temperatura. (b)- Peso de las tierras. (c)- Empuje de las tierras.

La Figura 6.31 muestra la malla deformada sobre la geometría inicial sin deformar (indicada con trazas verdes y puntos rojos), para las tres acciones descritas anteriormente: 


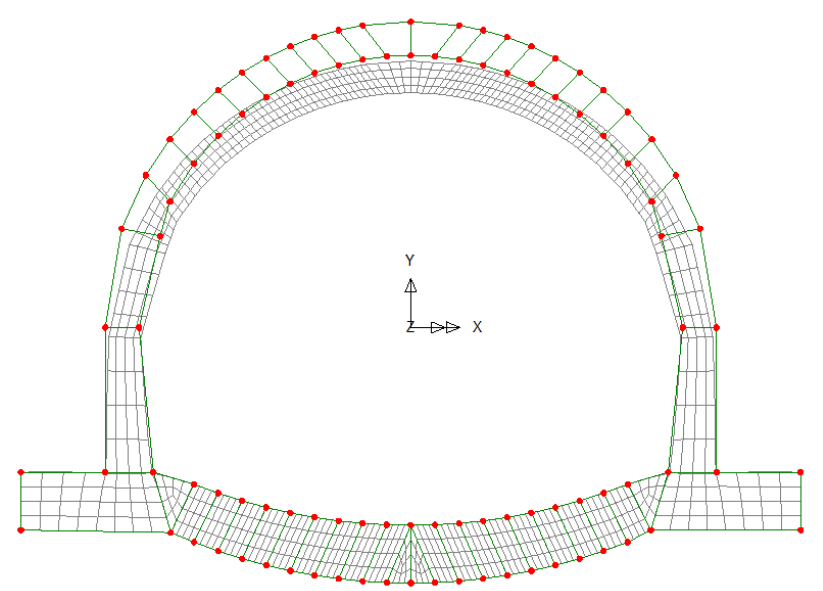

(a)

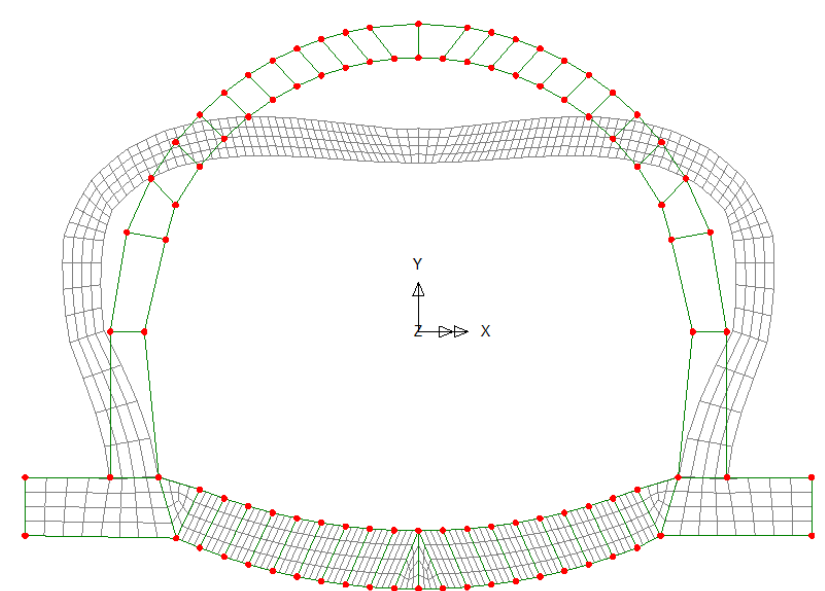

(b)

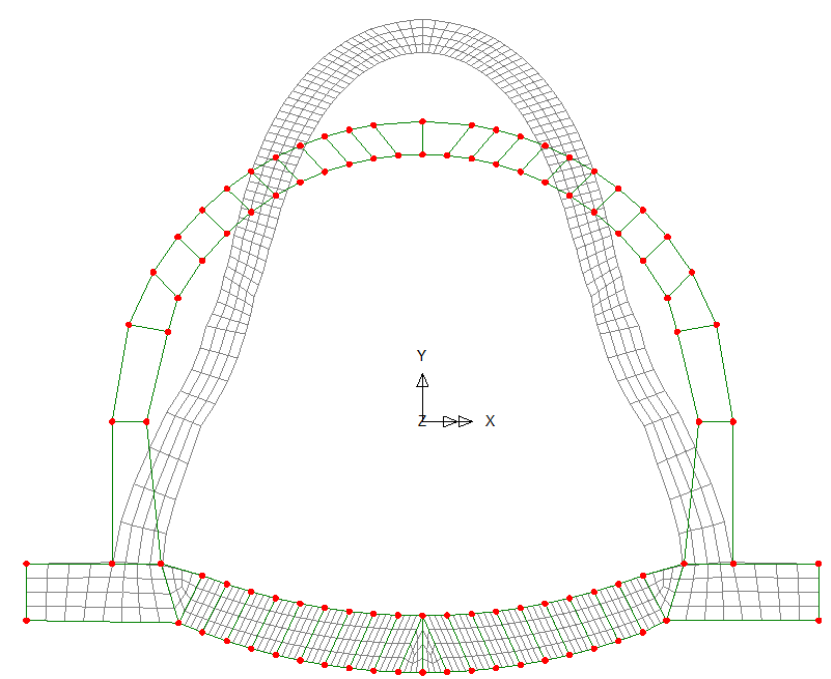

(c)

Figura 6.31 Malla deformada. (a)- Variaciones térmicas. (b)- Peso de las tierras. (c)- Empuje de las tierras. 
Monitorización estructural.

Aplicación con sensores de fibra óptica.

Realizando una combinación de las anteriores acciones se han obtenido los valores de las deformaciones teóricas a partir del modelo numérico simplificado. La Tabla 6.6 muestra los resultados medidos con los sensores ópticos (que se mostraron en la anterior Tabla 6.5) y los resultados teóricos:

\begin{tabular}{|c|c|c|c|c|c|c|c|}
\hline & \multicolumn{7}{|c|}{ Ubicación de los sensores } \\
\hline & 1 & 2 & 3 & 4 & 5 & 6 & 7 \\
\hline $\begin{array}{c}\text { Sensor de } \\
\text { longitud }(\mu \varepsilon) .\end{array}$ & 24.1 & -245.3 & -165.0 & - & -118.1 & -305.3 & -32.7 \\
\hline $\begin{array}{c}\text { Sensor } \\
\text { puntual }(\mu \varepsilon) .\end{array}$ & - & -198.9 & -106.6 & -78.3 & -146.6 & - & -106.8 \\
\hline $\begin{array}{c}\text { Deformación } \\
\text { teórica }(\mu \varepsilon) \text {. }\end{array}$ & -28 & -189.6 & -96.2 & 17 & -96.2 & -189.6 & -28 \\
\hline
\end{tabular}

Tabla 6.6 Valores de deformación medidos y teóricos entre la puesta a cero y la fecha de medición.

Respecto a los valores mostrados en la Tabla 6.6, es importante destacar algunos aspectos:

- Tal y como se indicó en el apartado 6.5.3.b, las mediciones obtenidas con los sensores ópticos indicaron, en general, un comportamiento simétrico del falso túnel. Los resultados teóricos, dadas las hipótesis de temperaturas adoptadas y las acciones debidas al relleno de tierras, ofrecen un comportamiento totalmente simétrico, como era de esperar.

- Las deformaciones teóricas obtenidas en los puntos 1 y 7 son de $-28 \mu \varepsilon$. Se trata de un valor pequeño de deformación de compresión, en comparación con el resto de valores alcanzados en los otros puntos instrumentados. Esto mismo sucedía con las mediciones obtenidas con sensores ópticos, salvo el sensor puntual del punto 7 que su medición se justificó errónea en el apartado 6.5.3.b.

- Las mayores deformaciones de compresión se han obtenido en los puntos 2 y 6 , con valores de $-189.6 \mu \varepsilon$. Las mediciones obtenidas con los sensores ópticos indicaron que los puntos 2 y 6 eran los que mayores deformaciones de compresión alcanzaban, coincidiendo con la pauta general de comportamiento en este punto instrumentado.

- Las deformaciones obtenidas en los puntos 3 y 5 también marcan la pauta general de comportamiento del falso túnel. A partir del modelo numérico, su valor de $-81.9 \mu \varepsilon$ indica una deformación de compresión inferior a la de los puntos 2 y 6, pero mayor a la obtenida en los hastiales de la estructura (puntos 1 y 7), tal y como indicaron las mediciones realizadas con los sensores ópticos.

- Finalmente se ha obtenido una deformación de $17 \mu \varepsilon$ en la clave de la estructura. Este valor representa una deformación de tracción que viene provocada porque la deformación debida al peso de las tierras es superior a la deformación que provoca el empuje. Comparando con el valor medido por el sensor puntual, este valor es el que presenta una mayor dispersión en relación con la pauta general de comportamiento. 
- En general y a pesar de las diferencias lógicas debidas a las incertidumbres y simpleza del modelo teórico, las pautas generales de comportamiento observadas coinciden notablemente bien con las que predice el modelo teórico.

Basándose en los resultados obtenidos del modelo numérico simplificado, la Tabla 6.7 indica qué parte de la deformación obtenida es debida a las variaciones térmicas, y qué parte es debida al efecto de las tierras sobre la bóveda de la estructura.

\begin{tabular}{|c|c|c|c|c|c|c|c|}
\hline & \multicolumn{7}{|c|}{ Ubicación de los sensores } \\
\hline & 1 & 2 & 3 & 4 & 5 & 6 & 7 \\
\hline $\begin{array}{l}\text { Relleno de } \\
\text { tierras (\%) }\end{array}$ & 42.8 & 92.3 & 85.3 & - & 85.3 & 92.3 & 42.8 \\
\hline $\begin{array}{l}\text { Variaciones } \\
\text { térmicas (\%) }\end{array}$ & 57.2 & 7.7 & 14.7 & - & 14.7 & 7.7 & 57.2 \\
\hline
\end{tabular}

Tabla 6.7 Porcentajes sobre la deformación total originados por el relleno de tierras y por las variaciones térmicas.

Tal y como se observa en la Tabla 6.7, el peso y el empuje de las tierras provocan gran parte de la deformación medida en los puntos instrumentados 2 y 6,3 y 5 , con porcentajes de $92.3 \%$ y $85.3 \%$ respectivamente, siendo estos puntos donde se han alcanzado los mayores valores de deformación de compresión. En los hastiales esta influencia es menor, causando un $42.8 \%$ del valor de las deformaciones. Los hastiales, tal y como se explicó anteriormente, son las zonas de menor valor de deformación de compresión.

\subsection{Conclusiones.}

En este capítulo se han presentado los trabajos de monitorización de una sección de falso túnel de la Línea de Alta Velocidad de Levante a su paso por Mogente (Valencia).

Los sensores han sido instalados siguiendo el perímetro de una sección transversal del túnel, y son sensores puntuales de deformación y temperatura, sensores de longitud y sensores distribuidos. El objetivo de este trabajo consiste en la aplicación de los sensores de longitud propuestos para su monitorización en una estructura real sometidos a las condiciones que en ella existen, y validar su aplicación. Para ello se ha realizado un modelo numérico simplificado 2D de elementos finitos, y se ha obtenido la pauta general de comportamiento de la sección modelada, para compararla con la pauta general de comportamiento que indican los sensores.

Las principales conclusiones obtenidas tras este trabajo han sido:

1) En el punto monitorizado 2 se instalaron dos sensores de deformación, de longitudes 1 y 0.6 metros. Los resultados de ambos sensores han mostrado un buen grado de ajuste entre ellos.

2) Es muy importante la monitorización de la temperatura en las monitorizaciones de obra. La temperatura tiene una afección importante sobre los sistemas de medida, y ésta debe ser compensada para obtener unos resultados aceptables. En este trabajo ha sido necesario la instalación de, al menos, un sensor de longitud que se encuentre anclado en un extremo y libre 
en el otro. Este sensor ha permitido llevar a cabo la compensación térmica que exige el sensado óptico. En esta aplicación, este sensor se ha instalado en la clave de la estructura. Para posibles futuras aplicaciones, sería necesario la instalación de más sensores de este tipo que permitan una compensación térmica más exacta. Otra posibilidad sería la de calibrar previamente la respuesta de este sensor frente a variaciones térmicas para su directa aplicación en la compensación térmica.

3) Durante las jornadas del 26 y 27/09/2011, la sección de la estructura se encuentra sometida a gradientes térmicos importantes entre el exterior y el interior de la bóveda, provocados por las diferencias de temperaturas diurnas y nocturnas. El gradiente de temperatura máximo medido se ha localizado en la cara exterior del hastial izquierdo, resultando ser de $18.17^{\circ} \mathrm{C}$.

4) Ante estas variaciones térmicas, la respuesta de la estructura es proporcional a las mismas, traccionándose y comprimiéndose ante incrementos y decrementos de temperatura. El valor máximo de estas deformaciones medidas por los sensores han sido de, aproximadamente, 40 $\mu \varepsilon$ en la rama ascendente de temperatura, y de $-15 \mu \varepsilon$ para valores de compresión. Este comportamiento ha sido comparado con los resultados de un modelo numérico simplificado 2D de elementos finitos, y en general, los sensores han reproducido notablemente bien la pauta general de comportamiento de la estructura.

5) Para niveles tan bajos de deformación, los sensores puntuales y de longitud han reproducido notablemente bien el comportamiento general de la estructura. Los valores de deformación de tracción han sido inferiores a la deformación última de fisuración del hormigón (estimada en torno a 50-100 $\mu \varepsilon$ ) y por este motivo, los sensores puntuales han mostrado resultados similares a los sensores de longitud, aunque con las lógicas diferencias motivadas por la heterogeneidad del material instrumentado.

6) Durante la toma de datos continua durante los días 26 y 27/09/2011 se ha observado un ligero comportamiento asimétrico de la bóveda del túnel (Figura 6.21), debido a las diferentes variaciones térmicas que tienen lugar entre los hastiales del túnel. Mientras que los sensores situados en el hastial izquierdo miden en torno a $40 \mu \varepsilon$, los sensores del hastial derecho miden en torno a $25 \mu \varepsilon$.

7) Una vez se ha realizado todo el relleno de tierras, se ha llevado a cabo una nueva medición, esta vez instantánea. En esta situación, los resultados medidos con los sensores y los resultados teóricos del modelo numérico simplificado han mostrado que la estructura presenta cierta simetría en su comportamiento. En estas mediciones existen mayores diferencias entre los sensores de longitud y los sensores puntuales. En este aspecto, es importante destacar que los sensores puntuales se ven fuertemente influenciados por los defectos locales que presente el material, en este caso un material heterogéneo como es el hormigón. Sin embargo, en la mayoría de puntos instrumentados existe una clara similitud entre las pautas generales de comportamiento que marcan ambos tipos de sensores.

8) Las mediciones del día 14/05/2012 han mostrado que la bóveda del falso túnel se encontraba comprimida con respecto a la fecha inicial de toma de datos el día 26/09/2011. Este comportamiento también fue obtenido con el modelo numérico simplificado de elementos finitos, salvo en la clave de la estructura, donde los valores teóricos indicaron que existen pequeñas tracciones de valor $16 \mu \varepsilon$. 
9) Algunos sensores ópticos puntuales han mostrado un comportamiento anómalo, como es el sensor situado en la posición 7 durante la toma de datos del día 14/05/2012. La causa más probable de su resultado fue que el sensor resultó dañado durante las operaciones de construcción de la sobrelevación, aceras y bordillos. De la misma forma, los sensores puntuales de la posición 1 y 6 sufrieron algún tipo de rotura durante estos trabajos que imposibilitó su medición. Todo esto indica la importancia de una mayor protección de la red de sensores en los trabajos de monitorización de obra.

10) En general, los sensores puntuales y de longitud han reproducido notablemente bien la pauta general de comportamiento de la estructura:

a. En general se ha observado la viabilidad y condiciones del uso de los sensores puntuales y de los sensores de longitud diseñados en condiciones de obra. Se ha observado que son capaces de medir con fiabilidad incluso deformaciones muy reducidas.

b. Se ha comprobado que, aunque sería preciso mejorar algunas protecciones, en general han resistido bastante bien las condiciones agresivas que impone una obra de construcción.

c. A pesar de que no es recomendable el empleo de los sensores puntuales para la monitorización de materiales heterogéneos, cuando estos elementos trabajan a compresión sería posible su empleo. Sin embargo, es muy importante evitar su instalación en zonas de coqueras, reparaciones y otras irregularidades puesto que sus mediciones podrían verse fuertemente alteradas. 



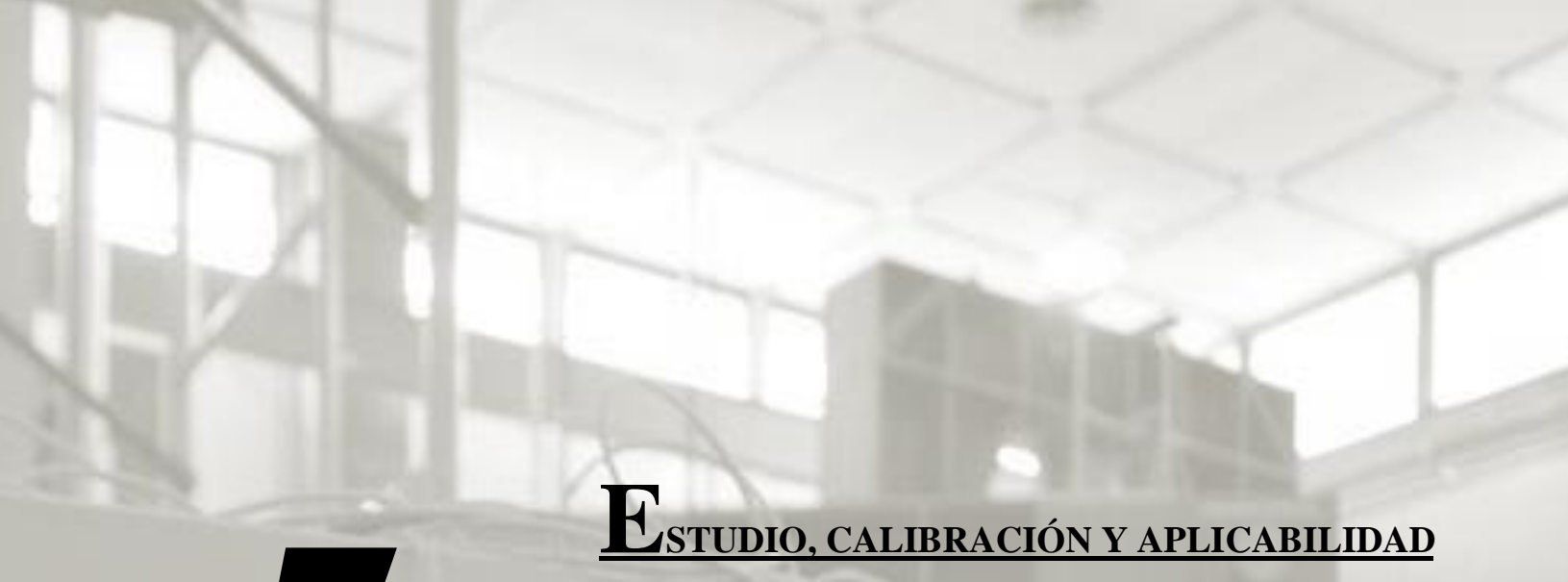

\section{DE SENSORES DISTRIBUIDOS MEDIANTE LA}

\section{DISPERSIÓN ESTIMULADA DE BRILLOUIN (SBS).}

El Capítulo 7 de esta Tesis Doctoral presenta los trabajos de caracterización de sensores ópticos distribuidos basados en el fenómeno de dispersión de Brillouin (en inglés, Brillouin Scattering). A diferencia de los sensores estudiados en los Capítulos 4 y 6 , este sensor proporciona una medida continua a lo largo de una longitud de fibra óptica, y es capaz de ofrecer valores "punto a punto".

La caracterización de estos sensores se ha llevado a cabo a partir de campañas experimentales, y se ha obtenido la respuesta de los mismos ante variaciones de temperatura y de deformación. Las variaciones térmicas han sido aplicadas empleando una cámara climática de la marca Feutron, mientras que la caracterización frente a deformaciones se ha realizado instalando los sensores sobre un elemento de hormigón al que se le aplicaron ciclos de carga y descarga con un pórtico metálico.

Estos estudios se han llevado a cabo sobre distintos tipos de fibra óptica, obteniendo sus diferencias y estudiando cuál de todas ellas presenta un mejor comportamiento para su empleo como sensores distribuidos.

Los resultados obtenidos muestran que los sensores distribuidos propuestos presentan una respuesta lineal con la carga aplicada, con la deformación y con la temperatura. Esta respuesta lineal presenta un elevado grado de ajuste y de esta forma, ha sido posible la calibración de los mismos y la obtención de las temperaturas y deformaciones en los ensayos realizados. 



\subsection{Introducción.}

En los Capítulos anteriores se han estudiado los diseños y las aplicaciones de dos tipos sensores ópticos para la instrumentación de estructuras. Ambas tipologías de sensores, puntuales y de longitud, responden a las necesidades que plantea cada enfoque de monitorización y sus aplicaciones han ofrecido resultados satisfactorios. Tal y como se citó en los correspondientes apartados, el principio de funcionamiento de estos sensores está basado en FBG's.

Sin embargo existen determinadas aplicaciones, tipologías estructurales y objetivos de monitorización donde el empleo de sensores puntuales o de longitud no es adecuado. Un claro ejemplo de esta situación consiste en la monitorización de elementos estructurales lineales de grandes longitudes: Una tubería, el tablero de un puente de gran luz, el cable portante de un puente colgante...

Particularicemos el ejemplo anterior al caso de un oleoducto. Imaginemos que se pretende monitorizar un tramo de 5 kilómetros de longitud de un oleoducto para conocer el valor de las deformaciones y temperaturas a las que está sometida la estructura y detectar posibles patologías que interrumpieran su correcto funcionamiento: Fisuras, pérdida de material transportado, calentamiento excesivo en un punto concreto...

El planteamiento de esta monitorización en base a sensores puntuales resultaría inabordable. Si se instalase un sensor puntual cada 5 metros, sería necesaria la instalación de 1000 sensores puntuales. Ello implicaría el empleo de 1000 máscaras de fase para la instalación de los sensores en serie y no repetir ninguna longitud de onda, además del empleo de equipos capaces de suministrar muy elevadas potencias ópticas para atravesar 1000 FBG's y que fuera posible la identificación de todos los sensores. Este planteamiento resultaría realmente inviable.

De una forma similar, una monitorización en base a sensores de longitud presentaría los mismos inconvenientes. En este caso, una mayor longitud de la estructura estaría monitorizada pero tampoco se solucionaría la problemática planteada.

Además con ambas estrategias no se cubriría la totalidad de la estructura, es decir, existirían zonas sin monitorizar. Por un lado, en caso del empleo de sensores puntuales sería prácticamente imposible detectar un daño, puesto que ello implicaría que éste debería producirse justo en el punto donde se encuentra el sensor. En el caso del empleo de sensores de longitud, si el estímulo (variación de temperatura o deformación) aparece en una zona no instrumentada no sería posible su detección. En cambio si el estímulo se da en el interior de la longitud del sensor, éste indicaría una deformación media mayor a la normal entre los dos puntos que delimitan su longitud. En este caso sería muy difícil la detección del daño durante su iniciación, y éste sólo sería apreciable en un estado avanzado de daño.

En cualquier caso, ambos tipos de sensores no presentan facilidades para su aplicación y no cubren las necesidades que se plantea en este ejemplo concreto.

Los sensores distribuidos basados en la dispersión de Brillouin ofrecen alternativas a este tipo de problemas. Como indica su nombre, se trata de un sensor continuo a través de la fibra. Esto significa que a lo largo del cable óptico empleado en la monitorización se dispondrá de tantos puntos de medida como permita la tecnología utilizada (actualmente se han conseguido resoluciones de centímetros). De esta forma y volviendo al ejemplo planteado del oleoducto, sobre éste se podría instalar 5 kilómetros de fibra óptica y tener miles de puntos de medida a lo largo de la misma, con la única limitación de la resolución antes mencionada. 
Monitorización estructural.

Aplicación con sensores de fibra óptica.

Con esta estrategia se da solución a la problemática planteada. En este caso, una patología o daño sería detectada y además se conocería el punto en el que se ha producido. Este aspecto es el que hace que los sensores distribuidos sean los más ideales para este tipo de aplicaciones.

\subsection{Principio de funcionamiento de sensores distribuidos mediante la dispersión estimulada de Brillouin (Stimulated Brillouin Scattering, SBS).}

El SBS (en inglés, Stimulated Brillouin Scattering) es un proceso no lineal que ocurre en la fibra cuando se inyecta en ella una potencia superior a una potencia umbral (que recibe el nombre de señal pump). Este hecho hace que una pequeña parte de la luz inyectada se refleje en cada punto de la fibra, lo que se conoce con el nombre de back-scattering y supone una difusión de la luz en sentido contra propagante de la potencia inyectada.

Debido a la poca potencia de este back-scattering, existen métodos que amplifican la señal reflejada. Esto se consigue a partir de la inyección desde el extremo opuesto de la fibra de otra señal, justo con la misma frecuencia (frecuencia Brillouin), que amplifique a la reflejada. Esta señal amplificada recibe el nombre de ganancia de Brillouin (Figura 7.1) y por este motivo, esta técnica recibe el nombre de "estimulada".

Este back-scattering de la luz presenta tres componentes que pueden ser identificadas y a través del estudio de sus variaciones puede obtenerse gran cantidad de información (Figura 7.1):

- Scattering Rayleigh. Este scattering se debe a imperfecciones macroscópicas. El scattering reflejado se detecta a la misma longitud de onda y frecuencia que la luz emitida.

- Scattering Brillouin. Es un scattering de menor intensidad que el anterior. Este fenómeno sirve para la medición de pequeñas fluctuaciones a escala microscópica como variaciones de deformación y temperatura. Este scattering se encuentra desplazado en frecuencia un valor aproximado de $11.1 \mathrm{GHz}$.

- Scattering Raman. Se trata de un fenómeno mucho menos probable y de mucha menor intensidad. Es capaz de detectar variaciones en la vibración de las moléculas del material, por lo que su uso se extiende a la identificación de materiales. Este scattering se encuentra desplazado en frecuencia un valor aproximado de $20 \mathrm{GHz}$. 


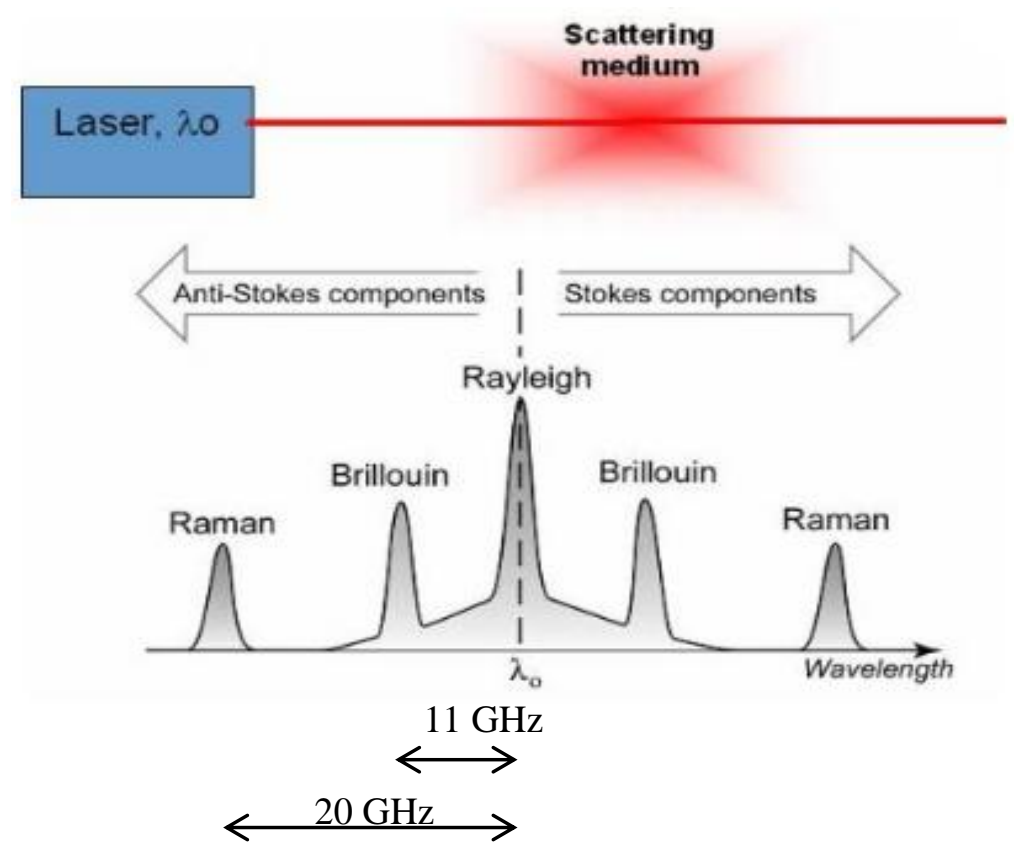

Figura 7.1 Back-scattering de la luz (Página web de la compañía FOS\&S).

En principio, se podría pensar que el SBS es un efecto perjudicial para transmitir por la fibra óptica y en efecto, en muchas aplicaciones es un efecto a mitigar para mejorar las prestaciones de la misma. Sin embargo es posible estimar la variación del SBS ante ciertos estímulos y calibrar esta variación para correlacionarla con algún parámetro estructural como son la deformación y la temperatura.

Esta correlación se realiza a partir de los desplazamientos de la frecuencia de Brillouin. Dado que ésta depende del índice de refracción y de la velocidad de las ondas acústicas en el interior de la fibra, ésta varía siempre que estos parámetros se vean afectados y por tanto, esta técnica puede ser usada para obtener la temperatura y la deformación a lo largo de la fibra óptica.

Para la obtención de las medidas se debe monitorizar la zona de ganancia antes mencionada, barriendo en sentido contra propagante a la señal pump inyectada con otra señal que abarque un ancho de banda suficiente. Esto se obtiene inyectando una señal separada del pump original $11 \mathrm{GHz}$. De esta forma, se obtiene una señal que corresponde con un pico separado $11 \mathrm{GHz}$ de la señal pump original.

Este pico separado $11 \mathrm{GHz}$ de la señal inyectada es el que experimenta variaciones en su frecuencia, llamada Frecuencia de Brillouin, de forma que frente a aumentos de temperatura la frecuencia de Brillouin también aumenta y viceversa. De una forma similar, la frecuencia de Brillouin aumenta en caso de tracciones en la fibra y viceversa.

Otro aspecto interesante que ofrece el sensado distribuido de Brillouin es el hecho de conocer en qué punto se sitúa la variación de frecuencia de Brillouin, y por tanto la variación del parámetro estructural estudiado. De esta forma se puede conocer la posición exacta donde se sitúa la variación.

En la Figura 7.2 se muestra el concepto de un análisis tridimensional de sensado distribuido de Brillouin. En los ejes se representa la variación de la frecuencia de Brillouin, la distancia de la fibra y la amplitud de la señal. 


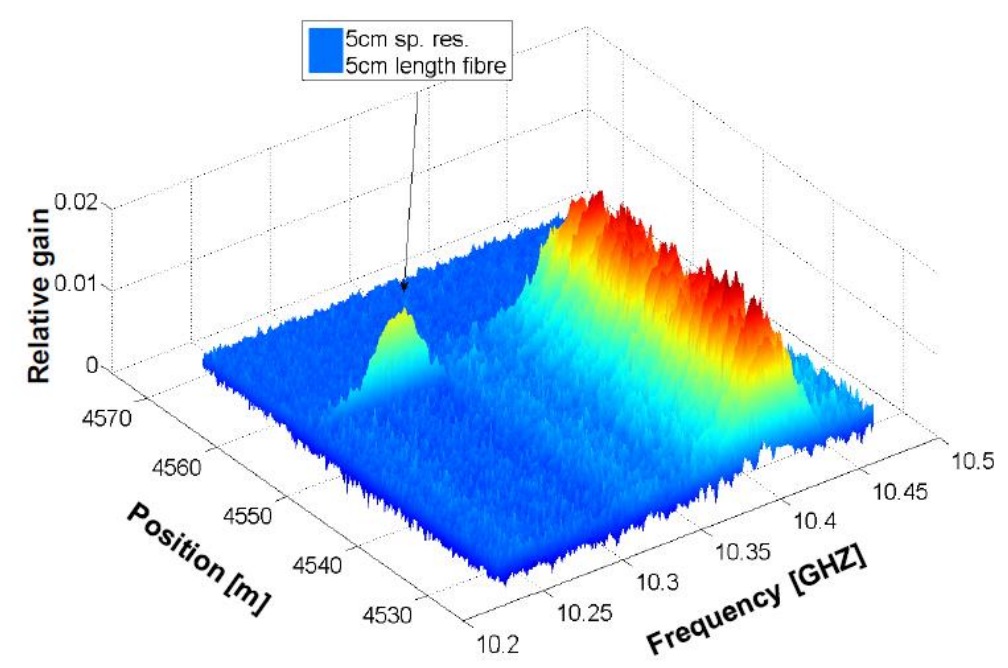

Figura 7.2 Análisis tridimensional basado en el Scattering de Brillouin (Luc et al. 2010).

En la Figura 7.2 se observa, aproximadamente a los 4560 metros de fibra óptica, un desplazamiento de la frecuencia de Brillouin. Como en este punto se ha producido una disminución de frecuencia, esto puede ser debido a un decremento de temperatura que sufre el material sobre el que está instalada la fibra o a deformaciones de compresión. De esta forma se identifica claramente el punto donde se produce la variación.

\subsection{Ventajas e inconvenientes del sensado distribuido.}

Como se ha citado en el apartado 7.1, los sensores distribuidos basados en el scattering de Brillouin son muy apropiados para algunas aplicaciones. Sin embargo, su naturaleza y principio de funcionamiento los hace inviables para otras. En general, el sensado distribuido presenta las siguientes particularidades:

- Tal y como se ha expuesto en el apartado 7.1, el sensado distribuido proporciona valores del parámetro a medir (deformación o temperatura) a lo largo de toda la longitud de la fibra óptica. Esto representa una clara ventaja respecto a las FBG's en determinados escenarios.

- La fibra óptica necesaria para llevar a cabo las mediciones es fibra óptica de comunicación estándar, sin necesidad de realizar ningún tratamiento. En el caso de las FGB's es necesario escribir sobre el núcleo de la fibra óptica una red de difracción, tal y como se explicó en el Capítulo 2. En este aspecto, el sensado distribuido presenta una clara ventaja económica con respecto a otras técnicas.

- Esta tecnología tiene muy bajas pérdidas por lo que su rendimiento máximo se alcanza en monitorizaciones de grandes distancias, del orden de kilómetros. De este modo, los sensores basados en SBS son particularmente atractivos para aplicaciones en las que se requieran grandes longitudes.

Además, el sensado distribuido de Brillouin presenta estos otros aspectos a tener en cuenta: 
- El sensado distribuido exige que la fibra óptica se encuentre totalmente adherida a la superficie de la estructura a monitorizar. Este proceso de instalación es más complejo que el de las FBG's, cuya adhesión es puntual.

- Esta técnica tiene una menor precisión que las FBG's. Sin embargo, en el campo de aplicación del sensado distribuido la precisión no juega un papel fundamental. Esa técnica alcanza su máximo rendimiento en la instrumentación de grandes distancias en las que sus resultados no exigen altas precisiones. A pesar de ello, es importante destacar que actualmente se han desarrollado técnicas que han conseguido alcanzar resoluciones del orden de $\mathrm{mm}$ con la técnica llamada "anti-pulsos" (Brown y Colpitts, 2007).

- Con los sensores distribuidos de Brillouin se tiene que trabajar en transmisión, no en reflexión como en el caso de los sensores FBG's, ya que es imprescindible inyectar potencia a la fibra por ambos extremos para que se pueda conseguir la ganancia de Brillouin, como se ha explicado en el apartado 8.2. Esto implica que en caso de rotura de la fibra no es posible seguir con la medida. En el caso de una monitorización en serie con FBG's, si se rompe la fibra en algún tramo siempre se podrá seguir monitorizando el tramo situado entre el interrogador y el punto de rotura.

- La unidad lectora del sensado distribuido es más compleja y de mayores dimensiones que la necesaria para la toma de datos con sensores FBG's, tal y como muestra la Figura 7.3. Sin embargo, actualmente existen investigadores como el Grupo de Comunicaciones Ópticas y Aplicaciones Electrónicas de la Universidad Pública de Navarra (UPNA) que están trabajando en el desarrollo de unidades lectoras de dimensiones más reducidas, más compactas y más económicas.

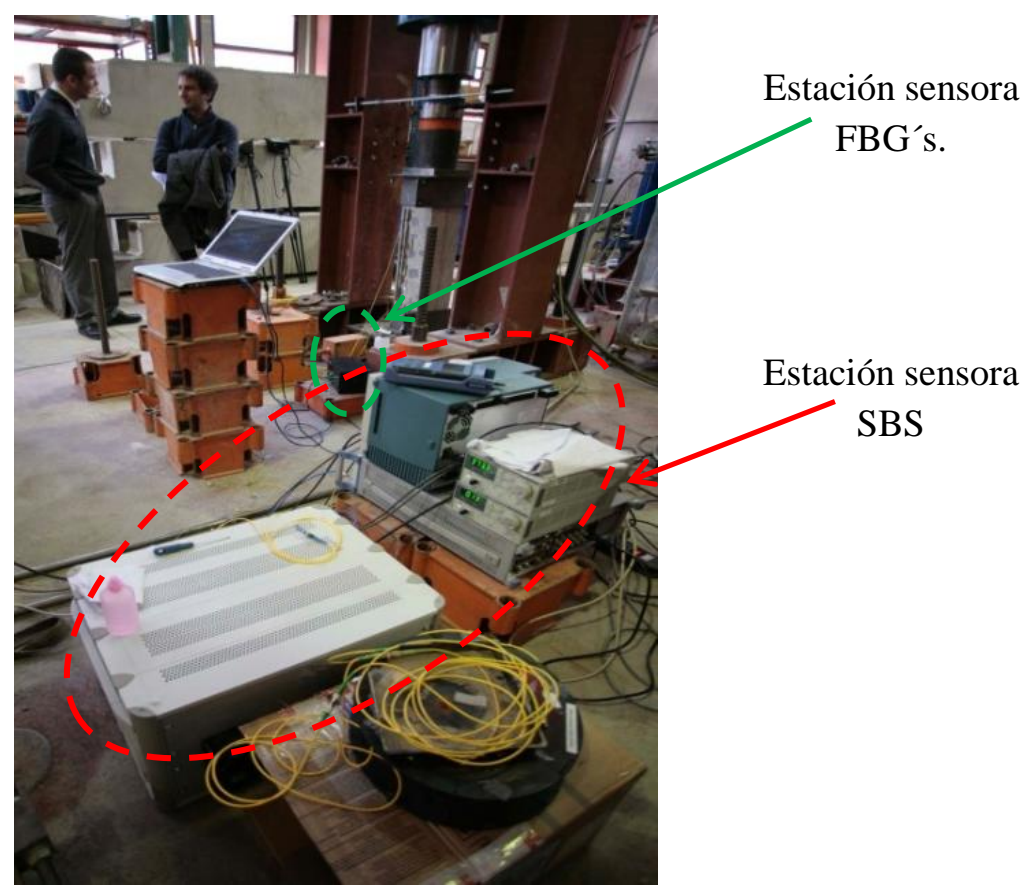

Figura 7.3 Imagen del ensayo. Unidades de lectura de los sensores basados en FBG's y sensores distribuidos. 
- Una de las grandes limitaciones comerciales que tienen los sistemas experimentales que se usan hoy en día es su complejidad y coste. Se deben controlar determinados parámetros que hacen al sistema más complejo. Además, son necesarios una gran cantidad de dispositivos de instrumentación y diversos aparatos de elevado coste. Esto lo hace poco atractivo desde el punto de vista comercial.

\subsubsection{Distancias de resolución del sensado distribuido de Brillouin.}

Como se ha explicado en los apartados anteriores, el sensado distribuido ofrece una medición continua del parámetro estudiado a lo largo de la longitud de la fibra óptica. Esta información se proporciona como datos en cualquier punto de la fibra. Estos datos son el resultado de la integración de las medidas en una determinada longitud de fibra óptica, llamada distancia de resolución.

La distancia de resolución $d_{B}$ se obtiene en función de la duración del pulso óptico emitido por el equipo de interrogación. Si por ejemplo se emite una señal óptica de duración $t_{B} 1$ milisegundo, teniendo en cuenta que la luz viaja a una velocidad $c$ de $310^{8} \mathrm{~m} / \mathrm{s}$ y que su índice de refracción n es aproximadamente 1.4, la distancia de resolución del sistema sería (ecuación 7.1):

$d_{B}=\frac{c t_{B}}{2 n}=\frac{310^{8} \mathrm{~m} / \mathrm{s} \times 110^{-3} \mathrm{~s}}{2 \times 1.4}=107000 \mathrm{~m}=107 \mathrm{~km}$

Esta distancia de resolución es demasiado grande no sólo para el ejemplo del oleoducto planteado en el apartado 7.1, sino para cualquier aplicación.

Si se disminuyera la duración de la señal óptica, por ejemplo a 1 nanosegundo, la nueva distancia de resolución sería de $10 \mathrm{~cm}$. Esto significa que en cada punto estudiado, el dato obtenido sería la integración de los valores en una distancia de $10 \mathrm{~cm}$. Teniendo en cuenta la tipología instrumentada, la tecnología empleada y los objetivos que impulsan a desarrollar una instrumentación de estas características, resulta evidente que la precisión de las medidas no es un aspecto de gran importancia.

En las campañas experimentales que engloban este capítulo, se ha empleado un pulso óptico de duración 10 nanosegundos, y a partir de la ecuación anterior la distancia de resolución ha sido de aproximadamente 1 metro.

\subsection{Calibración del sensado distribuido frente a temperaturas.}

El empleo de sensores distribuidos exige previamente su calibración, es decir, la obtención de un coeficiente que permita obtener el parámetro estructural estudiado a partir de las variaciones de la frecuencia de Brillouin obtenidas.

En este apartado se presentan los trabajos de calibración de los sensores distribuidos frente a temperaturas. El procedimiento para su calibración ha sido análogo al explicado en el Capítulo 4. La calibración se ha llevado a cabo a partir de campañas experimentales, sometiendo un tramo de fibra óptica a incrementos de temperatura y monitorizando la zona de ganancia de Brillouin con un analizador de redes (Figura 7.4.a). Los incrementos y decrementos de temperatura fueron aplicados con la cámara climática descrita en el Capítulo 4. Los tramos de fibra estudiados fueron de distintas longitudes, concretamente de $20 \mathrm{~km}, 2 \mathrm{~km}$ y $1.5 \mathrm{~km}$ (Figura 7.4.b). Es importante destacar la 
fragilidad y delicadeza del montaje y del ensayo. Las elevadas potencias de las señales empleadas exigían una limpieza muy escrupulosa de los elementos que intervinieron en el ensayo.

Los ensayos fueron realizados por personal del Instituto de Telecomunicaciones y Aplicaciones Multimedia (iTEAM) de la Universidad Politécnica de Valencia.

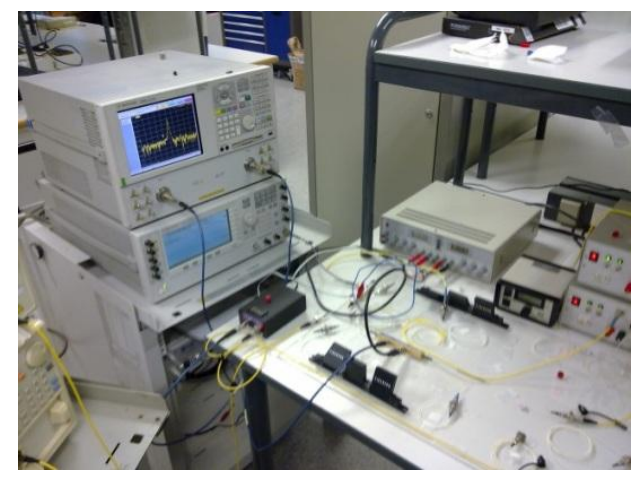

(a)

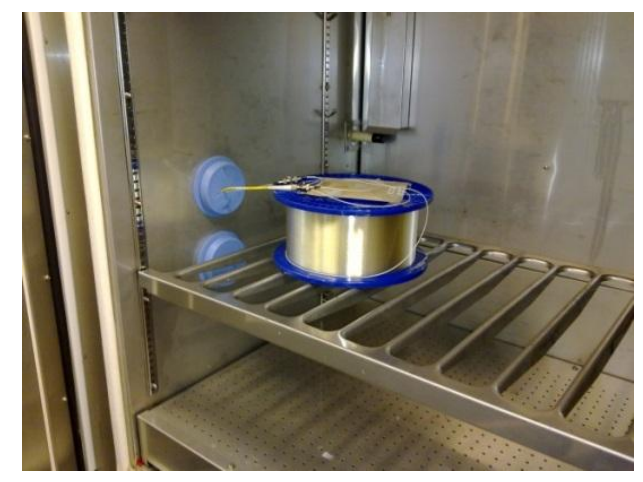

(b)

Figura 7.4 (a)-Montaje para la evaluación del SBS. En la pantalla del analizador de redes aparece el pico de la zona de ganancia de Brillouin. (b)- Tramo de $20 \mathrm{~km}$ de fibra óptica en la cámara climática.

\subsubsection{Tramo de fibra de $20 \mathrm{~km}$.}

Para la calibración frente a temperaturas se programó un ciclo de temperaturas como el indicado en la Figura 7.5. La temperatura inicial fue de $-20{ }^{\circ} \mathrm{C}$, incrementándose en $10^{\circ} \mathrm{C}$ hasta alcanzar $80^{\circ} \mathrm{C}$. Cada incremento se mantuvo constante 1 hora.

En la Figura 7.5 se muestra el ciclo térmico propuesto y las lecturas de la frecuencia de Brillouin.

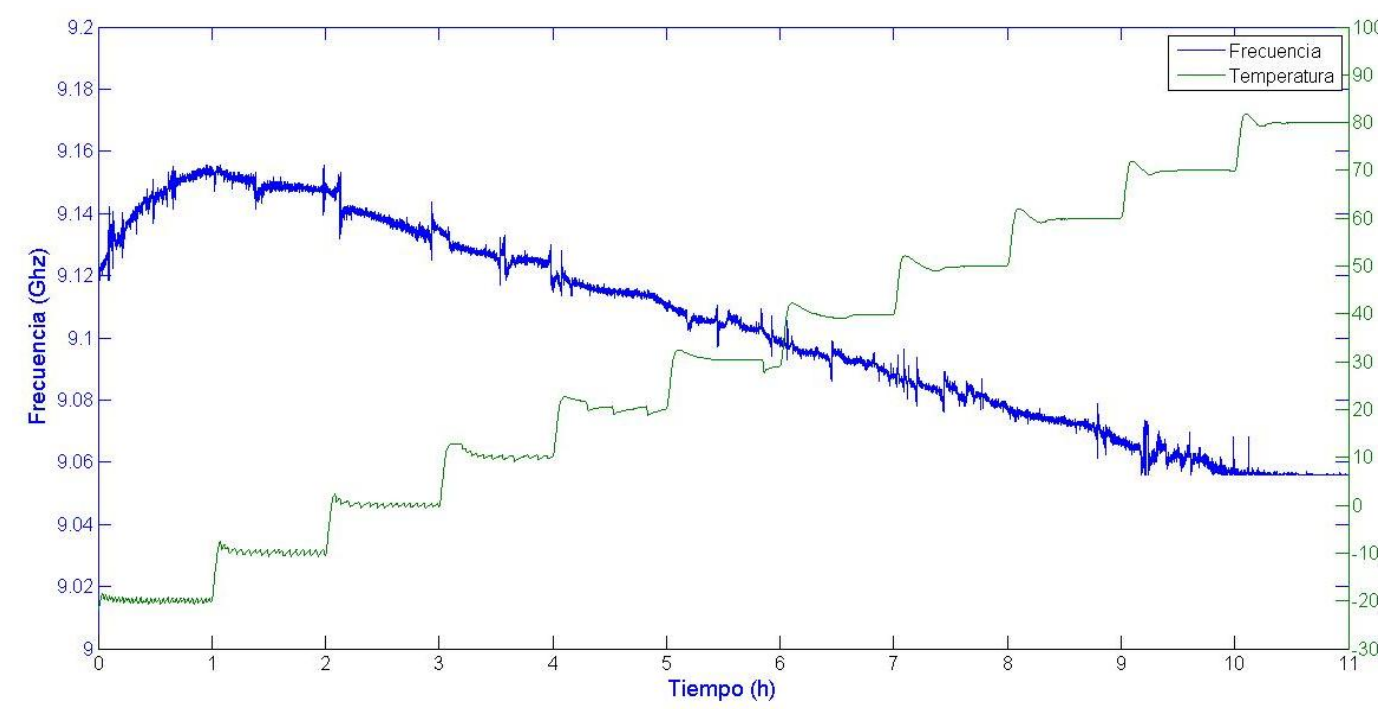

Figura 7.5 Frecuencia de Brillouin y temperaturas frente al tiempo. Tramo de fibra de $20 \mathrm{~km}$.

De los resultados mostrados en la Figura 7.5 anterior se deduce que a pesar de que gráficamente se observa una correlación directa entre frecuencia de Brillouin y temperatura, no se obtiene una estabilización en la frecuencia de Brillouin en cada intervalo de temperatura constante. Esto es debido a que para un carrete de $20 \mathrm{~km}, 1$ hora de exposición a temperatura constante es insuficiente para que 
Monitorización estructural.

Aplicación con sensores de fibra óptica.

todo el tramo de fibra llegue a las mismas condiciones. Como se observará en los siguientes apartados, esto no sucede con tramos de fibra de menor longitud.

Si de los anteriores valores de frecuencia de Brillouin se adoptan los últimos de cada tramo horizontal y se representan frente a la temperatura impuesta se obtiene una relación lineal (Figura 7.6). De esta relación, el coeficiente de sensibilidad obtenido es de $1.09 \mathrm{MHz} /{ }^{\circ} \mathrm{C}$.

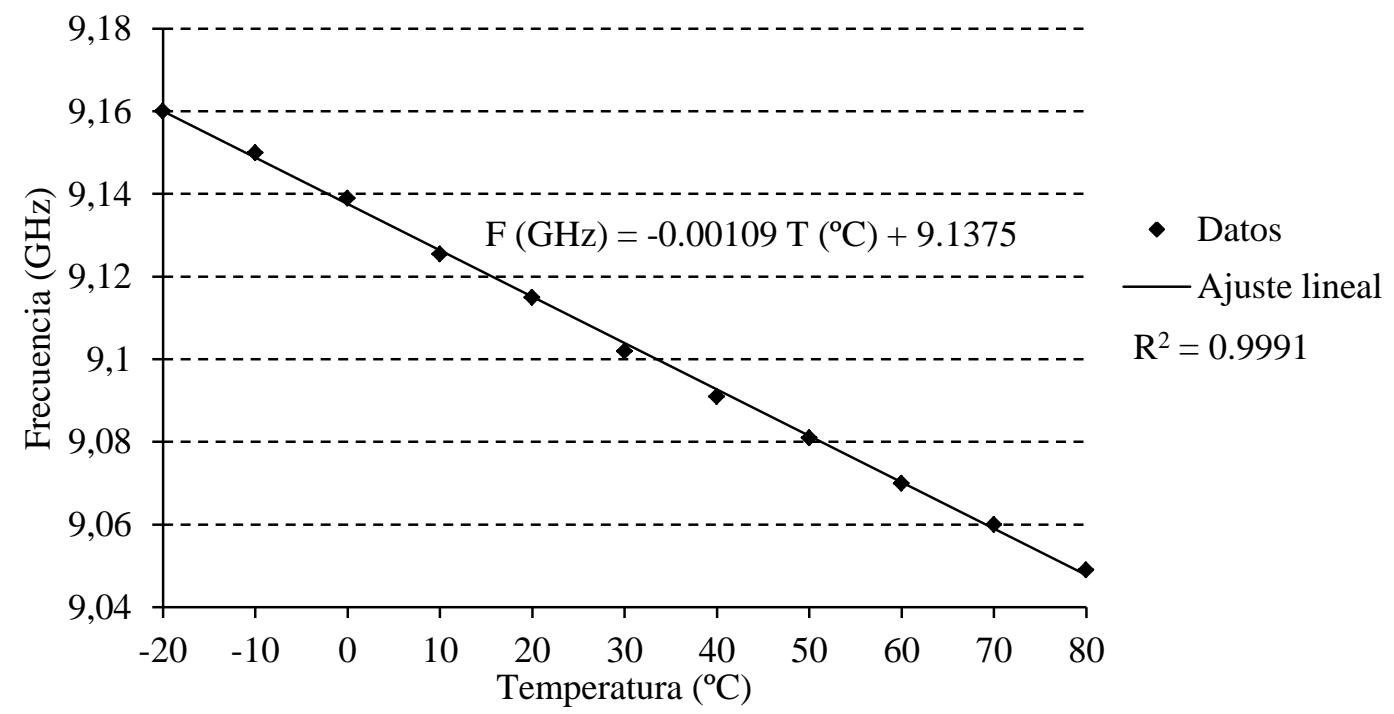

Figura 7.6 Frecuencia de Brillouin frente a temperatura impuesta. Tramo de fibra de $20 \mathrm{~km}$.

\subsubsection{Tramo de fibra de $1.5 \mathrm{~km}$.}

Los nuevos ensayos experimentales englobaron ciclos térmicos como el indicado en la Figura 7.7. La temperatura inicial fue de $-20{ }^{\circ} \mathrm{C}$ y la final de $40^{\circ} \mathrm{C}$, incrementándose en valores de $10^{\circ} \mathrm{C}$ y manteniéndose constante durante 2 horas (el doble que en el ensayo anterior). Además, el tramo de fibra de menor longitud mejoró la respuesta y por tanto los datos obtenidos.

De esta forma la frecuencia de Brillouin obtenida sí que se estabilizó en cada uno de los intervalos, identificando claramente cada valor donde ésta permanecía constante (Figura 7.7).

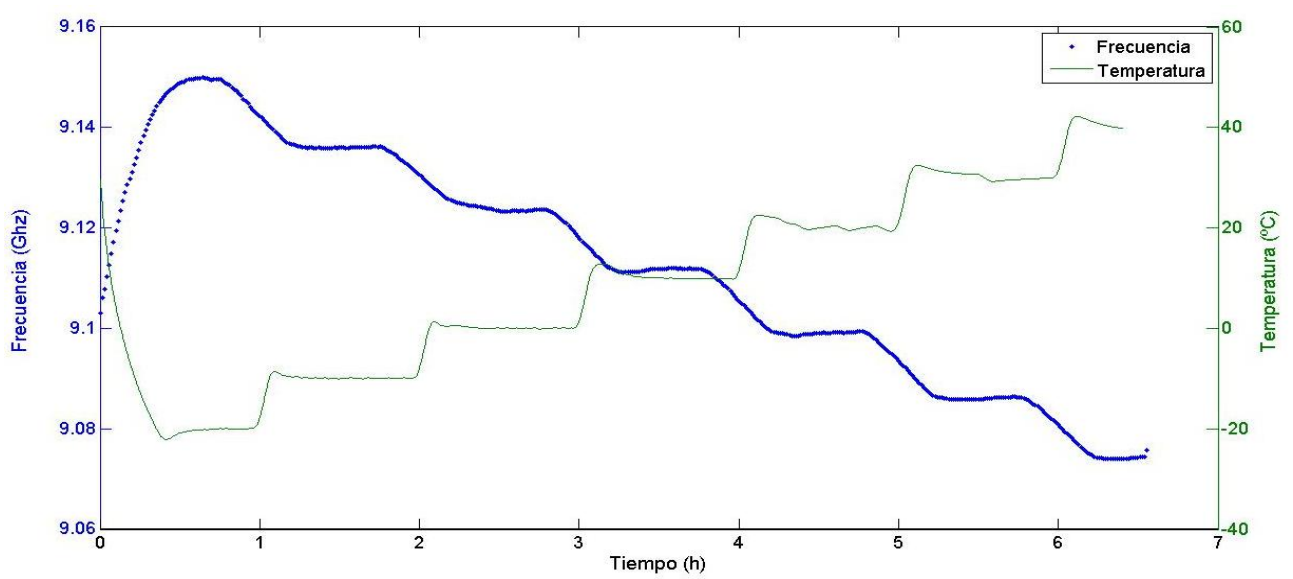

Figura 7.7 Frecuencia de Brillouin y temperaturas frente al tiempo. Tramo de fibra de $1.5 \mathrm{~km}$. 
Procediendo de una forma similar al apartado 7.4.1, se ha representado el valor de la frecuencia de Brillouin de cada intervalo frente a la temperatura impuesta, resultando ser de nuevo una relación lineal (Figura 7.8). El coeficiente de ajuste obtenido es de $1.256 \mathrm{MHz} /{ }^{\circ} \mathrm{C}$

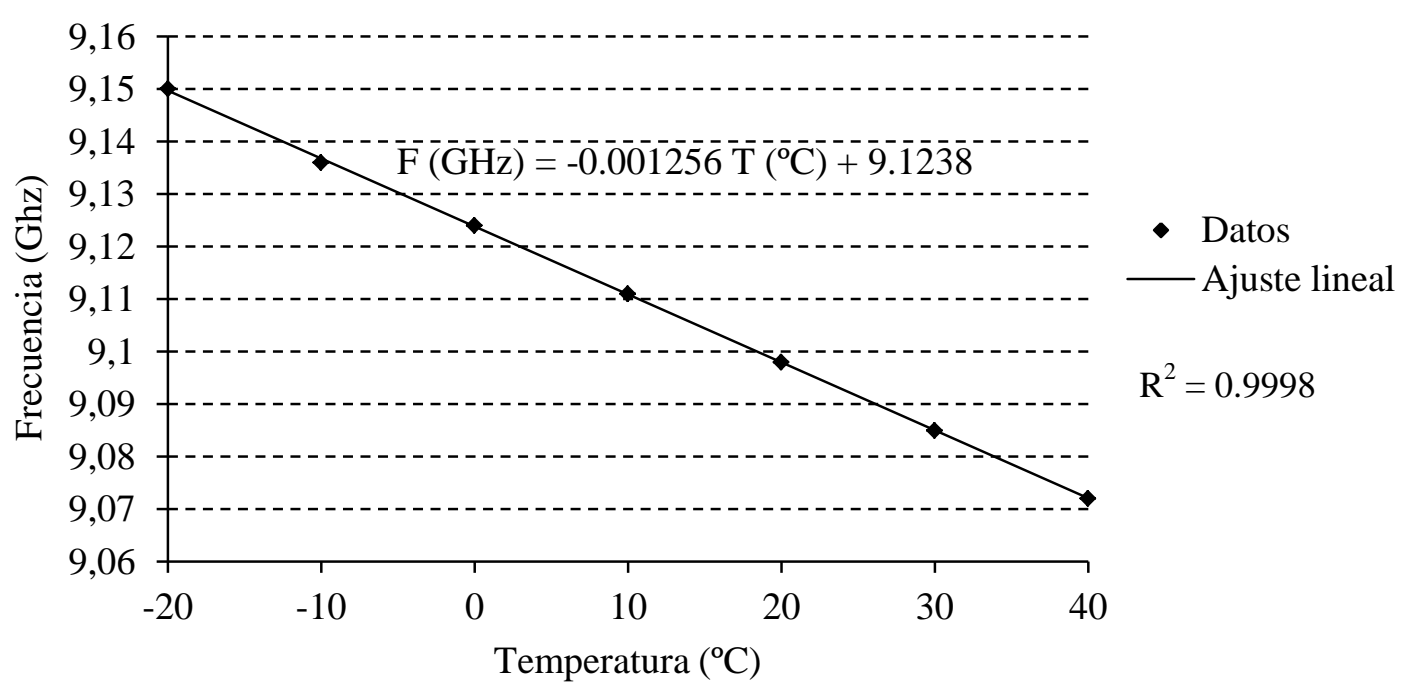

Figura 7.8 Frecuencia de Brillouin frente a temperatura impuesta. Tramo de fibra de $1.5 \mathrm{~km}$.

\subsubsection{Tramo de fibra de $2 \mathrm{~km}$.}

En esta tercera campaña de ensayos experimentales se propuso un ciclo térmico como el de la Figura 7.9. La temperatura inicial fue de $-20^{\circ} \mathrm{C}$, aumentando en incrementos de $10^{\circ} \mathrm{C}$ y de 30 minutos de duración hasta alcanzar $80{ }^{\circ} \mathrm{C}$, permaneciendo constante a esta temperatura durante 1 hora. Posteriormente, se programó el ciclo inverso. De esta forma se pretendió evaluar la posible histéresis asociada a la medida.

Las Figuras 7.9, 7.10.a y 7.10.b muestran los resultados del ensayo. En ellos se observa un peor ajuste que en los anteriores apartados debido a que la duración de cada intervalo propuesto es menor (30 minutos) y con ello, una peor estabilización de las medidas. No obstante el grado de ajuste sigue siendo muy elevado.

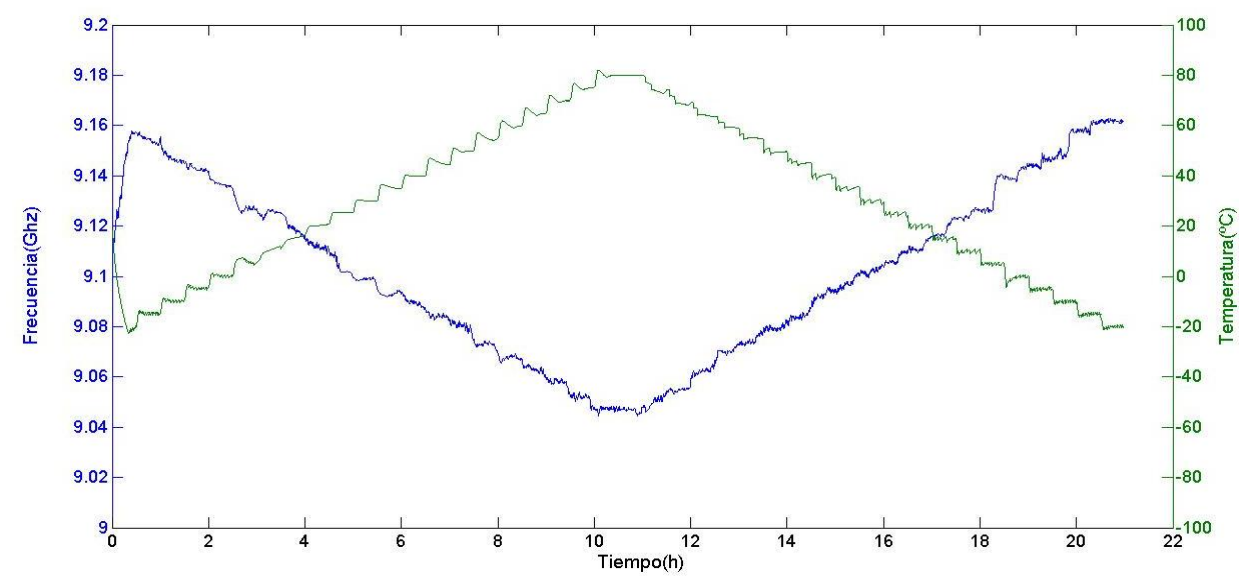

Figura 7.9 Frecuencia de Brillouin y temperaturas frente al tiempo. Tramo de fibra de $2 \mathrm{~km}$. 


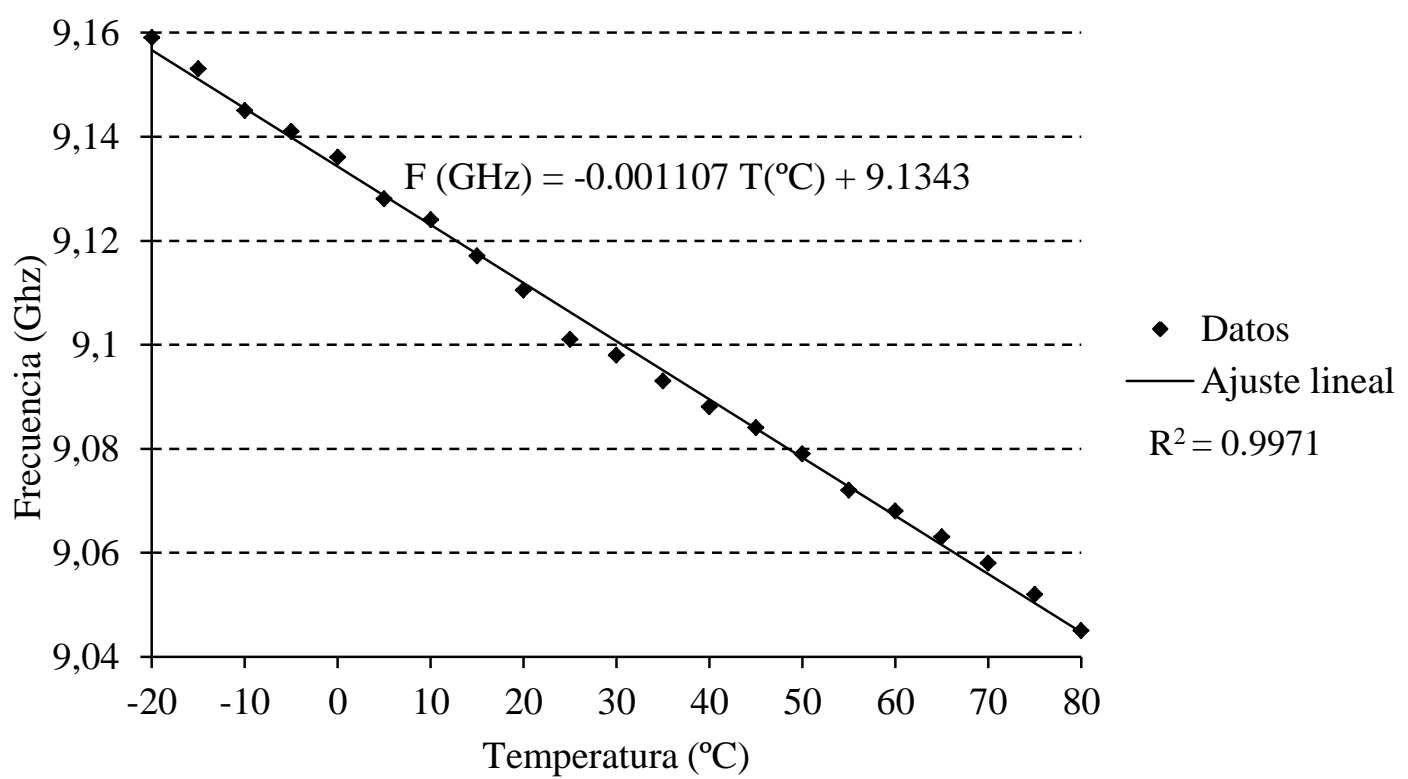

(a)

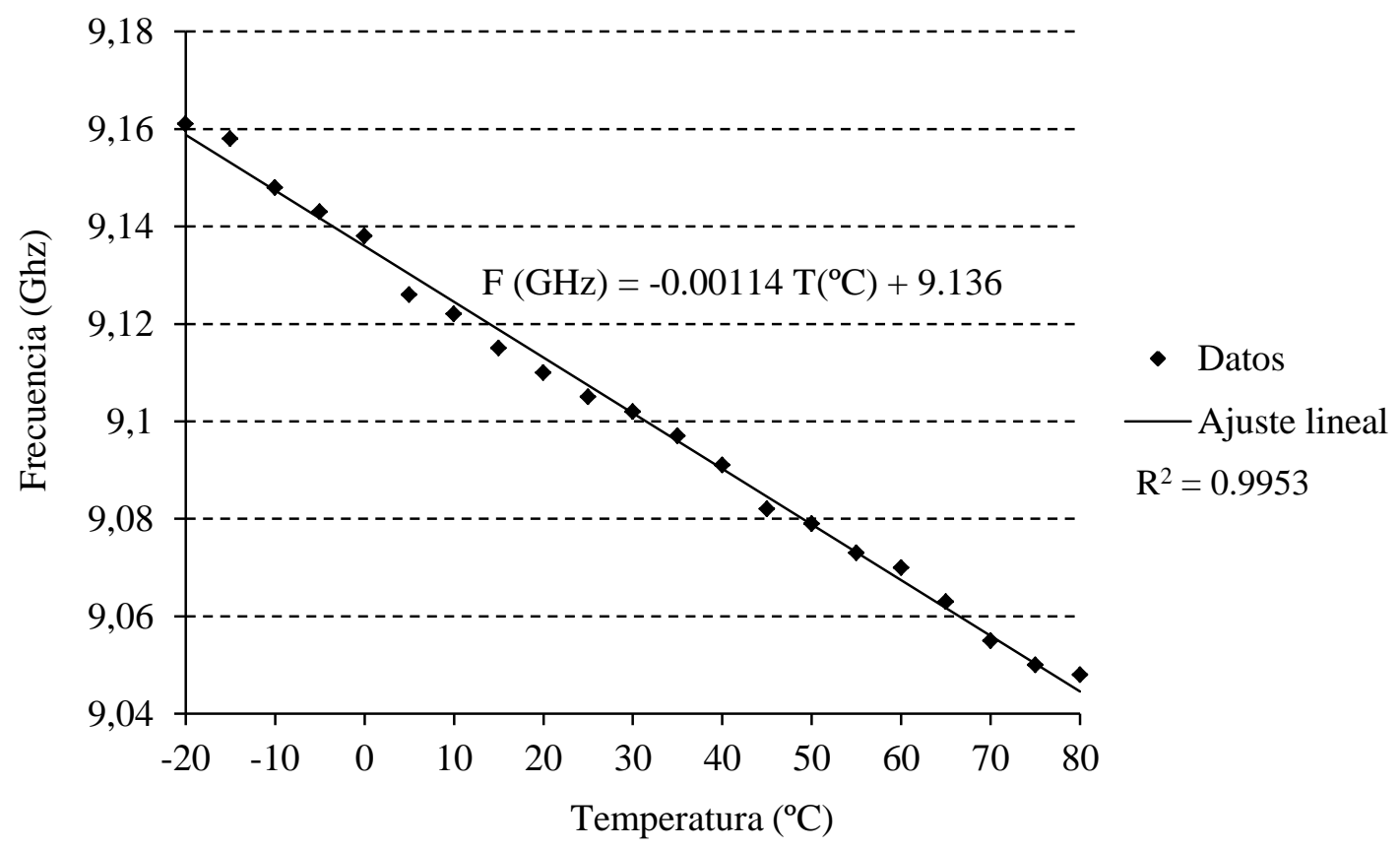

(b)

Figura 7.10 Frecuencia de Brillouin frente a temperatura impuesta. Tramo de fibra de $1.5 \mathrm{~km}$.

(a)- Rama ascendiente de temperatura. (b)- Rama descendiente de temperatura

Los coeficientes de sensibilidad obtenidos para la rama ascendente y descendente de temperatura son $1.107 \mathrm{y} 1.14 \mathrm{MHz} /{ }_{\mathrm{o}} \mathrm{C}$, respectivamente. Ambos coeficientes permiten afirmar que no se presentan problemas de histéresis. 


\subsubsection{Sensibilidades obtenidas.}

A la vista de los resultados anteriores, se observan pequeñas variaciones sobre el coeficiente de sensibilidad obtenido en cada uno de los ensayos (Tabla 7.1). Sin embargo se ha identificado el origen de estas variaciones y son debidas a los ciclos propuestos y a las longitudes de los tramos ensayados.

\begin{tabular}{ccc}
\hline Tramo de fibra óptica & Sensibilidad $\mathbf{M H z}^{\circ}{ }^{\mathrm{C}}$ & Coeficiente $\boldsymbol{R}^{2}$ \\
\hline $20 \mathrm{~km}$ & 1.09 & 0.999 \\
$1.5 \mathrm{~km}$ & 1.256 & 0.9998 \\
$2 \mathrm{~km}(R d, R a)$ & $1.14,1.107$ & $0.9971,0.9953$ \\
\hline
\end{tabular}

Tabla 7.1 Coeficientes de sensibilidad obtenidos en los ensayos y coeficientes $\mathrm{R}^{2}$. (Rd-Rama descendente, $R a$-Rama ascendente)

Para futuras aplicaciones (Capítulo 9) se adoptará el valor del coeficiente de sensibilidad correspondiente al tramo de fibra de $1.5 \mathrm{~km}$ y de valor $1.256 \mathrm{MHz} /{ }^{\circ} \mathrm{C}$. El motivo es que en este ensayo los ciclos propuestos son de mayor duración y la longitud del tramo ensayado es menor, por lo que las medidas obtenidas han sido más estables. Por otro lado, los resultados obtenidos en el tercer ensayo planteado han permitido observar que no ha habido posibles histéresis asociadas a los ciclos térmicos propuestos.

\subsection{Calibración del sensado distribuido frente a deformaciones.}

De una forma similar al apartado 7.4 se ha llevado a cabo la calibración del sensado distribuido de Brillouin para la medición de deformaciones en estructuras. El procedimiento adoptado consiste en la instalación de tramos de fibra óptica sobre un elemento de hormigón. Este elemento de hormigón es sometido a carga y durante el ensayo se monitoriza la variación de la frecuencia de Brillouin que experimenta la fibra óptica. La relación entre este parámetro y la deformación del elemento proporciona el coeficiente de sensibilidad.

Estos trabajos se han realizado conjuntamente con el Grupo de Comunicaciones Ópticas y Aplicaciones Electrónicas del Departamento de Ingeniería Eléctrica y Electrónica de la Universidad Pública de Navarra (UPNA).

Los doctores D. Alayn Loayssa, D. Mikel Sagues y D. Ander Zornoza se trasladaron hasta el laboratorio del Instituto y Ciencia de Tecnología del Hormigón (ICITECH) de la Universidad Politécnica de Valencia (UPV) con el equipo de adquisición de datos perteneciente al Grupo de Comunicaciones Ópticas y Aplicaciones Electrónicas de la Universidad Pública de Navarra. Este equipo recibe el nombre de "Estación Sensora SBS". Esta estación emplea la dispersión estimulada de Brillouin para obtener una medida distribuida de temperatura y/o deformación a lo largo de una fibra óptica. Concretamente, el parámetro de la fibra que se emplea para la calibración de la temperatura o deformación es el desplazamiento de la frecuencia de Brillouin, tal y como se explicó en el apartado 
Monitorización estructural.

Aplicación con sensores de fibra óptica.

7.2. La variación de este parámetro con la temperatura o deformación debe ser calibrado para cada tipo de fibra.

\subsubsection{Datos de las probetas empleadas en los ensayos y aplicación de la carga.}

Para la realización de los ensayos se fabricó un total de tres probetas prismáticas de hormigón, de base cuadrada de 0.2 metros de lado y 1 metro de altura.

Estas probetas fueron instrumentadas con sensores ópticos puntuales desarrollados en el Capítulo 4, con sensores ópticos de longitud desarrollados en el Capítulo 6 y con sensores distribuidos objeto de estudio en este Capítulo.

La probeta de hormigón fue sometida a cargas de compresión. La carga fue incrementándose progresivamente en incrementos de entre 10 y 15 toneladas hasta producir la rotura del hormigón. Cada incremento de carga permanecía constante durante 5 minutos, tiempo durante el cual se llevaron a cabo las mediciones del sensado distribuido (Figura 7.11). Las mediciones con los sensores puntuales y de longitud se realizaron de forma ininterrumpida en el tiempo. Posteriormente, se llevó a cabo la calibración y comparación del sensado distribuido (a partir de la medición de las variaciones en la frecuencia de Brillouin) en base a los resultados obtenidos por las otras tipologías de sensores.

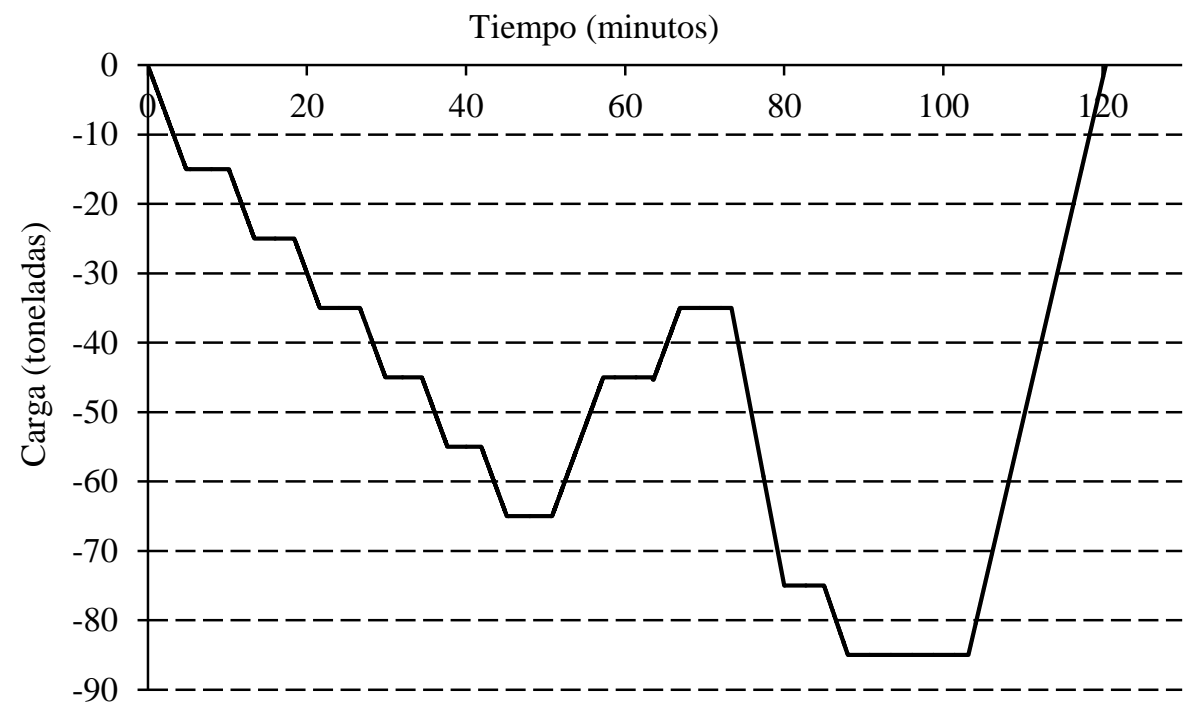

Figura 7.11 Ciclo de carga y descarga propuesto sobre la probeta de hormigón.

En las probetas propuestas ha sido necesario un refuerzo en las cabezas (zona de aplicación de la carga) para evitar la rotura de estas zonas por compresión localizada. Los refuerzos se han planteado en base a un encamisado metálico que confina al hormigón en esa zona (Figura 7.12). Con los refuerzos sobre la probeta, la distancia libre de hormigón resultó ser de $70 \mathrm{~cm}$. Los tres tipos de fibras ópticas han sido adheridas a lo largo de esta longitud. 


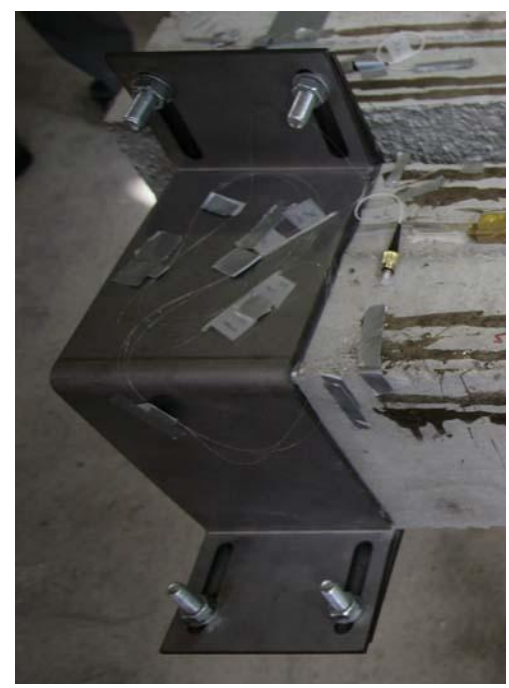

Figura 7.12 Imagen del refuerzo propuesto en las cabezas de las probetas.

La resistencia a compresión del hormigón de las probetas se determinó con ensayos de compresión simple sobre probetas normalizadas de $0.15 \mathrm{~m}$ de diámetro y $0.3 \mathrm{~m}$ de altura (UNE, 1996). Un total de 6 probetas cilíndricas fueron ensayadas, ofreciendo una resistencia a compresión de $39 \mathrm{MPa}$ a los 28 días. Estas mismas probetas cilíndricas dieron una resistencia a compresión de $28 \mathrm{MPa}$ a la edad en que se realizaron los ensayos, que fue a los 9 días. El límite elástico del acero de las armaduras fue $f_{y k}=500 \mathrm{MPa}$ y el del encamisado metálico de refuerzo de las cabezas $f_{y r}=265 \mathrm{MPa}$.

\subsubsection{Instrumentación de las probetas.}

Para la evaluación de los sensores distribuidos se instalaron tres tipos distintos de fibra óptica sobre la estructura, totalmente adherida a lo largo de la longitud de la pieza. Las fibras ópticas empleadas fueron

- Fibra óptica monomodo de la casa comercial Sumitomo.

- Fibra óptica monomodo con recubrimiento de acrilato de la casa comercial Corning, SMF-28 (Single Mode Fibre). Este tipo de fibra óptica es la empleada por el Grupo de Comunicaciones Ópticas y Aplicaciones Electrónicas de la Universidad Pública de Navarra (UPNA). Para los siguientes apartados, será referenciada como SMF-28 (UPNA).

- Fibra óptica monomodo con recubrimiento de poliamida de la casa comercial Corning, smf-28 (Single Mode Fibre). Este tipo de fibra óptica es la empleada por el Instituto de Telecomunicaciones y Aplicaciones Multimedia (iTEAM) de la Universidad Politécnica de Valencia (UPV). Para diferenciarla de la anterior será referenciada como smf-28 (UPV).

Dos tramos de cada tipo de fibra se encontraban adheridas a la estructura, de forma que cada tramo de fibra ha sido distinguida con subíndices 1 y 2 (Figura 7.13). 


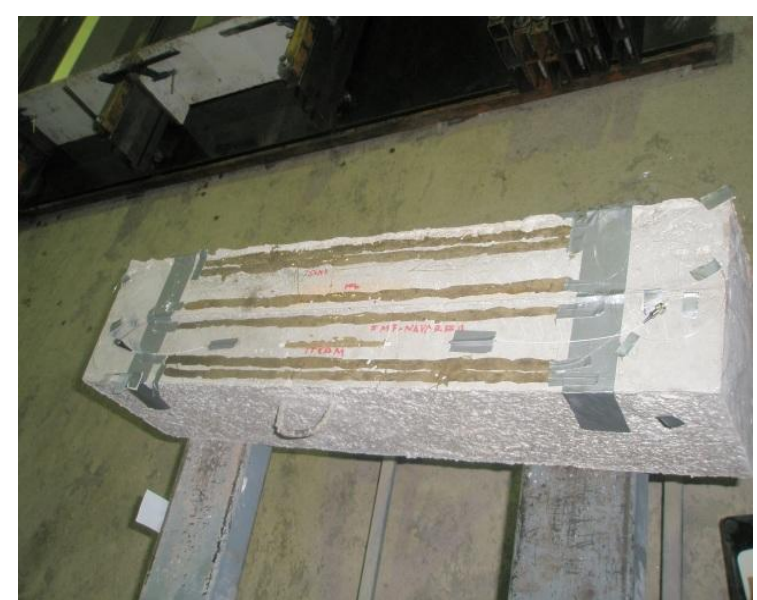

(a)

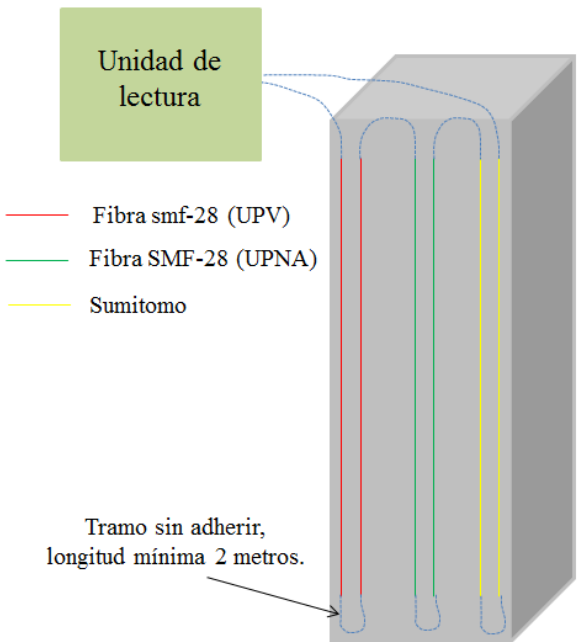

(b)

Figura 7.13 (a)- Probeta instrumentada con sensores distribuidos. (b)- Montaje de una probeta instrumentada con los tres tipos de fibras.

La instrumentación de las probetas se llevó a cabo siguiendo un cuidadoso procedimiento. Para ello se aplicó el procedimiento estudiado en el Capítulo 4 y en todo momento se intentó que la fibra óptica estuviera en contacto con la superficie de la estructura a monitorizar para permitir una correcta transferencia de deformaciones entre ambos elementos. Además y para conseguirlo, se empleó el adhesivo cuyas propiedades fueron estudiadas en el Capítulo 4.

\subsubsection{Resultados obtenidos de los ensayos.}

\section{a) Comportamiento general del sensado distribuido de Brillouin.}

Los resultados obtenidos con los sensores distribuidos se presentan en las Figuras 7.14 y 7.16. En ellos se presentan las distribuciones de la frecuencia de Brillouin a lo largo de la longitud de fibra óptica.

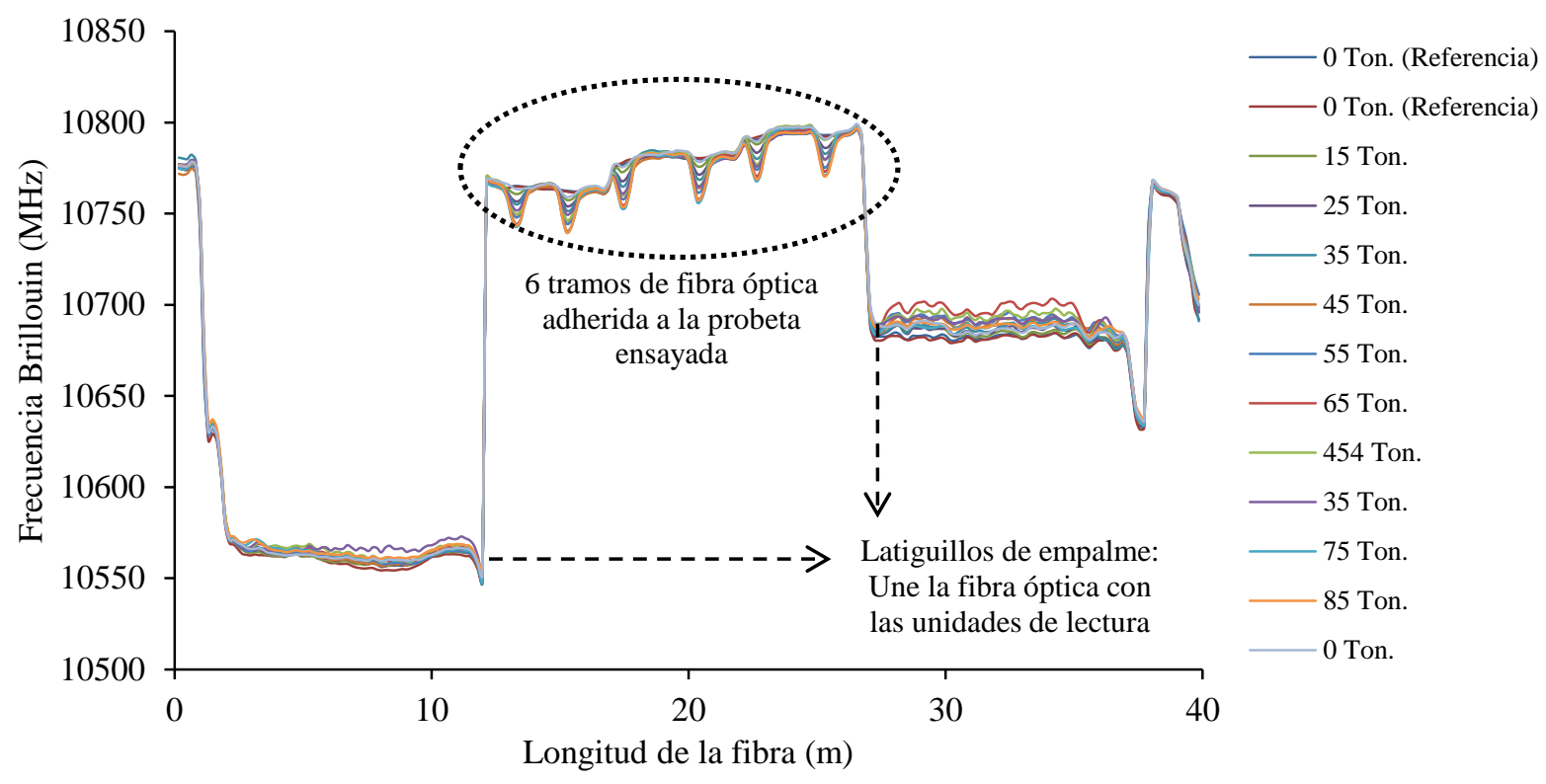

Figura 7.14 Evolución de la frecuencia Brillouin a lo largo de la fibra óptica en el tiempo en el que han sido tomadas las medidas del ensayo. La leyenda muestra la carga (toneladas) sobre la probeta en cada instante. 
En total se prepararon 40 metros de fibra óptica como se observa en la Figura 7.14. De los 40 metros, sólo 6 tramos de $70 \mathrm{~cm}$ de longitud cada uno se encuentran adheridos a la estructura, y por tanto sufren variaciones apreciables de la frecuencia de Brillouin. El resto de tramos responden con una frecuencia propia de Brillouin ya que este parámetro depende del tipo de fibra óptica empleada.

Concretamente, los tramos iniciales y finales de 10 metros de longitud cada uno, presentan una distribución casi uniforme de su frecuencia de Brillouin. Estas dos secciones corresponden a los tramos de fibra que no estaban adheridos a la probeta y que por tanto eran independientes de su deformación mecánica. Sin embargo, se observa que la frecuencia de Brillouin experimenta pequeñas variaciones en el tiempo a lo largo de estos dos tramos. Este hecho se debe a que estos tramos de fibra experimentaron aumentos de temperatura tal y como muestra la Figura 7.15 por la radiación solar incidente sobre ellos. Tal y como se comentó en el apartado 8.2 la frecuencia Brillouin es sensible a la temperatura y la respuesta ante este estímulo es una ligera variación de este parámetro.

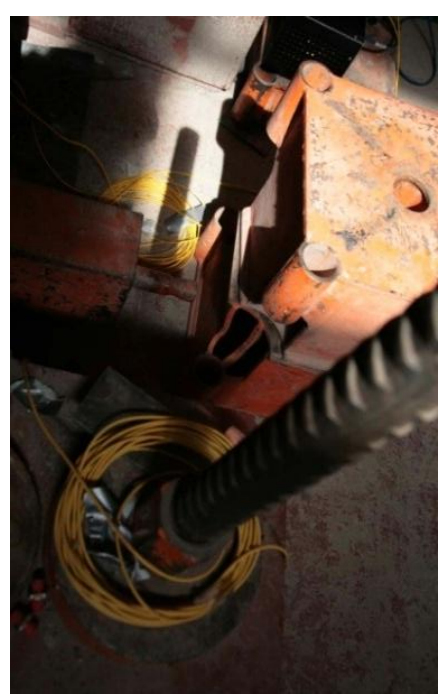

Figura 7.15 Latiguillos que unen la fibra óptica con la unidad de lectura. Durante el ensayo sufrió radiación directa de la luz solar.

La Figura 7.16 muestra el detalle de la evolución de la frecuencia Brillouin de los 6 tramos adheridos a la estructura y por tanto, sujetos a deformación mecánica. Cuando la estructura entra en carga los tramos de fibra instalados sobre ella experimentan una variación de la frecuencia Brillouin que se visualiza perfectamente en esta figura. La frecuencia Brillouin disminuye a medida que aumenta la deformación de compresión en la probeta.

Es importante destacar, que al tratarse de tres tipos de fibras distintas, cada una de ellas responde con una frecuencia de Brillouin distinta también. 


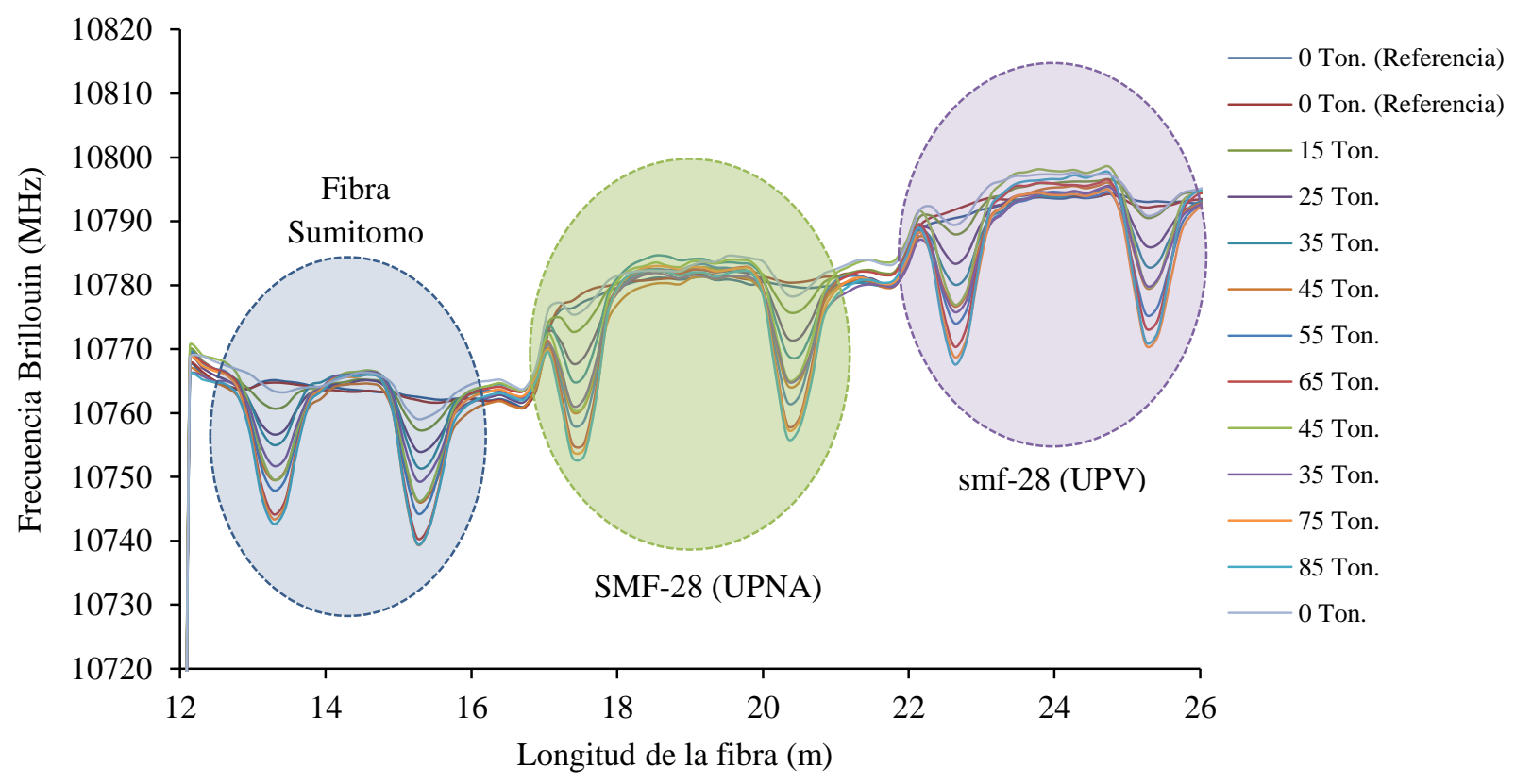

Figura 7.16 Variación de la frecuencia Brillouin en los 6 tramos de fibra adheridos a la estructura. La leyenda muestra la carga (toneladas) sobre la probeta en cada instante.

Por otro lado, la Figura 7.17 muestra la evolución de la frecuencia Brillouin en el tiempo durante el ensayo y aplicación de la carga. En esta figura y para todas las fibras ópticas se observan 4 tramos, dos ascendentes y otros dos descendentes.

En el primer tramo descendente disminuye la frecuencia de Brillouin debido a que aumenta la deformación de compresión de la probeta. A continuación existe un tramo ascendente, en torno al minuto 50 de iniciado el ensayo, donde se reduce la carga sobre la probeta y por tanto aumenta la frecuencia Brillouin. Finalmente, los otros dos tramos corresponden de nuevo con la aplicación de la carga y la liberación de la misma. Al finalizar el ensayo, el valor de la frecuencia de Brillouin vuelve al mismo valor inicial de antes de iniciar el ensayo.

La Figura 7.17 muestra tres grupos de curvas, cada una de ellas asociada a uno de los tipos de fibra que se instalaron sobre la probeta. Como se explicó en un apartado anterior, la frecuencia de Brillouin depende del tipo de fibra empleada. A pesar de que cada una responde con una frecuencia propia, todas responden de la misma manera ante el mismo estímulo.

Es importante destacar que las medidas distribuidas han sido tomadas durante el intervalo de tiempo en que la carga permanecía constante, adoptando un único valor de frecuencia Brillouin en cada intervalo. Por este motivo, la evolución de este valor en el tiempo (Figura 7.17) describe tramos que se ajustan en gran medida a tramos rectos. 


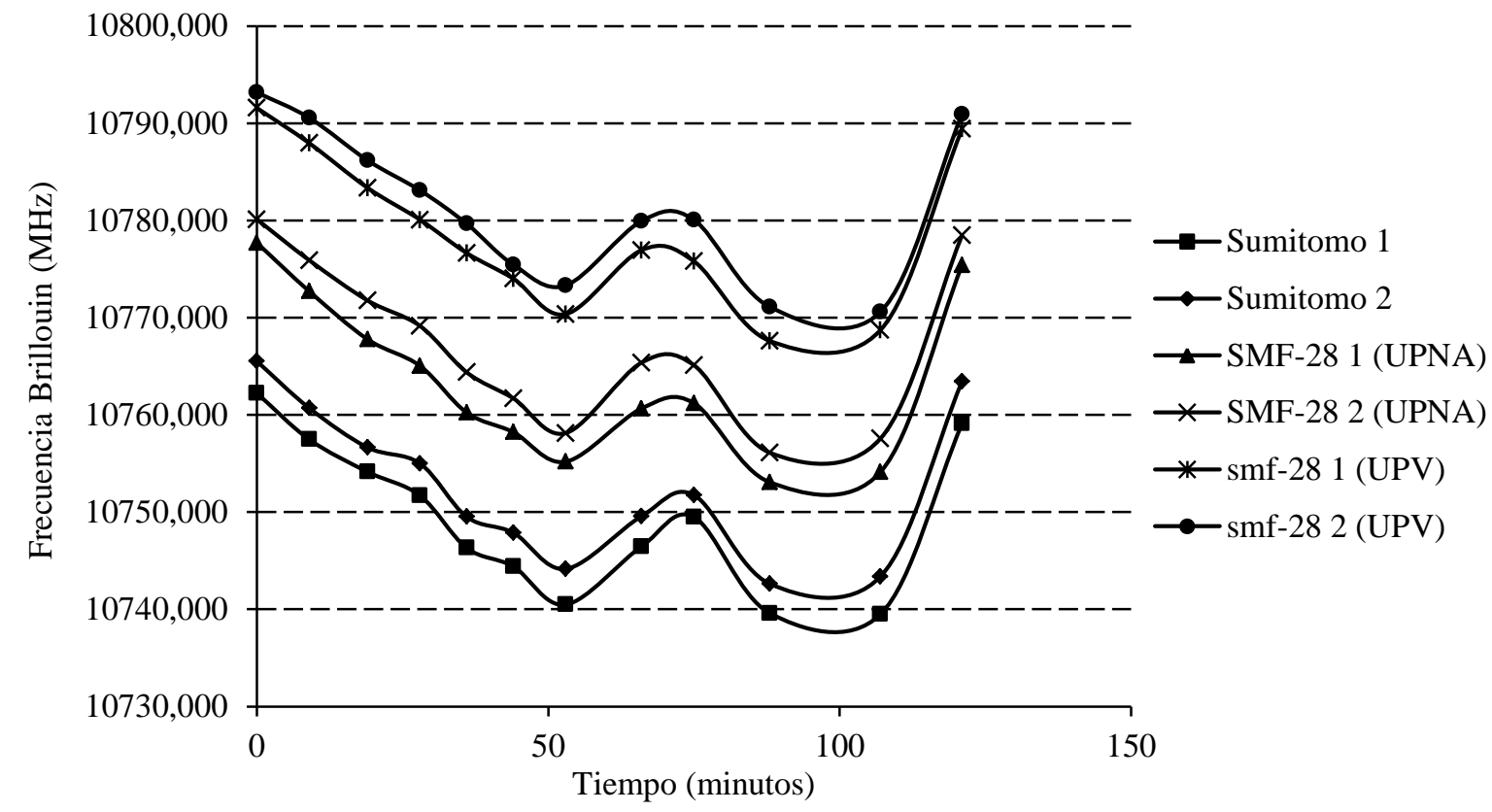

Figura 7.17 Evolución de la frecuencia Brillouin de las fibras instaladas en la probeta a lo largo del ensayo.

\section{b) Linealidad del sensado distribuido de Brillouin.}

La Figura 7.18 muestra la dependencia de la frecuencia Brillouin medida con la carga aplicada. La respuesta de la frecuencia Brillouin durante la aplicación de la carga muestra un comportamiento que se ajusta con un elevado grado a una recta. Los coeficientes de ajuste lineal $R^{2}$ se muestran en la Tabla 7.2 .

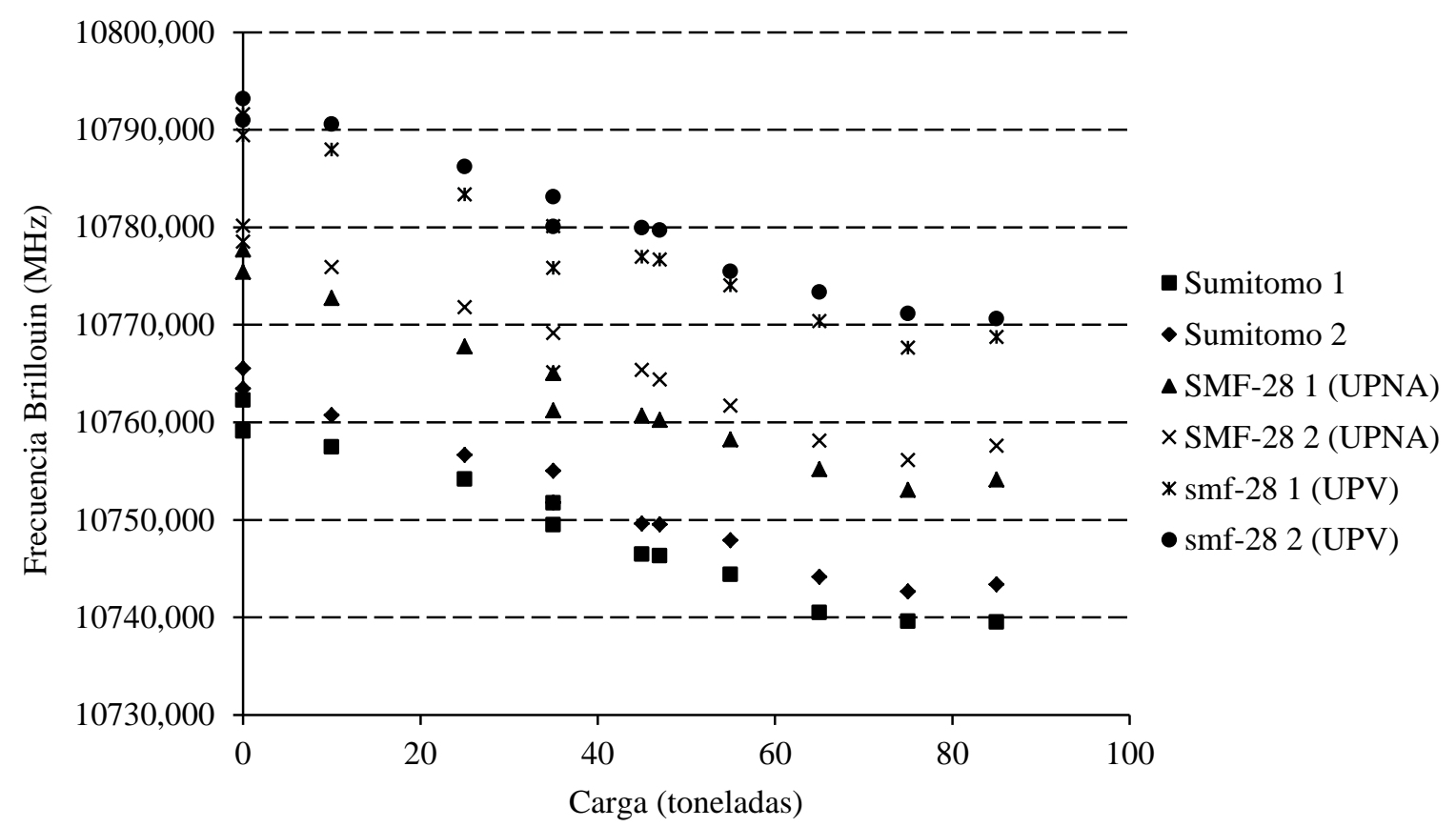

Figura 7.18 Evolución de la frecuencia Brillouin con la carga aplicada durante el ensayo. 
Monitorización estructural.

Aplicación con sensores de fibra óptica.

\begin{tabular}{cc}
\hline Tipo de fibra & $\boldsymbol{R}^{\mathbf{2}}$ \\
\hline Sumitomo 1 & 0.9667 \\
Sumitomo 2 & 0.9606 \\
SMF-28 1 (UPNA) & 0.9478 \\
SMF-28 2 (UPNA) & 0.9572 \\
Smf-28 1 (UPV) & 0.9548 \\
Smf-28 2 (UPV) & 0.9729 \\
\hline
\end{tabular}

Tabla 7.2 Coeficientes de ajuste $\mathrm{R}^{2}$ para cada tramo de fibra.

Como se observa en la Tabla 7.2 anterior, el grado de ajuste a una recta es muy elevado.

De una forma similar, la frecuencia Brillouin de una fibra es un parámetro que tiene una dependencia lineal con la deformación a la que está sometida. Las Figuras 7.19 y 7.20 muestra la relación existente entre la frecuencia de Brillouin y la deformación de la estructura, que ha sido medida por los sensores ópticos puntuales y de longitud de referencia. La relación entre frecuencia Brillouin y deformación proporcionará el coeficiente de sensibilidad, que permitirá obtener las deformaciones.

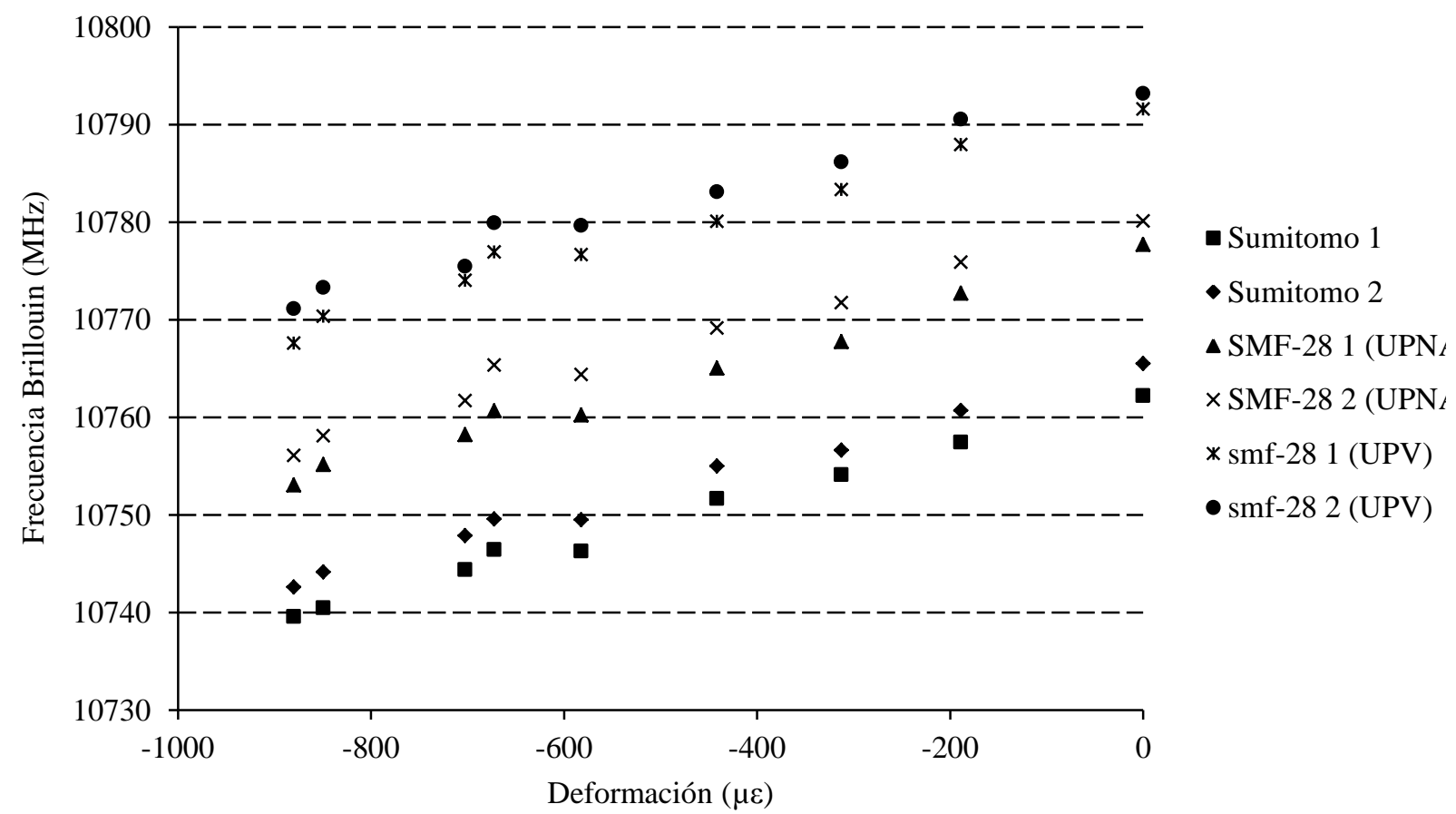

Figura 7.19 Relación entre la fecuencia Brillouin y la deformación de la probeta medida a partir de un sensor óptico puntual de referencia. 


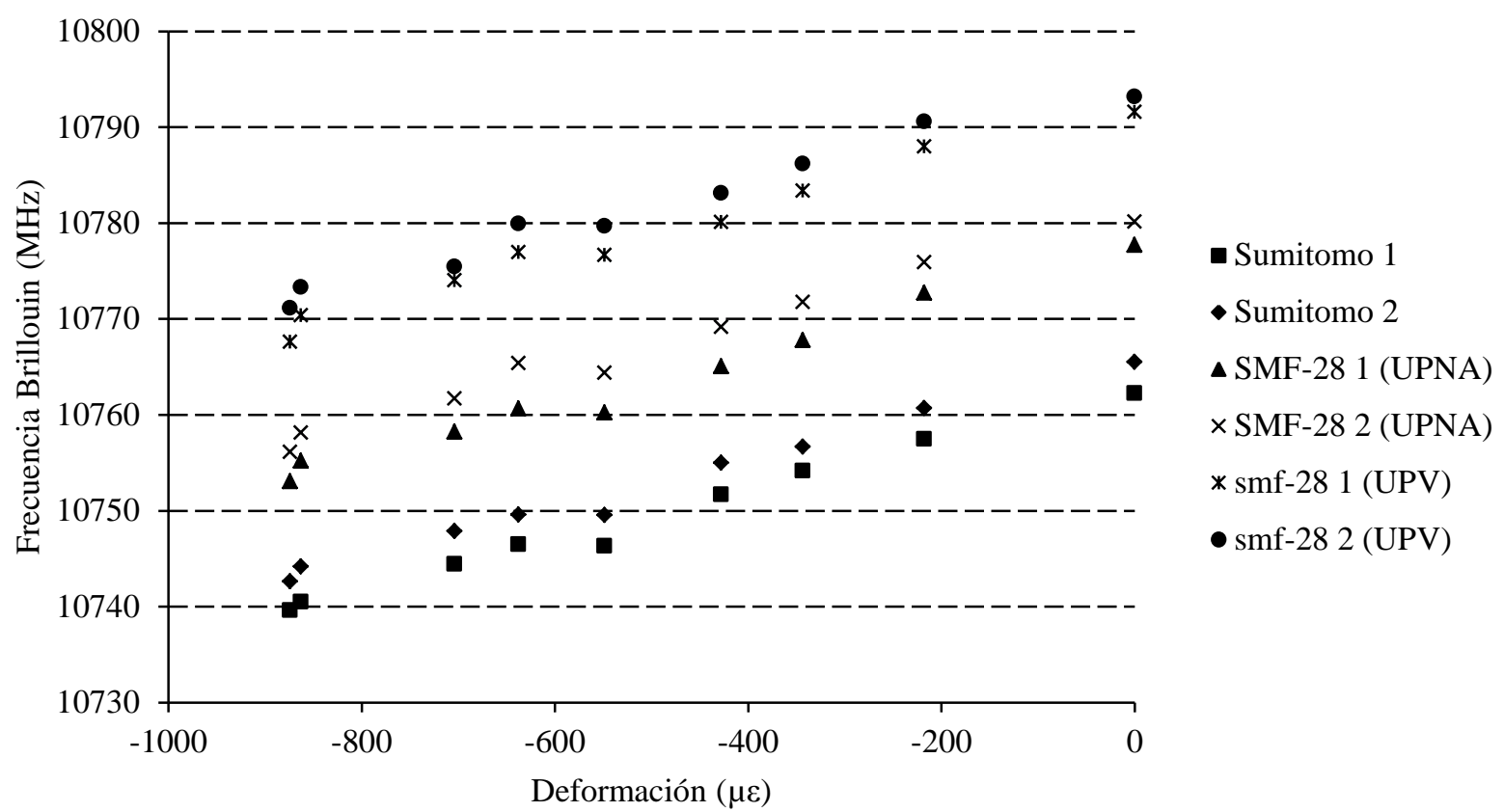

Figura 7.20 Relación entre la frecuencia Brillouin y la deformación de la probeta medida a partir de un sensor óptico de longitud de referencia.

Tal y como se observa en la Tabla 7.3, los grados de ajuste de los datos obtenidos a una recta son muy elevados. Las pequeñas desviaciones existentes se deben a los ajustes de la carga aplicada y fundamentalmente a las pequeñas diferencias de deformación medidas con los dos tipos de sensores.

\begin{tabular}{|c|c|c|}
\hline Tipo de fibra. & Ajuste $R^{2}$ sensor puntual. & Ajuste $R^{2}$ sensor de longitud. \\
\hline Sumitomo 1 & 0.9924 & 0.9892 \\
\hline Sumitomo 2 & 0.9904 & 0.9879 \\
\hline SMF-28 1 (UPNA) & 0.9874 & 0.9823 \\
\hline SMF-28 2 (UPNA) & 0.9816 & 0.9827 \\
\hline smf-28 1 (UPV) & 0.9803 & 0.9792 \\
\hline smf-28 2 (UPV) & 0.9751 & 0.9751 \\
\hline
\end{tabular}

Tabla 7.3 Coeficientes de ajuste $R^{2}$ para cada tipo de fibra y según sensor puntual y de longitud.

c) Sensibilidad de la deformación del sensado distribuido de Brillouin.

A partir de los valores anteriores es posible obtener la dependencia del desplazamiento en frecuencia Brillouin respecto a la deformación que sufre el elemento ensayado. Esta dependencia representa el coeficiente de sensibilidad de la deformación y es distinto para cada tipo de fibra óptica. De esta forma, a partir de la variación de la frecuencia Brillouin de una fibra óptica instalada en una estructura será posible la obtención de su deformación a lo largo de longitud de la fibra.

La Tabla 7.4 muestra los distintos coeficientes de sensibilidad calibrados empleando los sensores de referencia para los 6 tramos ensayados de fibra óptica (Sumitomo, SMF-28 (UPNA) y smf-28(UPV)). 


\begin{tabular}{|c|c|c|c|c|c|c|}
\hline $\begin{array}{l}\text { Sensibilidad } \\
\qquad \mathrm{MHz} / \mu \varepsilon\end{array}$ & $\begin{array}{c}\text { Sumitomo } \\
1\end{array}$ & $\begin{array}{c}\text { Sumitomo } \\
2\end{array}$ & $S M F-28,1$ & $S M F-28,2$ & $s m f-28,1$ & $s m f-28,2$ \\
\hline Sensor puntual & 0.0259 & 0.0262 & 0.0276 & 0.0274 & 0.0268 & 0.0258 \\
\hline Sensor de longitud & 0.0255 & 0.0258 & 0.0272 & 0.0270 & 0.0265 & 0.0255 \\
\hline Valor medio & 0.0257 & 0.0260 & 0.0274 & 0.0272 & 0.0266 & 0.0256 \\
\hline $\begin{array}{l}\text { Valor promedio por } \\
\text { tipo de fibra. }\end{array}$ & \multicolumn{2}{|c|}{0.0258} & \multicolumn{2}{|c|}{0.0273} & \multicolumn{2}{|c|}{0.0261} \\
\hline
\end{tabular}

Tabla 7.4 Coeficientes de sensibilidad de la deformación para el sensado distribuido de cada tipo de fibra óptica. Ajustes según el sensor óptico puntual, de longitud y valor medio.

Es importante destacar algunos aspectos que se deducen de la tabla anterior:

- Los coeficientes de sensibilidad obtenidos para un mismo tramo de fibra óptica deberían ser idénticos. Entre ellos existen algunas diferencias mínimas, que se deben a las pequeñas diferencias en las medidas de deformación empleando sensores puntuales y de longitud. Sin embargo estas diferencias son despreciables y por tanto los coeficientes de sensibilidad obtenidos presentan un buen ajuste.

- Los coeficientes de sensibilidad obtenidos difieren en mayor medida entre los tres tipos de fibra óptica obtenidos. Esto resulta evidente ya que como se explicó en un apartado anterior y en las Figuras 7.17, 7.18, 7.19 y 7.20 la frecuencia Brillouin depende del tipo de fibra óptica empleada.

- Los coeficientes de sensibilidad obtenidos en todas las fibras ópticas $(\sim 0.026 \mathrm{MHz} / \mu \varepsilon)$ son menores que el valor de referencia de la fibra SMF-28 (UPNA) $(\sim 0.04 \mathrm{MHz} / \mu \varepsilon)$. Esto se debe a que la longitud del tramo de fibra óptica que está instalado en la probeta y está sujeta a deformación es de $70 \mathrm{~cm}$, mientras que la resolución espacial del sensor Brillouin es de aproximadamente $100 \mathrm{~cm}$. Por tanto, la medida de deformación está integrada en $100 \mathrm{~cm}$, de los cuales sólo $70 \mathrm{~cm}$ sufren deformación.

\section{d) Comparación de los resultados obtenidos.}

La Figura 7.21 muestra las mediciones realizadas con el sensado distribuido de Brillouin. En esta figura se muestra las variaciones de deformación en función de la longitud de la fibra. Es importante tener en cuenta que el tramo desde los 0 hasta los 12 metros no está instalado en la probeta y por tanto no está sujeto a deformación, por lo que todas las variaciones medidas son debidas únicamente a los incrementos de temperatura que tuvieron lugar durante el tiempo del ensayo. De una forma similar, sucede lo mismo con el tramo que comprende desde los 26 hasta los 40 metros. Tal y como se observó en la Figura 7.15, la incidencia solar ha sido distinta según la hora y la posición de la fibra, de ahí que las mediciones obtenidas en estos dos tramos sean tan variables. 


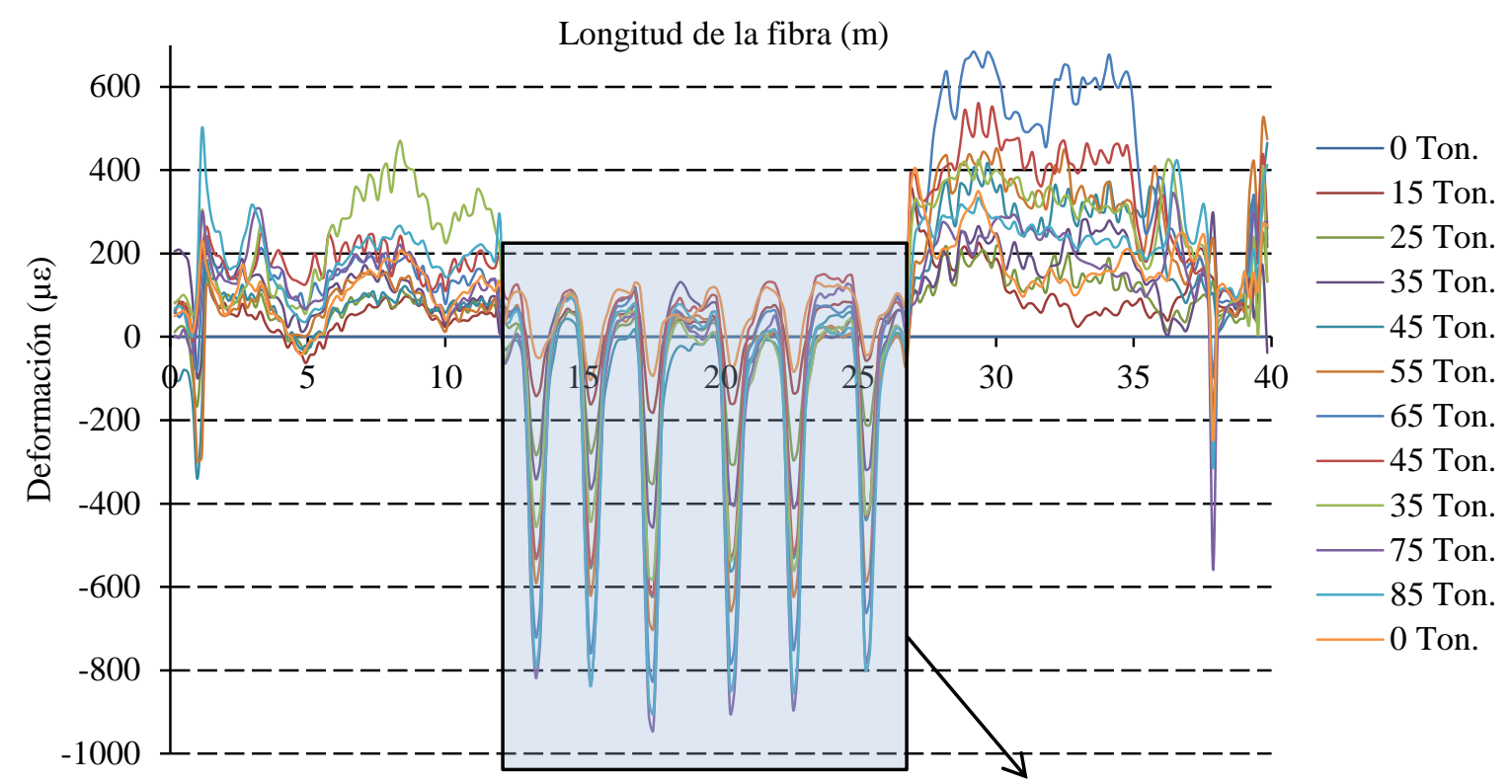

Tramo 12-26 metros: 6 tramos de fibra óptica instalados en la probeta y sujetos a deformación.

Figura 7.21 Deformación de la probeta en función de la longitud de la fibra óptica, medido por el sensado distribuido de Brillouin. (Deformaciones negativas corresponden con esfuerzos de compresión).

Longitud de la fibra (m)

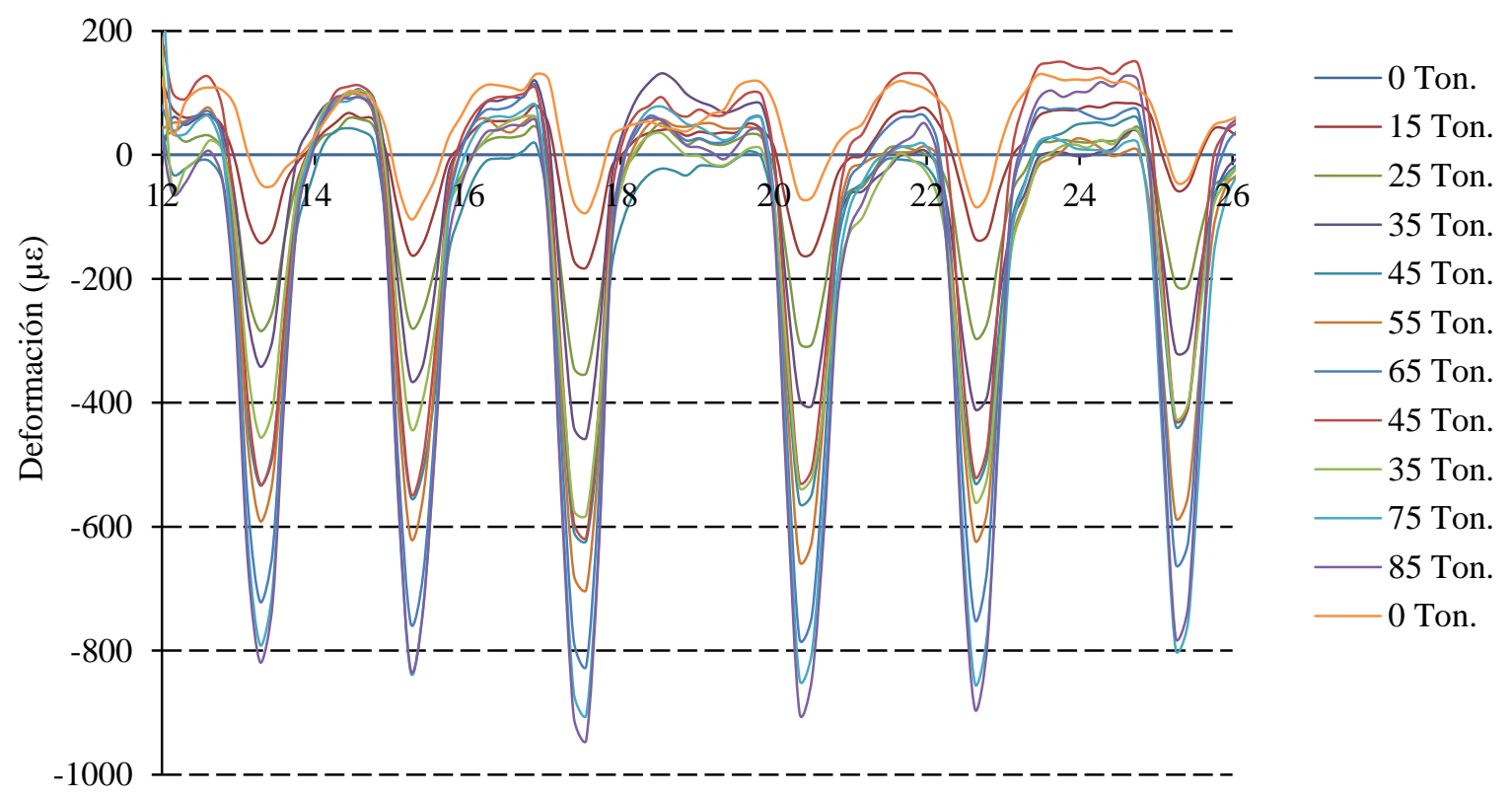

Figura 7.22 Deformación de la probeta en función de la longitud de la fibra óptica, medido por el sensado distribuido de Brillouin, para el tramo desde los 12 hasta los 26 metros. (Deformaciones negativas corresponden con esfuerzos de compresión). 
Monitorización estructural.

Aplicación con sensores de fibra óptica.

A partir de la Figura 7.21 se ha obtenido la Figura 7.22, donde se observa la deformación medida correspondiente al tramo desde los 12 hasta los 26 metros donde se encuentran las 6 secciones de fibra adheridas a la probeta. En esta figura se observa claramente el potencial de los sensores distribuidos en la monitorización de estructuras: se identifica claramente la posición donde se está produciendo una variación de deformación y es cuantificada.

Tal y como se observa en las anteriores figuras existen algunas diferencias entre los resultados de distintos tipos de fibras. Sin embargo esta desviación es relativamente pequeña, asumible y puede ser debida a distintas causas. Las más probables son los ajustes debidos a la carga aplicada por el gato a la probeta y la dificultad de aplicar sobre la probeta una carga totalmente centrada.

La Figura 7.23 muestra los resultados obtenidos de deformación en función del tiempo y su comparación con las distintas tipologías de sensado óptico (sensores puntuales, de longitud y 6 tramos de sensores distribuidos). Para la obtención de esta figura y las dos anteriores se han tenido en cuenta dos consideraciones:

- Se han empleado los valores de la Tabla 7.4 anterior para caracterizar la respuesta de los sensores distribuidos de Brillouin en las Figuras 7.21, 7.22 y 7.23.

- En la Figura 7.23, cada marcador de cada una de las series es un valor que se toma en cada uno de los escalones de carga que indica la Figura 7.11.

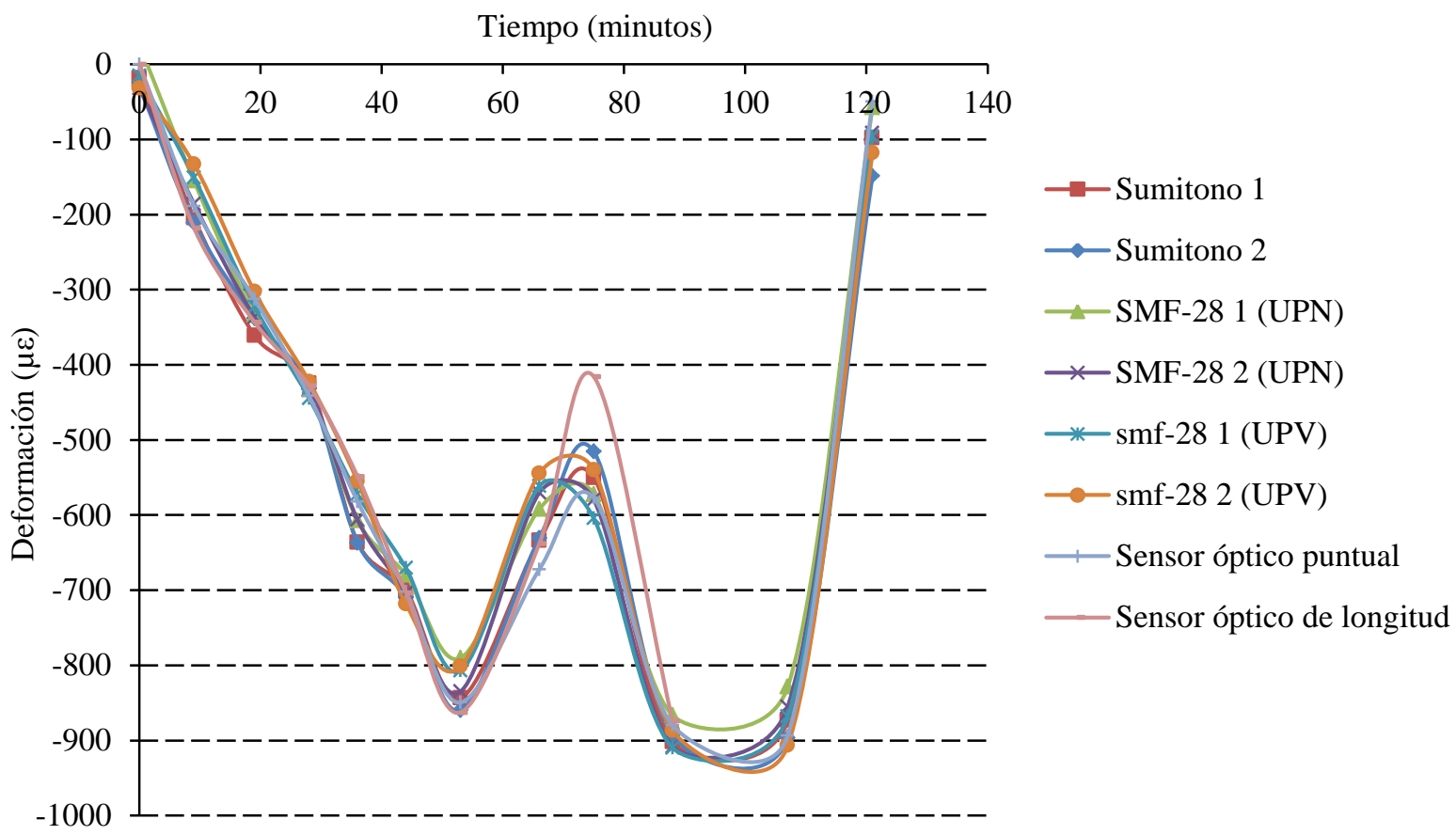

Figura 7.23 Deformación medida por el sensor puntual, de longitud y distribuidos durante el ensayo.

De nuevo en esta figura se observan diferencias entre los resultados obtenidos, que fueron comentadas anteriormente. 


\subsection{Conclusiones.}

En este apartado se ha estudiado el empleo de la fibra óptica como sensor distribuido para la aplicación a estructuras. Concretamente, el parámetro de la fibra óptica que se emplea para la obtención de la temperatura y deformación es la variación de la frecuencia Brillouin, explicada en el apartado 7.2. Esta técnica ofrece ventajas comparativas frente a otras técnicas en determinados escenarios, y su máximo rendimiento se obtiene en la instrumentación de grandes distancias como tuberías o puentes de grandes luces donde no se necesiten grandes precisiones en la resolución de la medida.

Siguiendo la línea planteada a lo largo de la Tesis Doctoral, se ha estudiado la calibración de esta técnica frente a temperaturas y deformaciones.

La calibración frente a temperaturas se ha llevado a cabo sometiendo a la fibra óptica a distintos ciclos térmicos. Con ellos, se ha medido la variación de la frecuencia Brillouin y se ha realizado un ajuste con los ciclos térmicos impuestos. La respuesta de la frecuencia de Brillouin frente a variaciones térmicas ha sido lineal con un elevado grado de ajuste. Las sensibilidades obtenidas muestran una pequeña dispersión que ha sido debida a los diferentes ciclos propuestos. Tras analizar los diferentes ensayos, la sensibilidad adoptada es la obtenida con el segundo ensayo, que corresponde al tramo de menor longitud de fibra ensayada y unos ciclos térmicos de mayor duración. Su valor es de 1.25 $\mathrm{MHz} /{ }^{\circ} \mathrm{C}$. Además no existen problemas de histéresis asociados a los ciclos ascendentes y descendentes de temperatura.

De una forma similar se ha llevado a cabo la calibración frente a deformaciones. Para ello, se fabricaron unas probetas de hormigón de base cuadrada de 0.2 metros de lado y 1 metro de altura, y sobre ella se instalaron sensores ópticos de referencia (puntuales y de longitud) y sensores distribuidos. La frecuencia Brillouin medida muestra una relación lineal con la carga aplicada y con la deformación del elemento y las sensibilidades obtenidas han permitido la obtención de las deformaciones en la pieza empleando sensores distribuidos. Las sensibilidades obtenidas dependen del tipo de fibra óptica empleada, ya que la frecuencia Brillouin varía con el tipo de fibra. Los valores medios obtenidos para las fibras tipo sumitomo, smf-28 (UPV) y SMF 28 (UPNA) han sido respectivamente $0.0258,0.0273$ y $0.0261 \mathrm{MHz} / \mu \varepsilon$. En general, este valor de sensibilidad obtenida es inferior al valor de referencia de la fibra SMF-28 (UPNA) $(\sim 0.04 \mathrm{MHz} / \mu \varepsilon)$ debido a que la longitud del tramo de fibra óptica que está sujeta a deformación es de $55 \mathrm{~cm}$, mientras que la resolución espacial del sensor Brillouin es de aproximadamente $80 \mathrm{~cm}$. Por tanto, la medida de deformación está integrada en $80 \mathrm{~cm}$, de los cuales sólo 55 sufren deformación.

Las tres fibras han permitido la correcta obtención de la deformación del elemento, mostrando en general un buen comportamiento.

Realizados los ensayos, es importante destacar las siguientes conclusiones:

1) Existen algunas diferencias entre las mediciones obtenidas con distintos tipos de fibras. Un motivo de estas pequeñas diferencias es que la carga sobre la probeta no se encuentra totalmente centrada por lo que la deformación del elemento no es perfectamente homogénea.

2) En el ciclo de carga propuesto se ha estabilizado la carga durante un tiempo de 5 minutos para poder tomar las medidas durante este intervalo y realizar un promediado. Durante este intervalo de tiempo se realiza un barrido de las frecuencias relativas del pulso óptico y de la 
onda continua, y a partir de aquí se ha obtenido el valor promedio. Este valor promedio es el que se ha presentado como valor de la medida. El empleo de estos valores promedios ha permitido una correcta estimación de las deformaciones en el elemento.

3) En estos ensayos se ha empleado un pulso óptico de duración 10 nanosegundos. Este pulso proporciona una distancia de resolución de aproximadamente 1 metro, es decir, los datos obtenidos son la integración de las medidas que tienen lugar en 1 metro de longitud. Atendiendo a las principales aplicaciones de este tipo de sensado, este aspecto no representa ningún inconveniente para la monitorización de grandes estructuras como puentes, tuberías o cables.

4) A pesar de que la precisión obtenida con los sistemas distribuidos de Brillouin ( $20 \mu \varepsilon$, Glisic e Inaudi, 2007) es menor que la precisión obtenida con los sensores FBG $(\sim 1 \mu \varepsilon$, Glisic e Inaudi, 2007), para deformaciones elevadas como las obtenidas en estos ensayos y a partir del promediado realizado en las medidas, los resultados obtenidos con los sensores distribuidos reproducen con un elevado grado de ajuste las deformaciones del elemento estructural.

5) La aplicación de la fibra óptica como sensor de deformación distribuido se puede observar claramente en las Figuras 7.21 y 7.22. En esta figura se observa la variación de deformación a lo largo de la longitud de la fibra óptica. De los 40 metros de fibra instrumentada se puede detectar claramente aquellos tramos que experimentan una variación de deformación, concretamente con los 6 tramos de fibra adherida a la probeta. A partir de este parámetro y con la calibración realizada a lo largo del capítulo, ha sido posible obtener el valor de la deformación en un tramo o punto en cuestión con un elevado grado de ajuste.

6) En el aspecto mostrado en el punto anterior 4), los sensores distribuidos presentan una clara ventaja frente a los sensores puntuales o de longitud. Tal y como se observa en la Figura 7.21, si se hubiera instalado un sensor puntual sobre el punto que corresponde a los $14.2 \mathrm{~m}$, el sensor apenas habría indicado variaciones en la medida. Y sin embargo, a ambos lados de este punto existen importantes incrementos de deformación.

7) Las sensibilidades obtenidas empleando sensores puntuales o de longitud son muy parecidas entre sí, debido a que las deformaciones obtenidas con los sensores puntuales y de longitud coinciden con un elevado grado de ajuste. A pesar de que no es recomendable el empleo de los sensores puntuales para materiales heterogéneos, cuando el elemento trabaja a compresión simple podrían emplearse ya que en este caso no existe fisuración y por tanto la medición media en una longitud es muy similar a la deformación de un punto interior a esa longitud.

8) En esta técnica es muy importante la correcta instalación de la fibra óptica en la estructura para permitir una correcta medición. Siguiendo las conclusiones obtenidas en el Capítulo 4, la instalación se debe realizar de forma que la fibra óptica se encuentre en contacto con la superficie del hormigón. Por otro lado es muy importante prestar atención al adhesivo empleado, que debe garantizar una correcta transferencia de deformaciones entre el hormigón y la fibra óptica.

Los ensayos realizados y resultados obtenidos muestran la validez de esta técnica para la obtención de temperaturas y deformaciones en estructuras. 


\section{A plicación de los Sensores distribuidos}

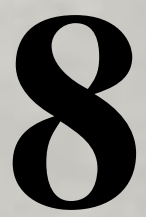

DESARROLLADOS EN EL CAPÍTULO 7 A LA

MONITORIZACIÓN DE UNA ESTRUCTURA REAL.

MONITORIZACIÓN DEL FALSO TÚNEL DE LA

LÍNEA DE ALTA VELOCIDAD DE LEVANTE A SU

PASO POR MOGENTE (VALENCIA).

Una vez caracterizada la respuesta de los sensores distribuidos en el Capítulo 7, este capítulo presenta los trabajos de monitorización de la estructura del falso túnel de la línea de alta velocidad de Levante a su paso por Mogente con sensores distribuidos de Brillouin.

Los trabajos realizados consistieron en la instalación de los sensores distribuidos sobre la bóveda del falso túnel y su posterior toma de medidas. Se instalaron dos sensores distribuidos. Uno de ellos medía las variaciones de temperatura, mientras que el otro era el encargado de medir la deformación de la estructura.

Los resultados obtenidos con los sensores distribuidos fueron comparados con los resultados experimentales obtenidos en el Capítulo 6 y se observó que, con la calibración de los sensores mostradas en el Capítulo 8 y la instalación propuesta, a pesar de sus limitaciones en cuanto a la resolución en la medida, los sensores ópticos distribuidos recogieron notablemente bien la pauta general de comportamiento del falso túnel. 



\subsection{Introducción.}

En el Capítulo 7 se han mostrado los estudios realizados para la caracterización de los sensores distribuidos basados en el desplazamiento de frecuencia en Brillouin. Allí se expusieron las calibraciones del sistema y su aplicación en trabajos experimentales de laboratorio para la obtención de temperaturas y deformaciones.

Siguiendo la línea planteada en esta Tesis Doctoral, el siguiente paso consiste en la aplicación de esta tecnología a la instrumentación de una obra real. En este caso, la obra es la misma que la analizada en el Capítulo 6: la estructura del falso túnel de la Línea de Alta Velocidad de Levante a su paso por Mogente.

Todos los datos relativos al calendario de medidas, geometría de la estructura y sistemas de monitorización se describen en el Capítulo 6 de este documento. En este Capítulo 8 se exponen:

- Los trabajos de instalación de los sensores distribuidos.

- Los principales resultados obtenidos empleando los sensores distribuidos.

- La comparación de los resultados de los sensores distribuidos con los resultados obtenidos con los sensores ópticos del Capítulo 6.

- Las principales conclusiones obtenidas de estos trabajos.

Los trabajos para la obtención de las temperaturas y deformaciones a partir de los sensores distribuidos también han sido llevados a cabo en colaboración con el Grupo de Comunicaciones Ópticas y Aplicaciones Electrónicas de la Universidad Pública de Navarra (UPNA).

\subsection{Instalación de los sensores ópticos distribuidos.}

La instalación de los sensores ópticos distribuidos se trata de una operación compleja. Su correcta instalación en la estructura requiere de unas obligadas operaciones previas:

- Preparación de la superficie. Esta operación exige un pulido previo de toda la superficie sobre la que va a estar adherida la fibra óptica. Este pulido tiene por objeto eliminar la lechada superficial del hormigón para permitir una buena adherencia con la fibra óptica.

- Limpieza de la superficie pulida con alcohol. Esta operación tiene por objeto eliminar las impurezas y suciedad de la superficie tras su preparación.

- Pegado de la fibra óptica. El pegado de la fibra óptica es una operación compleja y delicada. Hay que garantizar que la fibra óptica se encuentra totalmente adherida a la superficie de la estructura. No se puede producir la rotura de la fibra óptica, ya que ésta debe ser conectada por los dos extremos para producir la ganancia de Brillouin, tal y como se expuso en el Capítulo 7. Por este motivo el pegado de la fibra óptica se trata de una operación muy delicada. A todo esto hay que añadir la complejidad de la geometría de la estructura. Para su instalación, ha sido necesario el empleo de grúas elevadoras.

Para su instalación, la fibra óptica se coloca sobre una cinta adhesiva. Esta cinta adhesiva se coloca sobre la estructura, deslizándose a medida que se aplica el adhesivo bicomponente sobre la superficie 
Monitorización estructural.

Aplicación con sensores de fibra óptica.

previamente preparada y limpia. La fibra óptica instalada sobre la estructura es del tipo SMF-28, de la Universidad Pública de Navarra.

En total, se ha instalado una longitud total de fibra óptica de 49.9 metros sobre la estructura (Figura 8.1). La longitud total se divide en los tramos que se describen a continuación:

- Tramo A. El primer tramo corresponde con un patchcord (tramo de fibra óptica protegido de un recubrimiento de PVC) de 2,35 metros de longitud, que une las fibras ópticas instaladas con los equipos de medida.

- Tramo B. Este tramo, de 20.25 metros de longitud aproximadamente (de 2.35 a 22.6 metros), consiste en un latiguillo de fibra óptica sujeta a lo largo del perímetro del túnel con cinta adhesiva en una serie de puntos. Por tanto, mucha longitud de este tramo de fibra está colgando entre los puntos de unión de la fibra con el hormigón (Figura 8.2). Esta fibra tiene por objeto medir las variaciones de temperatura que experimenta la fibra para su posterior aplicación en la compensación térmica.

- Tramo C. Se trata del tramo de fibra óptica SMF-28 (UPNA) instalada con adhesivo bicomponente a lo largo del perímetro del falso túnel, con una longitud de19.5 metros, desde 25.5 a 45 metros aproximadamente (Figura 8.2). Esta fibra será la encargada de medir las variaciones de deformación que experimenta la estructura.

- Tramo D. Este último tramo corresponde al patchcord final que une la fibra óptica con el sistema de interrogación BOTDA (Brillouin Optical Time Domain Analysis), de 2,35 metros de longitud.

Con esta disposición de fibra óptica dispuesta, la lectura inicial proporciona el resultado indicado en la Figura 8.1, donde los cuatro tramos de fibra anteriormente descritos son claramente identificables. Cada uno de estos tramos responde con una frecuencia propia de Brillouin al tratarse de fibras distintas. 


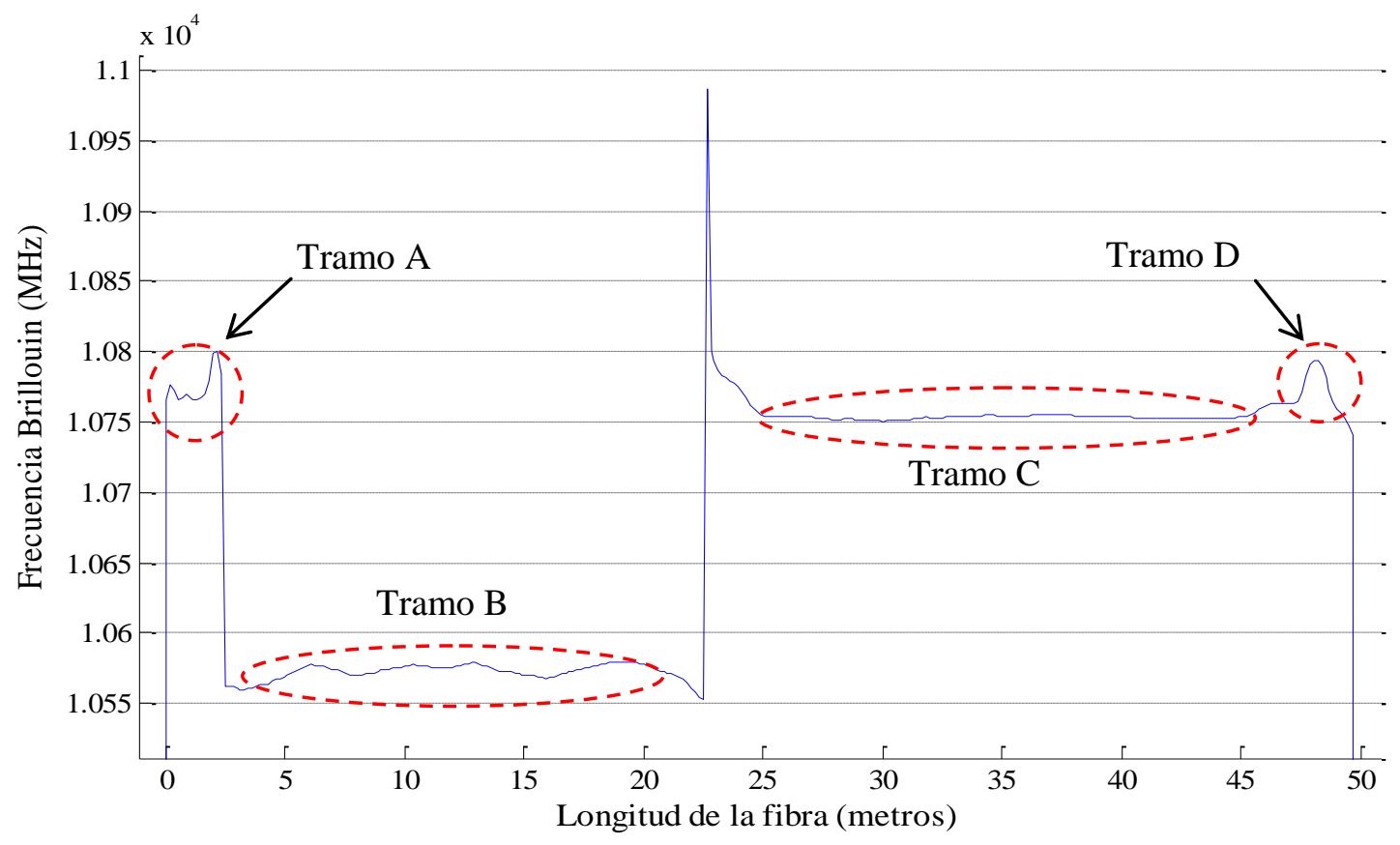

Figura 8.1 Variación de la frecuencia Brillouin a lo largo de la longitud de la fibra óptica.

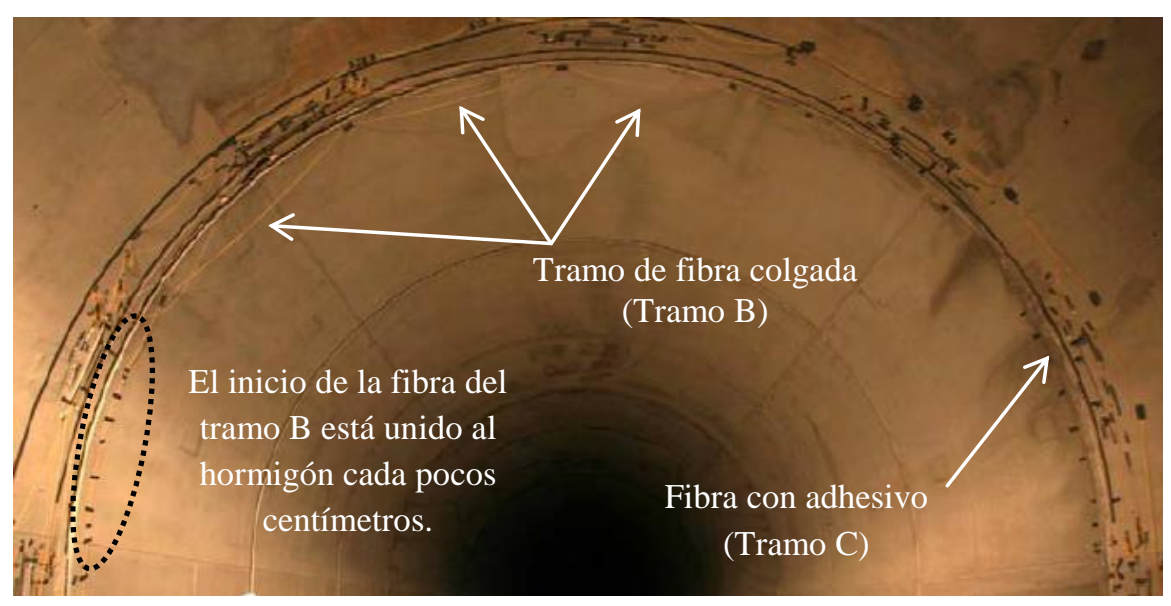

Figura 8.2 Sección del falso túnel instrumentada con distintas tecnologías.

Dado que los equipos de medida se encuentran ubicados en el lateral izquierdo de la Figura 8.3.a, el sentido creciente de la longitud de la fibra para el tramo B avanza en sentido horario, siguiendo el perímetro del túnel. En cambio, el sentido creciente de longitud para el tramo de fibra $\mathrm{C}$ avanza en sentido antihorario, ya que la fibra va conectada en sus dos extremos y éste es necesario que regrese a los equipos de medida (Figura 8.3.a y 8.3.b). 


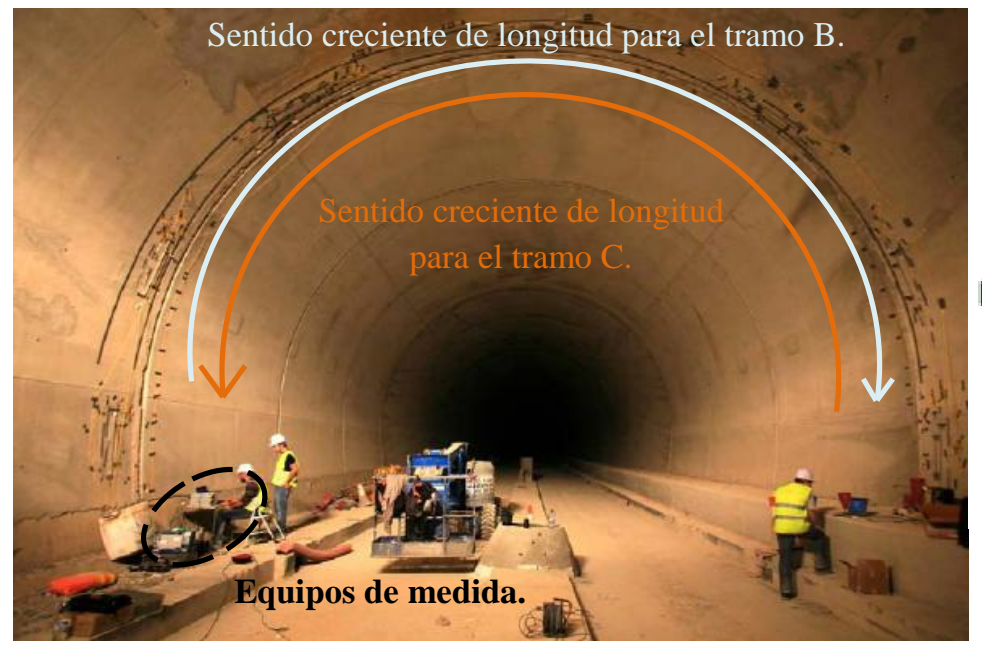

(a)

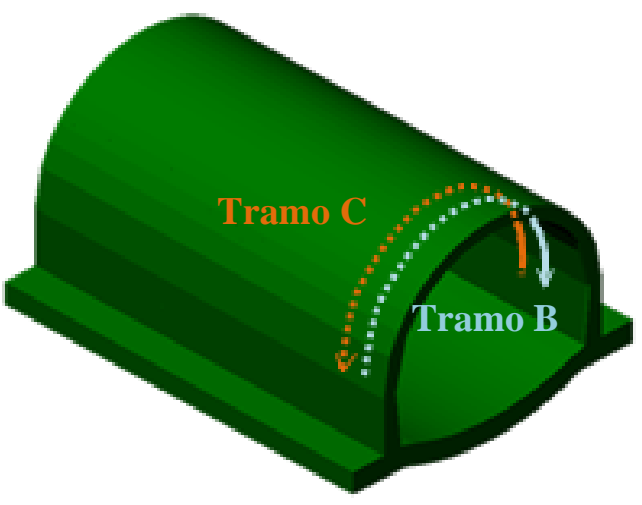

(b)

Figura 8.3 (a)- Sección transversal instrumentada, ubicación de los equipos de medida. (b)- Vista tridimensional de la estructura instrumentada con los tramos B y C de Brillouin.

Los datos que serán analizados se corresponden con los tramos B y C por ser las dos fibras que se encuentran sobre la estructura del túnel, una adherida mediante cinta adhesiva y la otra mediante adhesivo bicomponente.

\subsection{Análisis de los datos.}

A continuación se muestran los resultados obtenidos de la monitorización empleando el sensado distribuido de Brillouin. En los siguientes resultados la resolución del sistema es de 1 metro, lo que significa que cada resultado mostrado es la integración de los valores en cada metro de longitud de fibra. Las mediciones se han realizado cada 10 minutos.

\subsubsection{Mediciones continuas el día 26/09/2011.}

a) Tramo B. Tramo de fibra adherida a la estructura con cinta adhesiva en determinados puntos.

Como se indicó en el Capítulo 6, durante el día 26/09/2011 se llevaron a cabo mediciones continuas a lo largo de varias horas. En este apartado se van a mostrar las mediciones realizadas con el tramo B de fibra. Esta fibra está sujeta a variaciones de temperatura únicamente, ya que se encuentra aislada de la deformación del túnel.

Tal y como se mostró en la Figura 8.2, la fibra se encuentra adherida a la estructura sólo en unos puntos empleando para ello cinta adhesiva. En gran parte de su longitud, la fibra óptica se encuentra colgada entre los puntos de fijación de la fibra a la estructura. Esquemáticamente, esta disposición se muestra en la Figura 8.4 


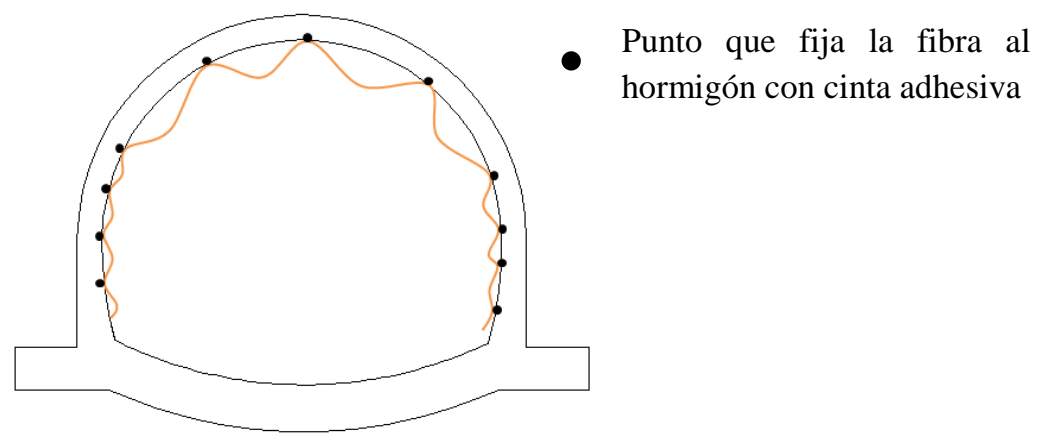

Figura 8.4 Disposición aproximada de la fibra que cuelga sobre la estructura del falso túnel.

Inicialmente, la primera lectura del tramo de fibra B correspondiente al día 26/09/2011 muestra el resultado indicado en la Figura 8.5.a. En esta figura se observa una frecuencia de Brillouin inicial muy variable dependiendo de la posición de la fibra óptica. La Figura 8.5.b muestra la evolución de la frecuencia de Brillouin a lo largo del tiempo, donde cada una de las trazas de color se corresponde con un instante de tiempo separado 10 minutos. Como se observa en la Figura 8.5.b, a lo largo de toda la longitud de fibra óptica se mide un aumento de la frecuencia de Brillouin, y este aumento no es igual para todos los puntos. Existen puntos de la fibra óptica que experimentan mayores aumentos en la frecuencia de Brillouin que otros, por lo que cabe esperar incrementos de temperaturas distintos en función del punto considerado.

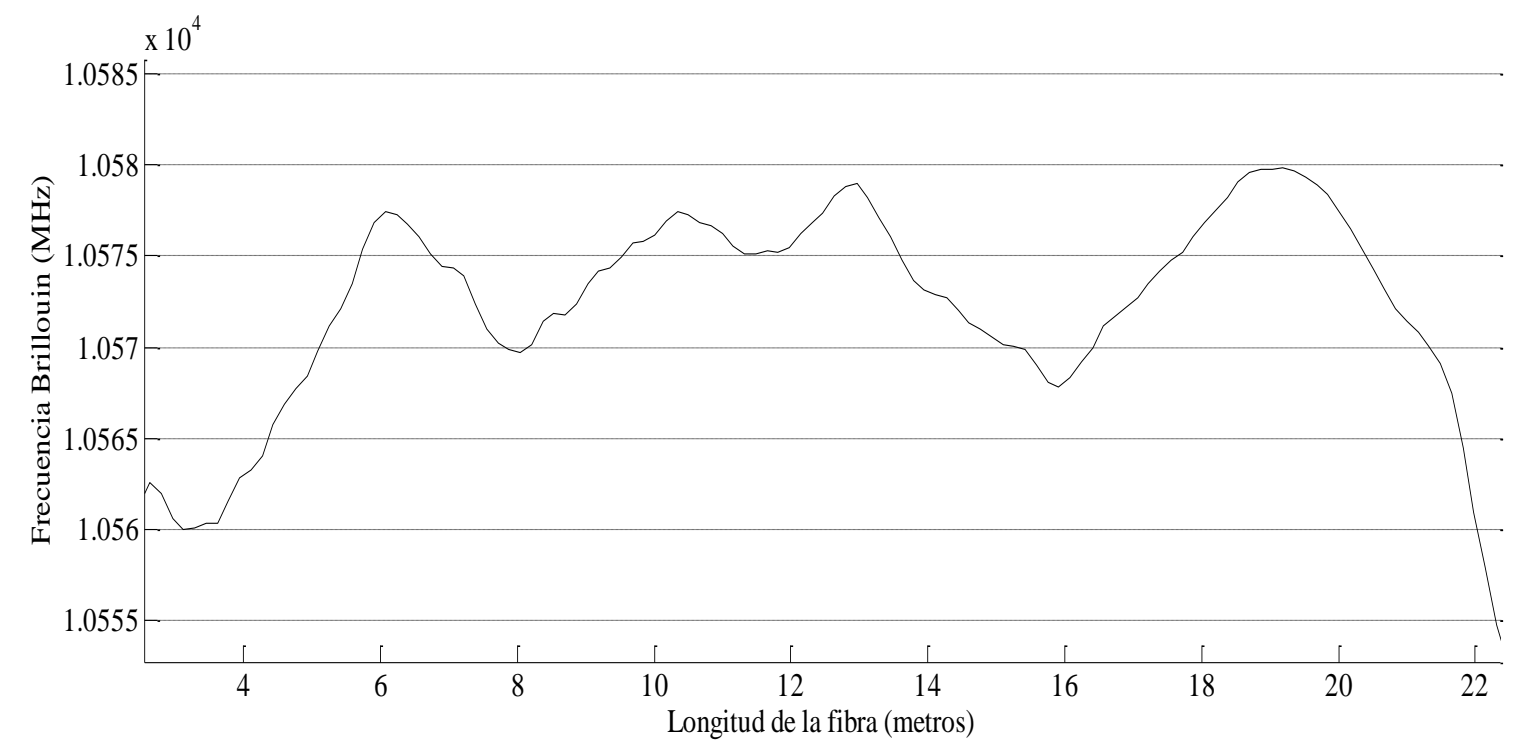

(a) 
Monitorización estructural.

Aplicación con sensores de fibra óptica.

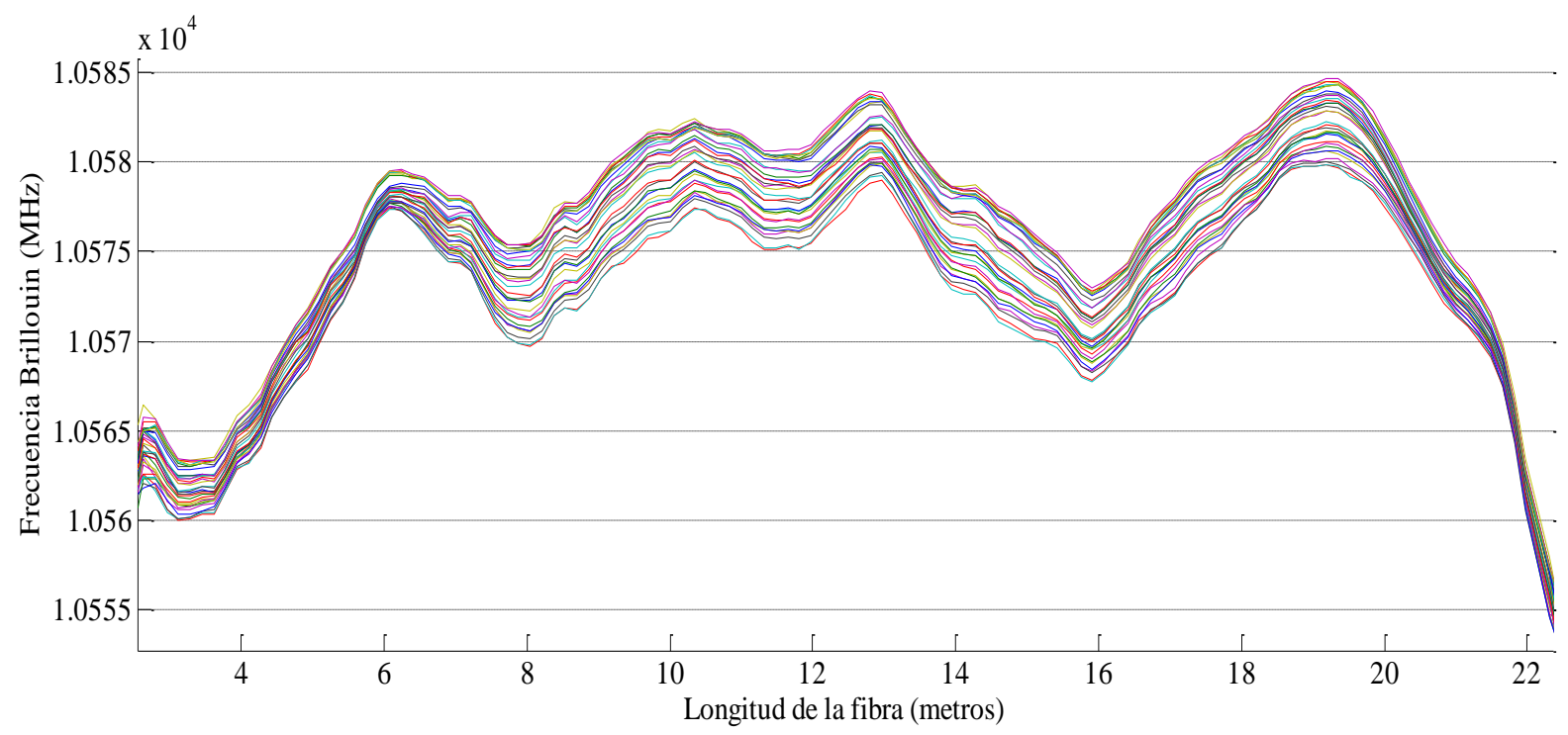

(b)

Figura 8.5 Distribución de la frecuencia de Brillouin en el tramo de fibra B durante el día 26/09/2011.

(a)- primera lectura del día, a las 09:34. (b). Medidas a lo largo del tiempo donde cada traza se corresponde con un instante de tiempo separados 10 minutos. Última lectura del día, a las 15:11.

La Figura 8.6 muestra la evolución de la frecuencia de Brillouin (tomando como ceros la primera medición del día indicada en la Figura 8.5.a) de un punto situado a 9 metros del inicio de la fibra que recae, aproximadamente, en el centro del tramo que cuelga en la parte izquierda de la estructura, de la forma que indica la Figura 8.6. En esta Figura se muestran 7 trazas en distinto color, que se corresponden con 7 puntos alrededor de los 9 metros, desde 8,5 hasta 9,5 metros separados 0,16 metros aproximadamente. Como se indicó anteriormente, cada una de estas trazas es el resultado de la integración en un metro de longitud.

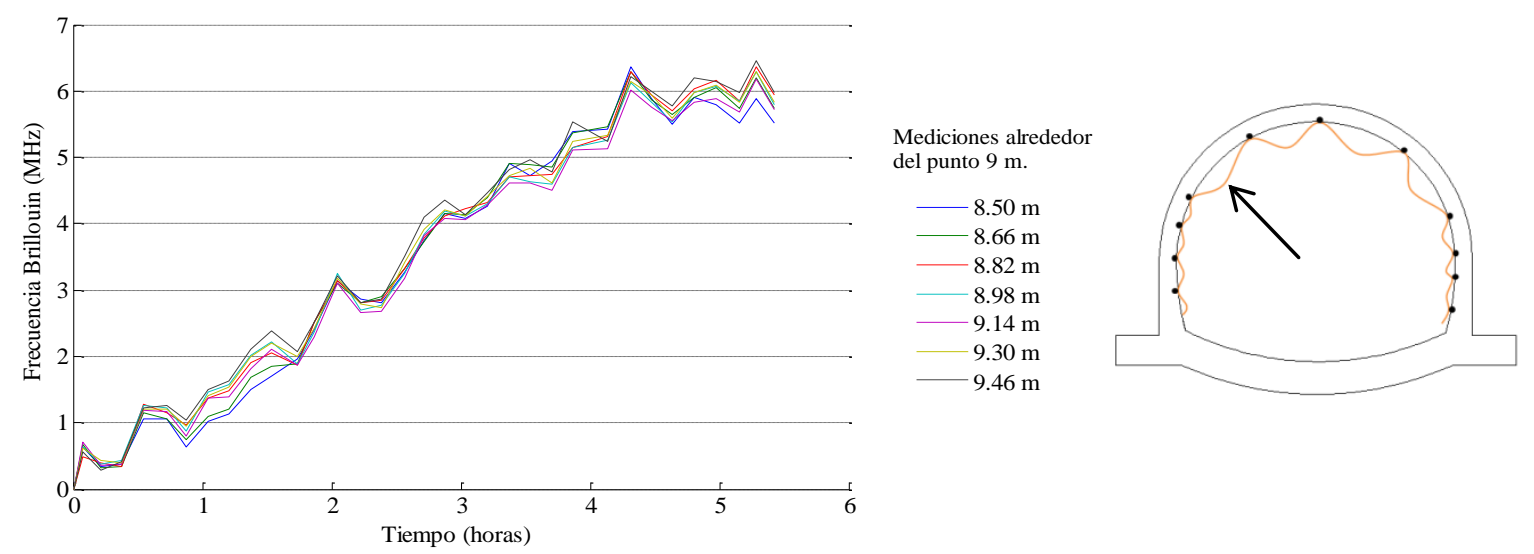

Figura 8.6 Evolución de la frecuencia de Brillouin en relación a la primera medida del día 26/09/2011, en un punto de la fibra óptica situado a 9 metros del inicio aproximadamente (indicado con una flecha en la Figura).

Tal y como se observa en la Figura 8.6, la frecuencia Brillouin tiene una tendencia ascendente a lo largo del tiempo. A partir de la calibración del Capítulo 7 se ha obtenido la evolución de temperaturas, que sigue esta misma ley (Figura 8.7). 


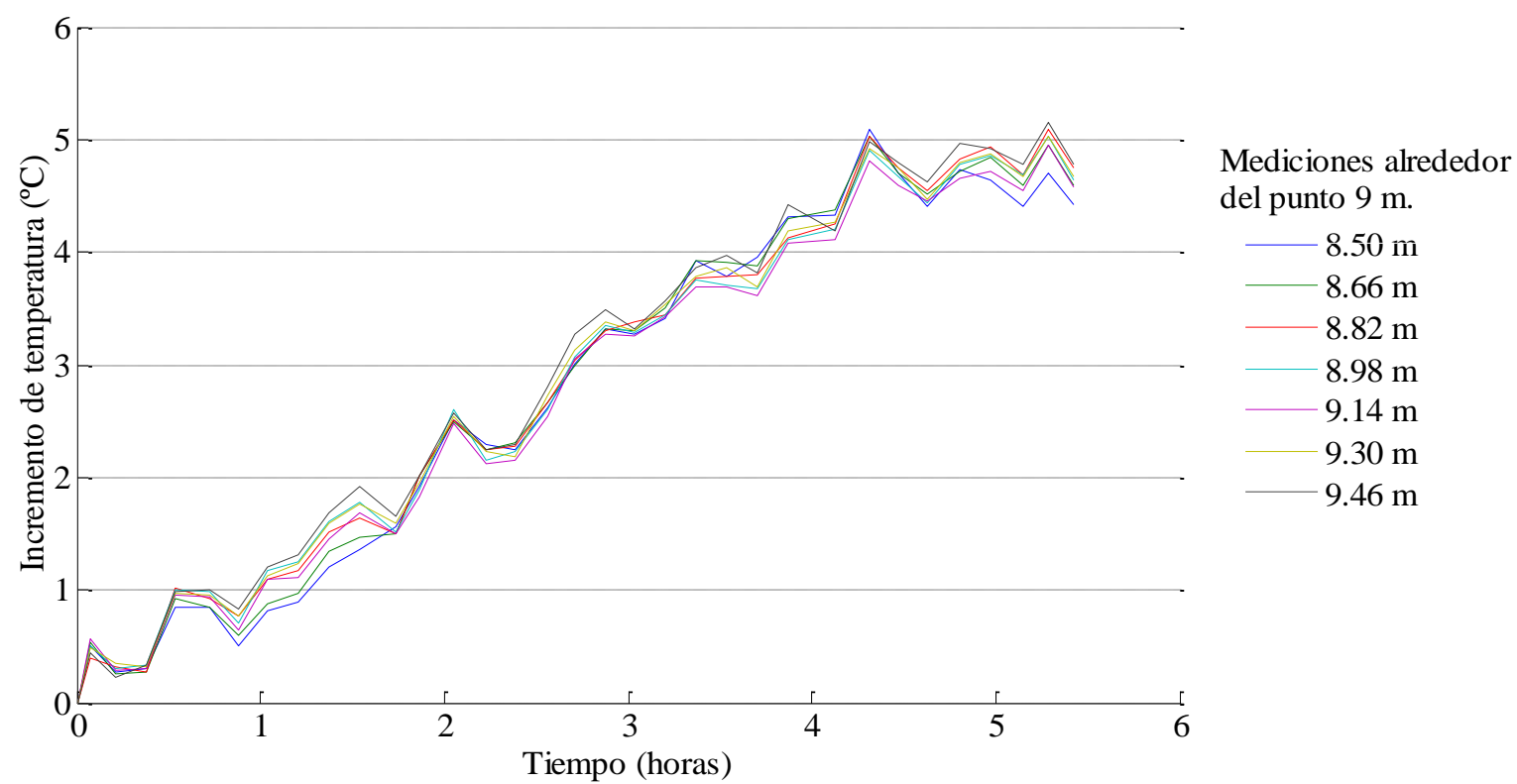

Figura 8.7 Evolución de la temperatura en relación a la primera medida del día 26/09/2011, en un punto de la fibra óptica situado a 9 metros del inicio aproximadamente.

Si se repite el anterior procedimiento para todos los puntos de la longitud de la fibra del tramo B, se obtiene la Figura 8.8, donde las medidas se encuentran normalizadas con respecto a la primera medida del día 26/09/2011 (que coincide con la línea horizontal a nivel 0) y cada una de las trazas de color se corresponde con una medición cada 10 minutos.

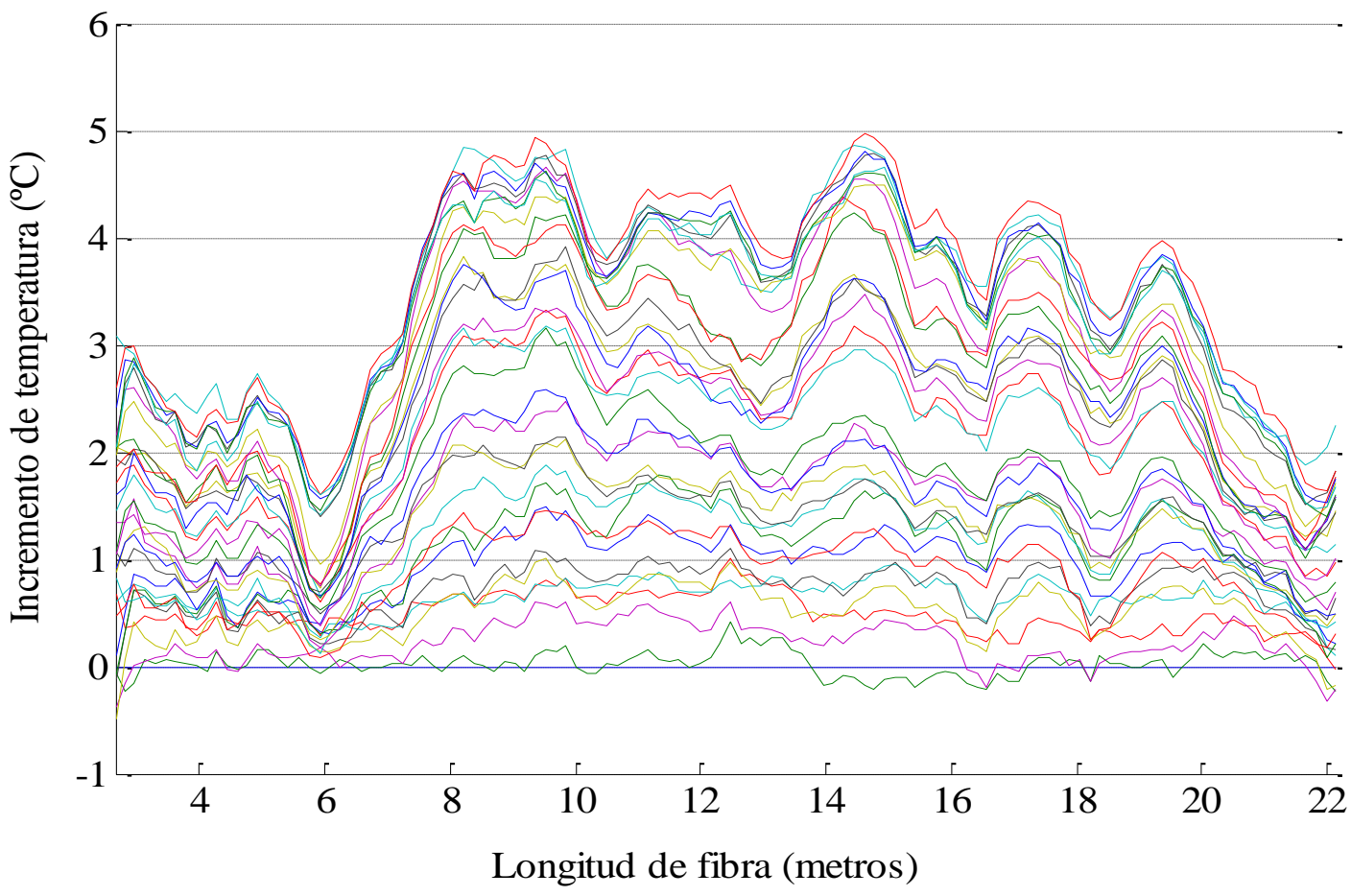

Figura 8.8 Incrementos de temperatura respecto a la primera medida del día 26/09/2011, a lo largo de la longitud de fibra óptica correspondiente al tramo B. 
Tras los resultados de la Figura 8.8, es importante destacar los siguientes aspectos:

- Todos los puntos de la fibra óptica sufren un aumento de temperatura, independientemente de la posición en la fibra. Sin embargo, este aumento no es uniforme en toda la longitud de la fibra, existiendo puntos con mayores variaciones térmicas que otros. Esto se traduce en una serie de picos y valles a lo largo de la longitud.

- Si se analiza la posición de los valles (6 m, 10.5m, 13.5m, $15.4 \mathrm{~m}, 16.6 \mathrm{~m}$ y $18,6 \mathrm{~m})$ se concluye que estos puntos se corresponden con aquellos en los que la fibra está adherida a la superficie de la bóveda del falso túnel con cinta adhesiva (puntos de color negro indicados en la Figura 8.4).

- Las variaciones térmicas que se han obtenido a lo largo de toda la longitud del tramo de fibra $\mathrm{B}$ muestran que existen puntos que han experimentado incrementos de hasta $5{ }^{\circ} \mathrm{C}$ y desde los 8 hasta los 20 metros aproximadamente, los incrementos medidos oscilan entre 3 y $5{ }^{\circ} \mathrm{C}$. Estos incrementos son muy superiores a los obtenidos a partir de las mediciones con sensores ópticos puntuales que se mostraron en el Capítulo 6. En aquel capítulo, el incremento de temperatura máximo alcanzado en el interior del falso túnel fue de $2.77{ }^{\circ} \mathrm{C}$ en el hastial izquierdo, y de $0.85^{\circ} \mathrm{C}$ en la clave de la estructura.

- Estas importantes diferencias han sido debidas a la forma de instalación del tramo de fibra B en la bóveda de la estructura. Los tramos que se encuentran colgados a lo largo de la bóveda no sólo sufren variaciones térmicas, sino que también experimentan movimientos debido a las corrientes de aire que circulan en el interior del túnel. Estos movimientos han provocado una variación de la frecuencia de Brillouin, que también ha sido medida.

- Este hecho justifica que en los puntos de contacto de la fibra con la estructura (valles en la Figura 8.4) la variación de la frecuencia de Brillouin haya sido inferior que en el resto de puntos. Sin embargo, la unión entre la fibra y el hormigón con cinta adhesiva no ha sido suficiente para evitar que el movimiento de la fibra debido a las corrientes de aire modifique la frecuencia de Brillouin.

- En los seis metros iniciales de fibra de la Figura 8.8 se observa un incremento de temperatura mucho menor que en el resto de longitud. Concretamente, en los cuatro primeros metros (correspondientes al hastial izquierdo del falso túnel de la Figura 8.2) se han medido unos incrementos de temperatura que oscilan entre 2.5 y $2.9^{\circ} \mathrm{C}$. Esto se debe claramente a la forma de sujeción del tramo de fibra B sobre el hormigón. Puede observarse en la Figura 8.2 cómo en esta zona, la fibra óptica se encuentra unida al hormigón con cinta adhesiva cada muy pocos centímetros. Esta unión impide que la fibra se mueva debido a las corrientes de aire y por tanto, su medición recoge únicamente las variaciones térmicas. En este punto, los incrementos de temperatura coinciden con los obtenidos en el Capítulo 6 por el sensor puntual del hastial izquierdo, que fue de $2.77^{\circ} \mathrm{C}$.

- En definitiva, tras observar los resultados obtenidos se deduce que el sensado distribuido para el tramo de fibra B se ve muy fuertemente afectado por la forma de instalación propuesta, por las variaciones térmicas existentes y por las corrientes de aire en el interior del túnel (que han provocado movimientos en los tramos de fibra colgantes y por tanto, una variación de la frecuencia Brillouin). 
- Este hecho ha imposibilitado realizar una compensación térmica sobre el tramo de fibra C (anclada a la estructura mediante adhesivo) ya que ambas fibras no se encuentran sometidas a las mismas variaciones térmicas. Sin embargo, es esperable que las variaciones térmicas sobre el tramo de fibra $\mathrm{C}$ sean relativamente pequeñas (ya que esta fibra se encuentra anclada al hormigón), por lo que la compensación térmica realizada no debería modificar en gran medida los resultados de deformación obtenidos para el tramo de fibra C.

- Para poder llevar a cabo una compensación térmica sería necesario que el tramo de fibra B se encontrara totalmente adherido a la estructura mediante cinta adhesiva, sin tramos intermedios donde cuelgue la fibra. De esta forma, esta fibra recogería únicamente los efectos térmicos.

\section{b) Tramo C. Tramo de fibra adherida a la estructura con adhesivo.}

En este apartado se van a mostrar las mediciones realizadas con el tramo de fibra C. Esta fibra está adherida a la superficie de la bóveda del túnel empleando un adhesivo bicomponente y por tanto, se encuentra sometida a variaciones térmicas (en el apartado anterior se dedujo que deben ser poco importantes) y a deformaciones del hormigón.

La Figura 8.9.a muestra la primera toma de datos para el tramo de fibra $C$ del día 26/09/2011. En esta figura se observa una frecuencia Brillouin a lo largo de la longitud de la fibra mucho más constante que en el caso del tramo de fibra B (Figura 8.5.a).

La Figura 8.9.b muestra la evolución de la frecuencia de Brillouin a lo largo del tiempo, donde cada una de las trazas de color se corresponde con un instante de tiempo separado 10 minutos. En esta figura se observa un incremento de la frecuencia Brillouin a lo largo de toda la longitud de fibra óptica correspondiente al tramo C, y este incremento no es igual para todos los puntos. Existen puntos de la fibra óptica que experimentan mayores aumentos en la frecuencia de Brillouin que otros, por lo que cabe esperar variaciones en la deformación distintas en función del punto considerado.

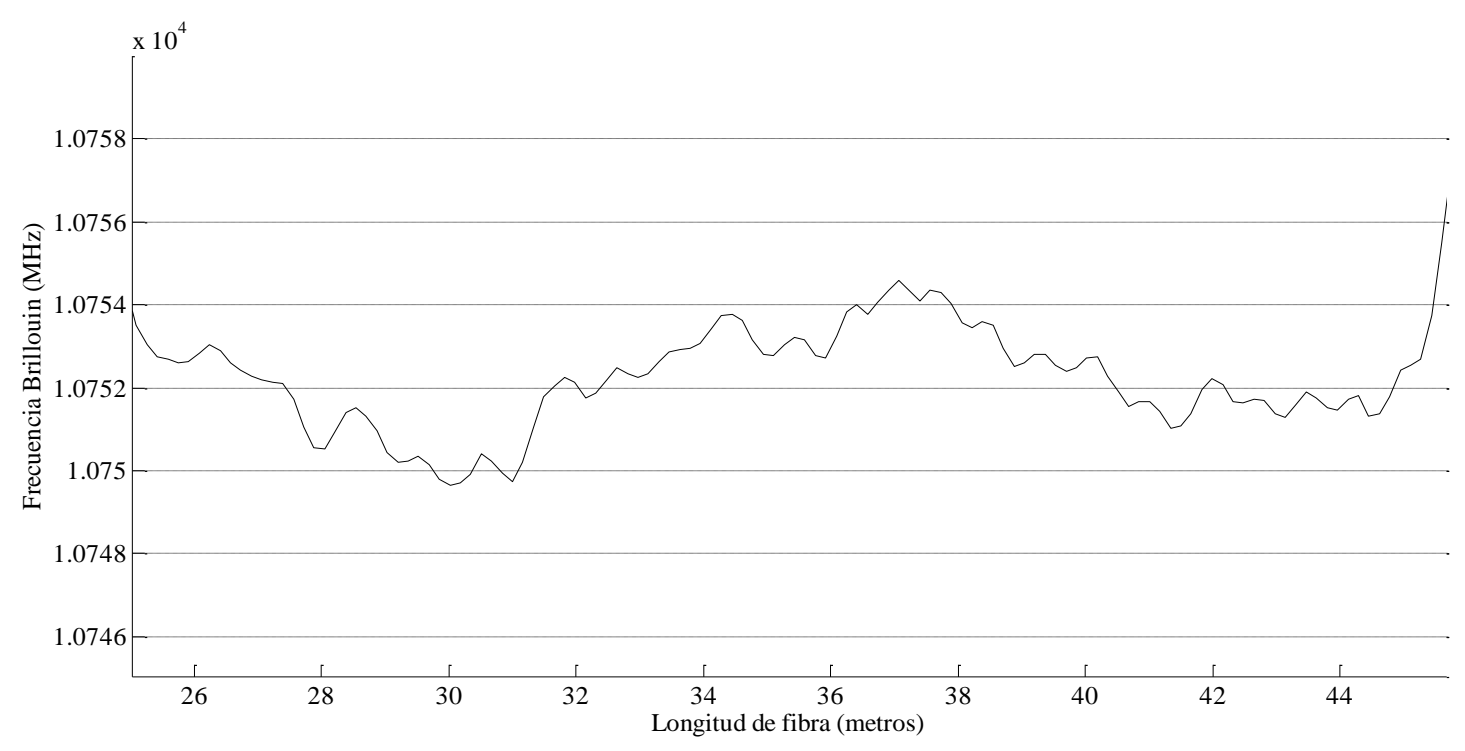


Monitorización estructural.

Aplicación con sensores de fibra óptica.

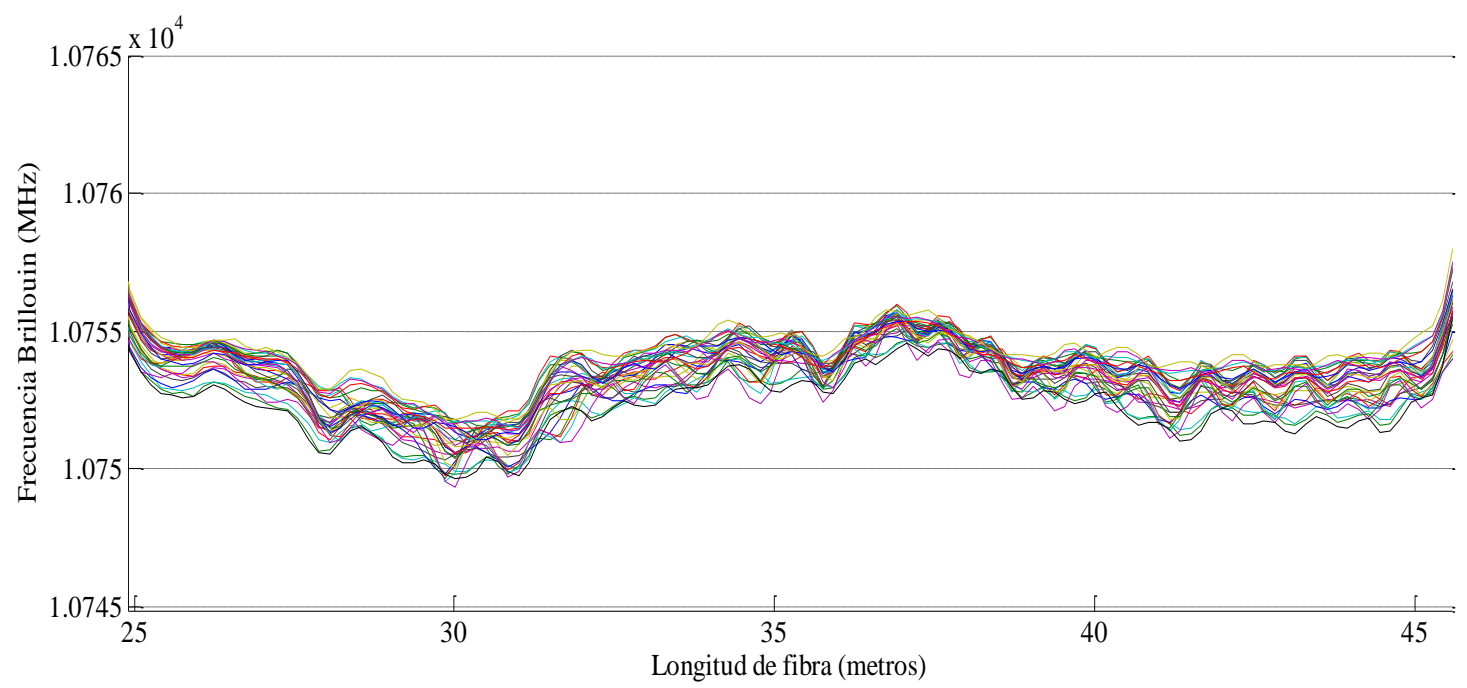

(b)

Figura 8.9 Distribución de la frecuencia de Brillouin en el tramo de fibra C durante el día 26/09/2011.

(a)- primera lectura del día, a las 09:34. (b). Medidas a lo largo del tiempo donde cada traza se corresponde con un instante de tiempo separados 10 minutos.

Tras los resultados de la Figura 8.9 es importante destacar los siguientes aspectos:

- En todos los puntos del tramo $\mathrm{C}$, independientemente de su posición, se produce un aumento de la frecuencia Brillouin. La Figura 8.10 muestra con una traza discontinua y marcadores de color negro la primera medida del día, por lo que esta línea puede considerarse el cero de las medidas. El resto de trazas se encuentran por encima de esta línea, lo que supone un aumento de la frecuencia de Brillouin en el tiempo (cada traza de color se corresponde con una medida separada 10 minutos). Esto indica que la bóveda del falso túnel sufre deformaciones de tracción, tal y como se presentó en el Capítulo 6.

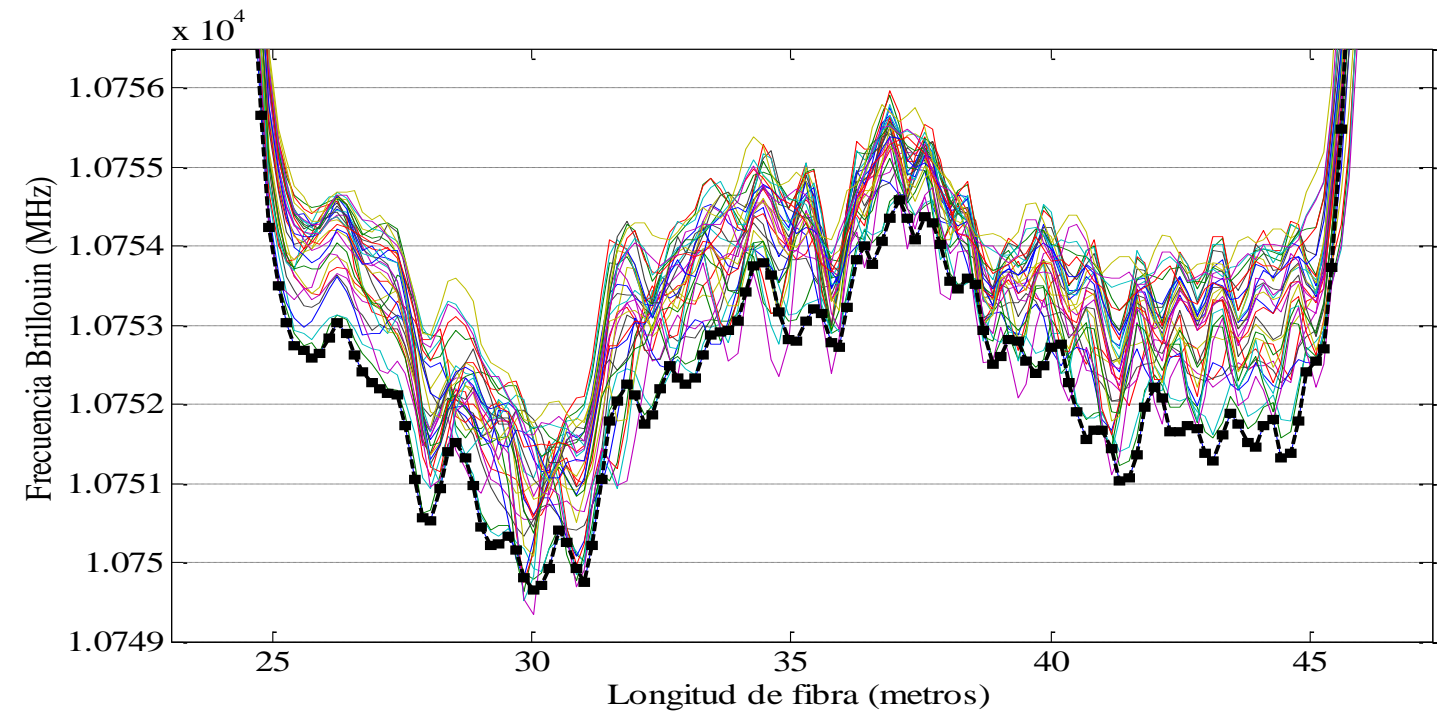

Figura 8.10 Evolución de la frecuencia Brillouin para el tramo de fibra C durante la jornada del 26/09/2011. En traza discontinua y marcadores de color se muestra la primera toma del día. 
- Las variaciones de la frecuencia de Brillouin en el primer instante del día, dadas por la traza discontinua y marcadores de color negro muestra un comportamiento variable (amplitud máxima $4 \mathrm{MHz}$ aproximadamente) a lo largo de la longitud de la fibra. Esto indica que la fibra tiene una pre-deformación que normalmente suele ir ligada al proceso de instalación de la fibra sobre la estructura.

Cada uno de los sensores expuestos en el Capítulo 6 han sido identificados sobre la longitud de la fibra óptica, y se han comparado los resultados que fueron expuestos en el Capítulo 6 (teóricos, sensores de longitud y sensores puntuales) con los resultados obtenidos a partir de los sensores distribuidos expuestos en este capítulo. Sólo ha sido posible la comparación de los sensores 2, 3, 4, 5 y 6 ya que el tramo $\mathrm{C}$ de fibra distribuida sólo alcanza esos sensores (Figura 8.11.a y Figura 8.11.b)

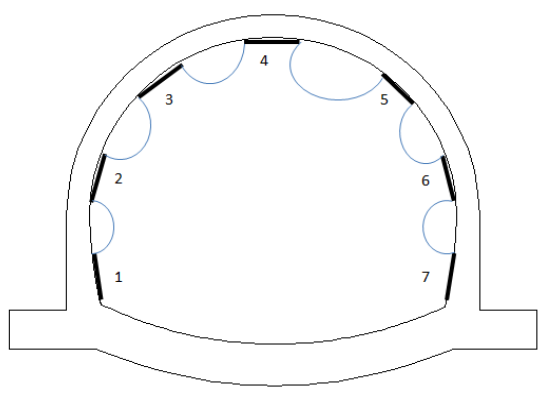

(a)

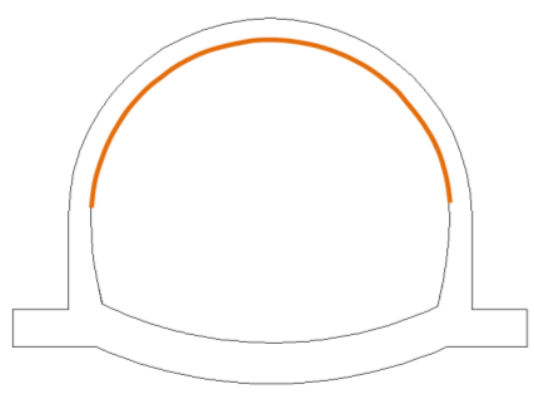

(b)

Figura 8.11 Ubicación de los sensores. (a)- Sensores de longitud. (b)- Sensor distribuido, tramo C de fibra.

Las Figuras 8.12, 8.13, 8.14, 8.15 y 8.16 muestran las comparaciones de los resultados para los sensores descritos.

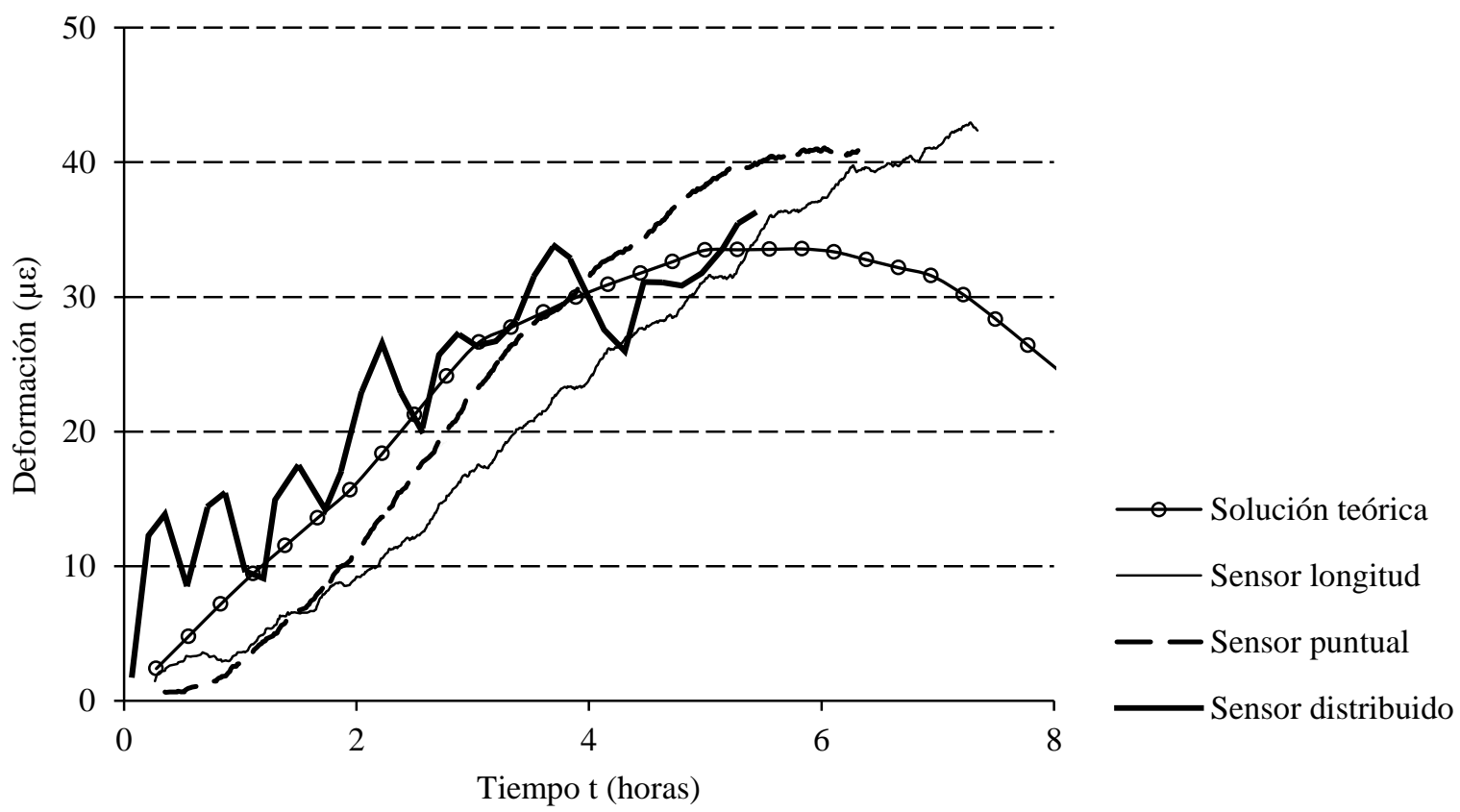

Figura 8.12 Evolución de las deformaciones para la posición 2. Comparación entre la solución teórica, sensores puntuales, sensores de longitud y sensores distribuidos. 
Monitorización estructural.

Aplicación con sensores de fibra óptica.

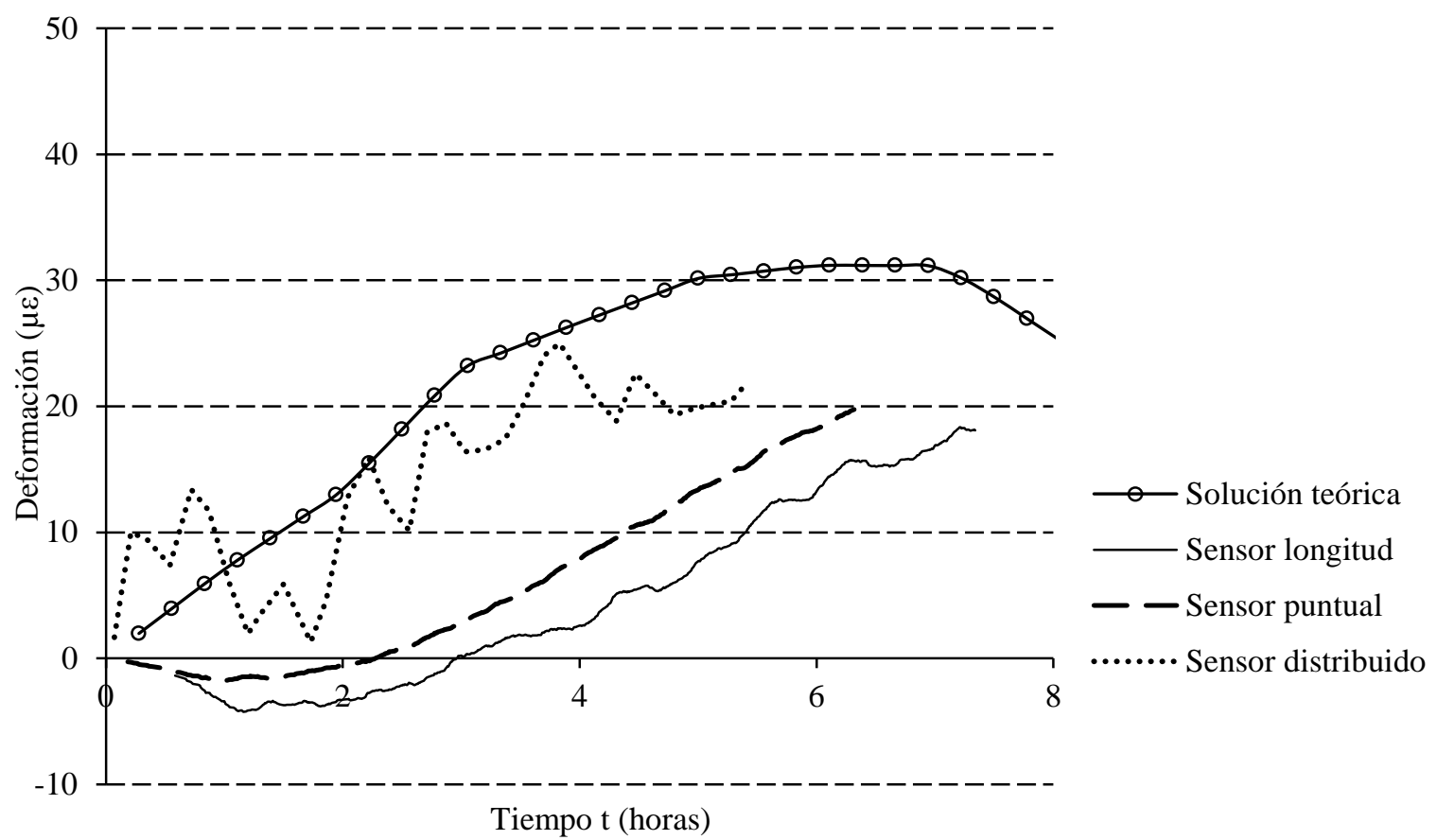

Figura 8.13 Evolución de las deformaciones para la posición 3. Comparación entre la solución teórica, sensores puntuales, sensores de longitud y sensores distribuidos.

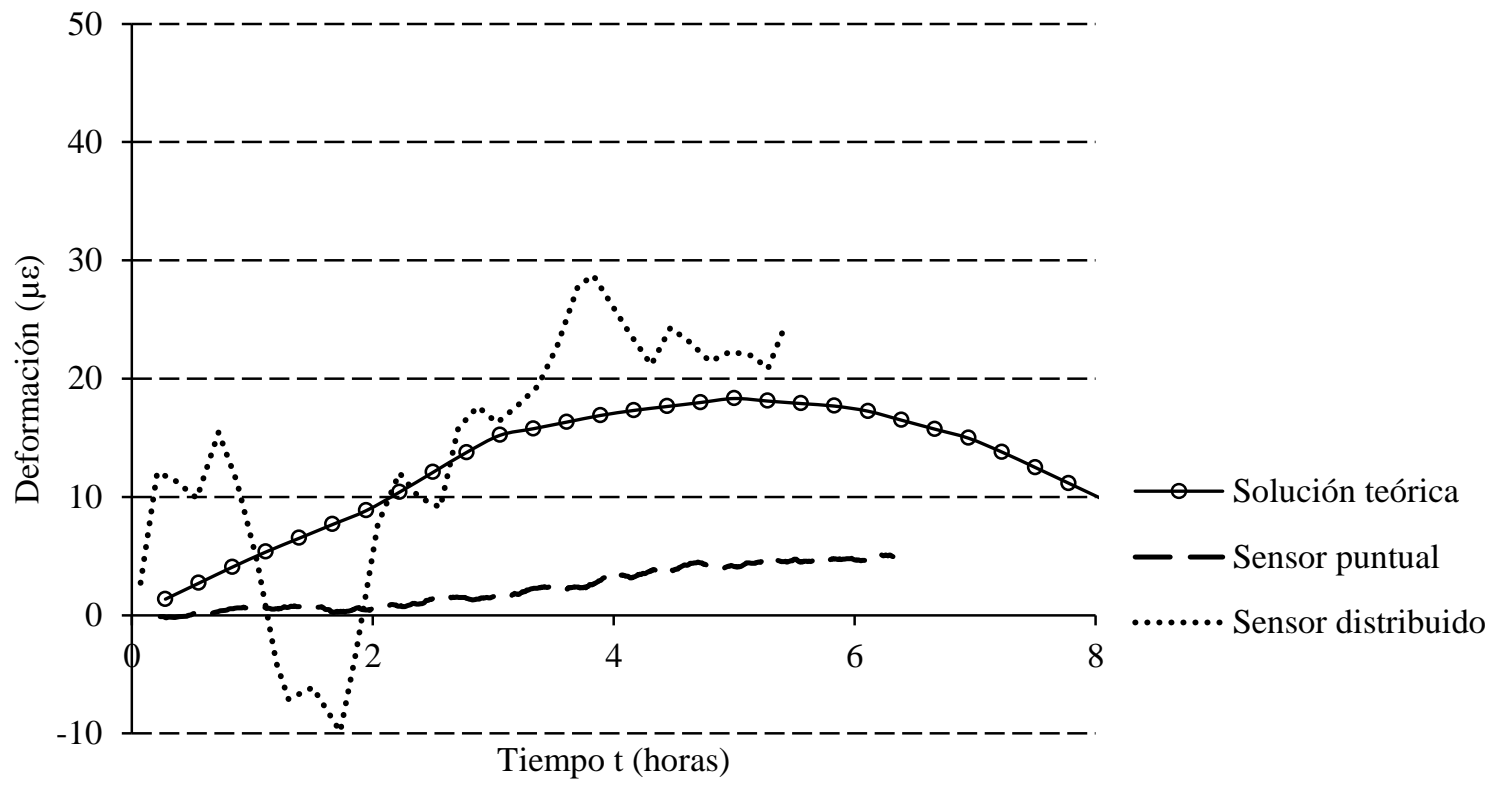

Figura 8.14 Evolución de las deformaciones para la posición 4. Comparación entre la solución teórica, sensores puntuales, sensores de longitud y sensores distribuidos. 


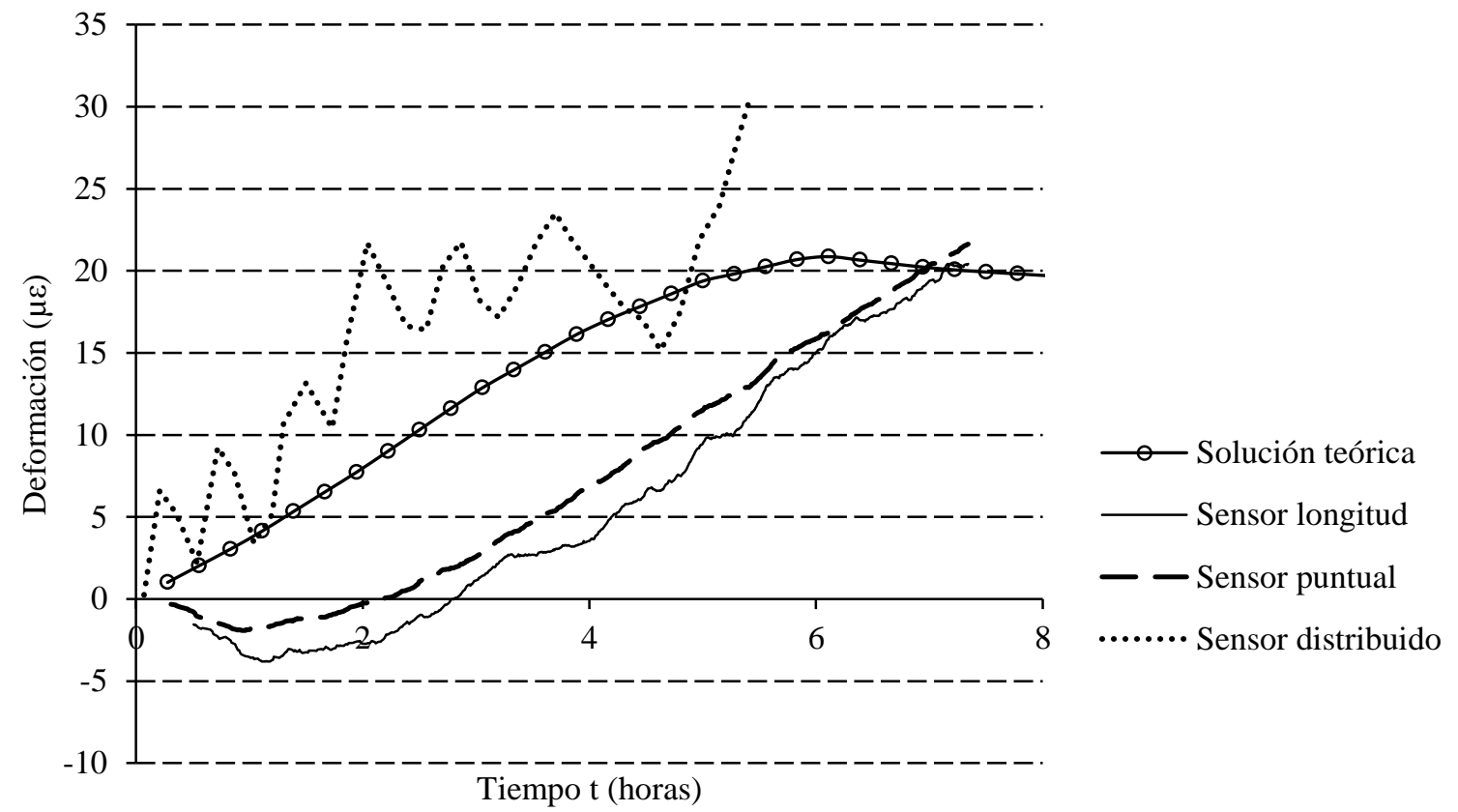

Figura 8.15 Evolución de las deformaciones para la posición 5. Comparación entre la solución teórica, sensores puntuales, sensores de longitud y sensores distribuidos.

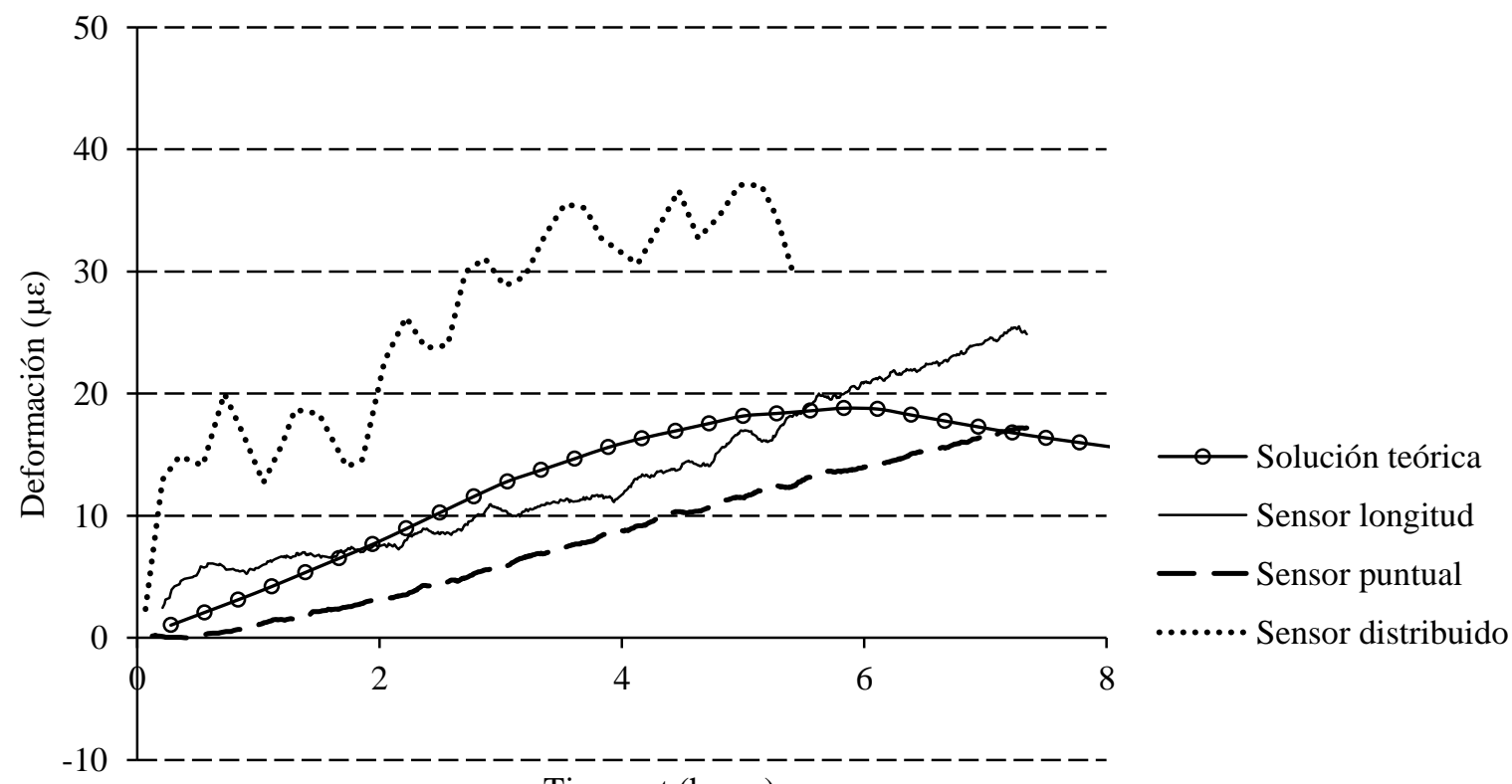

Tiempo t (horas)

Figura 8.16 Evolución de las deformaciones para la posición 6. Comparación entre la solución teórica, sensores puntuales, sensores de longitud y sensores distribuidos. 
Es importante señalar una serie de aspectos que se deducen de los resultados obtenidos:

- En los cinco puntos estudiados, los sensores distribuidos reproducen notablemente bien la pauta general de comportamiento que se presentó en el Capítulo 6 (Resultados teóricos del modelo numérico simplificado, sensores puntuales y sensores de longitud). En todos los puntos estudiados, los sensores distribuidos proporcionan deformaciones de tracción que tienen una tendencia creciente con el paso del tiempo.

- Como se observa, los resultados obtenidos con los sensores distribuidos presentan una mayor dispersión que los obtenidos con los sensores puntuales y de longitud. Hay que tener en cuenta que los sensores puntuales y de longitud están basados en FBG's y por tanto presentan una mayor precisión que los distribuidos. Por el contrario, los sensores distribuidos presentan una precisión del orden $20 \mu \varepsilon$ (Glisic e Inaudi, 2007), siendo éste uno de los motivos de su mayor dispersión.

- Los resultados de algunos de estos puntos estudiados presentan una mayor dispersión en comparación con los resultados proporcionados en el Capítulo 6. En cualquier caso, es importante destacar que con estos niveles de deformación tan pequeños, es esperable que existan diferencias en las medidas empleando distintas técnicas, sobre todo si una de ellas presenta una precisión del orden de $20 \mu \varepsilon$.

- Además es importante señalar que, al igual que en el Capítulo 7, la distancia de resolución empleada es de 1 metro y por tanto, los valores que se muestran aquí son el resultado de la integración en un metro de longitud de fibra. Resulta evidente que existan diferencias en las medidas obtenidas, ya que se están comparando medidas puntuales (fuertemente influenciadas por la posición), medidas con sensores de longitud y distribuidos (que integran las medidas que tienen lugar en una cierta distancia).

- Es importante recordar que sobre estos valores obtenidos sería necesario realizar una compensación térmica, pero ésta no ha sido posible debido a los resultados obtenidos en el apartado 8.3.1.a. Cabría esperar unos mejores resultados de los sensores distribuidos en caso de realizarse una correcta compensación térmica. Sin embargo, es de esperar que las variaciones térmicas sobre el tramo $\mathrm{C}$ de fibra tengan poca importancia en la compensación térmica, según los resultados obtenidos en el apartado 8.3.1.a.

\subsection{Conclusiones.}

En el Capítulo 6 se presentó los trabajos de monitorización de la estructura del falso túnel de la línea de Alta Velocidad de Levante a su paso por Mogente (Valencia) empleando sensores puntuales y de longitud de fibra óptica. En este Capítulo 8 se han mostrado los resultados obtenidos de la monitorización de esta estructura a partir de sensores ópticos distribuidos.

La tecnología distribuida se trata de un sistema de sensado óptico continuo que presenta multitud de ventajas en determinados escenarios. Su mayor rendimiento se alcanza en elevadas longitudes de monitorización y donde la precisión en la medida no sea un factor clave, dado que aproximadamente, la precisión de este sistema se encuentra en torno a los $20 \mu \varepsilon$, como además se indica en distintas publicaciones (Glisic e Inaudi, 2007). 
Los resultados que se han obtenido en este capítulo han permitido comprobar el funcionamiento de esta tecnología en la monitorización de una obra real, y teniendo en cuenta las limitaciones en cuanto a la precisión del sistema, en general los sensores distribuidos han reproducido notablemente bien la pauta general de comportamiento del falso túnel.

Por un lado se han monitorizado los incrementos de temperatura con el objetivo de llevar a cabo una posterior compensación térmica de las medidas. Sin embargo, ésta no ha sido posible debido a la instalación realizada de la fibra óptica. A partir de esta instalación, las mediciones de esta fibra óptica se vieron alteradas por los movimientos provocados por las corrientes de aire en el interior del falso túnel. Teniendo en cuenta esto, la fibra de temperatura (tramo B) no ha medido correctamente la temperatura a la que está sometida la fibra encargada de medir la deformación (tramo C) y no ha sido posible realizar una compensación térmica.

La solución al problema existente pasa por instalar la fibra óptica en contacto con el hormigón mediante una cinta adhesiva a lo largo de toda la longitud de la bóveda, sin tramos colgantes. De esta forma, esta fibra óptica se encontrará aislada de la deformación de la estructura, no sufrirá movimientos que modifiquen su medición, y la medida obtenida reproducirá notablemente bien las variaciones de temperatura en el hormigón. Es importante que ambas fibras ópticas (tramo B y C) se encuentren muy próximas entre sí.

Por otro lado, se han medido las deformaciones a lo largo de la bóveda de la estructura. A pesar de tener mucha menor precisión, los resultados de los sensores distribuidos han reproducido notablemente bien la pauta general de comportamiento expuesta en el Capítulo 6. En caso de realizarse una compensación térmica al tramo de fibra $\mathrm{C}$, cabría esperar una mejor aproximación de los resultados obtenidos.

A pesar de que con el empleo de sensores distribuidos se ha podido obtener la pauta general de comportamiento de la estructura, no es recomendable el empleo esta tecnología en aquellos trabajos de monitorización donde las deformaciones esperadas sean relativamente pequeñas. Por este motivo, el empleo de los sensores distribuidos ha alcanzado una gran aplicación en estructuras de grandes longitudes de monitorización como tuberías o puentes. Además, el empleo de este tipo de sensores es muy recomendable en situaciones de detección de fallos como sistema de alarma sin necesidad de grandes precisiones en sus mediciones. 



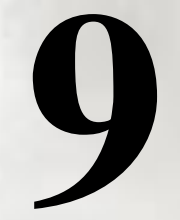

\section{ConCLUSIONS AND FUTURE RESEARCH.}

In this chapter the main conclusions of this $\mathrm{PhD}$ Thesis, its scientific production as well as some future research areas will be presented. 



\subsection{General conclusions.}

Material strain is one the most significant measurable parameters in Structural Health Monitoring. Strain sensors have, therefore, a particular importance in structural monitoring. They must be accurate enough to obtain significant data and reliable enough in order to ensure that their readings can be trusted. The measurement of temperature variations is also of capital importance in Structural monitoring. They affect not only structural stresses and strains but also its measurement systems. Temperature variations can alter significantly the strain sensors readings.

In this $\mathrm{PhD}$ Thesis the behavior of different types of optical sensors to measure strains and temperatures in structures has been studied as well as the conditions they should fulfill to provide accurate results.

The design of the strain sensor and the conditions for its installation in the structure are particularly important factors in order to obtain accurate results. On the other side, the design and installation conditions of temperature sensors are much simpler. In this case, the measuring optical fiber must be isolated mechanically from the structure, so that the variations measured by the sensor are due only to thermal variations.

The design and installation of strain sensors in a structure is a complex task that requires a special study in which the following topics need to be addressed:

- Study of the interaction of the sensors with the structure.

- Ensure a proper strain transfer between the structure and the measuring optical fiber.

- Consider the existence of strain variations happening in materials such as concrete due to cracking materials by using long gauge or distributed sensors.

Very little research has been undertaken related to the above mentioned topics despite its importance which justifies the studies carried out in this $\mathrm{PhD}$ Thesis.

This $\mathrm{PhD}$ Thesis starts with the study and the design of a point strain optical sensor based on FBG's. As indicated in some publications (Glisic and Inaudi, 1997), this type of sensor is suitable for the monitoring of structures built with homogeneous materials such as steel or built with composite materials if the sensor is placed in the direction of the composite fibers.

Many experimental tests have been carried out on concrete specimens to validate the performance of the proposed point strain optical sensors. These tests included compression tests where the measurements provided by the proposed sensor and strain gauges were in excellent agreement showing that it is possible to monitor concrete elements with point sensors if concrete works in compression. This is due to the absence of cracks during the loading process. These point sensors were also installed in a high speed railway tunnel structure near Mogente (Valencia). Results given by the point sensors were also in an excellent agreement with those obtained with other types of sensors and theoretical results (finite element models -FEM- of the structure), even if the vault of the structure was subjected to tensile stresses due to the existing temperature increases. This was explained because the strain level reached in the vault did not exceed the value of the cracking ultimate strain, estimated about 50-100 $\mu \varepsilon$ (Glisic and Inaudi, 2007). Therefore, it is possible say that point sensors should be 
applied to homogeneous materials such as steel, but can also be used in concrete structures submitted to compression stresses or to tensile strains below the cracking limit.

Sensor packaging is another important topic. Research conducted here has proved that the position of the optical fiber should be as close as possible to the surface of the monitored structure. Within this context, two topics deserve special attention:

(1) The thickness of the layer of material between the structure and the fiber. It is very important that the packaging of the sensors enables the optical fiber be as close as possible to the host structure. The point sensor developed for and presented in this $\mathrm{PhD}$ Thesis has a single layer packaging, so that the optical fiber is not embedded between two layers of material and only adhesive exists between optical fiber and structure. FEM studies carried out have proved the great accuracy of the proposed point sensor even if the sensor is installed deficiently (with a very thick layer of adhesive). The proposed strain sensor has been patented (patent with publication number 2329752 and called "optical sensor for structural monitoring").

(2) The mechanical properties of the adhesive used. It has been proved that these properties are very important to ensure accurate measurements. It is important that the adhesive has high values of transverse stiffness Modulus $G$ for improving strain transfer, or what is the same, high Young's Modulus values E and small Poisson's ratio values v.

The PhD Thesis continues with the design of a new long gauge strain fiber optic sensor based on FBG's to be installed in the surface of a monitored structure.

The use of long gauge sensors is necessary in non homogeneous material with irregularities or point defects such as cracks or air pockets, in order to avoid that these irregularities affect the sensor measurement. Currently, the design and operation of most of the commercial long gauge sensors that are being used in structural monitoring are based on the prestressing of the optical fiber which is attached to the host structure. However, prestressing is an operation with some degree of uncertainity because a prestressing level must be chosen and, sometimes, this level is difficult to define.

The design proposed in this $\mathrm{PhD}$ Thesis for a long gauge sensor does not require the prestressing of the optical fiber. The optical fiber is installed in a linear element and this element is anchored at two points of the host structure. To ensure the proper performance of the sensor, the following aspects should be considered:

(1) Anchor design must enable a good strain transfer between the host structure and the sensor. To reach this goal, the adhesive used must have a high Young Modulus. There are resins and adhesives that, despite being used in structural repairs, do not fulfill this condition and, therefore, should not be used to fix the sensor to the structure.

(2) The element supporting the optical fiber must not have a high EA value (E Young Modulus, A cross-sectional area). High EA values provoke a local modification of the strain field of the structural member and therefore a discrepancy between the actual and the sensor measured strain values. Furthermore, it has been observed that, given an EA value, sensors with greater length are more accurate.

(3) Since the proposed long gauge sensor might be subjected to compression stresses, it is necessary to avoid buckling. In this regard, this $\mathrm{PhD}$ Thesis has studied an element that inhibits this instability. Its effect causes an increase in the value of the flexural stiffness EI (E Young Modulus, I inertia moment) without changing the EA value. 
The performance of these long gauge sensors has been tested in experimental tests and in real worksite conditions (structural monitoring of a high speed railway tunnel near Mogente in Valencia).

Also, this long gauge design provides the basis for the development of a new long gauge sensor to measure strains and temperatures under high temperature conditions.

Finally, the PhD Thesis has studied the application of distributed optical sensors based on Brillouin scattering. These sensors provide data along the optical fiber length. Its main application consists on damage detection such as concrete cracking or pipes outflow.

Unlike previous sensors that are based on FBG's, this sensor detects the Brillouin frequency shift along the optical fiber length. For its structural engineering application, this Brillouin frequency shift is calibrated with temperature and strain.

This sensor has also been used in experimental tests and in the structural monitoring of a high speed railway tunnel near Mogente (Valencia). An optical fiber was installed around the perimeter of the tunnel vault, and measurements given by the sensor were compared to point and long gauge sensors results and tp results given by FEM analyses.

It is important to take into account some considerations related to the installation of the distributed optical sensor. First, the installation process is more complex than in the case of point or long gauge sensors. In this case, the optical fiber must be bonded along its entire length, making the installation operation difficult. This aspect should be carefully respected, because the sensor will give inaccurate measurements in areas where the fiber is not perfectly attached to the host structure.

Regarding the implementation of the sensor and the data recorded, it should be noted that these data are the integration in a distance, named resolution distance. This distance has been 1 meter (optical pulse duration used in the experimental works was 10 nanoseconds). This means that the values obtained are the integration of measures in 1 meter of optical fiber. It should be noted that this is not a problem for this type of optical sensing, given the main application of this type of sensors (long-span bridges, pipelines, cables ...). The results presented here coming from the distributed optical sensor were in a good agreement with those provided by other measurements systems.

As expected, the results obtained with this technology have a greater dispersion than those obtained with sensors based on FBG's. However, these results have shown the general pattern of the behavior of the structure due to thermal variations. Despite this, it is not recommended to use this technology in structural monitoring applications where the expected strain value is relatively small. This explains why the use of distributed sensors has a broad application in large structures such as pipes or long span bridges.

\subsection{Specific conclusions.}

As noted in section 9.1, in this $\mathrm{PhD}$ Thesis the design of new fiber optic sensors to measure strains and temperatures and their applications in Structural Health Monitoring has been studied.

This section presents the main specific conclusions obtained in each chapter of this PhD Thesis. Thus, the next sections will present:

- Section 9.2.1. Main conclusions related to the study and design of the point fiber optic sensor presented in Chapter 4. 
- Section 9.2.2. Main conclusions related to the study and design of a new long gauge fiber optic sensor presented in Chapter 5.

- Section 9.2.3. Main conclusions related to the application of these sensors to monitor a high railway tunnel near from Mogente (Valencia).

- Section 9.2.4. Main conclusions related to the study and calibration of distribuited Brillouin sensors.

- Section 9.2.5. Main conclusions related to the application of distribuited Brillouin sensors to monitor a high railway tunnel near from Mogente (Valencia).

\subsubsection{Main conclusions related to the study and design of the point fiber optic sensor presented in Chapter 4.}

In this chapter the main aspects of the design of the sensor packaging of the readings are studied, as well as the way in which they affect their accuracy and reliability.

- A sensor packaging for an FBG fiber optic point sensor has been developed and presented.

- The proposed sensor packaging has a single layer, so the optic fiber is practically in contact with the adhesive used to fix the sensor to the monitored surface.

- The readings of the optical sensor presented are hardly affected by the thickness of adhesive used to fix the device to the structure. Normal adhesive thickness values of 400 $\mu \mathrm{m}$ lead to reading errors below $2.5 \%$. Unusually high adhesive thickness values of around $1000 \mu \mathrm{m}$ yield a reading error below $6.5 \%$.

- To improve strain transfer is important that the adhesive has high values of the shear modulus G. This parameter depends on the Young's Modulus E and Poisson's ratio $v_{A}$. Since the Young's Modulus E is a known value (Table 4.1), the largest uncertainty occurs in the Poisson's ratio and for this reason a sensitivity study of this parameter has been studied.

- Low values (of the order of 0.1) of the adhesive Poisson's ratio $v_{A}$ improve strain transfer due to the higher value of the shear modulus G. On the other hand, high $v_{A}$ values cause greater errors in sensors readings. In all cases, and in the most unfavorable conditions $\left(\mathrm{t}=1000 \mu \mathrm{m}, v_{A}=0.4\right)$ maximum sensor error is below $6.5 \%$.

- The presence of a packaging layer below the sensor has a negative influence on its accuracy. For example, a layer of around $400 \mu \mathrm{m}$ thick can induce errors of up to $15 \%$. The precision of the two-layer sensor is thus highly sensitive to both manufacturing tolerances and to variations in packaging layer properties, e.g. changes in Poisson's ratio caused by temperature variations. This indicates that is preferable to use single-layer packaging, as in the model proposed in this Thesis.

- The importance of packaging temperature changes on the spectrum of the light reflected by the sensor has too been studied. The use of an appropriate packaging material was observed to enable the reflected light spectrum to maintain its shape during thermal variation cycles. 


\subsubsection{Main conclusions related to the study and design of the new long gauge fiber optic sensor presented in Chapter 5.}

Before the main conclusions of the chapter are presented, it is important to show that the scope of work and its results are based on the following considerations:

- The results obtained in this Chapter are applicable for the monitoring of hardened concrete structures.

- These results are valid for the range of elastic modulus in a hardened concrete. In this study we have tested concrete with Young's modulus values between 29450 and $34116 \mathrm{MPa}$ (even though its conclusions may be extended a bit further).

- These results are valid for usual dimensions used in concrete columns. In this study we have tested specimens with a square base with minimum and maximum dimensions of $0.1 \mathrm{~m}$ and $0.3 \mathrm{~m}$, respectively.

Once the scope of the work has been established, the main conclusions of the chapter can be enunciated:

- In this section the design of a new long gauge fiber optic sensor to measure strain in structures has been proposed. This new design has the particularity that the optical fiber does not need to be pretressed, avoiding this delicate and uncertain operation.

- In order to ensure a proper behavior, the packaging of the sensor must fulfill the conditions enunciated below.

- The design of the sensor anchoring pieces on the structure must allow a correct strain transfer between the host material (structure) and the sensor. In this section, the properties of the adhesive used have been studied, and the range of values that provide satisfactory results is given. It is shown that an adhesive with a Young's Modulus $E_{A}$ exceeding 400MPa will allow good strain transfer with ratios $\varepsilon_{S} / \varepsilon_{c}$ close to 1 . On the other side, $E_{A}$ values around $100 \mathrm{MPa}$ will provide differences between the host structure strains and the ones measured by the sensor of around $20 \%$.

- Low sensor $E_{m} A_{m}$ values will improve the strain measurement. High $E_{m} A_{m}$ values will give incorrect results because the sensors will oppose to the structure strain. For the concrete elements studied, $E_{m} A_{m}$ values below $5 \mathrm{MN}$ provide differences between host structure and measured strains of less than $4 \%$.

- The length of the sensor also influences the readings. As shown is Figure 5.12, the influence of the sensor $E_{m} A_{m}$ is less for sensors with longer lengths. For example, the ratio between concrete strain before installing the sensor and concrete strain once the sensor has been installed, ${ }^{\varepsilon_{C, 0}} / \varepsilon_{C}$, is around 0.89 when a $20 \mathrm{~cm}$ long and $20 \mathrm{MN} E_{m} A_{m}$ sensor is used,. It represents a measurement error around $11 \%$. By contrast, a ratio ${ }^{\varepsilon_{C, 0}} / \varepsilon_{C}$ of about 0.96 is obtained for the same sensor $\left(E_{m} A_{m}\right.$ value of $20 \mathrm{MN}$, but with a length of $60 \mathrm{~cm}$ (estimated measurement error of $4 \%$ ). 
- This study has not considered the relationship between the mechanical and geometrical properties of the proposed sensor with the host material Young's Modulus. However, the application ranges of the results have been obtained taking into account the scope of work (see comments above).

- On the other hand, high values of $E_{m} A_{m}$ cause high shear stresses in the anchor between sensor and structure, that may even cause the failure of the anchor. To avoid this failure mode, the use of a sensor with a $E_{m} A_{m}$ value below $700 \mathrm{KN}$, and the increase of the roughness in the contact between sensor and structure has been proposed.

- Since the sensor proposed can be subjected to compression, another possible failure mode is the sensor buckling. To avoid it, the use of an element that inhibits this instability has been proposed. This element will increase the $E_{m} I_{m}$ product but not the $E_{m} A_{m}$ value. A longitudinal element with a flexural stiffness $E_{m} I_{m}$ of $1 \mathrm{KNm}^{2}$ should solve this failure mode for sensors up to 1 meter length.

- Therefore, the proposed sensor design has the following characteristics:

Prismatic element with a cross section of $0.1 \mathrm{~cm}^{2}$.

$E_{m} A_{m}$ value of $700 \mathrm{KN}$.

$E_{m} I_{m}$ value of $58.310^{-6} \mathrm{KN} \mathrm{m}^{2}$. (buckling inhibitor not included)

Adhesive Young's Modulus value of $13000 \mathrm{MPa}$.

Long gauge between 20 and $100 \mathrm{~cm}$.

\subsubsection{Main conclusions related to the application of these sensors (point and long gauge sensors) to monitor a high railway tunnel near from Mogente (Valencia).}

The Structural Health Monitoring of a cross section high railway tunnel have been presented in this chapter.

Sensors have been installed around the perimeter of a tunnel cross section. Temperature, point and long gauge and distributed strain sensors have been used in order to check their validity and compare their behavior. The main goal of this work has been the validation of the performance and application of these sensors under real working conditions. A simplified 2D numerical model has been developed in order to compare the expected general pattern of behavior with the pattern of behavior indicated by the sensors.

The main conclusions of this chapter are presented below:

- Two 0.6 and $1.0 \mathrm{~m}$ long gauge strain sensors were installed in position named as "2". The results of both sensors have shown a good agreement between them.

- Temperature monitoring is very important. Temperature has an important influence in measurement systems, and it must be compensated in order to obtain good results. In this work the temperature effects have been compensated through the installation of one sensor that is anchored at one end and free at the other. In this application, this sensor is installed in the key of the structure. It is recommended that in future works more thermal compensation sensors be used in order to allow more accurate temperature compensation. Another 
possibility would be to calibrate this sensor response versus temperature variations for direct application in thermal compensation.

- During September 26 and 27, the structure was subjected to significant temperature changes between the outside and inside of the vault, caused by differences in daily and night temperatures. The maximum measured temperature change has been located in the left outer face, being about $18.17^{\circ} \mathrm{C}$. The measured time-temperature laws have been adjusted using linear and second and third parabolic equations, with an excellent degree of fit.

- The behavior of the structure has been proportional to these temperature changes. The maximum strain measured by the sensors have been of approximately $40 \mu \varepsilon$ during temperature increases and $-15 \mu \varepsilon$ when in compression. This behavior has been compared to the general pattern obtained using a 2D simplified numerical finite element model. In general, the sensors have reproduced notably well the expected general pattern of structure behavior.

- With low strains levels, point and long gauge sensors have reproduced notably well the general behavior of the structure. The tensile strain values were lower than the ultimate craking strain of the concrete (estimated about 50-100 $\mu \varepsilon$ ). For this reason, point and long gauge sensors have shown similar results, although with the logical differences motivated by the heterogeneity of the material.

- During the continuous data recording at this study, it has been observed an asymmetric behavior of the vault of the tunnel, due to temperature changes between outside and inside faces. While the sensors on the left side have measured maximum strains about $40 \mu \varepsilon$, sensors on the right side have shown around $25 \mu \varepsilon$.

- Once the landfill was completed, new instantaneous measurements have been carried out (on May $\left.14^{\text {th }}\right)$. In this situation, measured and theoretical results have shown that the structure has a certain symmetry in their behavior. For these measurements, there are major differences between long gauge and point sensors. In this way, it is important to highlight that the point sensors are strongly influenced by local defects of the material. However, the patterns of behavior observed in both types of sensors show a good agreement.

- Measurements on 14/05/2012 have shown that the tunnel was compressed with respect to the initial data collection on 26/09/2011. This behavior was also shown by the simplified numerical finite element model, except in the key of the structure, where the FEM model predicted small tensile stresses of around $16 \mu \varepsilon$.

- Some optical sensors have shown an anomalous behavior, like the sensor located at position 7 during data recording at 14/05/2012. The most likely cause for this result is that the sensor was damaged during construction of the sidewalks. Likewise, point sensors located at position 1 and 6 were broken during these works. These facts indicate the importance of greater protection of the sensor network during the construction phase.

- In general, both point and long gauge sensors gave sets of readings that were consistent with the expected behavior of the structure: 
a. In general it has been shown the feasability and conditions to use point and long gauge sensors designed under work conditions. It has been found that they are able to measure reliably even very small strains

b. It was found that, although some protections need to be improved, the sensors have resisted generally quite well the aggressive conditions imposed by a construction site.

c. Although is not recommended the use of point sensors for the monitorization of heterogeneous materials, when these elements work in compression it would be possible to use it. However, it is very important to avoid their installation in repaired areas and other irregularities because their measurements could be significantly altered.

\subsubsection{Main conclusions related to the study and calibration of distribuited Brillouin sensors.}

In this section, the use of distributed fiber optic sensors for structural monitoring has been studied. The sensor used measures the Brillouin Frequency Shift (BFS) reflected by the optical fiber to obtain temperature and strain. This technique offer advantages over other techniques in some scenarios, and its better performance is achieved in large distances such as pipes or large span bridges that do not require high precision in resolution measurements.

Temperature calibration has been carried out by subjecting the fiber to different thermal cycles. It has been observed that the relationship between BFS and temperature changes has been linear with an excellent fit. After some tests, a sensitivity of $1.25 \mathrm{MHz} /{ }^{\circ} \mathrm{C}$ has been adopted. Also, there aren't hysteresis cycles problems associated with temperature changes (increases and decreases).

Strain measurement has been calibrated through compression tests on a concrete column with a square cross secton of 0.2 meters side and 1 meter long, where distributed sensors were installed. The BFS shows a linear relationship between the applied load and the strain measured. The strain sensitivities are dependent on the type of fiber optic used because BFS is different in each optical fiber. The average values for Sumitomo, smf-28 (UPV) and SMF-28 (UPNA) were respectively 0.0258, 0.0273 and $0.0261 \mathrm{MHz} / \mu \varepsilon$.

In general, these values are lower than the reference sensitivity of the fiber SMF-28 (UPNA) $(\sim 0.04$ $\mathrm{MHz} /(\mu \varepsilon)$ ) because the length of optical fiber that was subjected to strain is $70 \mathrm{~cm}$, while the Brillouin sensor spatial resolution is approximately $100 \mathrm{~cm}$. Therefore, strain measurements are integrated into $100 \mathrm{~cm}$, but only $70 \mathrm{~cm}$ are subjected to strain.

The following conclusions can be highlighted from the tests:

- There were some differences between measurements from different types of fibers. One reason for these small differences is that the load on the specimen was not perfectly centered, so there was a small strain variation within the concrete section.

- Within the load cycle carried out during the tests the load was kept stable during a long time to make measurements and perform an average. This average value has been taken as the representative value of the reading. The use of these average values fitted correctly the strains in the concrete. 
- It is known that distributed Brillouin systems accuracy is smaller than FBG sensors accuracy (( $\sim 20 \mu \varepsilon$ versus $\sim 1 \mu \varepsilon$, Glisic and Inaudi, 2007). However, this degree of accuracy is more than enough when measuring high strain levels, that can be measured correctly with type of sensors.

- When using distributed sensors the installation of the sensor is of crucial importance in order to allow a correct measurement. As shown in Chapter 4, the installation must be performed in such a way that the optical fiber is in contact with the concrete surface. Also, the importance of the adhesive has to be highlighted in order to ensure a good strain transfer between concrete and optical fiber.

The tests performed and the results obtained have shown the validity and the limitations of this technique for the measurement of temperatures and strains in structures.

\subsubsection{Main conclusions related to the application of distribuited Brillouin sensors to monitor a high railway tunnel near from Mogente (Valencia).}

Chapter 8 presents the application of distributed fiber optic sensors for the monitoring of a high speed railway tunnel near Mogente (Valencia). This application has shown the performance of this technology under real construction site conditions, obtaining satisfactory results.

This technology has many advantages in certain scenarios. Their best performance is achieved when monitoring great lengths and where measurement accuracy is not a key factor (accuracy about $20 \mu \varepsilon$; Glisic and Inaudi, 2007).

From the works carried out in this chapter, the following conclusions can be extracted:

- The performance of this technology in a real monitoring process has been verified. Taking into account the limitations of the system (like its accuracy), distributed sensors have reproduced remarkably well the general patterns of behavior of the tunnel.

- Temperature changes in the structure have been monitored in order to carry out a thermal compensation. However, it hasn't been possible because temperature changes are strongly influenced by the position of the fiber. Due to the installation performed, the temperature fiber (section B, Figure 8.2) hasn't measured the temperature in the strain fiber (section C). For this reason it has been impossible to perform a correct thermal compensation.

- To solve this problem, it would be necessary to install optical fiber in contact with the concrete by an adhesive tape along the length of the vault, without hanging sections. Thus, this optical fiber will be isolated from the structure strain. It is important that both optical fibers (section B and C) are very close together.

- Strain has been measured along the tunnel vault. Despite having much less precision, distributed fiber optic sensors results have shown the tunnel structural behavior. The degree of fit between distributed sensor, long gauge sensors, point sensors and theoretical results was quite good. If it would be possible to make a thermal compensation, the results would significantly improve. 
- Although distributed sensors have been able to capture the main patterns of the tunnel behavior, it is not recommended to use this technology in structural monitoring where expected strains are relatively small. For this reason, use of distributed sensors has reached a great application in large structures such as pipes or long span bridges. Moreover, the use of distributed sensors is recommended in situations of failure detection and alarm system without the need for great precision in their measurements.

\subsection{Scientific production.}

As a result of the studies related to this Thesis, some scientific production like journal papers, congress conferences and research projects funded by public or private institutions has been obtained. The main results are shown below.

\subsubsection{Journal Papers.}

Benjamín Torres, Ignacio Payá-Zaforteza, Pedro A. Calderón, Jose M. Adam. "Analysis of the strain transfer in a new FBG sensor for Structural Health Monitoring". Engineering Structures 33 (2011) 539-548.

Antonio Bueno, Benjamín Torres, David Barrera, Pedro Antonio Calderón, José Manuel Lloris, María José López, Salvador Sales. "Optical fiber Sensors embedded in concrete for measurement of temperature in a real fire test". Optical Engineering, Volume 50 (12) SPIE - Dec 1, 2011.

\subsubsection{Conference Papers.}

Authors: Benjamín Torres, Ignacio Payá-Zaforteza, Antonio Bueno, Salvador Sales, Jose Miguel Adam, Pedro Antonio Calderón.

Title: Early age concrete temperature and shrinkage monitoring by using Fiber Bragg Grattings

Type: Paper

Congress: XXXVII IAHS World Congress on Housing Science

Place: Santander, Spain

Date: November, 2010.

Authors: Benjamín Torres, Ignacio Payá-Zaforteza, Antonio Bueno, Salvador Sales, Pedro Antonio Calderón, Raquel López.

Title: Aplicaciones de sensores de fibra óptica desarrollados por la U.P.V. para la medida de deformaciones y temperaturas en estructuras

Type: Paper

Congress: V Congreso de ACHE

Place: Barcelona, Spain

Date: October, 2011.

Authors: Antonio Bueno, Benjamín Torres, David Barrera, Salvador Sales.

Title: Monitoring of a steel incrementally launched bridge construction with strain and temperature FBGs sensors.

Type: Paper

Congress: SPIE Photonics Europe.

Place: Bruselas, Bélgica.

Date: Abril, 2010. 
Authors: Antonio Bueno, Benjamín Torres, David Barrera, Pedro A. Calderón, José Manuel Lloris, María José López, Salvador Sales.

Title: Fiber Bragg grating sensors embedded in concrete samples for a normalized fire test.

Type: Poster

Congress: 21st International Conference on Optical Fiber Sensors.

Place: Ottawa, Canada.

Date: May, 2011.

\subsubsection{Research Projects.}

Table 9.1 summarizes the research projects associated with this PhD Thesis, which have been funded by public or private institutions.

\begin{tabular}{|c|c|c|c|c|}
\hline Project. & Period. & $\begin{array}{l}\text { Funding } \\
\text { institution. }\end{array}$ & $\begin{array}{l}\text { Main } \\
\text { researcher. }\end{array}$ & Budget. \\
\hline $\begin{array}{l}\text { Desarrollo de técnicas de } \\
\text { implantación de sensores ópticos } \\
\text { en estructuras de hormigón } \\
\text { (PPI-05-05-5709-02) }\end{array}$ & $2005-2007$ & $\begin{array}{l}\text { Universidad } \\
\text { Politécnica de } \\
\text { Valencia. }\end{array}$ & $\begin{array}{l}\text { Pedro Antonio } \\
\text { Calderón García. }\end{array}$ & $9.000 €$ \\
\hline $\begin{array}{l}\text { Sensores ópticos aplicados a la } \\
\text { instrumentación de estructuras } \\
\text { para conocimiento de su } \\
\text { comportamiento en tiempo real } \\
\text { (Contrato I+D+i) }\end{array}$ & $2008-2011$ & SACYR S.A. & $\begin{array}{l}\text { Pedro Antonio } \\
\text { Calderón García. }\end{array}$ & $126.358 €$ \\
\hline $\begin{array}{l}\text { Desarrollo de sensores } \\
\text { avanzados de fibra óptica para } \\
\text { la determinación de propiedades } \\
\text { de materiales y salud estructural } \\
\text { (P41/08) }\end{array}$ & 2009-2011 & $\begin{array}{l}\text { Ministerio de } \\
\text { Fomento. }\end{array}$ & $\begin{array}{c}\text { Salvador Sales } \\
\text { Maicas }\end{array}$ & $152.890 €$ \\
\hline $\begin{array}{l}\text { Definición de condiciones de } \\
\text { monitorización, encapsulado y } \\
\text { fijación de sensores ópticos para } \\
\text { medir temperaturas y } \\
\text { deformaciones en estructuras a } \\
\text { altas temperaturas. }\end{array}$ & $2012-2015$ & $\begin{array}{l}\text { Ministerio de } \\
\text { Educación }\end{array}$ & $\begin{array}{l}\text { Ignacio Payá- } \\
\text { Zaforteza }\end{array}$ & $149.907 €$ \\
\hline
\end{tabular}

Table 9.1 Main Research Project Information.

\subsection{New and future research topics.}

Following the development of this $\mathrm{PhD}$ Thesis, there is a clear need for further research related to the structural health monitoring with fiber optic sensors. Some areas where additional research is required are: 
- In this $\mathrm{PhD}$ Thesis many types of sensors for structural monitoring have been studied. Calibration and design were tested in different scenarios, all under ambient temperatures. However, its design is not applicable to the monitoring of structures under high temperatures as it is the case of structures submitted to fire. In this regard, a new research topic to design strain and temperature sensors in the event of a fire has recently started (Table 9.1, section 9.3.3). The first experimental tests have already been made and results were satisfactory.

- Installing sensors during the construction has many advantages such as the possibility to place the sensors embedded in concrete. This means it would be possible to carry out a structural evaluation of the host structure at any moment of its lifecycle. However, this is not the case when the sensors were installed after construction of the structure. For this reason, it would be interesting to study a method or procedure for structural evaluation of existing structures monitored after its construction. Such a procedure would need an evaluation of the initial state of the structure (prior to its monitoring) 


\section{REFERENCIAS BIBLIOGRÁFICAS}

Adam Martínez JM (2007). "Contribución al estudio de soportes de hormigón armado reforzados con angulares y presillas metálicas. Análisis del soporte y del nudo viga-soporte sometidos a compresión centrada". Tesis Doctoral. Universidad Politécnica de Valencia.

Adam JM, Antonio Brencich, Tim G. Hughes, Tony Jefferson (2010). "Micromodelling of eccentrically loaded brickwork: Study of masonry wallettes". Engineering Structures 32, 1244-1251.

Alan DK, Michael AD, Heather JP, Michel LB, KP Koo, CG Askins, MA Putnam, EJ Friebele (1997). "Fiber Grating Sensors". Journal of Lightwave techbology. Vol. 15, № 8.

ALTRA Digital camera solf imaging system (1999). User manual. Shinjuku (Tokyo, Japan).

Ang J, Li HCH, Herszberg I, Bannister MK, Mouritz AP (2010). "Tensile fatigue properties of fibre Bragg grating optical fibre sensors”. International Journal of Fatigue 32, 762-768.

Ansari F, Libo Y (1998). "Mechanics of bond and interface shear transfer in optical fiber sensors". Journal of Engineering Mechanical 124, 4, 385-394.

Ansari F (2007). "State of the Art in the application of fiber optic sensor to cementitious composites". Cement \& Concrete composites. Vol 19. № 1, 3-19.

Araldit ${ }^{\circledR}$ Standard. Marca registrada de CIBA SPECIALITY CHEMICALS HOLDING INC. Ficha técnica del producto. Departamento Técnico CEYS S.A.

ASTM D 638-03 (2004a). “American Society for Testing Materials. Standard test method for tensile properties of plastics". Philadelphia (USA).

ASTM E 132 (2004b). “American Society for Testing Materials. Test method for Poisson's ratio at room temperature". Philadelphia (USA).

Baifeng JI, Weilian QU (2008). "The Research of Acoustic Emission Techniques for Non Destructive Testing and Health Monitoring on Civil Engineering Structures". International Conference on Condition Monitoring and Diagnosis, Beijing, China.

Barrera D, García R, Sales S, Gasch I, Ivorra S, Giménez E (2007). “Application of fiber Bragg grating optic sensors for material characterization”. $5^{\circ}$ Reunión Española de Optoelectrónica.

Barrera D (2008). "Diseño de sensores ópticos avanzados basados en FBG". Diploma de Estudios Avanzados (DEA). Universidad Politécnica de Valencia.

Bertholds A, Dändliker R (1986). "High-resolution photoelastic pressure sensor using lowbirefringence fiber". Applied Optics, Vol. 25, Issue 3, pp. 340-343.

Bertoncini A, Hammerschlag J.G (2000). Personal communication.

Billington DP (1985). The Tower and the Bridge: The new Art of Structural engineering. Princeton University Press. 
Biswas P, Bandyopadhyay S, Kesavan K, Parivallal S, Arun BS, Ravisankar K, Dasgupta K (2010). "Investigation on packages of fiber Bragg grating for use as embeddable strain sensor in concrete structure". Sensors and Actuators A, 157, 77-83.

Brown AW, Colpitts BG, and Brown K. "Dark-pulse Brillouin optical time-domain sensor with 20mm spatial resolution". Journal of Lightwave Technology, 25(1):381-386.

Bueno Martínez A (2008). "Diseño y caracterización de un sensor óptico autocompensado en temperatura para la medida de deformaciones en estructuras de ingeniería civil y desarrollo de un sistema de interrogación". Tesis Final de Máster. Escuela Técnica Superior de Ingenieros de Telecomunicaciones. Universidad Politécnica de Valencia.

Calderón PA (2010). "On the use of numerical methods for the design of deep excavations with diaphragm walls in urban areas: possibilities and limitations". Developments and applications in Computational Structures Technology. Computational Science, Engineering and Technology Series: 25. ISSN 1759-3158. Chapter 13.

Calderón PA, Glisic B (2012). Influence of mechanical and geometrical properties of embedded longgauge strain sensors on the accuracy of strain measurement. Meas. Sci. Technol. 23065604 (15pp).

Carosena M (2006). "Infrared thermography of masonry structures". Department of Energetics, Taiwan.

Chan THT, Yu L, Tam HY, Ni YQ, Liu SY, Chung WH, Cheng LK (2006). "Fiber Bragg grating sensors for structural health monitoring of Tsing Ma bridge: Background and experimental observation". Engineering Structures 28, 648-659.

Chang PC, Alison F, Liu SC (2003). "Review Paper: Health Monitoring of Civil Infrastructure". Structural Health Monitoring vol 2 (3), 257-267.

Chuan Li (2008). "Monitoring second lining of tunnel with mounted fiber bragg grating strain sensors". Automation in Construction. Vol. 17, 641- 644.

Clark MR, McCann DM, Forde MC (2003). "Application of infrared thermography to the nondestructive testing of concrete and masonry bridges". The King's Buildings, Edinburgh.

Davis MA, Bellemore DG, Kersey AD (1997). "Distributed Fiber Bragg Gratting strain sensing in reinforced concrete structural components". Cement \& Concrete Composites. Vol 19. N 1 . Pp 45-48.

Dawood TA, Shenoi RA, Sahin M (2007). "A procedure to embed fibre Bragg grating strain sensors into GFRP sandwich structures". Composites: Part A, 38, 217-226.

Del Groso A, Inaudi D, Bergmeister K, Santa U (2000). "Monitoring of Bridges and Concrete Structures with Fibre Optic Sensors in Europe”. Smartec S.A. Manno, Switzerland.

Del Groso A, Inaudi D (2004). "European Perspective on Monitoring-Based Maintenance". IABMAS, International Association for Bridge Maintenance and Safety. Kyoto, Japan. Page 1 of 5.

Del Grosso A, Torre A, Inaudi D (2005). "Monitoring system for a cable-stayed bridge using static and dynamic fiber optic sensors". 2nd International Conference on Structural Health Monitoring of Intelligent Infrastructure (SHMII 2), Shenzhen, China. 415-420. 
Donghoon K, Wonseok C (2009). "Integrated monitoring scheme for a maglev guideway using multiplexed FBG sensor arrays". NDT\&E International 42, 260-266.

Dongsheng L, Hongnan L, Liang R, Gangbing S (2006). "Strain transferring analysis of fiber Bragg grating sensors". Optical Engineering 45 (2).

Dongsheng L, Zhi Z, Jinping O (2012). "Development and sensing properties study of FRP-FBG smart stay cable for bridge health monitoring application". Measurement. Volumen 44. Issue 4. Pages $722-729$

Duck G, LeBlanc M (2000). “Arbitrary strain transfer from a host to an embedded fiber-optic sensor”. Smart Material and Structure, 9, 492-497.

Exequiel R, Analía V (2003). "Mechanical properties of natural fiber composite materials obtained by the technique of Infusion Vacuum". Jornadas SAM/ CONAMET/SIMPOSIO MATERIA. 2003.

Fei L, Jingyuan L, Naibing M, Morse TF (1999). "A fiber optic microbend sensor for distributed sensing application in the structural strain monitoring". Sensors and Actuators 75 1999, 41-44.

Frangopol DM, Estes AC, Augusti G, Ciampoli M (1998). "Optimal bridge management based on lifetime reliability and life-cycle cost". Short course on the Safety of Existing Bridges. ICOM\&MCS, pp 112-120, EPFL, Lausanne, Switzerland.

Fresvig T, Ludvigsen P, Steen H, Reikeras O (2008). "Fibre optic Bragg grating sensors: An alternative method to strain gauges for measuring deformation in bone". Medical Engineering \& Physics 30 104-108.

Fu-Zhen, Hongwei T, Shan-Tung T (2009). "In situ monitoring on prestress losses in the reinforced structure with fiber-optic sensors". Measurement 42 107-111.

Gerard F (2005). "Fibre optic sensor systems for monitoring composite structures". 0034-3617/05 Elsevier Ltd.

Glišić B, Inaudi D, Kronenberg P, Vurpillot S (1999). Dam monitoring using long SOFO sensor. Hydropower into the Next Century, Gmunden, Austria. 709-717.

Glišić B (2000). "Fibre Optic Sensors and Behaviour in Concrete at Early Age". Doctoral Thesis. Ecole Polytechnique Federale de Lausanne.

Glišić B, Inaudi D, Nan C (2002). "Piles monitoring during the axial compression, pullout and flexure test using fiber optic sensors". Transportation Research Record (TRR), Journal of TRB No. 1808 'Soil Mechanics 2002', paper N. 02-2701, Washington, DC, pp. 11-20.

Glišić B, Inaudi D, Lau JM (2005). "Long-term monitoring of high-rise buildings using long-gage fiber optic sensors". 7th International Conference on Multi-Purpose High-Rise Towers and Tall Buildings (IFHS), Dubai, United Arab Emirates. (on conference CD, paper \#0416).

Glišić B, Inaudi D (2007). "Fibre Optics Methods for Structural Health Monitoring”. Chichester: John Wiley \& Sons Ltd.

Gu X, Chen Z, Ansari F (2000). "Embedded fiber optic crack sensor for reinforced concrete structures". ACI Structural Journal, nº 97. Pag 468-476. 
HBM Measurements with confidence (2006). Material safety data sheet. www.hbm.com.

Hong-Nan L, Dong-Sheng L, Gang-Bing S (2004). "Recent applications of fiber optic sensors to health monitoring in civil engineering". Engineering Structures 26 1647-1657.

Idriss RL, Liang Z (2006). "Monitoring an interstate highway bridge with a built-in fiber-optic sensing system". IABMAS. Third International Conference on Bridge Maintenance, Safety and Management, Porto, Portugal. (on conference CD).

Inaudi D, Casanova N, Vurpillot S (1996). "Bridge Monitoring by fiber optic deformation sensors: design, emplacement and results". Society of Photo-Optical Instrumentation Engineers. Smart Structures and Materials. SPIE Vol 2719. Pag. 141-149.

Inaudi D (1997). "Fiber Optic Sensor Network for the Monitoring of Civil Engineering Structures". Doctoral Thesis. Ecole Polytechnique Federale de Lausanne.

Inaudi D, Casanova N, Steinmann G (1998). "SOFO: tunnel monitoring with fiber optic sensors". Reducing Risk in Tunnel Design and Construction, ITC, Basel, Switzerland. 25-36.

Inaudi D, Kronenberg P, Vurpillot S (1999a). "Long-term Monitoring of a Concrete Bridge with 100 Fiber Optic Long-gage Sensor". SPIE's International Symposium on Nondestructive Evaluation Techniques for Aging Infrastructure \& Manufacturing. Vol. 3587, Newport Beach, USA, 50-59.

Inaudi D, Vurpillo, S, Martinola G, Steinmann G (1999b). "SOFO: Structural Monitoring with Fiber Optic Sensors", Fib Commission Meeting, Vol. 40, No. 9, Institute or Structural Engineering, University of Applied Sciences, Vienna, Austria.

Inaudi D, Casanova N, Glišić B (2001). "Long-Term deformation monitoring of historical constructions with fiber optic sensors". 3rd International Seminar on Structural Analysis of Historical Constructions, Guimaraes, Portugal. 421-430.

Inaudi D, Glišić B (2002). "Long-Gage Sensor Topologies for Structural Monitoring”. TheFirst fib Congress on Concrete Structures in the 21st Century, Vol. 2, Session 15, Osaka, Japan. 15-16. (on conference $\mathrm{CD}$ ).

Inaudi D, Glišić B (2006). "Reliability and field testing of distributed strain and temperature sensors". 13th SPIE's Annual International Symposium on Smart Structures and Materials, Vol. SSM02, (Smart Sensor Monitoring Systems and Applications), Vol. 6167, San Diego, USA.

Inaudi D (2009). "Overview of 40 Bridge Structural Health Monitoring Projects". SMARTEC SA, Switzerland, Roctest Ltd, Canada. IBC 09-45.

Inaudi D (2011). "Long-gauge strain sensors for underwater and deep-water applications". 21st International Conference on Optical Fiber Sensors.

Jin W, Zhou Y, Chan PKC, Xu HG (2000). "A fibre-optic grating sensor for the study of flow-induced vibrations". Sensors and Actuators 79 36-45.

Joachim N (2003). "NDT Methods for the inspection of highway structures". Federal Highway Research Institute, Bergisch Gladbach, Germany. 
Jochen M, Tobias K (2011). "Sensing of Surface Strain with Flexible Fiber Bragg Strain Gages". HBM GmbH. Germany.

Jun Y, Yuan L (2009). "Package and installation of embeddable fiber optic sensors". Optics and Lasers in Engineering 47 1085-1090.

Junqi G, Bin S, Wei Z, Hong Z (2006). "Monitoring the stress of the post-tensioning cable using fiber optic distributed strain sensors". Measurement. Volumen 39. Issue 5. Pages 420-428.

Junqi G, Jin W, Jun L, Xinming Z (2011). "Monitoring of corrosion in reinforced concrete structures using Bragg gratting sensing”. NDT\&E International. Volume 44. Issue 2. Pages 202-205.

Kai Tai W, Christopher KY, Noah GO (2008). "Investigation of the strain transfer for surface-attached optical fiber strain sensors". Smart Materials and Structures, 17.

Kesavan K, Ravisankar K, Parivallal S, Sreeshylam P, Sridhar S (2010). "Experimental studies on fiber optic sensors embedded in concrete". Measurement 43 157-163.

Kin-Tak L, Chi-chiu Chan, Li-min Zhou, Wei Jin (2001). "Strain monitoring in compositestrengthened concrete structures using optical fibre sensors". Composites: Part B, 32, 33-45.

Kister G, Winter D, Badcock RA, Gebremichael YM, Boyle WJO, Meggitt BT, Grattan KTV, Fernando GF (2007a). "Structural health monitoring of a composite bridge using Bragg grating sensors. Part 1: Evaluation of adhesives and protection systems for the optical sensors". Engineering Structures 29 440-448.

Kister G, Badcock RA, Gebremichael YM, Boyle WJO, Grattan KTV, Fernando GF, Canning L (2007b). "Monitoring of an all-composite bridge using Bragg grating sensors". Construction and Building Materials 21 1599-1604.

Knecht A, Manetti L (2001). "Using GPS in structural health monitoring". Spie's 8th Annual International Symposium on Smart Structures and Materials. 4-8 march. Newport Beach (CA), USA.

Ko JM, Ni YQ (2005). "Technology developments in structural health monitoring of large-scale bridges". Engineering Structures 27, 1715-1725.

Kuang KSC, Tan CY, Chew SH, Quek ST (2011). "Monitoring of large strains in submerged geotextile tubes using plastic optical fibre sensors". Sensors and Actuators A: Physical. ISSN: 09244247. DOI: 10.1016/j.sna.2011.03.013

Lange J, Benning W, Siering K (2006). "Crack detection at concrete construction units from photogrammetric data using image processing procedures". ISPRS Commission VII Mid-term Symposium "Remote Sensing: From Pixels to Processes", Enschede, the Netherlands, 8-11 May.

Lee B (2003). "Review of present status of optical fiber sensors". Opt. Fiber Technol. 9, 57-79.

Leng JS, Asundi A (2002). "NDE of Smart structures using multimode fiber optic vibration sensors". NDT\&E International 35 (2002) 45-51 .

León J (2009). "Elementos que componen un sistema de gestión de puentes. Revisión crítica”. Jornada sobre Ingeniería de Mantenimiento para la gestión de los Puentes. Valencia, 16 Diciembre. 
Monitorización estructural.

Aplicación con sensores de fibra óptica.

Leung CKY, Wang X, Olson N (2000). "Debonding and calibration shift of optical fiber sensors in concrete". J. Eng. Mech. 126, 6, 300-307.

Li D, Li H, Ren L, Song GB (2006). "Strain transferring analysis of fiber Bragg grating sensors". Optical Engineering. 45 (2):1-8.

Li WY, Cheng CC, Lo YL(2009). "Investigation of strain transmission of surface-bonded FBGs used as strain sensors". Sensors and Actuators A. 149 201-207.

Liang R, Hong-Nan L, Dongsheng L (2009). "Cracking monitoring by FBG strain sensor in the small scale dam model”. Sensors and Smart Structures Technologies for Civil, Mechanical, and Aerospace Systems. Proc. of SPIE Vol. 7292. doi: 10.1117/12.804491

López JM, Jáuregui C, Quintela A, Echevarría J (2005). "Fiber optic civil structure monitoring system". Optical Engineering 44(4). April.

Luc Thévenaz, Stella Foaleng-Mafang, Kwang-Yong Song, Sanghoon Chin, Jean-Charles Beugnot, Nikolay Primerov, Moshe Tur (2010). "Recent Progress Towards Centimetric Spatial Resolution in Distributed Fibre Sensing”. Fourth European Workshop on Optical Fibre Sensors. Proc. of SPIE. Vol. 7653, 765309. doi: 10.1117/12.868432.

Lusas (2010). Lusas Reference Manual. Surrey (UK).

Maaskant R, Alavie T, Mesures RM, Tadros G, Rizhalla RH, Guha-Takurta A (1997). "Fiber Optic Bragg Gratting Sensor for Bridge Monitoring”. Cement \& Concrete Composites. Vol 19. №1. Pp 2123.

Magalhães F, Cunha A, Caetano E (2008). "Dynamic monitoring of a long span arch bridge". Engineering Structures 30 3034-3044.

Majumder M, Gangopadhyay TK, Chakraborty AK, Dasgupta K, Bhattacharya DK (2008). "Fibre Bragg gratings in structural health monitoring: Present status and applications". Sensors and Actuators A: Physical, Volume 147, Issue 1, Pages 150-164.

Molignoni M (2011). "Monitoraggio del ponte strallato sull'Adige attraverso sensori elastomagnetici e a fibra ottica: analisi del funzionamento dei sensori e del comportamento della struttura". Università degli studi di Trento. Facoltà di Ingegneria. Corso di Laurea Specialistica in Ingegneria Civile.

Moyo P, Brownjohn JMW, Suresh R, Tjin SC (2005). "Development of fiber Bragg grating sensors for monitoring civil Infrastructure”. Engineering Structures 27 1828-1834.

Nikles M, Vogel B, Briffod F (2004). "Leakage detection using fiber optics distributed temperature monitoring". 11th SPIE Annual International Symposium on Smart Structures and Materials, San Diego, USA. 18-25.

Norma UNE 83316. Ensayos de Hormigón. Determinación del módulo de elasticidad en compresión (1996).

Otsuka K, Mihashi H, Kiyota M, Mori S, Kawamata A (2003). "Observation of multiple cracking in Hybrid FRCC at Micro and Meso Levels". Journal of Advanced Concrete Technology Vol. 1.

Olympus corporation user manual SZ61 (2006). Shinjuku (Tokyo, Japan). 
Qingbin L, Guang L, Guanglun W (2003). "Effect of the plastic coating on strain measurement of concrete by fiber optic sensor". Measurement 34 215-227.

Quintela A, Jáuregui C, Echevarría J, López-Higuera JM (2002). "Embedded temperature- strain fibre Bragg grating sensor system validation for concrete structures". Journal of Optics A: Pure and Applied Optics. S387-S390.

Rajan G, Semenova Y, Mathew J, Farrell G (2010). "Experimental analysis and demonstration of a low cost fibre optic temperature sensor system for engineering applications". Sensors and Actuators A $16388-95$.

Radojicic A, Bailey S, Brühwiler E (1999). "Consideration of the Serviceability Limit State in a Time Dependant Probabilistic Cost Model". Application of Statistics and Probability. Vol. 2, pp 605-612, Balkema, Rotterdam, Netherlands.

Ramos CA, Oliveira R, Marques AT (2009). "Design of an optical fibre sensor patch for longitudinal strain measurement in structures". Materials and Design 30 2323-2331.

Rodrigo ASM, Lopez-Anido RA (2009). "Structural health monitoring of marine composite structural joints using embedded fiber Bragg grating strain sensors". Composite Structures. Volume: 89, Issue: 2, Publisher: Elsevier Ltd, Pages: 224-234. ISSN: 02638223. DOI: 10.1016/j.compstruct.2008.07.027

Rodrigues C, Félix C, Lage A, Figueiras J (2010). "Development of a long-term monitoring system based on FBG sensors applied to concrete bridges". Engineering Structures Volume: 32, Issue: 8, Publisher: ELSEVIER SCI LTD.

Samir NS, Mourad YR, Gergis W (2009). "Long term sensor-based monitoring of an LRFD designed steel girder bridge”. Engineering Structures, Volume 31, Issue 12, Pages 2954-2965.

Sans D, Gutiérrez C, MayugoL JA, Frovel M, Rams J (2011). "Analysis of embedding conditions of fibre Bragg grating sensors in composite laminates under longitudinal loading for reliable measurements". Submitted to Smart Mater. Struct. for peer review 27 September.

Shin CS, Chiang CC (2006). "Embedded Fibre Bragg Grating Sensors for Internal Fatigue Damage Monitoring in Polymeric Composites". Key Engineering Materials (Volumes 321 - 323). Pages 230233.

Shiuh HC, Chang-Yu T (2009). "Strain measurement of fiber optic sensor surface bonding on host material". Transactions of nonferreous Metals society of China 19. 143-149.

Shiuh-Chuan Her, Chih Ying Huang (2011). "Effect of Coating on the Strain Transfer of Optical Fiber Sensors". Sensors, 11, 6926-6941; doi:10.3390/s110706926.

Shu M, Haruka T, Hidehiko B, Nobuo T (2011). "Hierarchical fiber-optic-based sensing system: impact damage monitoring of large-scale CFRP structures". Smart Mater. Struct. 20085029 doi:10.1088/0964-1726/20/8/085029.

Sunaryo S, Takuji O, Inaudi D (2004). "Intelligent Sensory Technology for Health Monitoring Based Maintenance of Infrastructures". Proc of Smart Sensor Technology and Measurement Systems, SPIE, 5384-19, San Diego, California, Mar. 14-18. 
Takeda S, Aoki Y, Ishikawa T, Takeda N, Kikukawa H (2007). "Structural health monitoring of composite wing structure during durability test". Composite Structures. Volumen 79, Issue 1. Pages 133-139.

Torres Górriz, B (2008). Estudio experimental de sensors ópticos tipo FBG para medir deformaciones y temperaturas en estructuras. Máster Universitario en Ingeniería del Hormigón. Universidad Politécnica de Valencia.

Torres B, Payá-Zaforteza I, Bueno A, Sales S, Adam JM, Calderón PA (2010). "Early age concrete temperature and shrinkage monitoring by using Fiber Bragg Grattings". XXXVII IAHS. World Congress on Housing. October 26 - 29, Santander, Spain.

Torres B, Payá-Zaforteza I, Calderón PA, Adam JM (2011a). "Analysis of the strain transfer in a new FBG sensor for Structural Health Monitoring”. Engineering Structures 33, 539-548.

Torres Górriz B, Payá-Zaforteza I, Bueno Martínez A, Sales Maicas S, Calderón García P, López-Tola R (2011b). "Aplicaciones de los sensores de fibra óptica desarrollados por la Universidad Politécnica de Valencia para la medida de deformaciones y temperaturas en estructuras". V Congreso de Ache. Barcelona, 25-27 Octubre de 2011.

Villalba Herrero, Vicens (2006). "Monitorización de la corrosión en estructuras postensadas mediante sensores de fibra óptica". Universidad Politécnica de Cataluña. Departamento de Ingeniería de la Construcción. Marzo, 2006.

Vurpillot S, Krueger G, Benouaich D, Clément D, Inaudi D (1998). "Vertical Deflection of a PreStressed Concrete Bridge Obtained Using Deformation Sensors and Inclinometer Measurements", ACI Structural Journal, Vol. 95, No. 5, Sptember- October 1998.

Vurpillot S (1999). "Analyse automatis'ee des syst'emes de mesure de d'eformation pour l'auscultation des structures”. Ph.D. Thesis No 1982, EPFL, Lausanne, Switzerland.

Wendy W (2006). "Fiber Bragg Grating strain sensor for Railway applications". Doctoral Thesis. University of Auckland.

Whiteman T, Lichti DD, Chandler I (2002). "Measurement of deflections in concrete beams by closerange digital photogrammetry". Symposium on Geospatial theory Processing and Applications, Ottawa.

Wonseok C, Sungil K, Nam-Sik K, Hee-up L (2008). "Deflection estimation of a full scale prestressed concrete girder using long-gauge fiber optic sensors". Construction and Building Materials 22 394401.

Wosniok A, Nother N, Krebber K (2009). "Distributed Fibre Optic Sensor System for Temperature and Strain Monitoring Based on Brillouin Optical-Fibre Frequency-Domain Analysis". Procedia Chemistry 1, 397-400.

Xiaowei D, Ruifeng Z (2010). "Detection of liquid-level variation using a side-polished fiber Bragg grating". Optics \& Laser Technology 42 214-218.

Yang Z, Ansari F (2002). "Embedded fiber optic sensor for characterization of interface strains in FRP composite". Sensors and Actuators A 100 247-251. 
Yeo TL, Eckstein D, McKinley B, Boswell LF, Sun T, VGrattan KT (2006). "Demonstration of a fibre-optic sensing technique for the measurement of moisture absorption in concrete". Smart Mater. Struct. 15, N40-N45.

Yuan L, Qingbin L, Yijun L, Jun Y, Zhihai L (2001). "Fiber optic 2D sensor for measuring the strain inside the concrete specimen". Sensors and Actuators A 94. 25-31.

Yung BL, Chih LP, Yuan HK, Kuo CC, Jenn CC (2005). "Online monitoring of highway bridge construction using fiber Bragg grating sensors". Smart Materials and Structures 14 1075-1082.

Yuyama S, Yokoyama K, Niitani K, Ohtsu M, Uomoto T (2007). "Detection and evaluation of failures in high-strength tendon of prestressed concrete bridges by acoustic emission". Construction and Building Materials 21 491-500.

Zhi Z, Minghua H, Jianping H, Genda C, Jinping O (2010). "Ice Structure Monitoring with an Optical Fiber Sensing System”. Cold Regions Science and Technology, Vol. 61, No. 1, pp.1-5.

Zhishen W, Bin X, Keiji H, Atsuhiko M (2006). "Distributed optic fiber sensing for a full-scale PC girder strengthened with prestressed PBO sheets". Engineering Structures 28 1049-1059.

Zonta D, Glisic B, Pozzi M, Inaudi D, Lao JM, Chor CF (2011). "Analysis of High Rise Building Lifespan Monitoring data using Bayesian logic”. SPIE Smart Structures / NDE. San Diego, March 10.

\section{Páginas web visitadas:}

- www.ishmii.org

- www.nytimes.com

- www.hbm.com

- www.wikipedia.es

- Página web de la compañía FOS \& S. Fibre Optic Sensing. http://foss.browsbox.be/index/been/926/. 



\section{APÉNDICE I. PROYECTOS DE INVESTIGACIÓN FINANCIADOS POR ADMINISTRACIONES PÚBLICAS O EMPRESAS PRIVADAS.}

En el presente apéndice se muestran los proyectos de investigación realizados y relacionados con la temática estudiada en la presente Tesis Doctoral.

\begin{tabular}{|c|c|c|c|c|}
\hline Nombre del proyecto & Periodo & Financiador & $\begin{array}{c}\text { Investigador } \\
\text { principal }\end{array}$ & Presupuesto. \\
\hline $\begin{array}{l}\text { Desarrollo de técnicas de } \\
\text { implantación de sensores ópticos } \\
\text { en estructuras de hormigón } \\
\text { (PPI-05-05-5709-02) }\end{array}$ & $2005-2007$ & $\begin{array}{l}\text { Universidad } \\
\text { Politécnica de } \\
\text { Valencia. }\end{array}$ & $\begin{array}{l}\text { Pedro Antonio } \\
\text { Calderón García. }\end{array}$ & $9000 €$ \\
\hline $\begin{array}{l}\text { Sensores ópticos aplicados a la } \\
\text { instrumentación de estructuras } \\
\text { para conocimiento de su } \\
\text { comportamiento en tiempo real } \\
\text { (Contrato } \mathrm{I}+\mathrm{D}+\mathrm{i})\end{array}$ & $2008-2011$ & SACYR S.A. & $\begin{array}{l}\text { Pedro Antonio } \\
\text { Calderón García. }\end{array}$ & $126.358 €$ \\
\hline $\begin{array}{c}\text { Desarrollo de sensores } \\
\text { avanzados de fibra óptica para } \\
\text { la determinación de propiedades } \\
\text { de materiales y salud estructural } \\
\text { (P41/08) }\end{array}$ & $2009-2011$ & $\begin{array}{l}\text { Ministerio de } \\
\text { Fomento. }\end{array}$ & $\begin{array}{c}\text { Salvador Sales } \\
\text { Maicas }\end{array}$ & $152.890 €$ \\
\hline $\begin{array}{c}\text { Definición de condiciones de } \\
\text { monitorización, encapsulado y } \\
\text { fijación de sensores ópticos para } \\
\text { medir temperaturas y } \\
\text { deformaciones en estructuras a } \\
\text { altas temperaturas. }\end{array}$ & $2012-2015$ & $\begin{array}{l}\text { Ministerio de } \\
\text { Educación }\end{array}$ & $\begin{array}{l}\text { Ignacio Payá- } \\
\text { Zaforteza }\end{array}$ & $149.907 €$ \\
\hline
\end{tabular}

Tabla A1. Información de los proyectos de investigación 



\section{APÉNDICE II. PUBLICACIONES EN CONGRESOS Y REVISTAS}

En el presente apéndice se muestran las distintas publicaciones en revistas y en congresos que han sido generadas como consecuencia de la investigación realizada.

Las publicaciones que aquí se engloban son:

\section{a) Publicaciones en revistas:}

Benjamín Torres, Ignacio Payá-Zaforteza, Pedro A. Calderón, Jose M. Adam. "Analysis of the strain transfer in a new FBG sensor for Structural Health Monitoring". Engineering Structures 33 (2011) 539-548.

Antonio Bueno, Benjamín Torres, David Barrera, Pedro Antonio Calderón, José Manuel Lloris, María José López, Salvador Sales. "Optical fiber Sensors embedded in concrete for measurement of temperature in a real fire test". Optical Engineering, Volume 50 (12) SPIE - Dec 1, 2011.

\section{b) Publicaciones en Congresos:}

Authors: Benjamín Torres, Ignacio Payá-Zaforteza, Antonio Bueno, Salvador Sales, Jose Miguel Adam, Pedro Antonio Calderón.

Title: Early age concrete temperature and shrinkage monitoring by using Fiber Bragg Grattings

Type: Paper

Congress: XXXVII IAHS World Congress on Housing Science

Place: Santander, Spain

Date: November, 2010.

Authors: Benjamín Torres, Ignacio Payá-Zaforteza, Antonio Bueno, Salvador Sales, Pedro Antonio Calderón, Raquel López.

Title: Aplicaciones de sensores de fibra óptica desarrollados por la U.P.V. para la medida de deformaciones y temperaturas en estructuras

Type: Paper

Congress: V Congreso de ACHE

Place: Barcelona, Spain

Date: October, 2011.

Authors: Antonio Bueno, Benjamín Torres, David Barrera, Salvador Sales.

Title: Monitoring of a steel incrementally launched bridge construction with strain and temperature FBGs sensors.

Type: Paper

Congress: SPIE Photonics Europe.

Place: Bruselas, Bélgica. Date: Abril, 2010.

Authors: Antonio Bueno, Benjamín Torres, David Barrera, Pedro A. Calderón, José Manuel Lloris, María José López, Salvador Sales.

Title: Fiber Bragg grating sensors embedded in concrete samples for a normalized fire test.

Type: Poster

Congress: 21st International Conference on Optical Fiber Sensors.

Place: Ottawa, Canada.

Date: May, 2011. 

Provided for non-commercial research and education use. Not for reproduction, distribution or commercial use.

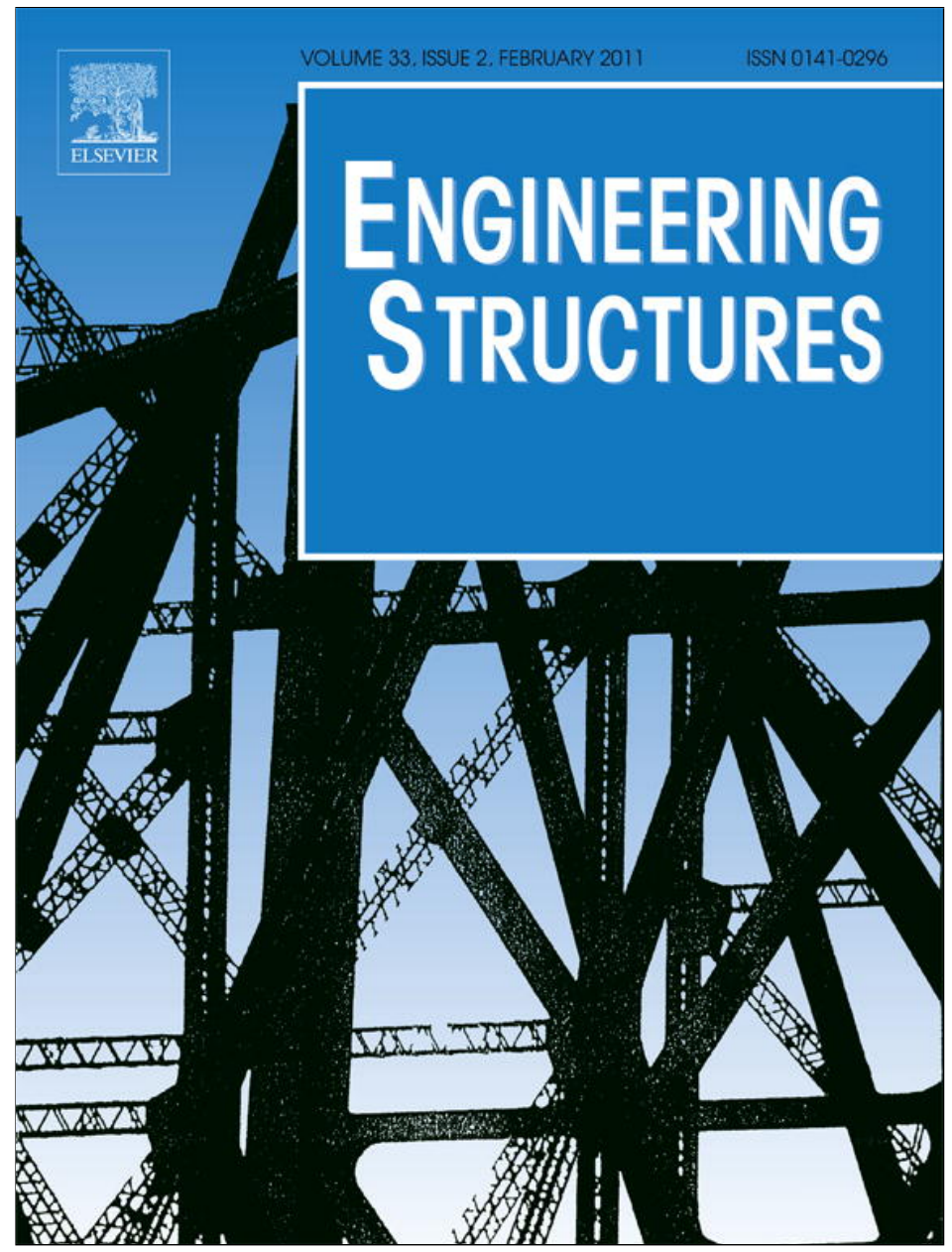

This article appeared in a journal published by Elsevier. The attached copy is furnished to the author for internal non-commercial research and education use, including for instruction at the authors institution and sharing with colleagues.

Other uses, including reproduction and distribution, or selling or licensing copies, or posting to personal, institutional or third party websites are prohibited.

In most cases authors are permitted to post their version of the article (e.g. in Word or Tex form) to their personal website or institutional repository. Authors requiring further information regarding Elsevier's archiving and manuscript policies are encouraged to visit:

http://www.elsevier.com/copyright 



\title{
Analysis of the strain transfer in a new FBG sensor for Structural Health Monitoring
}

\author{
Benjamin Torres, Ignacio Payá-Zaforteza*, Pedro A. Calderón, Jose M. Adam \\ ICITECH, Departamento de Ingeniería de la Construcción, Universidad Politécnica de Valencia. Camino de Vera s/n, 46071 Valencia, Spain
}

\section{A R T I C L E I N F O}

Article history:

Received 19 May 2010

Received in revised form

2 September 2010

Accepted 2 November 2010

Available online 30 November 2010

Keywords:

Fiber Bragg grating sensors

Fiber optic sensors

Strain transfer

Structural Health Monitoring

\begin{abstract}
A B S T R A C T
Structural Health Monitoring (SHM) is a topic of great interest in structural engineering due to the ageing of the built infrastructures and the growing use of innovative structural systems and construction materials. Although several sensing technologies have been developed for use in SHM systems, fiber optic sensors, especially Fiber Bragg grating sensors (FBGs), are now attracting much attention due to their advantages over other types of sensors.

This paper presents a new FBG strain sensor with an unsymmetrical packaging configuration designed to be fixed to the surface of the monitored structure. A 3D Finite Element numerical analysis of the sensor, packaging and adhesive used to fix the system to the host structure was conducted to study the influence of (1) the thickness and mechanical properties of the adhesive, and (2) the configuration of the packaging on the accuracy of the sensor. The results obtained from the numerical models show that the strains measured by the proposed system and the actual strains in the host structure differ by less than $2.5 \%$ due to the fact that the packaging contains only one layer of composite material in an unsymmetrical configuration. The proposed design can thus be said to be an improvement compared to the typical configurations of optical fiber surface strain sensors.
\end{abstract}

(c) 2010 Elsevier Ltd. All rights reserved.

\section{Introduction}

Structural Health Monitoring (SHM) has been defined by Li et al. [1] as the use of in situ, continuous or regular (routine) measurement and analyses of key structural and environmental parameters under operating conditions, for the purpose of warning of impending abnormal states or accidents at an early stage, as well as giving maintenance and rehabilitation advice. Although the formal establishment of this discipline is fairly recent (the International Society for Structural Health Monitoring of Intelligent Infrastructure was created in 2003 and the first journal devoted exclusively to this field appeared in 1993) the history of systems to monitor the behavior of bridges and buildings is much older, as engineers such as Torroja and Nervi monitored some of their innovative designs during the first half of the XXth century (see $[2,3]$ respectively).

A monitoring system is composed of three elements (see $[1,4-6])$ : a sensor system, a data processing system and a health evaluation system. Sensors that detect the transmission of electrical signals have traditionally been used in SHM, but in recent years the use of fiber optic sensors (FOSs) has attracted a great deal of attention in research and development, as reflected in both review

\footnotetext{
* Corresponding author. Tel.: +34 963877562; fax: +34 963877568.

E-mail addresses: bentorgo@upvnet.upv.es (B. Torres), igpaza@cst.upv.es (I. Payá-Zaforteza), pcaldero@cst.upv.es (P.A. Calderón), joadmar@cst.upv.es (J.M. Adam).
}

and application articles [1,7-12]. The main reasons for this interest are the falling prices of FOSs added to their advantages over conventional sensors. These advantages relate mainly to their flexibility, embeddability, multiplexity, small size and immunity to electrical or magnetic interference (see $[4,13])$. FOSs detect variations in the properties of light travelling through the optical fiber. The fiber usually consists of three layers: the core, which is a thin glass fiber, the cladding that confines the propagation of the light within the fiber core, and an outer coating or jacket that provides the fiber mechanical strength and protects it from damage and moisture absorption. Although there are three different FOS technologies (point, long gauge and distributed sensors), the most frequently used system at the present time is the Fiber Bragg grating (FBG) point sensor [8].

FBG sensors cannot be installed bare in a structural element as they are very fragile and the harsh environment of a construction site and building structures would affect their durability and behavior. Therefore several encapsulation or packaging techniques have been developed to protect them (see $[4,8,14]$ ). It is common practice to assume that the strain values recorded by FBG sensors are the actual strains in the host structure, but this is not always the case. In the common case of sensors that are first embedded in a material or fixed to a plate and then glued to the surface of the host structure, a discrepancy appears related to the thickness of the embedding material or fixing plate and the thickness and mechanical properties of the adhesive. 


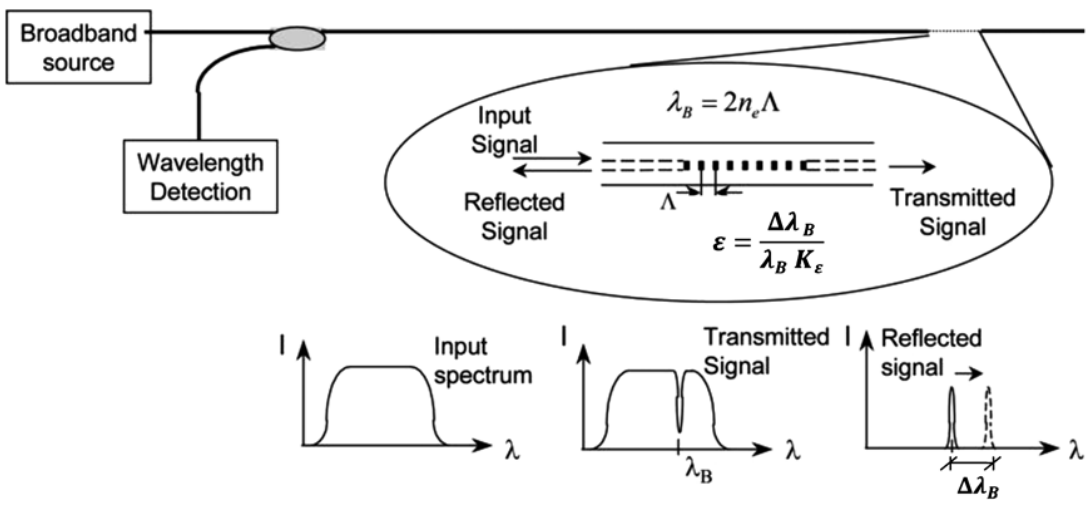

Fig. 1. Basic Bragg grating-based sensor system with transmissive or reflective detection options. Source: [11]. Reproduced with the kind permission of the authors.

This paper describes the design and experimental evaluation of a new surface FBG strain sensor system developed at the Universidad Politecnica de Valencia (UPV). Unlike many commonly used strain sensors in which the strain perceived by the sensor may differ from that on the surface on which it is placed, the packaging of the new sensor has been designed in such a way that the discrepancy between the recorded and real strain values is negligible. Following a description of the basic principles of FBG sensors, this paper explains the main features of the proposed packaging and describes the numerical studies carried out to evaluate its behavior and compare it to other similar systems. Finally, conclusions are drawn on the behavior of the packaging system, based on the previous analyses.

\section{Principle of FBG sensors}

An FBG is a reflector built in a short segment of the core of an optical fiber by exposing the fiber to an intense UV light. This exposure creates periodic alterations in the index of refraction of the fiber core, known as Bragg gratings, and makes the FBG (1) reflect only particular narrowband light wavelengths, of light known as Bragg wavelengths, and (2) transmit all others (see Fig. 1). The Bragg wavelength condition is given by Eq. (1), where $\lambda_{B}$ is the wavelength of the FBG, $n_{\text {eff }}$ is the effective refractive index of the fiber core, and $\Lambda$ is the Bragg grating period or distance between two consecutive alterations of the fiber core.

$\lambda_{B}=2 n_{\mathrm{eff}} \Lambda$.

As external perturbations such as strains $(\varepsilon)$ and temperature $(T)$ changes induce modifications in the Bragg grating period $\Lambda$, they also induce a shift $\Delta \lambda_{B}$ in the Bragg wavelength. This shift is given by Eq. (2), when only the dominant linear effects of $\varepsilon$ and $T$ on an FBG are considered and higher order cross-sensitivities are neglected (see [15]).

$\frac{\Delta \lambda_{B}}{\lambda_{B}}=K_{\varepsilon} \Delta \varepsilon+K_{T} \Delta T$.

In Eq. (2), $K_{\varepsilon}$ and $K_{T}$ are the coefficients of wavelength sensitivity to strain and temperature for an FBG whose values are given by:

$K_{\varepsilon}=\left[1-0,5 n_{\text {eff }}\left(\rho_{12}-v\left(\rho_{11}-\rho_{12}\right)\right)\right] \lambda_{B}$

$K_{T}=[1+\xi] \lambda_{B}$

where $\rho_{11}$ and $\rho_{12}$ are the components of the fiber optic strain tensor and $v$ is the fiber Poisson's ratio, $\xi$ is the fiber thermo-optic coefficient and $n_{\text {eff }}$ is the refractive index of the fiber (see $[15,16]$ for more details).

$K_{\varepsilon}$ and $K_{T}$ are obtained experimentally, $K_{T}$ being dependent on the material of the host structure. Once $K_{\varepsilon}$ and $K_{T}$ are known,

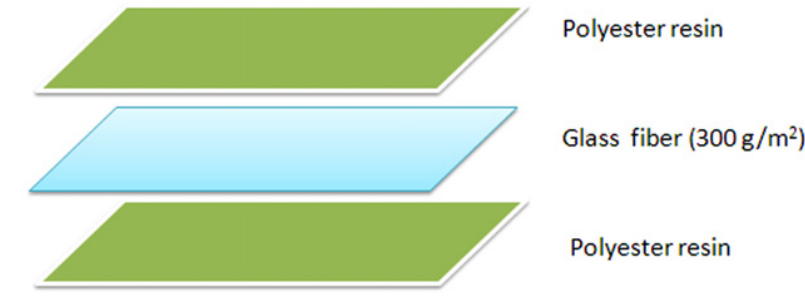

Optical fiber

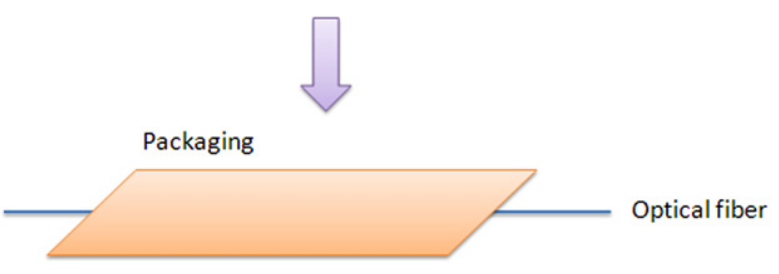

Fig. 2. UPV sensor. 3D view of the FBG and the packaging.

it is possible to obtain the values of the increments in strains and temperatures corresponding to a certain shift of the Bragg wavelength by using Eqs. (5) and (6) (see [17]). It should be pointed out that two sensors must be used when measuring to avoid strain misreading due to thermal effects. One of them is tightly fixed to the structure and is therefore affected by both strains and temperature changes, while the second is designed in such a way that it is only affected by temperature changes. In Eqs. (5) and (6), subscript 1 relates to the optical fiber sensor that is fixed to the structure and subscript 2 relates to the sensor that only measures temperature changes.

$\Delta \varepsilon=\frac{1}{K_{\varepsilon}}\left(\frac{\Delta \lambda_{B 1}}{\lambda_{B 1}}\right)-\frac{K_{T 1}}{K_{\varepsilon 1} K_{T 2}}\left(\frac{\Delta \lambda_{B 2}}{\lambda_{B 2}}\right)$

$\Delta T=\frac{1}{K_{T 2}}\left(\frac{\Delta \lambda_{B 2}}{\lambda_{B 2}}\right)$.

For the FBG strain sensor described in Section $3, K_{\varepsilon 1}$ is equal to $1.15 \mathrm{pm} / \mu \varepsilon$ whereas $K_{T 1}$ is equal to $17.5 \mathrm{pm} /{ }^{\circ} \mathrm{C}$ or $20.5 \mathrm{pm} /{ }^{\circ} \mathrm{C}$, depending on whether the host structure is made of concrete or steel, respectively, as explained in [16,18]. $K_{T 2}$ depends on the specific sensor used to measure temperature changes and is equal to $8 \mathrm{pm} /{ }^{\circ} \mathrm{C}$ for the system used in this study.

\section{FBG strain sensor}

The FBG strain sensor consists of an FBG protected by layers of composite material made of glass fiber reinforced polyester resin with a weight of $300 \mathrm{~g} / \mathrm{m}^{2}$ (see Fig. 2). The sensor system is 
Table 1

Adhesive specifications.

\begin{tabular}{|c|c|c|}
\hline Properties & Units & Value \\
\hline Coefficient of thermal expansion, $\alpha$ & ${ }^{\circ} \mathrm{C}^{-1}$ & $3.5-4 \times 10^{-6}$ \\
\hline Thermal conductivity & $\mathrm{K}_{\mathrm{cal}}$ & 0.17 (between 0 and $50{ }^{\circ} \mathrm{C}$ ) \\
\hline Young's modulus after hardening (approximate value) & $\mathrm{N} / \mathrm{mm}^{2}$ & 13000 \\
\hline Resistance & $\Omega$ & $>10^{15}$ \\
\hline Shrinkage during curing, depends on the ratio of the mixture & $\%$ & \\
\hline $1: 4$ & & 4 \\
\hline $1: 2$ & & 6 \\
\hline $1: 1$ & & 10 \\
\hline \multicolumn{3}{|l|}{ Temperature limits for strain measurements: } \\
\hline For zero-point related measurements & ${ }^{\circ} \mathrm{C}$ & $-200 \cdots+60$ \\
\hline For non zero-point related measurements & ${ }^{\circ} \mathrm{C}$ & $-200 \cdots+80$ \\
\hline
\end{tabular}

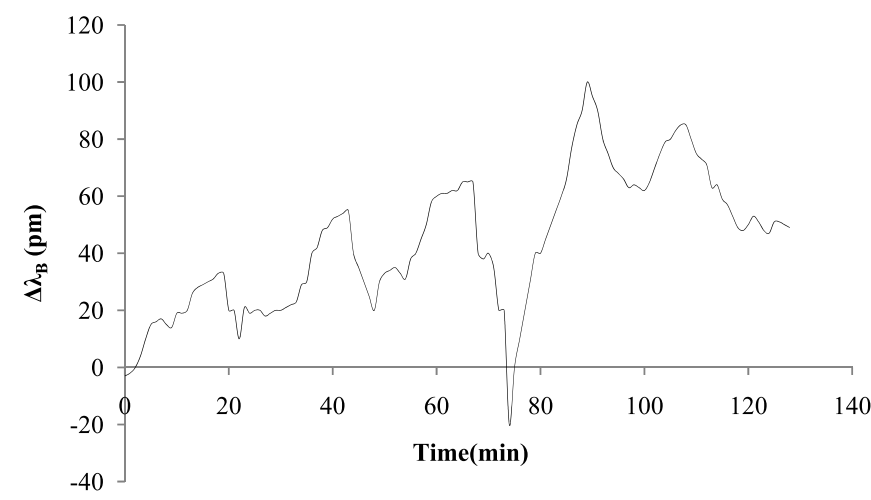

Fig. 3. Shift $\Delta \lambda_{B}$ in the Bragg wavelength of the sensor during its packaging.

approximately $100 \mathrm{~mm}$ long, $10 \mathrm{~mm}$ wide and $0.6 \mathrm{~mm}$ thick, and is fixed to the host metal or concrete structure with a very thin layer of an adhesive whose technical specifications are detailed in Table 1 . The following details are worthy of note:

(1) The FBG is placed on one of the packaging surfaces to ensure that the sensor is as close as possible to the monitored surface.

(2) The heat generated during the hardening of the polyester resin produces a shift in the Bragg wavelength. For the sensor analyzed in this paper, the shift after $130 \mathrm{~min}$ of resin hardening is $100 \mathrm{pm}$ (Fig. 3). This value represents only a
$2 \%$ of the difference between the Bragg wavelengths of two consecutive sensors (with a value of around $5000 \mathrm{pm}$ ) and therefore does not affect the performance and precision of the system.

(3) The design of the packaging minimizes the variation of the coefficients of wavelength sensitivity due to the packaging process.

The accuracy of the sensor was checked with experimental tests in which the readings of the FBG sensor were compared with those provided by strain gauges. All the sensors were placed on the surface of both concrete and steel specimens subjected to uniform tension, compression and bending. The readings of the FBG sensors were very similar to those of the strain gauges, with a maximum difference of only $3 \%$. Details of the experimental tests and their results have been described elsewhere $[16,18]$ and are not provided here.

The mechanical properties of the packaging, especially its Young's modulus $\left(E_{P}\right)$ and Poisson's ratio $\left(v_{P}\right)$, are necessary to perform the numerical analysis shown in Section 4. However, these properties were not known a priori, as they depend on the characteristics of the glass fiber and polyester resin used for the packaging. Six laboratory tests (see Fig. 4(a)) were thus performed to obtain these data. In each one of the tests, a $200 \mathrm{~mm} \times 20 \mathrm{~mm} \times$ $0.5 \mathrm{~mm}$ packaging specimen was loaded until failure in a tension test. Vertical and horizontal strains as well as the applied forces were measured (see Fig. 4(b)). The slope of the linear part of the
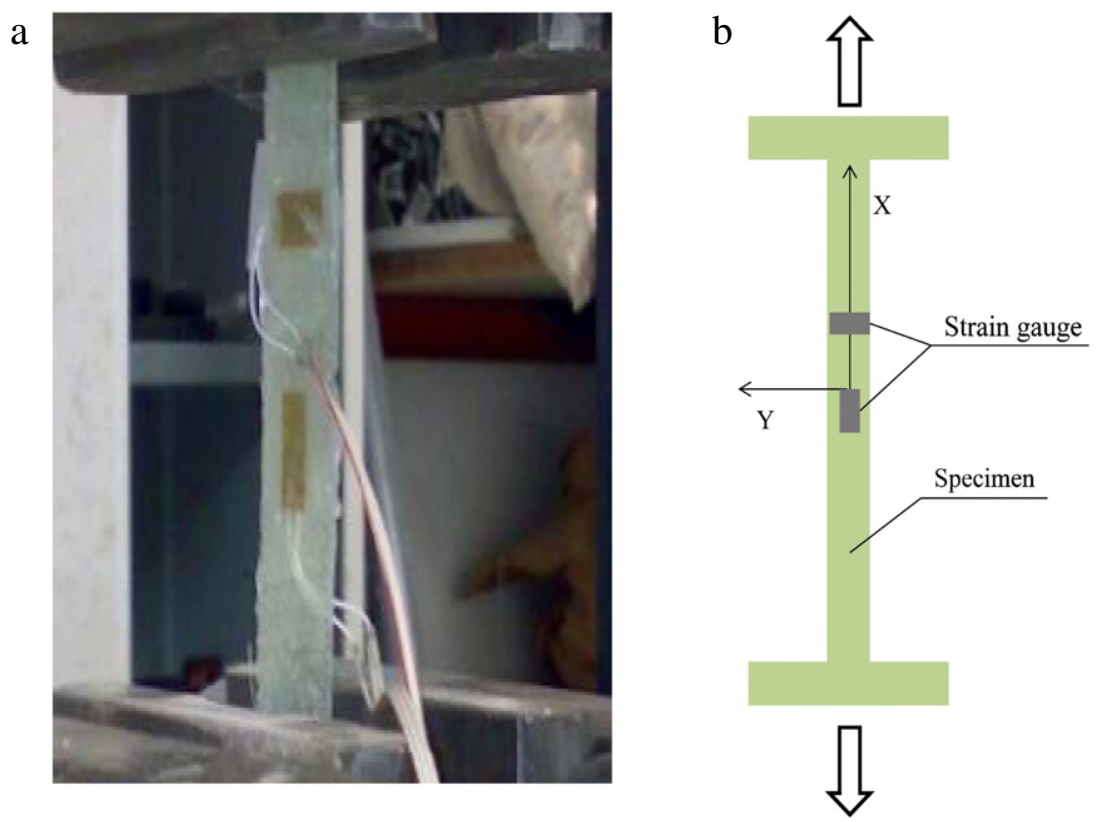

Fig. 4. (a) Tensile test of a packaging specimen. (b) Sketch showing the direction of the applied load and the positioning and orientation of the strain gauges. Note that the specimen has an I-shape, to make the load application easier. 
Table 2

Glass fiber reinforced polymer specimen mechanical properties.

\begin{tabular}{lll}
\hline Specimen & Young's modulus $E_{p}(\mathrm{GPa})$ & Poisson's ratio $v_{p}$ \\
\hline 1 & 16.6 & $0.36-0.39$ \\
2 & 13.4 & $0.29-0.4$ \\
3 & 12.8 & $0.31-0.38$ \\
4 & 11.3 & $0.33-0.4$ \\
5 & 13.1 & $0.29-0.36$ \\
6 & 12.7 & $0.33-0.4$ \\
\hline
\end{tabular}

vertical strain versus tension force plot gave a value of $E_{P}$ whereas $v_{P}$ was obtained according to Eqs. (7) and (8) (see $[19,20]$ ).

$\varepsilon_{y}=\frac{1}{E_{P}}\left\{\sigma_{y}-v_{p}\left(\sigma_{x}+\sigma_{z}\right)\right\}$

$v_{p}=-\frac{\sigma_{y}}{\sigma_{x}}$.

Table 2 provides the values of $E_{P}$ and $v_{P}$ of the packaging derived from the laboratory tests. These results are in good agreement with the values found in the literature [21] although Specimen 1 has a higher $E_{P}$ due to the fact that an amount of polyester resin bigger than the usual was used for its fabrication. For the numerical analysis carried out in Section 4, the values of $E_{P}$ and $v_{P}$ were taken as $11 \mathrm{GPa}$ and 0.4 respectively, as these values are the most unfavorable according to the analysis explained in Section 4.3.2.

\section{Numerical study}

\subsection{Introduction}

The readings provided by a sensor fixed to the monitored surface by an adhesive can be affected by the thickness and mechanical properties of the adhesive and therefore it is important to determine how these factors affect the measurements. This can be analyzed by an exhaustive experimental study, which would involve a considerable investment of time and money. In the present case, we opted for a numerical FEM study of the set formed by the sensor and adhesive. In these numerical models, a strain is imposed on the monitored surface and the strain transfer is then studied:

(1) Through the adhesive up to the optic fiber in the case of the sensor developed at the Universidad Politécnica de Valencia (UPV). This analysis is described in Sections 4.3.3 and 4.3.4.

(2) Through the adhesive and packaging up to the fiber when the numerical model is used to study sensor configuration, in which the optic fiber is wrapped in two layers of material (Fig. 5). This analysis is described in Section 4.3.5.
Table 3

Mechanical properties of the adhesive in the FE model. Source [23].

\begin{tabular}{lll}
\hline Properties & Units & Value \\
\hline Young's modulus $X$ & $\mathrm{GPa}$ & 13 \\
Young's modulus $Y$ & $\mathrm{GPa}$ & 13 \\
Shear modulus $X Y$ & & 0 \\
Poisson's ratio $X Y$ & & 0.2 \\
Angle of orthotropy & & 0 \\
Mass density & $\mathrm{GN} / \mathrm{m}^{3}$ & $2 \times 10^{-5}$ \\
Coefficient of thermal expansion $X$ & ${ }^{\circ} \mathrm{C}^{-1}$ & $0.035 \times 10^{-4}$ \\
Coefficient of thermal expansion $Y$ & & 0 \\
Coefficient of thermal expansion $Z$ & & 0 \\
\hline
\end{tabular}

4.2. Boundary conditions, description of the elements used and load application

The numerical study was carried out with Lusas software [22]. Due to the symmetry of loads and geometry, only a quarter of the packaging was analyzed in order to reduce computational costs. Fig. 6 shows the symmetry planes considered and the structural model. Besides the boundary conditions derived from symmetry, vertical movement was prevented at the base of the adhesive.

The optic fiber forming the sensor was modeled by type BRS3 linear elements (Fig. 7(a)) with three nodes, each with three degrees of freedom (translation along $X, Y, Z$ ). The adhesive and packaging were modeled with 3D HX20 solid elements (Fig. 7(b)). Each of these elements had 20 nodes, each one with three degrees of freedom (translations along $X, Y, Z$ ). All the model elements had linear elastic behavior.

The packaging and the adhesive had shared nodes on the contact surface. Contact elements were not considered on this interface since no relative movements were observed between these elements in the laboratory tests.

To study the strain transfer between the host structure and the fiber, an external deformation was applied on the lower adhesive plane in contact with the host structure. This deformation was imposed applying a temperature increase according to Eq. (9):

$\varepsilon=\alpha \Delta T$

where $\alpha$ is the coefficient of thermal expansion of the material and $\varepsilon$ is the imposed deformation.

To make the imposed deformation affect only the longitudinal axis of the sensor, the adhesive was considered to be an orthotropic material with the properties indicated in Table 3. The same Young's modulus was maintained in the three directions $(X, Y$ and $Z$ ) and the coefficient of thermal expansion was only considered in the longitudinal direction $X$. Since no data were supplied by the manufacturer on Poisson's ratio $v_{A}$, a study was carried out on the sensitivity to the influence of this parameter, as explained in Section 4.3.4. For the calculations in Sections 4.3.3 and 4.3.5 a value of $v_{A}=0.4$ was used.

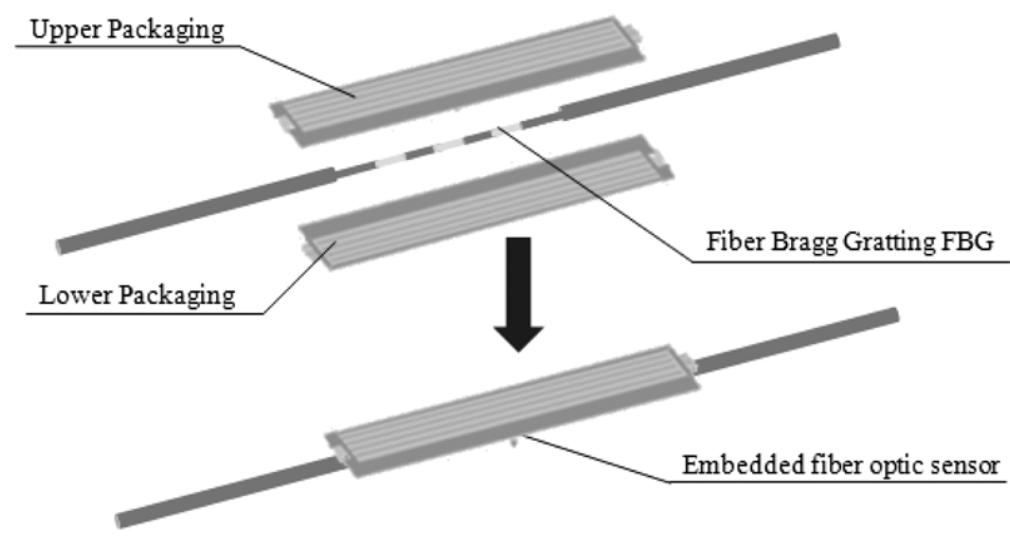

Fig. 5. Typical design of a generic sensor glued to the surface of a monitored structure. 
a

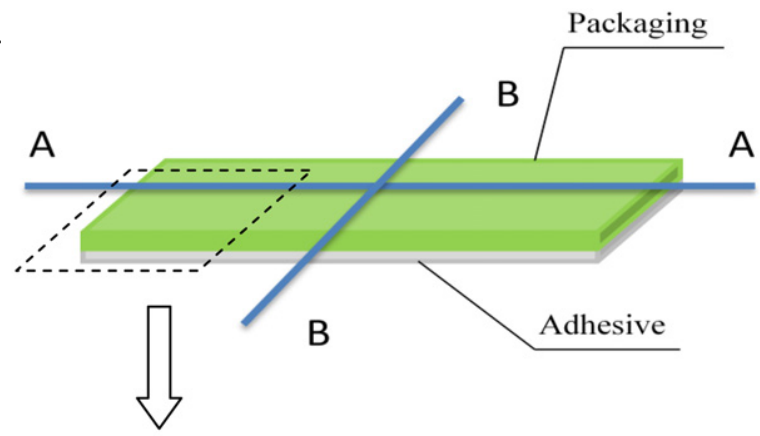

b

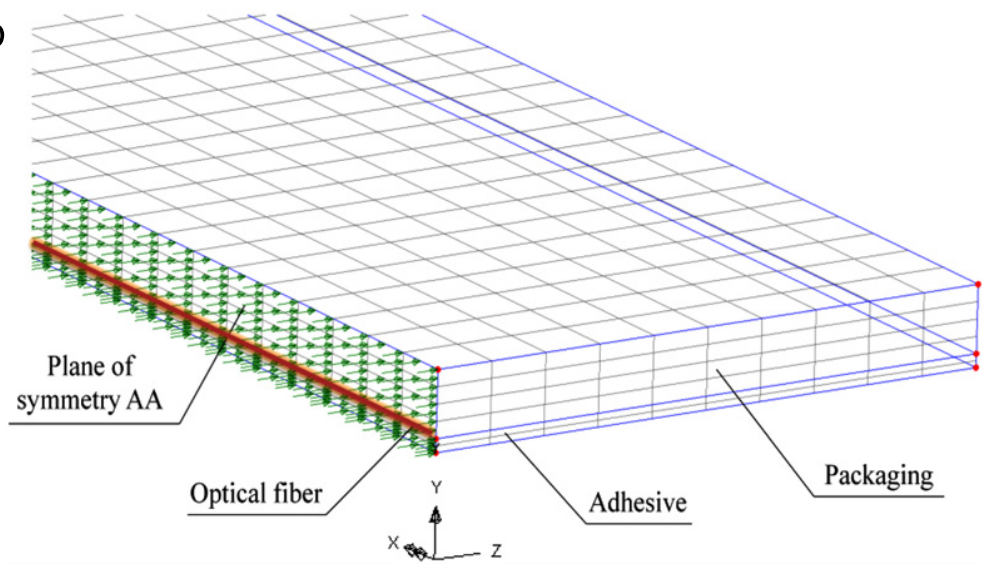

Fig. 6. (a) 3D view of the packaging and the adhesive showing its symmetry planes AA and BB. (b) View of part of the FE model.

a

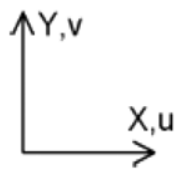

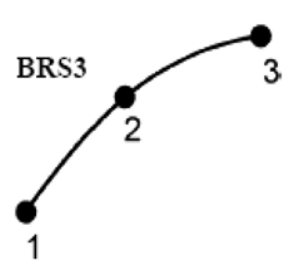

b

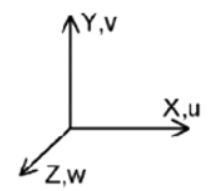

HX20

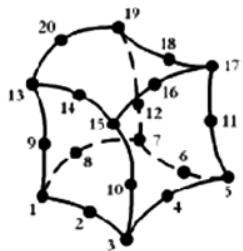

Fig. 7. BRS3 (a) and HX20 (b) elements. Source: Lusas [22].

\subsection{Results of the numerical study}

\subsubsection{General behavior}

The deformed mesh of all models followed the same behavior pattern and showed a strain gradient between the underside of the adhesive and the plane containing the optic fiber. As adhesive thickness is increased the gradient becomes steeper (Fig. 8) and is evaluated from the parameter $\Delta \varepsilon$ (\%) (Eq. (10)).

$\Delta \varepsilon(\%)=\frac{\varepsilon_{h}-\varepsilon_{f}}{\varepsilon_{h}} 100$.

In Eq. (10), $\varepsilon_{h}$ and $\varepsilon_{f}$ represent deformations on the surfaces of the host structure and the optic fiber, respectively. $\Delta \varepsilon(\%)$ is therefore a measure of the relative error of the strain sensor, values close to 0 indicating an almost perfect transfer of deformation from the host structure to the optic fiber.

4.3.2. Sensitivity analysis of Young's modulus $\left(E_{P}\right)$ and Poisson's ratio $\left(v_{P}\right)$ of the packaging

As the results of the experimental tests reported in Table 2 and related to $E_{P}$ and $v_{P}$ had some dispersion, the authors performed a sensitivity analysis that considered values of $E_{P}$ between 11 and $17 \mathrm{GPa}$, values of $v_{P}$ between 0.29 and 0.4 and two possible values of Poisson's ratio of the adhesive ( $v_{A}$ equal to 0.1 or 0.4 ). The goal of this analysis was to obtain the values of these parameters to be used in the studies discussed in Sections 4.3.3-4.3.5. Figs. 9 and 10 summarize the results. According to them, values of $E_{P}$ and of $v_{P}$ equal to $11 \mathrm{GPa}$ and $0.4 \mathrm{GPa}$ respectively should be used as they lead to the highest values of the relative error $\Delta \varepsilon$.

\subsubsection{Sensitivity analysis of the thickness of the adhesive ( $t$ )}

A sensitivity study was carried out on the influence of the adhesive thickness on the deformation as read by the optic fiber sensor. Fig. 11 shows an example of the deformations in the $X$-direction in a cross section of the sensor corresponding to an adhesive thickness of $100 \mu \mathrm{m}$. Fig. 12 represents the value of $\Delta \varepsilon(\%)$ as a function of the adhesive thickness. It can be seen that the errors made by the UPV sensor are quite small. For example, for the typical thickness of $400 \mu \mathrm{m}$ (see Fig. 13) the reading error is less than $3 \%$. It should be emphasized that even for large thicknesses (1000 $\mu \mathrm{m}$ ) UPV sensor errors are small (less than 6.5\%) compared to the errors of the two-layer sensors as discussed in Section 4.3.5. Values for thicknesses over $1000 \mu \mathrm{m}$ indicate very low adhesion and in such cases it is preferable to remove and re-locate the sensor. 
a

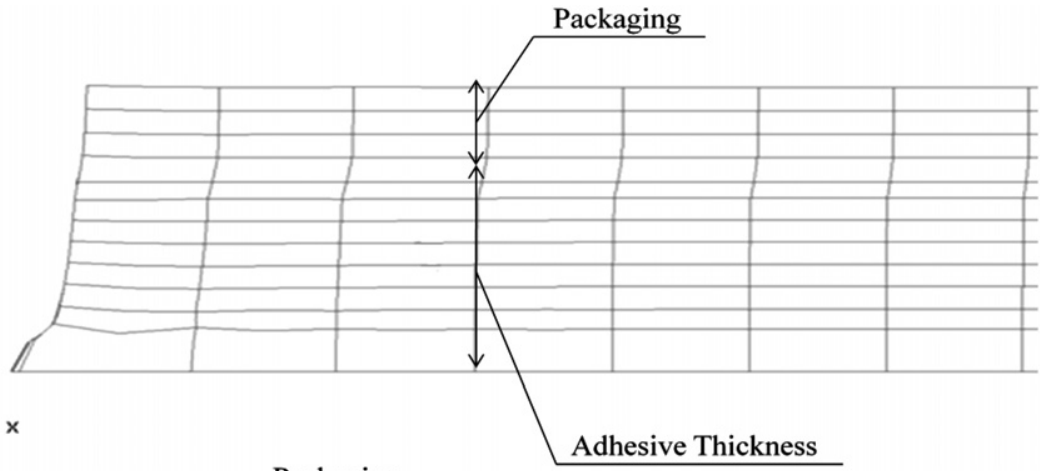

b

Packaging

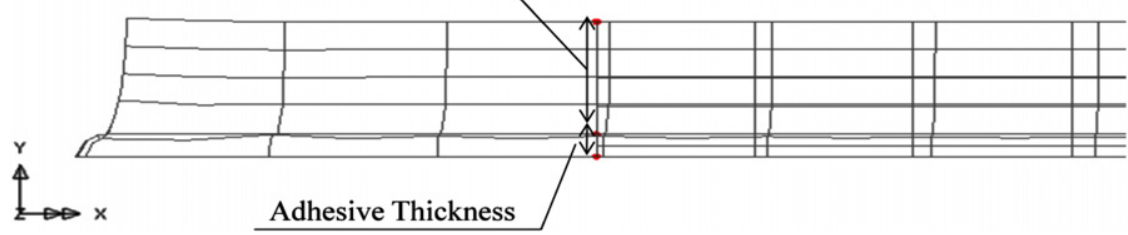

Fig. 8. Deformed mesh of the FE model for adhesive thicknesses of (a) $1000 \mu \mathrm{m}$ and (b) $100 \mu \mathrm{m}$.

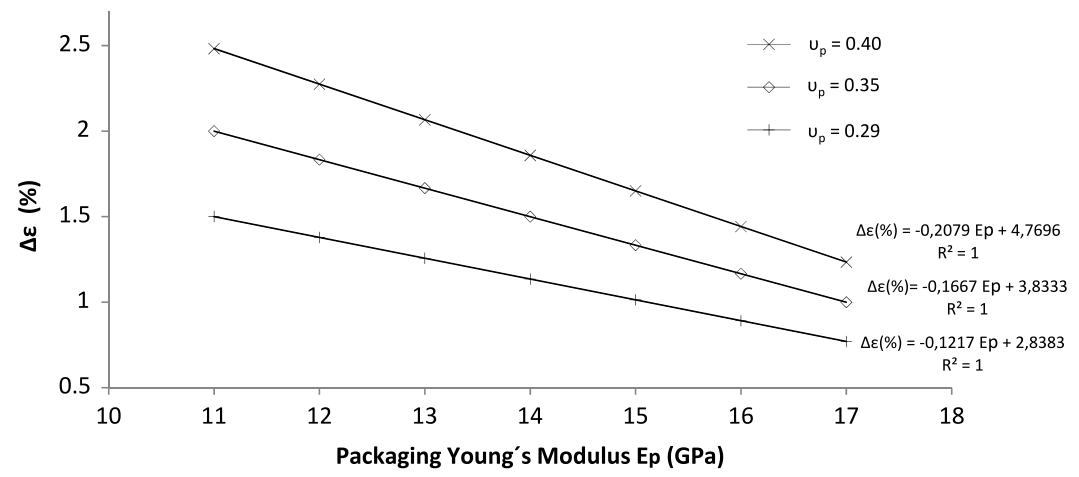

Fig. 9. Relation between the strain gradient $\Delta \varepsilon(\%)$ and the packaging Young's modulus $E_{P}$ (GPa) for different values of packaging Poisson's ratio $v_{p}$. Adhesive thickness $400(\mu \mathrm{m})$. Adhesive Poisson's ratio $v_{A}=0.4$.

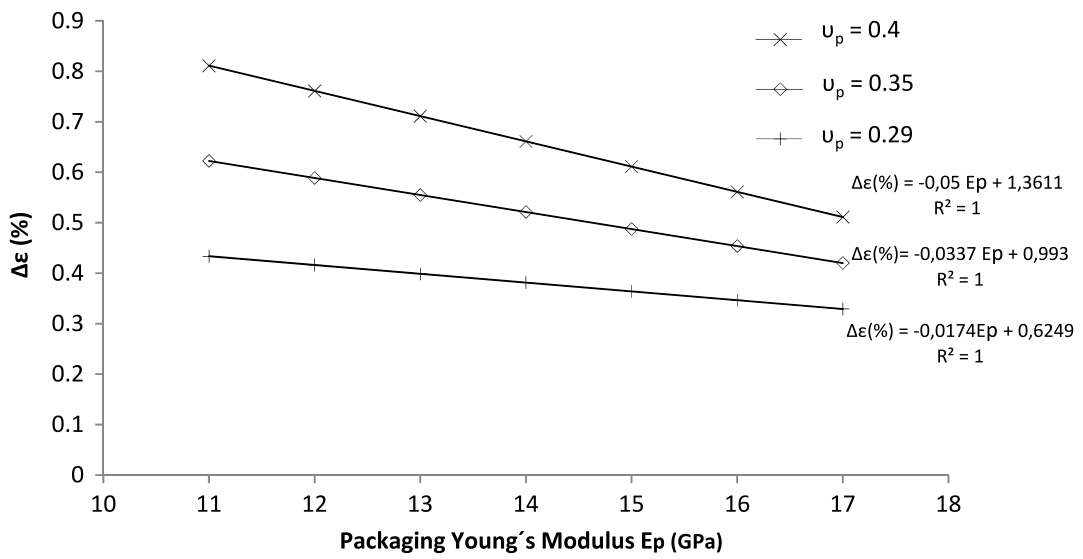

Fig. 10. Relation between the strain gradient $\Delta \varepsilon(\%)$ and the packaging Young's modulus $E_{P}(\mathrm{GPa})$ for different values of packaging Poisson's ratio $v_{p}$. Adhesive thickness $400(\mu \mathrm{m})$. Adhesive Poisson's ratio $v_{A}=0.1$. 


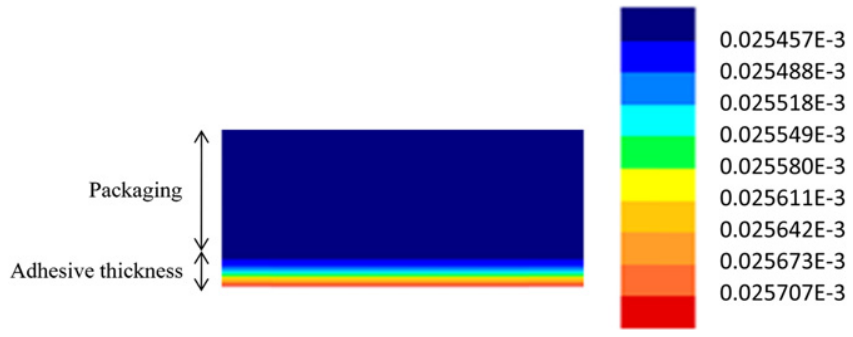

Fig. 11. Cross section of the sensor showing the strains in the $X$-direction. Adhesive thickness is $100 \mu \mathrm{m}$, adhesive Poisson's ratio is 0.4 .

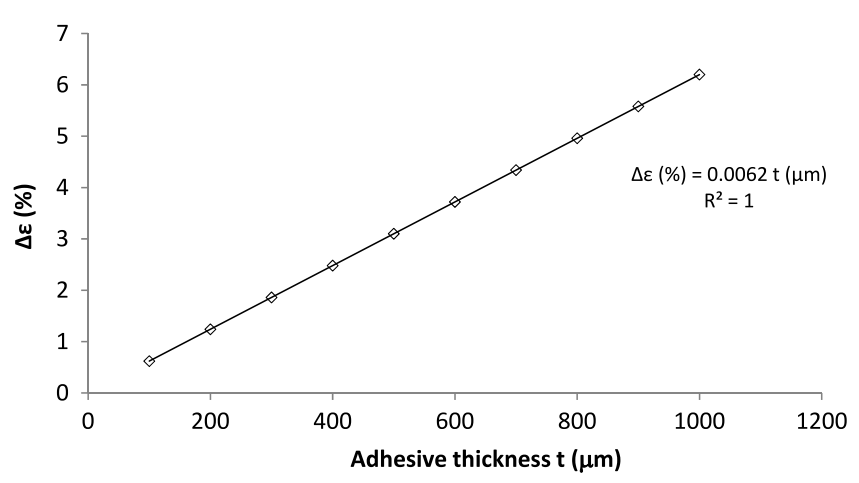

Fig. 12. Relation between strain gradient $\Delta \varepsilon$ (\%) and adhesive thickness $t(\mu \mathrm{m})$. Adhesive Poisson's ratio $v_{A}=0.4$.

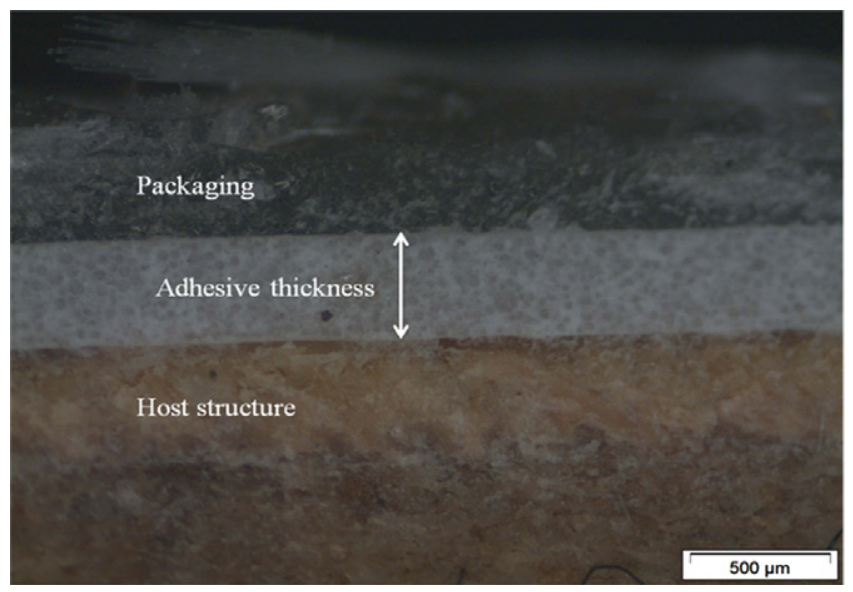

Fig. 13. Cross section of the UPV sensor glued to the host structure. The image was taken with a high resolution microscope StereoZoom SZ61 [24,25] and then processed with the software for image processing analySIS getIT Olympus [24,25].

\subsubsection{Sensitivity study of Poisson's ratio $\left(v_{A}\right)$ of the adhesive}

Table 1 contains the technical characteristics of the adhesive used in the study [23]. As the manufacturer provided no data on Poisson's ratio, a sensitivity analysis was carried out on the influence of this parameter on sensor error.

Fig. 14 shows how $\Delta \varepsilon(\%)$ varies with $v_{A}$ for an adhesive thickness of $400 \mu \mathrm{m}$. Eq. (11) represents the exponential law that fits the results and was obtained by a regression analysis. The correlation coefficient $R^{2}$ of the analysis is 0.9818 , which means that the exponential law fits the results very well.

$\Delta \varepsilon(\%)=0.5702 \mathrm{e}^{3.7322 v_{A}}$.

Fig. 15 shows the error variation of the sensor readings as a function of adhesive thickness of different Poisson's ratio values for the thicknesses commonly used to fix optical sensors. Maximum

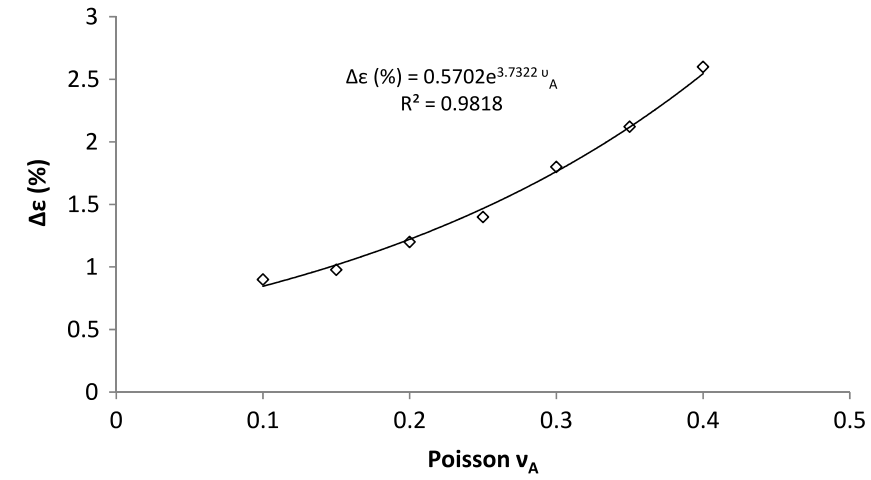

Fig. 14. Exponential correlation between the strain gradient $\Delta \varepsilon(\%)$ and the adhesive Poisson's ratio $v_{A}$. Adhesive thickness $t=400 \mu \mathrm{m}$.

$\Delta \varepsilon(\%)$ in the most unfavorable situation and for the usual adhesive thickness of $400 \mu \mathrm{m}$ does not exceed a value of $3 \%$.

\subsubsection{Comparison with other packaging systems}

In normal sensor packaging, the optic fiber is usually contained between two layers of material $[15,26]$, so that it was considered interesting to study strain transfer to the optic fiber for this type of sensor. To reach this goal, a numerical model of a generic two-layer sensor was created using the elements and mechanical properties described in Section 4.2 (see Fig. 16). Different numerical models were studied with adhesive thicknesses between 100 and $1000 \mu \mathrm{m}$ and bottom packaging layer thickness of between 100 and $700 \mu \mathrm{m}$. As can be seen in Fig. 17, the results in this case give $\Delta \varepsilon(\%)$ values much higher than in the case of the sensor described in Section 3. For example, at an adhesive thickness of $400 \mu \mathrm{m}$ the readings of the generic two-layer sensor give an error of $3.82 \%, 7.71 \%$ and $15.46 \%$ for bottom layer packaging thicknesses of 100,400 and $700 \mu \mathrm{m}$, respectively. These values are $35.1 \%, 67.8 \%$ and $83.9 \%$ higher than those obtained with the sensor described in Section 3. It should be emphasized that this behavior of the two-layer sensor means that the accuracy of its readings are highly influenced by manufacturing tolerances and variations in the properties of the packaging layers (e.g. changes in Poisson's ratio due to temperature variations). The packaging proposed in this study has no lower layer, which automatically rules out this source of error.

\section{Behavior of the packaging during thermal variations}

The fiber optic packaging process is crucial for the correct measurement of deformations since improper packaging can cause a reflected light spectrum with two or more peaks. As the FBG sensor is going to be exposed to thermal variations throughout the useful life of the structure to which it is fixed, its behavior during these events is especially important. A study was therefore carried out of the optic light spectrum reflected by the FBG sensor when subjected to a cycle of thermal variations produced by a Feutron KPK400 climatic chamber, as shown in Fig. 18.

Figs. 19 and 20 show the effects of temperature changes on the reflected light spectrum for two different packaging materials. Fig. 19 shows the effect on an unsuitable material, in which the light spectrum widens at top and bottom, shows a displacement towards the left and a tendency to deform at low temperatures. This distortion of the spectrum was caused by the random alignment of the glass fiber within the packaging. Fig. 20 shows the same test with the FBG sensor embedded in packaging made of a material that warranties a uniform distribution of the glass fibers. This material presents a better behavior under thermal variations as the shape of the reflected light spectrum remains constant during repeated thermal variations. 


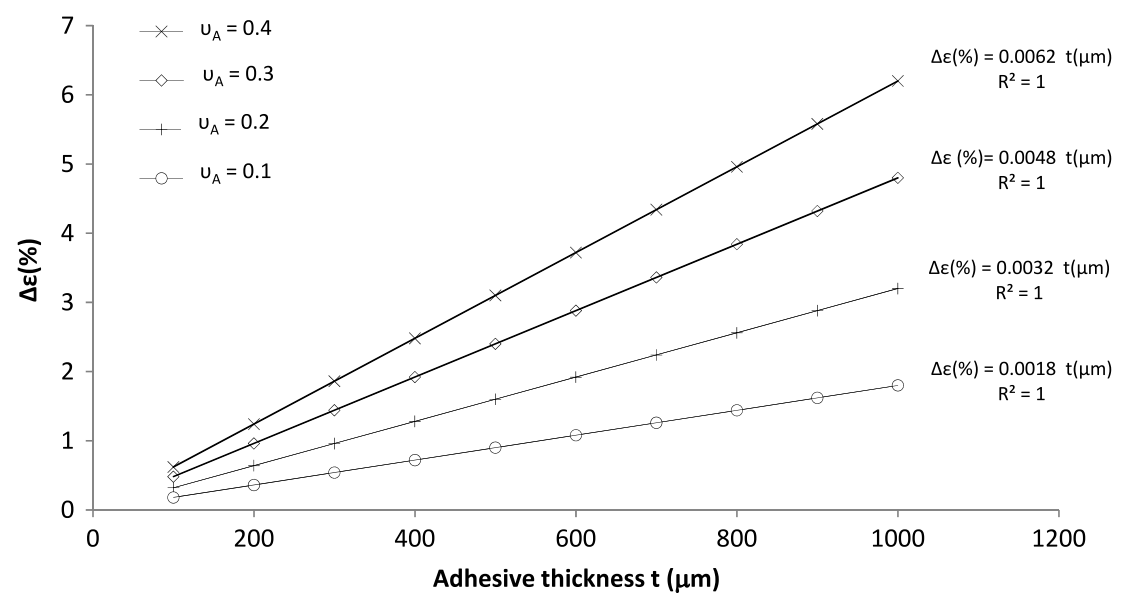

Fig. 15. Relation between the strain gradient $\Delta \varepsilon(\%)$ and the adhesive thickness $t(\mu \mathrm{m})$ for different values of the adhesive Poisson's ratio $v_{A}$.

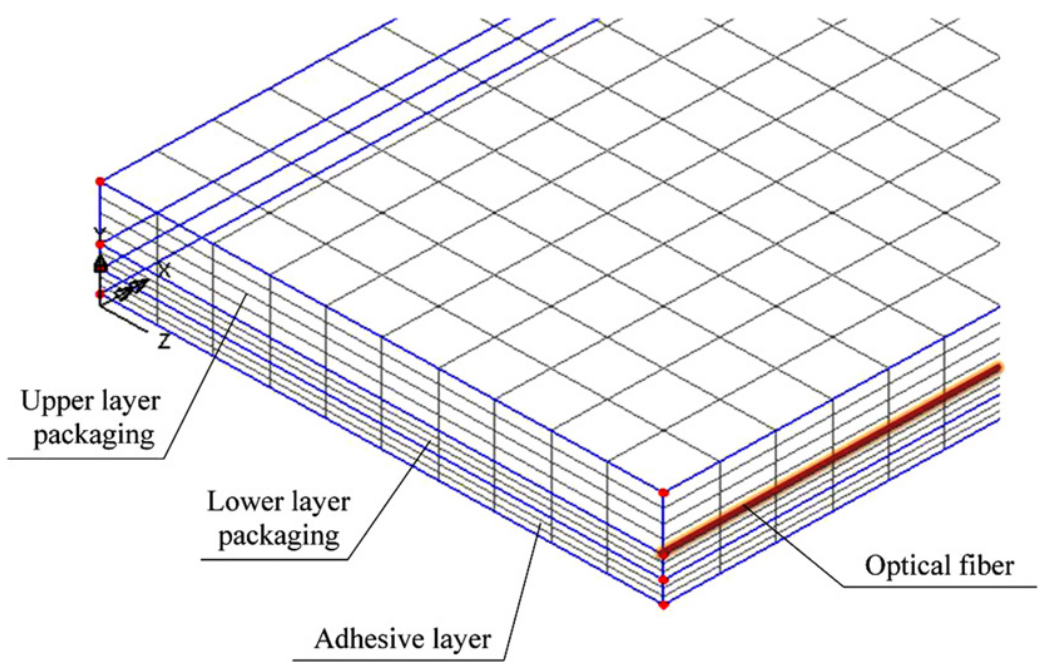

Fig. 16. 3D partial view of the FE model used for analyzing a generic two-layer sensor.

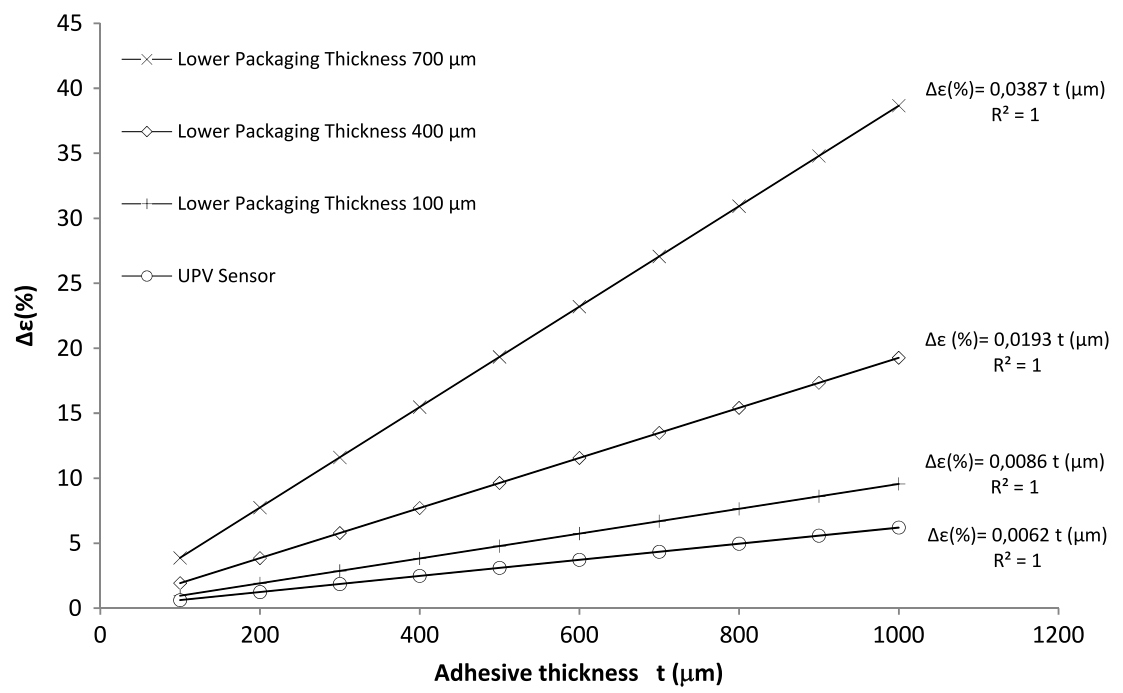

Fig. 17. Comparison of a UPV sensor and a two-layer sensor. Relation between the strain gradient $\Delta \varepsilon(\%)$ and the adhesive thickness $t$ ( $\mu$ m) for different values of thickness of the packaging lower layer $(\mu \mathrm{m})$. 


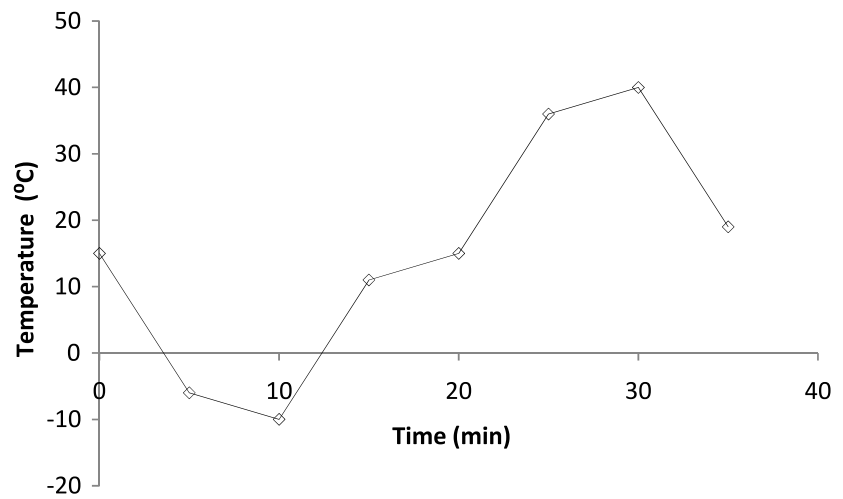

Fig. 18. Temperature cycle applied to the UPV sensor.

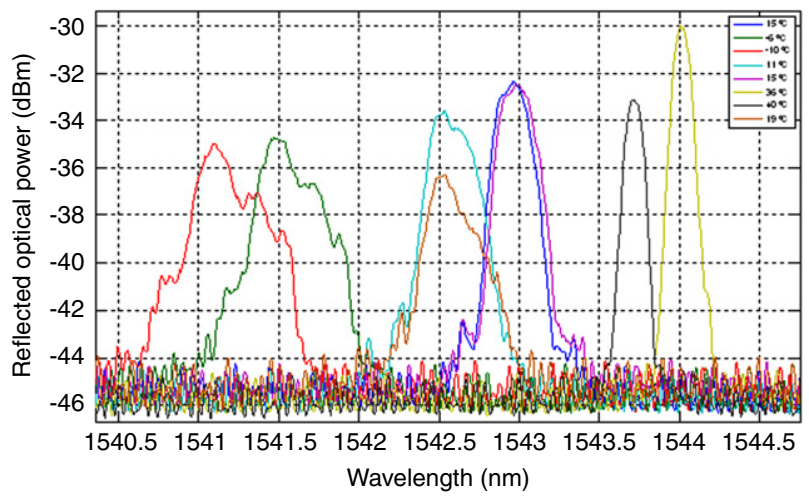

Fig. 19. Packaging made of an unsuitable material. Reflected light spectrum for different ambient temperatures.

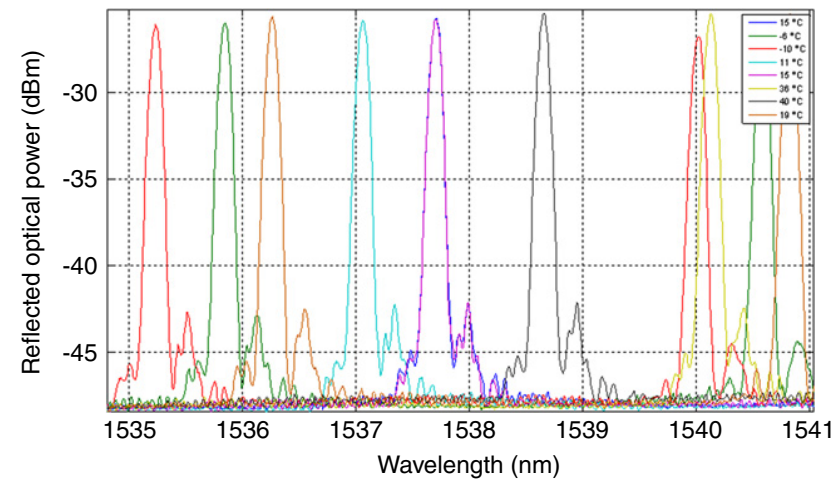

Fig. 20. Packaging made of a suitable material. Reflected light spectrum for different ambient temperatures.

\section{Conclusions}

This paper presents a new surface fiber optic sensor designed to measure strains in structures. The sensor packaging has a single layer so that the optic fiber is practically in contact with the adhesive used to fix the sensor to the monitored surface. The paper also contains details of a numerical study performed to characterize the sensor behavior and to obtain the reading errors $\Delta \varepsilon(\%)$ associated with parameters such as the adhesive thickness and the mechanical properties, which are subject to uncertainty. The numerical model was adapted to characterize the behavior of a sensor with two layers of packaging, in order to compare its behavior with that of the single-layer model. The following conclusions can be drawn from the results obtained:

(a) The configuration of the optical sensor proposed in this paper is hardly affected by the thickness of adhesive used to fix the device to the structure. Normal adhesive thickness values of $400 \mu \mathrm{m}$ lead to reading errors below 2.5\%. High thickness values around $1000 \mu \mathrm{m}$ yield a reading error below $6.5 \%$.

(b) Low values (of the order of 0.1) of the adhesive Poisson's ratio $v_{A}$ improve strain transfer due to the higher value of the shear modulus $G$. On the other hand, high $v_{A}$ values cause greater errors in sensor readings. In all cases, and in the most unfavorable conditions ( $\left.t=1000 \mu \mathrm{m}, v_{A}=0.4\right)$ maximum sensor error is below 6.5\%.

(c) The presence of a packaging layer below the sensor has a negative influence on its accuracy. For example, a thickness of around $400 \mu \mathrm{m}$ can induce errors of up to $15 \%$. The precision of the two-layer sensor is thus highly sensitive to both manufacturing tolerances and to variations in packaging layer properties, e.g. changes in Poisson's ratio caused by temperature variations. This indicates that it is preferable to use single-layer packaging, as in the model proposed in this paper.

(d) Also studied was the importance of packaging temperature changes on the spectrum of the light reflected by the sensor, which also affects its precision. The use of an appropriate packaging material was observed to enable the reflected light spectrum to maintain its shape during thermal variation cycles.

\section{Acknowledgements}

The authors wish to express their gratitude for the assistance provided by the ITEAM Institute of the UPV and Dr. L. Soriano and Dr. J. Payá of the ICITECH Institute of the UPV. This study was financed by a grant from the Sacyr Vallehermoso Construction Company within the framework of the project "Development of Optical Sensors Applied to Building Structures to Determine their Behavior in Real Time". Mr. Paya-Zaforteza got funding for this research from the Spanish Ministry of Education (Contract No. EX2008-0669 of the program for postdoctoral stays).

\section{References}

[1] Li HN, Li DS, Song GB. Recent applications of fiber optic sensors to health monitoring in civil engineering. Eng Struct 2004;26:1647-57.

[2] Fernández-Ordoñez JA, Navarro-Vera JR. Eduardo torroja ingeniero-engineer. Madrid: Pronaos; 1999. Bilingual edition Spanish-English.

[3] Desideri P, Nervi Jr PL, Positano G. Pier Luigi Nervi. Zurich: Patmos Verlag; 1982.

[4] Glisic B, Inaudi D. Fibre optic methods for structural health monitoring. Chichester: John Wiley \& Sons Ltd.; 2007.

[5] Orcesi AD, Frangopol DM, Kimb S. Optimization of bridge maintenance strategies based on multiple limit states and monitoring. Eng Struct 2010; 32(3):627-40.

[6] Shoukry SN, Riad MY, William GW. Longterm sensor-based monitoring of an LRFD designed steel girder bridge. Eng Struct 2009;31(12):2954-65.

[7] Ko JM, Ni YQ. Technology developments in structural health monitoring of large-scale bridges. Eng Struct 2005;27:1715-25.

[8] Majumder M, Gangopadhyay TM, Chakraborty AK. Fibre Bragg gratings in structural health monitoring-present status and applications. Sensors Actuators A 2008; 147:150-64.

[9] Chan THT, Yu L, Tam HY, Ni YQ, Liu SY, Chung WH, Cheng LK. Fiber Bragg grating sensors for structural health monitoring of tsing Ma bridge: background and experimental observation. Eng Struct 2006;28:648-59.

[10] Chan THT, Ashebo DB, Tam HY, Yu Y, Chan TF, Lee PC, Perez Gracia E. Vertical displacement measurements for bridges using optical fiber sensors and CCD cameras-a preliminary study. Struct Health Monit 2009;8:243-9.

[11] Chung W, Kang D. Full-scale test of a concrete box girder using FBG sensing system. Eng Struct 2008;30(3):643-52.

[12] Lin YB, Pan CL, Kuo YH, Chang KC, Chern JC. Online monitoring of highway bridge construction using fiber Bragg grating sensors. Smart Mater Struct 2005; $14: 1075-82$.

[13] Udd E. Fiber optic smart structures. New York: John Wiley \& Sons Inc.; 1995.

[14] Li D, Li H, Ren L, Song GB. Strain transferring analysis of fiber Bragg grating sensors. Opt Eng 2006;45(2):1-8.

[15] Moyo P, Brownjohn JMW, Suresh R, Tjin SC. Development of fiber Bragg grating sensors for monitoring civil infrastructure. Eng Struct 2005;27:1828-34.

[16] Barrera D, García R, Sales S, Gasch I, Ivorra S, Giménez E. Application of fiber Bragg grating optic sensors for material characterization. $5^{\circ}$ Reunión Española de Optoelectrónica. Optoel’07. 2007 [in Spanish]. 
[17] Wong Allan CL, Childs P, Berndt R, Macken T, Peng GD, Gowripalan N. Simultaneous measurement of shrinkage and temperature of reactive powder concrete at early-age using fibre Bragg grating sensors. Cem Concr Compos 2007;29(6):490-7.

[18] Torres Górriz B. Experimental study of FBG optical sensors to measure strain and temperatures in structures. Master thesis. Departamento de Ingeniería de la Construcción y Proyectos de Ingeniería Civil. Universidad Politécnica de Valencia. November 2009 [in Spanish].

[19] ASTM D 638-03. American Society for Testing Materials. Standard test method for tensile properties of plastics. Philadelphia (USA); April 2004.

[20] ASTM E132. American Society for Testing Materials. Test method for Poisson's ratio at room temperature. Philadelphia (USA); April 2004.
[21] Exequiel R, Analía V. Mechanical properties of natural fiber composite materials obtained by the technique of Infusion Vacuum. Jornadas SAM/ CONAMET/SIMPOSIO MATERIA. 2003 [in Spanish].

[22] Lusas. Lusas Reference Manual. Surrey (UK); 2010.

[23] HBM Measurements with confidence. Material safety data sheet. 2006 www.hbm.com.

[24] Olympus corporation user manual SZ61. Shinjuku (Tokyo, Japan); 2006.

[25] Digital camera ALTRA solf imaging system. User manual. Shinjuku (Tokyo, Japan); 1999.

[26] Gangopadhyay TK, Majumder M, Chakraborty AK, Dikshit AK, Bhattacharya DK. Fibre Bragg grating strain sensor and study of its packaging material for use in critical analysis on steel structure. Sensors Actuators A 2009;150(1): 78-86. 


\section{Optical Engineering}

\section{Optical fiber sensors embedded in concrete for measurement of temperature in a real fire test}

Antonio Bueno

BLтTjaInin Torres

David Barrera

Pedro Arstonio Calderon

José Manmel Lloris

Maria Jose Lomer

Sil Waulor Sales 



\title{
Optical fiber sensors embedded in concrete for measurement
}

\section{of temperature in a real fire test}

\author{
Antonio Bueno ${ }^{1, *}$, Benjamín Torres ${ }^{2}$, David Barrera ${ }^{1}$, Pedro Antonio Calderón ${ }^{2}$, José \\ Manuel Lloris ${ }^{3}$, María José López ${ }^{3}$, Salvador Sales ${ }^{1}$ \\ ${ }^{1}$ iTEAM Research Institute, Optical and Quantum Communications Group, Universidad \\ Politécnica de Valencia, Camino de Vera s/n, 46022 Valencia, Spain. \\ ${ }^{2}$ Instituto de Ciencia y Tecnolgía del Hormigón ICITECH, Universidad Politécnica de \\ Valencia, Camino de Vera s/n, 46022, Valencia, Spain \\ ${ }^{3}$ Instituto Tecnológico de la Construcción AIDICO, Avda. Benjamin Franklin 17, 46980, \\ Paterna, Valencia, Spain. \\ *Corresponding author: anbuemar@iteam.upv.es
}

We present the results of a real fire test using optical fiber sensors embedded in concrete samples. The temperature curve used in this experiment is described in the Spanish/European standard UNE-EN 1363-1 temperature profile for normalized concrete resistance to real fire tests, reaching temperatures of more than $1000^{\circ} \mathrm{C}$ inside the fire chamber and up to $600^{\circ} \mathrm{C}$ inside the concrete samples. Three types of optical sensors have been embedded in concrete: 1) standard fiber Bragg gratings (FBG) inscribed in photosensitive germanium-boron codoped fiber, 2) regenerated FBG (RFGB) inscribed in germanium doped fiber and 3) RFBG inscribed in germanium-boron codoped fiber. 
Keywords: Optical fiber temperature sensor; high temperature; fiber Bragg grating; regenerated fiber Bragg grating; fire test.

\section{Introduction}

The influence of fire on concrete structures has been reported in previous studies of structural damage because the strength of a concrete material decreases under the effect of high temperature $[1,2]$. To determine the damage produced by a fire to a concrete structure, some sensor elements need to be installed on the surface or inside the structure. Traditionally, electrical sensors have been used to obtain physical measurements of the structures, but recently some optical fiber sensors have been developed. Optical fiber sensors have the advantage that can be embedded in the concrete structures due to their small size.

In recent years optical fiber sensors for multiple applications have been widely used in the civil engineering field. Compared to the electrical sensors, the optical sensors present several advantages such as smaller and lighter, immunity to electromagnetic interference (EMI), high temperature tolerance and resistance to harsh environments. In addition, optical technology allows many sensors to be multiplexed in a single optical fiber in order to create a quasidistributed sensor system able to detect local damage.

In this test, two concrete samples were placed in a fire chamber to perform a real fire test using embedded optical fiber temperature sensors. A normalized UNE-EN 1363-1 fire temperature curve was programmed in to the fire chamber in order to obtain the heat transference inside the concrete samples. This real test is very helpful if the behavior of a concrete structure 
under fire is required, for example in a concrete tunnel, where the integrity of the structure has to be preserved in order to maintain the safety of the people inside the tunnel.

To date, previous publications concerning high temperature measurements in real structures using optical sensors have measured moderate high temperatures. In some works, embedded temperature sensors reached up to $150^{\circ} \mathrm{C}$ inside concrete specimens $[3,4]$. In another publication, external temperature sensors were employed to measure gas temperature, reaching a maximum temperature of $300{ }^{\circ} \mathrm{C}$ [5]. Higher temperatures were measured in the experiments carried out by Heiberg et al. [6], but in this case, embedding optical fiber Bragg grating sensors and thermocouples into aluminum alloys. To the best of our knowledge, this is the first experiment of a real test fire of concrete structures with embedded optical fiber Bragg grating sensors.

In the present test, maximum temperatures up to $600{ }^{\circ} \mathrm{C}$ inside the concrete beams are obtained corresponding to a chamber temperature close to $1100{ }^{\circ} \mathrm{C}$. Furthermore, three different types of optical temperature sensors, that will be described in the next section, have been used in the test to compare their features at high temperature.

\section{Sensors development}

All of the sensors used in this work are based on fiber Bragg gratings (FBG) [7]. The gratings were created through the phase mask method using an UV beam at $244 \mathrm{~nm}$ from a frequencydouble Argon laser. Up to 4 different phase masks were used to create gratings of central Bragg wavelengths at around $1529.4 \mathrm{~nm}, 1534.2 \mathrm{~nm}, 1539 \mathrm{~nm}$ and $1543.7 \mathrm{~nm}$. The lengths of the 
gratings were set to $2 \mathrm{~cm}$ with an apodized profile of the refractive index to decrease the secondary lobes of the reflection spectrum and to improve the response of the sensors.

Three different types of optical sensors based on FBGs have been developed. The first type is a standard FBG and the other two sensors have been created by specifically treating a FBG in a two steps process. The first step in this process consists in the loading of hydrogen into an optical fiber [8] with 25 bars pressure for 15 days at room temperature. This hydrogen loaded fiber is then exposed to UV light to create the FBG, achieving the formation of hydroxyl groups $(\mathrm{OH})$ in the core of the fiber. The second step in the creation of this type of sensor is the thermal treatment, which consists of a temperature ramp until the FBG is erased, then a steady state for several minutes in order to regenerate the grating and finally a cool down. The thermal annealing of the optical fiber leads to the creation of a much more stable FBG, being able to measure higher temperatures than a standard FBG. This new grating was called firstly as Chemical Composition Grating (CCG) [9] due to the chemical processes present in the creation of the grating, but recently the new term of Regenerated FBG (RFBG) is used [10], referring to the regeneration process of the grating during the thermal step.

Depending on the initial FBG (also known as "seed"), the resulting RFBG has different properties. Two different types of optical fibers were used in the grating inscription. The first one was a standard germanium (Ge) doped optical fiber and the other one was a germanium and boron $(\mathrm{Ge} / \mathrm{B})$ codoped photosensitive optical fiber. The main differences between them are the grating regeneration temperature (approximately $950{ }^{\circ} \mathrm{C}$ in the $\mathrm{Ge}$ optical fiber and $550{ }^{\circ} \mathrm{C}$ in the $\mathrm{Ge} / \mathrm{B}$ optical fiber approximately) and the maximum working temperature $\left(1200^{\circ} \mathrm{C}\right.$ in $\mathrm{Ge}$ fiber 
and around $800{ }^{\circ} \mathrm{C}$ in $\mathrm{Ge} / \mathrm{B}$ fiber). These thermal characteristics extend the temperature working limits of a standard FBG, established at a maximum temperature of $600{ }^{\circ} \mathrm{C}$. Besides, a standard FBG suffers from a highly significant reduction of the reflectivity when the temperature exceeds $300{ }^{\circ} \mathrm{C}$. It can be seen on Fig. 1 the setup made for the thermal treatment in order to achieve the grating regeneration. A tubular oven was used to heat the grating and an optical broadband source and an optical spectrum analyzer were used to monitor the grating's reflectivity.

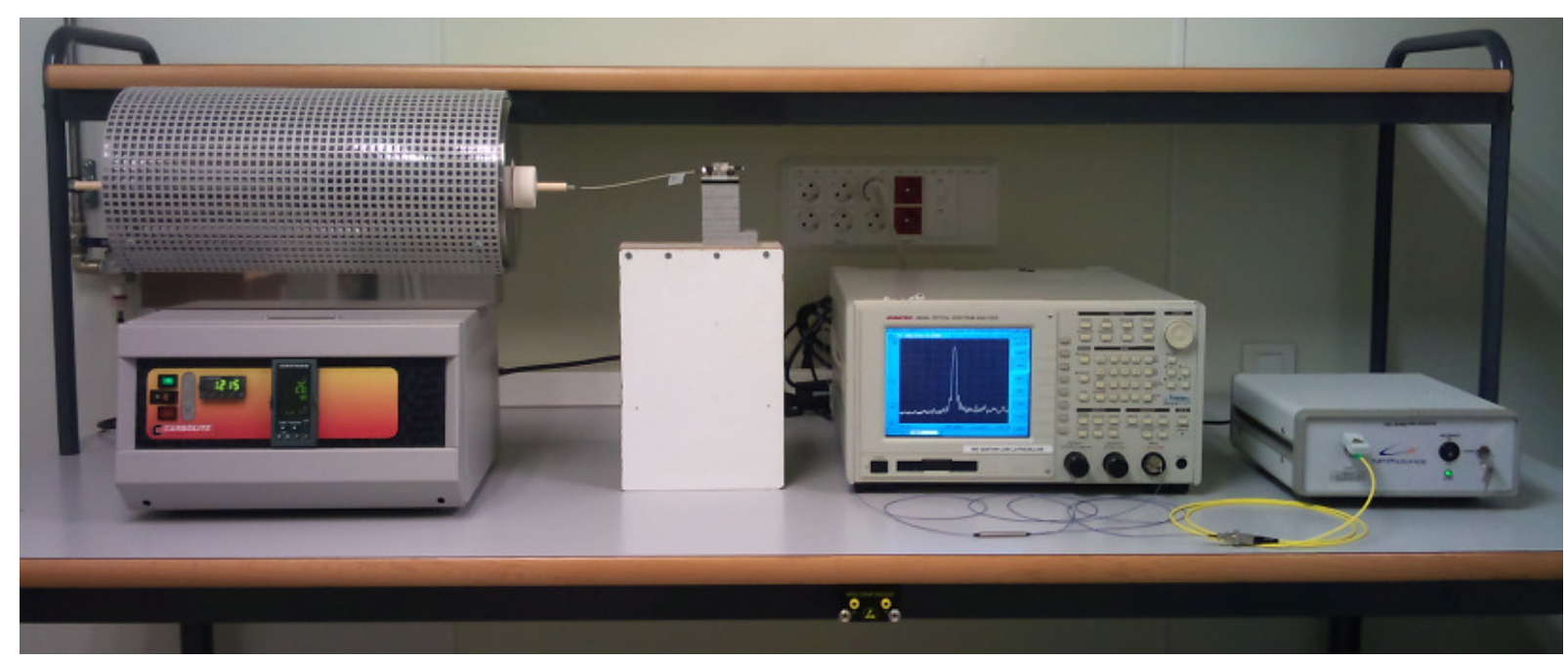

Fig. 1: Optical setup made for grating regeneration.

Once the FBGs and the RFBGs were created, it was necessary to develop a suitable protection to prevent damage to the optical fiber during the installation of the sensors. A simple sensor head was fabricated by introducing the optical fiber (containing an FBG or an RFBG) inside a hollow metal tube sealed at both ends. A good seal is helpful in the sensor transportation and it also prevents the accidental introduction of fresh concrete inside the tube during the installation. Therefore the ends of the metal tube were sealed with high temperature resistant glue. As a result the fiber remains loose inside the tube and is kept away from any external agent and is free from the strain applied to the tube (Fig. 2b) 
Electrical sensors were also placed, consisting of thermocouples type $\mathrm{K}$ able to measure temperatures up to $1000{ }^{\circ} \mathrm{C}$. These sensors were used to compare the measurements between optical and electrical sensors.

As a result of this process, 4 sets of sensors were fabricated, each one of them containing:

- 1 standard FBG sensor inscribed in a photosensitive Ge/B codoped optical fiber.

- 1 RFBG sensor inscribed in a Ge doped optical fiber.

- 1 RFBG sensor inscribed in a photosensitive Ge/B codoped fiber.

- 1 type K electrical thermocouple.

\section{Installation of sensors and concrete samples}

The fabricated sets of sensors mentioned in the previous section were installed inside two concrete samples. These samples were made in a cylindrical shape, having a size of $30 \mathrm{~cm}$ height and $15 \mathrm{~cm}$ of diameter. Two sets of sensors were embedded in each concrete specimen in two specific locations: the lateral side and the center of gravity $(7.5 \mathrm{~cm}$ from the lateral side and 15 cm height). Fig. 2a shows the schematic locations of the sensors in points L1 (lateral side) and $\mathrm{C} 1$ (center of gravity) in sample 1 and L2 (lateral side) and C2 (center of gravity) in sample 2. 


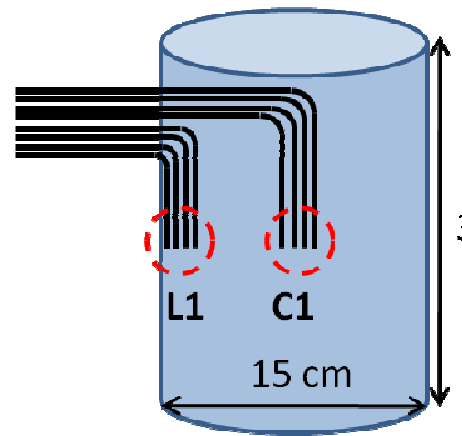

a) Sample 1

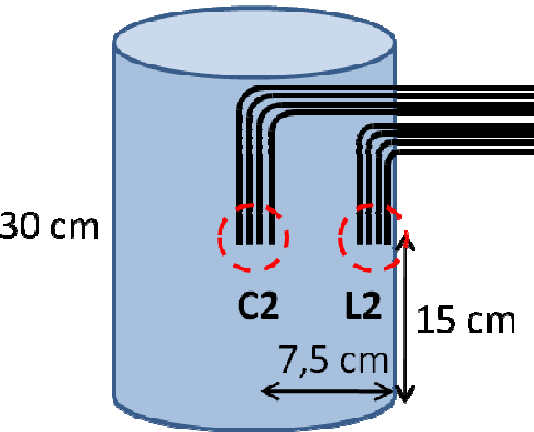

Sample 2

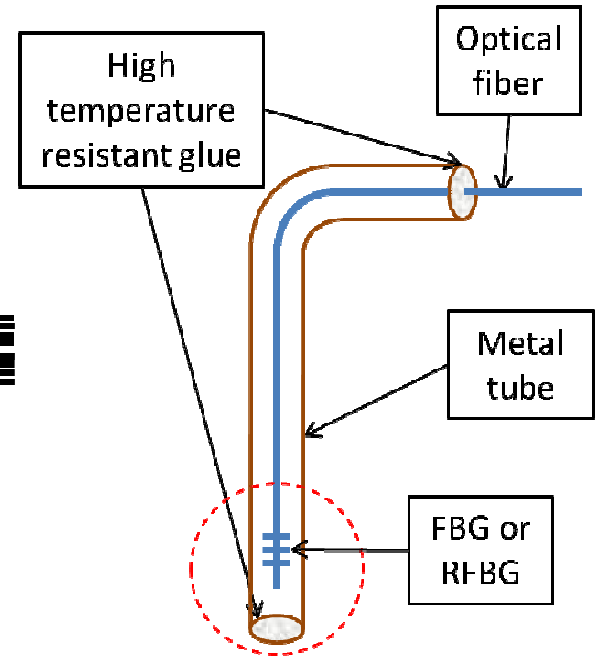

b)

Fig. 2: a) Sensor locations inside the concrete samples. b) Detailed view of the sensor package.

\begin{tabular}{|c|c|c|}
\hline Specimen & Location & Sensors installed \\
\hline \multirow{2}{*}{ Sample 1 } & $\mathrm{L} 1$ & $\mathrm{Ge} \# 1, \mathrm{Ge} / \mathrm{B} \# 1, \mathrm{FBG} \# 1, \mathrm{TC} \# 1$ \\
\cline { 2 - 3 } & $\mathrm{C} 1$ & $\mathrm{Ge} \# 2, \mathrm{Ge} / \mathrm{B} \# 2, \mathrm{FBG} \# 2, \mathrm{TC} \# 2$ \\
\hline \multirow{2}{*}{ Sample 2 } & $\mathrm{L} 2$ & $\mathrm{Ge} \# 3, \mathrm{Ge} / \mathrm{B} \# 3, \mathrm{FBG} \# 3, \mathrm{TC} \# 3$ \\
\cline { 2 - 3 } & $\mathrm{C} 2$ & $\mathrm{Ge} \# 4, \mathrm{Ge} / \mathrm{B} \# 4, \mathrm{FBG} \# 4, \mathrm{TC} \# 4$ \\
\hline
\end{tabular}

Table 1: Sensors installed inside the concrete samples.

The sensors were labeled as FBG\#1..4 for the FBG sensors, Ge\#1..4 for the RFBG inscribed in Ge doped fiber, Ge/B\#1..4 for the RFBG inscribed in Ge/B codoped fiber and TC\#1..4 for the thermocouples. It can be seen in Table 1 the locations of every single sensor inside the concrete samples after the installation. The sets of sensors were fixed to two cylindrical moulds, and then the concrete was carefully poured into each of them, completely covering the sensing heads of the sensors. In Fig. 3 it can be seen two sets of sensors fabricated on the left, and the location of these sets inside the cylindrical mould before the concrete pouring, on the right. The concrete samples were removed from their moulds after 48 hours, 
when the concrete had reached almost the $80 \%$ of its final strength. The next step was to move the samples to a specific concrete cure chamber where the samples were held for 34 days until they reached their maximum strength.

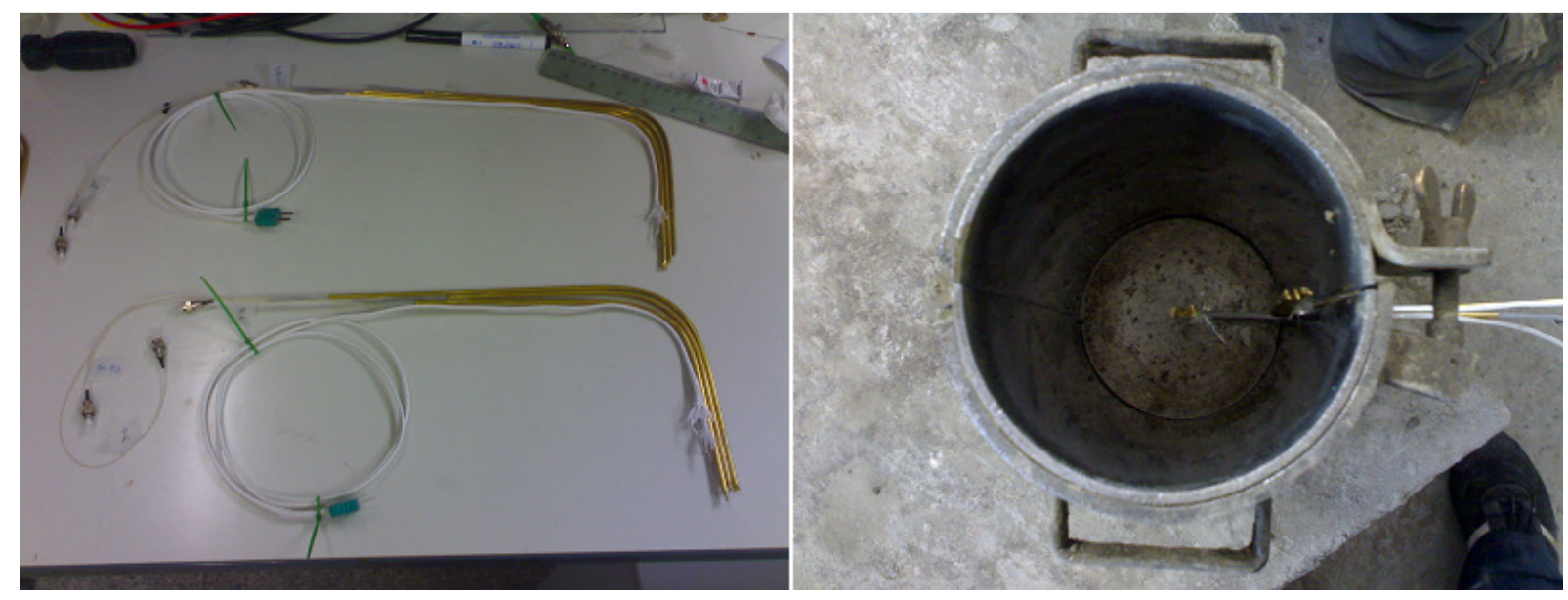

Fig. 3: Two sets of sensors fabricated, on the left. Location of these sets inside the cylindrical mould before concrete pouring, on the right.

The two concrete samples were moved to a fire chamber to perform the temperature test. The size of the fire chamber was $1.5 \mathrm{~m} \times 1.5 \mathrm{~m} \times 1.5 \mathrm{~m}$, containing 4 burners that were capable of increasing the temperature inside the chamber to above $1200{ }^{\circ} \mathrm{C}$. The burners were monitored by 4 different electrical thermocouples located inside the chamber. The concrete samples were positioned at the lateral sides of the floor inside the chamber. The metal tubes containing the sensors were extended about 30 centimeters and fed out of the fire chamber through a 2 centimeters diameter hole in order to allow the optical connections to the measurement equipment outside the chamber. A commercially available sensor interrogator was used to collect the data from the optical sensors during the test.

After the installation of the concrete samples, sensor named Ge/B\#1 appeared to be broken due to the transportation of the concrete specimens. Thus, the survival percentage during installation concerning the optical sensors was $93.75 \%$. 


\section{Real fire temperature test}

The Spanish/European standard UNE-EN 1363-1 temperature profile for normalized concrete resistance to real fire was used. The formula used for the temperature increment is:

$$
\Delta T=345 \log (8 t+1)
$$

where $t$ is time in minutes. According to equation (1), the target increment of temperature has a very fast initial response, followed by a slower temperature rise. The duration of the test was 120 minutes, when the temperature measured by the thermocouples inside the chamber was around $1060{ }^{\circ} \mathrm{C}$.

The data obtained by the optical sensor interrogator was converted to temperature by applying a second order temperature sensitivity of $8.6 \mathrm{pm} / \mathrm{K}$ and $7.97 \cdot 10^{-3} \mathrm{pm} /\left(\mathrm{K}^{2}\right)$ to the sensors inscribed in germanium-boron codoped fiber and $11.79 \mathrm{pm} / \mathrm{K}$ and $2.45 \cdot 10^{-3} \mathrm{pm} /\left(\mathrm{K}^{2}\right)$ to the sensors inscribed in germanium doped fiber. Sensitivity factors of the optical sensors were previously obtained through a calibration test. The spectrum of the gratings was also recorded in order to monitor the variation of the reflectivity with the temperature. In addition, the shape of the grating's spectrum changes. Indeed, it can be observed in Fig. $4 \mathrm{~b}$ that the peak power reflected by FBG\#1 decreases more than $20 \mathrm{~dB}$ and its spectrum become broader. On the other hand, the spectrum of RFBG Ge\#1 remains almost unchanged (Fig. 4a). 

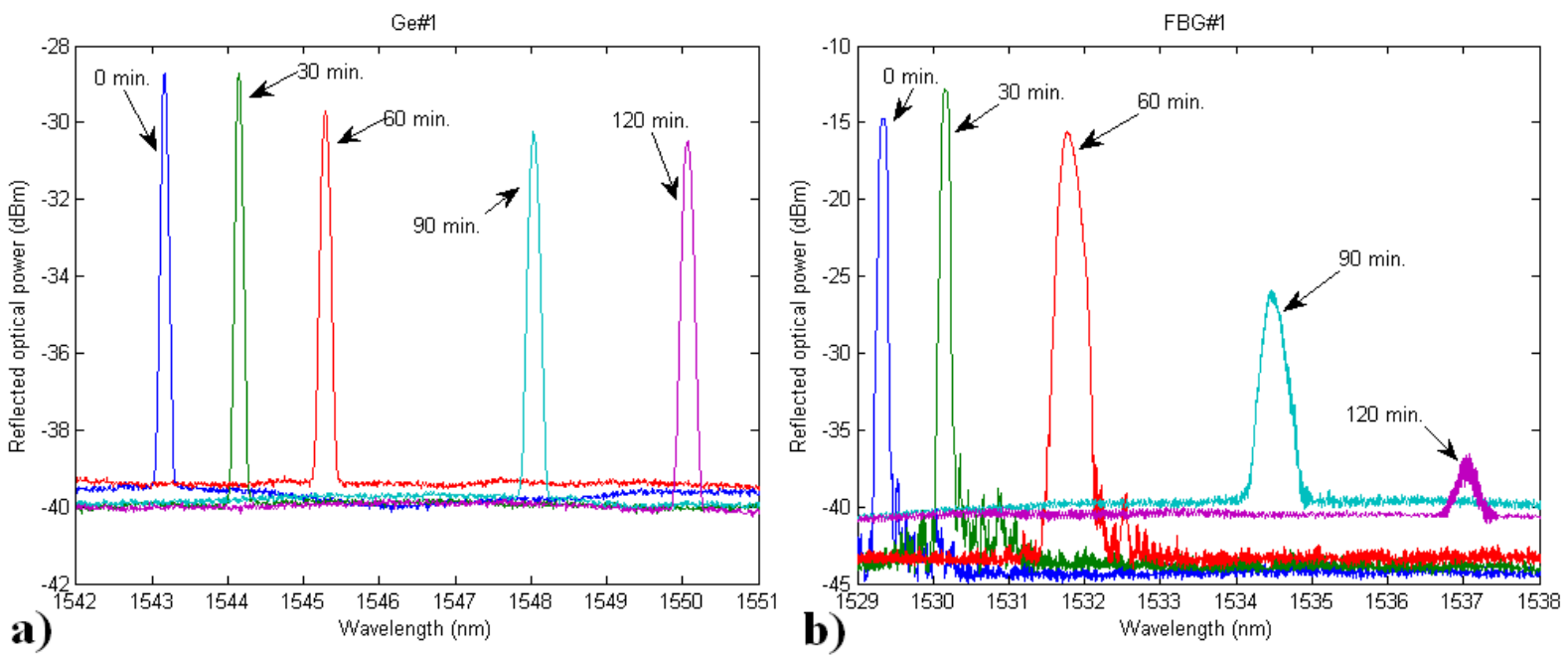

Fig. 4: Spectrum evolution at different times during the test of sensors a) Ge\#1 and b) FBG\#1.

In Figs. 5a-8a it can be seen the temperature evolution and in Fig. $5 b-8 b$ the normalized peak power reflected by the gratings during the fire test. It can be seen that at the center of gravity of both concrete samples (points $\mathrm{C} 1$ and $\mathrm{C} 2$ ) the temperature measured by the thermocouples and the optical sensors is very similar, whereas the temperature profile is rather different on the lateral surface (points L1 and L2). This is due to the location of the sensors during the installation and the temperature gradient inside the concrete sample. The thermocouples were the nearest sensors to the lateral side in both concrete samples, thus inside the chamber they were more exposed to the fire created by the burners. In addition, the temperature gradient is higher at the air-concrete interface during the heating process [1], and the thermocouples were also installed very close to that interface.

It can also be seen in Figs. 5a-8a a temperature ramp registered by the optical sensors at around 20 minutes. This is due to the thermal inertia of concrete [11] which consists of a time 
delay in the transference of heat through the concrete mass when the temperature changes from room temperature $\left(\sim 22^{\circ} \mathrm{C}\right)$ to a temperature higher than $500^{\circ} \mathrm{C}$ in only 5 minutes.
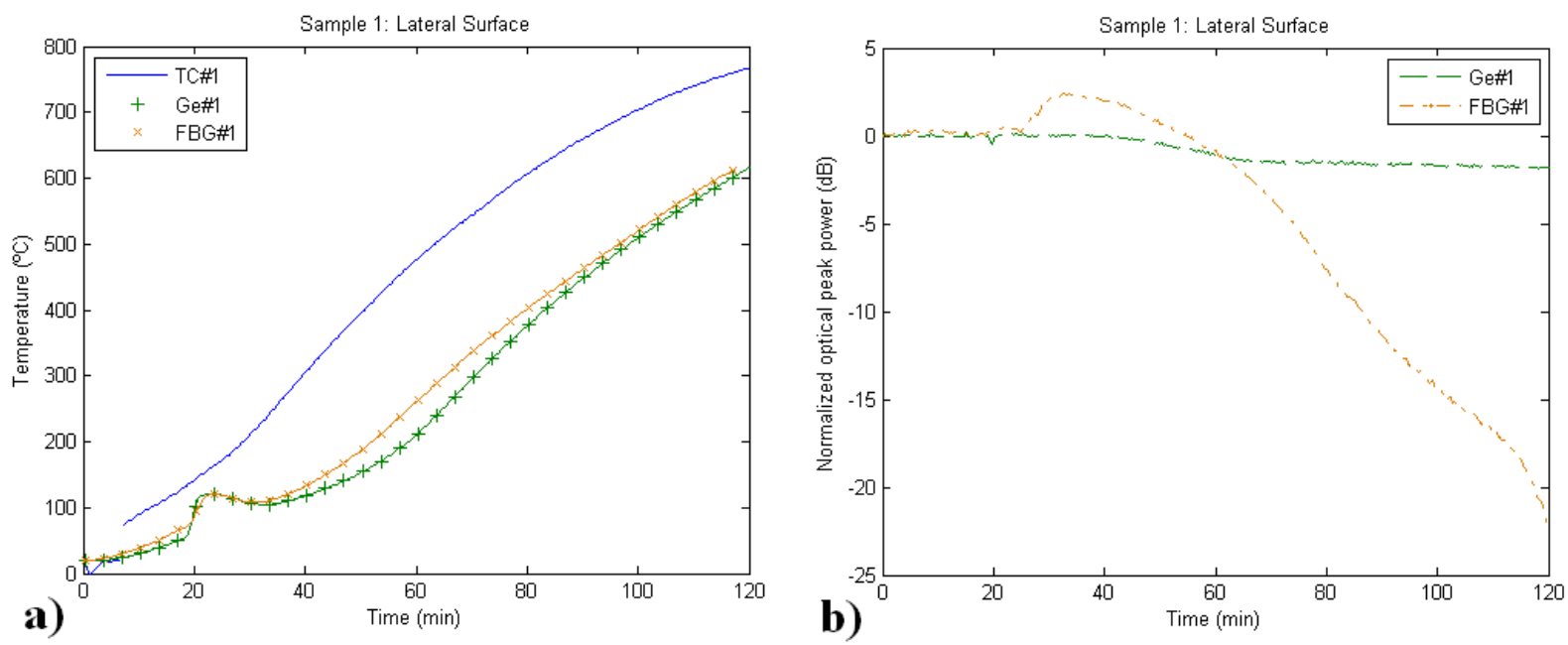

Fig. 5: Sample 1 at lateral surface (point L1): a) Temperature measured by optical and electrical sensors and b) Normalized optical peak power of the optical sensors.
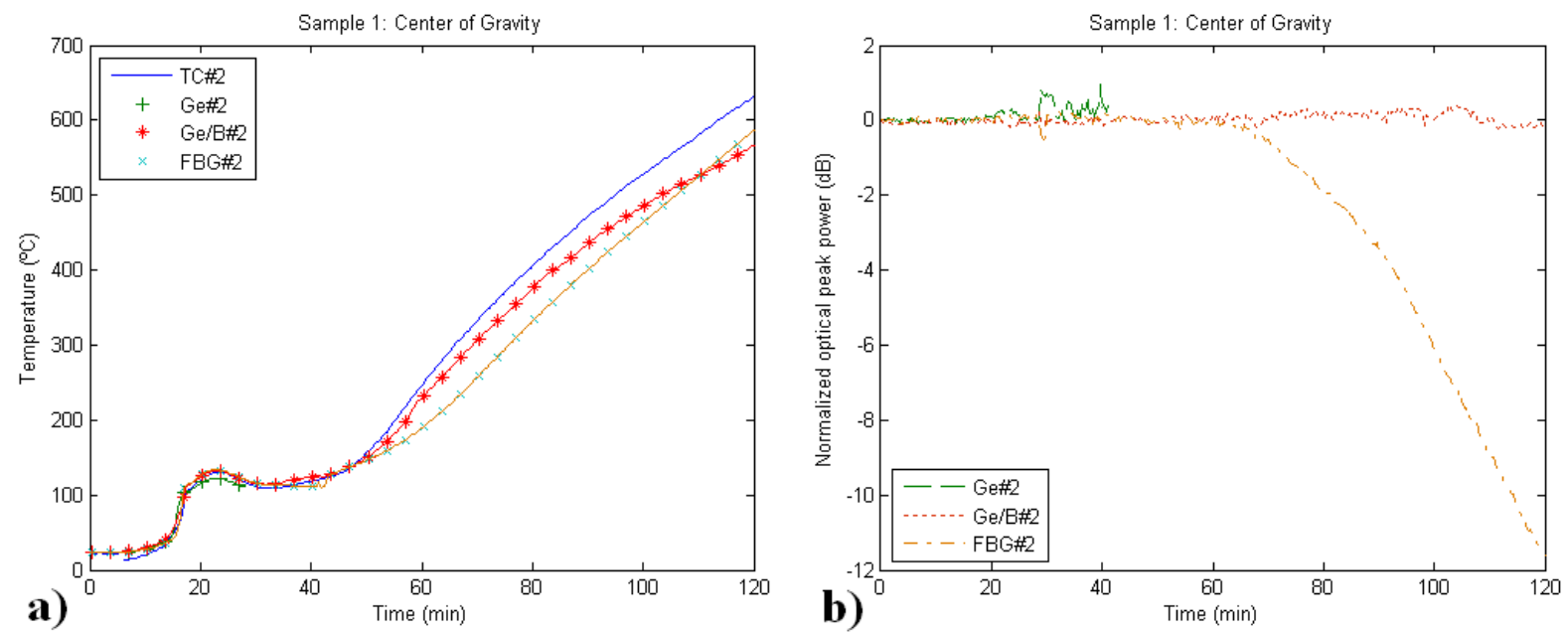

Fig. 6: Sample 1 at the center of gravity (point C1): a) Temperature measured by optical and electrical sensors and b) Normalized optical peak power of the optical sensors. 
Concerning sample 1 at point L1 (Fig. 5), the maximum temperature registered by FBG\#1 was $613.9^{\circ} \mathrm{C}$ although its reflectivity decreased $22.46 \mathrm{~dB}$ after 118 minutes from the start of the test, thus the Bragg wavelength was not clearly identified by the peak search algorithm used. At the same location, Ge\#1 shows a maximum temperature of $615.3^{\circ} \mathrm{C}$ after 120 minutes with only losses of $1.83 \mathrm{~dB}$. The thermocouple TC\#1 located at this point shows a maximum temperature of $767^{\circ} \mathrm{C}$.

Taking a look at point $\mathrm{C} 1$ in sample 1 (Fig. 6) a similar behavior can be seen in every sensor located at this point. Unfortunately, sensor Ge\#2 disappeared after 42 minutes. This is due to light reflections at the fiber splice since the noise floor increased above the reflected peak power of the grating, meaning that the peak search algorithm was unable to follow the wavelength shift. Ge/B\#2 sensor registered a maximum temperature of $566.5^{\circ} \mathrm{C}$ and $\mathrm{FBG} \# 2$ registered $587.4{ }^{\circ} \mathrm{C}$. Thermocouple $\mathrm{TC \# 2}$ showed a maximum temperature of $631{ }^{\circ} \mathrm{C}$. Concerning optical losses, Ge/B\#2 had only losses of $0.11 \mathrm{~dB}$ whereas FBG\#2 had losses of $11.55 \mathrm{~dB}$. 

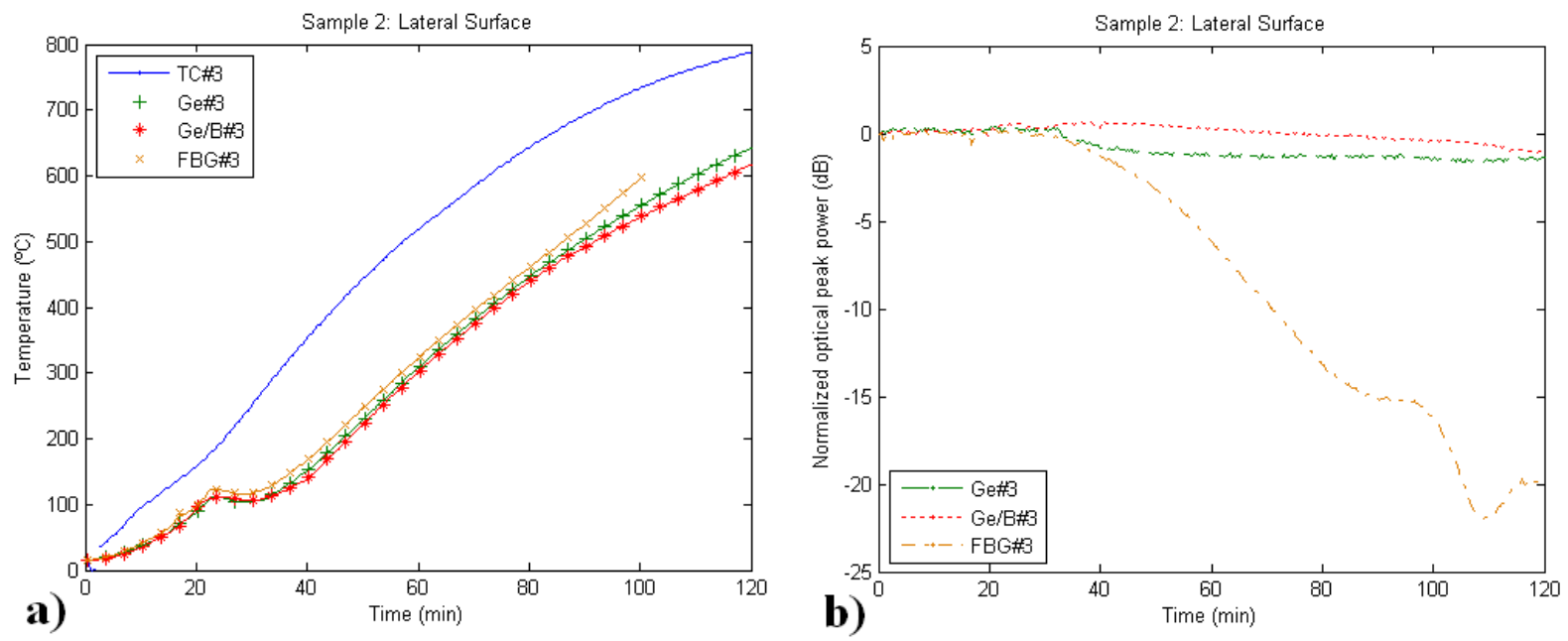

Fig. 7: Sample 2 at the lateral surface (point L2): a) Temperature measured by optical and electrical sensors and b) Normalized optical peak power of the optical sensors.
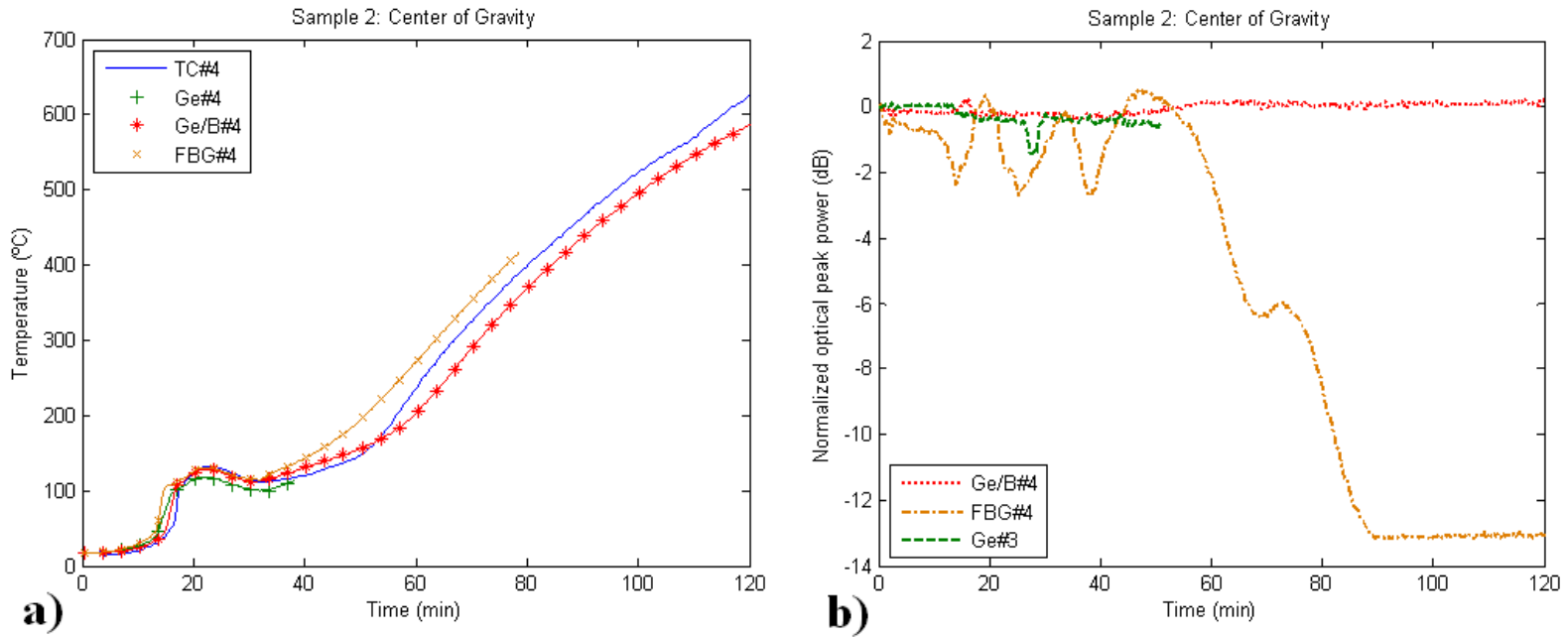

Fig. 8: Sample 2 at the center of gravity (point C2): a) Temperature measured by optical and electrical sensors and b) Normalized optical peak power of the optical sensors.

Moving on to sample 2 at point L2 (Fig. 7), the maximum temperatures measured by the optical sensors were: $641.7{ }^{\circ} \mathrm{C}$ by $\mathrm{Ge} \# 3,617^{\circ} \mathrm{C}$ by $\mathrm{Ge} / \mathrm{B} \# 3$ and $598.2{ }^{\circ} \mathrm{C}$ by $\mathrm{FBG} \# 3$ (although this sensor disappeared after 100 minutes) whereas termocoupler TC\#3 measured a maximum 
temperature of $788^{\circ} \mathrm{C}$. Optical losses experienced by the optical sensors were: $1.4 \mathrm{~dB}$ by Ge\#3 sensor, $1.01 \mathrm{~dB}$ by Ge/B\#3 sensor and more than $19.86 \mathrm{~dB}$ by FBG\#3 sensor.

At point $\mathrm{C} 2$ in sample 2 (Fig. 8), the results were similar to those obtained at point $\mathrm{C} 1$ in sample 1. Ge\#4 sensor disappeared at an early stage, after 52 minutes. This is due again to the problems originated in the splice. The maximum temperatures registered by the rest of the optical sensors were: $586.3{ }^{\circ} \mathrm{C}$ by Ge/B\#4 sensor and $417.9^{\circ} \mathrm{C}$ by FBG\#4 sensor (although its optical peak power disappeared in minute 79 due to the high losses). Thermocouple TC\#4 measured a maximum temperature of $625^{\circ} \mathrm{C}$. Optical losses experienced by the optical sensors were: $0.1 \mathrm{~dB}$ by Ge/B\#4 sensor and $13.03 \mathrm{~dB}$ by FBG\#4 sensor.

All the results extracted from this test are summarized in Table 2 concerning maximum temperature registered by the optical and electrical sensors and in Table 3 concerning the optical power losses in the peak power reflected by the gratings at the end of the test, normalized by the reflected peak power at the beginning of the test.

\begin{tabular}{|c|c|c|c|c|}
\hline & \multicolumn{2}{|c|}{ Sample 1} & \multicolumn{2}{|c|}{ Sample 2} \\
\hline & $\begin{array}{c}\# 1 \\
\text { (L1) }\end{array}$ & $\begin{array}{c}\# 2 \\
\text { (C1) }\end{array}$ & $\begin{array}{c}\# 3 \\
(\text { L2) }\end{array}$ & $\begin{array}{c}\# 4 \\
\text { (C2) }\end{array}$ \\
\hline Ge & $615.3^{\circ} \mathrm{C}$ & $\begin{array}{c}\text { Spectrum } \\
\text { disappearance }\end{array}$ & $641.7^{\circ} \mathrm{C}$ & $\begin{array}{c}\text { Spectrum } \\
\text { disappearance }\end{array}$ \\
\hline $\mathrm{Ge} / \mathrm{B}$ & not available & $566.5^{\circ} \mathrm{C}$ & $617^{\circ} \mathrm{C}$ & $586.3^{\circ} \mathrm{C}$ \\
\hline FBG & $613.9^{\circ} \mathrm{C}$ & $587.4^{\circ} \mathrm{C}$ & $\begin{array}{l}\text { Erased at around } \\
100 \text { minutes }\end{array}$ & $\begin{array}{l}\text { Erased at around } \\
\qquad 80 \text { minutes }\end{array}$ \\
\hline TC & $767^{\circ} \mathrm{C}$ & $631^{\circ} \mathrm{C}$ & $788^{\circ} \mathrm{C}$ & $625^{\circ} \mathrm{C}$ \\
\hline
\end{tabular}

Table 2: Maximum temperatures measured by optical and electrical sensors. 


\begin{tabular}{|c|c|c|c|c|}
\hline & \multicolumn{2}{|c|}{ Sample 1} & \multicolumn{2}{|c|}{ Sample 2} \\
\hline & $\begin{array}{c}\# 1 \\
\text { (L1) }\end{array}$ & $\begin{array}{c}\# 2 \\
(\mathrm{C} 1)\end{array}$ & $\begin{array}{c}\# 3 \\
\text { (L2) }\end{array}$ & $\begin{array}{c}\# 4 \\
\text { (C2) }\end{array}$ \\
\hline Ge & $1.83 \mathrm{~dB}$ & $\begin{array}{c}\text { Spectrum } \\
\text { dissapearance }\end{array}$ & $1.4 \mathrm{~dB}$ & $\begin{array}{c}\text { Spectrum } \\
\text { dissapearance }\end{array}$ \\
\hline Ge/B & not available & $0.11 \mathrm{~dB}$ & $1.01 \mathrm{~dB}$ & $0.1 \mathrm{~dB}$ \\
\hline FBG & $22.46 \mathrm{~dB}$ & $\begin{array}{l}\text { more than } \\
11.55 \mathrm{~dB}\end{array}$ & $\begin{array}{c}\text { more than } \\
19.86 \mathrm{~dB}\end{array}$ & $\begin{array}{l}\text { more than } \\
13.03 \mathrm{~dB}\end{array}$ \\
\hline
\end{tabular}

Table 3: Optical power losses of optical sensors after the test.

\section{Conclusions}

The optical sensors show a good agreement with the electrical ones at the center of gravity of both concrete samples. In the lateral surfaces there are some differences between the measurements obtained from the sensors located at this point. This is due to the position of the sensors during the installation and the temperature gradient inside the concrete sample. To be precise, the thermocouples were installed very close to the air-concrete interface, where the temperature gradient is higher. The optical sensor registered temperatures above $600{ }^{\circ} \mathrm{C}$ inside the concrete specimens whereas the temperature inside the chamber was around $1060{ }^{\circ} \mathrm{C}$ after 120 minutes.

The standard FBG sensors were completely erased during the test due to the high temperature, resulting in a decrease in the reflected peak power of the grating until the noise floor. Nevertheless, FBGs sensors can be used in high temperature tests if the time duration of 
the experiment is short enough, and they can be reusable in high temperature environment if the reflectivity losses of the grating during the previous tests are small enough.

The Ge and Ge/B doped fiber RFBG sensors have shown much less optical losses. However, the process of the fabrication of the RFBG is more complex. Thus, for each application can be choose one or other type of optical sensor. In our case, RFBG sensors appear to be useful sensors for high temperature applications, such as fire detection inside a tunnel, as they can withstand temperatures over $1000{ }^{\circ} \mathrm{C}$ [10] and the fire can be detected when the temperature extremely rises in the first minutes.

\section{Acknowledgements}

The authors gratefully acknowledge research funding by the Spanish Ministry of Science and Innovation through Project SOPROMAC P41/08.

\section{References}

[1] B.M. Luccioni, M.I. Figueroa and R.F. Danesi, "Thermo-mechanic model for concrete exposed to elevated temperatures", Engineering Structures 25, 729-742 (2003).

[2] H. Abdel-Fattah and S.A. Hamoush, "Variation of the fracture toughness of concrete with temperature", Construction and Building Materials, Vol. 11, No. 2, 105-108 (1997).

[3] J.C. Cardozo da Silva, C. Martelli, et al., "Dynamic analysis and temperature measurements of concrete cantilever beam using fibre Bragg gratings", Optics and Lasers in Engineering 45, 88-92 (2007). 
[4] Y.B. Lin, J.C. Chern, et al., "The utilization of fiber Bragg grating sensors to monitor high performance concrete at elevated temperature", Smart Materials and Structures 13, 784-790 (2004).

[5] A. Lönnermark, P.O. Hedekvist and H. Ingason, "Gas temperature measurements using fibre Bragg grating during fire experiments in a tunnel”, Fire Safety Journal 43, 119-126 (2008).

[6] G. Heiberg, J. Skaar, M. Fokine, L. Arnberg, "A new method for temperature measurement in solidifying aluminum alloys by use of optical fiber Bragg grating sensors", Transactions of the American Foundry Society, Vol. 110, Part 1, pp. 383-391 (2002).

[7] A.D. Kersey, et al., "Fiber grating sensors", Journal of Lightwave Technology, Vol. 15, No. 8, pp. 1442-1463 (1997).

[8] C.L. Liou, et al., "Characteristics of hydrogenated fiber Bragg gratings", Applied Physics A 64, pp. 191-197 (1997).

[9] M. Fokine, "Underlying mechanisms, applications, and limitations of chemical composition gratings in silica based fibers", Journal of Non-Crystaline Solids, No. 349, pp. 98-104 (2004).

[10] S. Bandyopadhyay, J. Canning, et al., "Ultrahigh-temperature regenerated gratings in boroncodoped germanosilicate optical fiber using 193 nm”, Optics Letters 33, pp. 1917-1919 (2008).

[11] L. Ropelewski and R. D. Neufeld, "Thermal inertia properties of autoclaved aerated concrete”, Journal of Energy Engineering, August 1999, pp. 59-75 (1999).

\section{Biographies}

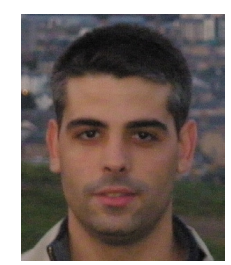

Antonio Bueno was born in Valencia (Spain) in 1981. He received the B.Sc. degree in Telecommunications in 2008 and the M.Sc. degree in Technologies, Systems and 
Networks of Communication in 2010 both from the Universidad Politécnica de Valencia (UPV), Spain. He is currently working towards the Ph. D. in optics at the Universidad Politécnica de Valencia in the Optical and Quantum Communications Group (OQCG) at iTEAM research institute under the supervision of Dr. Salvador Sales. His fields of interest are Fiber Bragg Grating and Brillouin fiber sensors.

Benjamín Torres was born in Valencia (Spain) in 1983. He received the B.Sc. degree in Civil Engineering in 2008 and the M.Sc. degree in Concrete Engineering in 2009 both from the Universidad Politécnica de Valencia (UPV), Spain. He is currently working towards the Ph. D. in civil engineering at the Universidad Politécnica de Valencia in the Concrete Science and Technology Research Group (ICITECH) under the supervision of Dr. Pedro A. Calderón. His fields of interest are fiber optic sensors for Structural Health Monitoring, forensic engineering and structural repairs.

David Barrera was born in Valencia (Spain) in 1981. He received the M. Sc. degree in Telecommunications Engineering from the Universidad Politécnica de Valencia (UPV) in 2006. Since then, he has been working at the Optical and Quantum Communications Group of the iTEAM research institute. His research interests include fibre Bragg grating, optical fiber sensing and polymer optical fibers. He is currently working towards the $\mathrm{Ph}$. D. degree at the Universidad Politécnica de Valencia and focusing on optical fiber sensing. 
Pedro Antonio Calderón (Melilla, Spain, 1962) is professor at the department of Civil Engineering of Universidad Politécnica de Valencia (Spain) since 1999. He obtained his B.Sc. in civil Engineering at the UPV in 1986, an M.Sc. degree in geotechnical engineering at the university of California, Davis, in 1990, and his Ph.D. at the UPV in 1992. He has been the director of the technical department of the company GIA (mainly dedicated to structural and geotechnical monitoring and assessment) from 1991 to 1999 . He is currently the director of the "building structures" research group at the Institute of Concrete Science and Technology (ICITECH) where he is currently involved in the topic "structural health monitoring". Author of more than 50 international papers and communications.

José Manuel Lloris owns a PhD in Chemistry from the Universidad Politécnica de Valencia in 2000 working towards the development of new electrochemical sensors. Since 2005 he is a Senior Researcher in The Materials Research Unit of AIDICO, Technological Research Center for Construction Materials. His work is focused on the Nanomateriales area, synthesis of nanoparticles, functional materials and smart systems. He has experience in the development of new materials with more than 40 papers. From 2001-2004 he gained a post-doctoral stage in the University of Cordoba. He worked on solid state chemistry to develop new lithium and sodium insertion materials.

Salvador Sales is professor at the Departamento de Comunicaciones, Universidad Politécnica de Valencia, Spain. He is also working in the iTEAM research institute. He received the M. Sc. and the Ph. D. in Telecomunicación from the Universidad Politécnica de Valencia. He is currently the coordinator of some Ph. D. Telecomunicación students at Universidad Politécnica de 
Valencia. He is co-author of more than 60 journal papers and 100 international conferences. He has been collaborating and leading some national and European research projects since 1997. His main research interests include fiber Bragg gratings, WDM and SCM lightwave systems and semiconductor optical amplifiers. 


\title{
XXXVII IAHS - Early age concrete temperature and shrinkage monitoring by using Fiber Bragg Grattings
}

\author{
Benjamín Torres $^{1 *}$, Ignacio Payá-Zaforteza ${ }^{1}$, Antonio Bueno ${ }^{2}$, Salvador Sales ${ }^{2}$ \\ Jose M. Adam ${ }^{1}$, Pedro A. Calderón ${ }^{1}$ \\ 1. ICITECH, Universidad Politécnica de Valencia, Camino de Vera s/n, 46022, Valencia. \\ 2: iTEAM, Universidad Politénica de Valencia, Camino de Vera s/n, 46022, Valencia \\ *email: bentorgo@upvnet.upv.es
}

Key words: Monitoring, fiber optic sensor, Shrinkage, temperature, early age concrete.

\begin{abstract}
Behaviour and durability of structural concrete is heavily influenced by its reology at very early ages and, specially, by the evolution of concrete temperatures and shrinkage. Therefore, it is of outermost importance to monitor both parameters.

This paper introduces the technologies developed by the research institutes ICITECH (Instituto y Ciencia de Tecnología del Hormigón) and ITEAM (Instituto de Telecomunicaciones y Aplicaciones Multimedia) of the Universidad Politécnica de Valencia in Spain for the measurement of temperature and shrinkage strain in early age concrete. The technology is based on the use of Fiber Bragg Gratings and both experimental works and applications on real structures will be presented. Specifically, the paper will explain the use of the sensors for the monitoring of the pile cap of a bridge foundation of $264 \mathrm{~m}^{3}$ of volume that was poured in one phase. The paper compares the measurements obtained by the fiber optic sensors with those given by a conventional electrical sensor and with values found in the literature for similar foundation works. The analysis of the results confirms the reliability of the developed fiber optic sensors and their advantages when compared to more traditional technologies.
\end{abstract}

\section{Introduction}

Concrete is the most used construction material in civil engineering. It is essential to have a good knowledge about their mechanical properties and behavior since the initial time that is created [1].

Concrete is created from a mixture of materials such as cement, aggregates, water and additives. After this mixing, the cement hydration process is activated and the life of concrete begins. During the chemical hydration process, the plastic state concrete changes into a hardened state. As a result of these chemical reactions concrete deforms: it expands due to temperature rise during hydration and shrinks due to water evaporation consumed in chemical reactions (endogenous shrinkage). 
This concrete behavior is considered the early age concrete and finishes when the thermal processes terminate. The concrete deformation at early age is called plastic shrinkage and therefore, residual tensile and compressive stresses are generated.

Studies and research ([2] - [4]) have shown that early age cracking of concrete can significantly increase the vulnerability of concrete structures decreasing its durability. These cracking form "open doors" to the infiltration and penetration of noxious substances that attack the concrete and rebars, such as chlorides and sulphate water [5]. Figure 1 [6] shows the influence of early age cracking on durability for a concrete structure. Even small gains in concrete performance during very early age, have a consequence of extending considerably the life span of the structure [6].

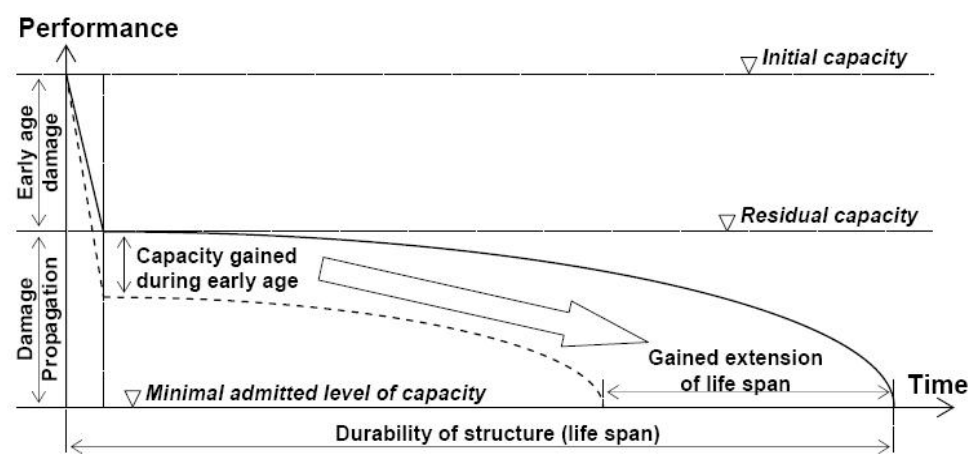

Figure 1: Influence of the early age damage on the durability of a concrete structure (Ref. [6]).

One way that allows us to evaluate the behavior of concrete during early age is to monitor temperature and shrinkage parameters. This provides a reliable source of information about its real behavior. For this reason it is recommended to start deformation monitoring of concrete structures from the moment of concrete pouring. It is the only way to collect the whole history of deformation and temperatures. For this purpose, it is necessary to provide the monitoring system with sensors that are capable of such measurements.

This paper presents the optical fiber technology developed at Universidad Politecnica de Valencia (Spain) by ICITECH and ITEAM Institutes to monitor deformations and temperatures during the early age in a concrete structure. This technique provides very satisfactory results and advantages over other conventional system, wich will be presented in this paper.

\section{Principle of FBG Sensor.}

Fiber optic technology has been used to carry out monitoring during the concrete early age. In recent years the use of fibre optic sensors (FOSs) has attracted much attention in research and development as shown by both review and application articles (e.g. [7-11]). The main reasons for this interest are the decreasing price of FOSs as well as the advantages of FOSs over conventional sensors:
a) Immunity to electrical or magnetic interference (EMI).
b) Embeddability.
c) Multiplexity.

Of all the types of fiber optic sensors, has decided to use the type FBG (Fiber Bragg Gratting), for its simplicity of manufacture, installation and inspection. The operating principle of FBG sensor is based on diffraction gratings.

A diffraction grating is an optical device that is created by altering, periodically or quasiperiodic, the refraction index of the optical fiber core.

For Bragg diffraction gratings of uniform period, greater interaction and coupling between modes, the incident and the reflected, occurs at the Bragg wavelength $\lambda_{B}$ (Figure 2) which is 
determined by the equation (1), where $n_{e f f}$ is the effective refractive index of the fiber core and $\Lambda$ is the Bragg grating period or distance between two consecutive alterations of the fiber core [12].

$$
\lambda_{B}=2 n_{\text {eff }} \Lambda
$$

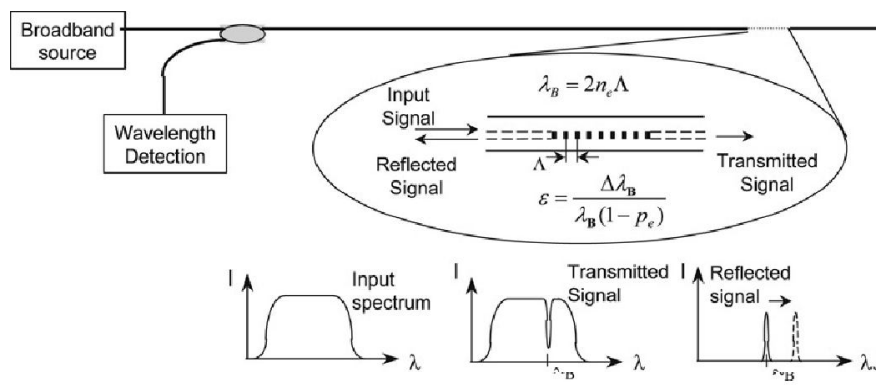

Figure 2: Input Spectrum, transmitted and reflected signal (Ref. [12]).

Both the Bragg grating period $\Lambda$ and the effective refractive index $n_{e f f}$ induced in the fiber core depend on the temperature $\Delta T$ and the strain $\Delta \varepsilon$ in the optical fiber. Therefore, they induce a shift in the Bragg wavelength. This shift is given by Equation 2:

$$
\frac{\Delta \lambda_{B}}{\lambda_{B}}=K_{\varepsilon} \Delta \varepsilon+K_{T} \Delta T
$$

$K_{\varepsilon}$ and $K_{T}$ are the coefficients of sensitivity of the deformation and temperature, respectively.

To measure the strain and temperature, these responses need to be separated and distinguished. For a pure temperature sensor, it is necessary to isolate the optical fiber mechanical deformation. Thus the variation of Bragg wavelength is proportional to the temperature variations (3):

$$
\frac{\Delta \lambda_{B}}{\lambda_{B}}=K_{T} \Delta T
$$

If 1 is the optical fiber sensor that is completely fixed to the structure and 2 relates to the sensor that only measures temperature changes, it is possible to write equations (4) and (5).

$$
\begin{gathered}
\Delta \lambda_{B 1} /_{\lambda_{B 1}}=K_{\varepsilon 1} \Delta \varepsilon+K_{T 1} \Delta T \\
\Delta \lambda_{B 2} / \lambda_{B 2}=K_{T 2} \Delta T
\end{gathered}
$$

Solving these equations system, we obtain the equations (6) and (7) that allow separation of the two responses.

$$
\begin{gathered}
\Delta \varepsilon=\frac{1}{k_{\varepsilon 1}}\left(\frac{\Delta \lambda_{B 1}}{\lambda_{B 1}}\right)-\frac{K_{T 1}}{K_{\varepsilon 1} K_{T 2}}\left(\frac{\Delta \lambda_{B 2}}{\lambda_{B 2}}\right) \\
\Delta T=\frac{1}{K_{T 2}}\left(\frac{\Delta \lambda_{B 2}}{\lambda_{B 2}}\right)
\end{gathered}
$$

The coefficients $k_{\varepsilon 1}, K_{T 1}, K_{T 2}$ have been obtained experimentally, as shown in [13]. 


\section{Optical Sensor Interrogation System.}

The interrogator system consists of a FBG Micron Optics sm125-500 model and a computer that is running a software developed specifically for this application (Figure 3).

The optical sensor interrogator sm125-500 is composed of a laser source with a Fabry-Perot wavelength range from 1510 to $1590 \mathrm{~nm}$, and 4 optical output channels. The maximum capture data frequency is $1 \mathrm{~Hz}$ and a measurement accuracy is $1 \mathrm{pm}[14]$.

\section{Experimental Campaign.}

\subsection{Geometry, materials and monitoring.}

To carry out experimental campaigns have been prepared a concrete cylindrical and prismatic specimens. The cylindrical specimens have a height of $30 \mathrm{~cm}$ and a diameter of $15 \mathrm{~cm}$. The prismatic specimens have dimensions of $10 \mathrm{~cm} \times 10 \mathrm{~cm} \times 40 \mathrm{~cm}$.

\subsubsection{Temperature.}

To measure the temperature has been used a conventional concrete with compressive strength $f_{c k} 25 \mathrm{MPa}$, and have been prepared a total of 3 cylindrical specimens.

Each cylindrical specimen was instrumented with an optical temperature sensor and a conventional electrical sensor (thermocouple), both embedded in order to validate the results. The pair of sensors are located in the center of gravity of the specimen, and soon each other. (Figure 3)

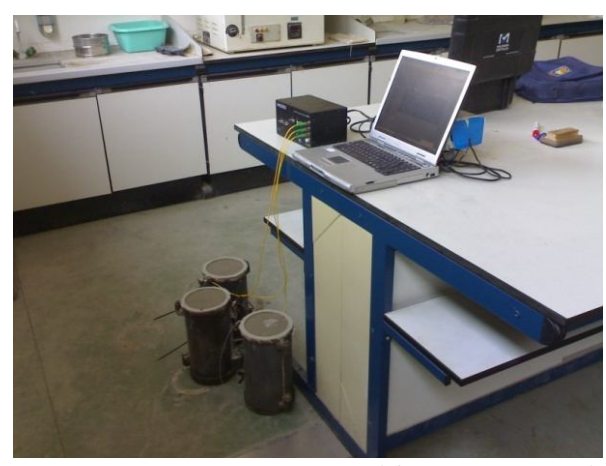

Figure 3: Interrogator sm125-500, computer and instrumented specimens with Optical temperature sensors and termocouples.

Monitoring began at the moment of concrete pouring and continued during the next hours in order to monitor temperature changes that are generated during the curing process of concrete

\subsubsection{Very early age deformation}

Concrete with recycled aggregates has been used to measure plastic shrinkage. The aim is to achieve greater shrinkage due to increased aggregates absorption with asphalt and ceramic provenance [15].

We have used a prismatic specimen that has been instrumented with two embedded optical sensors. One of them is used for temperature compensation explained in section 2 above. The other sensor measures the early age concrete deformation. To comparison, a conventional system has been used as a benchmarks. 


\subsection{Discussion of the experimental results.}

\subsubsection{Temperature.}

Monitoring has been carried out since the pouring and has lasted for the next 72 hours.

Since the release of hydration heat of concrete, an increase of temperature occurs during the first 10 hours. From this instant, the temperature evolution is governed by the change of day and night temperature (Figure 4a).

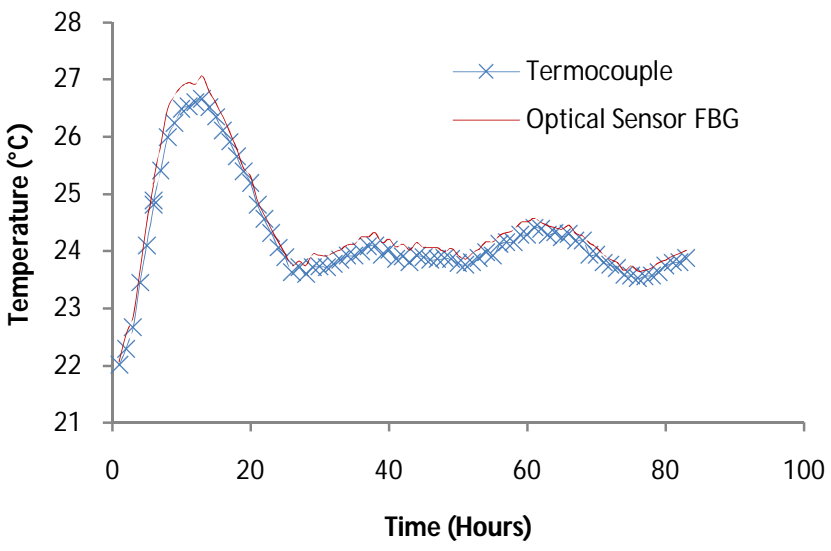

Figure 4a: Evolution of the law of temperatures in concrete.

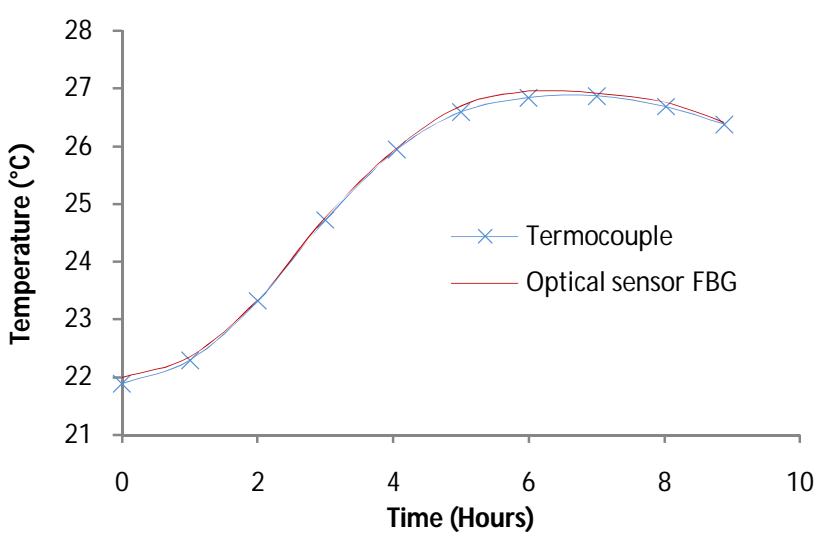

Figure 4b: Comparative measurements of optical sensors and termocouples.

The results show a strong agreement (Figure 4b) between the two measurements system, thereby demonstrating the validity of FBG optical sensors to measure temperature in concrete.

\subsubsection{Very early age deformation.}

Just as for the temperature measurement, this monitoring was carried out from the concrete pouring and continued for the next hours.

During the first hours the concrete is in a plastic state. In this way, it is important to determine the hardening time, defined as the time when the concrete has reached a consistency that is compatible with strain of the optical sensor.

During the early hours due to the plastic state of concrete in not possible to know the deformations suffered by the concrete. However, the deformations due to plastic shrinkage can be known from the three hours after pouring (Figure 5). Very early age deformation are measured in microstrain $(\mu \varepsilon)$.

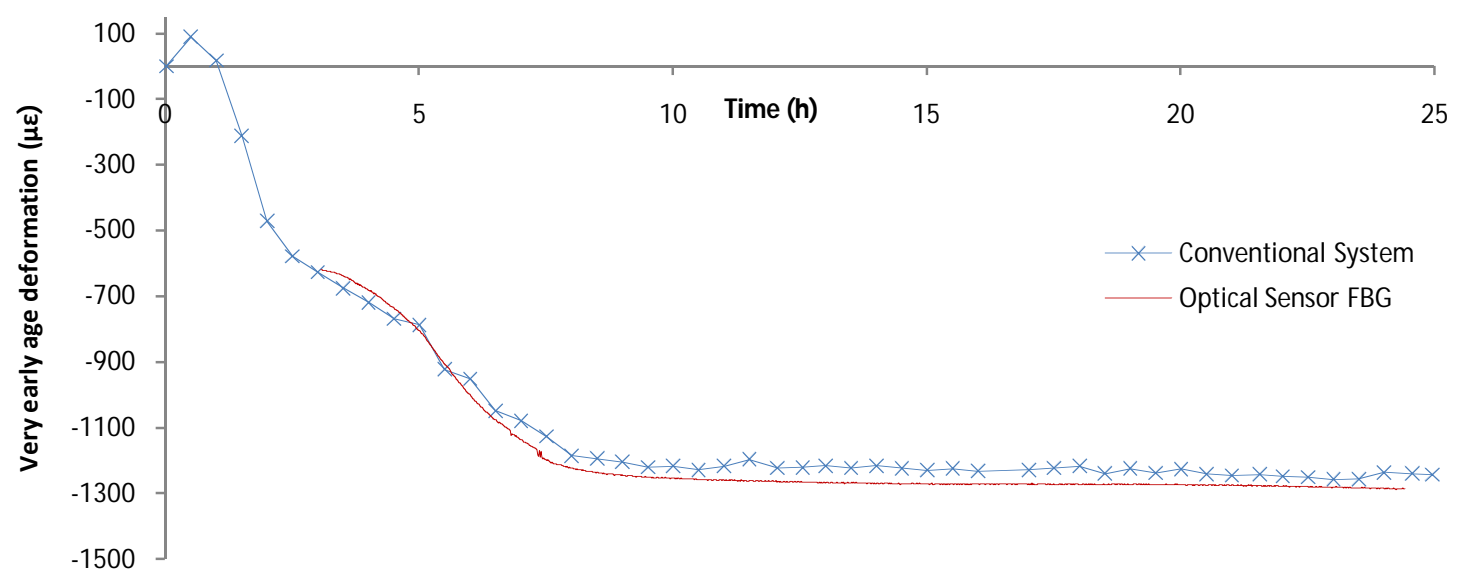

Figure 5: Early age concrete deformation. Comparative between optical and conventional system. 


\section{Example of monitoring of a real concrete structure.}

\subsection{Description of the structure.}

This section shows the work to carry out monitoring of the evolution of temperature on a reinforced concrete structure. This structure is a reinforced concrete pile cap of a cable-stayed bridge foundation. The cable-stayed bridge pylon has 164.28 meters height.

The pile cap has a volume of $264 \mathrm{~m}^{3}$. Gaida [16] defines a mass concrete structure as "any volume of concrete with dimensions large enough to require that measures be taken to cope with generation of heat from hydration of the cement and attendant volume change, to minimize cracking". For a concrete volume of its kind and according to this definition, it is important to monitor the development of concrete temperatures generated during the early ages.

\subsection{Structure monitoring.}

The pile cap was monitored with three optical sensors. One of them was located in the center of gravity of the pile cap (COG sensor). Another sensor was placed $15 \mathrm{~cm}$ away from the lateral pile cap formwork (Lateral sensor) but at the same depth than COG sensor (Figure 6a). The third optical sensor was used to monitor changes in environment temperature (environment sensor).

This monitoring strategy allows to knows the maximum temperature gradient generated between the pile cap centre and the side, and environment temperature.

Data collections took place form the concrete pouring and continued for the next 60 hours. The optical sensor data were compared with data obtained by a conventional electrical system

\subsection{Results.}

Figure $6 \mathrm{~b}$ shows the temperature laws obtained by optical sensors. COG sensor shows a greater temperature than Lateral sensor due to the greater difficulty of this point to dispel the concrete hydration heat. It can be seen in the evolution of the curves the moment the concrete reaches the optical sensor. Approximately 2 hours after pouring there is an abrupt change of slope in the curve describing the temperature evolution. This abrupt change represents the moment when the concrete reaches optical sensors (Figure 6b).
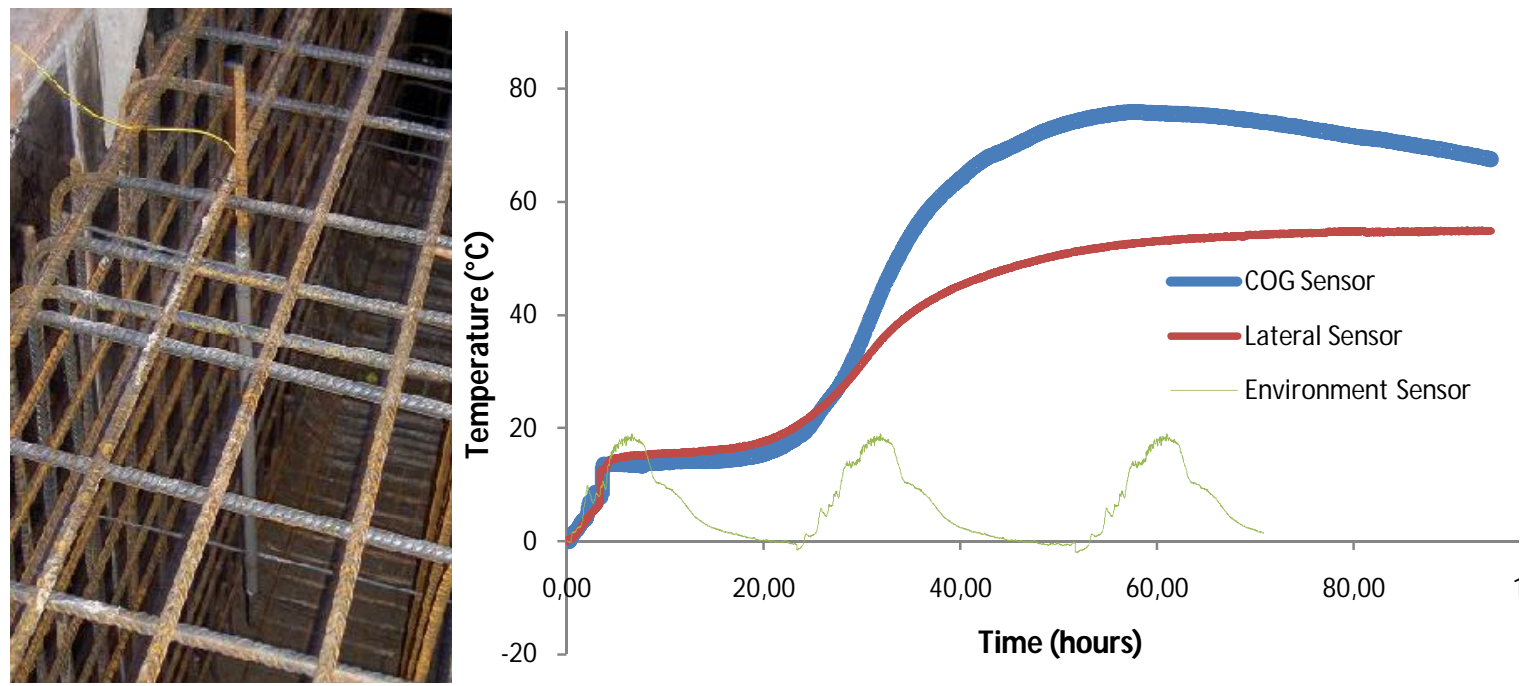

Figure 6a: Lateral Sensor. Figure 6b: Pile cap evolution temperature by optical sensors 
Figures $7 \mathrm{a}$ and $7 \mathrm{~b}$ compare the temperatures measured by conventional electrical sensors with those measured with optical sensors. There are data loss by electrical sensors that hinder the reading and interpretation of results.

It should be noted that other studies on the evolution of concrete temperatures have been carried out, and have shown similar results to those measured with optical sensors (see [17], [18]).

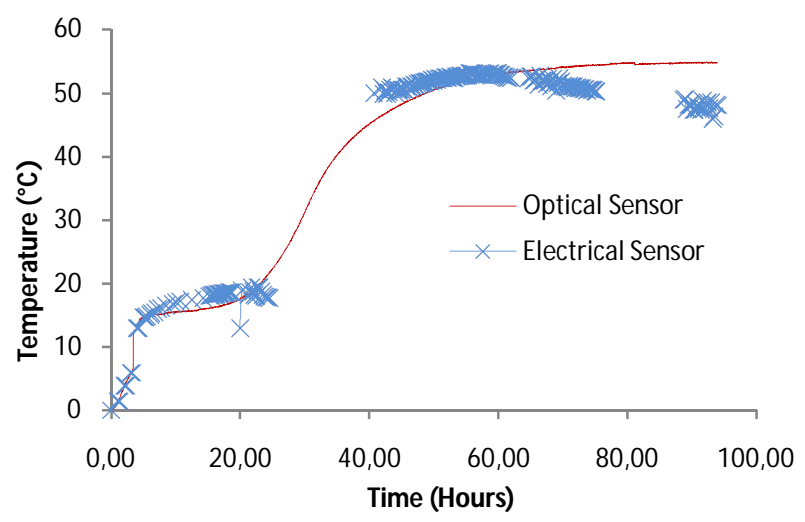

Figure 7a). Temperatures measured by lateral sensors.

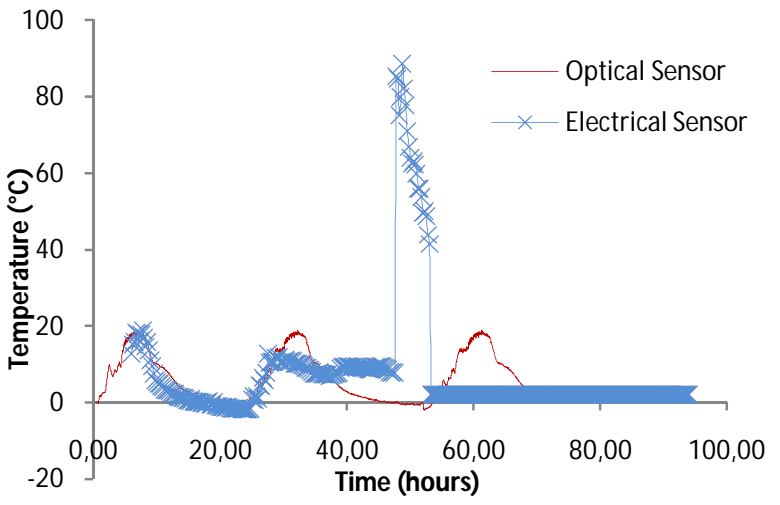

Figure 7b) Environment temperature.

\section{Conclusions.}

This paper shows the optical technology developed by the research institutes ICITECH and ITEAM of Universidad Politécnica de Valencia in Spain for the measurement of temperature and shrinkage strain in early age concrete.

It has proved the validity of optical sensors to measures the temperature in early age concrete, giving very satisfactory results in the experimental campaigns carried out.

For the shrinkage strain measurement is necessary to know the hardening time, from wich are made compatible concrete and optical sensors strains. This time has been found out to be three hours. From these three hours, we can know shrinkage strain concrete with great reliability.

On the other hand, developed and validated optical technology has been applied to obtain the temperatures developed in a structural element of a cable-stayed bridge. The results show the optical sensors reliability, demonstrating the guarantee that they presented in their application in the construction sector.

\section{Acknowledgements}

This work has been funded by the Spanish Ministry of Science and Innovation through Plan Nacional I+D TEC 2007-68065-C03-01. Thanks the Construction Company Sacyr Vallehermoso to allow performing the measurements in the reinforced concrete structure.

\section{References.}

[1] Glisic B., Simon N. Monitoring of concrete at very early age using stiff SOFO sensor. Cement \& Concrete Composites 22 (2000) 115-119

[2] Tazawa E., Iida K. Mechanism of thermal stress generation due to hydration heat of concrete, Transactions of the Japan Concrete Institute, 1983, vol. 5, p.p. 119-126.

[3] Thermal Cracking in Concrete at Early Age, RILEM International Symposium, Munich, Germany, 1994. 
[4] Limitation de la fissuration au jeune age du béton dans des structures hybrides. Research report. MCS, IMAC et ICOM, EPFL, Lausanne, Switzerland, 2000.

[5] Nagesh M., Bhattacharjee B. Modelling of Chloride Diffusion in Concrete and Determination of Diffusion Coefficients, ACI Materials Journal, Vol. 95, №2, April 1998.

[6] Glisic B., Inaudi D. Monitoring of Early and Very Early Age Deformation of Concrete Using Fiber Optic Sensors. Fédération Internationale du Béton. Proceedings of the 2nd International Congress: Monitoring, maintenance, repair, residual life and failures. Napoles, Italy. June 2006.

[7] Ko J.M., Ni Y.Q. Technology developments in structural health monitoring of large-scale bridges. Engineering Structures 2005; 27: 1715-25.

[8] Majumder M., Gangopadhyay T.M., Chakraborty A.K. Fibre Bragg gratings in structural health monitoring - Present status and applications. Sensors and Actuators A: Physical 2008; 147:150-64.

[9] Chan T.H.T., Yu L., Tam H.Y., Ni Y.Q., Liu S.Y., Chung W.H., Cheng L.K. Fiber Bragg grating sensors for structural health monitoring of Tsing Ma bridge: Background and experimental observation. Engineering Structures 2006; 28: 648-59.

[10] Chung W., Kang D. Full-scale test of a concrete box girder using FBG sensing system. Engineering Structures, 2008; 30(3): 643-52.

[11] Lin Y.B., Pan C.L., Kuo YH., Chang K.C., Chern J.C. Online monitoring of highway bridge construction using fiber Bragg grating sensors. Smart Mater. Struct. 2005; 14:1075-82.

[12] Chung W., Kang D. Full-scale test of a concrete box girder using FBG sensing system. Engineering Structures 30 (2008) 643-652.

[13] Torres B. Estudio experimental de sensores ópticos tipo FBG para medir deformaciones y temperaturas en estructuras. Tesina final de Máster en Ingeniería del Hormigón. Departamento de Ingeniería de la Construcción y Proyectos de Ingeniería Civil. Universidad Politécnica de Valencia. Noviembre 2009. In Spanish.

[14] Bueno Martínez A. Diseño y caracterización de un sensor óptico autocompensado en temperatura para la medida de deformaciones en estructuras de ingeniería civil y desarrollo de un sistema de interrogación. Escuela Técnica Superior de Ingenieros de Telecomunicaciones. Universidad Politécnica de Valencia. Septiembre 2008. In Spanish.

[15] Domingo A., Lázaro C., López F., Serrano M.A., Serna P., Castaño J.O., Creep and shrinkage of recycled aggregate concrete. Construction and Building Materials, 2009; Vol 23; pp. 2545-2553.

[16] Gaida J. and Alsamsam, E. "Engineering Mass Concrete Structures". Structural Engineer Professional Development Series. Portland Cement Association. 2006.

[17] Hermoso J. G., Ramírez L. A., Luna J. A. Colegio de Caminos, Canales y Puertos. Asociación Científico-Técnica del Hormigón Estructural. Estructuras y Edificación. Construcción de edificios altos. "Control" (2008). 314-316. In Spanish.

[18] Calzón J. M., Navarro M. G. Asociación Científico-técnica del Hormigón Estructural. Volumen 59. N N $^{\mathrm{o}}$ 249. "Torres Espacio. Building Structure” (2008). 24-26. In Spanish. 


\section{APLICACIONES DE LOS SENSORES DE FIBRA ÓPTICA DESARROLLADOS POR LA UNIVERSIDAD POLITÉCNICA DE VALENCIA (U.P.V.) PARA LA MEDIDA DE DEFORMACIONES Y TEMPERATURAS EN ESTRUCTURAS.}

\author{
Benjamín TORRES GÓRRIZ \\ Titulación: Ingeniero de C.C.P. \\ Entidad en la que trabaja: U.P.V.-ICITECH \\ Cargo: Investigador \\ bentorgo@upvnet.upv.es
}

\section{Antonio BUENO MARTÍNEZ \\ Titulación: Ingeniero de Telecomunicaciones \\ Entidad en la que trabaja: U.P.V.-ITEAM \\ Cargo: Investigador \\ anbuemar@iteam.upv.es}

\section{Pedro CALDERÓN GARCÍA}

Titulación: Ingeniero de C.C.P.

Entidad en la que trabaja: U.P.V. -ICITECH

Cargo: Titular de Universidad PDI

pcaldero@cst.upv.es

\author{
Ignacio PAYÁ-ZAFORTEZA \\ Titulación: Ingeniero de C.C.P. \\ Entidad en la que trabaja: U.P.V. -ICITECH \\ Cargo: Profesor Contratado Doctor PDI \\ igpaza@cst.upv.es
}

\author{
Salvador SALES MAICAS \\ Titulación: Ingeniero de Telecomunicaciones \\ Entidad en la que trabaja: U.P.V.-ITEAM \\ Cargo: Catedrático \\ ssales@dcom.upv.es
}

\author{
Raquel María LÓPEZ-TOLA SEGUÍN \\ Titulación: Arquitecto Técnico. \\ Entidad en la que trabaja: Sacyr-Vallehermoso \\ Cargo: Coordinadora en I+D+i en Edificación \\ rltola@gruposyv.com
}

\section{RESUMEN}

Este artículo presenta los resultados de los estudios llevados a cabo por el Instituto ICITECH (Instituto y Ciencia de Tecnología del Hormigón) e ITEAM (Instituto de Telecomunicaciones y Aplicaciones Multimedia) de la U.P.V. (Universidad Politécnica de Valencia) sobre el diseño y desarrollo de nuevos sensores de fibra óptica para la medida de deformaciones y temperaturas en estructuras. Habitualmente, la monitorización estructural ha empleado los sistemas basados en transmisión de señales eléctricas como elementos sensores. Sin embargo, estos sistemas presentan una serie de inconvenientes cuando la instrumentación de una estructura desea realizarse de forma continuada en el tiempo. Estos nuevos sensores han sido probados satisfactoriamente en diferentes aplicaciones, y ofrecen grandes ventajas sobre otros sistemas empleados tradicionalmente. Este artículo presenta la tecnología empleada, las diferentes aplicaciones y sus resultados.

PALABRAS CLAVE: Monitorización de estructuras, sensores de fibra óptica, Fiber Bragg Gratting, microdeformación, transferencia de deformaciones, variación de longitud de onda. 


\section{Introducción.}

La monitorización de estructuras (structural health monitoring -o SHM- en inglés) es una rama de la ingeniería estructural dedicada al desarrollo de sistemas que proporcionen información sobre cualquier cambio significativo o daño que se produzca en una estructura (www.ishmii.org).

El establecimiento formal de esta disciplina es relativamente reciente. Por ejemplo, la International Society for Structural Health Monitoring of Intelligent Infrastructure fue fundada en el año 2003 y el primer número de la revista más antigua dedicada íntegramente a esta disciplina, el "Journal of Intelligent Material Systems \& Structures “, apareció en el año 1990. No obstante, el interés por conocer el comportamiento real de las estructuras es muy anterior. En España, probablemente sea Eduardo Torroja el gran pionero de la instrumentación estructural. Su deseo por desarrollar nuevas formas con estructuras laminares le llevó a crear modelos reducidos de las mismas, y en algunos casos, incluso a instrumentar obras reales.

La monitorización estructural tiene una gran importancia y desarrollo hoy en día. Algunas de las causas de este auge son:

- Los SHM permiten disponer de datos que ayuden a optimizar la gestión de infraestructuras y garantizar su seguridad.

- Los datos proporcionados por los SHM permiten comprobar que el comportamiento real de las estructuras se corresponde con el previsto en proyecto.

- Los accidentes recientes de algunas estructuras como el colapso del puente metálico en Minneapolis (USA) el 2 de agosto del año 2006 (Figura1), han sensibilizado a la opinión pública sobre la importancia de invertir en el mantenimiento y gestión de las infraestructuras.
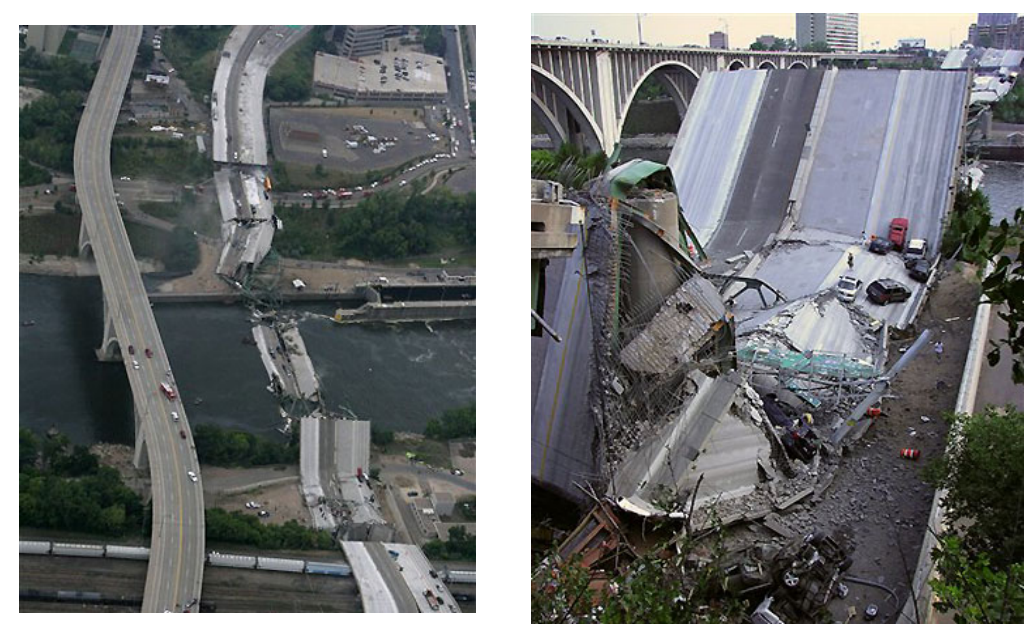

Figura 1. Colapso de un puente sobre el rio Missisipi en Mineapolis, USA. (www.nytimes.com)

Un sistema de monitorización se compone de los siguientes elementos [1]: Transductores, sistemas de procesado de datos y sistemas de evaluación de la seguridad de la estructura. Este documento se centra en uno de ellos: los sensores de fibra óptica (transductores), desarrollados por la Universidad Politécnica de Valencia (U.P.V. en lo sucesivo), empleados para la medida de deformaciones y temperaturas en estructuras. 


\section{Principio de funcionamiento de sensores ópticos tipo FBG.}

La fibra óptica representa una tecnología cuyo uso está cada vez más extendido. En los últimos años, el uso de los sensores de fibra óptica ha captado mucha atención en distintas investigaciones ([2-6]). Algunas de las principales razones de este interés son:

a) Inmunidad frente a las interferencias electromagnéticas.

b) Capacidad de ser embebidos en el hormigón

c) Capacidad de multiplexación.

De entre todos los tipos de sensores ópticos existentes, se ha decidido emplear los sensores FBG (Fiber Bragg Gratting) por su mayor simplicidad de funcionamiento, instalación e inspección. El principio de funcionamiento de los sensores FBG está basado en las redes de difracción.

Una red de difracción es un dispositivo óptico que se crea al alterar, de forma periódica o cuasiperiódica, el índice de refracción del núcleo de una fibra óptica. La luz que se propaga por el interior de la fibra óptica se encuentra con múltiples discontinuidades en el medio que provocan que parte de la luz incidente sea transmitida y otra parte sea reflejada debido a las sucesivas reflexiones (Figura 2)

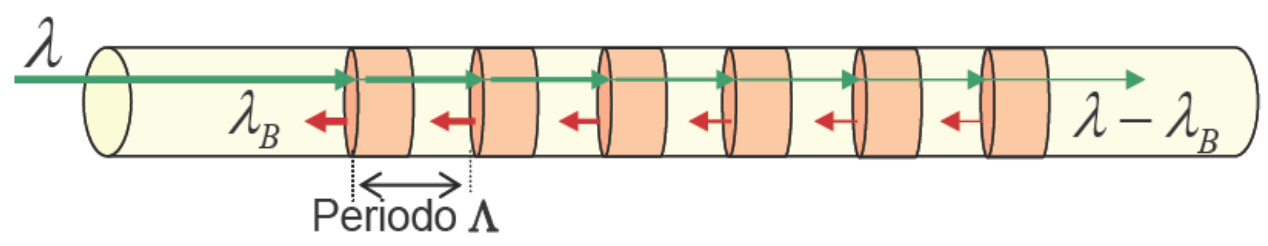

Figura 2. Esquema de una Red de difracción [7].

Para redes de difracción de Bragg de periodo uniforme, la mayor interacción o acoplamiento entre modos, el incidente y el reflejado, se produce a la longitud de onda conocida como longitud de onda de Bragg, $\lambda_{B}$ (Figura 3), la cual se determina a partir de la ecuación 1, donde $n_{\text {eff }}$ es el índice de refracción de la fibra óptica, y $\wedge$ es el periodo espacial de las perturbaciones inducidas en el índice de refracción del núcleo de la fibra óptica.

$\lambda_{B}=2 n_{e f f} \Lambda$

La longitud de onda reflejada de Bragg, se puede desplazar debido a variaciones de temperatura o bajo la influencia de tensiones aplicadas en la región donde se encuentra la FBG. Teniendo únicamente en cuenta los efectos termo-mecánicos, la variación de la longitud de onda en función del incremento de la temperatura y de la tensión axial se puede expresar mediante la ecuación (2):

$\frac{\Delta \lambda_{\mathrm{B}}}{\lambda_{B}}=K_{\varepsilon} \Delta \varepsilon+K_{T} \Delta T$

Donde $\varepsilon$ es la deformación unitaria en la región donde se encuentra la FBG, y $K_{\varepsilon}$ es una constante que indica la sensibilidad en deformación de la FBG, y que se obtiene de forma experimental. La segunda parte de la expresión, determina la deriva del máximo de reflectividad debido al incremento de temperatura [9]. 


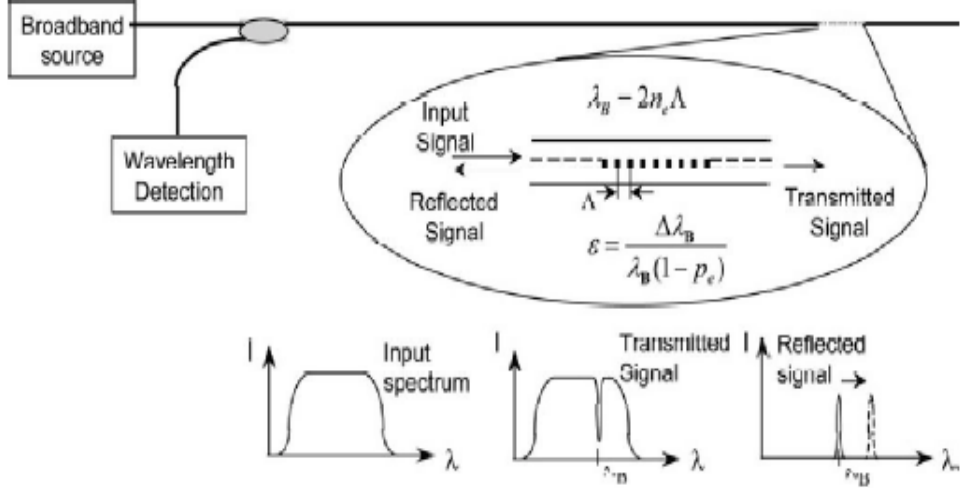

Figura 3. Espectro entrante, transmitido y reflejado. [8]

Observando la ecuación (2), las variaciones de la longitud de onda reflejada de Bragg responden a cambios de deformación y temperatura. Para poder medirlas, esta respuesta debe ser separada y distinguida. Si llamamos 1 a la fibra encargada de medir la deformación, y 2 a la fibra que compensa térmicamente a la anterior, se puede escribir las ecuaciones 3 y 4.

$\Delta \lambda_{B 1} \lambda_{B 1}=K_{\varepsilon 1} \Delta \varepsilon+K_{T 1} \Delta T$

$\Delta \lambda_{B 2} / \lambda_{B 2}=K_{T 2} \Delta T$

Resolviendo este sistema de ecuaciones, se obtiene las ecuaciones 5 y 6 , que permiten separar ambas respuestas.

$\Delta \varepsilon=\frac{1}{k_{\varepsilon 1}}\left(\frac{\Delta \lambda_{B 1}}{\lambda_{B 1}}\right)-\frac{K_{T 1}}{K_{\varepsilon 1} K_{T 2}}\left(\frac{\Delta \lambda_{B 2}}{\lambda_{B 2}}\right)$
$\Delta T=\frac{1}{K_{T 2}}\left(\frac{\Delta \lambda_{B 2}}{\lambda_{B 2}}\right)$

Los coeficientes $k_{\varepsilon 1}, K_{T 1}$ y $K_{T 2}$ han sido obtenidos experimentalmente como se indica en [1].

\section{Sistema de interrogación de sensores ópticos.}

El sistema de interrogación está compuesto por un interrogador de sensores basados en FBG de la marca Micron Optics, modelo sm125-500 y por un ordenador portátil en el que se ejecuta un software desarrollado específicamente para esta aplicación.

El interrogador de sensores ópticos sm125-500 tiene una frecuencia máxima de captura de datos de $1 \mathrm{~Hz}$, y tiene una precisión en la medida de $1 \mathrm{pm}$ (picometro).

El software utilizado para la monitorización de los sensores se ha desarrollado en el lenguaje de programación LabVIEW versión 8.5. [10] 


\section{Aplicaciones de los sensores ópticos desarrollados por la U.P.V.}

\subsection{Instrumentación de elementos a compresión.}

Se han realizado ensayos de compresión sobre elementos de hormigón y metálicos. Las probetas se han instrumentado con galgas extensométricas y sensores ópticos para comparar los resultados arrojados por ambos sistemas.

Los resultados obtenidos resultan ser muy satisfactorio en pruebas de compresión simple sobre probetas de hormigón y metálicas (Figura 4). Las pequeñas diferencias existentes pueden ser debidas a descentramientos de la carga debido a un refrentado no uniforme en la base de la probeta.

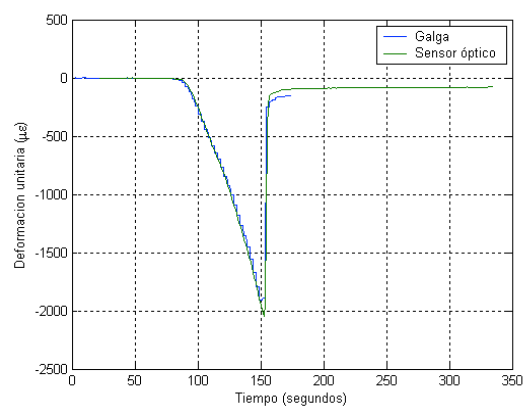

(a)

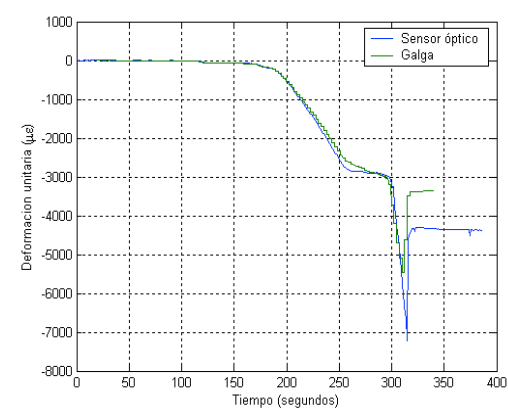

(b)

Figura 4. Resultados en pruebas de compresión simple para probetas de hormigón (a) y elementos metálicos (b)

\subsection{Instrumentación de elementos a flexión.}

Para llevar a cabo este ensayo se han fabricado una viga de hormigón armado que se ha ensayado a flexión en el laboratorio del Instituto ICITECH de la UPV.

Estas vigas se han instrumentado con los sensores ópticos tipo FBG desarrollados previamente por el ICITECH e ITEAM y con galgas extensométricas que han servido de referencia para validar los resultados arrojados por los sensores ópticos.

La geometría y dimensiones de las vigas se muestran en las figuras 5 y 6 .

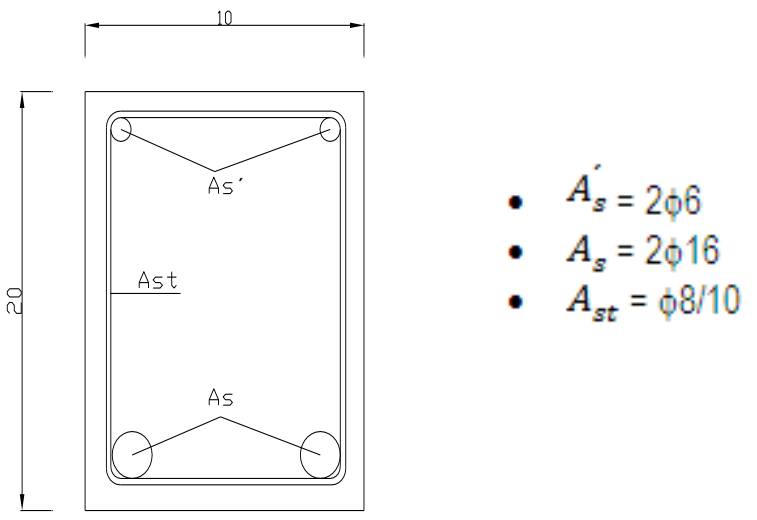

Figura 5. Sección transversal de las vigas a ensayar y disposición de armado.

Realizaciones: Gestión de estructuras: Edificación. 


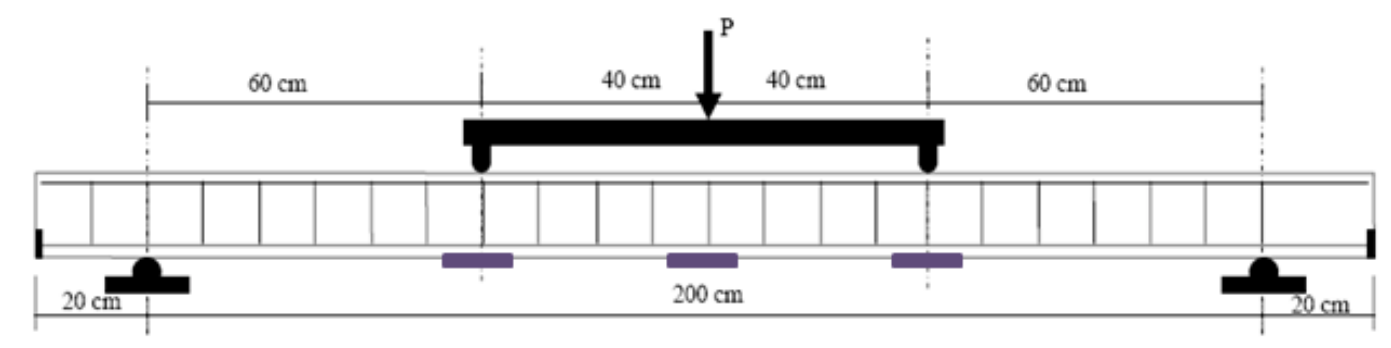

Figura 6. Esquema de ensayo.

Los resultados obtenidos han sido muy satisfactorios. La figura 7 muestra las deformaciones medidas por el sensor óptico y galga centrales, la carga aplicada $\mathrm{P}$ por el gato, y la deformación última de la sección $\varepsilon_{u}$.

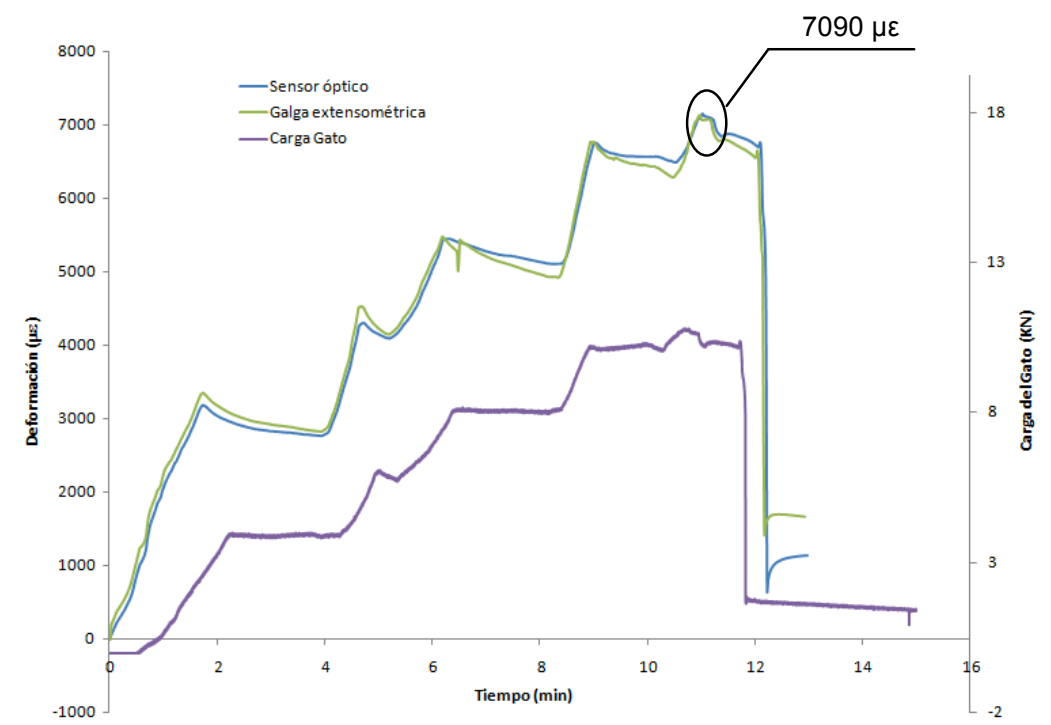

Figura 7. Leyes de deformaciones medidas por los sensores ópticos y galgas extensométricas, y carga vertical aplicada por el gato.

La deformación teórica que se alcanza en la cara inferior de la viga en el tramo central y en la situación de rotura es de $7.3 \%$ (figura 8) que se corresponde con un valor de $7300 \mu \varepsilon$. Ambas medidas, la teórica y las experimentales con galgas extensométricas y sensores de fibra óptica, presentan unas discrepancias máximas de un $2.8 \%$.

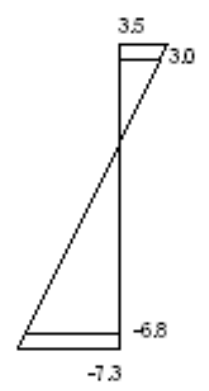

Figura 8. Plano de la deformada y valor de las deformaciones en las armaduras y en las caras superior e inferior de la viga ensayada en Estado Límite Último frente a solicitaciones normales. 


\subsection{Instrumentación de un nudo de edificación sometido a esfuerzos de flexo-compresión.}

El motivo que impulsa a proyectar este tipo de ensayos es conocer la deformación que sufren las armaduras de una estructura de hormigón armado durante un proceso de carga [11].

La estructura empleada en el ensayo simula un nudo de edificación de hormigón armado reforzado con presillas y angulares metálicos.

El ensayo consiste en colocar la probeta en el pórtico de ensayo (Figura 9) y aplicar inicialmente una carga horizontal de 12 toneladas. Tras la aplicación de la carga horizontal, se procede a la aplicación de la carga vertical hasta llevar la probeta a la rotura.

Se dispone durante el ensayo un sensor óptico en lar armaduras inferiores del nudo, y dos en las superiores.

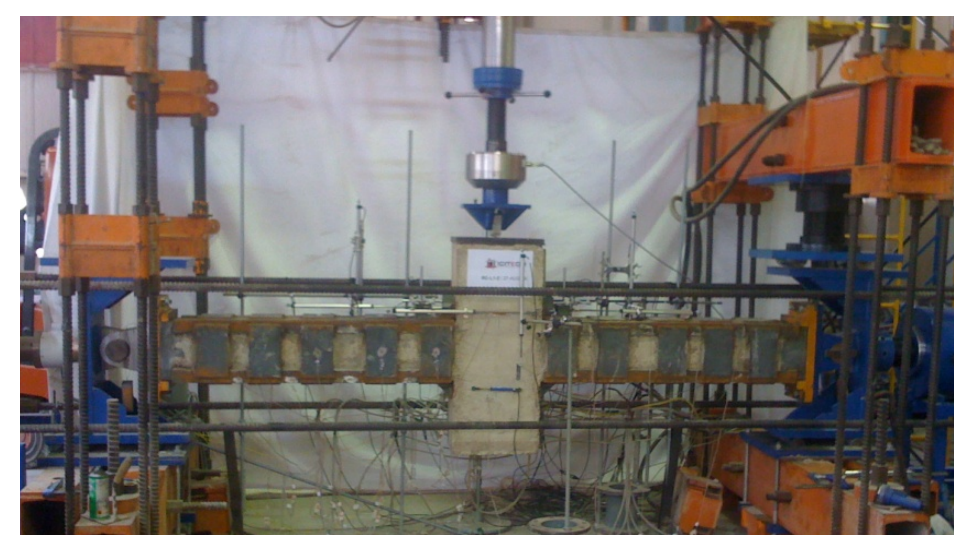

Figura 9. Imagen del nudo en el pórtico de ensayo.

Las leyes de deformaciones de las armaduras se muestran en la Figura 10.

Inicialmente y hasta el minuto 10 de comenzado el ensayo, los 3 sensores ópticos indican deformaciones de compresión hasta alcanzar un valor próximo a $350 \mu \varepsilon$ (fue medido al aplicar una carga horizontal de 12 toneladas).

Una vez aplicada la carga horizontal, se aplica la carga vertical que aumenta progresivamente hasta la rotura. El sensor instalado en la armadura inferior indica tracciones, que alcanzan un valor de $2000 \mu \varepsilon$. Los sensores ópticos situados en las armaduras superiores indican compresiones, con valores máximos de $1100 \mu \varepsilon$.

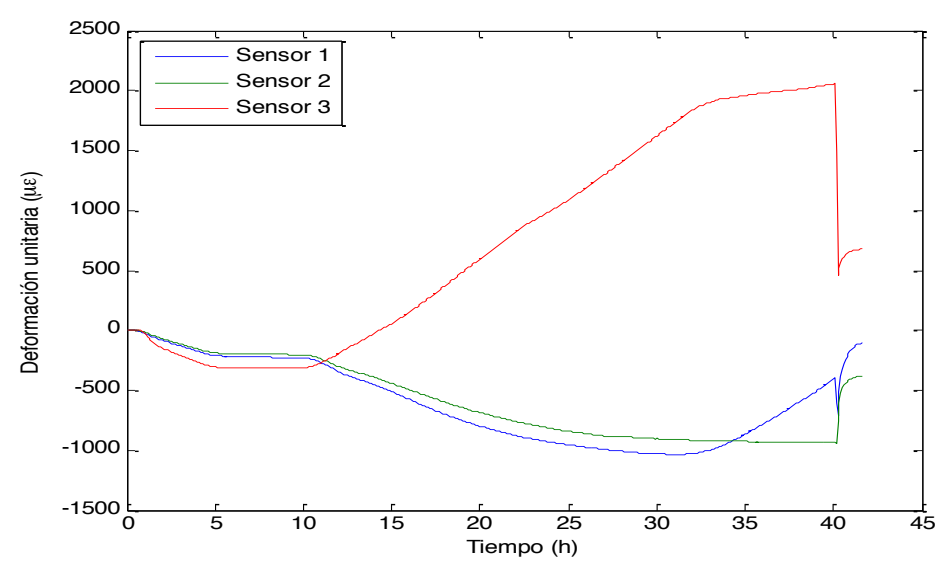

Figura 10. Leyes de deformaciones de los sensores ópticos instalados en las armaduras. 
No ha sido posible llevar a cabo la comparativa con los valores obtenidos por las galgas extensométricas, debido a que éstos no han sido captados correctamente, arrojando valores erróneos.

\subsection{Instrumentación de una obra real: Puente mixto de sección en cajón metálico construido por empuje.}

El objeto de este trabajo es el de conocer las deformaciones que se producen en las diferentes secciones de un cajón metálico durante su proceso constructivo(Figura 11). El puente ha sido ejecutado por la empresa constructora Sacyr Vallehermoso en el término de Alonsotegui (Bilbao).

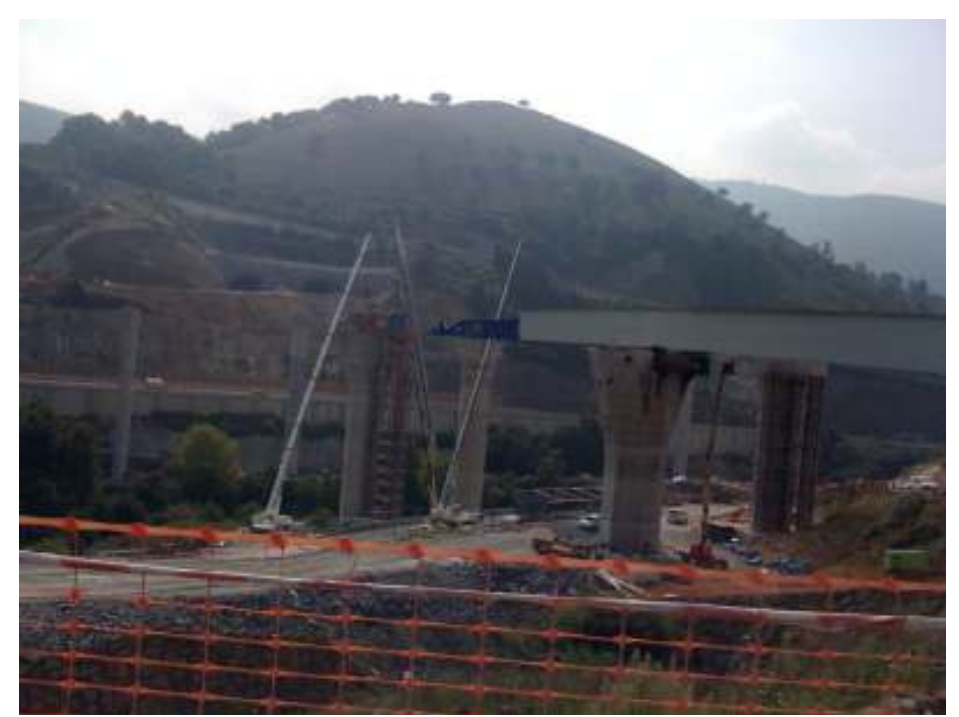

Figura 11. Vista del cajón metálico que forma la variante Sur Metropolitana de Bilbao, en el instante de su lanzamiento.

La instalación de los sensores ópticos se lleva a cabo en 5 secciones transversales diferentes del modo indicado en la figura 12. La instrumentación fue llevada a cabo al mismo tiempo que se producía el lanzamiento del cajón, ininterrumpidamente durante toda la operación. El control de las deformaciones se efectuaba in situ y en tiempo real. Los resultados obtenidos de la monitorización se muestran en la figura 4.9.

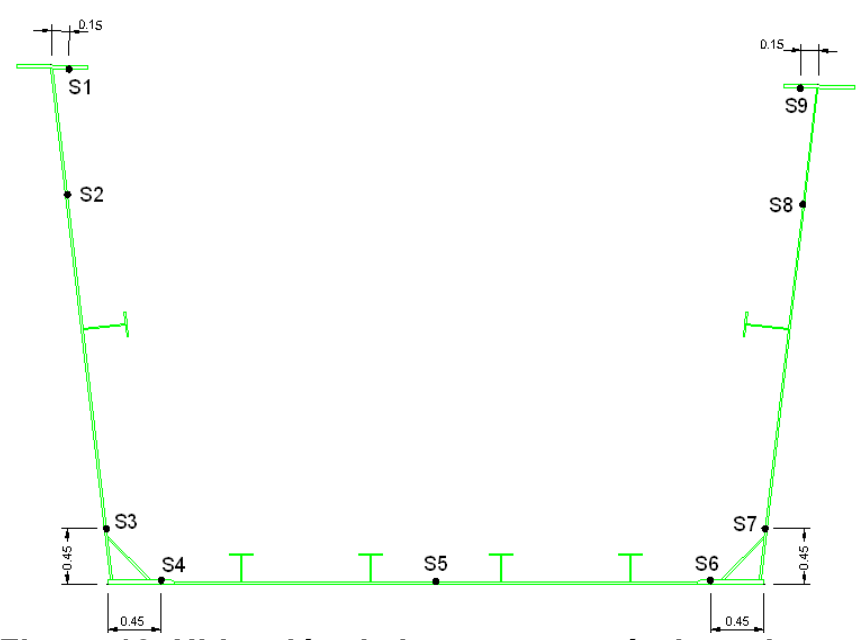

Figura 12. Ubicación de los sensores ópticos dentro de la sección transversal del cajón. 
A partir de la hora 8 de iniciada la operación de lanzamiento se produce en todas las secciones un efecto de estabilización de las deformaciones en el cajón (Figura 13). Este efecto tiene una duración de aproximadamente 15 horas, y es debido a que la monitorización se llevó a cabo durante la noche, en el que el cajón no es lanzado y por tanto sus deformaciones permanecen constantes.

A partir de la hora 31 de iniciada la operación de lanzamiento se produce un cambio brusco y repentino en la tendencia de las leyes que describen las deformaciones del cajón (Figura 13). Ello se debe a la operación de recuperación de flecha, cuyo objetivo es alzar la punta de la nariz del cajón hasta la cota de apoyo de las pilas.

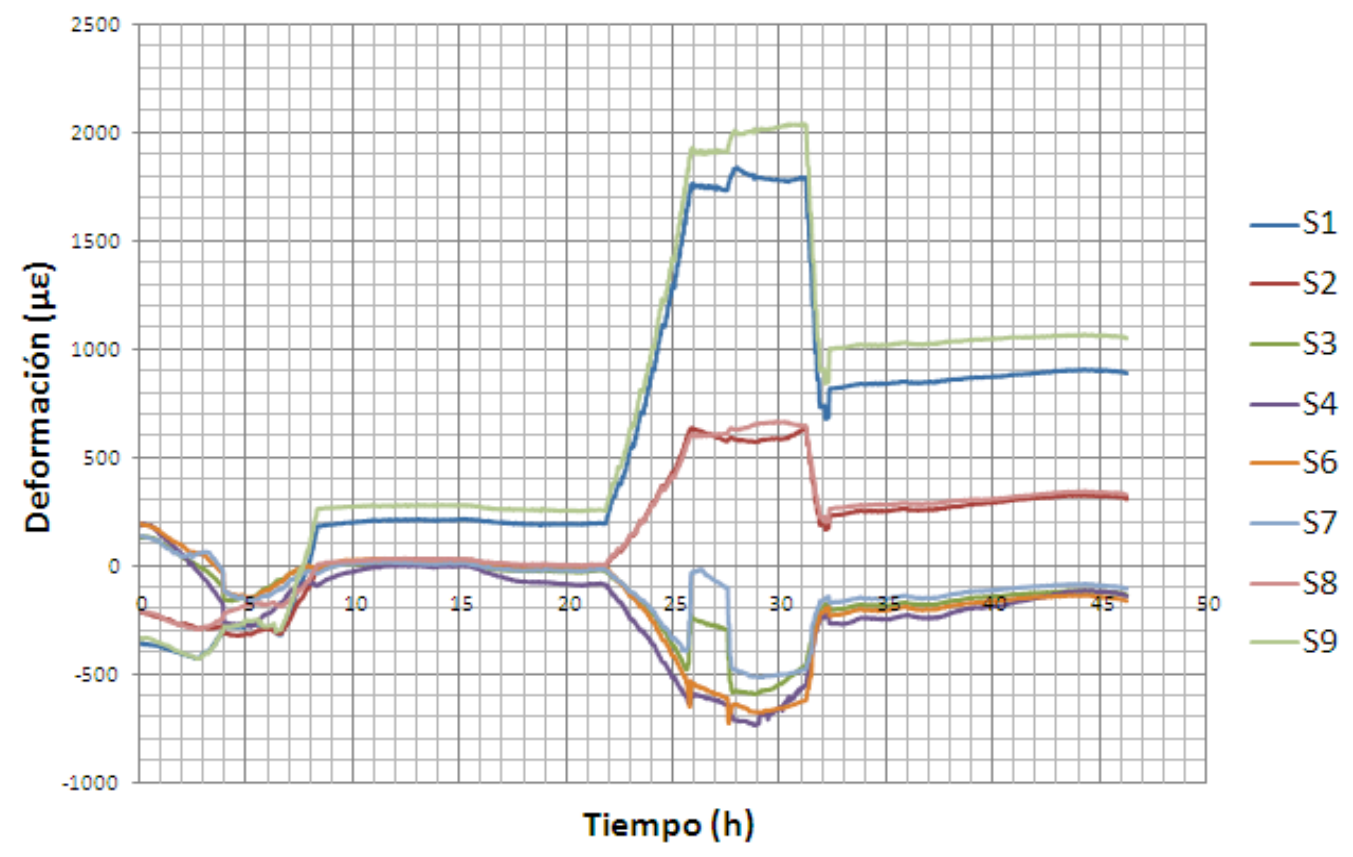

Figura 13. Leyes de deformaciones frente al tiempo en una de las secciones instrumentadas.

\section{Conclusiones}

Este artículo muestra la tecnología óptica desarrollada por los Institutos ICITECH e ITEAM de la Universidad Politécnica de Valencia para la medida de temperaturas y deformaciones en estructuras. Ha quedado demostrada la validez de los sensores ópticos para la medida de deformaciones en distintos elementos estructurales y distintas tipologías de cargas. La validez de los sensores ópticos para la medida de temperaturas en distintos elementos estructurales puede consultarse en [9].

Además, esta tecnología óptica se ha aplicado en la instrumentación de un cajón metálico construido por la técnica del "empuje", obteniendo las deformaciones en la fase crítica coincidente con su voladizo máximo.

Los resultados ofrecen la fiabilidad de los sensores ópticos, demostrando las garantías que éstos presentan en el sector de la construcción. 


\section{Agradecimientos:}

Los autores quieren agradecer la ayuda prestada a la Unidad Docente de Hormigón de la Universidad Politécnica de Valencia por permitir la instrumentación de sus estructuras empleadas en distintas investigaciones. Estos trabajos han sido financiados por la empresa constructora Sacyr Vallehermoso, en el marco del Proyecto "Desarrollo de sensores ópticos aplicados a la instrumentación de estructuras para conocimiento de su comportamiento en tiempo real", y por el proyecto SOPROMAC (Desarrollo de Sensores Ópticos Avanzados Basados en Fibra Óptica para la Determinación de las Propiedades de los Materiales Constructivos y la Salud Estructural de Infraestructuras), financiado en el marco de las ayudas del Programa Nacional de Cooperación Público-Privado del Plan Nacional de Investigación Científica, Desarrollo e Innovación Tecnológica 2008-2011.

\section{Referencias:}

[1] TORRES B. "Estudio experimental de sensores ópticos tipo FBG para medir deformaciones y temperaturas en estructuras". Tesina final de Máster en Ingeniería del Hormigón. Departamento de Ingeniería de la Construcción y Proyectos de Ingeniería Civil. Universidad Politécnica de Valencia. Noviembre 2009

[2] HO J.M., NI Y.Q. "Technology developments in structural health monitoring of large-scale bridges". Engineering Structures 2005; 27: 1715-25.

[3] MAJUMDER M., GANGOPADHYAY T.M. CHAKRABORTY A.K. "Fibre Bragg gratings in structural health monitoring - Present status and applications". Sensors and Actuators A: Physical 2008; 147:150-64.

[4] CHAN T.H.T., YU L., TAM H.Y., NI Y.Q., LIU S.Y., CHUNG W.H. CHENG L.K. "Fiber Bragg grating sensors for structural health monitoring of Tsing Ma bridge: Background and experimental observation". Engineering Structures 2006; 28: 648-59.

[5] CHUNG W., KAND D. Full-scale test of a concrete box girder using FBG sensing system. Engineering Structures, 2008; 30(3): 643-52.

[6] LIN Y.B., PAN C.L. KUO Y.H. CHANG K.C. CHERN J.C. "Online monitoring of highway bridge construction using fiber Bragg grating sensors". Smart Mater. Struct. 2005; 14:107582.

[7] BARRERA D. "Diseño de sensores ópticos avanzados basados en FBG". Diploma de Estudios Avanzados (DEA). Universidad Politécnica de Valencia. Septiembre 2008.

[8] TORRES B., PAYÁ-ZAFORTEZA I., CALDERÓN PA., ADAM JM. "Analysis of the strain transfer in a new FBG sensor for Structural Health Monitoring". Engineering Structures 33 (2011) 539-548.

[9] TORRES B., PAYÁ-ZAFORTEZA I., BUENO A., SALES S., ADAM JM., CALDERÓN PA. "Early age concrete temperature and shrinkage monitoring by using Fiber Bragg Grattings". XXXVII IAHS World Congress on Housing. October 26 - 29, 2010, Santander, Spain

[10] BUENO MARTÍNEZ A. "Diseño y caracterización de un sensor óptico autocompensado en temperatura para la medida de deformaciones en estructuras de ingeniería civil y desarrollo de un sistema de interrogación". Tesina Final de Máster. Escuela Técnica Superior de Ingenieros de Telecomunicaciones. Universidad Politécnica de Valencia. Septiembre 2008.

[11] GARZÓN-ROCA, J., ADAM, JM., CALDERÓN PA. Behaviour of RC columns strengthened by steel caging under combined bending and axial loads. Construction and Buildings Materials 25 (2011) 2402-2412. 


\title{
Monitoring of a steel incrementally launched bridge construction with strain and temperature FBGs sensors
}

\author{
Antonio Bueno ${ }^{1, *}$, Benjamín Torres ${ }^{2}$, David Barrera ${ }^{1}$, Pedro Calderón $^{2}$, Salvador Sales $^{1}$ \\ ${ }^{1}$ : iTEAM, Universidad Politénica de Valencia, Camino de Vera s/n, 46022, Valencia \\ 2: ICITECH, Universidad Politécnica de Valencia, Camino de Vera s/n, 46022, Valencia. \\ *anbuemar@iteam.upv.es
}

\begin{abstract}
We present in this paper the results of monitoring the construction process of a steel incrementally launched bridge located at the Kadagua Valley in Bilbao (Spain) with FBG sensors. The installation of FBG strain and temperature sensors was done in order to obtain deformation and temperature variations during the launching operation. The deflection recovery process was also monitored. The setup carried out in the sensors installation process consists of five optical channels (one for each cross section monitored) and a multiplexed structure of nine strain sensor in each optical channel. Temperature sensors were also installed in order to measure temperature variation of the steel structure but also for thermal compensation for the FBG strain sensors. The installation of the optical sensors is explained in detail including cleaning, bonding and connection of the almost fifty sensors installed in this structure. We also are going to explain the behaviour of the steel structure by presenting several figures showing the strain values for each sensor taken in real time during the launching of the bridge.
\end{abstract}

Keywords: FBG sensor, strain optical sensor, temperature optical sensor, incrementally launched bridge

\section{INTRODUCTION}

The monitoring of damage in civil structures has always been an interesting field for civil engineers. In fact, they created a new term called "Structural Health Monitoring" (SHM) to define a system that provides information on demand about any significant change or damage occurring in the structure. Such an interest is due to the high cost of the maintenance of a structure since monitoring methods used today are based on periodical visual inspections trying to detect cracks or physical degradations. Thus, a source of information is needed to achieve a real structural health monitoring system.

To obtain this information from the structure, sensor elements have to been installed all over the structure to collect the data that will be processed in order to obtain the information about the structure behavior. Traditionally, electrical sensors have been used for structural monitoring, but optical technology has playing an important role in the past two decades, particularly since fiber Bragg gratings (FBG) were firstly used as a strain and temperature sensor ${ }^{1}$. Optical fiber sensor have many advantages compared with electrical sensors: they are light weight, immune to electromagnetic interference (EMI) and resistant to harsh environments. Specifically, FBG sensors have also some advantages from other optical fiber sensors like easy fabrication and installation and high speed of interrogation.

Recently, many structures have been successfully monitored with FBG sensors. Structures such as foundation piles of a building $^{2}$, railway bridges ${ }^{3}$ or even a nuclear reactor core ${ }^{4}$. In this paper, the authors are going to show the health monitoring system of a civil bridge with FBG sensors during the bridge construction process.

\section{PROJECT DESCRIPTION}

The structure monitored in this work is a steel incrementally launched bridge located at Kadagua Valley near the city of Bilbao in Spain. This bridge is placed above Kadagua river and some other existing roads and railways and it will be used for terrestrial vehicles to cross the valley. The construction of the bridge started in April of 2008 and the estimated total duration is 24 months. 
Total length of the bridge will be 364,12 meters and it is going to be launched in phases due to the reduced working area that allows to construct only 50 meters at once. Monitoring phase was the bridge launch between piles P1-2 and P1-3. An additional structure, known as "nose" was constructed at the end of the bridge in order to help the bridge to lean on the piles as only 50 meters can be constructed at once and the distance between piles are higher than this. Initial and final launch situations are shown in figure 1.

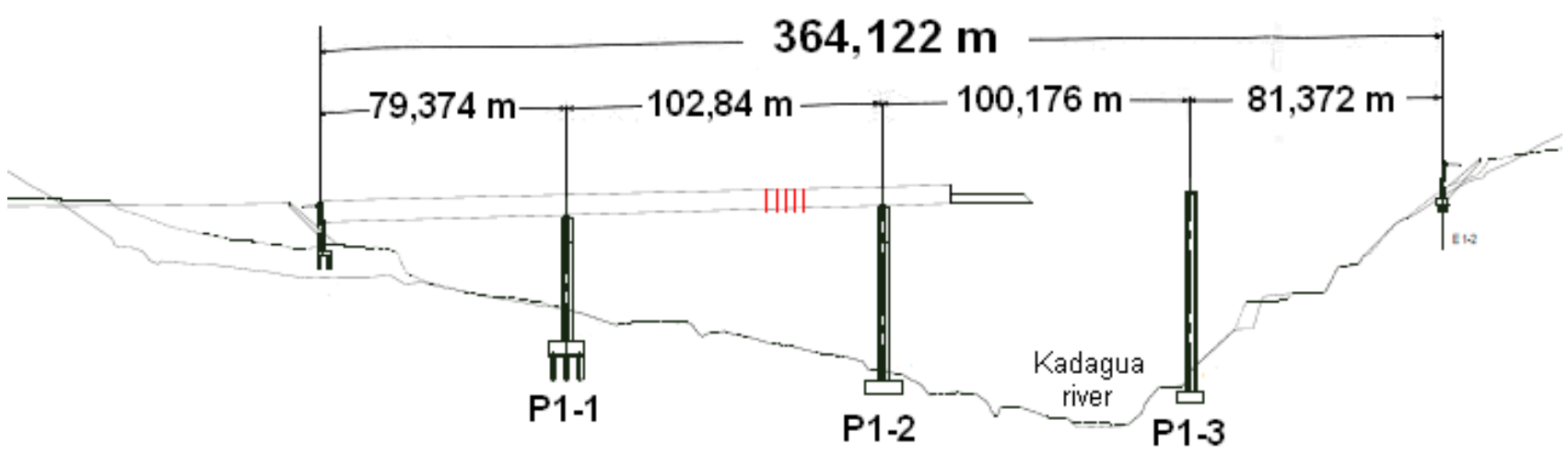

INITIAL SITUATION

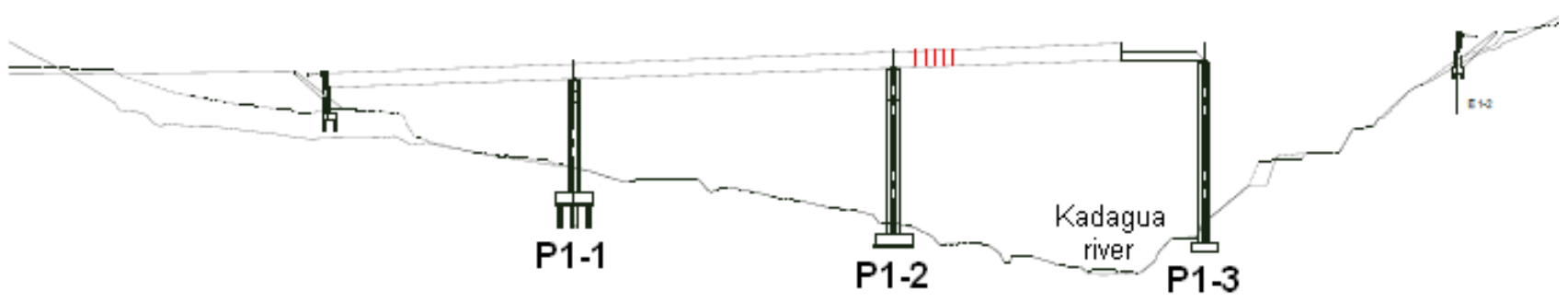

FINAL SITUATION

Figure 1: Initial and final situation for second and third bridge launch.

In the initial situation of this launch, the bridge nose lean on the pile P1-2 as it can be seen on the upper part in figure 1. The lower part of this figure shows the final situation, where the bridge is projected in a 100,176 meters long cantilever, including nose. Final situation in this launch is the worst case for the bridge in the whole launching operation. The main reason is that in this situation there is a 100,176 meters long cantilever and in addition the length of the constructed bridge before the support in the pile P1-2 is more than 180 meters. Once the bridge reaches the pile P1-3 it starts a deflection recovery process to compensate the existing cantilever and to lean the bridge on this pile.

The objective of this project is to monitor up to 5 cross-sections of the bridge during the launching operation. These sections can be seen depicted (vertical lines) in figure 1 for initial and final situations. They are the nearest sections to the pile P1-2 when the bridge is reaching pile P1-3. The main reason to monitor these sections is because the sections just over the pile P1-2 have more stiffness than the others ones. In each section 9 FBG strain sensors were installed in order to obtain strain values in those locations where the strain measurements allow us to understand the behavior of the bridge. In figure 2 it can be seen on the left the schematic cross-section of the bridge with the sensor locations and on the right a real picture of the bridge's cross-section. 


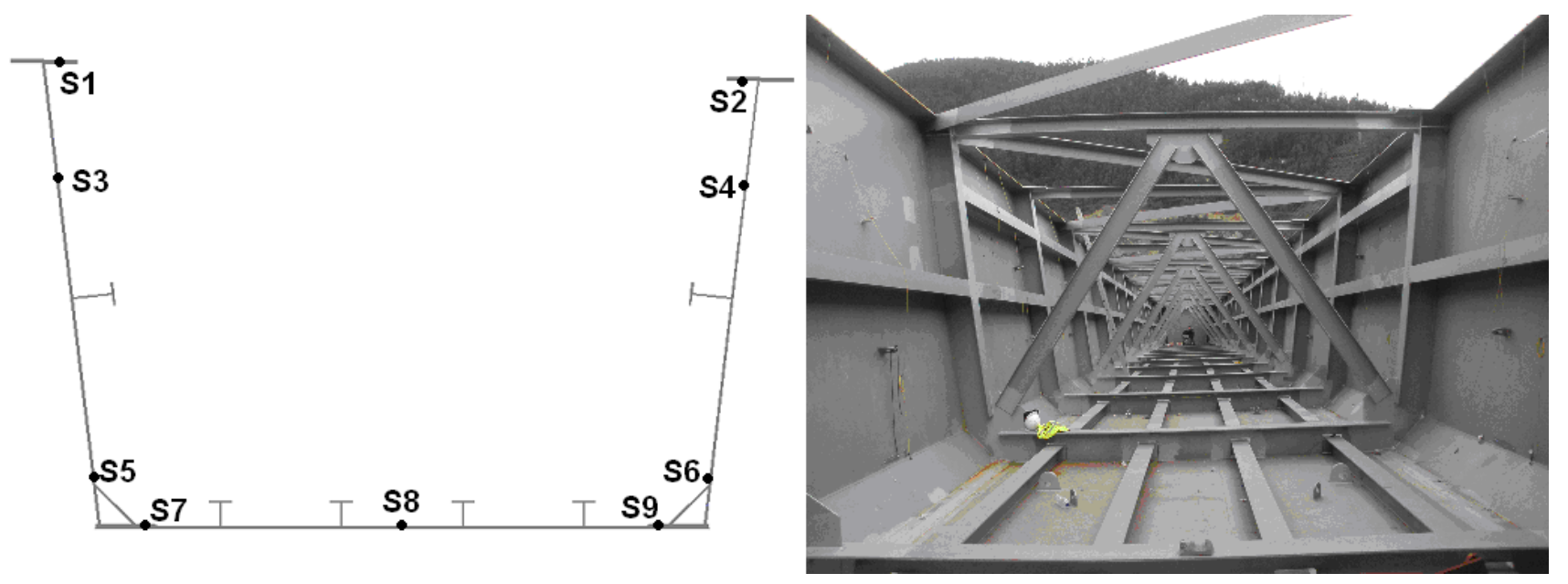

Figure 2: Cross-section of the bridge. Left: Schematic with sensor locations. Right: Real picture.

The sensors installed are supposed to measure strain values of compression and traction depending on the sensor location. For example, sensors 1 to 4 (see figure 2 on the left) will obtain traction strain values as the structure is supported on its base between piles P1-1 and P1-2 and the monitored sections are also located between these piles. On the other hand, sensors 5 to 9 will obtain compressive strain values. Once the structure reaches the pile P1-3, it starts a deflection recovery process that will compensate the cantilever, and so an inversion in the strain (compression to tensile and vice versa) will occur.

It is important to note that this is a steel structure, thus a maximum value of strain has not to be reached in order to keep safe the bridge construction process. Strain value for steel yield is around $2000 \mu \varepsilon$ therefore strain values measured from the sensor monitoring system would not exceed this limit value. That's why it is a key factor to monitor in real time strain values from the bridge during launching operation in order to avoid the collapse of the structure and to assure physical integrity of the construction workers.

\section{ENGINEERING DESCRIPTION}

The sensors installed on the structure were based on Fiber Bragg Gratings (FBG). These FBG sensors were fabricated at iTEAM research institute with the phase mask technique ${ }^{5}$ with up to 12 different phase masks. A Gaussian apodization was applied in FBG fabrication process. The central wavelengths for the fabricated gratings were located between 1518 and $1573 \mathrm{~nm}$ inclusive, and spaced $5 \mathrm{~nm}$ one between each other. The first nine central wavelength sensors were fabricated as strain sensors, and the rest of them as temperature sensors.

Sensor installation strategy was to use a single optical fiber to connect sensors in each cross-section, taking advantage of FBG wavelength multiplexing possibility. Since up to 9 strain sensors were installed in each section, we installed one of each central wavelength FBG strain sensor and 3 temperature compensation sensors in key locations per section.

For sensor interrogation, we used a commercially available optical sensor interrogator. This device has up to 4 optical channels with a wavelength range of 1510 to $1590 \mathrm{~nm}$. Since we needed to monitor 5 bridge sections and each section need an exclusive use of a single optical channel, we finally used a channel extender in order to obtain a total number of 16 optical channels.

Another important issue to take into account is that once the bridge launching operation starts, it is absolutely forbidden for any person to stay inside the bridge steel structure. That's why we connected the optical sensor interrogator to a wireless router to access sensors data from outside the bridge structure. Wireless coverage of the router was around 500 meters, long enough to access the sensor monitoring system information from a safe location. In addition, a personal computer was wired to the router acting as a local data storage device. The final setup for the monitoring system is depicted in figure 3. 


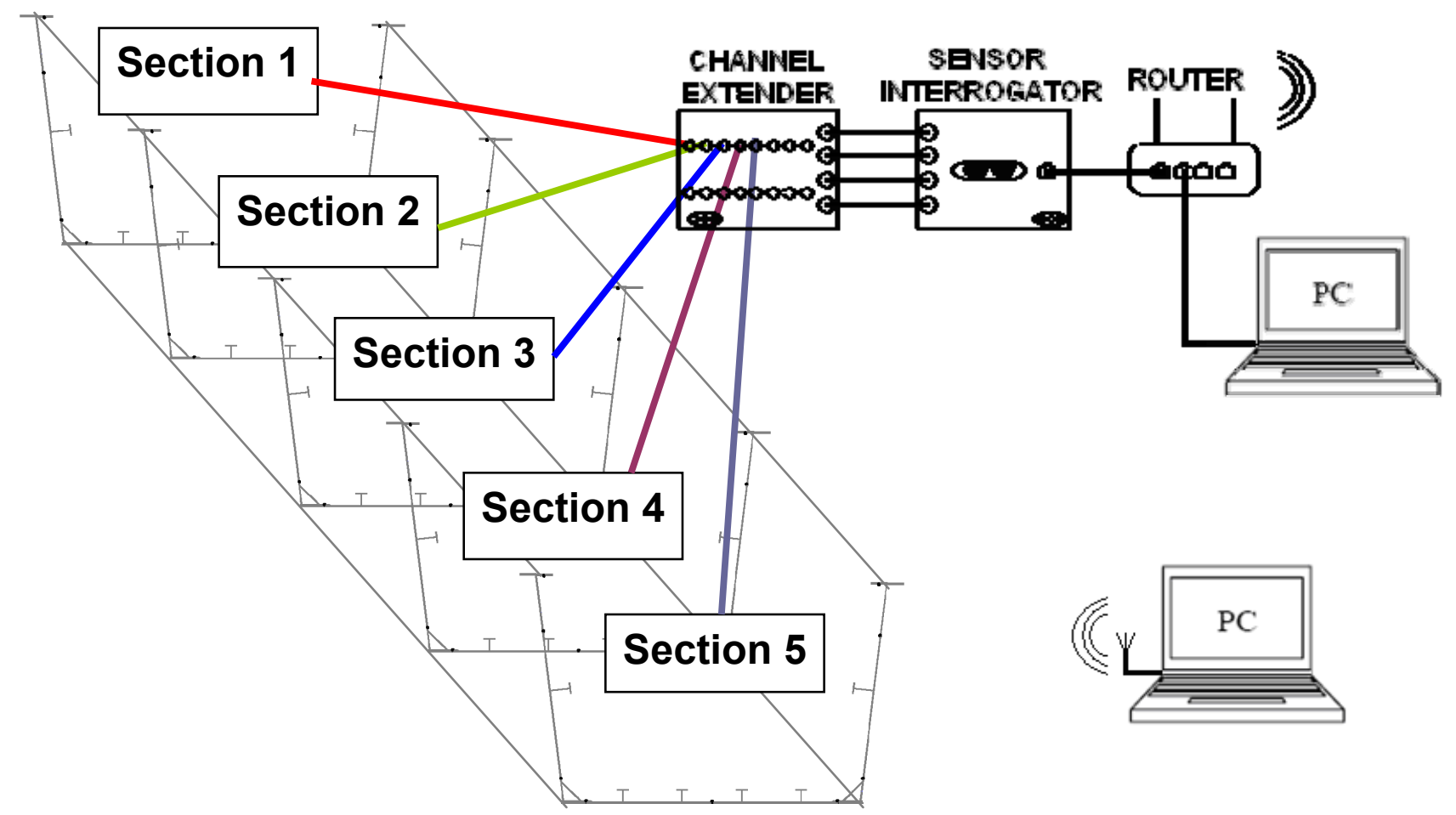

Figure 3: Final setup for structural health optical sensor monitoring system developed to monitor the bridge launch.

In figure 3, the section 5 is the nearest section to the end of the structure and section 1 is the nearest section to the pile P1-2 in the launch final situation. That is the reason why section 1 is expected to measure higher strain values than the other ones.

Strain and temperature optical sensors were installed in two different days due to the high number of sensor required for the entire monitoring system. Procedure of sensor installation was as follows:

- Preparation of the bonding surface: Steel surface of the bridge was painted during its construction to avoid metal oxidation. It was necessary to remove the huge paint layer with a radial saw to assure the maximum strain transfer from the steel structure to the optical sensor ${ }^{6}$. In addition, a flat surface is obtained from the metal erosion and thus we achieve an improvement in the later bonding process.

- Adhesive and sensor preparation: Once the surface is ready, the next step is to prepare the sensor. Firstly the sensor is transferred to a $15 \mathrm{~cm}$ length tape with the bonding to the steel structure side up. Adhesive preparation requires mixing two compounds vigorously for a couple of minutes, and then it is ready to apply to the sensor surface. It is important to apply enough adhesive to provide sufficient coverage once the sensor is bonded for proper adhesion. Insufficient adhesive will lead to an incorrect strain measurement but on the other hand, too much adhesive will decrease the strain transfer form the steel structure to the sensor. Right quantity of adhesive was obtained from several trial and error iterations in laboratory tests.

- Sensor bonding to the surface: The final step is to bond the prepared sensor to the steel surface. The sensor has to be placed in the desired point very smoothly and gently. Once the sensor is in contact with the surface, it must be applied firm and steady pressure in the whole sensor length for 10 seconds until the sensor is well consolidated. Later, pressing the tape surrounding the sensor helps to fix it during the adhesive curing. This adhesive curing depends on the environmental conditions (temperature mainly) but entire process takes no longer than 12 of hours. The slow curing time of this adhesive avoid residual stress that usually led to an optical spectrum deformation ${ }^{7}$. 
When all the sensors are properly bonding, they can be connected as mentioned before. Taking advantage of wavelength multiplexing, it is possible to connect sensors serially in the same section. As a result, five optical channels coming from five structure cross-sections carrying information of more than 50 sensors were available. Optical spectrum of every single sensor is shown in figure 4.

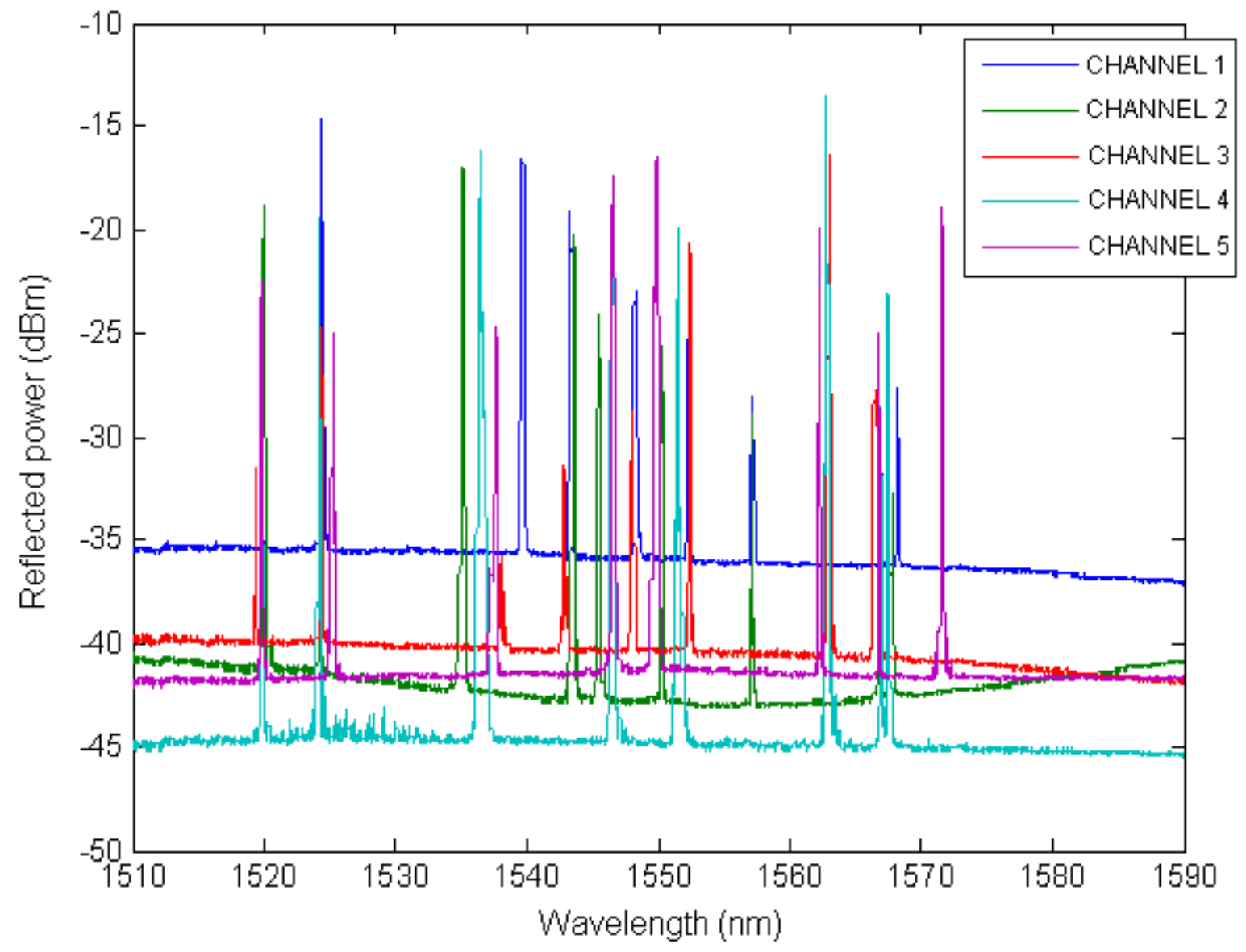

Figure 4: Optical spectrum of every single sensor installed in the steel bridge.

\section{RESULTS AND DISCUSSION}

The data collected from launching operation was extremely big, as information from more than 50 sensors was retrieved for more than 46 hours. Data save interval was set to 1 minute, time enough to detect substantial strain changes in the structure because launching speed was around 5 meters per hour due to the delicate nature of the opertation. We are going to show only the results coming from sections 1 and 5 of the bridge since results obtained from the rest of the sections are similar.

It can be seen in figure 5 the strain curves obtained from the sensors installed in section 1 . Sensor numeration is based in the schematic of the cross-section of the structure shown in figure 1 on the left. Thus, sensors numbers 1 and 2 are installed in the upper part of the structure, sensors 3 and 4 are the sensors installed at a medium structure height and sensors 5 to 9 were installed at the bottom of the structure. The monitoring system was continuously collecting data, night included. Night monitoring periods are clearly identifiable because strain values remain constant for several hours. In particular these night periods are between hours 8,5 and 21,8 for the first night and between hours 32,3 and 46,2 . It can be proved that the sensors are working properly as no strain change has been recorded when the launching operation was stopped. 


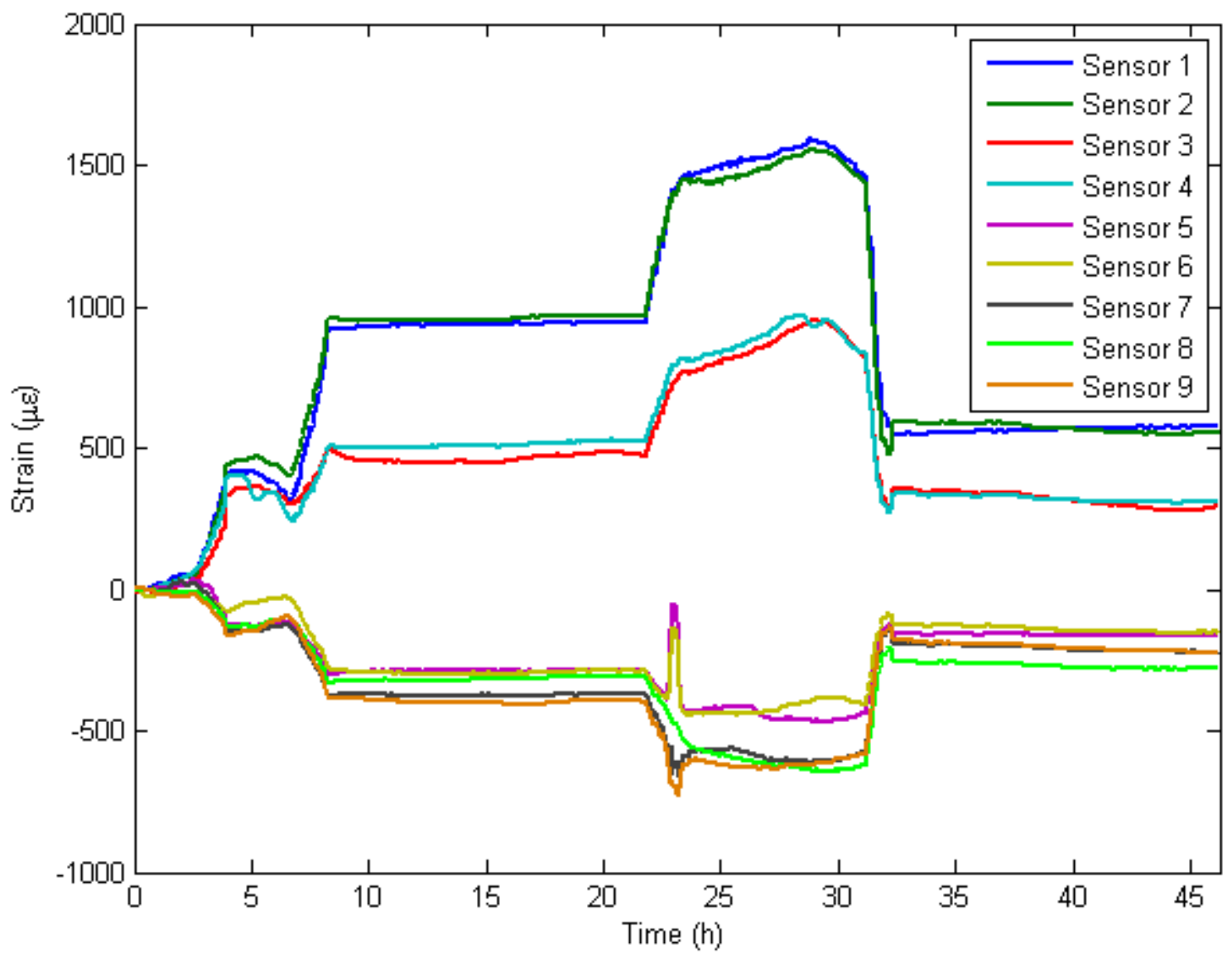

Figure 5: Strain curves obtained for the sensors installed in section 1 during the bridge launch (nights included)

Sensors 1 to 4 obtained tensile strain values and the sensors that registered the maximum strain values were sensors 1 and 2 as expected. Maximum strain value for sensor 1 was 1594,3 $\mu \varepsilon$ and for sensor 2 was $1556 \mu \varepsilon$. These two sensors registered the maximum strain due to their location because the upper part of the structure has the maximum deflection when the structure is projected in a cantilever over the pile P1-2, as the structure is leaned on its base in this pile. Sensors 3 and 4 also registered high strain values but lower than sensors 1 and 2. Maximum strain value for sensor 3 was 954,1 $\mu \varepsilon$ and for sensor 4 was $970,3 \mu \varepsilon$.

Regarding the sensors 5 to 9 , it can be seen compressive strain values and the sensors that registered the maximum compressive strain values were sensors 7,8 and 9 because they were installed at the bottom of the structure. Maximum compressive strain value for sensors 7,8 and 9 were $658,5 \mu \varepsilon, 645,9 \mu \varepsilon$ and $728,1 \mu \varepsilon$ respectively. Sensors 5 and 6 recorded a maximum compressive strain value of $467,9 \mu \varepsilon$ and $448,3 \mu \varepsilon$ respectively. Concerning sensors installed at the bottom of the structure, it can be seen in figure 4, anomaly behaviour around hour 23. Sensors 5 and 6 experienced a traction strain peak whereas sensors 7 and 9 experienced a compression peak. It is due to the support of the base of this monitored section on the hydraulic jacks located on the pile P1-2.

Finally, figure 5 shows around hour 31 the result of the deflection recovery process. In such a process the structure is raised up in order to be leaned on the pile P1-3. The previous deformation of the structure is compensated by recovering the initial deflection. Upper sensors ( 1 to 4 ) get compressed whereas lower sensors (5 to 9) get tensiled. Not all the strain is compensated as the structure is now supported on piles P1-2 and P1-3 and the structure is deformed due to its own weight. 
As a remark, it is important to notice that the maximum strain value registered by any sensor is less than $2000 \mu \varepsilon$. Maximum strain value was $1594,3 \mu \varepsilon$ and it was obtained by sensor 1 in section 1 . This strain value is the $79,7 \%$ of the steel yield strain, and that means that in fact this bridge launch phase was a delicate maneuver.

Depicted in figure 6 are the strain curves for the sensors installed in section 5, which is the first monitored section from the end of the structure. The shapes of the curves are quite similar to the curves shown in figure 5 although maximum strain values of compression and tensile are lower.

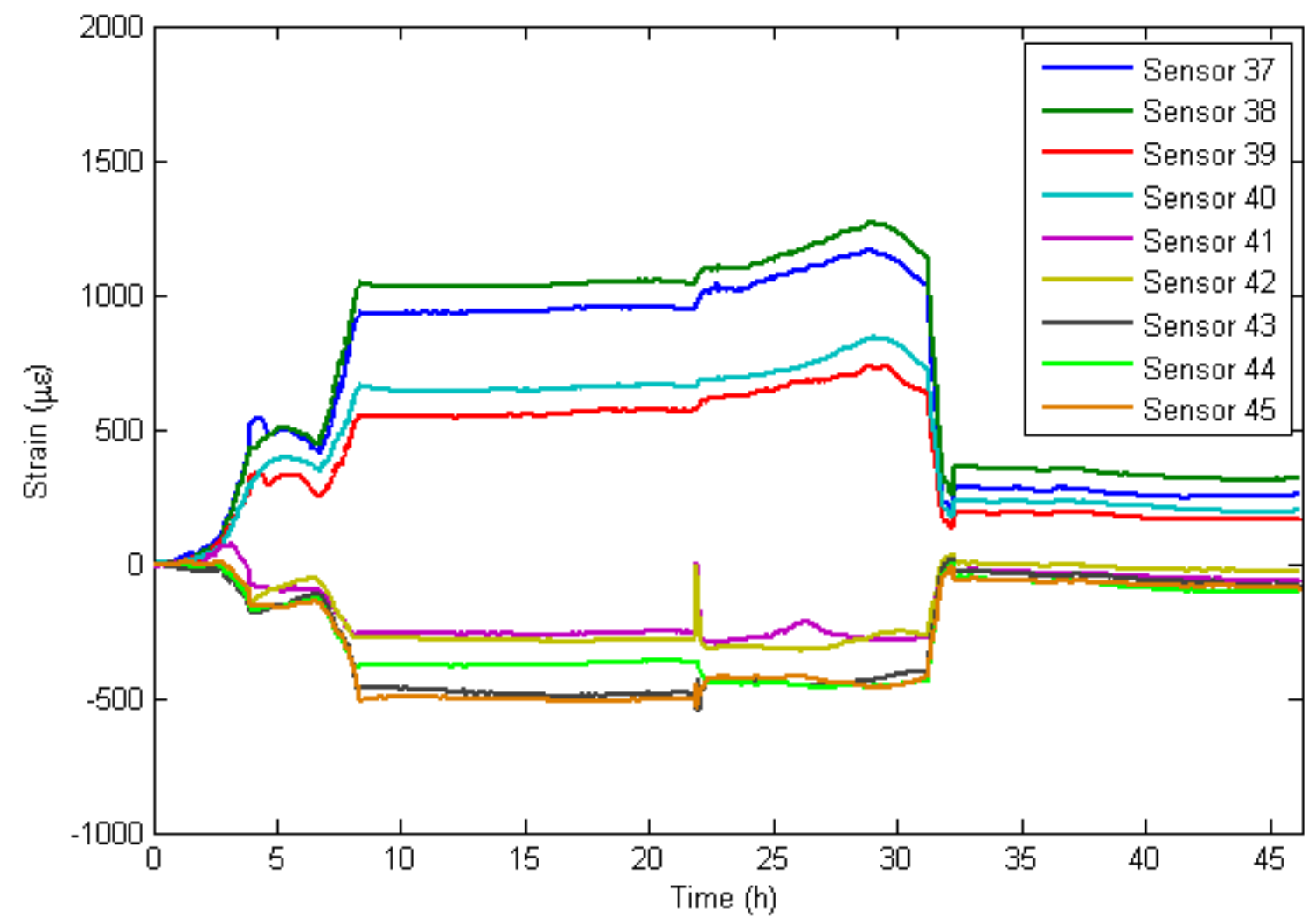

Figure 6: Strain curves obtained for the sensors installed in section 5 during the bridge launch (nights included)

\section{CONCLUSIONS}

A large number of FBG sensors were successfully installed in the surface of a steel structure. Sensor preparation and bonding were first tested in laboratory in order to make them controllable and repeatable for sensor installation in a real construction. The FGB wavelength multiplexing is very suitable to save tenths of meters of optical fiber cable, allowing a simple connection setup. Optical interrogator and channel extender are simple handling instruments because they only need a personal computer connected to them in order to collect de data. An additional feature was added to the monitoring system consisting in the wireless connection with a personal computer to enable real time data consulting from outside the structure.

As a result of the data extracted from the strain monitoring system and compared with theoretical assumptions we can conclude that the FBG optical sensors installed in the steel structure worked properly. The results show a good agreement with the theoretic behavior of the cross-section of a steel structure during its launching. The sensor reliability was demonstrated by maintaining the data storage during the night since no strain changes were observed. 
The structure health monitoring system resulted as a very useful tool to control the whole launching operation by taking real time data from the sensors installed. A really high number of strain values were recorded by the sensors net installed in the critical sections in order to avoid the structure collapse. Strain values were higher than expected but the structure integrity was always kept.

\section{REFERENCES}

[1] Meltz, G., Morey, W. W., Glenn, W. H., Farina, J.D., "In-fiber Bragg-grating sensors", Proc. OFS'88, London, England, 163-166 (1988).

[2] Kister, G., et al., "Methodology and integrity monitoring of foundation concrete piles using Bragg grating optical fibre sensors", Engineering Structures 29, 2048-2055 (2007).

[3] Kerrouche, A., et al., "Field tests of fibre Bragg grating sensors incorporated into CFRP for railway bridge strengthening condition monitoring", Sensors and Actuators A 148, 68-74 (2008).

[4] Fernandez, A., et al., "Temperature monitoring of nuclear reactor cores with multiplexed fiber Bragg grating sensors", Opt. Eng. 41(6), 1246-1254 (2002).

[5] Hill, K. O.,"Bragg gratings fabricated in monomode photosensitive optical fiber by UV exposure through a phase mask,” Appl. Phys. Lett., vol. 62, 1035-1037 (1993).

[6] Wan, K. T., Leung, C., Olson, N., "Investigation of the strain transfer for surface-attached optical fiber strain sensors", Smart Mater. Struct. 17, 1-12 (2008).

[7] Kuang, K.S., et al., "Embedded fibre Bragg grating sensors in advanced composite materials", Composites Science and Technology 61, 1379-1387 (2001). 


\title{
Fiber Bragg Grating Sensors Embedded in Concrete Samples for a Normalized Fire Test
}

\author{
Antonio Bueno $^{1}$, Benjamín Torres ${ }^{2}$, David Barrera ${ }^{1}$, Pedro Calderón ${ }^{2}$,José Manuel Lloris ${ }^{3}$, María \\ José López ${ }^{3}$ and Salvador Sales ${ }^{1}$ \\ ${ }^{1}$ iTEAM research institute, Optical and Quantum Communications Group, Universidad Politécnica \\ de Valencia, Camino de Vera s/n, 46022, Valencia, Spain. \\ ${ }^{2}$ Instituto de Ciencia y Tecnología del Hormigón ICITECH, Universidad Politécnica de Valencia, \\ Camino de Vera de s/n, 46022, Valencia, Spain. \\ ${ }^{3}$ Instituto Tecnológico de la Construcción AIDICO, Avda. Benjamin Franklin 17, 46980, Paterna, \\ Valencia, Spain.
}

\begin{abstract}
Optical fiber sensors based on Fiber Bragg Gratings (FBG) have been embedded in concrete samples for temperature measurement. Three different types of gratings have been used in this experiment: FBGs inscribed in photosensitive germanium-boron codoped fiber and Regenerated Fiber Bragg Gratings (RFBG) inscribed in germanium doped and in germanium-boron codoped fiber. The concrete samples were placed inside a fire chamber where the temperature was increased above $1000^{\circ} \mathrm{C}$ as described in the Spanish/European standard UNE-EN 1363-1 temperature profile for concrete resistance to real fire. The temperature was monitored in real time. We have compared the performance of the optical sensors and electrical thermocouples. The RFBGs have shown a very good performance while the FBGs are able to monitor high-temperatures until their disappearance.
\end{abstract}

Keywords: regenerated fiber Bragg grating, embedded optical sensors, high temperature, concrete, fire test.

\section{INTRODUCTION}

Optical fiber sensors have been widely used in recent years for multiple applications in the civil engineering field. One of the main applications is the so called Health Monitoring System (HMS), consisting in the monitoring of physical parameters (such as temperature, strain, $\mathrm{pH}$, etc.) in a structure in order to know in real time the safety condition and avoid personal damage. Compared to the electrical sensors, the optical fiber sensors present several advantages such as: immunity to electromagnetic interference (EMI), small size, light weight, high temperature tolerance and resistance in harsh environments. In addition, optical technology allows tens of sensors to be multiplexed in a single optical fiber in order to create a quasi-distributed sensor system able to detect local damage detection.

In structures made of concrete, the monitoring of temperature becomes a key factor as it is known that the strength in a concrete material decreases under the effect of high temperature ${ }^{1}$. To date, previous publications concerning high temperature measurements in real structures using optical sensors reached to moderate high temperatures. In some works, embedded temperature sensors reached a temperature of $150^{\circ} \mathrm{C}$ inside concrete specimens ${ }^{2,3}$. In other publication, external temperature sensor was employed to measure temperature, reaching to a maximum temperature of almost 300 ${ }^{\circ} \mathrm{C}^{4}$. In this paper, a fire situation in a concrete structure is reproduced using a fire chamber, placing inside two concrete samples and exposing them to temperatures of above $1000^{\circ} \mathrm{C}$. Three different types of optical fiber-based temperature 
sensors were embedded inside the concrete specimens to compare their features in the high temperature measurements, obtaining maximum temperatures up to $600{ }^{\circ} \mathrm{C}$. This real test aims to reach the possibility of monitoring concrete structures, like tunnels, where the integrity of the structure has to be preserved in order to maintain the safety of the people.

\section{SENSOR DEVELOPMENT AND INSTALLATION}

As mentioned in the introduction, three different types of optical sensors were developed. All of them are based in fiber Bragg gratings $(\mathrm{FBG})^{5}$. The first type is an apodized FBG, inscribed in commercial photosensitive fiber, intrinsically capable to measure temperature as its Bragg wavelength shifts following the change in temperature. The other two types of sensors were created specifically treating a FBG in a two steps process: hydrogen load into the optical fiber before the grating inscription ${ }^{6}$ and thermal anneal after the grating inscription. This new grating was firstly known as Chemical Composition Grating $(\mathrm{CCG})^{7}$ due to the chemical processes present in the creation of the grating, but recently the new term of Regenerated FBG (RFBG) is used ${ }^{8}$, referring to the regeneration process during the thermal step. Depending on the initial FBG (also known as "seed"), the resulting RFBG has different properties. Two different types of optical fibers were used in the grating inscription of RFBG. The first one was a standard germanium $(\mathrm{Ge})$ doped optical fiber and the other one was a photosensitive germanium and boron $(\mathrm{Ge} / \mathrm{B})$ codoped optical fiber. The main differences between them are the grating regeneration temperature $\left(950{ }^{\circ} \mathrm{C}\right.$ in the $\mathrm{Ge}$ optical fiber and $550{ }^{\circ} \mathrm{C}$ in the $\mathrm{Ge} / \mathrm{B}$ optical fiber approximately) and the maximum working temperature (more than $1200{ }^{\circ} \mathrm{C}$ in $\mathrm{Ge}$ fiber and until $800{ }^{\circ} \mathrm{C}$ in $\mathrm{Ge} / \mathrm{B}$ fiber). These thermal characteristics extends the temperature working limits of a standard $\mathrm{FBG}$, established in a maximum of around $600^{\circ} \mathrm{C}$, suffering however a highly significant reduction of the optical peak power from $300{ }^{\circ} \mathrm{C}$ and above.

In addition to the optical sensors, electrical sensors were also placed, consisting in thermocouples type $\mathrm{K}$ which are able to measure temperatures above $1000^{\circ} \mathrm{C}$. In conclusion, four sets of sensors were installed, containing each one of them: 1 FBG sensor inscribed in a photosensitive Ge/B codoped optical fiber, 1 RFBG sensor inscribed in a standard Ge doped optical fiber, 1 RFBG sensor inscribed in a photosensitive Ge/B codoped fiber and $1 \mathrm{~K}$-type electrical thermocouple. The sets of sensors were installed inside two concrete samples. These concrete samples were cylindrical having a size of 30 $\mathrm{cm}$ height and $15 \mathrm{~cm}$ of diameter. Two sets of sensors were embedded in each concrete specimen in two specific locations: The lateral side (in fact, around to $1 \mathrm{~cm}$ from the lateral side) and the center of gravity $(7,5 \mathrm{~cm}$ from the lateral side), both locations at $15 \mathrm{~cm}$ height. Figure 1 shows the schematic view of the locations of the sensors. The sensors were labeled as FBG\#1..4 for the FBG sensors, Ge\#1..4 for the RFBG sensors inscribed in Ge doped fiber, Ge/B\#1..4 for the RFBG sensors inscribed in Ge/B codoped fiber and TC\#1..4 for the thermocouples.

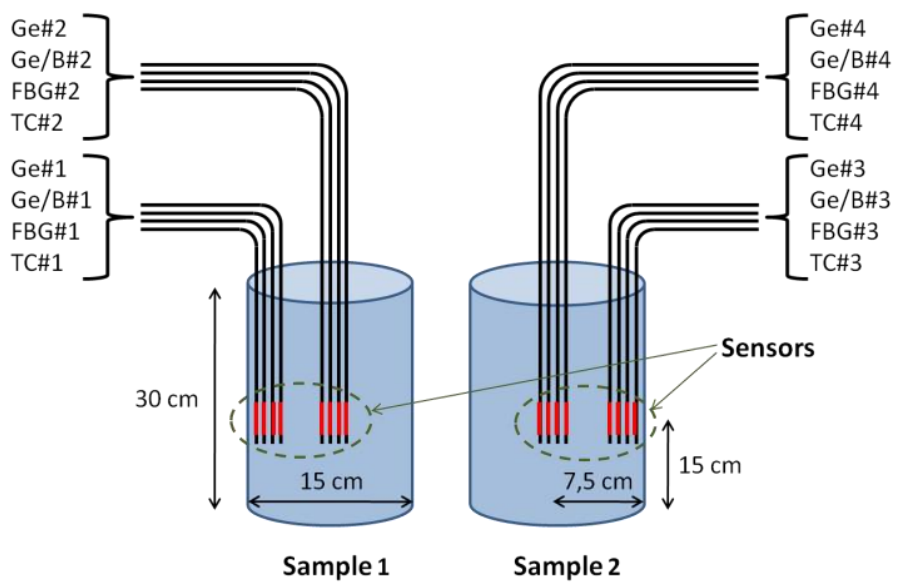

Figure 1: Schematic sensor locations inside the concrete samples. 
The sets of sensors were fixed to the mould for cylindrical samples, and then the concrete was carefully poured into the mould, completely covering this way the sensing heads of the sensors. The concrete samples were dismoulded after 48 hours, when the concrete had reached almost the $80 \%$ of their final strength. The next step was to move them to a specific concrete cure chamber where the samples stayed for 34 days until they reach their maximum strength.

\section{NORMALIZED FIRE TEMPERATURE TEST}

The two concrete samples were moved to AIDICO (Technological Institute of Construction) facilities to carry out the temperature test in a fire chamber placed in there. That fire chamber's size is $1,5 \mathrm{~m} \times 1,5 \mathrm{~m} \times 1,5 \mathrm{~m}$ and was fed by 4 burners being able to increase the temperature inside the chamber up above $1200^{\circ} \mathrm{C}$. The burners are controlled by 4 different electrical thermocouples located inside the chamber. The target temperature can be set at any time in order to create a specific temperature target profile. In this test, the Spanish/European standard UNE-EN 1363-1 temperature profile for normalized concrete resistance to real fire was used. The expression for the temperature increment is:

$$
\Delta T=345 \cdot \log (8 t+1)
$$

where $t$ is the time in minutes. The concrete samples were placed inside the chamber onto the floor and in lateral sides. At the beginning of the test, sensor named Ge/B\#1 appeared to be broken probably due to the transportation of the concrete specimens. Thus, survival percentage during installation concerning the optical sensors was $91,67 \%$.

The duration of the test was 120 minutes, when the temperature measured by the thermocouples inside the chamber was around $1060^{\circ} \mathrm{C}$. The temperature registered inside the concrete specimens by the optical sensors and the reference thermocouples are shown in figure 2 . The data obtained by the optical sensors was converted in temperature by applying a second order temperature sensibility of $8,6 \mathrm{pm} /{ }^{\circ} \mathrm{C}$ and $7,97 \cdot 10^{-3} \mathrm{pm} /\left({ }^{\circ} \mathrm{C}^{2}\right)$ to the sensors inscribed in germanium-boron codoped fiber and $11,79 \mathrm{pm} /{ }^{\circ} \mathrm{C}$ and $2,45 \cdot 10^{-3} \mathrm{pm} /\left({ }^{\circ} \mathrm{C}^{2}\right)$ to the sensors inscribed in germanium doped fiber. Sensibility factors of the optical sensors were obtained previously to the experiment.
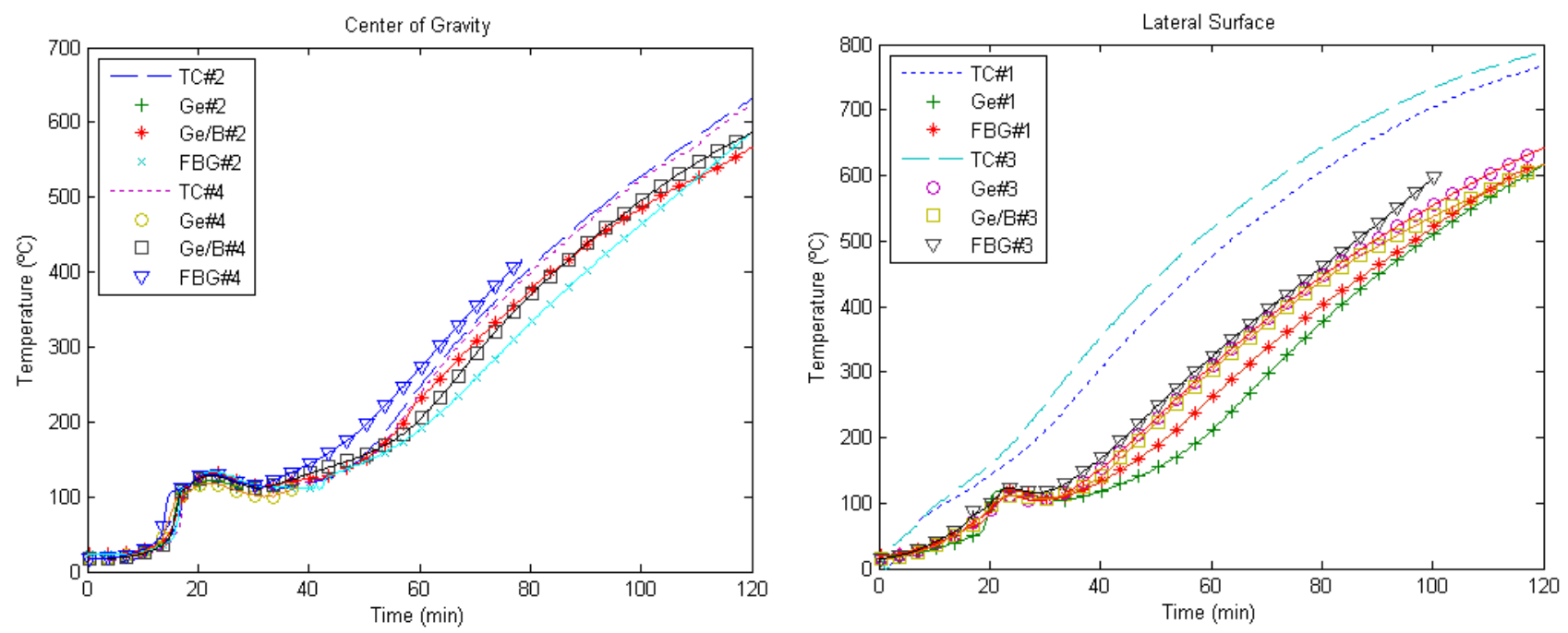

Figure 2: Temperature curves registered by the sensors installed in the center of gravity (left) and in the lateral surface (right) of both concrete samples. 
It can be seen that in the center of gravity of both concrete samples the temperature measured by the thermocouples and the optical sensors is very similar whereas it is a bit different at the lateral surface. The latter is due to the fact that thermocouples located at this point are a bit displaced and the gradient of temperature is quite high. At the center of gravity of both samples, the sensors based in RFBG Ge doped suddenly disappeared probably due to light reflections at the fiber splice as the noise floor increased above the power of the peak level, being the peak search algorithm unable to follow the wavelength shift. Standard FBG sensors almost disappeared during the test, due to the decrease of peak power until the noise floor. The RFBG sensors based on Ge doped fiber and Ge/B codoped fiber have shown negligible optical losses during the experiment, less than $1 \mathrm{~dB}$, whereas the FBG sensors inscribed in $\mathrm{Ge} / \mathrm{B}$ codoped fiber presented more than $20 \mathrm{~dB}$ of optical losses.

\section{CONCLUSIONS}

The optical sensors have shown a good agreement with the electrical ones, in particular at the center of gravity. Besides, the optical sensors can be multiplexed, they have small size and they are easy to embed. Optical sensors have confirmed very similar behavior between them even though they were inscribed in different optical fibers. The RFBG sensors have proved that they are very suitable for very high temperature applications, in example for fire detection inside a tunnel, since they can withstand temperatures over $1000^{\circ} \mathrm{C}^{7,8}$. On the other hand, we have realized that the reflectivity of the sensors based on standard FBG almost disappear at higher temperatures. Nevertheless, FBGs sensors can be used in high temperature experiments if the time duration of the experiment is short enough. It is important to note that one of the sensors did not survive during the transportation and two more sensors did not survive until the end of the test thus it is necessary to improve the sensor packaging.

\section{Agreements}

The authors gratefully acknowledge research funding by the Spanish Ministry of Science and Innovation through Project SOPROMAC P41/08.

\section{References}

[1] Abdel-Fattah, H., Hamoush, S.A., "Variation of the fracture toughness of concrete with temperature", Construction and Building Materials, 11 (2), 105-108 (1997).

[2] Cardozo da Silva, J.C., Martelli, C., et al., "Dynamic analysis and temperature measurements of concrete cantilever beam using fibre Bragg gratings", Optics and Lasers in Engineering, 45, 88-92 (2007).

[3] Lin, Y.B., Chern, J.C., et al., "The utilization of fiber Brag grating sensors to monitor high performance concrete at elevated temperature", Smart Materials and Structures, 13, 784-790 (2004).

[4] Lönnenmark, A., Hedekvist, P.O., Ingason, H., "Gas temperature measurements using fibre Bragg grating during fire experiments in a tunnel”, Fire Safety Journal, 43, 119-126 (2008).

[5] Kersey, A.D. et al., "Fiber grating sensors", Journal of Lightwave Technology, 15 (8), 1442-1463 (1997).

[6] Liou, C.L. et al., “Characteristics of hydrogenated fiber Bragg gratings”, Applied Physics A, 64, 191-197 (1997).

[7] Fokine, M., "Formation of thermally stable chemical composition gratings in optical fibers", Journal of the Optical Society of America B, 19 (8), 1759-1765 (2002).

[8] Bandyopadhyay, S., Canning J., et al., "Ultrahigh-temperature regenerated gratings in boron-codoped germanosilicate optical fiber using 193 nm”, Optics Letters, 33, 1917-1919 (2008). 